\title{
A Copper(I)-Catalyzed Radical-Relay Reaction Enabling the Intermolecular 1,2-Alkylborylation of Unactivated Olefins
}

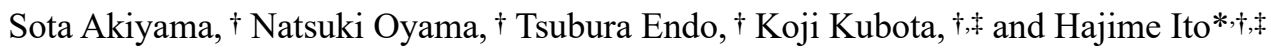 \\ ${ }^{\dagger}$ Division of Applied Chemistry, Graduate School of Engineering, Hokkaido University, \\ Sapporo, Hokkaido, 060-8628, Japan. \\ ¥Institute for Chemical Reaction Design and Discovery (WPI-ICReDD), Hokkaido University, \\ Sapporo, Hokkaido 060-8628, Japan.
}

e-mail:hajito@eng.hokudai.ac.jp

Table of Contents
1. Instrumentation and Chemicals
S2
2. Substrate Preparation Procedures
S3-6
3. General Borylation Procedures
S7-8
4. Borylation Product Characterizations
S9-36
5. Derivatization of Alkyl Boronates
S37-44
6. Reaction with a Chiral Ligand
S45
7. Single Crystal X-ray Structural Analysis
S46-47
8. Mechanistic Investigations
S48-49
9. The Details of Optimization Conditions for $4 \mathbf{a a}$
S50-53
10. Comparison between the Reaction with $\mathrm{ZnBr}_{2}$ and without $\mathrm{ZnBr}_{2}$
S54
11. References
S55
12. NMR Spectra
S56-230
13. HPLC Chart of $\mathbf{4 b d}$
S231-232 


\section{Instrumentation and Chemicals}

Materials were obtained from commercial suppliers and purified by standard procedures unless otherwise noted. Solvents were also purchased from commercial suppliers, degassed via three freeze-pump-thaw cycles, and further dried over molecular sieves (MS 4 $\AA$ ). NMR spectra were recorded on JEOL JNM-ECX400P and JNM-ECS400 spectrometers $\left({ }^{1} \mathrm{H}: 400 \mathrm{MHz},{ }^{13} \mathrm{C}\right.$ : $100 \mathrm{MHz}$, and $\left.{ }^{19} \mathrm{~F}: 373 \mathrm{MHz}\right)$. Tetramethylsilane $\left({ }^{1} \mathrm{H}\right), \mathrm{CDCl}_{3}\left({ }^{1} \mathrm{H},{ }^{13} \mathrm{C}\right)$, and fluorobenzene $\left({ }^{19} \mathrm{~F}\right.$, $\delta-113.60)$ were employed as the external standards. Multiplicity was recorded as follows: $\mathrm{s}=$ singlet, $\mathrm{d}=$ doublet, $\mathrm{t}=$ triplet, $\mathrm{q}=$ quartet, $\mathrm{m}=$ multiplet. GLC analyses were conducted with a Shimadzu GC-2014 or GC-2025 equipped with a ULBON HR-1 glass capillary column (Shinwa Chemical Industries) and an FID detector. Recycle preparative gel permeation chromatography (GPC) was conducted with a JAI LC-9101 using $\mathrm{CHCl}_{3}$ as the eluent. Highresolution mass spectra were recorded at the Global Facility Center, Hokkaido University. The reactions with Blue LED were carried out by using Kessil A 150WE TUNA Blue. Enantiomeric ratios were determined by HPLC analysis (high-performance liquid chromatography) with a Hitachi Chromaster HPLC [Chiral Technologies Chiralpak IBN-3 (4.6 x 250 mm)]. Specific optical rotations were measured with HORIBA SEPA-300 and a Rudolph Research Analytical Autopol IV Polarimeter. Single crystal X-ray structural analysis was carried out on a Rigaku XtaLAB PRO MM007 diffractometer using graphite monochromated $\mathrm{Cu}-\mathrm{K} \alpha$ radiation. The structure was solved by direct methods and expanded using Fourier techniques. Non-hydrogen atoms were refined anisotropically. Hydrogen atoms were refined using the riding model. All calculations were performed using the Olex 2 crystallographic software package except for refinement, which was performed using SHELXL-2013. 


\section{Substrate Preparation Procedures}

The fluorinated alkyl bromides $1 \mathbf{a}^{1}, \mathbf{1} \mathbf{c}^{2}, \mathbf{1} \mathbf{f}^{3}, \mathbf{1} \mathbf{g}^{1}, \mathbf{1 i}^{4}, \mathbf{1 j}^{3}, \mathbf{1 \mathbf { l } ^ { 5 }}$, and $\mathbf{1 \mathbf { m } ^ { 6 }}$ are known compounds and were prepared in accordance to references. The fluorinated halides $\mathbf{1 n}-\mathbf{1} \mathbf{p}$ were purchased from commercial suppliers. The new compounds $1 \mathbf{h}, \mathbf{1 k}$ and $1 \mathrm{ae}$ were synthesized according to references and characterized described below. The tertiary or secondary alkyl bromides 1b, 1d, 1e, 1q, 1r, 1v-1ad were purchased from commercial suppliers. Tertiary alkyl bromides $\mathbf{1} \mathbf{s}^{7}, \mathbf{1} \mathbf{t}^{7}$, and $\mathbf{1} \mathbf{u}^{8}$ are known compounds and were prepared according to references. All spectroscopic data were matched in those reported.

Table S1. Alkyl bromides used in this study.<smiles>CC(Br)(c1ccccc1)C(F)(F)Br</smiles>

$1 \mathrm{a}$<smiles>BrC12CC3CC(C1)CC(Br)(C3)C2</smiles>

$1 b$<smiles>CCC(C)Br</smiles>

1c<smiles>BrC1CCOCC1</smiles>

$1 d$<smiles>BrC(Br)c1ccccc1</smiles>

$1 \mathrm{e}$<smiles>CCOC(=O)CCC(F)(F)Br</smiles>

$1 f$

19

$1 \mathrm{~h}$

$1 \mathrm{i}$

1j<smiles>CC(=O)OC(C)(F)Br</smiles>

$1 \mathrm{k}$<smiles>FC(F)(Br)CO[Mg]</smiles>

11<smiles>CC(C)(C)Br</smiles>

$1 q$<smiles>BrC1CCCCC1</smiles>

1v<smiles>CCC(C)(C)Br</smiles>

$1 \mathrm{r}$

$1 w$<smiles>CC(C)(C)ON1CCC(Br)CC1</smiles><smiles>CCC(Br)CC</smiles>

1 aа<smiles>FC(F)(Br)COBr</smiles>

$1 \mathrm{~m}$<smiles>CCOC(=O)C(F)(F)Br</smiles>

1n<smiles>CCOC(=O)C(F)Br</smiles>

10<smiles>FC(F)(F)C(F)(F)C(F)(F)I</smiles>

$1 p$<smiles>CC(C)(Br)CCc1ccccc1</smiles>

$1 s$<smiles>BrC1CC2CCC1C2</smiles>

$1 x$<smiles>CCOC(=O)C(C)Br</smiles>

1 ac<smiles>CC1(Br)CCCCC1</smiles>

1t<smiles>BrC1C2CC3CC(C2)CC1C3</smiles>

$1 y$<smiles>[N+]=[W]</smiles><smiles>O=C(CCC(F)(F)Br)c1ccccc1</smiles>

$1 \mathrm{ad}$<smiles>CCCCC(C)(Br)CCCC</smiles>

1u<smiles>BrC1CCC1</smiles>

$1 z$ 
The olefins $\mathbf{2} \mathbf{a}-\mathbf{2 g}, \mathbf{2 i}, \mathbf{2} \mathbf{l}-\mathbf{2} \mathbf{p} \mathbf{2} \mathbf{s}, \mathbf{2} \mathbf{u}-\mathbf{2} \mathbf{w}$ were purchased from commercial suppliers. The functionalized olefins $\left(\mathbf{2} \mathbf{h}^{9}, \mathbf{2} \mathbf{j}^{10}, \mathbf{2} \mathbf{k}^{11}, \mathbf{2} \mathbf{q}^{12}, \mathbf{2} \mathbf{r}^{13}\right.$, and $\left.\mathbf{2} \mathbf{t}^{14}\right)$ are known compounds and were prepared according to references. All spectroscopic data were matched in those reported.

Table S2. Olefins used in this study.

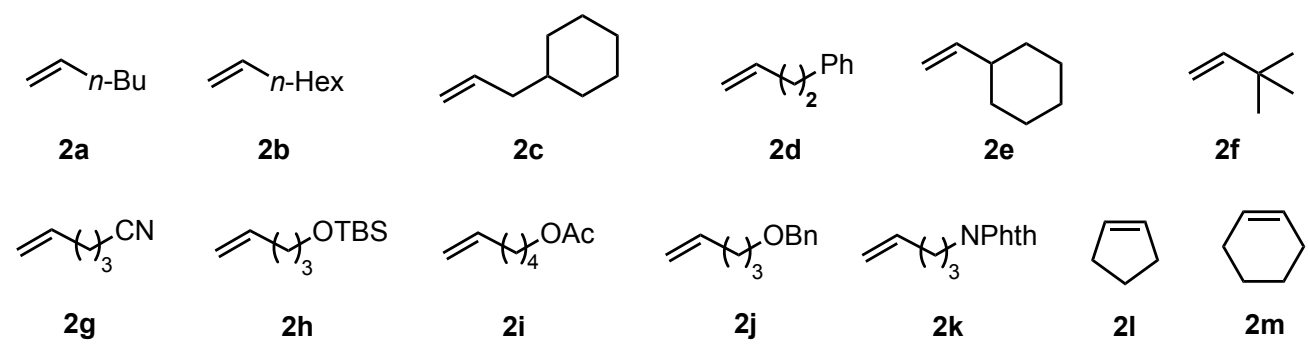<smiles>C=CCCCOC(=O)Cc1c(C)n(C(=O)c2ccc(Cl)cc2)c2ccc(OC)cc12</smiles><smiles>C=CCNC(=O)OCC</smiles>

2s

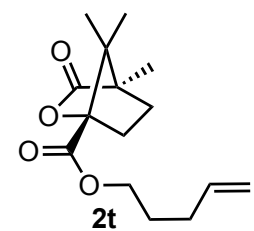

From camphanic acid

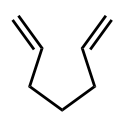

$2 \mathrm{u}$<smiles>C=CCC(CC=C)(OCC)OCC</smiles>

$2 v$<smiles>C=CC1CC1</smiles>

2w 
Preparation of (5-bromo-5,5-difluoropentyl)benzene (1a). ${ }^{3}$

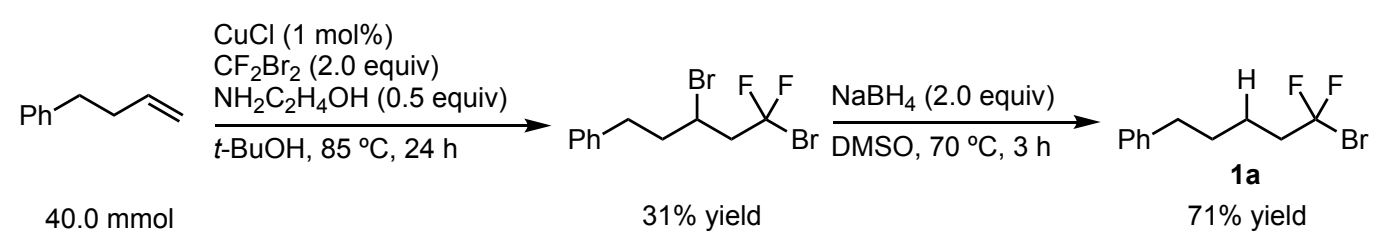

$\mathrm{A} \mathrm{CuCl}(40.0 \mathrm{mg}, 0.4 \mathrm{mmol}), 1$-aminoethanol $(1.2 \mathrm{~mL}, 20.0 \mathrm{mmol})$ and 4-phenyl-1-butene $(1.0 \mathrm{~g}, 40.0 \mathrm{mmol})$ were dissolved in anhydrous $t-\mathrm{BuOH}(6.6 \mathrm{~mL})$, then the $\mathrm{CF}_{2} \mathrm{Br}_{2}(7.4 \mathrm{~mL}$, $60.0 \mathrm{mmol}$ ) was added to the reaction mixture in one portion at $0{ }^{\circ} \mathrm{C}$ under a nitrogen atmosphere. The mixture was allowed to warm up to $85{ }^{\circ} \mathrm{C}$ and stir for $24 \mathrm{~h}$. After that, the reaction mixture was passed through a silica gel short column to remove the $\mathrm{Cu}$ catalyst, and the volatiles were removed in vacuo. The brown oil residue was purified by Kugelrohr distillation under a reduced pressure $\left(6.0 \times 10^{-1} \mathrm{hPa}, 120{ }^{\circ} \mathrm{C}\right)$ to give target difluoro alkyl bromide ( $4.3 \mathrm{~g}, 31 \%$ yield) as a colorless oil.

The resultant product $(4.3 \mathrm{~g}, 12.6 \mathrm{mmol})$ was dissolved in DMSO $(17.5 \mathrm{~mL})$ and then $\mathrm{NaBH}_{4}$ was added to this reaction mixture $(951 \mathrm{mg}, 25.2 \mathrm{mmol})$ in several portions under a nitrogen atmosphere. The mixture was allowed to stir at $70{ }^{\circ} \mathrm{C}$ for $3 \mathrm{~h}$. After that, the mixture was diluted with $\mathrm{Et}_{2} \mathrm{O}$ at $0{ }^{\circ} \mathrm{C}$, quenched by $3 \mathrm{M} \mathrm{HCl}_{\mathrm{aq}}$, and the mixture was extracted with $\mathrm{Et}_{2} \mathrm{O}$ three times. The combined organic layer was washed with water three times. Then the organic layer was dried over $\mathrm{MgSO}_{4}$, filtered, and concentrated in vacuo. The brown oil residue was purified by Kugelrohr distillation under a reduced pressure $\left(6.0 \times 10^{-1} \mathrm{hPa}, 10{ }^{\circ} \mathrm{C}\right)$ to give $1 \mathrm{a}$ ( $2.4 \mathrm{~g}, 71 \%$ yield) as a colorless oil. The spectroscopic data was matched with the literature. ${ }^{1}$

\section{Preparation of (2-bromo-2,2-difluoroethyl)cyclohexane (1h). ${ }^{1}$}

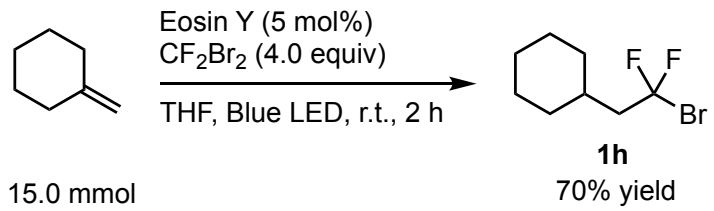

A $100 \mathrm{~mL}$ two-necked flask was charged with Eosin Y (48.6 mg, $0.075 \mathrm{mmol})$ and the olefin $(180.3 \mu \mathrm{L}, 1.5 \mathrm{mmol})$ in THF $(45 \mathrm{~mL})$. Then $\mathrm{CF}_{2} \mathrm{Br}_{2}(554 \mu \mathrm{L}, 6.0 \mathrm{mmol})$ was added in one portion at $0{ }^{\circ} \mathrm{C}$ to the reaction mixture before the mixture was degassed three times by the freeze-pump-thaw procedure. The flask was placed at a distance of $2 \mathrm{~cm}$ from the blue LEDs, and the mixture was stirred under a nitrogen atmosphere with irradiation by blue LEDs for $2 \mathrm{~h}$. After the volatiles were removed in vacuo, the oily residue was subjected to silica gel chromatography (pentane) to give $\mathbf{1 h}(237 \mathrm{mg}, 70 \%$ yield) as a colorless oil.

${ }^{1} \mathrm{H}$ NMR $\left(399 \mathrm{MHz}, \mathrm{CDCl}_{3}\right): \delta 0.96-1.36(\mathrm{~m}, 5 \mathrm{H}), 1.61-1.87(\mathrm{~m}, 6 \mathrm{H}), 2.28(\mathrm{td}, J=6.0$, $15.6 \mathrm{~Hz}, 2 \mathrm{H}) .{ }^{13} \mathrm{C}$ NMR $\left(100 \mathrm{MHz}, \mathrm{CDCl}_{3}\right): \delta 25.9(\mathrm{CH}), 33.1(\mathrm{CH}), 34.2(\mathrm{CH}), 51.3(\mathrm{t}, J=$ 
$\left.19.9 \mathrm{~Hz}, \mathrm{CH}_{2}\right), 123.1$ (t, $\left.J=308.3 \mathrm{~Hz}, C F_{2}\right) .{ }^{19} \mathrm{~F} \mathrm{NMR}\left(373 \mathrm{MHz}, \mathrm{CDCl}_{3}\right): \delta-41.0$ (t, $J=15.3$ $\mathrm{Hz}, 2 \mathrm{~F})$. HRMS-FI $(\mathrm{m} / \mathrm{z})$ : [M] $]^{+}$calcd for $\mathrm{C}_{8} \mathrm{H}_{13} \mathrm{BrF}_{2}, 226.0169$; found, 226.0177 .

Preparation of 5-bromo-5,5-difluoropentyl acetate (1k). ${ }^{1}$

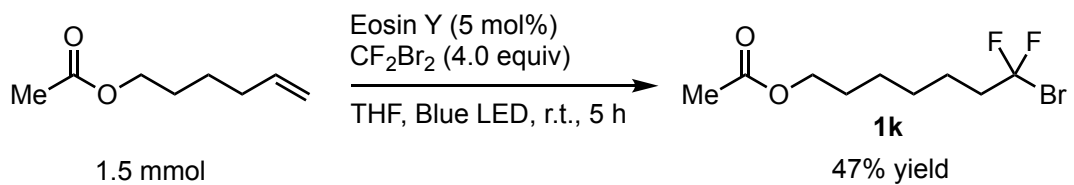

$1 \mathbf{k}$ was prepared from the corresponding olefin $(214.4 \mathrm{mg}, 1.5 \mathrm{mmol})$ under the blue LED conditions according to the procedure described above. The product $1 \mathbf{k}$ was obtained in $47 \%$ yield (191.2 mg).

${ }^{1} \mathrm{H}$ NMR (392 MHz, $\left.\mathrm{CDCl}_{3}\right): \delta 1.32-1.47(\mathrm{~m}, 4 \mathrm{H}), 1.59-1.72(\mathrm{~m}, 4 \mathrm{H}), 2.05(\mathrm{~s}, 3 \mathrm{H}), 2.27-$ $2.43(\mathrm{~m}, 2 \mathrm{H}), 4.06(\mathrm{t}, J=6.5 \mathrm{~Hz}, 2 \mathrm{H}) .{ }^{13} \mathrm{C} \mathrm{NMR}\left(99 \mathrm{MHz}, \mathrm{CDCl}_{3}\right): \delta 20.9\left(\mathrm{CH}_{3}\right), 23.8\left(\mathrm{CH}_{2}\right)$, $25.6\left(\mathrm{CH}_{2}\right), 28.0\left(\mathrm{CH}_{2}\right), 28.3\left(\mathrm{CH}_{2}\right), 44.1(\mathrm{t}, J=21.3 \mathrm{~Hz}, \mathrm{CH}), 64.2\left(\mathrm{CH}_{2}\right), 123.0(\mathrm{t}, J=306.9$ $\left.\mathrm{Hz}, \mathrm{CF}_{2}\right), 171.1(\mathrm{C}) .{ }^{19} \mathrm{~F}$ NMR (373 MHz, $\left.\mathrm{CDCl}_{3}\right): \delta-44.1$ to $-43.9(\mathrm{~m}, 2 \mathrm{~F})$. HRMS-ESI $(\mathrm{m} / \mathrm{z})$ : $[\mathrm{M}+\mathrm{Na}]^{+}$calcd for $\mathrm{C}_{9} \mathrm{H}_{15} \mathrm{O}_{2} \mathrm{BrF}_{2} \mathrm{Na}, 295.0121$; found, 295.0109.

\section{Preparation of (7-bromo-7,7-difluoroheptyl)benzene (1 ae). ${ }^{1}$}

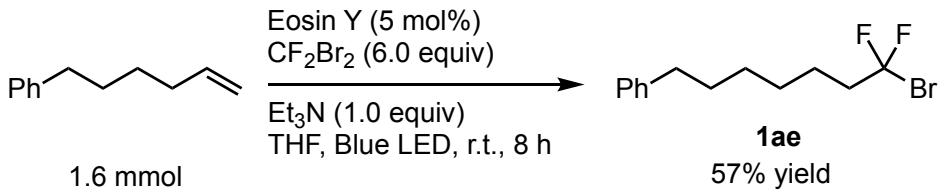

A $100 \mathrm{~mL}$ two-necked flask was charged with Eosin Y (48.6 mg, $0.075 \mathrm{mmol}), \mathrm{Et}_{3} \mathrm{~N}$ (209.1 $\mu \mathrm{L}, 1.5 \mathrm{mmol}$ ), and the olefin (251.6 mg, $1.6 \mathrm{mmol})$ in THF (45 mL). Then $\mathrm{CF}_{2} \mathrm{Br}_{2}(554 \mu \mathrm{L}$, $6.0 \mathrm{mmol}$ ) was added in one portion at $0{ }^{\circ} \mathrm{C}$ to the reaction mixture before the mixture was degassed three times by the freeze-pump-thaw procedure. The flask was placed at a distance of $2 \mathrm{~cm}$ from the blue LEDs and the mixture was stirred under a nitrogen atmosphere and irradiated by blue LEDs for $3 \mathrm{~h}$. After that, the second portion of $\mathrm{CF}_{2} \mathrm{Br}_{2}(250 \mu \mathrm{L}, 3.0 \mathrm{mmol})$ was added to the reaction mixture. Then the mixture was stirred for another $5 \mathrm{~h}$. The volatiles were removed in vacuo, and the oily residue was subjected to silica gel chromatography (hexane) to give 1ae (260.8 $\mathrm{mg}, 57 \%$ yield) as a colorless oil.

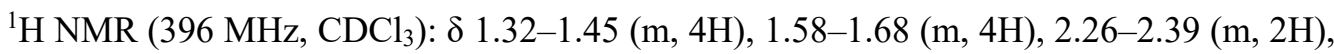
$2.61(\mathrm{t}, J=7.7 \mathrm{~Hz}, 2 \mathrm{H}), 7.15-7.21(\mathrm{~m}, 3 \mathrm{H}), 7.25-7.31(\mathrm{~m}, 2 \mathrm{H}) .{ }^{13} \mathrm{C} \mathrm{NMR}\left(100 \mathrm{MHz}, \mathrm{CDCl}_{3}\right)$ : $\delta 23.8\left(\mathrm{CH}_{2}\right), 28.3\left(\mathrm{CH}_{2}\right), 28.8\left(\mathrm{CH}_{2}\right), 31.2\left(\mathrm{CH}_{2}\right), 35.8\left(\mathrm{CH}_{2}\right), 44.2\left(\mathrm{t}, J=21.1 \mathrm{~Hz}, \mathrm{CH}_{2}\right), 123.2$ (t, $\left.J=307.1 \mathrm{~Hz}, C \mathrm{~F}_{2}\right), 125.7(\mathrm{CH}), 128.3(\mathrm{CH}), 128.4(\mathrm{CH}), 142.5(C) .{ }^{19} \mathrm{~F}$ NMR $(373 \mathrm{MHz}$, $\left.\mathrm{CDCl}_{3}\right): \delta-43.9$ to $-43.7(\mathrm{~m}, 2 \mathrm{~F})$. HRMS-EI $(\mathrm{m} / \mathrm{z})$ : $[\mathrm{M}]^{+}$calcd for $\mathrm{C}_{13} \mathrm{H}_{17} \mathrm{BrF}_{2}, 290.0482$; found, 290.0479 . 


\section{General Borylation Procedures}

\section{Procedure of the copper(I)-catalyzed 1,2-alkyl borylation for 4aa (Condition A).}

An oven-dried reaction vial was charged with bis(pinacolato)diboron (3) $(38.1 \mathrm{mg}, 0.15$ $\mathrm{mmol})$, IMes $\bullet \mathrm{HCl}(2.5 \mathrm{mg}, 0.0075 \mathrm{mmol})$ and transferred to an argon-filled glove box. $\left[\mathrm{Cu}(\mathrm{MeCN})_{4}\right] \mathrm{BF}_{4}(1.5 \mathrm{mg}, 0.005 \mathrm{mmol}), \mathrm{ZnBr}_{2}(4.5 \mathrm{mg}, 0.02 \mathrm{mmol})$, and $\mathrm{K}(\mathrm{O}-t-\mathrm{Bu})(20.2 \mathrm{mg}$, $0.18 \mathrm{mmol}$ ) were placed in the reaction vial, and then the vial was capped with a rubber septum and removed from the glovebox. The vial with reagents was refilled with a nitrogen atmosphere and the solids were suspended in 1,4-dioxane/DMF (200 $\mu \mathrm{L} / 50 \mu \mathrm{L})$, then the suspension was allowed to stir for $1 \mathrm{~min}$ at r.t. After that, olefin $\mathbf{2 a}(100.8 \mu \mathrm{L}, 0.80 \mathrm{mmol})$ and alkyl electrophile $1 \mathrm{a}(26.3 \mathrm{mg}, 0.10 \mathrm{mmol})$ were subsequently added to the suspension at r.t., then the mixture was allowed to stir at $50{ }^{\circ} \mathrm{C}$. After completing the reaction, the mixture was passed through a short plug of silica gel $(\Phi: 10 \mathrm{~mm}$, the height of the silica-gel column: $30 \mathrm{~mm})$, eluted with $\mathrm{Et}_{2} \mathrm{O}$, and the volatiles were removed in vacuo. The resulting oily residue was subjected to silica gel chromatography ( $\mathrm{Et}_{2} \mathrm{O} /$ hexane, typically $\left.0: 100-4: 96\right)$ to give the corresponding alkylborylation product $\mathbf{4 a a}$ as a colorless oil.

\section{Procedure of the copper(I)-catalyzed 1,2-alkyl borylation for 4 ba (Condition B).}

An oven-dried reaction vial was charged with bis(pinacolato)diboron (3) (76.2 mg, 0.3 mmol), IMes $\bullet \mathrm{HCl}(10.2 \mathrm{mg}, 0.03 \mathrm{mmol})$ and transferred to an argon-filled glove box. $\left[\mathrm{Cu}(\mathrm{MeCN})_{4}\right] \mathrm{BF}_{4}(6.3 \mathrm{mg}, 0.02 \mathrm{mmol}), \mathrm{ZnBr}_{2}(9.0 \mathrm{mg}, 0.04 \mathrm{mmol})$, and $\mathrm{K}(\mathrm{O}-t-\mathrm{Bu})(40.4 \mathrm{mg}$, $0.36 \mathrm{mmol}$ ) were placed in the reaction vial, then the vial was capped with a rubber septum and removed from the glovebox. The vial with reagents was refilled with a nitrogen atmosphere and the solids were suspended in 1,4-dioxane/DMF $(300 \mu \mathrm{L} / 100 \mu \mathrm{L})$, then the suspension was allowed to stir for $1 \mathrm{~min}$ at r.t. After that, olefin $\mathbf{2 a}(100.5 \mu \mathrm{L}, 0.80 \mathrm{mmol})$ and alkyl electrophile 1b $(43.1 \mathrm{mg}, 0.20 \mathrm{mmol})$ in 1,4-dioxane $(300 \mu \mathrm{L})$ were subsequently added to the suspension at r.t., then the mixture was allowed to stir at $50^{\circ} \mathrm{C}$. After completing the reaction, the mixture was passed through a short plug of silica gel ( $\Phi: 10 \mathrm{~mm}$, the height of the silica-gel column: 30 $\mathrm{mm}$ ), eluted with $\mathrm{Et}_{2} \mathrm{O}$, and volatiles were removed in vacuo. The resulting oily residue was subjected to silica gel chromatography $\left(\mathrm{Et}_{2} \mathrm{O} /\right.$ hexane, typically $\left.0: 100-4: 96\right)$ to give the corresponding alkylborylation product $\mathbf{4 b a}$ as a colorless oil.

\section{Procedure of the copper(I)-catalyzed 1,2-alkyl borylation for 4ca (Condition C).}

An oven-dried reaction vial was charged with bis(pinacolato)diboron (3) (190.5 mg, 0.75 $\mathrm{mmol})$, IMes $\bullet \mathrm{HCl}(25.6 \mathrm{mg}, 0.075 \mathrm{mmol})$ and transferred to an argon-filled glove box. $\left[\mathrm{Cu}(\mathrm{MeCN})_{4}\right] \mathrm{BF}_{4}(15.7 \mathrm{mg}, 0.05 \mathrm{mmol}), \mathrm{ZnBr}_{2}(22.5 \mathrm{mg}, 0.10 \mathrm{mmol})$, and $\mathrm{K}(\mathrm{O}-t-\mathrm{Bu})(101.1$ 
$\mathrm{mg}, 0.90 \mathrm{mmol}$ ) were placed in the reaction vial, then the vial was capped with a rubber septum and removed from the glovebox. The vial with reagents was refilled with a nitrogen atmosphere and the solids were suspended in 1,4-dioxane/DMF (500 $\mu \mathrm{L} / 100 \mu \mathrm{L})$, then the suspension was allowed to stir vigorously for $1 \mathrm{~min}$ at r.t. After that, olefin $\mathbf{2 a}(1.25 \mathrm{~mL}, 10.0 \mathrm{mmol})$ and alkyl electrophile $1 \mathrm{c}(113.3 \mathrm{mg}, 0.50 \mathrm{mmol})$ were subsequently added to the suspension at r.t., then the mixture was allowed to stir vigorously at $50{ }^{\circ} \mathrm{C}$. After completing the reaction, the mixture was passed through a short plug of silica gel ( $\Phi: 10 \mathrm{~mm}$, the height of the silica-gel column: 30 $\mathrm{mm}$ ), eluted with $\mathrm{Et}_{2} \mathrm{O}$, and volatiles were removed in vacuo. The resulting oily residue was subjected to silica gel chromatography $\left(\mathrm{Et}_{2} \mathrm{O} /\right.$ hexane, typically $\left.0: 100-4: 96\right)$ to give the corresponding alkylborylation product $\mathbf{4} \mathbf{c a}$ as a colorless oil.

\section{Procedure of the copper(I)-catalyzed 1,2-alkyl borylation for 4da (Condition D).}

An oven-dried reaction vial was charged with bis(pinacolato)diboron (3) (190.5 mg, 0.75 mmol), IMes $\bullet \mathrm{HCl}(25.6 \mathrm{mg}, 0.075 \mathrm{mmol})$ and transferred to an argon-filled glove box. $\left[\mathrm{Cu}(\mathrm{MeCN})_{4}\right] \mathrm{BF}_{4}(15.7 \mathrm{mg}, 0.05 \mathrm{mmol}), \mathrm{ZnBr}_{2}(22.5 \mathrm{mg}, 0.10 \mathrm{mmol})$, and $\mathrm{K}(\mathrm{O}-t-\mathrm{Bu})(101.1$ $\mathrm{mg}, 0.90 \mathrm{mmol}$ ) were placed in the reaction vial, then the vial was capped with a rubber septum and removed from the glovebox. The vial with reagents was refilled with a nitrogen atmosphere and the solids were suspended in 1,4-dioxane/DMF (500 $\mu \mathrm{L} / 100 \mu \mathrm{L})$, then the suspension was allowed to stir vigorously for $1 \mathrm{~min}$ at r.t. After that, olefin $\mathbf{2 a}(1.25 \mathrm{~mL}, 10.0 \mathrm{mmol})$ and alkyl electrophile $1 \mathbf{d}(83.7 \mathrm{mg}, 0.51 \mathrm{mmol})$ were subsequently added to the suspension at r.t., then the mixture was allowed to stir vigorously at $50{ }^{\circ} \mathrm{C}$. After completing the reaction, the mixture was passed through a short plug of silica gel $(\Phi: 10 \mathrm{~mm}$, the height of the silica-gel column: 30 $\mathrm{mm}$ ), eluted with $\mathrm{Et}_{2} \mathrm{O}$, and volatiles were removed in vacuo. The resulting oily residue was subjected to silica gel chromatography $\left(\mathrm{Et}_{2} \mathrm{O} /\right.$ hexane, typically $\left.0: 100-4: 96\right)$ and further purified by GPC to give the corresponding alkylborylation product $\mathbf{4 d a}$ as a colorless oil. 


\section{Borylation Product Characterizations}

2-(7,7-Difluoro-11-phenylundecan-5-yl)-4,4,5,5-tetramethyl-1,3,2-dioxaborolane (4aa).

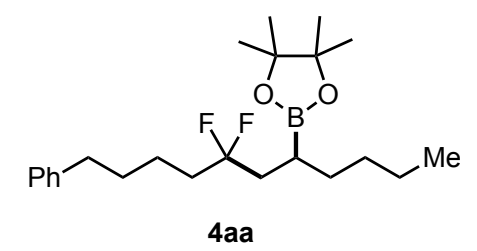

Prepared according to condition A described above. The reaction was conducted for $2 \mathrm{~h}$ with $53.6 \mathrm{mg}(0.20 \mathrm{mmol})$ of $\mathbf{1 a}$ and $100.8 \mu \mathrm{L}(0.80 \mathrm{mmol})$ of $\mathbf{2 a}$. The product $4 \mathbf{a a}$ was obtained in $73 \%$ yield $(58.5 \mathrm{mg})$.

${ }^{1} \mathrm{H}$ NMR $\left(392 \mathrm{MHz}, \mathrm{CDCl}_{3}\right): \delta 0.88(\mathrm{t}, J=6.9 \mathrm{~Hz}, 3 \mathrm{H}), 1.13-1.37(\mathrm{~m}, 7 \mathrm{H}), 1.24(\mathrm{~s}, 12 \mathrm{H})$, 1.39-1.54 (m, 3H), 1.60-1.69 (m, 2H), 1.75-1.91 (m, 2H), 1.93-2.10 (m, 1H), 2.62 (t, $J=7.6$ $\mathrm{Hz}, 2 \mathrm{H}), 7.14-7.21(\mathrm{~m}, 3 \mathrm{H}), 7.24-7.31(\mathrm{~m}, 2 \mathrm{H}) .{ }^{13} \mathrm{C} \mathrm{NMR}\left(99 \mathrm{MHz}, \mathrm{CDCl}_{3}\right): \delta 14.0\left(\mathrm{CH}_{3}\right)$, 17.3 (br, B-CH), 22.0 (t, $\left.J=4.7 \mathrm{~Hz}, \mathrm{CH}_{2}\right), 22.8\left(\mathrm{CH}_{2}\right), 24.70\left(\mathrm{CH}_{3}\right), 24.74\left(\mathrm{CH}_{3}\right), 31.1\left(\mathrm{CH}_{2}\right)$, $31.2\left(\mathrm{CH}_{2}\right), 31.4\left(\mathrm{CH}_{2}\right), 35.7\left(\mathrm{CH}_{2}\right) 36.5\left(\mathrm{t}, J=25.5 \mathrm{~Hz}, C \mathrm{H}_{2}\right), 38.4\left(\mathrm{t}, J=25.1 \mathrm{~Hz}, \mathrm{CH}_{2}\right), 83.1$ (C), $125.4\left(\mathrm{t}, J=241.7 \mathrm{~Hz}, \mathrm{CF}_{2}\right), 125.7(\mathrm{CH}), 128.27(\mathrm{CH}), 128.33(\mathrm{CH}), 142.2(\mathrm{C}) .{ }^{19} \mathrm{~F}$ NMR $\left(369 \mathrm{MHz}, \mathrm{CDCl}_{3}\right): \delta-97.8$ to $-95.6(\mathrm{~m}, 2 \mathrm{~F})$. HRMS-ESI $(\mathrm{m} / \mathrm{z}):[\mathrm{M}+\mathrm{Na}]^{+}$calcd for $\mathrm{C}_{23} \mathrm{H}_{37}{ }^{11} \mathrm{BF}_{2} \mathrm{O}_{2} \mathrm{Na}, 417.2751$; found, 417.2747.

\section{2-(5,5-Difluoro-1-phenyltridecan-7-yl)-4,4,5,5-tetramethyl-1,3,2-dioxaborolane (4ab).}

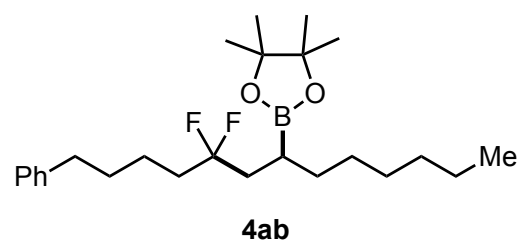

Prepared according to condition A described above, except the catalyst loading $\{10 \mathrm{~mol} \%$ of $\left[\mathrm{Cu}(\mathrm{MeCN})_{4}\right] \mathrm{BF}_{4}$ and $15 \mathrm{~mol} \%$ of $\mathrm{IMes} \bullet \mathrm{HCl}$ were used.\}. The reaction was conducted for 2 $\mathrm{h}$ with $52.6 \mathrm{mg}(0.20 \mathrm{mmol})$ of $\mathbf{1 a}$ and $125.2 \mu \mathrm{L}(0.80 \mathrm{mmol})$ of $\mathbf{2} \mathbf{b}$. The product $4 \mathbf{a b}$ was obtained in $66 \%$ yield $(55.6 \mathrm{mg})$.

${ }^{1} \mathrm{H}$ NMR (392 MHz, CDCl $): \delta 0.87(\mathrm{t}, J=6.9 \mathrm{~Hz}, 3 \mathrm{H}), 1.13-1.37(\mathrm{~m}, 11 \mathrm{H}), 1.23(\mathrm{~s}, 12 \mathrm{H})$, $1.37-1.55$ (m, 3H), 1.60-1.69 (m, 2H), 1.74-1.91 (m, 2H), 1.93-2.09 (m, 1H), 2.62 (t, $J=7.6$ $\mathrm{Hz}, 2 \mathrm{H}), 7.14-7.21(\mathrm{~m}, 3 \mathrm{H}), 7.24-7.31(\mathrm{~m}, 2 \mathrm{H}) .{ }^{13} \mathrm{C} \mathrm{NMR}\left(100 \mathrm{MHz}, \mathrm{CDCl}_{3}\right): \delta 14.1\left(\mathrm{CH}_{3}\right)$, 17.3 (br, B-CH), 22.1 (t, $\left.J=4.3 \mathrm{~Hz}, \mathrm{CH}_{2}\right), 22.6\left(\mathrm{CH}_{2}\right), 24.72\left(\mathrm{CH}_{3}\right), 24.75\left(\mathrm{CH}_{3}\right), 28.8\left(\mathrm{CH}_{2}\right)$, $29.4\left(\mathrm{CH}_{2}\right), 31.2\left(\mathrm{CH}_{2}\right), 31.7\left(\mathrm{CH}_{2}\right), 31.8\left(\mathrm{CH}_{2}\right), 35.7\left(\mathrm{CH}_{2}\right), 36.5\left(\mathrm{t}, J=25.1 \mathrm{~Hz}, \mathrm{CH}_{2}\right), 38.4(\mathrm{t}$, $\left.J=25.1 \mathrm{~Hz}, C_{2}\right), 125.4\left(\mathrm{t}, J=239.0 \mathrm{~Hz}, C \mathrm{~F}_{2}\right), 125.7(\mathrm{CH}), 128.28(\mathrm{CH}), 128.34(\mathrm{CH}), 142.2$ (C). ${ }^{19} \mathrm{~F}$ NMR (373 MHz, $\left.\mathrm{CDCl}_{3}\right): \delta-97.8$ to $-95.6(\mathrm{~m}, 2 \mathrm{~F})$. HRMS-ESI $(\mathrm{m} / \mathrm{z}):[\mathrm{M}+\mathrm{Na}]^{+}$calcd for $\mathrm{C}_{25} \mathrm{H}_{41} \mathrm{O}_{2}{ }^{11} \mathrm{BF}_{2} \mathrm{Na}$, 445.3064; found, 445.3053 . 


\section{2-(1-Cyclohexyl-4,4-difluoro-8-phenyloctan-2-yl)-4,4,5,5-tetramethyl-1,3,2-}

dioxaborolane (4ac).

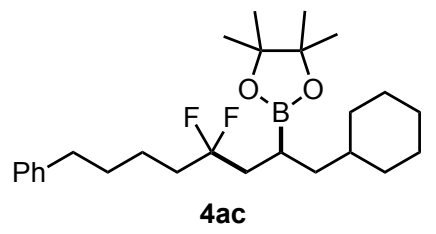

Prepared according to condition A described above. The reaction was conducted for $2 \mathrm{~h}$ with $52.6 \mathrm{mg}(0.20 \mathrm{mmol})$ of $\mathbf{1 a}$ and $123.8 \mu \mathrm{L}(0.80 \mathrm{mmol})$ of $\mathbf{2 c}$. The product $4 \mathbf{a c}$ was obtained in $65 \%$ yield $(56.6 \mathrm{mg})$.

${ }^{1} \mathrm{H}$ NMR (401 MHz, $\left.\mathrm{CDCl}_{3}\right): \delta 0.84(\mathrm{q}, J=6.7 \mathrm{~Hz}, 2 \mathrm{H}), 1.11-1.21(\mathrm{~m}, 4 \mathrm{H}), 1.23(\mathrm{~s}, 12 \mathrm{H})$, $1.28-1.37(\mathrm{~m}, 2 \mathrm{H}), 1.45-1.55(\mathrm{~m}, 2 \mathrm{H}), 1.60-1.72(\mathrm{~m}, 7 \mathrm{H}), 1.73-2.06(\mathrm{~m}, 5 \mathrm{H}), 2.62(\mathrm{t}, J=7.6$ $\mathrm{Hz}, 2 \mathrm{H}), 7.14-7.21(\mathrm{~m}, 3 \mathrm{H}), 7.25-7.30(\mathrm{~m}, 2 \mathrm{H}) .{ }^{13} \mathrm{C} \mathrm{NMR}\left(100 \mathrm{MHz}, \mathrm{CDCl}_{3}\right): \delta 14.3$ (br, B$\mathrm{CH}), 22.1\left(\mathrm{CH}_{2}\right), 24.7\left(\mathrm{CH}_{3}\right), 24.8\left(\mathrm{CH}_{3}\right), 26.3\left(\mathrm{CH}_{2}\right), 26.4\left(\mathrm{CH}_{2}\right), 26.6\left(\mathrm{CH}_{2}\right), 31.2\left(\mathrm{CH}_{2}\right), 33.1$ $\left(\mathrm{CH}_{2}\right), 33.6\left(\mathrm{CH}_{2}\right), 35.7\left(\mathrm{CH}_{2}\right), 36.5\left(\mathrm{t}, J=25.4 \mathrm{~Hz}, \mathrm{CH}_{2}\right), 36.7(\mathrm{CH}), 38.7\left(\mathrm{t}, J=25.4 \mathrm{~Hz}, \mathrm{CH}_{2}\right)$, $39.4\left(\mathrm{CH}_{2}\right), 83.0(\mathrm{C}), 125.4\left(\mathrm{t}, J=241.9 \mathrm{~Hz}, \mathrm{CF}_{2}\right), 125.7(\mathrm{CH}), 128.28(\mathrm{CH}), 128.34(\mathrm{CH})$, $142.2(C) .{ }^{19} \mathrm{~F} \mathrm{NMR}\left(369 \mathrm{MHz}, \mathrm{CDCl}_{3}\right): \delta-97.7$ to -95.3 (m, 2F). HRMS-ESI $(\mathrm{m} / \mathrm{z}):[\mathrm{M}+\mathrm{Na}]^{+}$ calcd for $\mathrm{C}_{26} \mathrm{H}_{41} \mathrm{O}_{2}{ }^{11} \mathrm{BF}_{2} \mathrm{Na}, 457.3065$; found, 457.3054 .

\section{7,7-Difluoro-11-phenyl-5-(4,4,5,5-tetramethyl-1,3,2-dioxaborolan-2-yl)undecanenitrile} (4ad).

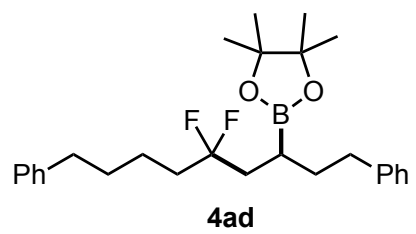

Prepared according to condition A described above, except the catalyst loading $\{10 \mathrm{~mol} \%$ of $\left[\mathrm{Cu}(\mathrm{MeCN})_{4}\right] \mathrm{BF}_{4}$ and $15 \mathrm{~mol} \%$ of $\mathrm{IMes} \cdot \mathrm{HCl}$ were used.\}. The reaction was conducted for 3 $\mathrm{h}$ with $52.6 \mathrm{mg}(0.20 \mathrm{mmol})$ of $\mathbf{1 a}$ and $120.1 \mu \mathrm{L}(0.80 \mathrm{mmol})$ of $\mathbf{2 d}$. The product $4 \mathbf{a d}$ was obtained in $58 \%$ yield $(50.9 \mathrm{mg})$.

${ }^{1} \mathrm{H}$ NMR $\left(392 \mathrm{MHz}, \mathrm{CDCl}_{3}\right): \delta 1.26(\mathrm{~s}, 12 \mathrm{H}), 1.45-1.55(\mathrm{~m}, 2 \mathrm{H}), 1.58-1.71(\mathrm{~m}, 4 \mathrm{H}), 1.72$ $1.94(\mathrm{~m}, 4 \mathrm{H}), 1.98-2.15(\mathrm{~m}, 1 \mathrm{H}), 2.61(\mathrm{t}, J=7.8 \mathrm{~Hz}, 4 \mathrm{H}), 7.13-7.21(\mathrm{~m}, 6 \mathrm{H}), 7.23-7.31(\mathrm{~m}$, 4H). ${ }^{13} \mathrm{C}$ NMR (100 MHz, $\left.\mathrm{CDCl}_{3}\right): \delta 17.3(\mathrm{br}, \mathrm{B}-\mathrm{CH}), 22.0\left(\mathrm{t}, J=4.3 \mathrm{~Hz}, \mathrm{CH}_{2}\right), 24.8\left(\mathrm{CH}_{3}\right)$, $31.2(\mathrm{CH}), 33.8(\mathrm{CH}), 35.3(\mathrm{CH}), 35.7(\mathrm{CH}), 36.6\left(\mathrm{t}, J=25.4 \mathrm{~Hz}, \mathrm{CH}_{2}\right), 38.1(\mathrm{t}, J=24.9 \mathrm{~Hz}$, $\left.\mathrm{CH}_{2}\right), 83.2(\mathrm{C}), 125.4\left(\mathrm{t}, J=241.9 \mathrm{~Hz}, \mathrm{CF}_{2}\right), 125.7(\mathrm{CH}), 128.29(\mathrm{CH}), 128.34(\mathrm{CH}), 142.2(\mathrm{C})$, $142.4(C) .{ }^{19} \mathrm{~F} \mathrm{NMR}\left(373 \mathrm{MHz}, \mathrm{CDCl}_{3}\right): \delta-97.7$ to $-95.5(\mathrm{~m}, 2 \mathrm{~F})$. HRMS-ESI $(\mathrm{m} / \mathrm{z}):[\mathrm{M}+\mathrm{Na}]^{+}$ calcd for $\mathrm{C}_{27} \mathrm{H}_{37} \mathrm{O}_{2}{ }^{11} \mathrm{BF}_{2} \mathrm{Na}$, 465.2752; found, 465.2741. 


\section{2-(1-Cyclohexyl-3,3-difluoro-7-phenylheptyl)-4,4,5,5-tetramethyl-1,3,2-dioxaborolane}

(4ae).

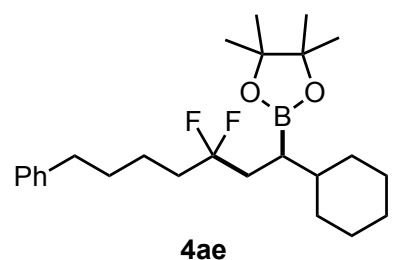

Prepared according to condition A described above, except the catalyst loading $\{10 \mathrm{~mol} \%$ of $\left[\mathrm{Cu}(\mathrm{MeCN})_{4}\right] \mathrm{BF}_{4}$ and $15 \mathrm{~mol} \%$ of IMes $\bullet \mathrm{HCl}$ were used.\}. The reaction was conducted for 3 $\mathrm{h}$ with $52.1 \mathrm{mg}(0.20 \mathrm{mmol})$ of $\mathbf{1 a}$ and $109.5 \mu \mathrm{L}(0.80 \mathrm{mmol})$ of $2 \mathrm{e}$. The product $4 \mathrm{ae}$ was obtained in $64 \%$ yield $(54.0 \mathrm{mg})$.

${ }^{1} \mathrm{H}$ NMR (392 MHz, $\left.\mathrm{CDCl}_{3}\right): \delta$ 0.91-1.28 (m, 6H), $1.24(\mathrm{~s}, 12 \mathrm{H}), 1.29-1.41(\mathrm{~m}, 1 \mathrm{H}), 1.45-$ $1.55(\mathrm{~m}, 2 \mathrm{H}), 1.60-1.92(\mathrm{~m}, 10 \mathrm{H}), 2.00-2.14(\mathrm{~m}, 1 \mathrm{H}), 2.62(\mathrm{t}, J=7.6 \mathrm{~Hz}, 2 \mathrm{H}), 7.14-7.21(\mathrm{~m}$, $3 \mathrm{H}), 7.24-7.32(\mathrm{~m}, 2 \mathrm{H}) .{ }^{13} \mathrm{C} \mathrm{NMR}\left(99 \mathrm{MHz}, \mathrm{CDCl}_{3}\right): \delta 22.0\left(\mathrm{t}, J=4.1 \mathrm{~Hz}, \mathrm{CH}_{2}\right), 23.8$ (br, B$\mathrm{CH}), 24.8\left(\mathrm{CH}_{3}\right), 24.9\left(\mathrm{CH}_{3}\right), 26.6\left(\mathrm{t}, J=5.6 \mathrm{~Hz}, \mathrm{CH}_{2}\right), 31.2\left(\mathrm{CH}_{2}\right), 32.0\left(\mathrm{CH}_{2}\right), 32.6\left(\mathrm{CH}_{2}\right)$, $35.7\left(\mathrm{CH}_{2}\right), 35.9$ (t, $\left.J=24.6 \mathrm{~Hz}, C \mathrm{H}_{2}\right), 36.5\left(\mathrm{t}, J=26.0 \mathrm{~Hz}, \mathrm{CH}_{2}\right), 40.0(\mathrm{CH}), 83.1(C), 125.68$ (t, $\left.J=241.7 \mathrm{~Hz}, C \mathrm{~F}_{2}\right), 125.69(\mathrm{CH}), 128.26(\mathrm{CH}), 128.33(\mathrm{CH}), 142.2(\mathrm{C}) .{ }^{19} \mathrm{~F}$ NMR $(373 \mathrm{MHz}$, $\mathrm{CDCl}_{3}$ ): $\delta-98.8$ to $-95.6(\mathrm{~m}, 2 \mathrm{~F})$. HRMS-ESI $(\mathrm{m} / \mathrm{z})$ : $[\mathrm{M}+\mathrm{Na}]^{+}$calcd for $\mathrm{C}_{25} \mathrm{H}_{39} \mathrm{O}_{2}{ }^{11} \mathrm{BF}_{2} \mathrm{Na}$, 443.2908; found, 443.2900 .

\section{2-(5,5-Difluoro-2,2-dimethyl-9-phenylnonan-3-yl)-4,4,5,5-tetramethyl-1,3,2}

dioxaborolane (4af).

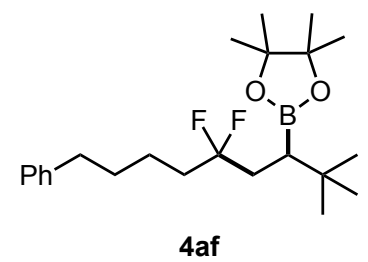

Prepared according to condition A described above, except the catalyst loading $\{10 \mathrm{~mol} \%$ of $\left[\mathrm{Cu}(\mathrm{MeCN})_{4}\right] \mathrm{BF}_{4}$ and $15 \mathrm{~mol} \%$ of $\mathrm{IMes} \cdot \mathrm{HCl}$ were used.\}. The reaction was conducted for 2 h with $52.6 \mathrm{mg}(0.20 \mathrm{mmol})$ of $\mathbf{1 a}$ and $103.6 \mu \mathrm{L}(0.80 \mathrm{mmol})$ of $\mathbf{2 f}$. The product 4 af was obtained in $49 \%$ yield $(38.9 \mathrm{mg}$ ) with small amounts of unidentified byproducts.

${ }^{1} \mathrm{H}$ NMR (396 MHz, $\mathrm{CDCl}_{3}$ )): $\delta 0.94$ (s, 9H), 1.24 (s, 12H), 1.48-1.55 (m, 2H), 1.60-1.92 (m, 6H), 1.96-2.14 (m, 1H), $2.62(\mathrm{t}, J=7.7 \mathrm{~Hz}, 2 \mathrm{H}), 7.14-7.22(\mathrm{~m}, 3 \mathrm{H}), 7.24-7.32(\mathrm{~m}, 2 \mathrm{H})$. ${ }^{13} \mathrm{C} \mathrm{NMR}\left(99 \mathrm{MHz}, \mathrm{CDCl}_{3}\right): \delta 22.0\left(\mathrm{t}, J=4.8 \mathrm{~Hz}, \mathrm{CH}_{2}\right), 24.8\left(\mathrm{CH}_{3}\right), 25.0\left(\mathrm{CH}_{3}\right), 29.2\left(\mathrm{CH}_{3}\right)$, $31.2\left(\mathrm{CH}_{2}\right), 31.7(\mathrm{C}), 34.2\left(\mathrm{t}, J=24.6 \mathrm{~Hz}, C \mathrm{H}_{2}\right), 35.7\left(\mathrm{CH}_{2}\right), 36.5\left(\mathrm{t}, J=25.5 \mathrm{~Hz}, C \mathrm{H}_{2}\right), 83.1$ 
(C), $125.72(\mathrm{CH}), 125.76\left(\mathrm{t}, J=235.1 \mathrm{~Hz}, \mathrm{CF}_{2}\right), 128.28(\mathrm{CH}), 128.34(\mathrm{CH}), 142.2(C)$. The carbon directly attached to the boron atom was not detected. ${ }^{19} \mathrm{~F}$ NMR $\left(373 \mathrm{MHz}, \mathrm{CDCl}_{3}\right): \delta-$ 97.9 to -95.1 (m, 2F). HRMS-ESI $(m / z)$ : $[\mathrm{M}+\mathrm{Na}]^{+}$calcd for $\mathrm{C}_{23} \mathrm{H}_{37} \mathrm{O}_{2}{ }^{11} \mathrm{BF}_{2} \mathrm{Na}, 417.2751$; found, 417.2742 .

\section{7,7-Difluoro-11-phenyl-5-(4,4,5,5-tetramethyl-1,3,2-dioxaborolan-2-yl)undecanenitrile} (4ag).

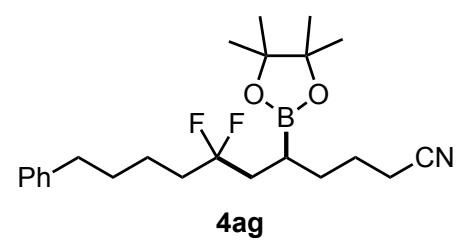

Prepared according to condition A described above. The reaction was conducted for $3 \mathrm{~h}$ with $53.2 \mathrm{mg}(0.20 \mathrm{mmol})$ of $\mathbf{1 a}$ and $90.9 \mu \mathrm{L}(0.80 \mathrm{mmol})$ of $\mathbf{2 g}$. The product $4 \mathbf{a g}$ was obtained in $52 \%$ yield (42.9 mg).

${ }^{1} \mathrm{H}$ NMR (401 MHz, CDCl $): \delta 1.19-1.29(\mathrm{~m}, 2 \mathrm{H}), 1.23(\mathrm{~s}, 12 \mathrm{H}), 1.47-1.54(\mathrm{~m}, 2 \mathrm{H}), 1.60-$ $1.71(\mathrm{~m}, 5 \mathrm{H}), 1.75-1.91(\mathrm{~m}, 3 \mathrm{H}), 1.96-2.14(\mathrm{~m}, 1 \mathrm{H}), 2.34(\mathrm{t}, J=7.0 \mathrm{~Hz}, 2 \mathrm{H}), 2.62(\mathrm{t}, J=7.6$ $\mathrm{Hz}, 2 \mathrm{H}), 7.14-7.21(\mathrm{~m}, 3 \mathrm{H}), 7.24-7.31(\mathrm{~m}, 2 \mathrm{H}) .{ }^{13} \mathrm{C} \mathrm{NMR}\left(100 \mathrm{MHz}, \mathrm{CDCl}_{3}\right): \delta 16.5$ (br, B$\mathrm{CH}), 17.2\left(\mathrm{CH}_{2}\right), 22.1$ (t, $\left.J=4.3 \mathrm{~Hz}, \mathrm{CH}_{2}\right), 24.7\left(\mathrm{CH}_{3}\right), 24.7\left(\mathrm{CH}_{2}\right), 30.6\left(\mathrm{CH}_{2}\right), 31.1\left(\mathrm{CH}_{2}\right)$, $35.7\left(\mathrm{CH}_{2}\right), 36.8$ (t, $\left.J=25.9 \mathrm{~Hz}, \mathrm{CH}_{2}\right), 38.0$ (t, $\left.J=25.4 \mathrm{~Hz}, \mathrm{CH}_{2}\right), 83.4(\mathrm{C}), 119.5(\mathrm{C}), 125.3$ (t, $\left.J=242.4 \mathrm{~Hz}, C_{2}\right), 125.7(C \mathrm{H}), 128.28(\mathrm{CH}), 128.33(\mathrm{CH}), 142.1(C) .{ }^{19} \mathrm{~F}$ NMR $(369 \mathrm{MHz}$, $\left.\mathrm{CDCl}_{3}\right): \delta-98.2$ to $-96.1(\mathrm{~m}, 2 \mathrm{~F})$. HRMS-ESI $(\mathrm{m} / \mathrm{z})$ : $[\mathrm{M}+\mathrm{Na}]^{+}$calcd for $\mathrm{C}_{23} \mathrm{H}_{34} \mathrm{O}_{2} \mathrm{~N}^{11} \mathrm{BF}_{2} \mathrm{Na}$, 428.2547; found, 428.2538 .

tert-Butyl[(6,6-difluoro-10-phenyl-4-(4,4,5,5-tetramethyl-1,3,2-dioxaborolan-2yl)decyl)oxy]dimethylsilane (4ah).

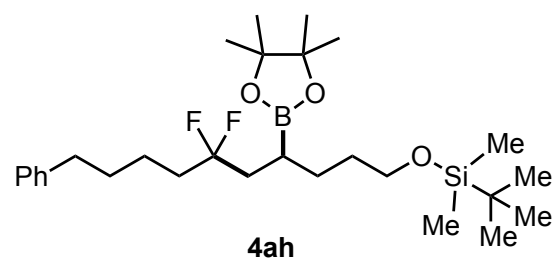

Prepared according to condition A described above, except the catalyst loading $\{10 \mathrm{~mol} \%$ of $\left[\mathrm{Cu}(\mathrm{MeCN})_{4}\right] \mathrm{BF}_{4}$ and $15 \mathrm{~mol} \%$ of IMes $\bullet \mathrm{HCl}$ was used.\}. The reaction was conducted for $2 \mathrm{~h}$ with $52.8 \mathrm{mg}(0.20 \mathrm{mmol})$ of $\mathbf{1 a}$ and $197.0 \mu \mathrm{L}(0.80 \mathrm{mmol})$ of $\mathbf{2 h}$. The product $4 \mathbf{a h}$ was obtained in $61 \%$ yield $(62.6 \mathrm{mg})$. 
${ }^{1} \mathrm{H}$ NMR (401 MHz, $\left.\mathrm{CDCl}_{3}\right): \delta 0.04(\mathrm{~s}, 6 \mathrm{H}), 0.88(\mathrm{~s}, 9 \mathrm{H}), 1.14-1.29(\mathrm{~m}, 2 \mathrm{H}), 1.23(\mathrm{~s}, 12 \mathrm{H})$, $1.31-1.55(\mathrm{~m}, 6 \mathrm{H}), 1.58-1.71(\mathrm{~m}, 2 \mathrm{H}), 1.72-1.92(\mathrm{~m}, 2 \mathrm{H}), 1.93-2.15(\mathrm{~m}, 1 \mathrm{H}), 2.62(\mathrm{t}, J=7.6$ $\mathrm{Hz}, 2 \mathrm{H}), 3.59$ (t, $J=6.4 \mathrm{~Hz}, 2 \mathrm{H}), 7.14-7.21(\mathrm{~m}, 3 \mathrm{H}), 7.23-7.31(\mathrm{~m}, 2 \mathrm{H}) .{ }^{13} \mathrm{C}$ NMR $(99 \mathrm{MHz}$, $\left.\mathrm{CDCl}_{3}\right): \delta-5.3\left(\mathrm{CH}_{3}\right), 17.0(\mathrm{br}, \mathrm{B}-\mathrm{CH}), 18.3(\mathrm{C}), 22.1\left(\mathrm{t}, J=4.3 \mathrm{~Hz}, \mathrm{CH}_{2}\right), 24.7\left(\mathrm{CH}_{3}\right), 26.0$ $\left(\mathrm{CH}_{3}\right), 27.9\left(\mathrm{CH}_{2}\right), 31.2\left(\mathrm{CH}_{2}\right), 32.1\left(\mathrm{CH}_{2}\right), 35.7\left(\mathrm{CH}_{2}\right), 36.6\left(\mathrm{t}, J=26.0 \mathrm{~Hz}, \mathrm{CH}_{2}\right), 38.4(\mathrm{t}, J=$ $\left.25.0 \mathrm{~Hz}, \mathrm{CH}_{2}\right), 63.2\left(\mathrm{CH}_{2}\right), 83.1(\mathrm{C}), 125.4\left(\mathrm{t}, J=241.7 \mathrm{~Hz}, \mathrm{CF}_{2}\right), 125.7(\mathrm{CH}), 128.3(\mathrm{CH})$, $128.4(\mathrm{CH}), 142.2(\mathrm{C}) .{ }^{19} \mathrm{~F}$ NMR (373 MHz, $\left.\mathrm{CDCl}_{3}\right): \delta-98.2$ to $-95.4(\mathrm{~m}, 2 \mathrm{~F})$. HRMS-ESI (m/z): $[\mathrm{M}+\mathrm{Na}]^{+}$calcd for $\mathrm{C}_{28} \mathrm{H}_{49}{ }^{11} \mathrm{BF}_{2} \mathrm{O}_{3} \mathrm{SiNa}$, 533.3410; found, 533.3404 .

\section{7,7-Difluoro-11-phenyl-5-(4,4,5,5-tetramethyl-1,3,2-dioxaborolan-2-yl)undecyl acetate} (4ai).

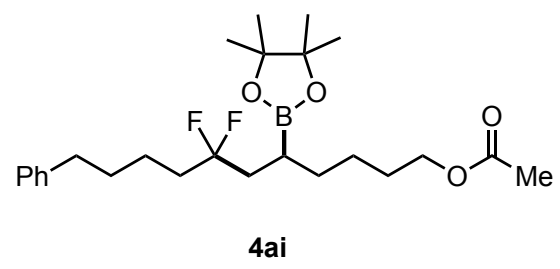

Prepared according to condition A described above, except the catalyst loading \{10 mol\% of $\left[\mathrm{Cu}(\mathrm{MeCN})_{4}\right] \mathrm{BF}_{4}$ and $15 \mathrm{~mol} \%$ of $\mathrm{IMes} \cdot \mathrm{HCl}$ was used.\} with 1.2 equiv of $\mathrm{B}_{2}(\mathrm{pin})_{2}$. The reaction was conducted for $2 \mathrm{~h}$ with $52.8 \mathrm{mg}(0.20 \mathrm{mmol})$ of $1 \mathrm{a}$ and $130.0 \mu \mathrm{L}(0.82 \mathrm{mmol})$ of 2i. The product 4 ai was obtained in $55 \%$ yield $(49.5 \mathrm{mg})$.

${ }^{1} \mathrm{H}$ NMR (401 MHz, $\left.\mathrm{CDCl}_{3}\right): \delta 1.18-1.28(\mathrm{~m}, 2 \mathrm{H}), 1.23(\mathrm{~s}, 12 \mathrm{H}), 1.33-1.42(\mathrm{~m}, 2 \mathrm{H}), 1.45-$ $1.55(\mathrm{~m}, 2 \mathrm{H}), 1.58-1.69(\mathrm{~m}, 5 \mathrm{H}), 1.75-1.91(\mathrm{~m}, 3 \mathrm{H}), 1.94-2.11(\mathrm{~m}, 1 \mathrm{H}), 2.03(\mathrm{~s}, 3 \mathrm{H}), 2.62(\mathrm{t}$, $J=7.4 \mathrm{~Hz}, 2 \mathrm{H}), 4.04(\mathrm{t}, J=6.6 \mathrm{~Hz}, 2 \mathrm{H}), 7.14-7.21(\mathrm{~m}, 3 \mathrm{H}), 7.24-7.30(\mathrm{~m}, 2 \mathrm{H}) .{ }^{13} \mathrm{C} \mathrm{NMR}(99$ $\left.\mathrm{MHz}, \mathrm{CDCl}_{3}\right): \delta 17.3(\mathrm{br}, \mathrm{B}-\mathrm{CH}), 20.9\left(\mathrm{CH}_{3}\right), 22.0\left(\mathrm{t}, J=4.3 \mathrm{~Hz}, \mathrm{CH}_{2}\right), 24.69\left(\mathrm{CH}_{3}\right), 24.72$ $\left(\mathrm{CH}_{3}\right), 25.2\left(\mathrm{CH}_{2}\right), 28.6\left(\mathrm{CH}_{2}\right), 31.2\left(\mathrm{CH}_{2}\right), 31.3\left(\mathrm{CH}_{2}\right), 35.7\left(\mathrm{CH}_{2}\right), 36.6\left(\mathrm{t}, J=25.5 \mathrm{~Hz}, \mathrm{CH}_{2}\right)$, 38.2 (t, $\left.J=25.0 \mathrm{~Hz}, \mathrm{CH}_{2}\right), 64.4\left(\mathrm{CH}_{2}\right), 83.2(C), 125.3$ (t, $\left.J=241.7 \mathrm{~Hz}, \mathrm{CF}_{2}\right), 125.7(\mathrm{CH})$, $128.26(\mathrm{CH}), 128.32(\mathrm{CH}), 142.2(\mathrm{C}), 171.1(\mathrm{C}) .{ }^{19} \mathrm{~F}$ NMR (373 MHz, $\left.\mathrm{CDCl}_{3}\right): \delta-98.0$ to 95.5 (m, 2F). HRMS-ESI $(\mathrm{m} / \mathrm{z})$ : $[\mathrm{M}+\mathrm{Na}]^{+}$calcd for $\mathrm{C}_{25} \mathrm{H}_{39}{ }^{11} \mathrm{BF}_{2} \mathrm{O}_{4} \mathrm{Na}, 475.2806$; found, 475.2803 .

\section{2-[1-(Benzyloxy)-6,6-difluoro-10-phenyldecan-4-yl]-4,4,5,5-tetramethyl-1,3,2-} dioxaborolane (4aj). 


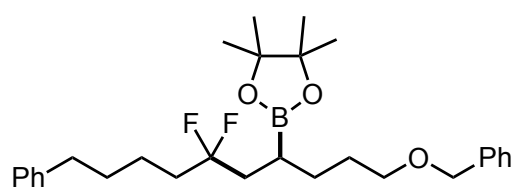

4aj

Prepared according to condition A described above, except the catalyst loading $\{10 \mathrm{~mol} \%$ of $\left[\mathrm{Cu}(\mathrm{MeCN})_{4}\right] \mathrm{BF}_{4}$ and $15 \mathrm{~mol} \%$ of IMes $\bullet \mathrm{HCl}$ was used. $\}$. The reaction was conducted for 3 $\mathrm{h}$ with $53.1 \mathrm{mg}(0.20 \mathrm{mmol})$ of $\mathbf{1 a}$ and $150.0 \mu \mathrm{L}(0.80 \mathrm{mmol})$ of $\mathbf{2} \mathbf{j}$. The product 4 aj was obtained in $30 \%$ yield $(29.2 \mathrm{mg})$.

${ }^{1} \mathrm{H}$ NMR (401 MHz, $\left.\mathrm{CDCl}_{3}\right): \delta 1.23(\mathrm{~s}, 12 \mathrm{H}), 1.37-1.57$ (m, 4H), 1.59-1.68 (m, 5H), 1.73$1.91(\mathrm{~m}, 3 \mathrm{H}), 1.94-2.11(\mathrm{~m}, 1 \mathrm{H}), 2.61(\mathrm{t}, J=7.8 \mathrm{~Hz}, 2 \mathrm{H}), 3.46(\mathrm{t}, J=6.4 \mathrm{~Hz}, 2 \mathrm{H}), 4.49(\mathrm{~s}, 2 \mathrm{H})$, 7.14-7.20 (m, 3H), 7.24-7.27 (m, 3H), 7.30-7.35 (m, 4H). ${ }^{13} \mathrm{C}$ NMR (99 MHz, $\left.\mathrm{CDCl}_{3}\right): \delta 17.1$ (br, B-CH), $22.0\left(\mathrm{t}, J=4.2 \mathrm{~Hz}, \mathrm{CH}_{2}\right), 24.8\left(\mathrm{CH}_{3}\right), 28.2\left(\mathrm{CH}_{2}\right), 29.0\left(\mathrm{CH}_{2}\right), 31.2\left(\mathrm{CH}_{2}\right), 35.7$ $\left(\mathrm{CH}_{2}\right), 36.6$ (t, $\left.J=25.4 \mathrm{~Hz}, \mathrm{CH}_{2}\right), 38.3\left(\mathrm{t}, J=24.9 \mathrm{~Hz}, \mathrm{CH}_{2}\right), 70.3\left(\mathrm{CH}_{2}\right), 72.8\left(\mathrm{CH}_{2}\right), 83.2(\mathrm{C})$, 125.3 (t, $\left.J=240.4 \mathrm{~Hz}, \mathrm{CF}_{2}\right), 125.7(\mathrm{CH}), 127.4(\mathrm{CH}), 127.6(\mathrm{CH}), 128.30(\mathrm{CH}), 128.34(\mathrm{CH})$, 138.6 (C), $142.2(C) .{ }^{19} \mathrm{~F}$ NMR (373 MHz, $\left.\mathrm{CDCl}_{3}\right): \delta-98.1$ to $-95.4(\mathrm{~m}, 2 \mathrm{~F})$. HRMS-ESI $(\mathrm{m} / \mathrm{z})$ : $[\mathrm{M}+\mathrm{Na}]^{+}$calcd for $\mathrm{C}_{29} \mathrm{H}_{41}{ }^{11} \mathrm{BF}_{2} \mathrm{O}_{3} \mathrm{Na}, 509.3014$; found, 509.3009.

\section{2-(6,6-Difluoro-10-phenyl-4-(4,4,5,5-tetramethyl-1,3,2-dioxaborolan-2-}

yl)decyl)isoindoline-1,3-dione (4ak).

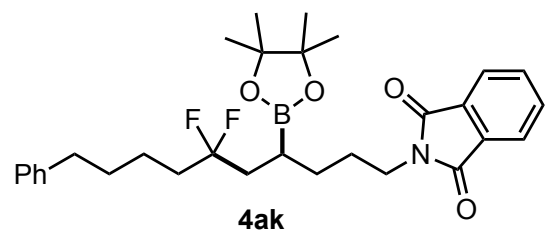

Prepared according to condition A described above, except the catalyst loading $\{10 \mathrm{~mol} \%$ of [ $\left.\mathrm{Cu}(\mathrm{MeCN})_{4}\right] \mathrm{BF}_{4}$ and $15 \mathrm{~mol} \%$ of IMes $\bullet \mathrm{HCl}$ was used. $\}$ with 1,4-dioxane/DMF $(500 \mu \mathrm{L} / 200$ $\mu \mathrm{L})$. The reaction was conducted for $4 \mathrm{~h}$ with $52.6 \mathrm{mg}(0.20 \mathrm{mmol})$ of $1 \mathrm{a}$ and $174.7 \mathrm{mg}(0.81$ $\mathrm{mmol}$ ) of $\mathbf{2 k}$. The product $\mathbf{4 a k}$ was obtained in $28 \%$ yield $(23.0 \mathrm{mg})$.

${ }^{1} \mathrm{H}$ NMR (392 MHz, $\left.\mathrm{CDCl}_{3}\right): \delta 1.21(\mathrm{~s}, 12 \mathrm{H}), 1.35-1.55(\mathrm{~m}, 4 \mathrm{H}), 1.58-1.746(\mathrm{~m}, 5 \mathrm{H})$, $1.749-1.90$ (m, 3H), 1.92-2.10 (m, 1H), 2.61 (t, $J=7.8 \mathrm{~Hz}, 2 \mathrm{H}), 3.67$ (t, $J=7.1 \mathrm{~Hz}, 2 \mathrm{H}), 7.13-$ 7.20 (m, 3H), 7.23-7.30 (m, 2H), 7.67-7.73 (m, 2H), 7.80-7.86 (m, 2H). ${ }^{13} \mathrm{C}$ NMR (99 MHz, $\left.\mathrm{CDCl}_{3}\right): \delta 17.0(\mathrm{br}, \mathrm{B}-\mathrm{CH}), 22.0\left(\mathrm{t}, J=4.7 \mathrm{~Hz}, \mathrm{CH}_{2}\right), 24.7\left(\mathrm{CH}_{3}\right), 27.9\left(\mathrm{CH}_{2}\right), 28.9\left(\mathrm{CH}_{2}\right), 31.2$ $\left(\mathrm{CH}_{2}\right), 35.7\left(\mathrm{CH}_{2}\right), 36.5\left(\mathrm{t}, J=24.9 \mathrm{~Hz}, \mathrm{CH}_{2}\right), 38.0\left(\mathrm{CH}_{2}\right), 38.2\left(\mathrm{t}, J=25.4 \mathrm{~Hz}, \mathrm{CH}_{2}\right), 83.3(\mathrm{C})$, $123.1(\mathrm{CH}), 125.3\left(\mathrm{t}, J=240.5 \mathrm{~Hz}, \mathrm{CF}_{2}\right), 125.7(\mathrm{CH}), 128.27(\mathrm{CH}), 128.34(\mathrm{CH}), 132.1(C)$, $133.8(\mathrm{CH}), 142.2(\mathrm{C}), 168.3(\mathrm{C}) .{ }^{19} \mathrm{~F}$ NMR (373 MHz, $\left.\mathrm{CDCl}_{3}\right)$ : $\delta-98.3$ to $-95.4(\mathrm{~m}, 2 \mathrm{~F})$. HRMS-ESI $(m / z)$ : $[\mathrm{M}+\mathrm{Na}]^{+}$calcd for $\mathrm{C}_{30} \mathrm{H}_{38}{ }^{11} \mathrm{BF}_{2} \mathrm{NO}_{4} \mathrm{Na}, 548.2760$; found, 548.2757. 
2-[(trans)-2-(1,1-Difluoro-5-phenylpentyl)cyclopentyl]-4,4,5,5-tetramethyl-1,3,2dioxaborolane (4al)

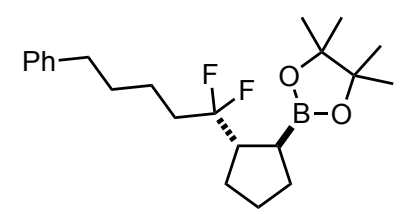

4al

Prepared according to condition A described above, except the catalyst loading $\{10 \mathrm{~mol} \%$ of $\left[\mathrm{Cu}(\mathrm{MeCN})_{4}\right] \mathrm{BF}_{4}$ and $15 \mathrm{~mol} \%$ of IMes $\bullet \mathrm{HCl}$ was used. $\}$. The reaction was conducted for 4 $\mathrm{h}$ with $52.6 \mathrm{mg}(0.20 \mathrm{mmol})$ of $1 \mathrm{a}$ and $70.8 \mu \mathrm{L}(0.80 \mathrm{mmol})$ of $2 \mathrm{l}$. The product $4 \mathrm{al}$ was obtained in $44 \%$ yield $(33.4 \mathrm{mg})$ with d.r. $=>95: 5$.

${ }^{1} \mathrm{H}$ NMR $\left(392 \mathrm{MHz}, \mathrm{CDCl}_{3}\right): \delta 1.23(\mathrm{~s}, 12 \mathrm{H}), 1.28-1.40(\mathrm{~m}, 1 \mathrm{H}), 1.42-1.92(\mathrm{~m}, 12 \mathrm{H}), 2.35-$ $2.52(\mathrm{~m}, 1 \mathrm{H}), 2.62(\mathrm{t}, J=7.6 \mathrm{~Hz}, 2 \mathrm{H}), 7.14-7.21(\mathrm{~m}, 3 \mathrm{H}), 7.23-7.30(\mathrm{~m}, 2 \mathrm{H}) .{ }^{13} \mathrm{C} \mathrm{NMR}(100$ $\left.\mathrm{MHz}, \mathrm{CDCl}_{3}\right): \delta 21.7$ (t, $\left.J=3.8 \mathrm{~Hz}, \mathrm{CH}_{2}\right), 22.7$ (br, B-CH), $24.56\left(\mathrm{CH}_{3}\right), 24.63\left(\mathrm{CH}_{3}\right), 26.9$ $\left(\mathrm{CH}_{2}\right), 27.5\left(\mathrm{CH}_{2}\right), 29.4\left(\mathrm{CH}_{2}\right), 31.4\left(\mathrm{CH}_{2}\right), 35.7\left(\mathrm{t}, J=25.4 \mathrm{~Hz}, \mathrm{CH}_{2}\right), 35.8\left(\mathrm{CH}_{2}\right), 48.2(\mathrm{t}, J=$ $24.0 \mathrm{~Hz}, \mathrm{CH}), 83.1(\mathrm{C}), 125.7(\mathrm{CH}), 126.2\left(\mathrm{t}, J=243.4 \mathrm{~Hz}, \mathrm{CF}_{2}\right), 128.27(\mathrm{CH}), 128.34(\mathrm{CH})$, $142.3(C) .{ }^{19} \mathrm{~F}$ NMR (373 MHz, $\mathrm{CDCl}_{3}$ ): $\delta-106.5$ to -105.6 (m, $1 \mathrm{~F}$ ), -103.8 to -102.9 (m, $1 \mathrm{~F}$ ). HRMS-ESI $(m / z)$ : $[\mathrm{M}+\mathrm{Na}]^{+}$calcd for $\mathrm{C}_{22} \mathrm{H}_{33} \mathrm{O}_{2}{ }^{11} \mathrm{BF}_{2} \mathrm{Na}$, 401.2438; found, 401.2429.

\section{2-[(trans)-2-(1,1-Difluoro-5-phenylpentyl)cyclohexyl]-4,4,5,5-tetramethyl-1,3,2-} dioxaborolane (4am).

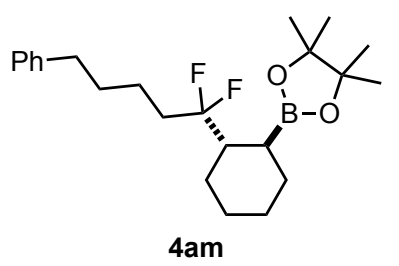

Prepared according to condition A described above, except the catalyst loading $\{10 \mathrm{~mol} \%$ of $\left[\mathrm{Cu}(\mathrm{MeCN})_{4}\right] \mathrm{BF}_{4}$ and $15 \mathrm{~mol} \%$ of IMes $\bullet \mathrm{HCl}$ was used. $\}$. The reaction was conducted for 5 h with $52.9 \mathrm{mg}(0.20 \mathrm{mmol})$ of $1 \mathbf{a}$ and $81.0 \mu \mathrm{L}(0.80 \mathrm{mmol})$ of $\mathbf{2} \mathbf{m}$. The product $4 \mathbf{a m}$ was obtained in $18 \%$ yield $(14.1 \mathrm{mg})$ with d.r. $=>95: 5$. The relative stereochemistry of 4 am was determined by X-ray crystallographic analysis.

${ }^{1} \mathrm{H}$ NMR (392 MHz, $\left.\mathrm{CDCl}_{3}\right): \delta$ 0.92-1.33 (m, 6H), $1.22(\mathrm{~s}, 12 \mathrm{H}), 1.57-2.04(\mathrm{~m}, 10 \mathrm{H}), 2.62$ (t, $J=7.6 \mathrm{~Hz}, 2 \mathrm{H}), 7.12-7.21(\mathrm{~m}, 3 \mathrm{H}), 7.22-7.31(\mathrm{~m}, 2 \mathrm{H}) .{ }^{13} \mathrm{C} \mathrm{NMR}\left(100 \mathrm{MHz}, \mathrm{CDCl}_{3}\right): \delta 21.5$ $\left(\mathrm{CH}_{2}\right), 24.58\left(\mathrm{CH}_{3}\right), 24.63\left(\mathrm{CH}_{3}\right), 25.5\left(\mathrm{CH}_{2}\right), 26.3\left(\mathrm{CH}_{2}\right), 27.1\left(\mathrm{t}, J=4.3 \mathrm{~Hz}, \mathrm{CH}_{2}\right), 27.5\left(\mathrm{CH}_{2}\right)$, $31.2\left(\mathrm{CH}_{2}\right), 33.4$ (t, $\left.J=25.9 \mathrm{~Hz}, \mathrm{CH}_{2}\right), 35.7\left(\mathrm{CH}_{2}\right), 44.7(\mathrm{t}, J=23.0 \mathrm{~Hz}, \mathrm{CH}), 82.8(C), 125.7$ 
$(C \mathrm{H}), 126.7\left(\mathrm{t}, J=243.9 \mathrm{~Hz}, \mathrm{CF}_{2}\right), 128.3(\mathrm{CH}), 128.4(\mathrm{CH}), 142.3(\mathrm{C})$. The carbon directly attached to the boron atom was not detected. ${ }^{19} \mathrm{~F}$ NMR $\left(373 \mathrm{MHz}, \mathrm{CDCl}_{3}\right): \delta-104.6$ to -103.7 (m, 1F), -99.2 to $-98.3(\mathrm{~m}, 1 \mathrm{~F})$. HRMS-ESI $(\mathrm{m} / \mathrm{z})$ : $[\mathrm{M}+\mathrm{Na}]^{+}$calcd for $\mathrm{C}_{23} \mathrm{H}_{35} \mathrm{O}_{2}{ }^{11} \mathrm{BF}_{2} \mathrm{Na}$, 415.2595; found, 415.2589 .

2-(7,7-Difluoropentadecan-5-yl)-4,4,5,5-tetramethyl-1,3,2-dioxaborolane (4fa).

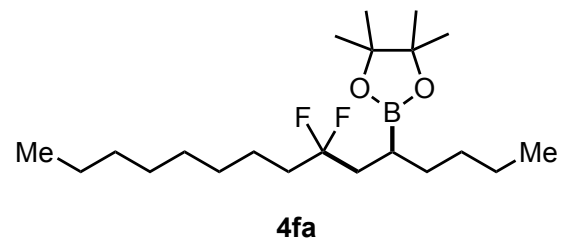

Prepared according to condition A described above. The reaction was conducted for $4 \mathrm{~h}$ with $48.6 \mathrm{mg}(0.20 \mathrm{mmol})$ of $\mathbf{1 f}$ and $100.8 \mu \mathrm{L}(0.80 \mathrm{mmol})$ of $\mathbf{2 a}$. The product $4 \mathbf{f a}$ was obtained in $71 \%$ yield $(53.3 \mathrm{mg})$.

${ }^{1} \mathrm{H}$ NMR $\left(401 \mathrm{MHz}, \mathrm{CDCl}_{3}\right): \delta 0.88(\mathrm{t}, J=6.8 \mathrm{~Hz}, 6 \mathrm{H}), 1.15-1.36(\mathrm{~m}, 18 \mathrm{H}), 1.24(\mathrm{~s}, 12 \mathrm{H})$, $1.39-1.51(\mathrm{~m}, 2 \mathrm{H}), 1.71-1.89(\mathrm{~m}, 2 \mathrm{H}), 1.94-2.10(\mathrm{~m}, 1 \mathrm{H}) .{ }^{13} \mathrm{C} \mathrm{NMR}\left(99 \mathrm{MHz}, \mathrm{CDCl}_{3}\right): \delta 14.0$ $\left(\mathrm{CH}_{3}\right), 14.1\left(\mathrm{CH}_{3}\right), 17.4$ (br, B-CH), 22.3 (t, $\left.J=4.7 \mathrm{~Hz}, \mathrm{CH}_{2}\right), 22.6\left(\mathrm{CH}_{2}\right), 22.8\left(\mathrm{CH}_{2}\right), 24.72$ $\left(\mathrm{CH}_{3}\right), 24.74\left(\mathrm{CH}_{3}\right), 29.1\left(\mathrm{CH}_{2}\right), 29.36\left(\mathrm{CH}_{2}\right), 29.38\left(\mathrm{CH}_{2}\right), 31.1\left(\mathrm{CH}_{2}\right), 31.5\left(\mathrm{CH}_{2}\right), 31.8\left(\mathrm{CH}_{2}\right)$, 36.7 (t, $\left.J=25.5 \mathrm{~Hz}, C \mathrm{H}_{2}\right), 38.4\left(\mathrm{t}, J=25.0 \mathrm{~Hz}, C \mathrm{H}_{2}\right), 83.1(C), 125.6$ (t, $J=241.2 \mathrm{~Hz}, C \mathrm{~F}_{2}$ ). ${ }^{19} \mathrm{~F}$ NMR (369 MHz, $\left.\mathrm{CDCl}_{3}\right): \delta-97.7$ to $-95.5(\mathrm{~m}, 2 \mathrm{~F})$. HRMS-ESI $(\mathrm{m} / \mathrm{z})$ : $[\mathrm{M}+\mathrm{Na}]^{+}$calcd for $\mathrm{C}_{21} \mathrm{H}_{41} \mathrm{O}_{2}{ }^{11} \mathrm{BF}_{2} \mathrm{Na}, 397.3064$; found, 397.3061 .

\section{2-(1-Cyclohexyl-3,3-difluorononan-5-yl)-4,4,5,5-tetramethyl-1,3,2-dioxaborolane (4ga).}

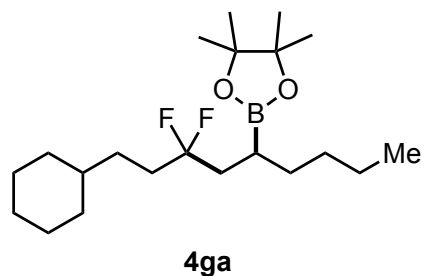

Prepared according to condition A described above, except the catalyst loading \{10 mol\% of $\left[\mathrm{Cu}(\mathrm{MeCN})_{4}\right] \mathrm{BF}_{4}$ and $15 \mathrm{~mol} \%$ of IMes $\bullet \mathrm{HCl}$ was used. $\}$. The reaction was conducted for 3 $\mathrm{h}$ with $49.0 \mathrm{mg}(0.20 \mathrm{mmol})$ of $\mathbf{1 g}$ and $100.8 \mu \mathrm{L}(0.80 \mathrm{mmol})$ of 2a. The product 4 ga was obtained in $69 \%$ yield $(51.9 \mathrm{mg})$.

${ }^{1} \mathrm{H}$ NMR $\left(392 \mathrm{MHz}, \mathrm{CDCl}_{3}\right): \delta 0.81-0.97(\mathrm{~m}, 2 \mathrm{H}), 0.88(\mathrm{t}, J=6.5 \mathrm{~Hz}, 3 \mathrm{H}), 1.07-1.51(\mathrm{~m}$, $13 \mathrm{H}), 1.24(\mathrm{~s}, 12 \mathrm{H}), 1.60-1.90(\mathrm{~m}, 8 \mathrm{H}), 1.92-2.10(\mathrm{~m}, 1 \mathrm{H}) .{ }^{13} \mathrm{C} \mathrm{NMR}\left(100 \mathrm{MHz}, \mathrm{CDCl}_{3}\right): \delta$ $14.0\left(\mathrm{CH}_{3}\right), 17.3$ (br, B-CH), $22.8\left(\mathrm{CH}_{2}\right), 24.7\left(\mathrm{CH}_{3}\right), 26.3\left(\mathrm{CH}_{2}\right), 26.6\left(\mathrm{CH}_{2}\right), 29.7\left(\mathrm{CH}_{2}\right), 31.1$ $\left(\mathrm{CH}_{2}\right), 31.5\left(\mathrm{CH}_{2}\right), 33.1\left(\mathrm{~d}, J=5.7 \mathrm{~Hz}, \mathrm{CH}_{2}\right), 34.2\left(\mathrm{t}, J=25.4 \mathrm{~Hz}, \mathrm{CH}_{2}\right), 37.4(\mathrm{CH}), 38.4(\mathrm{t}, J$ 
$\left.=25.4 \mathrm{~Hz}, \mathrm{CH}_{2}\right), 83.1(\mathrm{C}), 125.8\left(\mathrm{t}, J=241.4 \mathrm{~Hz}, C \mathrm{~F}_{2}\right) .{ }^{19} \mathrm{~F} \mathrm{NMR}\left(373 \mathrm{MHz}, \mathrm{CDCl}_{3}\right): \delta-97.9$ to -95.7 (m, 2F). HRMS-ESI $(m / z)$ : $[\mathrm{M}+\mathrm{Na}]^{+}$calcd for $\mathrm{C}_{21} \mathrm{H}_{39} \mathrm{O}_{2}{ }^{11} \mathrm{BF}_{2} \mathrm{Na}, 395.2907$; found, 395.2904 .

2-(1-Cyclohexyl-2,2-difluorooctan-4-yl)-4,4,5,5-tetramethyl-1,3,2-dioxaborolane (4ha).

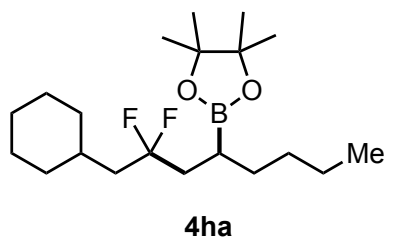

Prepared according to condition A described above, except the catalyst loading $\{10 \mathrm{~mol} \%$ of $\left[\mathrm{Cu}(\mathrm{MeCN})_{4}\right] \mathrm{BF}_{4}$ and $15 \mathrm{~mol} \%$ of IMes $\bullet \mathrm{HCl}$ was used. $\}$. The reaction was conducted for 3 $\mathrm{h}$ with $45.4 \mathrm{mg}(0.20 \mathrm{mmol})$ of $\mathbf{1 h}$ and $100.8 \mu \mathrm{L}(0.80 \mathrm{mmol})$ of $\mathbf{2 a}$. The product 4 ha was obtained in $63 \%$ yield $(45.2 \mathrm{mg})$.

${ }^{1} \mathrm{H}$ NMR $\left(392 \mathrm{MHz}, \mathrm{CDCl}_{3}\right): \delta 0.88(\mathrm{t}, J=6.7 \mathrm{~Hz}, 3 \mathrm{H}), 0.92-1.03(\mathrm{~m}, 2 \mathrm{H}), 1.07-1.50(\mathrm{~m}$, $11 \mathrm{H}), 1.24(\mathrm{~s}, 12 \mathrm{H}), 1.58-1.89(\mathrm{~m}, 8 \mathrm{H}), 1.94-2.12(\mathrm{~m}, 1 \mathrm{H}) .{ }^{13} \mathrm{C} \mathrm{NMR}\left(100 \mathrm{MHz}, \mathrm{CDCl}_{3}\right): \delta$ $14.0\left(\mathrm{CH}_{3}\right), 17.3$ (br, B-CH), $22.8\left(\mathrm{CH}_{2}\right), 24.7\left(\mathrm{CH}_{3}\right), 26.2\left(\mathrm{CH}_{2}\right), 31.1\left(\mathrm{CH}_{2}\right), 31.4\left(\mathrm{CH}_{2}\right), 32.4$ $(\mathrm{CH}), 33.8\left(\mathrm{CH}_{2}\right), 34.0\left(\mathrm{CH}_{2}\right), 39.1\left(\mathrm{t}, J=25.4 \mathrm{~Hz}, \mathrm{CH}_{2}\right), 43.9\left(\mathrm{t}, J=24.4 \mathrm{~Hz}, \mathrm{CH}_{2}\right), 83.0(C)$, $125.8\left(\mathrm{t}, J=242.4 \mathrm{~Hz}, C \mathrm{~F}_{2}\right) .{ }^{19} \mathrm{~F}$ NMR $\left(373 \mathrm{MHz}, \mathrm{CDCl}_{3}\right): \delta-94.9$ to $-92.8(\mathrm{~m}, 2 \mathrm{~F})$. HRMSESI $(m / z):[\mathrm{M}+\mathrm{Na}]^{+}$calcd for $\mathrm{C}_{20} \mathrm{H}_{37} \mathrm{O}_{2}{ }^{11} \mathrm{BF}_{2} \mathrm{Na}, 381.2751$; found, 381.2747 .

\section{2,2-Difluoro-4-(4,4,5,5-tetramethyl-1,3,2-dioxaborolan-2-yl)octyl pentanoate (4ia).}

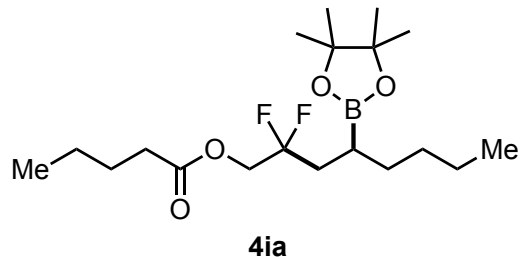

Prepared according to condition A described above, except the catalyst loading $\{10 \mathrm{~mol} \%$ of $\left[\mathrm{Cu}(\mathrm{MeCN})_{4}\right] \mathrm{BF}_{4}$ and $15 \mathrm{~mol} \%$ of IMes $\bullet \mathrm{HCl}$ was used. $\}$. The reaction was conducted for 2 $\mathrm{h}$ with $50.4 \mathrm{mg}(0.21 \mathrm{mmol})$ of $1 \mathbf{i}$ and $100.8 \mu \mathrm{L}(0.80 \mathrm{mmol})$ of $\mathbf{2 a}$. The product $4 \mathbf{i a}$ was obtained in $59 \%$ yield $(45.3 \mathrm{mg})$.

${ }^{1} \mathrm{H}$ NMR $\left(399 \mathrm{MHz}, \mathrm{CDCl}_{3}\right): \delta 0.88(\mathrm{t}, J=7.2 \mathrm{~Hz}, 3 \mathrm{H}), 0.92(\mathrm{t}, J=7.4 \mathrm{~Hz}, 3 \mathrm{H}), 1.23(\mathrm{~s}$, $12 \mathrm{H}), 1.25-1.52(\mathrm{~m}, 10 \mathrm{H}), 1.59-1.68(\mathrm{~m}, 2 \mathrm{H}), 1.82-2.17(\mathrm{~m}, 1 \mathrm{H}), 2.38(\mathrm{t}, J=7.6 \mathrm{~Hz}, 2 \mathrm{H}), 4.24$ (dd, $J=8.0,13.6 \mathrm{~Hz}, 2 \mathrm{H}) .{ }^{13} \mathrm{C} \mathrm{NMR}\left(100 \mathrm{MHz}, \mathrm{CDCl}_{3}\right): \delta 13.6\left(\mathrm{CH}_{3}\right), 13.9\left(\mathrm{CH}_{3}\right), 16.9(\mathrm{br}$, B-CH), $22.2\left(\mathrm{CH}_{2}\right), 22.7\left(\mathrm{CH}_{2}\right), 24.68\left(\mathrm{CH}_{3}\right), 24.74\left(\mathrm{CH}_{3}\right), 26.9\left(\mathrm{CH}_{2}\right), 31.0\left(\mathrm{CH}_{2}\right), 31.3\left(\mathrm{CH}_{2}\right)$, $33.6\left(\mathrm{CH}_{2}\right), 35.7\left(\mathrm{t}, J=23.5 \mathrm{~Hz}, C \mathrm{H}_{2}\right), 63.8\left(\mathrm{t}, J=33.1 \mathrm{~Hz}, C \mathrm{H}_{2}\right), 83.2(C), 121.8(\mathrm{t}, J=243.4$ 
$\left.\mathrm{Hz}, \mathrm{CF}_{2}\right), 172.7(\mathrm{C}) .{ }^{19} \mathrm{~F}$ NMR (373 MHz, $\left.\mathrm{CDCl}_{3}\right): \delta-106.3$ to $-103.2(\mathrm{~m}, 2 \mathrm{~F})$. HRMS-ESI (m/z): $[\mathrm{M}+\mathrm{Na}]^{+}$calcd for $\mathrm{C}_{19} \mathrm{H}_{35} \mathrm{O}_{4}{ }^{11} \mathrm{BF}_{2}, 399.2492$; found, 399.2489 .

\section{2,2-Difluoro-4-(4,4,5,5-tetramethyl-1,3,2-dioxaborolan-2-yl)octyl pentanoate (4ja).}

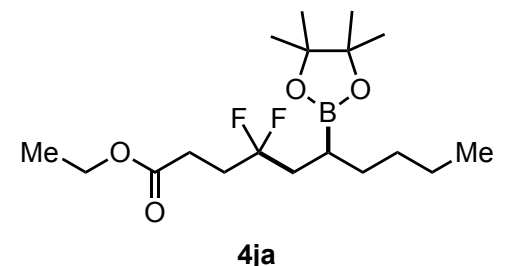

Prepared according to condition A described above, except the catalyst loading $\{10 \mathrm{~mol} \%$ of $\left[\mathrm{Cu}(\mathrm{MeCN})_{4}\right] \mathrm{BF}_{4}$ and $15 \mathrm{~mol} \%$ of IMes $\bullet \mathrm{HCl}$ was used.\}. The reaction was conducted for 4 $\mathrm{h}$ with $46.2 \mathrm{mg}(0.20 \mathrm{mmol})$ of $\mathbf{1 j}$ and $100.8 \mu \mathrm{L}(0.80 \mathrm{mmol})$ of $\mathbf{2 a}$. The product $\mathbf{4 j a}$ was obtained in $42 \%$ yield $(30.5 \mathrm{mg})$.

${ }^{1} \mathrm{H}$ NMR (392 MHz, $\left.\mathrm{CDCl}_{3}\right): \delta 0.88(\mathrm{t}, J=6.9 \mathrm{~Hz}, 3 \mathrm{H}), 1.15-1.37(\mathrm{~m}, 11 \mathrm{H}), 1.24(\mathrm{~s}, 12 \mathrm{H})$, $1.75-1.91(\mathrm{~m}, 1 \mathrm{H}), 1.97-2.28$ (m, 2H), 2.50 (t, $J=7.8 \mathrm{~Hz}, 2 \mathrm{H}), 4.14$ (q, $J=4.8 \mathrm{~Hz}, 2 \mathrm{H}) .{ }^{13} \mathrm{C}$ NMR (100 MHz, $\left.\mathrm{CDCl}_{3}\right): \delta 14.0\left(\mathrm{CH}_{3}\right), 14.2\left(\mathrm{CH}_{3}\right), 17.2(\mathrm{br}, \mathrm{B}-\mathrm{CH}), 22.8\left(\mathrm{CH}_{2}\right), 24.70\left(\mathrm{CH}_{3}\right)$, $24.75\left(\mathrm{CH}_{3}\right), 27.4\left(\mathrm{t}, J=4.3 \mathrm{~Hz}, \mathrm{CH}_{2}\right), 31.0\left(\mathrm{CH}_{2}\right), 31.4\left(\mathrm{CH}_{2}\right), 32.0\left(\mathrm{t}, J=25.9 \mathrm{~Hz}, \mathrm{CH}_{2}\right), 38.6$ (t, $\left.J=24.5 \mathrm{~Hz}, C \mathrm{H}_{2}\right), 60.6\left(\mathrm{CH}_{2}\right), 83.2(C), 124.5\left(\mathrm{t}, J=242.4 \mathrm{~Hz}, C \mathrm{~F}_{2}\right), 172.5(C) .{ }^{19} \mathrm{~F}$ NMR $\left(373 \mathrm{MHz}, \mathrm{CDCl}_{3}\right): \delta-99.9$ to $-97.8(\mathrm{~m}, 2 \mathrm{~F})$. HRMS-ESI $(\mathrm{m} / \mathrm{z}):[\mathrm{M}+\mathrm{Na}]^{+}$calcd for $\mathrm{C}_{18} \mathrm{H}_{33} \mathrm{O}_{4}{ }^{11} \mathrm{BF}_{2} \mathrm{Na}$, 385.2336; found, 385.2331.

\section{7,7-Difluoro-9-(4,4,5,5-tetramethyl-1,3,2-dioxaborolan-2-yl)tridecyl acetate (4ka).}

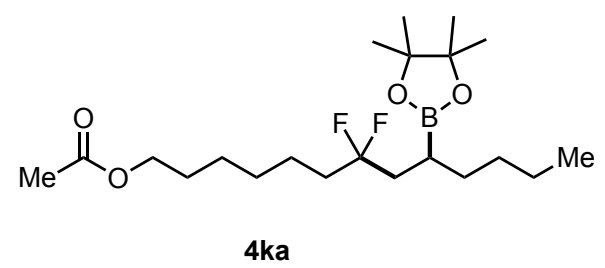

Prepared according to condition A described above, except the catalyst loading $\{10 \mathrm{~mol} \%$ of $\left[\mathrm{Cu}(\mathrm{MeCN})_{4}\right] \mathrm{BF}_{4}$ and $15 \mathrm{~mol} \%$ of IMes $\bullet \mathrm{HCl}$ was used.\} with 1.2 equiv of $\mathrm{B}_{2}(\mathrm{pin})_{2}$. The reaction was conducted for $3 \mathrm{~h}$ with $53.0 \mathrm{mg}(0.19 \mathrm{mmol})$ of $1 \mathrm{k}$ and $100.0 \mu \mathrm{L}(0.80 \mathrm{mmol})$ of 2a. The product $4 \mathrm{ka}$ was obtained in $69 \%$ yield $(54.6 \mathrm{mg})$.

${ }^{1} \mathrm{H} \mathrm{NMR}\left(401 \mathrm{MHz}, \mathrm{CDCl}_{3}\right): \delta 0.88(\mathrm{t}, J=7.2 \mathrm{~Hz}, 3 \mathrm{H}), 1.16-1.40(\mathrm{~m}, 10 \mathrm{H}), 1.24(\mathrm{~s}, 12 \mathrm{H})$, $1.42-1.51(\mathrm{~m}, 2 \mathrm{H}), 1.59-1.67$ (m, 3H), 1.73-1.90 (m, 3H), 1.94-2.10 (m, 1H), 2.05 (s, 3H), $4.05(\mathrm{t}, J=6.6 \mathrm{~Hz}, 2 \mathrm{H}) .{ }^{13} \mathrm{C}$ NMR $\left(99 \mathrm{MHz}, \mathrm{CDCl}_{3}\right): \delta 14.0\left(\mathrm{CH}_{3}\right), 17.3(\mathrm{br}, \mathrm{B}-\mathrm{CH}), 21.0$ $\left(\mathrm{CH}_{3}\right), 22.2\left(\mathrm{t}, J=4.7 \mathrm{~Hz}, \mathrm{CH}_{2}\right), 22.8\left(\mathrm{CH}_{2}\right), 24.70\left(\mathrm{CH}_{3}\right), 24.73\left(\mathrm{CH}_{3}\right), 25.7\left(\mathrm{CH}_{2}\right), 28.4\left(\mathrm{CH}_{2}\right)$, $29.0\left(\mathrm{CH}_{2}\right), 31.1\left(\mathrm{CH}_{2}\right), 31.4\left(\mathrm{CH}_{2}\right), 36.6\left(\mathrm{t}, J=25.5 \mathrm{~Hz}, C \mathrm{H}_{2}\right), 38.4$ (t, $\left.J=25.0 \mathrm{~Hz}, \mathrm{CH}_{2}\right), 64.4$ 
$\left(\mathrm{CH}_{2}\right), 83.1(C), 125.4$ (t, $\left.J=241.7 \mathrm{~Hz}, C \mathrm{~F}_{2}\right), 171.2(C) .{ }^{19} \mathrm{~F}$ NMR $\left(373 \mathrm{MHz}, \mathrm{CDCl}_{3}\right): \delta-98.2$ to $-95.4(\mathrm{~m}, 2 \mathrm{~F})$. HRMS-ESI $(\mathrm{m} / \mathrm{z})$ : $[\mathrm{M}+\mathrm{Na}]^{+}$calcd for $\mathrm{C}_{21} \mathrm{H}_{39} \mathrm{O}_{4}{ }^{11} \mathrm{BF}_{2} \mathrm{Na}, 427.2806$; found, 427.2800 .

tert-Butyl\{[2,2-difluoro-4-(4,4,5,5-tetramethyl-1,3,2-dioxaborolan-2 yl)octyl]oxy\}dimethylsilane (4la).

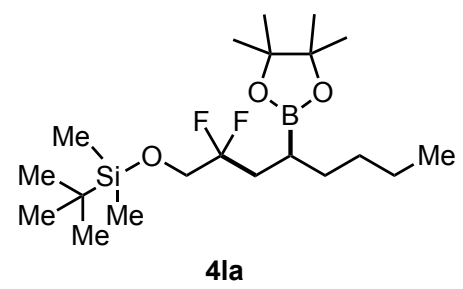

Prepared according to condition A described above, except the catalyst loading $\{10 \mathrm{~mol} \%$ of $\left[\mathrm{Cu}(\mathrm{MeCN})_{4}\right] \mathrm{BF}_{4}$ and $15 \mathrm{~mol} \%$ of IMes $\cdot \mathrm{HCl}$ was used. $\}$. The reaction was conducted for 5 $\mathrm{h}$ with $55.0 \mathrm{mg}(0.20 \mathrm{mmol})$ of $\mathbf{1 l}$ and $100.8 \mu \mathrm{L}(0.80 \mathrm{mmol})$ of $\mathbf{2 a}$. The product $4 \mathbf{l a}$ was obtained in $57 \%$ yield $(46.6 \mathrm{mg})$.

${ }^{1} \mathrm{H}$ NMR (396 MHz, $\left.\mathrm{CDCl}_{3}\right): \delta 0.07$ (s, 6H), 0.81-0.97 (m, 12H), $1.24(\mathrm{~s}, 12 \mathrm{H}), 1.27-1.52$ (m, 8H), 1.84-2.15 (m, 1H), 3.65-3.79 (m, 2H). $\left.{ }^{13} \mathrm{C} \mathrm{NMR} \mathrm{(100} \mathrm{MHz,} \mathrm{CDCl}_{3}\right): \delta-5.52\left(\mathrm{CH}_{3}\right)$, -5.50 $\left(\mathrm{CH}_{3}\right), 14.0\left(\mathrm{CH}_{3}\right), 16.7$ (br, B-CH), $18.2(\mathrm{C}), 22.7\left(\mathrm{CH}_{2}\right), 24.67\left(\mathrm{CH}_{3}\right), 24.72\left(\mathrm{CH}_{3}\right)$, $25.7\left(\mathrm{CH}_{3}\right), 31.1\left(\mathrm{CH}_{2}\right), 31.4\left(\mathrm{CH}_{2}\right), 34.9\left(\mathrm{t}, J=24.2 \mathrm{~Hz}, C \mathrm{H}_{2}\right), 64.5\left(\mathrm{t}, J=34.5 \mathrm{~Hz}, \mathrm{CH}_{2}\right), 83.0$ (C), 123.7 (t, $\left.J=248.4 \mathrm{~Hz}, C \mathrm{~F}_{2}\right) .{ }^{19} \mathrm{~F}$ NMR (373 MHz, $\left.\mathrm{CDCl}_{3}\right): \delta-108.3$ to $-107.4(\mathrm{~m}, 1 \mathrm{~F}),-$ 104.7 to -103.8 (m, 1F). HRMS-ESI $(\mathrm{m} / \mathrm{z})$ : $[\mathrm{M}+\mathrm{Na}]^{+}$calcd for $\mathrm{C}_{20} \mathrm{H}_{41} \mathrm{O}_{3}{ }^{11} \mathrm{BF}_{2} \mathrm{NaSi}, 429.2782$; found, 429.2779 .

2-[1-(Benzyloxy)-2,2-difluorooctan-4-yl]-4,4,5,5-tetramethyl-1,3,2-dioxaborolane (4ma).

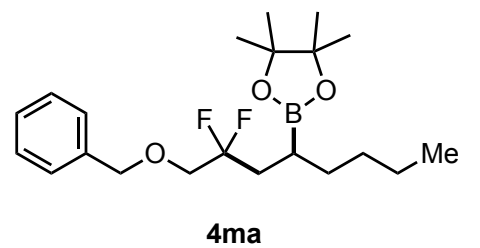

Prepared according to condition A described above, except the catalyst loading \{10 mol\% of $\left[\mathrm{Cu}(\mathrm{MeCN})_{4}\right] \mathrm{BF}_{4}$ and $15 \mathrm{~mol} \%$ of IMes $\bullet \mathrm{HCl}$ was used. $\}$. The reaction was conducted for 2 $\mathrm{h}$ with $51.5 \mathrm{mg}(0.21 \mathrm{mmol})$ of $\mathbf{1 m}$ and $100.8 \mu \mathrm{L}(0.80 \mathrm{mmol})$ of $\mathbf{2 a}$. The product $4 \mathbf{m a}$ was obtained in $28 \%$ yield $(21.8 \mathrm{mg})$.

${ }^{1} \mathrm{H}$ NMR $\left(392 \mathrm{MHz}, \mathrm{CDCl}_{3}\right): \delta 0.88(\mathrm{t}, J=6.9 \mathrm{~Hz}, 3 \mathrm{H}), 1.23(\mathrm{~s}, 12 \mathrm{H}), 1.25-1.52(\mathrm{~m}, 8 \mathrm{H})$, 1.89-2.19 (m, 1H), 3.53-3.72 (m, 2H), 4.55-4.65 (m, 2H), 7.27-7.40 (m, 5H). ${ }^{13} \mathrm{C}$ NMR (100 $\left.\mathrm{MHz}, \mathrm{CDCl}_{3}\right): \delta 14.0\left(\mathrm{CH}_{3}\right), 16.7(\mathrm{br}, \mathrm{B}-\mathrm{CH}), 22.8\left(\mathrm{CH}_{2}\right), 24.69\left(\mathrm{CH}_{3}\right), 24.75\left(\mathrm{CH}_{3}\right), 31.1$ $\left(\mathrm{CH}_{2}\right), 31.4\left(\mathrm{CH}_{2}\right), 35.6\left(\mathrm{t}, J=23.5 \mathrm{~Hz}, \mathrm{CH}_{2}\right), 70.7\left(\mathrm{t}, J=32.1 \mathrm{~Hz}, \mathrm{CH}_{2}\right), 73.7\left(\mathrm{CH}_{2}\right), 83.1(\mathrm{C})$, 
$123.4\left(\mathrm{t}, J=243.9 \mathrm{~Hz}, C_{2}\right), 127.7(\mathrm{CH}), 127.8(\mathrm{CH}), 128.4(\mathrm{CH}), 137.4(C) .{ }^{19} \mathrm{~F}$ NMR $(369$ $\left.\mathrm{MHz}, \mathrm{CDCl}_{3}\right): \delta-105.6$ to $-101.6(\mathrm{~m}, 2 \mathrm{~F})$. HRMS-ESI $(\mathrm{m} / \mathrm{z}):[\mathrm{M}+\mathrm{Na}]^{+}$calcd for $\mathrm{C}_{21} \mathrm{H}_{33} \mathrm{O}_{3}{ }^{11} \mathrm{BF}_{2} \mathrm{Na}, 405.2387$; found, 405.2381 .

\section{2-(7-Fluoropentadecan-5-yl)-4,4,5,5-tetramethyl-1,3,2-dioxaborolane (4ca).}

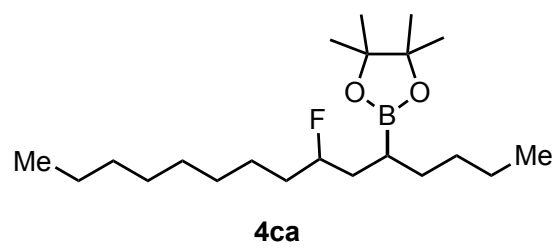

Prepared according to condition $\mathbf{C}$ described above. The reaction was conducted for $2 \mathrm{~h}$ with $113.3 \mathrm{mg}(0.50 \mathrm{mmol})$ of $\mathbf{1 c}$ and $1.25 \mathrm{~mL}(10.0 \mathrm{mmol})$ of $\mathbf{2 a}$. The product $4 \mathbf{c a}$ was obtained in $43 \%$ yield $(76.3 \mathrm{mg})$ with d.r. $=67: 33$, which was determined by ${ }^{19} \mathrm{~F}$ NMR analysis of the crude reaction mixture.

${ }^{1} \mathrm{H}$ NMR $\left(401 \mathrm{MHz}, \mathrm{CDCl}_{3}\right): \delta 0.88(\mathrm{t}, J=6.8 \mathrm{~Hz}, 6 \mathrm{H}), 1.14-1.50(\mathrm{~m}, 20 \mathrm{H}), 1.24(\mathrm{~s}, 12 \mathrm{H})$, $1.52-1.84(\mathrm{~m}, 3 \mathrm{H}), 4.37-4.60(\mathrm{~m}, 1 \mathrm{H}) .{ }^{13} \mathrm{C}$ NMR $\left(99 \mathrm{MHz}, \mathrm{CDCl}_{3}\right.$, *indicates signals of the diastereomers): $\delta 14.0\left(\mathrm{CH}_{3}\right), 14.1\left(\mathrm{CH}_{3}\right), 19.4(\mathrm{br}, \mathrm{B}-\mathrm{CH}),{ }^{*} 20.5(\mathrm{br}, \mathrm{B}-\mathrm{CH}), 22.6\left(\mathrm{CH}_{2}\right), 22.9$ $\left(\mathrm{CH}_{2}\right), * 24.68\left(\mathrm{CH}_{3}\right), 24.73\left(\mathrm{CH}_{3}\right), 24.8\left(\mathrm{CH}_{3}\right), * 25.1\left(\mathrm{~d}, J=5.6 \mathrm{~Hz}, \mathrm{CH}_{2}\right), 25.2(\mathrm{~d}, J=4.8 \mathrm{~Hz}$, $\left.\mathrm{CH}_{2}\right), 29.2\left(\mathrm{CH}_{2}\right), 29.4\left(\mathrm{CH}_{2}\right), 29.5\left(\mathrm{CH}_{2}\right), 31.1\left(\mathrm{CH}_{2}\right), * 31.20\left(\mathrm{CH}_{2}\right), 31.25\left(\mathrm{CH}_{2}\right), * 31.3\left(\mathrm{CH}_{2}\right)$, $31.8\left(\mathrm{CH}_{2}\right), 35.37\left(\mathrm{~d}, J=20.8 \mathrm{~Hz}, C \mathrm{H}_{2}\right), * 35.42\left(\mathrm{~d}, J=20.8 \mathrm{~Hz}, C_{\mathrm{H}}\right), 36.9(\mathrm{~d}, J=20.8 \mathrm{~Hz}$, $\left.C \mathrm{H}_{2}\right), * 37.3\left(\mathrm{~d}, J=20.7 \mathrm{~Hz}, C \mathrm{H}_{2}\right), * 82.7(\mathrm{~d}, J=5.7 \mathrm{~Hz}, C), 82.9(C), 94.1(\mathrm{~d}, J=167.1 \mathrm{~Hz}$, $C \mathrm{H}), * 94.5(\mathrm{~d}, J=168.1 \mathrm{~Hz}, C \mathrm{H}) .{ }^{19} \mathrm{~F}$ NMR $\left(377 \mathrm{MHz}, \mathrm{CDCl}_{3}\right): \delta-180.1$ to $-179.7(\mathrm{~m}, 0.67 \mathrm{~F})$, -177.8 to -177.3 (m, 0.33F). HRMS-ESI $(\mathrm{m} / \mathrm{z})$ : $[\mathrm{M}+\mathrm{Na}]^{+}$calcd for $\mathrm{C}_{21} \mathrm{H}_{42}{ }^{11} \mathrm{BO}_{2} \mathrm{FNa}, 379.3158$; found, 379.3153 .

\section{2-(1-Cyclohexyl-4-fluorododecan-2-yl)-4,4,5,5-tetramethyl-1,3,2-dioxaborolane (4cc).}

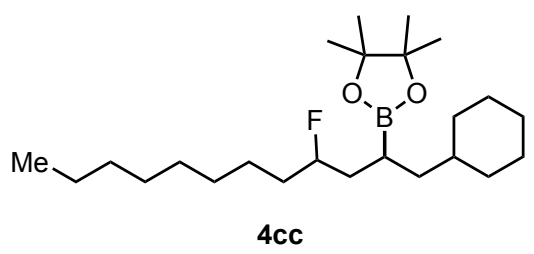

Prepared according to condition $\mathbf{C}$ described above. The reaction was conducted for $2 \mathrm{~h}$ with $113.5 \mathrm{mg}(0.50 \mathrm{mmol})$ of $1 \mathbf{c}$ and $1.54 \mathrm{~mL}(10.0 \mathrm{mmol})$ of $2 \mathbf{c}$. The product $4 \mathbf{c c}$ was obtained in $50 \%$ yield $(100.6 \mathrm{mg})$ with d.r. $=65: 35$, which was determined by ${ }^{19} \mathrm{~F}$ NMR analysis of the crude reaction mixture.

${ }^{1} \mathrm{H}$ NMR $\left(392 \mathrm{MHz}, \mathrm{CDCl}_{3}\right): \delta 0.76-0.93(\mathrm{~m}, 5 \mathrm{H}), 1.06-1.50(\mathrm{~m}, 20 \mathrm{H}), 1.24(\mathrm{~s}, 12 \mathrm{H}), 1.52-$ $1.83(\mathrm{~m}, 8 \mathrm{H}), 4.36-4.58(\mathrm{~m}, 1 \mathrm{H}) .{ }^{13} \mathrm{C}$ NMR $\left(100 \mathrm{MHz}, \mathrm{CDCl}_{3}, *\right.$ indicates signals of the diastereomers): $\delta 14.1\left(\mathrm{CH}_{3}\right), 16.4$ (br, B-CH), *17.7 (br, B-CH), $22.6\left(\mathrm{CH}_{2}\right), 24.6\left(\mathrm{CH}_{3}\right), 24.8$ 
$\left(\mathrm{CH}_{3}\right), * 25.1\left(\mathrm{~d}, J=3.8 \mathrm{~Hz}, \mathrm{CH}_{2}\right), 25.2\left(\mathrm{~d}, J=4.8 \mathrm{~Hz}, \mathrm{CH}_{2}\right), * 26.3\left(\mathrm{CH}_{2}\right), 26.4\left(\mathrm{CH}_{2}\right), 26.6$ $\left(\mathrm{CH}_{2}\right), 29.2\left(\mathrm{CH}_{2}\right), * 29.4\left(\mathrm{CH}_{2}\right), 29.5\left(\mathrm{CH}_{2}\right), 31.8\left(\mathrm{CH}_{2}\right), * 33.0\left(\mathrm{CH}_{2}\right), 33.3\left(\mathrm{CH}_{2}\right), 33.5\left(\mathrm{CH}_{2}\right)$, *33.8 $\left(\mathrm{CH}_{2}\right), 35.3\left(\mathrm{~d}, J=21.0 \mathrm{~Hz}, \mathrm{CH}_{2}\right), * 35.4\left(\mathrm{~d}, J=21.0 \mathrm{~Hz}, \mathrm{CH}_{2}\right), 36.6(\mathrm{CH}), * 36.9(\mathrm{CH})$, $37.0\left(\mathrm{~d}, J=21.0 \mathrm{~Hz}, C \mathrm{H}_{2}\right)$, *37.7 (d, $\left.J=21.0 \mathrm{~Hz}, \mathrm{CH}_{2}\right), 38.9\left(\mathrm{CH}_{2}\right), * 39.3\left(\mathrm{CH}_{2}\right), * 82.83(C)$, $82.85(C), 94.1$ (d, $J=166.2 \mathrm{~Hz}, C H), * 94.6(\mathrm{~d}, J=165.3 \mathrm{~Hz}, C H) .{ }^{19} \mathrm{~F}$ NMR $(377 \mathrm{MHz}$, $\left.\mathrm{CDCl}_{3}\right): \delta-179.8$ to $-179.4(\mathrm{~m}, 0.65 \mathrm{~F}),-177.3$ to $-176.9(\mathrm{~m}, 0.35 \mathrm{~F})$. HRMS-ESI $(\mathrm{m} / \mathrm{z})$ : $[\mathrm{M}+\mathrm{Na}]^{+}$calcd for $\mathrm{C}_{24} \mathrm{H}_{46}{ }^{11} \mathrm{BO}_{2} \mathrm{FNa}, 419.3472$; found, 419.3466 .

$(8 R, 9 S, 13 S, 14 S)-3-(\{7-[($ tert-Butyldimethylsilyl)oxy]-6,6-difluoro-4-(4,4,5,5-tetramethyl1,3,2-dioxaborolan-2-yl)heptyl\}oxy)-13-methyl-6,7,8,9,11,12,13,14,15,16-decahydro-17Hcyclopenta $[a]$ phenanthren-17-one (4lq).

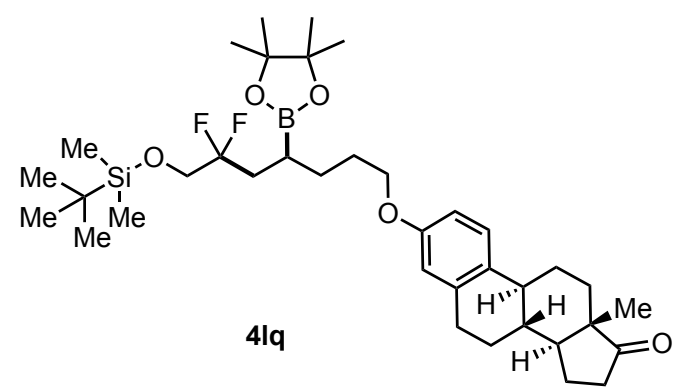

Prepared according to condition A described above, except the catalyst loading $\{10 \mathrm{~mol} \%$ of $\left[\mathrm{Cu}(\mathrm{MeCN})_{4}\right] \mathrm{BF}_{4}$ and $15 \mathrm{~mol} \%$ of IMes $\bullet \mathrm{HCl}$ was used. $\}$ with 1,4-dioxane/DMF $(500 \mu \mathrm{L} / 150$ $\mu \mathrm{L})$. The reaction was conducted for $4 \mathrm{~h}$ with $56.5 \mathrm{mg}(0.21 \mathrm{mmol})$ of 11 and $270.7 \mathrm{mg}(0.80$ mmol) of $\mathbf{2 q}$. The product $4 \mathbf{l q}$ was obtained in $33 \%$ yield $(44.2 \mathrm{mg})$. The d.r. ratio was not determined.

${ }^{1} \mathrm{H}$ NMR (392 MHz, $\mathrm{CDCl}_{3}$ ): $\delta 0.07$ (s, 6H), 0.90 (s, 9H), 0.91 (s, 3H), 1.20-1.35 (m, 3H), $1.23(\mathrm{~s}, 12 \mathrm{H}), 1.37-1.69(\mathrm{~m}, 6 \mathrm{H}), 1.72-1.84(\mathrm{~m}, 2 \mathrm{H}), 1.92-2.20(\mathrm{~m}, 6 \mathrm{H}), 2.21-2.29(\mathrm{~m}, 1 \mathrm{H})$, 2.35-2.44 (m, 1H), 2.50 (dd, $J=5.6,19.0 \mathrm{~Hz}, 1 \mathrm{H}), 2.82-2.92(\mathrm{~m}, 2 \mathrm{H}), 3.65-3.77(\mathrm{~m}, 2 \mathrm{H}), 3.92$ (t, $J=6.5 \mathrm{~Hz}, 2 \mathrm{H}), 6.63(\mathrm{~d}, J=2.7 \mathrm{~Hz}, 1 \mathrm{H}), 6.70(\mathrm{dd}, J=1.7,8.6 \mathrm{~Hz}, 1 \mathrm{H}), 7.18(\mathrm{~d}, J=8.6 \mathrm{~Hz}$, 1H). ${ }^{13} \mathrm{C} \mathrm{NMR}\left(100 \mathrm{MHz}, \mathrm{CDCl}_{3}\right): \delta-5.49\left(\mathrm{CH}_{3}\right), 13.8\left(\mathrm{CH}_{3}\right), 16.5(\mathrm{br}, \mathrm{B}-\mathrm{CH}), 18.2(\mathrm{C}), 21.6$ $\left(\mathrm{CH}_{2}\right), 24.7\left(\mathrm{CH}_{3}\right), 25.7\left(\mathrm{CH}_{3}\right), 25.9\left(\mathrm{CH}_{2}\right), 26.6\left(\mathrm{CH}_{2}\right), 28.1\left(\mathrm{CH}_{2}\right), 28.6\left(\mathrm{CH}_{2}\right), 29.6\left(\mathrm{CH}_{2}\right)$, $31.6\left(\mathrm{CH}_{2}\right), 34.8\left(\mathrm{t}, J=23.5 \mathrm{~Hz}, C \mathrm{H}_{2}\right), 35.8\left(\mathrm{CH}_{2}\right), 38.4(\mathrm{CH}), 44.0(\mathrm{CH}), 48.0(\mathrm{C}), 50.4(\mathrm{CH})$, $64.6\left(\mathrm{t}, J=34.5 \mathrm{~Hz}, C \mathrm{H}_{2}\right), 67.8\left(\mathrm{CH}_{2}\right), 83.2(\mathrm{C}), 112.2(\mathrm{CH}), 114.5(\mathrm{CH}), 123.6(\mathrm{t}, J=243.3$ $\left.\mathrm{Hz}, C \mathrm{~F}_{2}\right), 126.2(\mathrm{CH}), 131.8(C), 137.6(C), 157.0(C), 220.9(C) .{ }^{19} \mathrm{~F} \mathrm{NMR}\left(369 \mathrm{MHz}, \mathrm{CDCl}_{3}\right)$ : $\delta-108.2$ to $-104.2(\mathrm{~m}, 2 \mathrm{~F})$. HRMS-ESI $(\mathrm{m} / \mathrm{z})$ : $[\mathrm{M}+\mathrm{Na}]^{+}$calcd for $\mathrm{C}_{37} \mathrm{H}_{59} \mathrm{O}_{5}{ }^{11} \mathrm{BF}_{2} \mathrm{NaSi}$, 683.4092; found, 683.4083 .

11-Acetoxy-5,5-difluoro-3-(4,4,5,5-tetramethyl-1,3,2-dioxaborolan-2-yl)undecyl 2-[1-(4- 
chlorobenzoyl)-2-methyl-1 $H$-indol-3-yl]acetate (4kr).

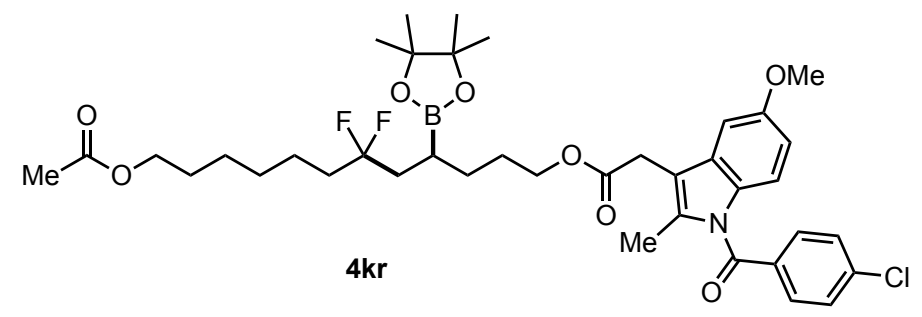

Prepared according to condition A described above, except the catalyst loading $\{10 \mathrm{~mol} \%$ of $\mathrm{Cu}(\mathrm{MeCN})_{4} \mathrm{BF}_{4}$ and $15 \mathrm{~mol} \%$ of IMes $\bullet \mathrm{HCl}$ was used. $\}$ with 1,4-dioxane/DMF $(500 \mu \mathrm{L} / 150$ $\mu \mathrm{L})$. The reaction was conducted for $4 \mathrm{~h}$ with $54.4 \mathrm{mg}(0.20 \mathrm{mmol})$ of $1 \mathbf{k}$ and $344.9 \mathrm{mg}(0.81$ $\mathrm{mmol}$ ) of $\mathbf{2 r}$. The product $\mathbf{4} \mathbf{k r}$ was obtained in $25 \%$ yield $(37.3 \mathrm{mg}$ ) with unidentified small amounts of byproducts.

${ }^{1} \mathrm{H}$ NMR $\left(396 \mathrm{MHz}, \mathrm{CDCl}_{3}\right): \delta 1.21(\mathrm{~s}, 12 \mathrm{H}), 1.31-1.51(\mathrm{~m}, 8 \mathrm{H}), 1.59-1.68(\mathrm{~m}, 5 \mathrm{H}), 1.69-$ $1.86(\mathrm{~m}, 3 \mathrm{H}), 1.88-2.08(\mathrm{~m}, 1 \mathrm{H}), 2.05(\mathrm{~s}, 3 \mathrm{H}), 2.39(\mathrm{~s}, 3 \mathrm{H}), 3.65(\mathrm{~s}, 2 \mathrm{H}), 3.83(\mathrm{~s}, 3 \mathrm{H}), 4.07(\mathrm{dt}$, $J=2.7,10.5 \mathrm{~Hz}, 4 \mathrm{H},), 6.66(\mathrm{dd}, J=2.8,9.1 \mathrm{~Hz}, 1 \mathrm{H}), 6.85(\mathrm{~d}, J=9.1 \mathrm{~Hz}, 1 \mathrm{H}), 6.96(\mathrm{~d}, J=2.4$ $\mathrm{Hz}, 1 \mathrm{H}), 7.48(\mathrm{~d}, J=7.1 \mathrm{~Hz}, 2 \mathrm{H}), 7.67(\mathrm{~d}, J=8.7 \mathrm{~Hz}, 2 \mathrm{H}) .{ }^{13} \mathrm{C} \mathrm{NMR}\left(100 \mathrm{MHz}, \mathrm{CDCl}_{3}\right): \delta$ $13.3\left(\mathrm{CH}_{3}\right), 16.9$ (br, B-CH), $21.0\left(\mathrm{CH}_{3}\right), 22.1\left(\mathrm{t}, J=3.8 \mathrm{~Hz}, \mathrm{CH}_{2}\right), 24.7\left(\mathrm{CH}_{3}\right), 25.7\left(\mathrm{CH}_{2}\right)$, $27.88\left(\mathrm{CH}_{2}\right), 27.91\left(\mathrm{CH}_{2}\right), 28.4\left(\mathrm{CH}_{2}\right), 28.9\left(\mathrm{CH}_{2}\right), 30.3\left(\mathrm{CH}_{2}\right), 36.7\left(\mathrm{t}, J=25.2 \mathrm{~Hz}, \mathrm{CH}_{2}\right), 38.2$ (t, $\left.J=24.8 \mathrm{~Hz}, \mathrm{CH}_{2}\right), 55.6\left(\mathrm{CH}_{3}\right), 64.4\left(\mathrm{CH}_{2}\right), 65.0\left(\mathrm{CH}_{2}\right), 83.2(\mathrm{C}), 101.2(\mathrm{CH}), 111.6(\mathrm{CH})$, $112.7(C), 114.9(C H), 125.2$ (t, $\left.J=240.7 \mathrm{~Hz}, C F_{2}\right), 129.1(C \mathrm{H}), 130.6(C), 130.7(C), 131.1$ (CH), 133.9 (C), 135.9 (C), $139.2(C), 156.0(C), 168.2(C), 170.8(C), 171.2(C) .{ }^{19} \mathrm{~F}$ NMR $\left(373 \mathrm{MHz}, \mathrm{CDCl}_{3}\right): \delta-98.5$ to $-96.1(\mathrm{~m}, 2 \mathrm{~F})$. HRMS-EI $(\mathrm{m} / \mathrm{z}):[\mathrm{M}]^{+}$calcd for $\mathrm{C}_{39} \mathrm{H}_{51}{ }^{11} \mathrm{BClF}_{2} \mathrm{NO}_{8}, 745.3371$; found, 745.3377 . 


\section{2-\{1-[(3r,5r,7r)-Adamantan-1-yl]hexan-2-yl\}-4,4,5,5-tetramethyl-1,3,2-dioxaborolane}

(4ba).

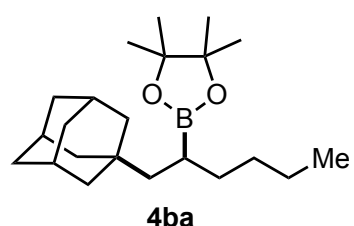

Prepared according to condition $\mathbf{B}$ described above. The reaction was conducted for $2 \mathrm{~h}$ with $43.1 \mathrm{mg}(0.20 \mathrm{mmol})$ of $\mathbf{1 b}$ and $100.5 \mu \mathrm{L}(0.80 \mathrm{mmol})$ of $\mathbf{2 a}$. The product $\mathbf{4 b a}$ was obtained in $71 \%$ yield (49.0 mg).

${ }^{1} \mathrm{H}$ NMR $\left(401 \mathrm{MHz}, \mathrm{CDCl}_{3}\right): \delta 0.87(\mathrm{t}, J=4.8 \mathrm{~Hz}, 3 \mathrm{H}), 0.93-1.04(\mathrm{~m}, 2 \mathrm{H}), 1.19-1.31(\mathrm{~m}$, $5 \mathrm{H}), 1.24(\mathrm{~s}, 6 \mathrm{H}), 1.25(\mathrm{~s}, 6 \mathrm{H}), 1.34-1.53(\mathrm{~m}, 8 \mathrm{H}), 1.62-1.76(\mathrm{~m}, 6 \mathrm{H}), 1.87-1.96(\mathrm{~m}, 3 \mathrm{H}) .{ }^{13} \mathrm{C}$ NMR (99 MHz, CDCl $)$ : $\delta 14.0\left(\mathrm{CH}_{3}\right), 16.9(\mathrm{br}, \mathrm{B}-\mathrm{CH}), 22.9\left(\mathrm{CH}_{2}\right), 24.8\left(\mathrm{CH}_{3}\right), 24.9\left(\mathrm{CH}_{3}\right)$, $28.8(\mathrm{CH}), 31.3\left(\mathrm{CH}_{2}\right), 32.8(\mathrm{C}), 33.3\left(\mathrm{CH}_{2}\right), 37.2\left(\mathrm{CH}_{2}\right) 42.7\left(\mathrm{CH}_{2}\right), 46.6\left(\mathrm{CH}_{2}\right), 82.7(\mathrm{C})$. HRMS-ESI $(\mathrm{m} / \mathrm{z})$ : $[\mathrm{M}+\mathrm{H}]^{+}$calcd for $\mathrm{C}_{22} \mathrm{H}_{40} \mathrm{O}_{2}{ }^{11} \mathrm{~B}, 347.3120$; found, 347.3117 .

\section{2-\{1-[(3r,5r,7r)-Adamantan-1-yl]-3-cyclohexylpropan-2-yl\}-4,4,5,5-tetramethyl-1,3,2-} dioxaborolane (4bc).

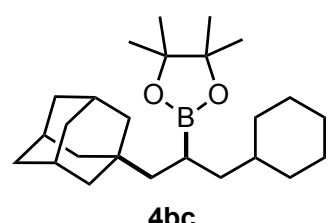

Prepared according to condition $\mathbf{B}$ described above. The reaction was conducted for $2 \mathrm{~h}$ with $43.3 \mathrm{mg}(0.20 \mathrm{mmol})$ of $\mathbf{1 b}$ and $122.2 \mu \mathrm{L}(0.80 \mathrm{mmol})$ of $\mathbf{2 c}$. The product $\mathbf{4 b c}$ was obtained in $54 \%$ yield ( $42.2 \mathrm{mg})$.

${ }^{1} \mathrm{H}$ NMR (401 MHz, $\left.\mathrm{CDCl}_{3}\right): \delta$ 0.75-0.90 (m, 2H), 0.95-1.36 (m, 11H), $1.24(\mathrm{~s}, 6 \mathrm{H}), 1.25$ (s, 6H), 1.37-1.52 (m, 7H), 1.59-1.69 (m, 8H), 1.88-1.96 (m, 3H). ${ }^{13} \mathrm{C} \mathrm{NMR}\left(99 \mathrm{MHz}, \mathrm{CDCl}_{3}\right)$ : $\delta 13.9$ (br, B-CH), $24.8\left(\mathrm{CH}_{3}\right), 26.45\left(\mathrm{CH}_{2}\right), 26.50\left(\mathrm{CH}_{2}\right), 26.7\left(\mathrm{CH}_{2}\right), 28.8(\mathrm{CH}), 32.9(\mathrm{C}), 33.5$ $\left(\mathrm{CH}_{2}\right), 33.7\left(\mathrm{CH}_{2}\right), 36.7(\mathrm{CH}), 37.2\left(\mathrm{CH}_{2}\right), 41.2\left(\mathrm{CH}_{2}\right), 42.7\left(\mathrm{CH}_{2}\right), 46.7\left(\mathrm{CH}_{2}\right), 82.7(\mathrm{C})$. HRMS-EI $(\mathrm{m} / \mathrm{z})$ : $[\mathrm{M}]^{+}$calcd for $\mathrm{C}_{25} \mathrm{H}_{43} \mathrm{O}_{2}{ }^{11} \mathrm{~B}, 386.3361$; found, 386.3350 .

2-\{1-[(3r,5r,7r)-Adamantan-1-yl]-4-phenylbutan-2-yl $\} 4,4,5,5-t e t r a m e t h y l-1,3,2-$ dioxaborolane (4bd). 


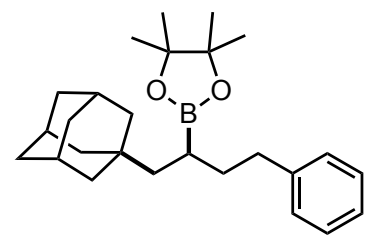

4bd

Prepared according to condition B described above. The reaction was conducted for $4 \mathrm{~h}$ with $43.0 \mathrm{mg}(0.20 \mathrm{mmol})$ of $\mathbf{1} \mathbf{b}$ and $120.2 \mu \mathrm{L}(0.80 \mathrm{mmol})$ of $\mathbf{2 d}$. The product $\mathbf{4 b d}$ was obtained in $71 \%$ yield $(56.1 \mathrm{mg})$.

${ }^{1} \mathrm{H}$ NMR $\left(396 \mathrm{MHz}, \mathrm{CDCl}_{3}\right): \delta 1.00-1.13(\mathrm{~m}, 2 \mathrm{H}), 1.26(\mathrm{~s}, 6 \mathrm{H}), 1.27(\mathrm{~s}, 6 \mathrm{H}), 1.38-1.77(\mathrm{~m}$, 15H), 1.88-1.96 (m, 3H), 2.53-2.68 (m, 2H), 7.12-7.21 (m, 3H), 7.23-7.31 (m, 2H). ${ }^{13} \mathrm{C}$ NMR (100 MHz, $\left.\mathrm{CDCl}_{3}\right): \delta 17.2(\mathrm{br}, \mathrm{B}-\mathrm{CH}), 24.8\left(\mathrm{CH}_{3}\right), 24.9\left(\mathrm{CH}_{3}\right), 28.7(\mathrm{CH}), 32.8(\mathrm{C}), 35.5\left(\mathrm{CH}_{2}\right)$, $35.7\left(\mathrm{CH}_{2}\right), 37.2\left(\mathrm{CH}_{2}\right), 42.7\left(\mathrm{CH}_{2}\right) 46.4\left(\mathrm{CH}_{2}\right), 82.9(\mathrm{C}), 125.5(\mathrm{CH}), 128.2(\mathrm{CH}), 128.3(\mathrm{CH})$, $143.0(C)$. HRMS-EI $(\mathrm{m} / \mathrm{z})$ : $[\mathrm{M}]^{+}$calcd for $\mathrm{C}_{26} \mathrm{H}_{39} \mathrm{O}_{2}{ }^{11} \mathrm{~B}, 394.3048$; found, 394.3036 .

\section{(\{6-[(3r,5r,7r)-Adamantan-1-yl]-5-(4,4,5,5-tetramethyl-1,3,2-dioxaborolan-2-} yl)hexyl\}oxy)(tert-butyl)dimethylsilane (4bh).

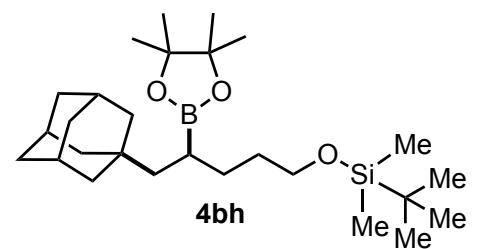

Prepared according to condition $\mathbf{B}$ described above. The reaction was conducted for $4 \mathrm{~h}$ with $43.0 \mathrm{mg}(0.20 \mathrm{mmol})$ of $\mathbf{1 b}$ and $197.5 \mu \mathrm{L}(0.80 \mathrm{mmol})$ of $\mathbf{2 h}$. The product $\mathbf{4 b h}$ was obtained in $67 \%$ yield $(61.3 \mathrm{mg})$.

${ }^{1} \mathrm{H}$ NMR (401 MHz, $\left.\mathrm{CDCl}_{3}\right): \delta 0.04$ (s, 6H), 0.89 (s, 9H), 0.92-1.06 (m, 2H), 1.24 (s, 6H), $1.25(\mathrm{~s}, 6 \mathrm{H}), 1.34-1.76(\mathrm{~m}, 17 \mathrm{H}), 1.86-1.96(\mathrm{~m}, 3 \mathrm{H}), 3.58(\mathrm{t}, J=6.4 \mathrm{~Hz}, 2 \mathrm{H}) .{ }^{13} \mathrm{C}$ NMR $(99$ $\left.\mathrm{MHz}, \mathrm{CDCl}_{3}\right): \delta-5.3(\mathrm{CH}), 16.7(\mathrm{br}, \mathrm{B}-\mathrm{CH}), 18.3(\mathrm{C}), 24.8\left(\mathrm{CH}_{3}\right), 24.9\left(\mathrm{CH}_{3}\right), 26.0\left(\mathrm{CH}_{3}\right)$, $28.7\left(\mathrm{CH}_{3}\right), 29.6\left(\mathrm{CH}_{2}\right), 32.3\left(\mathrm{CH}_{2}\right), 32.8(\mathrm{C}), 37.2\left(\mathrm{CH}_{2}\right), 42.7\left(\mathrm{CH}_{2}\right), 46.5\left(\mathrm{CH}_{2}\right), 63.5\left(\mathrm{CH}_{2}\right)$, $82.8(C)$. HRMS-ESI $(m / z)$ : $[\mathrm{M}+\mathrm{Na}]^{+}$calcd for $\mathrm{C}_{27} \mathrm{H}_{51}{ }^{11} \mathrm{BO}_{3} \mathrm{SiNa}, 485.3598$; found, 485.3596 .

\section{6-[(3r,5r,7r)-Adamantan-1-yl]-5-(4,4,5,5-tetramethyl-1,3,2-dioxaborolan-2-yl)hexyl} acetate (4bi).

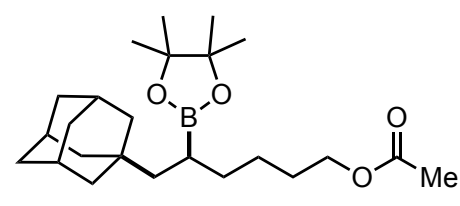

4bi

Prepared according to condition $\mathbf{B}$ described above with 1.2 equiv of $\mathrm{B}_{2}(\mathrm{pin})_{2}$. The reaction 
was conducted for $5 \mathrm{~h}$ with $43.1 \mathrm{mg}(0.20 \mathrm{mmol})$ of $\mathbf{1 b}$ and $113.7 \mu \mathrm{L}(0.80 \mathrm{mmol})$ of $\mathbf{2 i}$. The product $4 \mathrm{bi}$ was obtained in $62 \%$ yield $(50.4 \mathrm{mg})$.

${ }^{1} \mathrm{H}$ NMR (401 MHz, $\left.\mathrm{CDCl}_{3}\right): \delta 0.94-1.03(\mathrm{~m}, 2 \mathrm{H}), 1.24(\mathrm{~s}, 6 \mathrm{H}), 1.25(\mathrm{~s}, 6 \mathrm{H}), 1.34-1.53(\mathrm{~m}$, $11 \mathrm{H}), 1.59-1.72(\mathrm{~m}, 8 \mathrm{H}), 1.88-1.95(\mathrm{~m}, 3 \mathrm{H}), 2.03(\mathrm{~s}, 3 \mathrm{H}), 4.04(\mathrm{t}, J=6.6 \mathrm{~Hz}, 2 \mathrm{H}) .{ }^{13} \mathrm{C} \mathrm{NMR}$ (99 MHz, $\left.\mathrm{CDCl}_{3}\right): \delta 17.0(\mathrm{br}, \mathrm{B}-\mathrm{CH}), 21.0\left(\mathrm{CH}_{2}\right), 24.8\left(\mathrm{CH}_{3}\right), 24.9\left(\mathrm{CH}_{3}\right), 25.3\left(\mathrm{CH}_{2}\right), 28.7$ $(\mathrm{CH}), 28.8\left(\mathrm{CH}_{2}\right), 32.8(\mathrm{C}), 33.2\left(\mathrm{CH}_{2}\right), 37.2\left(\mathrm{CH}_{2}\right) 42.6\left(\mathrm{CH}_{2}\right), 46.5\left(\mathrm{CH}_{2}\right), 64.6\left(\mathrm{CH}_{2}\right), 82.8$ (C), $171.2(C)$. HRMS-ESI $(m / z)$ : $[\mathrm{M}+\mathrm{Na}]^{+}$calcd for $\mathrm{C}_{24} \mathrm{H}_{41}{ }^{11} \mathrm{BO}_{4} \mathrm{Na}, 427.2995$; found, 427.2993.

Ethyl 11-[(3r,5r,7r)-adamantan-1-yl]-10-(4,4,5,5-tetramethyl-1,3,2-dioxaborolan-2yl)undecanoate (4bs).

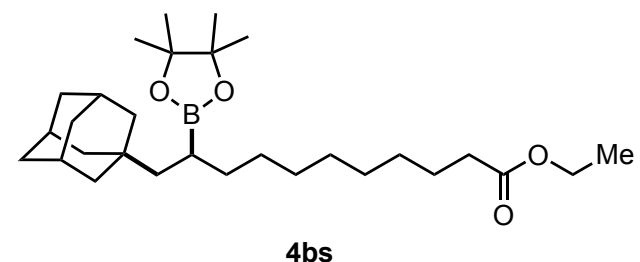

Prepared according to condition B described above. The reaction was conducted for $4 \mathrm{~h}$ with $43.6 \mathrm{mg}(0.20 \mathrm{mmol})$ of $\mathbf{1 b}$ and $193.0 \mu \mathrm{L}(0.80 \mathrm{mmol})$ of $\mathbf{2 s}$. The product $4 \mathbf{b s}$ was obtained in $63 \%$ yield $(60.3 \mathrm{mg})$.

${ }^{1} \mathrm{H}$ NMR (392 MHz, $\left.\mathrm{CDCl}_{3}\right): \delta 0.92-1.03(\mathrm{~m}, 2 \mathrm{H}), 1.22-1.30(\mathrm{~m}, 12 \mathrm{H}), 1.235(\mathrm{~s}, 6 \mathrm{H}), 1.244$ (s, 6H), 1.33-1.53 (m, 9H), 1.58-1.72 (m, 9H), 1.88-1.95 (m, 3H), 2.28 (t, $J=7.6 \mathrm{~Hz}, 2 \mathrm{H})$, 4.12 (q, $J=4.8 \mathrm{~Hz}, 2 \mathrm{H}) .{ }^{13} \mathrm{C}$ NMR $\left(100 \mathrm{MHz}, \mathrm{CDCl}_{3}\right): \delta 14.2\left(\mathrm{CH}_{3}\right), 17.0$ (br, B-CH), 24.8 $\left(\mathrm{CH}_{3}\right), 24.9\left(\mathrm{CH}_{3}\right), 25.0\left(\mathrm{CH}_{2}\right), 28.7(\mathrm{CH}), 29.0\left(\mathrm{CH}_{2}\right), 29.1\left(\mathrm{CH}_{2}\right), 29.2\left(\mathrm{CH}_{2}\right), 29.3\left(\mathrm{CH}_{2}\right)$, $29.8\left(\mathrm{CH}_{2}\right), 32.8(\mathrm{C}), 33.6\left(\mathrm{CH}_{2}\right), 34.4\left(\mathrm{CH}_{2}\right), 37.2\left(\mathrm{CH}_{2}\right), 42.6\left(\mathrm{CH}_{2}\right), 46.6\left(\mathrm{CH}_{2}\right), 60.1\left(\mathrm{CH}_{2}\right)$, $82.7(C), 173.9(C)$. HRMS-EI $(m / z)$ : $[\mathrm{M}]^{+}$calcd for $\mathrm{C}_{29} \mathrm{H}_{51} \mathrm{O}_{4}{ }^{11} \mathrm{~B}, 474.3886$; found, 474.3882 .

\section{2-(2,2-Dimethyloctan-4-yl)-4,4,5,5-tetramethyl-1,3,2-dioxaborolane (4qa).}

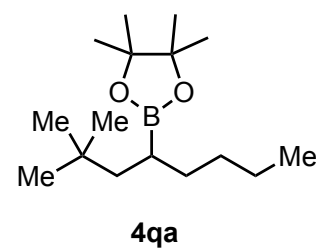

Prepared according to condition A described above, except the catalyst loading $\{10 \mathrm{~mol} \%$ of $\left[\mathrm{Cu}(\mathrm{MeCN})_{4}\right] \mathrm{BF}_{4}$ and $15 \mathrm{~mol} \%$ of IMes $\bullet \mathrm{HCl}$ was used. $\}$. The reaction was conducted for 2 $\mathrm{h}$ with $54.8 \mathrm{mg}(0.40 \mathrm{mmol})$ of $\mathbf{1 q}$ and $201.0 \mu \mathrm{L}(1.60 \mathrm{mmol})$ of $\mathbf{2 a}$. The product $\mathbf{4 q a}$ was purified by GPC and obtained in 32\% yield $(33.9 \mathrm{mg})$.

${ }^{1} \mathrm{H}$ NMR (396 MHz, $\mathrm{CDCl}_{3}$ ): $\delta$ 0.80-0.98 (m, 14H), 1.236 (s, 6H), 1.244 (s, 6H), 1.25-1.44 
(m, 6H), 1.49-1.54 (m, 1H). $\left.{ }^{13} \mathrm{C} \mathrm{NMR} \mathrm{(99} \mathrm{MHz,} \mathrm{CDCl}_{3}\right): \delta 14.0\left(\mathrm{CH}_{3}\right), 19.2(\mathrm{br}, \mathrm{B}-\mathrm{CH}), 23.0$ $\left(\mathrm{CH}_{2}\right), 24.8\left(\mathrm{CH}_{3}\right), 24.9\left(\mathrm{CH}_{3}\right), 29.7\left(\mathrm{CH}_{3}\right), 31.0(\mathrm{C}), 31.3\left(\mathrm{CH}_{2}\right), 33.3\left(\mathrm{CH}_{2}\right), 45.9\left(\mathrm{CH}_{2}\right), 82.7$ (C). HRMS-EI $(\mathrm{m} / \mathrm{z})$ : [M] ${ }^{+}$calcd for $\mathrm{C}_{16} \mathrm{H}_{33} \mathrm{O}_{2}{ }^{10} \mathrm{~B}, 267.2610$; found, 267.2620 .

\section{2-(3,3-Dimethylnonan-5-yl)-4,4,5,5-tetramethyl-1,3,2-dioxaborolane (4ra).}

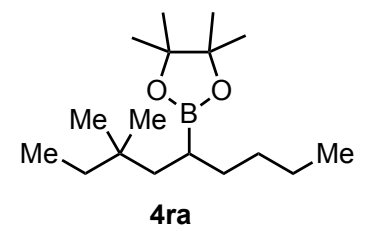

Prepared according to condition A described above, except the catalyst loading $\{10 \mathrm{~mol} \%$ of $\left[\mathrm{Cu}(\mathrm{MeCN})_{4}\right] \mathrm{BF}_{4}$ and $15 \mathrm{~mol} \%$ of IMes $\bullet \mathrm{HCl}$ was used. $\}$. The reaction was conducted for 4 $\mathrm{h}$ with $60.4 \mathrm{mg}(0.40 \mathrm{mmol})$ of $1 \mathbf{r}$ and $201.0 \mu \mathrm{L}(0.80 \mathrm{mmol})$ of $\mathbf{2 a}$. The product $4 \mathbf{r a}$ was purified by GPC and obtained in 35\% yield (39.8 mg).

${ }^{1} \mathrm{H}$ NMR $\left(392 \mathrm{MHz}, \mathrm{CDCl}_{3}\right): \delta 0.76-0.83(\mathrm{~m}, 9 \mathrm{H}), 0.87(\mathrm{t}, J=6.7 \mathrm{~Hz}, 3 \mathrm{H}), 0.90-0.97(\mathrm{~m}$, 1H), 1.10-1.44 (m, 9H), $1.236(\mathrm{~s}, 6 \mathrm{H}), 1.244(\mathrm{~s}, 6 \mathrm{H}), 1.49$ (dd, $J=6.9,13.8 \mathrm{~Hz}, 1 \mathrm{H}) .{ }^{13} \mathrm{C}$ NMR (99 MHz, $\left.\mathrm{CDCl}_{3}\right): \delta 8.4\left(\mathrm{CH}_{3}\right), 14.0\left(\mathrm{CH}_{3}\right), 18.7(\mathrm{br}, \mathrm{B}-\mathrm{CH}), 23.0\left(\mathrm{CH}_{2}\right), 24.8\left(\mathrm{CH}_{3}\right), 24.9$ $\left(\mathrm{CH}_{3}\right), 26.67\left(\mathrm{CH}_{3}\right), 26.72\left(\mathrm{CH}_{3}\right), 31.3\left(\mathrm{CH}_{2}\right), 33.3(\mathrm{C}), 33.4\left(\mathrm{CH}_{2}\right), 34.5\left(\mathrm{CH}_{2}\right), 43.7\left(\mathrm{CH}_{2}\right)$, 82.7 (C). HRMS-ESI $(m / z):[\mathrm{M}+\mathrm{H}]^{+}$calcd for $\mathrm{C}_{17} \mathrm{H}_{36} \mathrm{O}_{2}{ }^{11} \mathrm{~B}, 283.2812$; found, 283.2816

\section{2-(3,3-Dimethyl-1-phenylnonan-5-yl)-4,4,5,5-tetramethyl-1,3,2-dioxaborolane (4sa).}

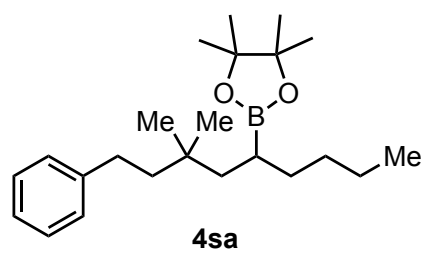

Prepared according to condition A described above, except the catalyst loading $\{10 \mathrm{~mol} \%$ of $\left[\mathrm{Cu}(\mathrm{MeCN})_{4}\right] \mathrm{BF}_{4}$ and $15 \mathrm{~mol} \%$ of IMes $\bullet \mathrm{HCl}$ was used. $\}$. The reaction was conducted for 3 $\mathrm{h}$ with $90.7 \mathrm{mg}(0.40 \mathrm{mmol})$ of $2 \mathrm{~s}$ and $201.0 \mu \mathrm{L}(1.6 \mathrm{mmol})$ of $\mathbf{2 a}$. The product $4 \mathbf{s a}$ was obtained in $26 \%$ yield $(37.1 \mathrm{mg})$.

${ }^{1} \mathrm{H}$ NMR $\left(392 \mathrm{MHz}, \mathrm{CDCl}_{3}\right): \delta 0.88(\mathrm{t}, J=6.9 \mathrm{~Hz}, 3 \mathrm{H}), 0.91(\mathrm{~s}, 3 \mathrm{H}), 0.92(\mathrm{~s}, 3 \mathrm{H}), 0.94$ $1.06(\mathrm{~m}, 2 \mathrm{H}), 1.19(\mathrm{~s}, 6 \mathrm{H}), 1.20(\mathrm{~s}, 6 \mathrm{H}), 1.24-1.34(\mathrm{~m}, 6 \mathrm{H}), 1.37-1.62(\mathrm{~m}, 3 \mathrm{H}), 2.53(\mathrm{t}, J=8.6$ $\mathrm{Hz}, 2 \mathrm{H}), 7.12-7.20(\mathrm{~m}, 3 \mathrm{H}), 7.22-7.28(\mathrm{~m}, 2 \mathrm{H}) .{ }^{13} \mathrm{C} \mathrm{NMR}\left(99 \mathrm{MHz}, \mathrm{CDCl}_{3}\right): \delta 14.0\left(\mathrm{CH}_{3}\right)$, 18.7 (br, B-CH), $23.0\left(\mathrm{CH}_{2}\right), 24.8\left(\mathrm{CH}_{3}\right), 27.0\left(\mathrm{CH}_{3}\right), 27.2\left(\mathrm{CH}_{3}\right), 30.9\left(\mathrm{CH}_{2}\right), 31.3\left(\mathrm{CH}_{2}\right), 33.4$ $\left(\mathrm{CH}_{2}\right), 33.6(\mathrm{C}), 44.2\left(\mathrm{CH}_{2}\right), 44.9\left(\mathrm{CH}_{2}\right), 82.8(\mathrm{C}), 125.4(\mathrm{CH}), 128.2(\mathrm{CH}), 128.4(\mathrm{CH}), 143.7$ (C). HRMS-EI $(m / z)$ : $[\mathrm{M}]^{+}$calcd for $\mathrm{C}_{23} \mathrm{H}_{39} \mathrm{O}_{2}{ }^{11} \mathrm{~B}, 358.3047$; found, 358.3040 .

4,4,5,5-Tetramethyl-2-[1-(1-methylcyclohexyl)hexan-2-yl]-1,3,2-dioxaborolane (4ta). 


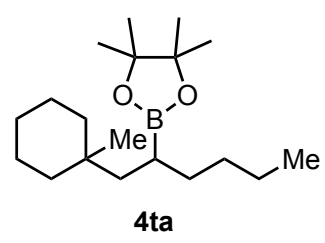

Prepared according to condition A described above, except the catalyst loading \{10 mol\% of $\left[\mathrm{Cu}(\mathrm{MeCN})_{4}\right] \mathrm{BF}_{4}$ and $15 \mathrm{~mol} \%$ of IMes $\bullet \mathrm{HCl}$ was used. $\}$. The reaction was conducted for 4 $\mathrm{h}$ with $70.6 \mathrm{mg}(0.40 \mathrm{mmol})$ of $1 \mathrm{t}$ and $201.0 \mu \mathrm{L}(1.6 \mathrm{mmol})$ of $2 \mathrm{a}$. The product $4 \mathrm{ta}$ was obtained in $24 \%$ yield $(29.7 \mathrm{mg})$.

${ }^{1} \mathrm{H}$ NMR (401 MHz, $\left.\mathrm{CDCl}_{3}\right): \delta 0.83(\mathrm{~s}, 3 \mathrm{H}), 0.87(\mathrm{t}, J=7.0 \mathrm{~Hz}, 3 \mathrm{H}), 0.91-0.98(\mathrm{~m}, 1 \mathrm{H})$, $1.15-1.30(\mathrm{~m}, 10 \mathrm{H}), 1.23(\mathrm{~s}, 6 \mathrm{H}), 1.25(\mathrm{~s}, 6 \mathrm{H}), 1.34-1.47$ (m, 6H), 1.49-1.55 (m, 2H). ${ }^{13} \mathrm{C}$ NMR (99 MHz, $\left.\mathrm{CDCl}_{3}\right): \delta 14.0\left(\mathrm{CH}_{3}\right), 17.9$ (br, B-CH), $22.2\left(\mathrm{CH}_{2}\right), 23.0\left(\mathrm{CH}_{2}\right), 24.8\left(\mathrm{CH}_{3}\right), 24.9$ $\left(\mathrm{CH}_{3}\right), 26.6\left(\mathrm{CH}_{2}\right), 31.3\left(\mathrm{CH}_{2}\right), 33.2(\mathrm{C}), 33.4\left(\mathrm{CH}_{2}\right), 38.0\left(\mathrm{CH}_{2}\right), 44.8\left(\mathrm{CH}_{2}\right), 82.7(\mathrm{C})$. HRMS$\mathrm{EI}(\mathrm{m} / \mathrm{z})$ : [M] $]^{+}$calcd for $\mathrm{C}_{19} \mathrm{H}_{37}{ }^{11} \mathrm{BO}_{2}, 308.2890$; found, 308.2901 .

\section{2-(7-Butyl-7-methylundecan-5-yl)-4,4,5,5-tetramethyl-1,3,2-dioxaborolane (4ua).}

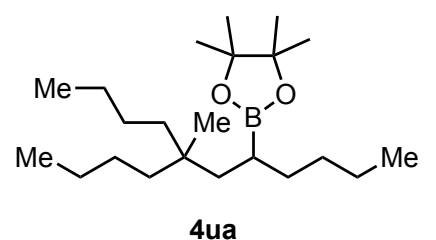

Prepared according to condition A described above, except the catalyst loading \{10 mol\% of $\left[\mathrm{Cu}(\mathrm{MeCN})_{4}\right] \mathrm{BF}_{4}$ and $15 \mathrm{~mol} \%$ of $\mathrm{IMes} \bullet \mathrm{HCl}$ were used.\}. The reaction was conducted for 3 $\mathrm{h}$ with $88.0 \mathrm{mg}(0.40 \mathrm{mmol})$ of $\mathbf{1 u}$ and $201.0 \mu \mathrm{L}(1.6 \mathrm{mmol})$ of $\mathbf{2 a}$. The product $4 \mathbf{u a}$ was obtained in $21 \%$ yield $(29.4 \mathrm{mg})$.

${ }^{1} \mathrm{H}$ NMR $\left(396 \mathrm{MHz}, \mathrm{CDCl}_{3}\right): \delta 0.77$ (s, 3H), 0.84-0.91 (m, 9H), 1.09-1.18 (m, 9H), 1.20$1.41(\mathrm{~m}, 11 \mathrm{H}), 1.24(\mathrm{~s}, 6 \mathrm{H}), 1.25(\mathrm{~s}, 6 \mathrm{H}), 1.46(\mathrm{dd}, J=10.5,13.7 \mathrm{~Hz}, 1 \mathrm{H}) .{ }^{13} \mathrm{C} \mathrm{NMR}(99 \mathrm{MHz}$, $\left.\mathrm{CDCl}_{3}\right)$ : $\delta 14.0\left(\mathrm{CH}_{3}\right), 14.3\left(\mathrm{CH}_{3}\right), 18.0(\mathrm{br}, \mathrm{B}-\mathrm{CH}), 23.0\left(\mathrm{CH}_{2}\right), 23.7\left(\mathrm{CH}_{2}\right), 24.70\left(\mathrm{CH}_{3}\right), 24.73$ $\left(\mathrm{CH}_{3}\right), 24.8\left(\mathrm{CH}_{3}\right), 24.9\left(\mathrm{CH}_{3}\right), 25.1\left(\mathrm{CH}_{3}\right), 25.90\left(\mathrm{CH}_{2}\right), 25.96\left(\mathrm{CH}_{2}\right), 31.3\left(\mathrm{CH}_{2}\right), 33.6\left(\mathrm{CH}_{2}\right)$, $35.5(\mathrm{C}), 39.1\left(\mathrm{CH}_{2}\right), 39.3\left(\mathrm{CH}_{2}\right), 42.1\left(\mathrm{CH}_{2}\right), 82.7(\mathrm{C})$. HRMS-ESI $(\mathrm{m} / \mathrm{z}):[\mathrm{M}+\mathrm{H}]^{+}$calcd for $\mathrm{C}_{22} \mathrm{H}_{46} \mathrm{O}_{2}{ }^{11} \mathrm{~B}, 353.3589$; found, 353.3585 .

5-[(3R,5R)-Adamantan-1-y]]-4-(4,4,5,5-tetramethyl-1,3,2-dioxaborolan-2-yl)pentyl $(1 S, 4 R)-4,7,7$-trimethyl-3-oxo-2-oxabicyclo[2.2.1] heptane-1-carboxylate (4bt). 


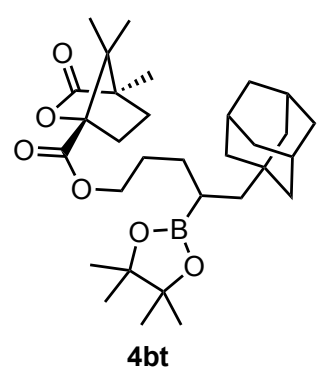

Prepared according to condition B described above. The reaction was conducted for $4 \mathrm{~h}$ with $43.1 \mathrm{mg}(0.20 \mathrm{mmol})$ of $\mathbf{1 b}$ and $213.0 \mathrm{mg}(0.80 \mathrm{mmol})$ of $\mathbf{2 t}$. The product $4 \mathbf{b t}$ was purified by GPC and obtained in $52 \%$ yield $(55.5 \mathrm{mg})$. The d.r. ratio was not determined.

${ }^{1} \mathrm{H}$ NMR (396 MHz, CDCl 3 ): $\delta 0.96$ (s, 3H), 0.98-1.03 (m, 2H), 1.05 (s, 3H), $1.12(\mathrm{~s}, 3 \mathrm{H})$, 1.235 (s, 6H), 1.243 (s, 6H), 1.36-1.52 (m, 8H), 1.56-1.78 (m, 10H), 1.87-1.97 (m, 4H), 2.03 (ddd, $J=4.6,9.4,13.8 \mathrm{~Hz}, 1 \mathrm{H}), 2.42(\mathrm{ddd}, J=3.4,9.9,14.2 \mathrm{~Hz}, 1 \mathrm{H}), 4.15-4.27(\mathrm{~m}, 2 \mathrm{H}) .{ }^{13} \mathrm{C}$ NMR (100 MHz, $\left.\mathrm{CDCl}_{3}\right): \delta 9.7\left(\mathrm{CH}_{3}\right), 16.5(\mathrm{br}, \mathrm{B}-\mathrm{CH}), 16.7\left(\mathrm{CH}_{3}\right), 16.8\left(\mathrm{CH}_{3}\right), 24.76\left(\mathrm{CH}_{3}\right)$, $24.85\left(\mathrm{CH}_{3}\right), 28.0(\mathrm{C}), 28.6(\mathrm{CH}), 28.9\left(\mathrm{CH}_{2}\right), 29.59\left(\mathrm{CH}_{2}\right), 29.63\left(\mathrm{CH}_{2}\right), 30.6\left(\mathrm{CH}_{2}\right), 32.7$ $\left(\mathrm{CH}_{2}\right), 37.1\left(\mathrm{CH}_{2}\right), 42.6\left(\mathrm{CH}_{2}\right), 46.3\left(\mathrm{CH}_{2}\right), 54.0(\mathrm{C}), 54.7(\mathrm{C}), 65.9\left(\mathrm{CH}_{2}\right), 82.9(\mathrm{C}), 91.1(\mathrm{C})$, $167.4(C), 178.1(C)$. HRMS-EI $(m / z)$ : [M] ${ }^{+}$calcd for $\mathrm{C}_{31} \mathrm{H}_{49} \mathrm{O}_{6}{ }^{11} \mathrm{~B}, 528.3628$; found, 528.3612 .

\section{4,4,5,5-Tetramethyl-2-\{1-(tetrahydro-2H-pyran-4-yl)hexan-2-yl\}-1,3,2-dioxaborolane} (4da).

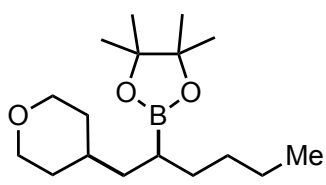

4da

Prepared according to condition $\mathbf{D}$ described above. The reaction was conducted for $4 \mathrm{~h}$ with $83.7 \mathrm{mg}(0.51 \mathrm{mmol})$ of $\mathbf{1 d}$ and $1.25 \mathrm{~mL}$ ( $10.0 \mathrm{mmol})$ of $\mathbf{2 a}$. The product $4 \mathbf{d a}$ was purified by GPC and obtained in $31 \%$ yield ( $46.3 \mathrm{mg})$.

${ }^{1} \mathrm{H}$ NMR $\left(392 \mathrm{MHz}, \mathrm{CDCl}_{3}\right): \delta 0.88(\mathrm{t}, J=7.1 \mathrm{~Hz}, 3 \mathrm{H}), 1.21-1.29(\mathrm{~m}, 8 \mathrm{H}), 1.25(\mathrm{~s}, 12 \mathrm{H})$, $1.31-1.68$ (m, 6H), 3.37 (tdd, $J=2.2,4.8,11.7 \mathrm{~Hz}, 2 \mathrm{H}), 3.90-3.98(\mathrm{~m}, 2 \mathrm{H}) .{ }^{13} \mathrm{C} \mathrm{NMR}(99 \mathrm{MHz}$, $\left.\mathrm{CDCl}_{3}\right): \delta 14.0\left(\mathrm{CH}_{3}\right), 20.4(\mathrm{br}, \mathrm{B}-\mathrm{CH}), 22.9\left(\mathrm{CH}_{2}\right), 24.7\left(\mathrm{CH}_{3}\right), 24.8\left(\mathrm{CH}_{3}\right), 31.3\left(\mathrm{CH}_{2}\right), 31.4$ $\left(\mathrm{CH}_{2}\right), 33.1\left(\mathrm{CH}_{2}\right), 33.5\left(\mathrm{CH}_{2}\right), 34.4(\mathrm{CH}), 38.5\left(\mathrm{CH}_{2}\right), 68.2\left(\mathrm{CH}_{2}\right), 82.8(\mathrm{C})$. HRMS-ESI $(\mathrm{m} / \mathrm{z})$ : $[\mathrm{M}+\mathrm{H}]^{+}$calcd for $\mathrm{C}_{17} \mathrm{H}_{34}{ }^{11} \mathrm{BO}_{3}, 297.2599$; found, 297.2595 .

2-(1,3-Dicyclohexylpropan-2-yl)-4,4,5,5-tetramethyl-1,3,2-dioxaborolane (4ve). 


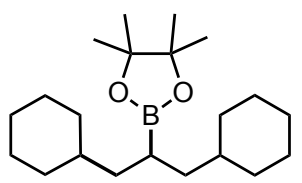

$4 \mathrm{vc}$

Prepared according to condition $\mathbf{D}$ described above. The reaction was conducted for $3 \mathrm{~h}$ with $82.0 \mathrm{mg}(0.50 \mathrm{mmol})$ of $\mathbf{1 v}$ and $1.54 \mathrm{~mL}(10.0 \mathrm{mmol})$ of $\mathbf{2 c}$. The product $4 \mathbf{v e}$ was purified by GPC and obtained in $30 \%$ yield ( $49.8 \mathrm{mg})$.

${ }^{1} \mathrm{H}$ NMR (392 MHz, $\left.\mathrm{CDCl}_{3}\right): \delta$ 0.72-0.95 (m, 4H), 1.04-1.35 (m, 13H), $1.24(\mathrm{~s}, 12 \mathrm{H}), 1.57-$ $1.80(\mathrm{~m}, 10 \mathrm{H}) .{ }^{13} \mathrm{C}$ NMR $\left(99 \mathrm{MHz}, \mathrm{CDCl}_{3}\right): \delta 17.9(\mathrm{br}, \mathrm{B}-\mathrm{CH}), 24.7\left(\mathrm{CH}_{3}\right), 26.4\left(\mathrm{CH}_{2}\right), 26.5$ $\left(\mathrm{CH}_{2}\right), 26.7\left(\mathrm{CH}_{2}\right), 33.2\left(\mathrm{CH}_{2}\right), 33.8\left(\mathrm{CH}_{2}\right), 37.1(\mathrm{CH}), 39.5\left(\mathrm{CH}_{2}\right), 82.7(\mathrm{C})$. HRMS-APCI $(\mathrm{m} / \mathrm{z}):[\mathrm{M}+\mathrm{H}]^{+}$calcd for $\mathrm{C}_{21} \mathrm{H}_{40}{ }^{11} \mathrm{BO}_{2}, 335.3120$; found, 335.3118 .

\section{2-(1-Cyclohexylhexan-2-yl)-4,4,5,5-tetramethyl-1,3,2-dioxaborolane (4va).}

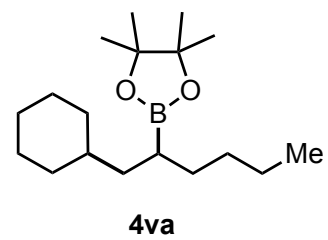

Prepared according to condition $\mathbf{D}$ described above. The reaction was conducted for $3 \mathrm{~h}$ with $81.5 \mathrm{mg}(0.50 \mathrm{mmol})$ of $1 \mathbf{v}$ and $625 \mu \mathrm{L}(5.0 \mathrm{mmol})$ of $\mathbf{2 a}$ in $1,4-$ dioxane $/ \mathrm{DMF}=(500 \mu \mathrm{L} / 125$ $\mu \mathrm{L})$. The product $4 \mathbf{v a}$ was purified by GPC and obtained in $25 \%$ yield $(37.1 \mathrm{mg})$.

${ }^{1} \mathrm{H}$ NMR $\left(392 \mathrm{MHz}, \mathrm{CDCl}_{3}\right): \delta 0.74-0.93(\mathrm{~m}, 5 \mathrm{H}), 1.02-1.42(\mathrm{~m}, 13 \mathrm{H}), 1.24(\mathrm{~s}, 12 \mathrm{H}), 1.58$ $1.78(\mathrm{~m}, 5 \mathrm{H}) .{ }^{13} \mathrm{C}$ NMR $\left(100 \mathrm{MHz}, \mathrm{CDCl}_{3}\right): \delta 14.1\left(\mathrm{CH}_{3}\right), 20.9(\mathrm{br}, \mathrm{B}-\mathrm{CH}), 23.0\left(\mathrm{CH}_{2}\right), 24.7$ $\left(\mathrm{CH}_{3}\right), 24.8\left(\mathrm{CH}_{3}\right), 26.4\left(\mathrm{CH}_{2}\right), 26.5\left(\mathrm{CH}_{2}\right), 26.7\left(\mathrm{CH}_{2}\right), 31.5\left(\mathrm{CH}_{2}\right), 31.6\left(\mathrm{CH}_{2}\right), 33.3\left(\mathrm{CH}_{2}\right)$, $33.8\left(\mathrm{CH}_{2}\right), 37.1(\mathrm{CH}), 39.2\left(\mathrm{CH}_{2}\right), 82.7(\mathrm{C})$. HRMS-APCI $(\mathrm{m} / \mathrm{z})$ : $[\mathrm{M}+\mathrm{H}]^{+}$calcd for $\mathrm{C}_{18} \mathrm{H}_{36}{ }^{11} \mathrm{BO}_{2}, 295.2806$; found, 295.2804.

tert-Butyl 4-\{2-(4,4,5,5-tetramethyl-1,3,2-dioxaborolan-2-yl)hexyl\}piperidine-1carboxylate (4wa).

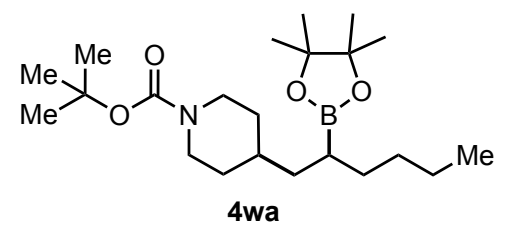

Prepared according to condition $\mathbf{D}$ described above. The reaction was conducted for $3 \mathrm{~h}$ with $132.2 \mathrm{mg}(0.50 \mathrm{mmol})$ of $\mathbf{1} \mathbf{w}$ and $1.25 \mathrm{~mL}(10.0 \mathrm{mmol})$ of $\mathbf{2 a}$. The product $4 \mathbf{w a}$ was purified by GPC and obtained in $25 \%$ yield $(49.1 \mathrm{mg})$. 
${ }^{1} \mathrm{H}$ NMR (392 MHz, $\left.\mathrm{CDCl}_{3}\right): \delta 0.87(\mathrm{t}, J=6.9 \mathrm{~Hz}, 3 \mathrm{H}), 0.98-1.11(\mathrm{~m}, 2 \mathrm{H}), 1.17-1.42(\mathrm{~m}$, $10 \mathrm{H}), 1.24$ (s, 12H), 1.45 (s, 9H), 1.58-1.74 (m, 2H), 2.56-2.71 (m, 2H), 3.93-4.14 (m, 2H). ${ }^{13} \mathrm{C} \mathrm{NMR}\left(99 \mathrm{MHz}, \mathrm{CDCl}_{3}\right): \delta 14.0\left(\mathrm{CH}_{3}\right), 20.7(\mathrm{br}, \mathrm{B}-\mathrm{CH}), 22.9\left(\mathrm{CH}_{2}\right), 24.7\left(\mathrm{CH}_{3}\right), 24.8\left(\mathrm{CH}_{3}\right)$, $28.4\left(\mathrm{CH}_{3}\right), 31.3\left(\mathrm{CH}_{2}\right), 31.4\left(\mathrm{CH}_{2}\right), 32.0\left(\mathrm{CH}_{2}\right), 32.5\left(\mathrm{CH}_{2}\right), 35.4(\mathrm{CH}), 38.1\left(\mathrm{CH}_{2}\right), 44.1\left(\mathrm{CH}_{2}\right)$, 79.0 (C) $82.8(C), 154.9(C)$. HRMS-ESI $(m / z):[\mathrm{M}+\mathrm{Na}]^{+}$calcd for $\mathrm{C}_{22} \mathrm{H}_{42}{ }^{11} \mathrm{BNO}_{4} \mathrm{Na}$, 418.3103; found, 418.3097.

\section{2-[1-\{(1S,2R,4R)-Bicyclo[2.2.1]heptan-2-yl\}hexan-2-yl]-4,4,5,5-tetramethyl-1,3,2-}

dioxaborolane (4xa).

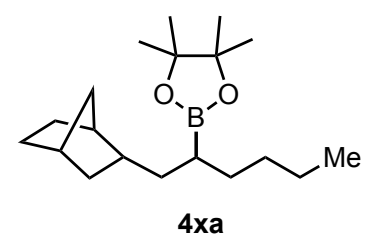

Prepared according to condition $\mathbf{D}$ described above. The reaction was conducted for $3 \mathrm{~h}$ with $87.3 \mathrm{mg}(0.50 \mathrm{mmol})$ of $\mathbf{1} \mathbf{x}$ and $1.25 \mathrm{~mL}(10.0 \mathrm{mmol})$ of $\mathbf{2 a}$. The product $\mathbf{4} \mathbf{x a}$ was purified by GPC and obtained in $31 \%$ yield $(47.8 \mathrm{mg})$. The d.r. ratio was not determined.

${ }^{1} \mathrm{H}$ NMR (392 MHz, $\left.\mathrm{CDCl}_{3}\right): \delta 0.87(\mathrm{t}, J=6.3 \mathrm{~Hz}, 3 \mathrm{H}), 0.93-1.15(\mathrm{~m}, 5 \mathrm{H}), 1.19-1.50(\mathrm{~m}$, $13 \mathrm{H}), 1.25(\mathrm{~s}, 12 \mathrm{H}), 1.89-2.00(\mathrm{~m}, 1 \mathrm{H}), 2.12-2.18(\mathrm{~m}, 1 \mathrm{H}) .{ }^{13} \mathrm{C} \mathrm{NMR}\left(99 \mathrm{MHz}, \mathrm{CDCl}_{3}\right.$ *indicates signals of the diastereomers): $\delta 14.1\left(\mathrm{CH}_{3}\right), 23.0\left(\mathrm{CH}_{2}\right), 24.8\left(\mathrm{CH}_{3}\right), 28.8\left(\mathrm{CH}_{2}\right)$, *28.9 $\left(\mathrm{CH}_{2}\right), 30.1\left(\mathrm{CH}_{2}\right), * 30.2\left(\mathrm{CH}_{2}\right), 31.3\left(\mathrm{CH}_{2}\right), * 31.5\left(\mathrm{CH}_{2}\right), 31.56\left(\mathrm{CH}_{2}\right), * 31.58\left(\mathrm{CH}_{2}\right)$, $35.0\left(\mathrm{CH}_{2}\right), * 35.2\left(\mathrm{CH}_{2}\right), 36.4(\mathrm{CH}), * 36.6(\mathrm{CH}), 38.2\left(\mathrm{CH}_{2}\right), * 38.5\left(\mathrm{CH}_{2}\right), 38.6\left(\mathrm{CH}_{2}\right), * 38.7$ $\left(\mathrm{CH}_{2}\right), 40.5(\mathrm{CH}), 41.6(\mathrm{CH}), * 41.8(\mathrm{CH}), 82.7(\mathrm{C})$. The carbon directly attached to the boron atom was not detected. HRMS-APCI $(\mathrm{m} / \mathrm{z}):[\mathrm{M}+\mathrm{H}]{ }^{+}$calcd for $\mathrm{C}_{19} \mathrm{H}_{36}{ }^{11} \mathrm{BO}_{2}, 307.2806$; found, 307.2804 .

\section{2-[1-\{(1r,3r,5r,7r)-Adamantan-2-yl\}hexan-2-yl]-4,4,5,5-tetramethyl-1,3,2-dioxaborolane} (4ya).

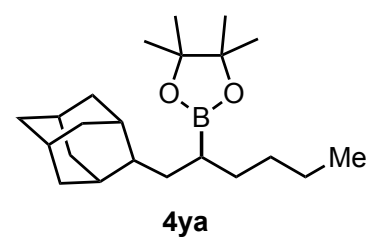

Prepared according to condition $\mathbf{D}$ described above. The reaction was conducted for $5 \mathrm{~h}$ with $107.6 \mathrm{mg}(0.50 \mathrm{mmol})$ of $\mathbf{1 y}$ and $625 \mu \mathrm{L}(5.0 \mathrm{mmol})$ of $\mathbf{2 a}$ in 1,4 -dioxane/DMF $=(1 \mathrm{~mL} / 250$ $\mu \mathrm{L})$. The product 4 ya was purified by GPC and obtained in $26 \%$ yield $(44.5 \mathrm{mg})$.

${ }^{1} \mathrm{H}$ NMR $\left(392 \mathrm{MHz}, \mathrm{CDCl}_{3}\right): \delta 0.84-0.91(\mathrm{~m}, 4 \mathrm{H}), 0.93-1.03(\mathrm{~m}, 2 \mathrm{H}), 1.12-1.50(\mathrm{~m}, 11 \mathrm{H})$, $1.24(\mathrm{~s}, 12 \mathrm{H}), 1.60-1.94(\mathrm{~m}, 10 \mathrm{H}) .{ }^{13} \mathrm{C} \mathrm{NMR}\left(99 \mathrm{MHz}, \mathrm{CDCl}_{3}\right): \delta 14.7\left(\mathrm{CH}_{3}\right), 21.7(\mathrm{br}, \mathrm{B}-\mathrm{CH})$, 
$23.0\left(\mathrm{CH}_{2}\right), 24.7\left(\mathrm{CH}_{3}\right), 24.8\left(\mathrm{CH}_{3}\right), 28.1(\mathrm{CH}), 28.3(\mathrm{CH}), 31.1(\mathrm{CH}), 31.5\left(\mathrm{CH}_{2}\right), 31.6\left(\mathrm{CH}_{2}\right)$, 31.7 $\left(\mathrm{CH}_{2}\right), 31.8\left(\mathrm{CH}_{2}\right), 32.6(\mathrm{CH}), 34.6\left(\mathrm{CH}_{2}\right), 38.4\left(\mathrm{CH}_{2}\right), 39.3\left(\mathrm{CH}_{2}\right), 39.4\left(\mathrm{CH}_{2}\right), 43.9(\mathrm{CH})$, 82.7 (C). HRMS-APCI $(m / z)$ : $[\mathrm{M}+\mathrm{H}]^{+}$calcd for $\mathrm{C}_{22} \mathrm{H}_{40}{ }^{11} \mathrm{BO}_{2}, 347.3120$; found, 347.3118 .

\section{2-(1-Cyclobutylhexan-2-yl)-4,4,5,5-tetramethyl-1,3,2-dioxaborolane (4za).}

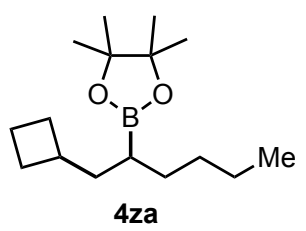

Prepared according to condition $\mathbf{D}$ described above. The reaction was conducted for $7 \mathrm{~h}$ with $67.7 \mathrm{mg}(0.50 \mathrm{mmol})$ of $\mathbf{1 z}$ and $1.25 \mathrm{~mL}$ ( $10.0 \mathrm{mmol})$ of $\mathbf{2 a}$. The product $4 \mathbf{z a}$ was purified by GPC and obtained in $20 \%$ yield $(26.8 \mathrm{mg})$.

${ }^{1} \mathrm{H}$ NMR $\left(392 \mathrm{MHz}, \mathrm{CDCl}_{3}\right): \delta 0.84-0.91(\mathrm{~m}, 3 \mathrm{H}), 1.17-1.44(\mathrm{~m}, 9 \mathrm{H}), 1.24(\mathrm{~s}, 12 \mathrm{H}), 1.47-$ $1.87(\mathrm{~m}, 4 \mathrm{H}), 1.92-2.06(\mathrm{~m}, 2 \mathrm{H}), 2.27(\mathrm{dt}, J=7.7,15.4 \mathrm{~Hz}, 1 \mathrm{H}) .{ }^{13} \mathrm{C} \mathrm{NMR}\left(100 \mathrm{MHz}, \mathrm{CDCl}_{3}\right)$ : $\delta 14.1\left(\mathrm{CH}_{3}\right), 18.4\left(\mathrm{CH}_{2}\right), 23.0\left(\mathrm{CH}_{2}\right), 24.78\left(\mathrm{CH}_{3}\right), 24.83\left(\mathrm{CH}_{3}\right), 28.7\left(\mathrm{CH}_{2}\right), 28.8\left(\mathrm{CH}_{2}\right), 31.2$ $\left(\mathrm{CH}_{2}\right), 31.5\left(\mathrm{CH}_{2}\right), 36.0(\mathrm{CH}), 38.8\left(\mathrm{CH}_{2}\right), 82.7(\mathrm{C})$. The carbon directly attached to the boron atom was not detected. HRMS-APCI $(m / z):[\mathrm{M}+\mathrm{H}]^{+}$calcd for $\mathrm{C}_{16} \mathrm{H}_{32}{ }^{11} \mathrm{BO}_{2}, 267.2493$; found, 267.2490 .

2-(3-Ethylnonan-5-yl)-4,4,5,5-tetramethyl-1,3,2-dioxaborolane (4aaa).

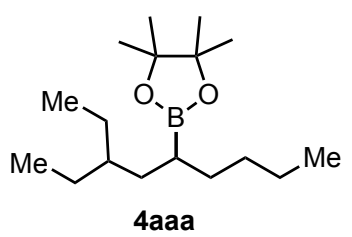

Prepared according to condition $\mathbf{D}$ described above. The reaction was conducted for $4 \mathrm{~h}$ with $76.2 \mathrm{mg}(0.50 \mathrm{mmol})$ of $\mathbf{1 a a}$ and $1.25 \mathrm{~mL}(10.0 \mathrm{mmol})$ of $\mathbf{2 a}$. The product $4 \mathbf{a a a}$ was purified by GPC and obtained in $18 \%$ yield $(26.1 \mathrm{mg})$.

${ }^{1} \mathrm{H}$ NMR (392 MHz, $\left.\mathrm{CDCl}_{3}\right): \delta$ 0.79-0.91 (m, 9H), 0.98-1.09 (m, 1H), 1.11-1.45 (m, 13H), 1.24 (s, $12 \mathrm{H}) .{ }^{13} \mathrm{C}$ NMR $\left(99 \mathrm{MHz}, \mathrm{CDCl}_{3}\right): \delta 10.5\left(\mathrm{CH}_{3}\right), 11.0\left(\mathrm{CH}_{3}\right), 14.1\left(\mathrm{CH}_{3}\right), 21.3(\mathrm{br}, \mathrm{B}-$ $\mathrm{CH}), 23.0\left(\mathrm{CH}_{2}\right), 24.8\left(\mathrm{CH}_{3}\right), 25.1\left(\mathrm{CH}_{2}\right), 25.8\left(\mathrm{CH}_{2}\right), 31.6\left(\mathrm{CH}_{2}\right), 31.7\left(\mathrm{CH}_{2}\right), 35.1\left(\mathrm{CH}_{2}\right)$, $39.9(\mathrm{CH}), 82.7(\mathrm{C})$. HRMS-EI $(\mathrm{m} / \mathrm{z})$ : $[\mathrm{M}+\mathrm{H}]^{+}$calcd for $\mathrm{C}_{17} \mathrm{H}_{35}{ }^{11} \mathrm{BO}_{2}, 282.2733$; found, 282.2743

\section{4,4,5,5-Tetramethyl-2-(7-methyltridecan-5-yl)-1,3,2-dioxaborolane (4aba).}




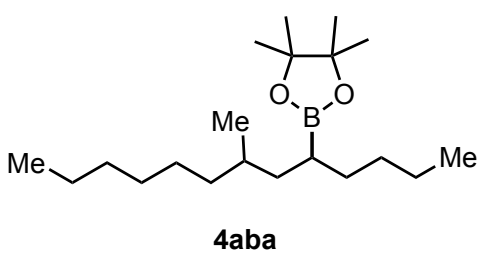

Prepared according to condition $\mathbf{D}$ described above. The reaction was conducted for $3 \mathrm{~h}$ with $94.6 \mathrm{mg}(0.49 \mathrm{mmol})$ of $\mathbf{1} \mathbf{a b}$ and $1.25 \mathrm{~mL}(10.0 \mathrm{mmol})$ of $\mathbf{2 a}$. The product $\mathbf{4 a b a}$ was purified by GPC and obtained in $16 \%$ yield $(26.0 \mathrm{mg})$. The d.r. ratio was not determined.

${ }^{1} \mathrm{H}$ NMR (392 MHz, $\left.\mathrm{CDCl}_{3}\right): \delta 0.80-0.92(\mathrm{~m}, 9 \mathrm{H}), 1.00-1.10(\mathrm{~m}, 2 \mathrm{H}), 1.17-1.35(\mathrm{~m}, 18 \mathrm{H})$, 1.24 (s, 12H). ${ }^{13} \mathrm{C}$ NMR (99 MHz, $\mathrm{CDCl}_{3} *$ indicates signals of the diastereomers): $\delta 14.09$ $\left(\mathrm{CH}_{3}\right), 14.13\left(\mathrm{CH}_{3}\right), 19.8\left(\mathrm{CH}_{3}\right), * 19.9\left(\mathrm{CH}_{3}\right), 21.4(\mathrm{br}, \mathrm{B}-\mathrm{CH}), 22.7\left(\mathrm{CH}_{2}\right), 22.97\left(\mathrm{CH}_{2}\right), * 23.01$ $\left(\mathrm{CH}_{2}\right), 24.75\left(\mathrm{CH}_{3}\right), 24.80\left(\mathrm{CH}_{3}\right), 26.98\left(\mathrm{CH}_{2}\right), * 27.01\left(\mathrm{CH}_{2}\right), 29.6\left(\mathrm{CH}_{2}\right), * 29.7\left(\mathrm{CH}_{2}\right), 31.1$ $\left(\mathrm{CH}_{2}\right), 31.5\left(\mathrm{CH}_{2}\right),{ }^{*} 31.6\left(\mathrm{CH}_{2}\right), 31.86\left(\mathrm{CH}_{2}\right), 31.93(\mathrm{CH}),{ }^{*} 32.0\left(\mathrm{CH}_{2}\right), * 32.4(\mathrm{CH}), 36.8\left(\mathrm{CH}_{2}\right)$, *37.7 $\left(\mathrm{CH}_{2}\right), 38.9\left(\mathrm{CH}_{2}\right), * 39.3\left(\mathrm{CH}_{2}\right), 82.7(\mathrm{C})$. HRMS-APCI $(\mathrm{m} / \mathrm{z}):[\mathrm{M}+\mathrm{H}]^{+}$calcd for $\mathrm{C}_{20} \mathrm{H}_{42}{ }^{11} \mathrm{BO}_{2}, 325.3276$; found, 325.3274 . 
2-[1-(Benzyloxy)-2,2-difluorooctan-4-yl]-4,4,5,5-tetramethyl-1,3,2-dioxaborolane (4aeb).

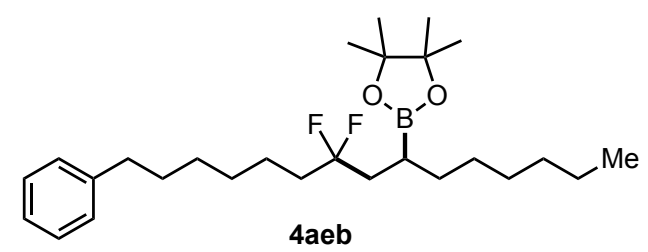

Prepared according to condition A described above, except the catalyst loading $\{10 \mathrm{~mol} \%$ of $\left[\mathrm{Cu}(\mathrm{MeCN})_{4}\right] \mathrm{BF}_{4}$ and $15 \mathrm{~mol} \%$ of $\mathrm{IMes} \cdot \mathrm{HCl}$ were used.\}. The reaction was conducted for 3 $\mathrm{h}$ with $114.7 \mathrm{mg}(0.39 \mathrm{mmol})$ of $\mathbf{1 a e}$ and $251.1 \mu \mathrm{L}(0.80 \mathrm{mmol})$ of $\mathbf{2} \mathbf{b}$. The product $\mathbf{4 a e b}$ was obtained in $74 \%$ yield $(131.9 \mathrm{mg})$.

${ }^{1} \mathrm{H}$ NMR $\left(396 \mathrm{MHz}, \mathrm{CDCl}_{3}\right): \delta 0.87(\mathrm{t}, J=6.7 \mathrm{~Hz}, 3 \mathrm{H}), 1.21-1.38(\mathrm{~m}, 15 \mathrm{H}), 1.23(\mathrm{~s}, 12 \mathrm{H})$, $1.40-1.51(\mathrm{~m}, 3 \mathrm{H}), 1.59-1.67(\mathrm{~m}, 2 \mathrm{H}), 1.71-1.88(\mathrm{~m}, 2 \mathrm{H}), 1.93-2.10(\mathrm{~m}, 1 \mathrm{H}), 2.60(\mathrm{t}, J=7.7$ $\mathrm{Hz}, 2 \mathrm{H}), 7.14-7.21(\mathrm{~m}, 3 \mathrm{H}), 7.24-7.31(\mathrm{~m}, 2 \mathrm{H}) .{ }^{13} \mathrm{C} \mathrm{NMR}\left(100 \mathrm{MHz}, \mathrm{CDCl}_{3}\right): \delta 14.0\left(\mathrm{CH}_{3}\right)$, 17.3 (br, B-CH), 22.3 (t, $\left.J=4.3 \mathrm{~Hz}, \mathrm{CH}_{2}\right), 22.6\left(\mathrm{CH}_{2}\right), 24.70\left(\mathrm{CH}_{3}\right), 24.73\left(\mathrm{CH}_{3}\right), 28.8\left(\mathrm{CH}_{2}\right)$, $29.0\left(\mathrm{CH}_{2}\right), 29.2\left(\mathrm{CH}_{2}\right), 29.4\left(\mathrm{CH}_{2}\right), 29.6\left(\mathrm{CH}_{2}\right), 31.3\left(\mathrm{CH}_{2}\right), 31.7\left(\mathrm{CH}_{2}\right), 31.8\left(\mathrm{CH}_{2}\right), 35.9\left(\mathrm{CH}_{2}\right)$, 36.7 (t, $\left.J=25.4 \mathrm{~Hz}, C \mathrm{H}_{2}\right), 38.4$ (t, $\left.J=24.9 \mathrm{~Hz}, C \mathrm{H}_{2}\right), 83.0(C), 125.5$ (t, $J=241.5 \mathrm{~Hz}, C \mathrm{~F}_{2}$ ), $125.6(\mathrm{CH}), 128.2(\mathrm{CH}), 128.3(\mathrm{CH}), 142.7(\mathrm{C}) .{ }^{19} \mathrm{~F} \mathrm{NMR}\left(369 \mathrm{MHz}, \mathrm{CDCl}_{3}\right): \delta-97.7$ to -95.1 (m, 2F). HRMS-ESI $(\mathrm{m} / \mathrm{z})$ : $[\mathrm{M}+\mathrm{Na}]^{+}$calcd for $\mathrm{C}_{27} \mathrm{H}_{45} \mathrm{O}_{2}{ }^{11} \mathrm{BF}_{2} \mathrm{Na}$, 473.3378; found, 473.3373 .

\section{2-\{[2-(2,2-Difluoro-6-phenylhexyl)cyclopentyl]methyl\}-4,4,5,5-tetramethyl-1,3,2-}

dioxaborolane (7a).

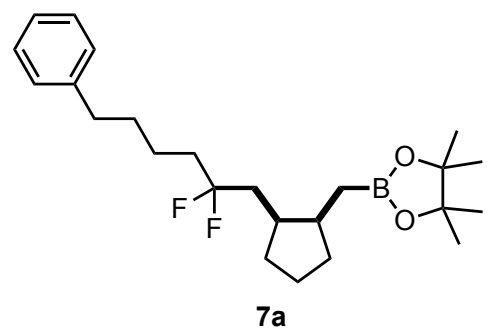

Prepared according to condition A described above, except the catalyst loading $\{10 \mathrm{~mol} \%$ of $\mathrm{Cu}(\mathrm{MeCN})_{4} \mathrm{BF}_{4}$ and $15 \mathrm{~mol} \%$ of IMes $\bullet \mathrm{HCl}$ was used.\}. The reaction was conducted for $2 \mathrm{~h}$ with $53.2 \mathrm{mg}(0.20 \mathrm{mmol})$ of $\mathbf{1 a}$ and $108.4 \mu \mathrm{L}(0.80 \mathrm{mmol})$ of $\mathbf{2} \mathbf{u}$. The product $7 \mathbf{a}$ was obtained in $79 \%$ yield $(64.6 \mathrm{mg})$ with cis:trans $=83: 17$, which was determined by GC-analysis of the crude reaction mixture.

${ }^{1} \mathrm{H}$ NMR (401 MHz, $\left.\mathrm{CDCl}_{3}\right): \delta 0.51-0.81(\mathrm{~m}, 2 \mathrm{H}), 1.17-1.53(\mathrm{~m}, 6 \mathrm{H}), 1.24(\mathrm{~s}, 12 \mathrm{H}), 1.60-$ $2.20(\mathrm{~m}, 10 \mathrm{H}), 2.62(\mathrm{~d}, J=7.6 \mathrm{~Hz}, 2 \mathrm{H}), 7.14-7.21(\mathrm{~m}, 3 \mathrm{H}), 7.23-7.31(\mathrm{~m}, 2 \mathrm{H}) .{ }^{13} \mathrm{C}$ NMR $(100$ $\left.\mathrm{MHz}, \mathrm{CDCl}_{3}\right): \delta 11.5\left(\mathrm{br}, \mathrm{B}-\mathrm{CH}_{2}\right), 22.1\left(\mathrm{t}, J=4.8 \mathrm{~Hz}, \mathrm{CH}_{2}\right), 22.3\left(\mathrm{CH}_{2}\right), 24.7\left(\mathrm{CH}_{3}\right), 24.9$ $\left(\mathrm{CH}_{3}\right), 30.1\left(\mathrm{CH}_{2}\right), 31.2\left(\mathrm{CH}_{2}\right), 32.7\left(\mathrm{CH}_{2}\right), 35.7\left(\mathrm{CH}_{2}\right), 36.7\left(\mathrm{t}, J=24.9 \mathrm{~Hz}, \mathrm{CH}_{2}\right), 36.8(\mathrm{t}, J=$ $25.9 \mathrm{~Hz}, \mathrm{CH}_{2}$ ), $37.7(\mathrm{CH}), 38.7(\mathrm{CH}), 82.9(\mathrm{C}), 125.68$ (t, $\left.J=234.3 \mathrm{~Hz}, \mathrm{CF}_{2}\right), 125.72(\mathrm{CH})$, 
128.28 (CH), $128.32(\mathrm{CH}), 142.2(\mathrm{C}) .{ }^{19} \mathrm{~F} \mathrm{NMR}\left(373 \mathrm{MHz}, \mathrm{CDCl}_{3}\right): \delta-97.6$ to $-95.1(\mathrm{~m}, 2 \mathrm{~F})$. HRMS-ESI $(\mathrm{m} / \mathrm{z})$ : $[\mathrm{M}+\mathrm{Na}]^{+}$calcd for $\mathrm{C}_{24} \mathrm{H}_{37} \mathrm{O}_{2}{ }^{11} \mathrm{BF}_{2} \mathrm{Na}, 429.2751$; found, 429.2746 .

\section{2-\{[2-(2,2-Difluorodecyl)cyclopentyl]methyl\}-4,4,5,5-tetramethyl-1,3,2-dioxaborolane}

(7b).

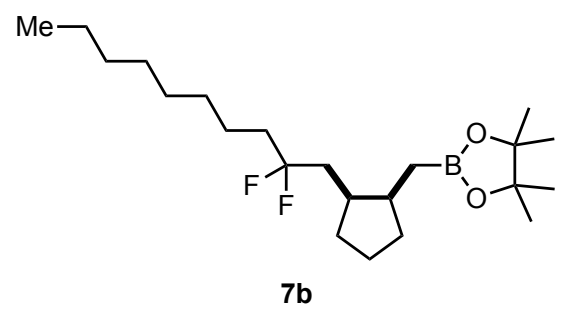

Prepared according to condition A described above, except the catalyst loading $\{10 \mathrm{~mol} \%$ of $\left[\mathrm{Cu}(\mathrm{MeCN})_{4}\right] \mathrm{BF}_{4}$ and $15 \mathrm{~mol} \%$ of IMes $\cdot \mathrm{HCl}$ was used. $\}$. The reaction was conducted for 2 $\mathrm{h}$ with $48.6 \mathrm{mg}(0.20 \mathrm{mmol})$ of $\mathbf{1} \mathbf{f}$ and $108.4 \mu \mathrm{L}(0.80 \mathrm{mmol})$ of $\mathbf{2} \mathbf{u}$. The product $7 \mathbf{b}$ was obtained in $67 \%$ yield $(51.9 \mathrm{mg})$ with cis:trans $=83: 17$, which was determined by GC-analysis of the crude reaction mixture.

${ }^{1} \mathrm{H}$ NMR (396 MHz, $\left.\mathrm{CDCl}_{3}\right): \delta 0.52-0.81(\mathrm{~m}, 2 \mathrm{H}), 0.88(\mathrm{t}, J=7.1 \mathrm{~Hz}, 3 \mathrm{H}), 1.08-1.33(\mathrm{~m}$, $13 \mathrm{H}), 1.24(\mathrm{~s}, 6 \mathrm{H}), 1.25(\mathrm{~s}, 6 \mathrm{H}), 1.35-1.57(\mathrm{~m}, 4 \mathrm{H}), 1.60-2.21(\mathrm{~m}, 7 \mathrm{H}) .{ }^{13} \mathrm{C} \mathrm{NMR}(100 \mathrm{MHz}$, $\left.\mathrm{CDCl}_{3}\right): \delta 11.5\left(\mathrm{br}, \mathrm{B}-\mathrm{CH}_{2}\right), 22.3\left(\mathrm{CH}_{2}\right), 22.4\left(\mathrm{t}, J=4.8 \mathrm{~Hz}, \mathrm{CH}_{2}\right), 22.6\left(\mathrm{CH}_{2}\right), 24.7\left(\mathrm{CH}_{3}\right), 24.9$ $\left(\mathrm{CH}_{3}\right), 29.1\left(\mathrm{CH}_{2}\right), 29.3\left(\mathrm{CH}_{2}\right), 29.4\left(\mathrm{CH}_{2}\right), 30.1\left(\mathrm{CH}_{2}\right), 31.8\left(\mathrm{CH}_{2}\right), 32.7\left(\mathrm{CH}_{2}\right), 36.6(\mathrm{t}, J=$ $\left.24.5 \mathrm{~Hz}, C \mathrm{H}_{2}\right), 37.0$ (t, $\left.J=25.4 \mathrm{~Hz}, C \mathrm{H}_{2}\right), 37.7(\mathrm{CH}), 38.7(\mathrm{CH}), 82.9(\mathrm{C}), 125.9$ (t, $J=241.9$ $\left.\mathrm{Hz}, \mathrm{CF}_{2}\right) .{ }^{19} \mathrm{~F}$ NMR $\left(373 \mathrm{MHz}, \mathrm{CDCl}_{3}\right): \delta-97.4$ to $-94.6(\mathrm{~m}, 2 \mathrm{~F})$. HRMS-ESI $(\mathrm{m} / \mathrm{z}):[\mathrm{M}+\mathrm{Na}]^{+}$ calcd for $\mathrm{C}_{22} \mathrm{H}_{41} \mathrm{O}_{2} \mathrm{BF}_{2} \mathrm{Na}, 409.3064$; found, 409.3060 .

Diethyl 3-(2,2-difluorodecyl)-4-[(4,4,5,5-tetramethyl-1,3,2-dioxaborolan-2yl)methyl]cyclopentane-1,1-dicarboxylate (7c).

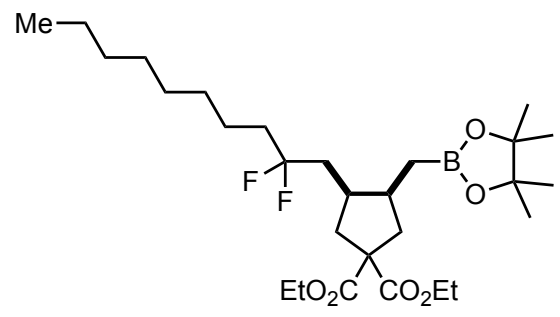

7c

Prepared according to condition A described above, except the catalyst loading $\{10 \mathrm{~mol} \%$ of $\left[\mathrm{Cu}(\mathrm{MeCN})_{4}\right] \mathrm{BF}_{4}$ and $15 \mathrm{~mol} \%$ of IMes $\bullet \mathrm{HCl}$ was used. $\}$. The reaction was conducted for 2 $\mathrm{h}$ with $49.3 \mathrm{mg}(0.20 \mathrm{mmol})$ of $\mathbf{1}$ and $193.4 \mu \mathrm{L}(0.80 \mathrm{mmol})$ of $\mathbf{2 v}$. The product $7 \mathbf{c}$ was obtained in $59 \%$ yield $(63.6 \mathrm{mg})$ with cis:trans $=91: 9$, which was determined by GC-analysis of the crude reaction mixture. 
${ }^{1} \mathrm{H}$ NMR (401 MHz, $\left.\mathrm{CDCl}_{3}\right): \delta 0.60-0.80(\mathrm{~m}, 2 \mathrm{H}), 0.88(\mathrm{t}, J=6.6 \mathrm{~Hz}, 3 \mathrm{H}), 1.20-1.34(\mathrm{~m}$, $15 \mathrm{H}), 1.24(\mathrm{~s}, 12 \mathrm{H}), 1.38-1.50(\mathrm{~m}, 2 \mathrm{H}), 1.61-1.99(\mathrm{~m}, 5 \mathrm{H}), 2.01-2.17(\mathrm{~m}, 2 \mathrm{H}), 2.24-2.36(\mathrm{~m}$, 2H), 2.43 (ddd, $J=6.2,13.6,19.2 \mathrm{~Hz}, 2 \mathrm{H}), 4.17$ (q, $J=4.7 \mathrm{~Hz}, 4 \mathrm{H}) .{ }^{13} \mathrm{C}$ NMR $(100 \mathrm{MHz}$, $\left.\mathrm{CDCl}_{3}\right): \delta 11.5$ (br, B-CH 2$), 13.97\left(\mathrm{CH}_{3}\right), 14.02\left(\mathrm{CH}_{3}\right), 22.3\left(\mathrm{CH}_{2}\right), 22.6\left(\mathrm{CH}_{2}\right), 24.7\left(\mathrm{CH}_{3}\right)$, $24.8\left(\mathrm{CH}_{3}\right), 29.1\left(\mathrm{CH}_{2}\right), 29.30\left(\mathrm{CH}_{2}\right), 29.33\left(\mathrm{CH}_{2}\right), 31.8\left(\mathrm{CH}_{2}\right), 36.0\left(\mathrm{t}, J=24.9 \mathrm{~Hz}, \mathrm{CH}_{2}\right)$, $37.031(\mathrm{CH}), 37.034\left(\mathrm{t}, J=25.9 \mathrm{~Hz}, \mathrm{CH}_{2}\right), 38.4(\mathrm{CH}), 38.6\left(\mathrm{CH}_{2}\right), 41.0\left(\mathrm{CH}_{2}\right), 58.8(\mathrm{C}), 61.3$ $\left(\mathrm{CH}_{2}\right), 83.0(\mathrm{C}), 125.4$ (t, $\left.J=242.4 \mathrm{~Hz}, C \mathrm{~F}_{2}\right), 172.7$ (C), $172.8(C) .{ }^{19} \mathrm{~F}$ NMR (373 MHz, $\left.\mathrm{CDCl}_{3}\right): \delta-98.0$ to $-95.6(\mathrm{~m}, 2 \mathrm{~F})$. HRMS-ESI $(\mathrm{m} / \mathrm{z})$ : $[\mathrm{M}+\mathrm{Na}]^{+}$calcd for $\mathrm{C}_{28} \mathrm{H}_{49} \mathrm{O}_{6}{ }^{11} \mathrm{BF}_{2} \mathrm{Na}$, 553.3488; found, 553.3486 .

2-[(2-\{[(3r,5r,7r)-Adamantan-1-yl]methyl\}cyclopentyl)methyl]-4,4,5,5-tetramethyl-1,3,2dioxaborolane (7d).

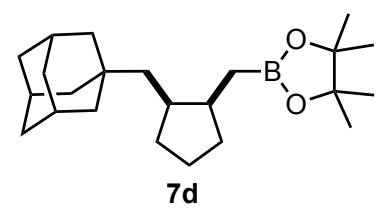

Prepared according to condition $\mathbf{B}$ described above, except the catalyst loading $\{10 \mathrm{~mol} \%$ of $\left[\mathrm{Cu}(\mathrm{MeCN})_{4}\right] \mathrm{BF}_{4}$ and $15 \mathrm{~mol} \%$ of IMes $\bullet \mathrm{HCl}$ was used. $\}$. The reaction was conducted for 3 h with $43.0 \mathrm{mg}(0.20 \mathrm{mmol})$ of $\mathbf{1} \mathbf{b}$ and $108.4 \mu \mathrm{L}(0.80 \mathrm{mmol})$ of $\mathbf{2} \mathbf{u}$. The product $\mathbf{7 d}$ was obtained in $74 \%$ yield $(53.2 \mathrm{mg})$ with cis:trans $=80: 20$, which was determined by GC-analysis of the crude reaction mixture.

${ }^{1} \mathrm{H}$ NMR $\left(399 \mathrm{MHz}, \mathrm{CDCl}_{3}\right): \delta$ 0.47-0.57 (m, 1H), 0.76-0.92 (m, 2H), 1.19-1.34 (m, 4H), $1.24(\mathrm{~s}, 12 \mathrm{H}), 1.37-1.54(\mathrm{~m}, 7 \mathrm{H}), 1.57-1.72(\mathrm{~m}, 8 \mathrm{H}), 1.77-1.87(\mathrm{~m}, 1 \mathrm{H}), 1.88-1.95(\mathrm{~m}, 3 \mathrm{H})$, 1.99-2.13 (m, 1H). ${ }^{13} \mathrm{C}$ NMR (100 MHz, $\left.\mathrm{CDCl}_{3}\right): \delta 11.2(\mathrm{br}, \mathrm{B}-\mathrm{CH}), 22.3\left(\mathrm{CH}_{2}\right), 24.7\left(\mathrm{CH}_{3}\right)$, $24.9\left(\mathrm{CH}_{3}\right), 28.8(\mathrm{CH}), 32.0\left(\mathrm{CH}_{2}\right), 32.8(\mathrm{C}), 32.9\left(\mathrm{CH}_{2}\right), 37.2\left(\mathrm{CH}_{2}\right), 38.0(\mathrm{CH}), 39.9(\mathrm{CH})$, $43.1\left(\mathrm{CH}_{2}\right), 45.3\left(\mathrm{CH}_{2}\right), 82.8(\mathrm{C})$. HRMS-EI $(\mathrm{m} / \mathrm{z})$ : $[\mathrm{M}]^{+}$calcd for $\mathrm{C}_{23} \mathrm{H}_{39} \mathrm{O}_{2}{ }^{11} \mathrm{~B}, 358.3047$; found, 358.3044 .

Diethyl 3-\{[(3r,5r,7r)-adamantan-1-yl]methyl\}-4-[(4,4,5,5-tetramethyl-1,3,2-

dioxaborolan-2-yl)methyl]cyclopentane-1,1-dicarboxylate (7e).

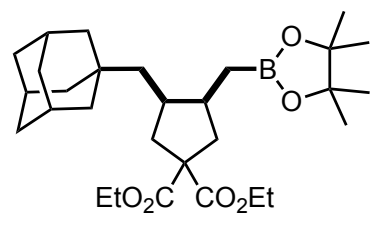

$7 \mathrm{e}$ 
Prepared according to condition B described above, except the catalyst loading \{10 mol\% of $\left[\mathrm{Cu}(\mathrm{MeCN})_{4}\right] \mathrm{BF}_{4}$ and $15 \mathrm{~mol} \%$ of IMes $\cdot \mathrm{HCl}$ was used. $\}$. The reaction was conducted for 3 $\mathrm{h}$ with $43.0 \mathrm{mg}(0.20 \mathrm{mmol})$ of $\mathbf{1 b}$ and $193.4 \mu \mathrm{L}(0.80 \mathrm{mmol})$ of $\mathbf{2 v}$. The product $7 \mathbf{e}$ was obtained in $71 \%$ yield $(71.7 \mathrm{mg})$ with cis:trans $=87: 13$, which was determined by GC-analysis of the crude reaction mixture.

${ }^{1} \mathrm{H}$ NMR (392 MHz, $\left.\mathrm{CDCl}_{3}\right): \delta 0.51-0.65(\mathrm{~m}, 1 \mathrm{H}), 0.76-0.98(\mathrm{~m}, 2 \mathrm{H}), 1.17-1.29(\mathrm{~m}, 8 \mathrm{H})$, $1.24(\mathrm{~s}, 12 \mathrm{H}), 1.39-1.53(\mathrm{~m}, 6 \mathrm{H}), 1.57-1.72(\mathrm{~m}, 6 \mathrm{H}), 1.88-1.95(\mathrm{~m}, 3 \mathrm{H}), 2.02-2.12(\mathrm{~m}, 2 \mathrm{H})$, $2.20(\mathrm{tt}, J=5.5,10.7 \mathrm{~Hz}, 1 \mathrm{H}), 2.35(\mathrm{ddd}, J=6.6,13.3,23.6 \mathrm{~Hz}, 2 \mathrm{H}), 4.17$ (q, $J=4.7 \mathrm{~Hz}, 4 \mathrm{H})$. ${ }^{13} \mathrm{C}$ NMR $\left(100 \mathrm{MHz}, \mathrm{CDCl}_{3}\right): \delta 11.4(\mathrm{br}, \mathrm{B}-\mathrm{CH}), 14.0\left(\mathrm{CH}_{3}\right), 24.7\left(\mathrm{CH}_{3}\right), 24.9\left(\mathrm{CH}_{3}\right), 28.7$ $(\mathrm{CH}), 32.6(\mathrm{C}), 37.1\left(\mathrm{CH}_{2}\right), 37.6(\mathrm{CH}), 39.6(\mathrm{CH}), 40.4\left(\mathrm{CH}_{2}\right), 41.1\left(\mathrm{CH}_{2}\right), 43.0\left(\mathrm{CH}_{2}\right), 44.3$ $\left(\mathrm{CH}_{2}\right), 59.0(\mathrm{C}), 61.1\left(\mathrm{CH}_{2}\right), 82.9(\mathrm{C}), 172.9(\mathrm{C}), 173.3(\mathrm{C})$. HRMS-ESI $(\mathrm{m} / \mathrm{z}):[\mathrm{M}+\mathrm{Na}]^{+}$calcd for $\mathrm{C}_{29} \mathrm{H}_{47} \mathrm{O}_{6}{ }^{11} \mathrm{BNa}, 525.3363$; found, 525.3356.

\section{2-(6,6-Difluorotetradec-3-en-1-yl)-4,4,5,5-tetramethyl-1,3,2-dioxaborolane (8).}

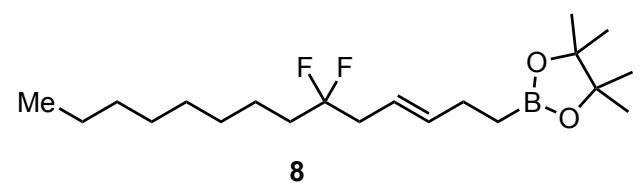

Prepared according to condition A described above, except the catalyst loading $\{10 \mathrm{~mol} \%$ of $\left[\mathrm{Cu}(\mathrm{MeCN})_{4}\right] \mathrm{BF}_{4}$ and $15 \mathrm{~mol} \%$ of IMes $\bullet \mathrm{HCl}$ was used. $\}$ at $30{ }^{\circ} \mathrm{C}$. The reaction was conducted for $3 \mathrm{~h}$ with $49.3 \mathrm{mg}(0.20 \mathrm{mmol})$ of $\mathbf{1 f}$ and $66.8 \mu \mathrm{L}(0.80 \mathrm{mmol})$ of $\mathbf{2} \mathbf{w}$. The product 8 was obtained in $63 \%$ yield $(46.0 \mathrm{mg})$ with $E / Z=78: 22$, which was determined by ${ }^{1} \mathrm{H}$ NMR analysis.

${ }^{1} \mathrm{H}$ NMR (396 MHz, $\mathrm{CDCl}_{3}$, *indicates a signal of the minor isomer): $\delta 0.81-0.94(\mathrm{~m}, 5 \mathrm{H})$, $1.20-1.34(\mathrm{~m}, 10 \mathrm{H}), 1.25$ (s, 12H), 1.38-1.51 (m, 2H), 1.69-1.87 (m, 2H), 2.15 (q, J = 7.3 Hz, $2 \mathrm{H}), 2.50(\mathrm{td}, J=7.3,15.4 \mathrm{~Hz}, 1.56 \mathrm{H}), * 2.61(\mathrm{td}, J=7.1,15.7 \mathrm{~Hz}, 0.44 \mathrm{H}), 5.30(\mathrm{dtt}, J=1.9$, $5.7,13.6 \mathrm{~Hz}, 1 \mathrm{H}), 5.57(\mathrm{dt}, J=7.0,14.4 \mathrm{~Hz}, 1 \mathrm{H}) .{ }^{13} \mathrm{C} \mathrm{NMR}\left(100 \mathrm{MHz}, \mathrm{CDCl}_{3}, *\right.$ indicates signals of the minor isomer): $\delta 10.6\left(\mathrm{br}, \mathrm{B}-\mathrm{CH}_{2}\right), 14.1\left(\mathrm{CH}_{3}\right), * 21.8\left(\mathrm{CH}_{2}\right), 22.0(\mathrm{t}, J=4.4 \mathrm{~Hz}$, $\left.\mathrm{CH}_{2}\right), 22.6\left(\mathrm{CH}_{2}\right), 24.8\left(\mathrm{CH}_{3}\right), 26.8\left(\mathrm{CH}_{2}\right), 29.1\left(\mathrm{CH}_{2}\right), 29.3\left(\mathrm{CH}_{2}\right), 31.8\left(\mathrm{CH}_{2}\right), 35.7(\mathrm{t}, J=25.0$ $\left.\mathrm{Hz}, \mathrm{CH}_{2}\right), 39.9\left(\mathrm{t}, J=26.4 \mathrm{~Hz}, C \mathrm{H}_{2}\right), 83.0(C),{ }^{*} 119.5(\mathrm{t}, J=5.2 \mathrm{~Hz}, C \mathrm{H}), 120.1$ (t, $J=6.2 \mathrm{~Hz}$, $\mathrm{CH}), 124.7\left(\mathrm{t}, J=242.4 \mathrm{~Hz}, C \mathrm{~F}_{2}\right), * 136.2(\mathrm{CH}), 137.8(\mathrm{CH}) .{ }^{19} \mathrm{~F}$ NMR $\left(373 \mathrm{MHz}, \mathrm{CDCl}_{3}\right): \delta$ -97.6 to -97.4 (m, 2F). HRMS-ESI $(\mathrm{m} / \mathrm{z})$ : $[\mathrm{M}+\mathrm{Na}]^{+}$calcd for $\mathrm{C}_{20} \mathrm{H}_{37} \mathrm{O}_{2}{ }^{11} \mathrm{BF}_{2} \mathrm{Na}, 381.2751$; found, 381.2747 . 


\section{Derivatization of Alkyl Boronates}

\section{Experimental Procedure of Homologation of 4aa.}
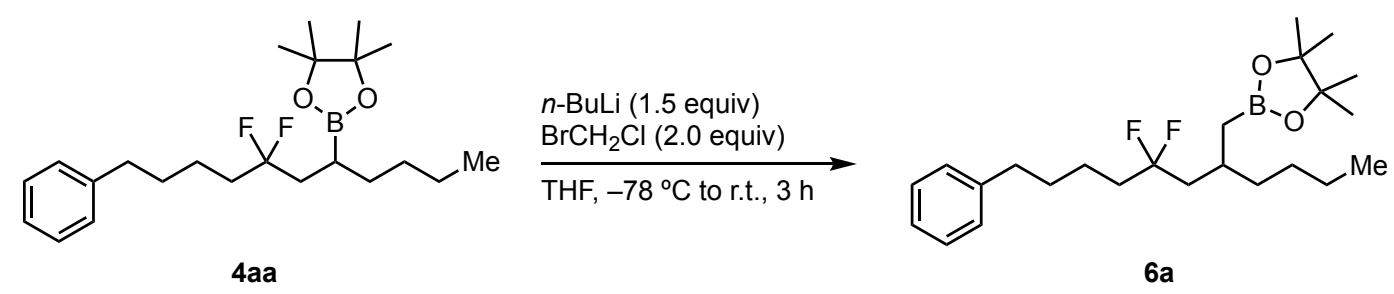

$6 a$

Homologation reaction of $4 \mathbf{a a}$ was performed according to the literature procedure. ${ }^{15}$ In an oven-dried reaction vial, $4 \mathbf{a a}(40.6 \mathrm{mg}, 0.10 \mathrm{mmol})$ and $\mathrm{BrCH}_{2} \mathrm{Cl}(13.4 \mu \mathrm{L}, 0.20 \mathrm{mmol})$ were dissolved in THF $(600 \mu \mathrm{L})$ in a nitrogen atmosphere and the mixture was cooled to $-78{ }^{\circ} \mathrm{C}$. A solution of $n-\mathrm{BuLi}$ in hexane $(1.6 \mathrm{M}, 94 \mu \mathrm{L}, 0.15 \mathrm{mmol})$ was then added dropwise to the reaction mixture, and the mixture was allowed to stir at r.t. for $3 \mathrm{~h}$. The mixture was then quenched by addition of saturated aqueous $\mathrm{NH}_{4} \mathrm{Cl}$ solution and extracted three times with $\mathrm{Et}_{2} \mathrm{O}$. The combined organic layer was washed with brine, dried over $\mathrm{MgSO}_{4}$ followed by filtration. After evaporation, the crude material was purified by silica gel chromatography $\left(\mathrm{Et}_{2} \mathrm{O} /\right.$ hexane, 0:100-8:92) to give the corresponding boronate $\mathbf{6 a}(36.5 \mathrm{mg}, 0.089 \mathrm{mmol}, 87 \%)$ as a colorless oil.

${ }^{1} \mathrm{H}$ NMR (392 MHz, $\left.\mathrm{CDCl}_{3}\right): \delta$ 0.84-0.92 (m, 5H), 1.17-1.32 (m, 7H), $1.23(\mathrm{~s}, 12 \mathrm{H}), 1.47-$ $1.54(\mathrm{~m}, 2 \mathrm{H}), 1.66(\mathrm{dt}, J=7.4,15.2 \mathrm{~Hz}, 2 \mathrm{H}), 1.73-1.95(\mathrm{~m}, 4 \mathrm{H}), 2.63(\mathrm{t}, J=7.6 \mathrm{~Hz}, 2 \mathrm{H}), 7.16-$ $7.21(\mathrm{~m}, 3 \mathrm{H}), 7.24-7.31(\mathrm{~m}, 2 \mathrm{H}) .{ }^{13} \mathrm{C} \mathrm{NMR}\left(100 \mathrm{MHz}, \mathrm{CDCl}_{3}\right): \delta 14.1\left(\mathrm{CH}_{3}\right), 17.4(\mathrm{br}, \mathrm{B}-\mathrm{CH})$, $22.0\left(\mathrm{t}, J=4.8 \mathrm{~Hz}, \mathrm{CH}_{2}\right), 22.9\left(\mathrm{CH}_{2}\right), 24.80\left(\mathrm{CH}_{3}\right), 24.84\left(\mathrm{CH}_{3}\right), 28.8\left(\mathrm{CH}_{2}\right), 29.1(\mathrm{CH}), 31.2$ $\left(\mathrm{CH}_{2}\right), 35.7\left(\mathrm{CH}_{2}\right), 36.72\left(\mathrm{CH}_{2}\right), 36.73\left(\mathrm{t}, J=25.4 \mathrm{~Hz}, \mathrm{CH}_{2}\right), 42.3\left(\mathrm{t}, J=24.0 \mathrm{~Hz}, \mathrm{CH}_{2}\right), 82.9$ (C), $125.7(\mathrm{CH}), 125.8\left(\mathrm{t}, J=245.8 \mathrm{~Hz}, C \mathrm{~F}_{2}\right), 128.29(\mathrm{CH}), 128.34(\mathrm{CH}), 142.2(\mathrm{C}) .{ }^{19} \mathrm{~F}$ NMR $\left(373 \mathrm{MHz}, \mathrm{CDCl}_{3}\right): \delta-95.1(\mathrm{t}, J=17.2 \mathrm{~Hz}, 2 \mathrm{~F})$. HRMS-ESI $(\mathrm{m} / \mathrm{z}):[\mathrm{M}+\mathrm{Na}]^{+}$calcd for $\mathrm{C}_{24} \mathrm{H}_{39} \mathrm{O}_{2}{ }^{11} \mathrm{BF}_{2} \mathrm{Na}, 431.2908$; found, 431.2906 . 


\section{Experimental Procedure of Oxidation of 4aa.}

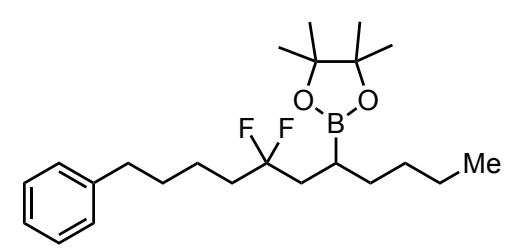

4 aa

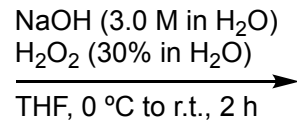

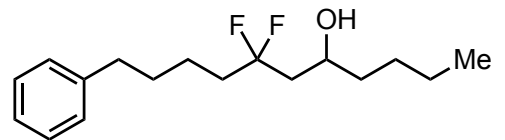

6b

In a reaction vial, 4aa $(40.4 \mathrm{mg}, 0.10 \mathrm{mmol})$ was dissolved in THF $(3 \mathrm{~mL})$, then the reaction mixture was cooled to $0{ }^{\circ} \mathrm{C} .3 .0 \mathrm{M} \mathrm{NaOH}_{\mathrm{aq}}$ and $30 \% \mathrm{H}_{2} \mathrm{O}_{2 \text { aq }}$ were subsequently added to the reaction mixture at $0{ }^{\circ} \mathrm{C}$ under air, and then the reaction mixture was allowed to stir at r.t. for 2 h. The reaction mixture was then quenched by addition of a saturated aqueous $\mathrm{Na}_{2} \mathrm{~S}_{2} \mathrm{O}_{3}$ solution and extracted three times with $\mathrm{Et}_{2} \mathrm{O}$. The combined organic layer was dried over $\mathrm{MgSO}_{4}$ followed by filtration. The crude material was purified by silica gel chromatography (EtOAc/hexane, 0:100-8:92) to give the corresponding alcohol 6b (25.8 $\mathrm{mg}, 0.091 \mathrm{mmol}, 91 \%)$ as a colorless oil.

${ }^{1} \mathrm{H}$ NMR (392 MHz, CDCl$)$ ): $\delta 0.91$ (t, $\left.J=7.3 \mathrm{~Hz}, 3 \mathrm{H}\right), 1.23-1.55(\mathrm{~m}, 8 \mathrm{H}), 1.67$ (dt, $J=7.6$, $15.4 \mathrm{~Hz}, 2 \mathrm{H}), 1.84-2.04(\mathrm{~m}, 4 \mathrm{H}), 2.63(\mathrm{t}, J=7.6 \mathrm{~Hz}, 2 \mathrm{H}), 3.96-4.04(\mathrm{~m}, 1 \mathrm{H}), 7.15-7.22(\mathrm{~m}$, $3 \mathrm{H}), 7.25-7.31(\mathrm{~m}, 2 \mathrm{H}) .{ }^{13} \mathrm{C} \mathrm{NMR}\left(100 \mathrm{MHz}, \mathrm{CDCl}_{3}\right): \delta 14.0\left(\mathrm{CH}_{3}\right), 21.9\left(\mathrm{t}, J=4.8 \mathrm{~Hz}, \mathrm{CH}_{2}\right)$, $22.6\left(\mathrm{CH}_{2}\right), 27.5\left(\mathrm{CH}_{2}\right), 31.1\left(\mathrm{CH}_{2}\right), 35.7\left(\mathrm{CH}_{2}\right), 37.0\left(\mathrm{t}, J=25.4 \mathrm{~Hz}, \mathrm{CH}_{2}\right), 37.3\left(\mathrm{CH}_{2}\right), 43.4(\mathrm{t}$, $\left.J=23.5 \mathrm{~Hz}, C \mathrm{H}_{2}\right), 66.8$ (t, $\left.J=4.3 \mathrm{~Hz}, C \mathrm{H}\right), 125.4$ (t, $\left.J=241.9 \mathrm{~Hz}, C F_{2}\right), 125.8(C \mathrm{H}), 128.32$ $(\mathrm{CH}), 128.34(\mathrm{CH}), 142.1(\mathrm{C}) .{ }^{19} \mathrm{~F} \mathrm{NMR}\left(373 \mathrm{MHz}, \mathrm{CDCl}_{3}\right): \delta-97.6$ to $-95.6(\mathrm{~m}, 2 \mathrm{~F})$. HRMS$\mathrm{EI}(\mathrm{m} / \mathrm{z}):[\mathrm{M}]^{+}$calcd for $\mathrm{C}_{17} \mathrm{H}_{26} \mathrm{OF}_{2}, 284.1952$; found, 284.1958.

\section{Experimental Procedure of Amination of 4aa.}

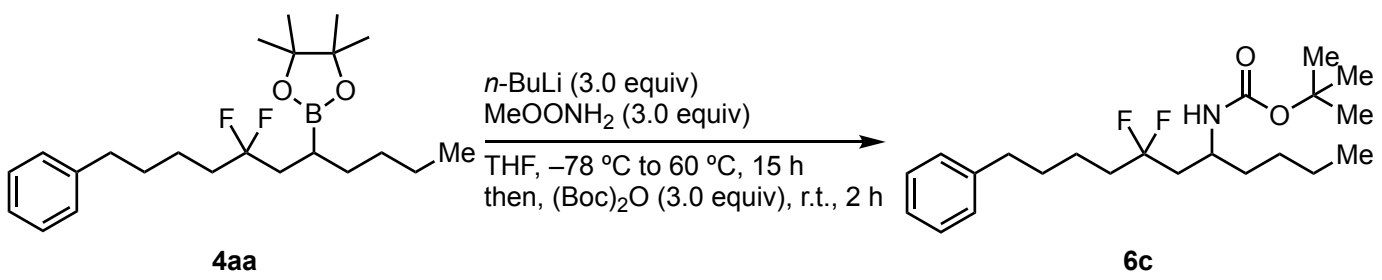

Amination of $4 \mathbf{a a}$ was performed according to the literature procedure. ${ }^{16}$ In an oven-dried reaction vial, $\mathrm{MeONH}_{2}(0.77 \mathrm{M}$ in THF, $392 \mu \mathrm{L}, 0.30 \mathrm{mmol})$ was dissolved in THF $(600 \mu \mathrm{L})$. After the mixture was cooled to $-78{ }^{\circ} \mathrm{C}$, a solution of $n$-BuLi in hexane $(1.6 \mathrm{M}, 188 \mu \mathrm{L}, 0.30$ mmol) was added dropwise under a nitrogen atmosphere. Then $4 \mathbf{a a}(40.0 \mathrm{mg}, 0.10 \mathrm{mmol})$ in THF $(340 \mu \mathrm{L})$ was added dropwise to the solution and stirred at $60{ }^{\circ} \mathrm{C}$. After $15 \mathrm{~h},(\mathrm{Boc})_{2} \mathrm{O}$ $(68.9 \mu \mathrm{L}, 0.30 \mathrm{mmol})$ was added to the mixture and stirred for $2 \mathrm{~h}$ at r.t. The mixture was then quenched by addition of $\mathrm{H}_{2} \mathrm{O}$ and extracted three times with EtOAc. The combined organic layer was dried over $\mathrm{MgSO}_{4}$ followed by filtration and evaporation. After purification by flash 
silica gel column chromatography $\left(\mathrm{Et}_{2} \mathrm{O} /\right.$ hexane, $\left.3: 97-12: 88\right), \mathbf{6}$ was further purified by GPC to give a pure product $(20.5 \mathrm{mg}, 0.053 \mathrm{mmol}, 53 \%$ ) as a white solid.

${ }^{1} \mathrm{H}$ NMR (392 MHz, $\mathrm{CDCl}_{3}$ ): $\delta 0.89$ (t, $\left.J=6.9 \mathrm{~Hz}, 3 \mathrm{H}\right), 1.22-1.37(\mathrm{~m}, 4 \mathrm{H}), 1.43(\mathrm{~s}, 9 \mathrm{H})$, $1.46-1.55$ (m, 4H), 1.66 (dt, $J=7.7,15.6 \mathrm{~Hz}, 2 \mathrm{H}), 1.81-2.04$ (m, 4H), $2.62(\mathrm{t}, J=7.6 \mathrm{~Hz}, 2 \mathrm{H})$, $3.70-3.88(\mathrm{~m}, 1 \mathrm{H}), 4.33-4.50(\mathrm{~m}, 1 \mathrm{H}), 7.14-7.21(\mathrm{~m}, 3 \mathrm{H}), 7.24-7.31(\mathrm{~m}, 2 \mathrm{H}) .{ }^{13} \mathrm{C}$ NMR $(100$ $\left.\mathrm{MHz}, \mathrm{CDCl}_{3}\right): \delta 14.0\left(\mathrm{CH}_{3}\right), 21.9\left(\mathrm{t}, J=4.3 \mathrm{~Hz}, \mathrm{CH}_{2}\right), 22.4\left(\mathrm{CH}_{2}\right), 27.9\left(\mathrm{CH}_{2}\right), 28.4\left(\mathrm{CH}_{3}\right)$, $31.1\left(\mathrm{CH}_{2}\right), 35.6\left(\mathrm{CH}_{2}\right), 35.7\left(\mathrm{CH}_{2}\right), 36.3\left(\mathrm{t}, J=25.4 \mathrm{~Hz}, \mathrm{CH}_{2}\right), 41.2\left(\mathrm{t}, J=23.0 \mathrm{~Hz}, \mathrm{CH}_{2}\right), 46.4$ (CH), $79.2(C), 124.8$ (t, $\left.J=241.5 \mathrm{~Hz}, \mathrm{CF}_{2}\right), 125.7(\mathrm{CH}), 128.3(\mathrm{CH}), 128.4(\mathrm{CH}), 142.1(\mathrm{C})$, $155.3(C) .{ }^{19} \mathrm{~F}$ NMR $\left(373 \mathrm{MHz}, \mathrm{CDCl}_{3}\right): \delta-95.2$ to $-94.9(\mathrm{~m}, 2 \mathrm{~F})$. HRMS-ESI $(\mathrm{m} / \mathrm{z}):[\mathrm{M}+\mathrm{Na}]^{+}$ calcd for $\mathrm{C}_{22} \mathrm{H}_{35} \mathrm{O}_{2} \mathrm{NF}_{2} \mathrm{Na}, 406.2528$; found, 406.2527 .

\section{Experimental Procedure of Fluorination of 4aa.}
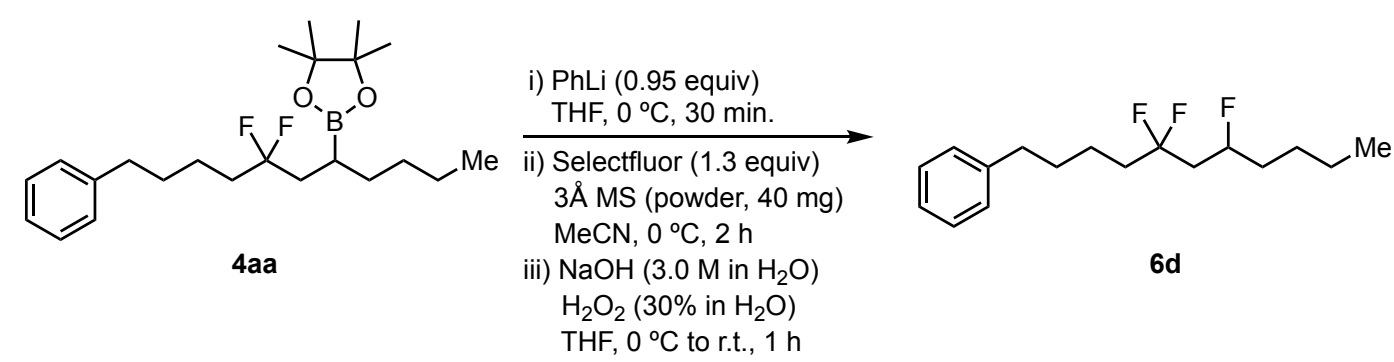

Fluorination of $\mathbf{4 a a}$ was performed according to the literature procedure. ${ }^{17}$ In an oven-dried reaction vial, 4aa (39.5 mg, $0.10 \mathrm{mmol})$ was dissolved in THF $(1 \mathrm{~mL})$. After the mixture was cooled to $0{ }^{\circ} \mathrm{C}$, a solution of PhLi in butyl ether $(1.6 \mathrm{M}, 59.4 \mu \mathrm{L}, 0.095 \mathrm{mmol})$ was added dropwise, and the reaction mixture was stirred at $0{ }^{\circ} \mathrm{C}$ for $30 \mathrm{~min}$. After that, $\mathrm{MeCN}(200 \mu \mathrm{L})$ was added to the reaction mixture, and the volatiles were removed in vacuo upon warming to r.t. A nitrogen atmosphere was re-established, and $\mathrm{MeCN}(1 \mathrm{~mL})$ was added. In a separate ovendried reaction vial, Selectfluor $(46.1 \mathrm{mg}, 0.13 \mathrm{mmol}$ ) and $3 \AA \mathrm{MS}$ (powder, $40 \mathrm{mg}$ ) were dissolved in $\mathrm{MeCN}(1 \mathrm{~mL})$ at $0{ }^{\circ} \mathrm{C}$ under a nitrogen atmosphere. The pre-prepared solution of 4aa with $\mathrm{PhLi}$ in $\mathrm{MeCN}$ was added dropwise to the separate reaction mixture of Selectfluor and $3 \AA \mathrm{MS}$ in $\mathrm{MeCN}$, and the mixture was stirred at r.t. for $2 \mathrm{~h}$. The mixture was then passed through a short plug of silica gel ( $\Phi: 10 \mathrm{~mm}$, height of the silica-gel column: $30 \mathrm{~mm})$, eluted with $\mathrm{Et}_{2} \mathrm{O}$ and volatiles were removed in vacuo. The crude material was purified by silica gel chromatography (hexane only), before the excess boronic ester and $\mathrm{PhB}$ (pin) were oxidized by the addition of $3 \mathrm{M} \mathrm{NaOH}_{\mathrm{aq}}(500 \mu \mathrm{L})$ and $30 \% \mathrm{H}_{2} \mathrm{O}_{2 \mathrm{aq}}(500 \mu \mathrm{L})$ in THF $(3 \mathrm{~mL})$. After $1 \mathrm{~h}$, the mixture was extracted three times with $\mathrm{Et}_{2} \mathrm{O}$, dried over $\mathrm{MgSO}_{4}$. The resultant mixture was purified again by silica gel chromatography $\left(\mathrm{Et}_{2} \mathrm{O} /\right.$ hexane, $\left.0: 100-2: 98\right)$ to give $\mathbf{6 d}(17.7 \mathrm{mg}$, $0.062 \mathrm{mmol}, 62 \%$ ) as colorless oil.

${ }^{1} \mathrm{H}$ NMR (392 MHz, $\left.\mathrm{CDCl}_{3}\right): \delta 0.92(\mathrm{t}, J=7.1 \mathrm{~Hz}, 3 \mathrm{H}), 1.20-1.47$ (m, 4H), 1.49-1.59 (m, 
2H), 1.61-1.75 (m, 4H), 1.84-2.32 (m, 4H), $2.63(\mathrm{t}, J=7.6 \mathrm{~Hz}, 2 \mathrm{H}), 4.75(\mathrm{dtt}, J=3.9,8.0,49.5$ $\mathrm{Hz}, 1 \mathrm{H}), 7.14-7.22(\mathrm{~m}, 3 \mathrm{H}), 7.24-7.31(\mathrm{~m}, 2 \mathrm{H}) .{ }^{13} \mathrm{C} \mathrm{NMR}\left(100 \mathrm{MHz}, \mathrm{CDCl}_{3}\right): \delta 13.9\left(\mathrm{CH}_{3}\right)$, 21.9 (t, $\left.J=4.3 \mathrm{~Hz}, C_{2}\right), 22.4\left(C_{2}\right), 26.9\left(\mathrm{~d}, J=4.8 \mathrm{~Hz}, C \mathrm{H}_{2}\right), 31.1\left(\mathrm{CH}_{2}\right), 35.3(\mathrm{~d}, J=21.1$ $\left.\mathrm{Hz}, \mathrm{CH}_{2}\right), 35.7\left(\mathrm{CH}_{2}\right), 36.6\left(\mathrm{dt}, J=1.1,24.9 \mathrm{~Hz}, \mathrm{CH}_{2}\right), 41.8\left(\mathrm{dt}, J=13.0,25.6 \mathrm{~Hz}, \mathrm{CH}_{2}\right), 89.1$ (d, $J=166.7 \mathrm{~Hz}, C \mathrm{H}-\mathrm{F}), 124.0\left(\mathrm{t}, J=242.4 \mathrm{~Hz}, \mathrm{CF}_{2}\right), 125.8(\mathrm{CH}), 128.3(\mathrm{CH}), 128.4(\mathrm{CH})$, 142.2 (C). ${ }^{19} \mathrm{~F}$ NMR (369 MHz, $\left.\mathrm{CDCl}_{3}\right): \delta-180.4$ to $-179.9(\mathrm{~m}, 1 \mathrm{~F}),-98.2$ to $-97.3(\mathrm{~m}, 1 \mathrm{~F})$, 95.0 to $-94.0(\mathrm{~m}, 1 \mathrm{~F})$. HRMS-EI $(\mathrm{m} / \mathrm{z})$ : $[\mathrm{M}]^{+}$calcd for $\mathrm{C}_{17} \mathrm{H}_{25} \mathrm{~F}_{3}, 286.1908$; found, 286.1911.

\section{Experimental Procedure of Protonation of 4aa.}

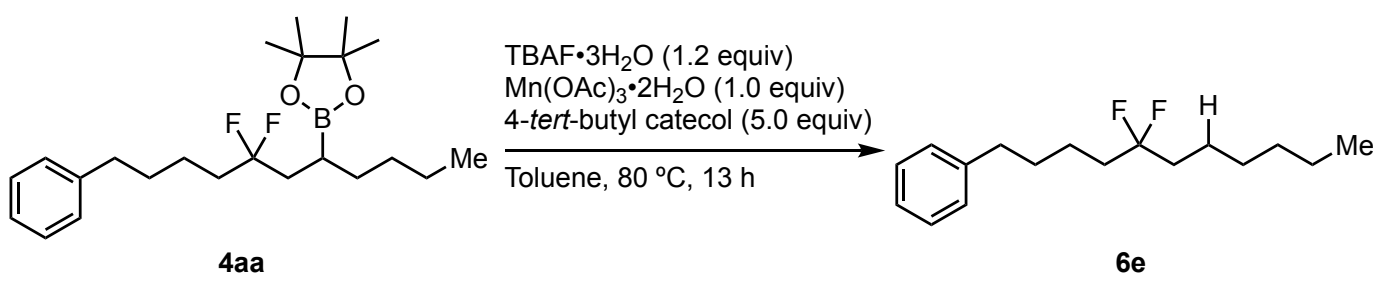

Protonation of 4aa was performed according to the literature procedure. ${ }^{18}$ In a reaction vial, $\mathrm{TBAF} \cdot 3 \mathrm{H}_{2} \mathrm{O}(37.9 \mathrm{mg}, 0.12 \mathrm{mmol}), \mathrm{Mn}(\mathrm{OAc})_{3} \cdot 2 \mathrm{H}_{2} \mathrm{O}(26.8 \mathrm{mg}, 0.1 \mathrm{mmol})$, and tert-butyl catechol (97.9 mg, $0.59 \mathrm{mmol})$ were dissolved in toluene $(600 \mu \mathrm{L})$. Then $4 \mathbf{a a}(39.7 \mathrm{mg}, 0.10$ $\mathrm{mmol})$ in toluene $(700 \mu \mathrm{L})$ was added dropwise to the mixture under a nitrogen atmosphere and stirred at $80^{\circ} \mathrm{C}$ for $13 \mathrm{~h}$. The mixture was then passed through a short plug of silica gel ( $\Phi: 10$ $\mathrm{mm}$, the height of the silica-gel column: $30 \mathrm{~mm}$ ), eluted with $\mathrm{Et}_{2} \mathrm{O}$, and volatiles were removed in vacuo. The crude material was purified by silica gel chromatography $\left(\mathrm{Et}_{2} \mathrm{O} /\right.$ hexane, 0:1002:98) to give 6 e ( $15.0 \mathrm{mg}, 0.056 \mathrm{mmol}, 56 \%)$ as a colorless oil.

${ }^{1} \mathrm{H}$ NMR (392 MHz, $\left.\mathrm{CDCl}_{3}\right): \delta 0.89(\mathrm{t}, J=6.7 \mathrm{~Hz}, 3 \mathrm{H}), 1.21-1.36(\mathrm{~m}, 6 \mathrm{H}), 1.38-1.477(\mathrm{~m}$, 2H), 1.480-1.54 (m, 2H), 1.61-1.90 (m, 6H), 2.63 (t, $J=8.0 \mathrm{~Hz}, 2 \mathrm{H}), 7.15-7.21$ (m, 3H), 7.24$7.31(\mathrm{~m}, 2 \mathrm{H}) .{ }^{13} \mathrm{C} \mathrm{NMR}\left(100 \mathrm{MHz}, \mathrm{CDCl}_{3}\right): \delta 14.0\left(\mathrm{CH}_{3}\right), 22.0\left(\mathrm{t}, J=4.8 \mathrm{~Hz}, \mathrm{CH}_{2}\right), 22.3(\mathrm{t}, J$ $\left.=4.8 \mathrm{~Hz}, \mathrm{CH}_{2}\right), 22.5\left(\mathrm{CH}_{2}\right), 29.1\left(\mathrm{CH}_{2}\right), 31.2\left(\mathrm{CH}_{2}\right), 31.6\left(\mathrm{CH}_{2}\right), 35.7\left(\mathrm{CH}_{2}\right), 36.1(\mathrm{t}, J=25.4$ $\left.\mathrm{Hz}, \mathrm{CH}_{2}\right), 36.3$ (t, $\left.J=25.4 \mathrm{~Hz}, \mathrm{CH}_{2}\right), 125.3$ (t, $\left.J=241.0 \mathrm{~Hz}, C_{2}\right), 125.8(\mathrm{CH}), 128.3(\mathrm{CH})$, $128.4(\mathrm{CH}), 142.2(\mathrm{C}) .{ }^{19} \mathrm{~F}$ NMR (369 MHz, $\left.\mathrm{CDCl}_{3}\right): \delta-98.1$ (t, $\left.J=16.9 \mathrm{~Hz}, 2 \mathrm{~F}\right)$. HRMS-EI (m/z): $[\mathrm{M}]^{+}$calcd for $\mathrm{C}_{17} \mathrm{H}_{26} \mathrm{~F}_{2}, 268.2003$; found, 268.2004. 


\section{Experimental Procedure of Arylation of 4aa.}

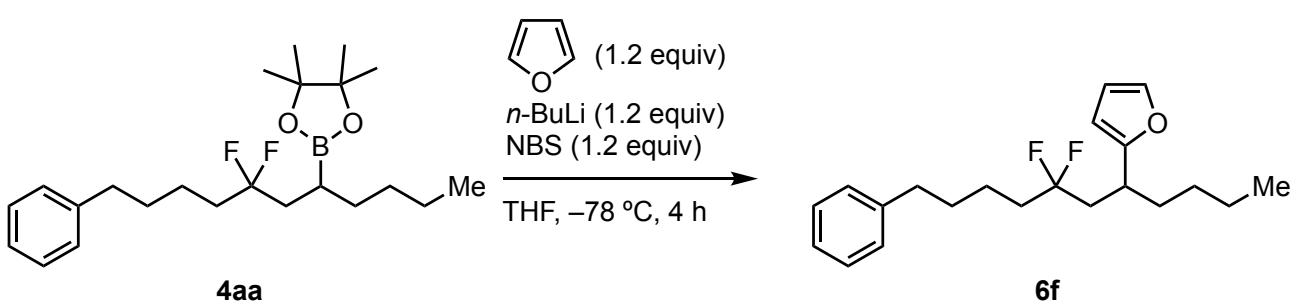

Arylation of 4aa was performed according to the literature procedure. ${ }^{19}$ To an oven-dried reaction vial, furan $(10.0 \mu \mathrm{L}, 0.13 \mathrm{mmol})$ in THF $(400 \mu \mathrm{L})$ was added, and the mixture was cooled to $-78{ }^{\circ} \mathrm{C}$ under a nitrogen atmosphere. A solution of $n$-BuLi in hexane $(1.6 \mathrm{M}, 75 \mu \mathrm{L}$, $0.12 \mathrm{mmol}$ ) was then added dropwise and stirred at r.t. for $30 \mathrm{~min}$. After that, $4 \mathbf{a a}(39.4 \mathrm{mg}, 0.1$ mmol) in THF $(200 \mu \mathrm{L})$ was added dropwise to the mixture at $-78^{\circ} \mathrm{C}$ and stirred for $1 \mathrm{~h}$, then the NBS (21.4 mg, $0.12 \mathrm{mmol})$ in THF $(400 \mu \mathrm{L})$ was added to this reaction mixture. After the reaction mixture was stirred at $-78^{\circ} \mathrm{C}$ for $2 \mathrm{~h}$, the mixture was quenched by addition of $\mathrm{Na}_{2} \mathrm{~S}_{2} \mathrm{O}_{3}$ and extracted three times with $\mathrm{Et}_{2} \mathrm{O}$. The combined organic layer was dried over $\mathrm{MgSO}_{4}$ followed by filtration. After evaporation, the remained crude material was purified by silica gel chromatography $\left(\mathrm{Et}_{2} \mathrm{O} /\right.$ hexane, 0:100-6:94) to give $\mathbf{6 f}(21.6 \mathrm{mg}, 0.065 \mathrm{mmol}, 65 \%)$ as a colorless oil.

${ }^{1} \mathrm{H}$ NMR $\left(392 \mathrm{MHz}, \mathrm{CDCl}_{3}\right): \delta 0.85(\mathrm{t}, J=7.5 \mathrm{~Hz}, 3 \mathrm{H}), 1.08-1.46(\mathrm{~m}, 6 \mathrm{H}), 1.58-1.76(\mathrm{~m}$, $6 \mathrm{H}), 1.98-2.13(\mathrm{~m}, 1 \mathrm{H}), 2.17-2.34(\mathrm{~m}, 1 \mathrm{H}), 2.56(\mathrm{t}, J=7.6 \mathrm{~Hz}, 2 \mathrm{H}), 2.92-3.02(\mathrm{~m}, 1 \mathrm{H}), 6.00$ $(\mathrm{d}, J=3.1 \mathrm{~Hz}, 1 \mathrm{H}), 6.27(\mathrm{dd}, J=1.3,2.7 \mathrm{~Hz}, 1 \mathrm{H}), 7.10-7.21(\mathrm{~m}, 3 \mathrm{H}), 7.23-7.32(\mathrm{~m}, 3 \mathrm{H}) .{ }^{13} \mathrm{C}$ NMR (100 MHz, $\left.\mathrm{CDCl}_{3}\right): \delta 13.9\left(\mathrm{CH}_{3}\right), 21.9\left(\mathrm{t}, J=4.8 \mathrm{~Hz}, \mathrm{CH}_{2}\right), 22.4\left(\mathrm{CH}_{2}\right), 29.2\left(\mathrm{CH}_{2}\right), 31.2$ $\left(\mathrm{CH}_{2}\right), 33.4(\mathrm{t}, J=3.8 \mathrm{~Hz}, \mathrm{CH}), 34.8\left(\mathrm{CH}_{2}\right), 35.7\left(\mathrm{CH}_{2}\right), 36.3\left(\mathrm{t}, J=25.4 \mathrm{~Hz}, \mathrm{CH}_{2}\right), 40.4(\mathrm{t}, J=$ $\left.25.4 \mathrm{~Hz}, \mathrm{CH}_{2}\right), 105.4(\mathrm{CH}), 110.0(\mathrm{CH}), 124.8\left(\mathrm{t}, J=242.4 \mathrm{~Hz}, \mathrm{CF}_{2}\right), 125.7(\mathrm{CH}), 128.29(\mathrm{CH})$, $128.34(\mathrm{CH}), 140.8(\mathrm{CH}), 142.2(\mathrm{C}), 157.4(\mathrm{C}) .{ }^{19} \mathrm{~F} \mathrm{NMR}\left(369 \mathrm{MHz}, \mathrm{CDCl}_{3}\right): \delta-98.4$ to -97.4 (m, 1F), -95.0 to $-94.1(\mathrm{~m}, 1 \mathrm{~F})$. HRMS-EI $(\mathrm{m} / \mathrm{z})$ : $[\mathrm{M}]^{+}$calcd for $\mathrm{C}_{21} \mathrm{H}_{28} \mathrm{OF}_{2}, 334.2108$; found, 334.2107. 

$(6 \mathrm{~g})$.

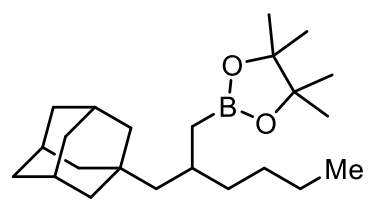

$6 \mathrm{~g}$

Prepared according to the described procedure as for the boronate 6a. The reaction was conducted for $3 \mathrm{~h}$ with $36.8 \mathrm{mg}(0.11 \mathrm{mmol})$ of $4 \mathbf{b a}$. The product $6 \mathrm{~g}$ was obtained in $87 \%$ yield (33.3 mg, $0.087 \mathrm{mmol})$.

${ }^{1} \mathrm{H}$ NMR (392 MHz, $\left.\mathrm{CDCl}_{3}\right): \delta 0.79(\mathrm{t}, J=6.3 \mathrm{~Hz}, 2 \mathrm{H}), 0.88(\mathrm{t}, J=6.9 \mathrm{~Hz}, 3 \mathrm{H}), 0.93-1.00$ (m, 1H), 1.05-1.13 (m, 1H), $1.16-1.37$ (m, 6H), $1.24(\mathrm{~s}, 12 \mathrm{H}), 1.45-1.53(\mathrm{~m}, 6 \mathrm{H}), 1.58-1.73$ (m, 7H), 1.88-1.97 (m, 3H). ${ }^{13} \mathrm{C}$ NMR (99 MHz, $\left.\mathrm{CDCl}_{3}\right): \delta 14.2\left(\mathrm{CH}_{3}\right), 20.1$ (br, $\left.\mathrm{B}-\mathrm{CH}_{2}\right), 23.1$ $\left(\mathrm{CH}_{2}\right), 24.8\left(\mathrm{CH}_{3}\right), 24.9\left(\mathrm{CH}_{3}\right), 28.5(\mathrm{CH}), 28.8(\mathrm{CH}), 29.3\left(\mathrm{CH}_{2}\right), 33.1(\mathrm{C}), 37.2\left(\mathrm{CH}_{2}\right), 38.8$ $\left(\mathrm{CH}_{2}\right), 43.1\left(\mathrm{CH}_{2}\right), 52.3\left(\mathrm{CH}_{2}\right), 82.7(\mathrm{C})$. HRMS-ESI $(\mathrm{m} / \mathrm{z})$ : $[\mathrm{M}+\mathrm{H}]^{+}$calcd for $\mathrm{C}_{23} \mathrm{H}_{42}{ }^{11} \mathrm{BO}_{2}$, 361.3277; found, 361.3272 .

\section{1-[(3r,5r,7r)-Adamantan-1-yl]hexan-2-ol (6h).}

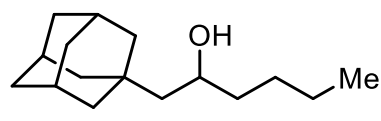

$6 h$

Prepared according to the described procedure as for the alcohol $\mathbf{6 b}$. The reaction was conducted for $3 \mathrm{~h}$ with $36.5 \mathrm{mg}(0.11 \mathrm{mmol})$ of $4 \mathbf{b a}$. The product $6 \mathrm{~h}$ was obtained in $92 \%$ yield (22.8 mg, $0.096 \mathrm{mmol}$ ).

${ }^{1} \mathrm{H}$ NMR (401 MHz, $\left.\mathrm{CDCl}_{3}\right): \delta 0.91(\mathrm{t}, J=7.0 \mathrm{~Hz}, 3 \mathrm{H}), 1.11-1.50(\mathrm{~m}, 12 \mathrm{H}), 1.52-1.58(\mathrm{~m}$, 2H), $1.66(\mathrm{t}, J=13.8 \mathrm{~Hz}, 3 \mathrm{H}), 1.69$ (t, $J=13.8 \mathrm{~Hz}, 3 \mathrm{H}), 1.91-1.99(\mathrm{~m}, 3 \mathrm{H}), 3.76-3.85$ (m, 1H). ${ }^{13} \mathrm{C}$ NMR (99 MHz, $\left.\mathrm{CDCl}_{3}\right): \delta 14.1\left(\mathrm{CH}_{3}\right), 22.7\left(\mathrm{CH}_{2}\right), 27.8\left(\mathrm{CH}_{2}\right), 28.7(\mathrm{CH}), 32.2(\mathrm{C}), 37.1$ $\left(\mathrm{CH}_{2}\right), 39.3\left(\mathrm{CH}_{2}\right), 43.1\left(\mathrm{CH}_{2}\right), 52.5\left(\mathrm{CH}_{2}\right), 68.0(\mathrm{CH})$. HRMS-ESI $(\mathrm{m} / \mathrm{z})$ : $[\mathrm{M}+\mathrm{Na}]^{+}$calcd for $\mathrm{C}_{16} \mathrm{H}_{28} \mathrm{ONa}$, 259.2038; found, 259.2032.

\section{Experimental Procedure of Olefination of $4 \mathrm{ba}$.}
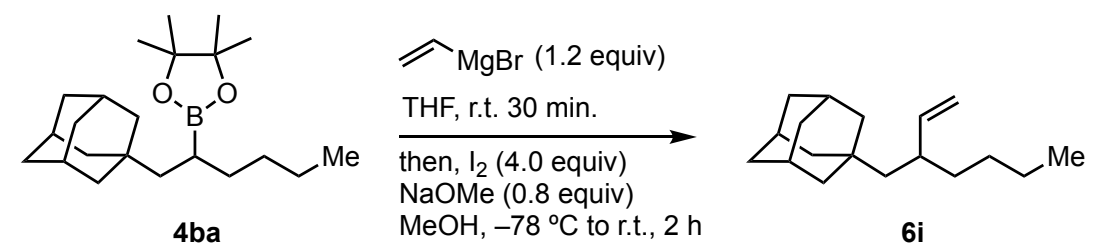

6i

Olefination of $4 \mathbf{b a}$ was performed according to the literature procedure. ${ }^{20}$ To an oven-dried 
reaction vial, $4 \mathbf{b a}(36.4 \mathrm{mg}, 0.1 \mathrm{mmol})$ in THF $(1 \mathrm{~mL})$ was added, then vinyl magnesium bromide (1.0 M in THF, $400 \mu \mathrm{L}, 0.4 \mathrm{mmol}$ ) was added to the reaction mixture at r.t. under a nitrogen atmosphere. The reaction mixture was stirred at r.t. for $30 \mathrm{~min}$., then cooled to $-78^{\circ} \mathrm{C}$. A solution of $\mathrm{I}_{2}(50.8 \mathrm{mg}, 0.4 \mathrm{mmol})$ in $\mathrm{MeOH}(1.3 \mathrm{~mL})$ was added dropwise to the reaction mixture and stirred for another $30 \mathrm{~min}$. After that, $\mathrm{NaOMe}(43.2 \mathrm{mg}, 0.8 \mathrm{mmol})$ in $\mathrm{MeOH}(1.6$ $\mathrm{mL}$ ) was added dropwise to the mixture. The mixture was warmed up to r.t. and stirred for $2 \mathrm{~h}$. The mixture was then quenched by addition of $\mathrm{Na}_{2} \mathrm{~S}_{2} \mathrm{O}_{3}$ and extracted three times with $\mathrm{Et}_{2} \mathrm{O}$. The combined organic layer was dried over $\mathrm{MgSO}_{4}$ followed by filtration. The crude material was purified by silica gel chromatography $\left(\mathrm{Et}_{2} \mathrm{O} /\right.$ hexane, $\left.0: 100-4: 96\right)$ to give $6 \mathbf{i}(17.5 \mathrm{mg}, 0.066$ $\mathrm{mmol}, 66 \%$ ) as a colorless oil.

${ }^{1} \mathrm{H}$ NMR (392 MHz, $\left.\mathrm{CDCl}_{3}\right): \delta 0.87(\mathrm{t}, J=7.1 \mathrm{~Hz}, 3 \mathrm{H}), 1.04-1.15(\mathrm{~m}, 2 \mathrm{H}), 1.17-1.32(\mathrm{~m}$, $6 \mathrm{H}), 1.41-1.53(\mathrm{~m}, 6 \mathrm{H}), 1.62(\mathrm{t}, J=14.4 \mathrm{~Hz}, 3 \mathrm{H}), 1.66(\mathrm{t}, J=14.6 \mathrm{~Hz}, 3 \mathrm{H}), 1.87-1.95(\mathrm{~m}, 3 \mathrm{H})$, 2.06-2.18 (m, 1H), 4.85-4.94 (m, 2H), 5.52-5.64 (m, 1H). ${ }^{13} \mathrm{C}$ NMR (99 MHz, $\left.\mathrm{CDCl}_{3}\right): \delta 14.1$ $\left(\mathrm{CH}_{3}\right), 22.8\left(\mathrm{CH}_{2}\right), 28.8(\mathrm{CH}), 29.3\left(\mathrm{CH}_{2}\right), 33.2(\mathrm{C}), 37.2\left(\mathrm{CH}_{2}\right), 37.4\left(\mathrm{CH}_{2}\right), 38.7(\mathrm{CH}), 43.1$ $\left(\mathrm{CH}_{2}\right), 50.1\left(\mathrm{CH}_{2}\right), 112.6\left(\mathrm{CH}_{2}\right), 146.1(\mathrm{CH})$. HRMS-EI $(\mathrm{m} / \mathrm{z})$ : [M] ${ }^{+}$calcd for $\mathrm{C}_{18} \mathrm{H}_{30}, 246.2348$; found, 246.2352 .

\section{9,9-Difluoro-15-phenylpentadecan-7-ol (6j).}

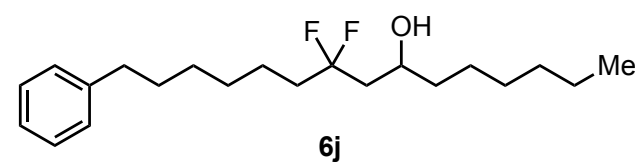

Prepared according to the described procedure as for the alcohol $\mathbf{6 b}$. The reaction was conducted for $3 \mathrm{~h}$ with $45.2 \mathrm{mg}(0.10 \mathrm{mmol})$ of $4 \mathbf{a e b}$. The product $\mathbf{6 j}$ was obtained in $87 \%$ yield (29.6 mg, $0.087 \mathrm{mmol})$.

${ }^{1} \mathrm{H}$ NMR $\left(392 \mathrm{MHz}, \mathrm{CDCl}_{3}\right): \delta 0.88(\mathrm{t}, J=6.5 \mathrm{~Hz}, 3 \mathrm{H}), 1.21-1.52(\mathrm{~m}, 16 \mathrm{H}), 1.59-1.67(\mathrm{~m}$, 2H), 1.79-2.03 (m, 4H), 2.60 (t, $J=7.6 \mathrm{~Hz}, 2 \mathrm{H}), 3.97-4.04$ (m, 1H), 7.14-7.20 (m, 3H), 7.24$7.31(\mathrm{~m}, 2 \mathrm{H}) .{ }^{13} \mathrm{C} \mathrm{NMR}\left(100 \mathrm{MHz}, \mathrm{CDCl}_{3}\right): \delta 14.0\left(\mathrm{CH}_{3}\right), 22.2\left(\mathrm{t}, J=4.8 \mathrm{~Hz}, \mathrm{CH}_{2}\right), 22.6\left(\mathrm{CH}_{2}\right)$, $25.3\left(\mathrm{CH}_{2}\right), 29.0\left(\mathrm{CH}_{2}\right), 29.16\left(\mathrm{CH}_{2}\right), 29.18\left(\mathrm{CH}_{2}\right), 31.3\left(\mathrm{CH}_{2}\right), 31.8\left(\mathrm{CH}_{2}\right), 35.9\left(\mathrm{CH}_{2}\right), 37.2(\mathrm{t}$, $\left.J=25.4 \mathrm{~Hz}, \mathrm{CH}_{2}\right), 37.6\left(\mathrm{CH}_{2}\right), 43.4\left(\mathrm{t}, J=24.0 \mathrm{~Hz}, \mathrm{CH}_{2}\right), 66.8(\mathrm{CH}), 125.5(\mathrm{t}, J=241.5 \mathrm{~Hz}$, $\left.\mathrm{CF}_{2}\right), 125.6(\mathrm{CH}), 128.2(\mathrm{CH}), 128.4(\mathrm{CH}), 142.6(\mathrm{C}) .{ }^{19} \mathrm{~F}$ NMR $\left(373 \mathrm{MHz}, \mathrm{CDCl}_{3}\right): \delta-97.6$ to -95.7 (m, 2F). HRMS-EI (m/z): [M] ${ }^{+}$calcd for $\mathrm{C}_{21} \mathrm{H}_{34} \mathrm{OF}_{2}, 340.2578$; found, 340.2568 .

(7,7-Difluoropentadecyl)benzene (6k).

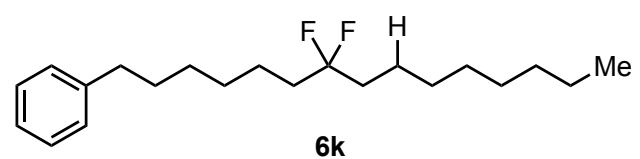


Prepared according to the described procedure as for $6 \mathrm{e}$. The reaction was conducted for 18 $\mathrm{h}$ with $45.0 \mathrm{mg}(0.10 \mathrm{mmol})$ of $\mathbf{4 a e b}$. The product $\mathbf{6 k}$ was obtained in $53 \%$ yield $(17.1 \mathrm{mg}$, $0.053 \mathrm{mmol})$.

${ }^{1} \mathrm{H}$ NMR (401 MHz, $\left.\mathrm{CDCl}_{3}\right): \delta 0.88(\mathrm{t}, J=7.0 \mathrm{~Hz}, 3 \mathrm{H}), 1.19-1.32(\mathrm{~m}, 10 \mathrm{H}), 1.33-1.38(\mathrm{~m}$, 4H), 1.40-1.50 (m, 4H), 1.59-1.68 (m, 2H), 1.70-1.85 (m, 4H), 2.60 (t, $J=7.8 \mathrm{~Hz}, 2 \mathrm{H}) 7.15-$ $7.21(\mathrm{~m}, 3 \mathrm{H}), 7.24-7.31(\mathrm{~m}, 2 \mathrm{H}) .{ }^{13} \mathrm{C} \mathrm{NMR}\left(100 \mathrm{MHz}, \mathrm{CDCl}_{3}\right): \delta 14.1\left(\mathrm{CH}_{3}\right), 22.28(\mathrm{t}, J=5.8$ $\left.\mathrm{Hz}, \mathrm{CH}_{2}\right), 22.34$ (t, $\left.J=5.8 \mathrm{~Hz}, \mathrm{CH}_{2}\right), 22.6\left(\mathrm{CH}_{2}\right), 29.0\left(\mathrm{CH}_{2}\right), 29.1\left(\mathrm{CH}_{2}\right), 29.3\left(\mathrm{CH}_{2}\right), 29.36$ $\left(\mathrm{CH}_{2}\right), 29.42\left(\mathrm{CH}_{2}\right), 31.3\left(\mathrm{CH}_{2}\right), 31.8\left(\mathrm{CH}_{2}\right), 35.9\left(\mathrm{CH}_{2}\right), 36.26\left(\mathrm{t}, J=25.9 \mathrm{~Hz}, \mathrm{CH}_{2}\right), 36.31(\mathrm{t}$, $\left.J=25.4 \mathrm{~Hz}, \mathrm{CH}_{2}\right), 125.4\left(\mathrm{t}, J=241.4 \mathrm{~Hz}, C \mathrm{~F}_{2}\right), 125.6(\mathrm{CH}), 128.2(\mathrm{CH}), 128.4(\mathrm{CH}), 142.7$ (C). ${ }^{19} \mathrm{~F}$ NMR (373 MHz, $\left.\mathrm{CDCl}_{3}\right): \delta-98.2$ to $-98.0(\mathrm{~m}, 2 \mathrm{~F})$. HRMS-EI $(\mathrm{m} / \mathrm{z}):[\mathrm{M}]^{+}$calcd for $\mathrm{C}_{21} \mathrm{H}_{34} \mathrm{~F}_{2}, 324.2629$; found, 324.2623 . 


\section{Reaction with a Chiral Ligand}

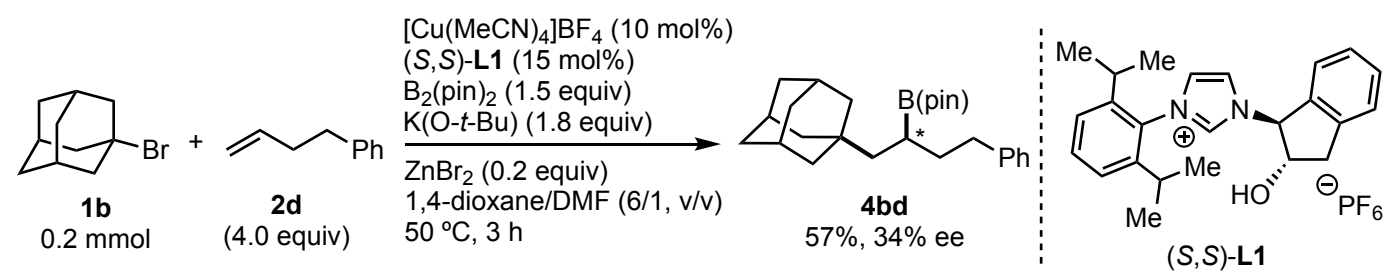

The reaction was conducted for $3 \mathrm{~h}$ according to condition $\mathbf{B}$ described above with $43.6 \mathrm{mg}$ $(0.20 \mathrm{mmol})$ of $\mathbf{1 b}, 120.0 \mu \mathrm{L}(0.80 \mathrm{mmol})$ of $\mathbf{2 d}$, and $15.2 \mathrm{mg}(0.03 \mathrm{mmol})$ of a chiral ligand $(S, S)$-L1. The product $4 \mathbf{b d}$ was obtained in $57 \%$ yield $(45.8 \mathrm{mg})$ with $34 \%$ ee.

Spectroscopic data were matched in the date of $\mathbf{4 b d}$ described above. The enantioselectivity was determined by HPLC analysis of the corresponding alcohol after oxidation of the boryl group. Daicel CHIRALPAK. IB-3, 2-PrOH/Hexane $=5 / 95,0.5 \mathrm{~mL} / \mathrm{min}, 40{ }^{\circ} \mathrm{C}$, retention time: 15.99 min [major enantiomer], and 12.83 min [minor enantiomer]. $[\alpha]_{\mathrm{D}}{ }^{26.1}+2.47(c) 0.91$, $\left.\mathrm{CHCl}_{3}\right)$. 


\section{Single Crystal X-ray Structural Analysis}

The relative stereochemistry of $\mathbf{4 a m}$ was determined by X-ray crystallographic analysis. The details were summarized in Figure S1 and Table S3.

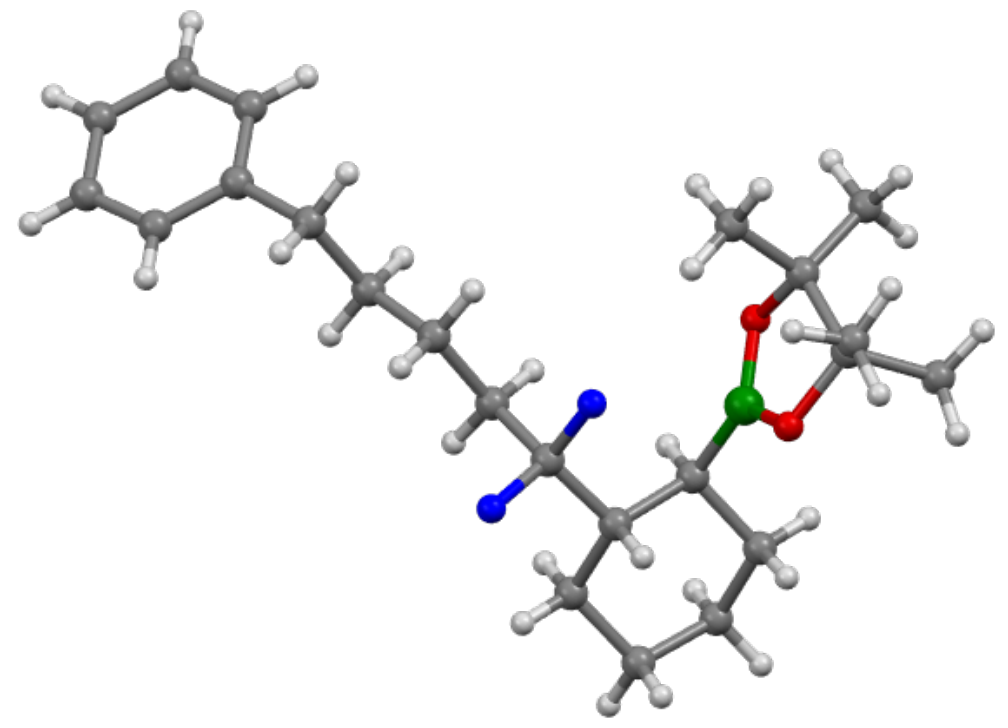

Figure S1. Molecular structure of 4am. 
Table S3. Summary of X-ray crystallographic data for 4am.

CCDC

Empirical formula

Formula weight

Temperature/K

Crystal system

Space group

$a / \AA$

$b / \AA$

$c / \AA$

$\alpha /{ }^{\circ}$

$\beta /{ }^{\circ}$

$\gamma /{ }^{\circ}$

Volume $/ \AA^{3}$

Z

$\rho_{\text {calc }} \mathrm{g} / \mathrm{cm}^{3}$

$\mu / \mathrm{mm}^{-1}$

$\mathrm{F}(000)$

Crystal size $/ \mathrm{mm}^{3}$

Radiation

$2 \theta$ range for data collection $/{ }^{\circ}$

Index ranges

Reflections collected

Independent reflections

Data/restraints/parameters

Goodness-of-fit on $\mathrm{F}^{2}$

Final $R$ indexes [I $>=2 \sigma(\mathrm{I})]$

Final $R$ indexes [all data]

Largest diff. peak/hole / e $\AA^{-3}$
2046556

$\mathrm{C}_{23} \mathrm{H}_{35} \mathrm{BF}_{2} \mathrm{O}_{2}$

392.32

123

monoclinic

$P 21 / \mathrm{n}$

$12.5781(6)$

$10.5058(4)$

$17.3733(9)$

90

$103.258(5)$

90

2234.57(18)

4

1.166

0.699

848.0

$0.27 \times 0.1 \times 0.07$

$\operatorname{CuK} \alpha(\lambda=1.54184)$

7.884 to 151.834

$-15 \leqq h \leqq 14,-8 \leqq k \leqq 12,-19 \leqq l \leqq 21$

12277

$4454\left[R_{\text {int }}=0.0305, R_{\text {sigma }}=0.0348\right]$

$4454 / 0 / 245$

1.044

$R_{1}=0.0626, w R_{2}=0.1733$

$R_{1}=0.0774, w R_{2}=0.1888$

$0.41 /-0.32$ 


\section{Mechanistic Investigations}

\subsection{The reaction without an olefin.}

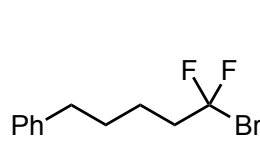

1a

$0.10 \mathrm{mmol}$
$\left[\mathrm{Cu}(\mathrm{MeCN})_{4}\right] \mathrm{BF}_{4}(10 \mathrm{~mol} \%)$

IMes $\cdot \mathrm{HCl}(15 \mathrm{~mol} \%)$

$\mathrm{B}_{2}(\operatorname{pin})_{2}(3)$ (1.5 equiv)

$\mathrm{K}(\mathrm{O}-\mathrm{t}$-Bu) (1.8 equiv)

$\mathrm{ZnBr}_{2}(20 \mathrm{~mol} \%)$

1,4-dioxane/DMF $(4 / 1, \mathrm{v} / \mathrm{v})$ $50^{\circ} \mathrm{C}, 4 \mathrm{~h}$

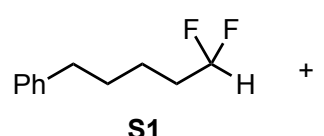

S1

$17 \%{ }^{19} \mathrm{~F}$ NMR yield

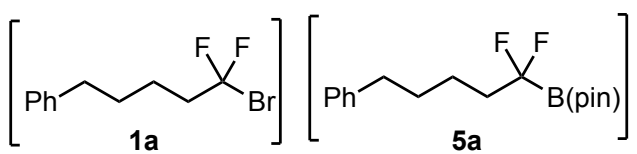

not detected

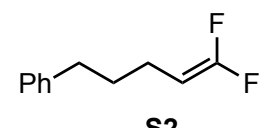

S2

$6 \%{ }^{19} \mathrm{~F}$ NMR yield

not detected

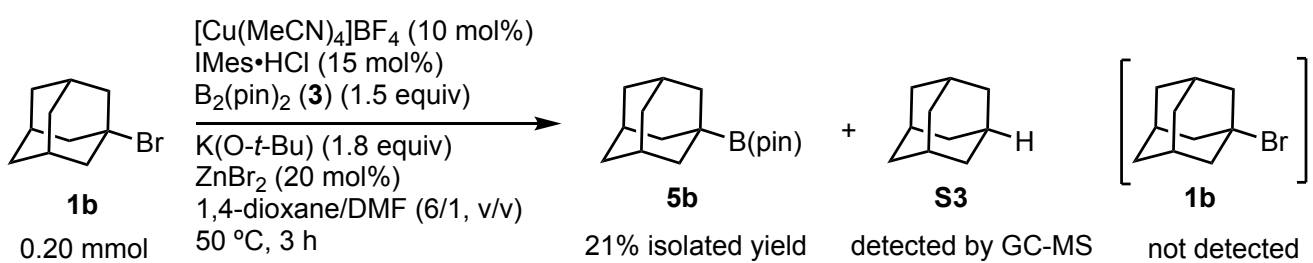

Contrast reactions without an olefin were conducted with $\mathbf{1 a}$ and $\mathbf{1 b}$. As a result, the reaction of $\mathbf{1 a}$ under our best condition gave no boryl substitution product $\mathbf{5 a}$, while the reaction of $\mathbf{1 b}$ gave a small amount of boronate $\mathbf{5 b}$ (21\% yield) with a several byproducts including protonation products (S1-S3). Notably, the starting materials 1a and 1b were fully consumed in both cases, which indicated that the electronic or steric repulsion inhibited a SET from the boryl copper (II) intermediate as we proposed in the main text.

\subsection{The reaction with a proton source.}

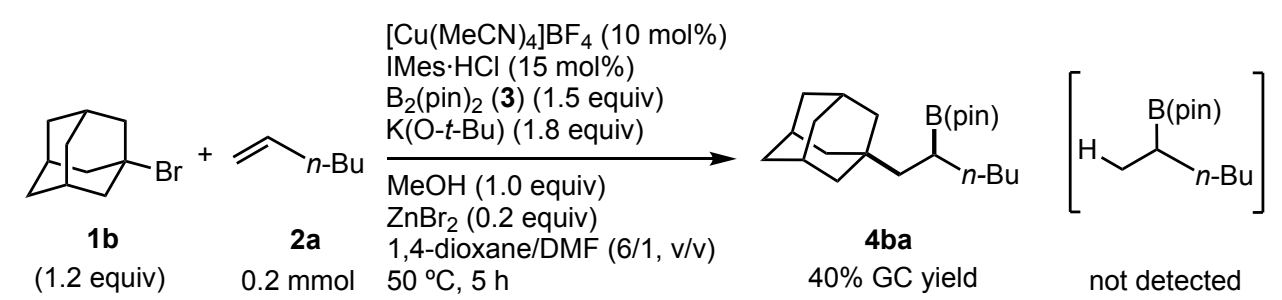

To eliminate a possible pathway via two electron mechanism in which boryl copper(I) intermediate initially reacts with an olefin followed by electrophilic substitution of an alkyl halide, we conducted the reaction with $\mathrm{MeOH}$ as a proton source. An oven-dried reaction vial was charged with bis(pinacolato)diboron (3) $(76.2 \mathrm{mg}, 0.3 \mathrm{mmol})$, IMes $\bullet \mathrm{HCl}(10.2 \mathrm{mg}, 0.03$ mmol) and transferred to an argon filled glove box. $\left[\mathrm{Cu}(\mathrm{MeCN})_{4}\right] \mathrm{BF}_{4}(6.3 \mathrm{mg}, 0.02 \mathrm{mmol})$, $\mathrm{ZnBr}_{2}(9.0 \mathrm{mg}, 0.04 \mathrm{mmol})$, and $\mathrm{K}(\mathrm{O}-t-\mathrm{Bu})(40.4 \mathrm{mg}, 0.36 \mathrm{mmol})$ were added to the reaction vial, and then the vial was capped with a rubber septum and removed from the glovebox. The solids were suspended in 1,4-dioxane/DMF (300 $\mu \mathrm{L} / 100 \mu \mathrm{L})$, and the suspension was allowed 
to stir for $1 \mathrm{~min}$ at r.t. After that, the olefin $\mathbf{2 a}(16.8 \mathrm{mg}, 0.20 \mathrm{mmol})$ and the alkyl electrophile $\mathbf{1 b}(51.7 \mathrm{mg}, 0.24 \mathrm{mmol})$ in 1,4-dioxane $(300 \mu \mathrm{L})$ were subsequently added to the suspension, followed by the addition of $\mathrm{MeOH}(8.1 \mu \mathrm{L}, 1.0$ equiv) at r.t., then the mixture was allowed to stir at $50{ }^{\circ} \mathrm{C}$ for $5 \mathrm{~h}$. The yield of $\mathbf{4 b a}$ was determined by GC analysis with $\mathrm{C}_{13} \mathrm{H}_{28}$ as an internal standard. Notably, the hydroborylation products were not detected in this reaction condition, which indicated that the reaction would proceed via a radical-relay mechanism. 


\section{The Details of Optimization Conditions for 4aa}

The reactions were conducted according to condition $\mathbf{A}$ described above. The reaction was conducted for $2 \mathrm{~h}$ with 1a and 2a. The yield of product $4 \mathbf{a a}$ was determined by GC analysis of the crude reaction mixture with $\mathrm{C}_{13} \mathrm{H}_{28}$ as an internal standard.

Table S4. Screening of Copper Catalysts.

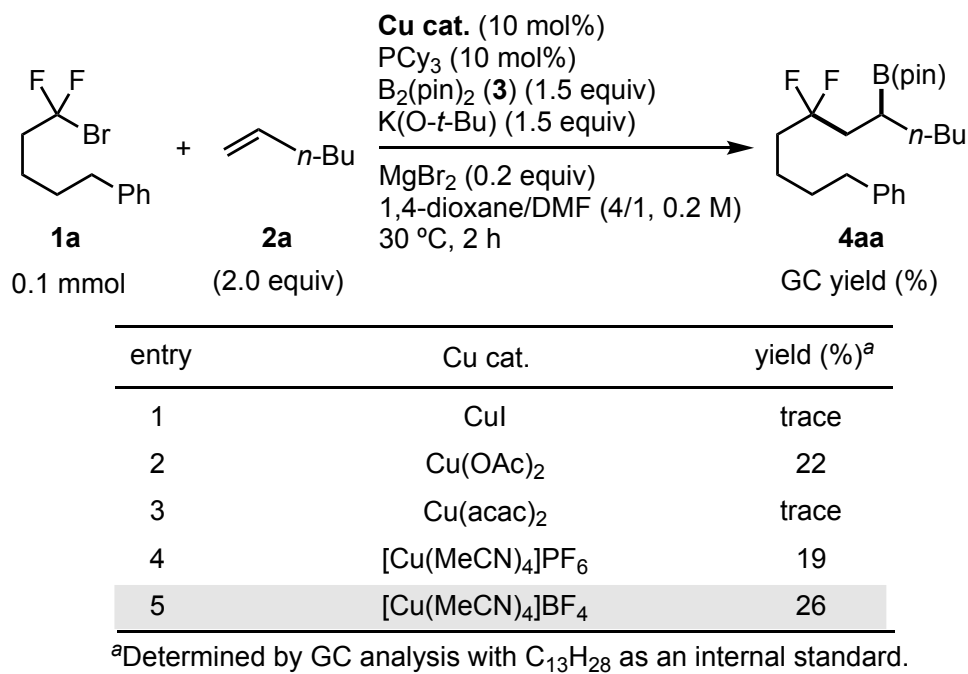

Table S5. Screening of Solvents.

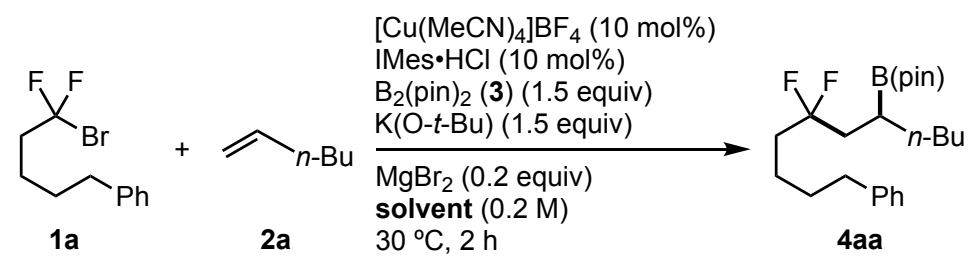

$0.1 \mathrm{mmol}$ (2.0 equiv) GC yield (\%)

\begin{tabular}{ccc}
\hline entry & solvent & yield $(\%)^{a}$ \\
\hline 1 & 1,4-dioxane & 19 \\
2 & DMF & 9 \\
3 & DMSO & 7 \\
4 & THF & complex mixture \\
5 & 1,4-dioxane/DMF (1:1) & 19 \\
6 & 1,4-dioxane/DMF $(4: 1)$ & 31 \\
\hline
\end{tabular}

Determined by $\mathrm{GC}$ analysis with $\mathrm{C}_{13} \mathrm{H}_{28}$ as an internal standard. 
Table S6. Screening of Ligands.

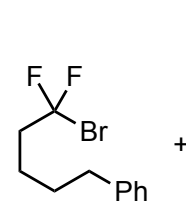

1 a

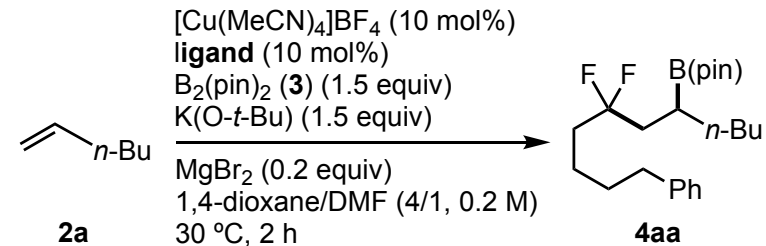

$0.1 \mathrm{mmol}$

(2.0 equiv)

\begin{tabular}{|c|c|c|}
\hline entry & ligand & yield $(\%)^{a}$ \\
\hline 1 & $\mathrm{PCy}_{3}$ & 26 \\
\hline 2 & $\mathrm{PtBu} \mathrm{u}_{3}$ & 15 \\
\hline 3 & Sphos & trace \\
\hline 4 & Xphos & trace \\
\hline 5 & CyJohnPhos & trace \\
\hline 6 & dppp & 8 \\
\hline 7 & dppf & 7 \\
\hline 8 & bpy & trace \\
\hline 9 & L1 & 6 \\
\hline 10 & Bphen & trace \\
\hline 11 & L2 & 10 \\
\hline 12 & IMes $\bullet \mathrm{HCl}$ & 31 \\
\hline 13 & SIMes $\bullet \mathrm{HCl}$ & trace \\
\hline 14 & IPr•HCl & 11 \\
\hline 15 & $\mathrm{ICy} \cdot \mathrm{HCl}$ & 23 \\
\hline 16 & L3 & 22 \\
\hline
\end{tabular}

a Determined by $\mathrm{GC}$ analysis with $\mathrm{C}_{13} \mathrm{H}_{28}$ as an internal standard.

Phosphine Ligands<smiles>C1CCC(P(C2CCCCC2)C2CCCCC2)CC1</smiles>

$\mathrm{PCy}_{3}$<smiles>CC(C)(C)P(C(C)(C)C)C(C)(C)C</smiles>

$\mathrm{PtBu}_{3}$<smiles>COc1cccc(OC)c1-c1ccccc1F</smiles>

SPhos<smiles>CC(C)c1cc(C(C)C)c(-c2ccccc2C(C)C)c(C(C)C)c1</smiles>

XPhos<smiles>CCCc1ccccc1-c1ccccc1</smiles>

CyJohnPhos<smiles>Pc1ccccc1</smiles>

dppp

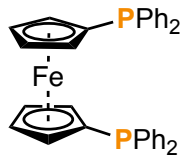

dppf 


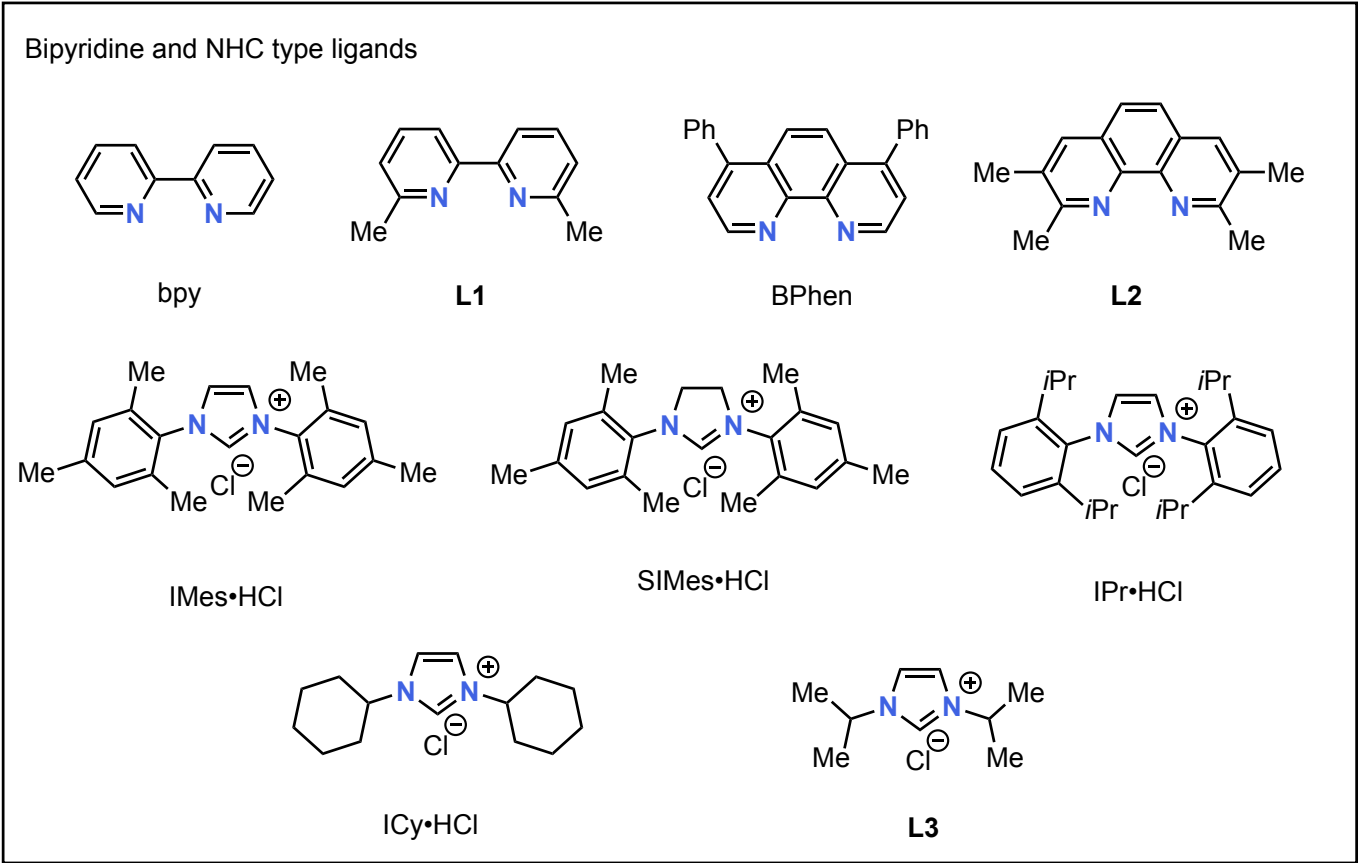

Table S7. Screening of Bases and Ligand Equivalents.

\begin{tabular}{|c|c|c|c|}
\hline & & $\begin{array}{l}{\left[\mathrm{Cu}(\mathrm{MeCN})_{4}\right] \mathrm{BF}_{4}(10 \mathrm{~mol} \%)} \\
\mathrm{IMes} \cdot \mathrm{HCl}(\mathbf{x} \text { mol} \%) \\
\mathrm{B}_{2}(\text { pin })_{2}(3)(1.5 \text { equiv }) \\
\text { base }(1.5 \text { equiv })\end{array}$ & $\mathrm{F} \quad \mathrm{l}_{\mathrm{l}}^{\mathrm{B} \text { (pin }}$ \\
\hline $0.1 \mathrm{mmol}$ & $\begin{array}{c}2 \mathbf{a} \\
(2.0 \text { equiv) }\end{array}$ & $\begin{array}{l}\mathrm{MgBr}_{2}(0.2 \text { equiv) } \\
1,4 \text {-dioxane/DMF (4/1, } 0.2 \mathrm{M}) \\
30^{\circ} \mathrm{C}, 2 \mathrm{~h}\end{array}$ & $\begin{array}{c}\text { 4aa } \\
\text { GC yield (\%) }\end{array}$ \\
\hline entry & base & x (ligand eq.) & yield $(\%)^{a}$ \\
\hline 1 & $\mathrm{~K}(\mathrm{O}-t-\mathrm{Bu})$ & $10 \mathrm{~mol} \%$ & 31 \\
\hline $2^{b}$ & $\mathrm{~K}(\mathrm{O}-\mathrm{t}-\mathrm{Bu})$ & $15 \mathrm{~mol} \%$ & 35 \\
\hline $2^{b}$ & $\mathrm{Na}(\mathrm{O}-t-\mathrm{Bu})$ & 15 mol\% & 18 \\
\hline $3^{b}$ & $\mathrm{Li}(\mathrm{O}-t-\mathrm{Bu})$ & $15 \mathrm{~mol} \%$ & 8 \\
\hline 4 & KOMe & $15 \mathrm{~mol} \%$ & 11 \\
\hline 5 & $\mathrm{KSiMe}_{3}$ & $15 \mathrm{~mol} \%$ & 27 \\
\hline
\end{tabular}

Determined by GC analysis with $\mathrm{C}_{13} \mathrm{H}_{28}$ as an internal standard. ${ }^{b}$ The reaction time was $12 \mathrm{~h}$. 
Table S8. Screening of Additives, Olefin Equivalents, and Temperatures.

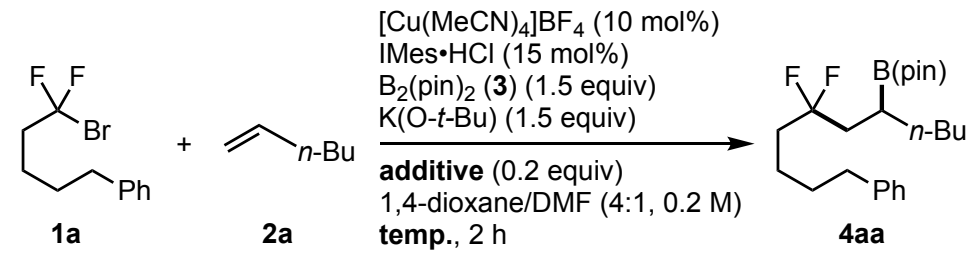

$0.1 \mathrm{mmol}$ (x equiv)

$\mathrm{GC}$ yield $(\%)$

\begin{tabular}{ccccc}
\hline entry & additive & x (olefin eq.) & temp. $\left({ }^{\circ} \mathrm{C}\right)$ & yield $(\%)^{a}$ \\
\hline 1 & $\mathrm{MgBr}_{2}$ & 2.0 & 30 & 35 \\
2 & $\mathrm{AgOTf}$ & 2.0 & 30 & 29 \\
$3^{b}$ & $\mathrm{MgBr}_{2}$ & 2.0 & 40 & 24 \\
4 & $\mathrm{ZnBr}_{2}$ & 2.0 & 40 & 48 \\
5 & $\mathrm{ZnBr}_{2}$ & 3.0 & 40 & 59 \\
\hline
\end{tabular}

${ }^{a}$ Determined by GC analysis with $\mathrm{C}_{13} \mathrm{H}_{28}$ as an internal standard. ${ }^{b}$ The reaction time was $12 \mathrm{~h}$

Table S9. Optimization toward the Best Condition.

\begin{tabular}{|c|c|c|c|c|}
\hline$F$ & & $\begin{array}{l}{[\mathrm{Cu}(\mathrm{MeCN}} \\
\mathrm{IMes} \bullet \mathrm{HCl}( \\
\mathrm{B}_{2}(\mathrm{pin})_{2}(3 \\
\mathrm{K}(\mathrm{O}-t-\mathrm{Bu})\end{array}$ & $\begin{array}{l}E_{4}(10 \mathrm{~mol} \%) \\
\text { ol } \%) \\
\text { s equiv) } \\
\text { equiv) }\end{array}$ & $\underbrace{\mathrm{F}(\mathrm{pin})}_{n-\mathrm{B}}$ \\
\hline $0.1 \mathrm{mmol}$ & $2 a$ & $\begin{array}{l}\mathrm{ZnBr}_{2}(0.2 \\
\text { 1,4-dioxan } \\
\text { temp, } 2 \mathrm{~h}\end{array}$ & v) & $\begin{array}{c}\text { Paa } \\
\text { GC yield (\%) }\end{array}$ \\
\hline entry & olefin eq. & temp. $\left({ }^{\circ} \mathrm{C}\right)$ & conc. (M) & yield $(\%)^{a}$ \\
\hline 1 & 3.0 & 40 & 0.2 & 59 \\
\hline 2 & 3.0 & 50 & 0.2 & 43 \\
\hline 3 & 3.0 & 60 & 0.2 & 49 \\
\hline $4^{b}$ & 3.0 & 40 & 0.2 & 67 \\
\hline $5^{b}$ & 3.0 & 50 & 0.2 & 61 \\
\hline $6^{b}$ & 3.0 & 40 & 0.4 & 62 \\
\hline $7^{b, c}$ & 3.0 & 50 & 0.4 & 71 \\
\hline $8^{b, c}$ & 4.0 & 50 & 0.4 & 79 \\
\hline
\end{tabular}

Determined by GC analysis with $\mathrm{C}_{13} \mathrm{H}_{28}$ as an internal standard. ${ }^{b} 1.8$ eq. of $\mathrm{K}(\mathrm{O}-\mathrm{t}-\mathrm{Bu})$ was used. ${ }^{C} 5 \mathrm{~mol} \%$ of $\mathrm{Cu}$ cat and $7.5 \mathrm{~mol} \%$ of $\mathrm{IMes} \cdot \mathrm{HCl}$ were used. 


\section{Comparison between the Reaction with $\mathrm{ZnBr}_{2}$ and without $\mathrm{ZnBr}_{2}$}

Although the effect of $\mathrm{ZnBr}_{2}$ is still not fully understood, we assume that this additive is of critical importance to increase the solubility of the excess base. ${ }^{21}$ For comparison, we have also conducted the reaction without $\mathrm{ZnBr}_{2}$. The reaction with $\mathrm{ZnBr}_{2}$ gave a non-sticky solution, while the reaction without $\mathrm{ZnBr}_{2}$ gave a slightly gooey solution (Figure S2). Further studies to uncover the precise role of $\mathrm{ZnBr}_{2}$ in this reaction are currently in progress in our laboratory.

Figure S2. Photo of the reaction mixtures after 15 minutes.

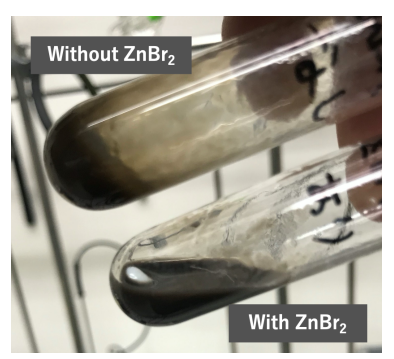




\section{References}

1. Lin, Q. Y.; Xu, X. H.; Qing, F. L. Org. Biomol. Chem. 2015, 13, 8740.

2. Dolbier, W. R.; Okamoto, M. J. Fluor. Chem. 2014, 179, 33.

3. Nihei, T.; Nishi, Y.; Ikeda, N.; Yokotani, S.; Ishihara, T.; Arimitsu, S.; Konno, T. Synthesis 2016, 48,865 .

4. Jodry, J. J.; Nagamori, M; Hagiwara, Y.; Fujiwara, M.; Narizuka, S. PCT Int. Appl. 2009, WO 2009038980 A1.

5. Xiao, Y. L.; Min, Q. Q.; Xu, C.; Wang, R. W.; Zhang, X. Angew. Chem., Int. Ed. 2016, 55, 5837.

6. Das, S.; Ko, N.; Lee, E.; Kim, S. E.; Lee, B. C. Chem. Commun. 2019, 55, 14355.

7. Shim, E.; Zakarian, A. Synlett. 2020, 31, 683.

8. Jadhav, A. R.; Thombal, R. S.; Nigam, P.; Jadhav, V. H. Tetrahedron Lett. 2015, 56, 5235.

9. Thaima, T.; Willis, A. C.; Pyne, S. G. Tetrahedron 2019, 75, 130476.

10. Srihari, P.; Kumaraswamy, B.; Somaiah, R.; Yadav, J. S. Synthesis 2010, 6, 1039.

11. Stang, E. M.; White, M. C. J. Am. Chem. Soc. 2011, 133, 14892.

12. Zhang, Z.; Zhu, L.; Li, C. Chinese J. Chem. 2019, 37, 452.

13. Lipshutz, B. H.; Petersen, T. B.; Abela, A. R. Org. Lett. 2008, 10, 1333.

14. Xiao, H.; Shen, H.; Zhu, L.; Li, C. J. Am. Chem. Soc. 2019, 141, 11440.

15. Sadhu, K. M.; Matteson, D. S. Organometallics 1985, 4, 1687.

16. Mlynarski, S. N.; Karns, A. S.; Morken, J. P. J. Am. Chem. Soc. 2012, 134, 16449.

17. Sandford, C.; Rasappan, R.; Aggarwal, V. K. J. Am. Chem. Soc. 2015, 137, 10100.

18. Rasappan, R.; Aggarwal, V. K. Nat. Chem. 2014, 6, 810.

19. Bonet, A.; Odachowski, M.; Leonori, D.; Essafi, S.; Aggarwal, V. K. Nat. Chem. 2014, 6, 584.

20. Armstrong, R. J.; Aggarwal, V. K. Synthesis 2017, 49, 3323.

21. Wang, J.; Shang, M.; Lundberg, H.; Feu, K. S.; Hecker, S. J.; Qin, T.; Blackmond, D. G.; Baran, P. S. ACS Catal. 2018, 8, 9537. 


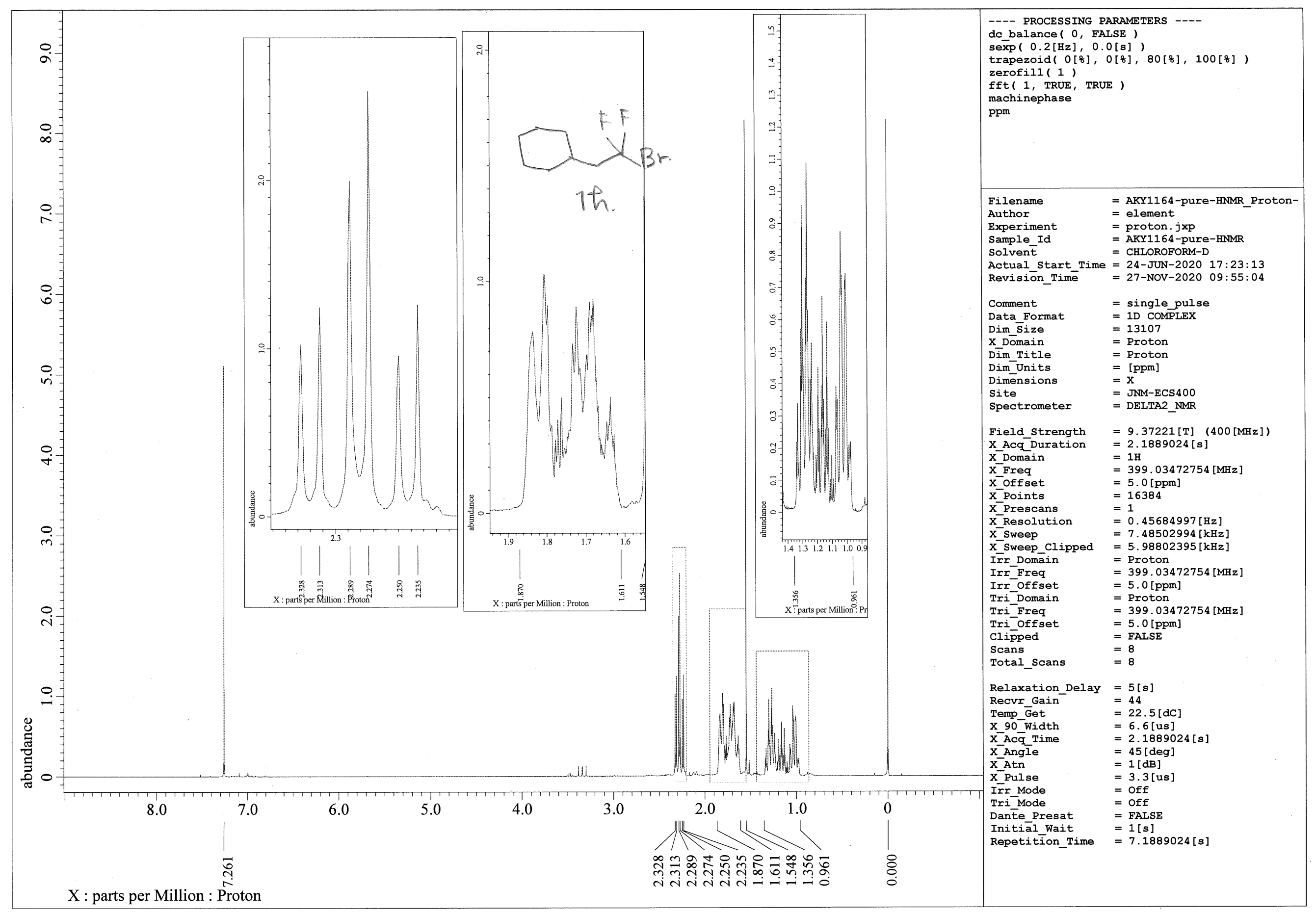




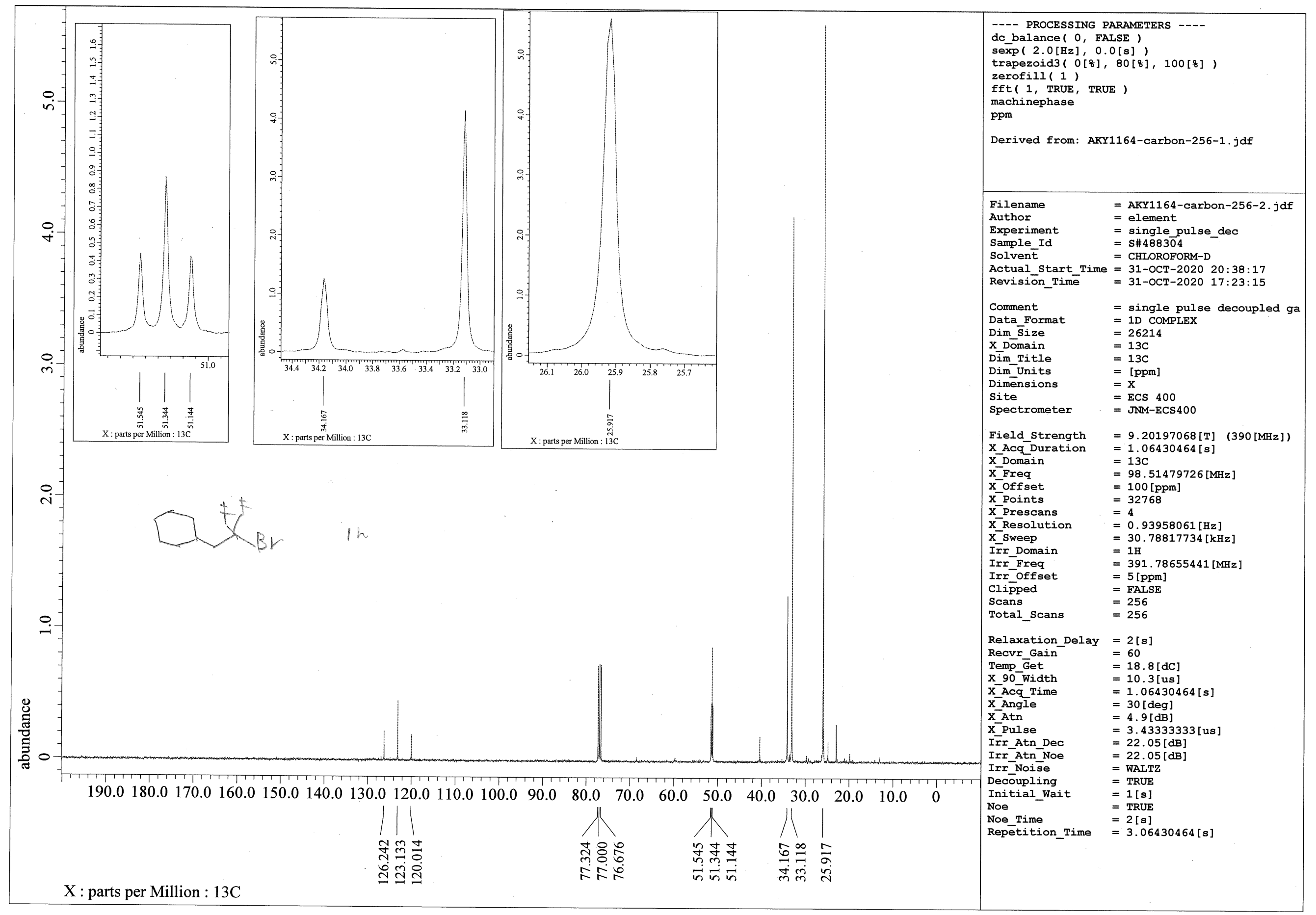




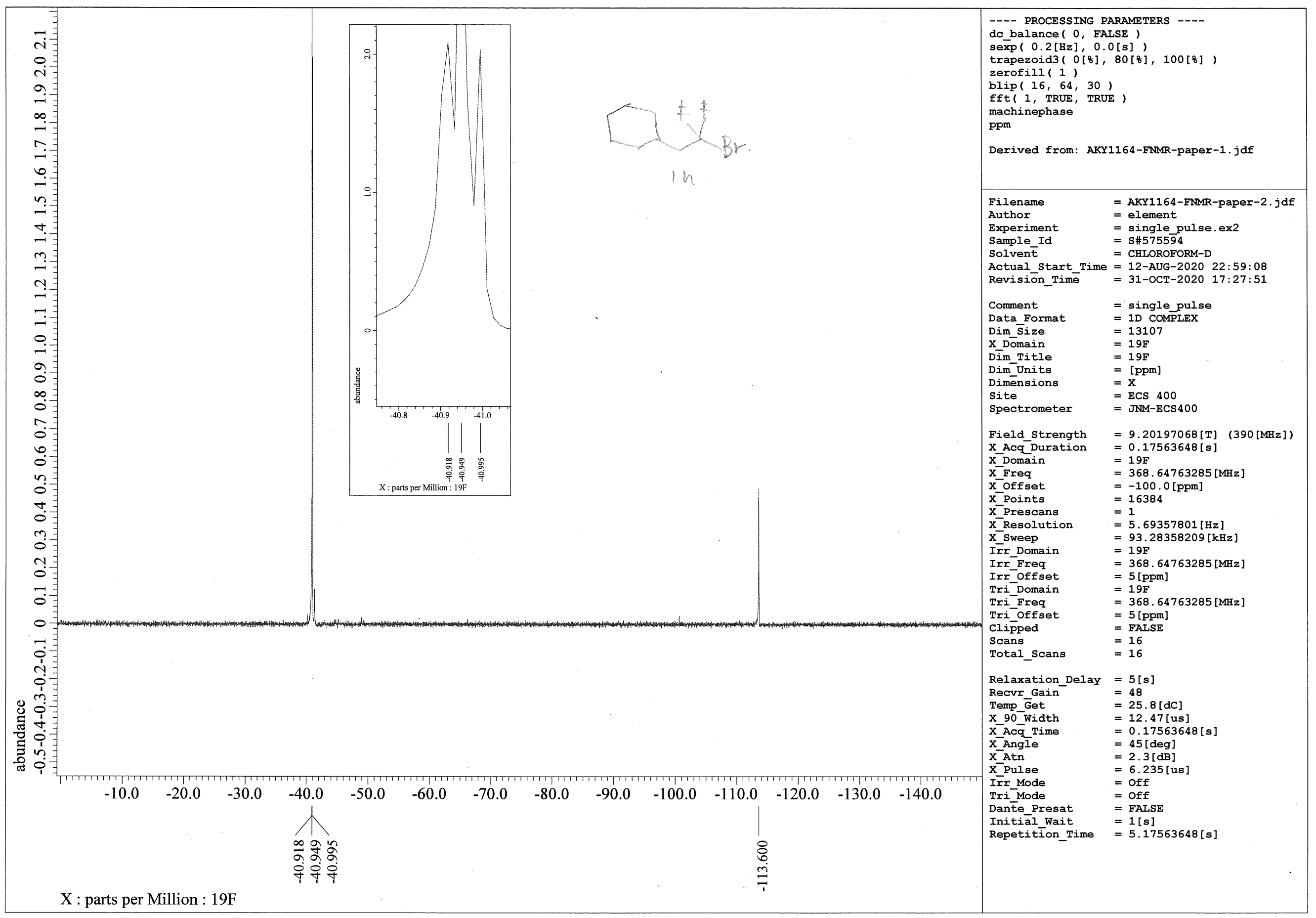




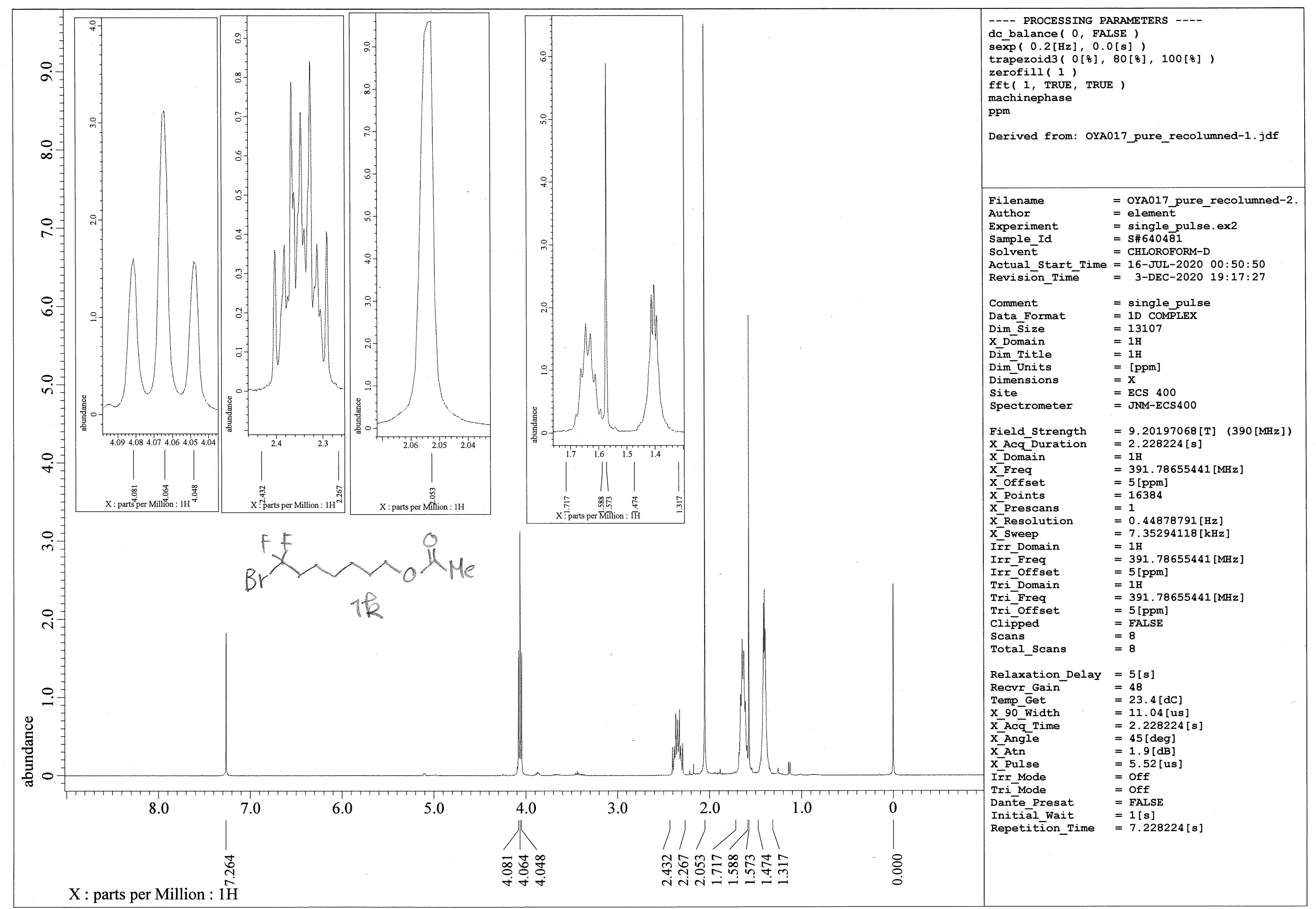




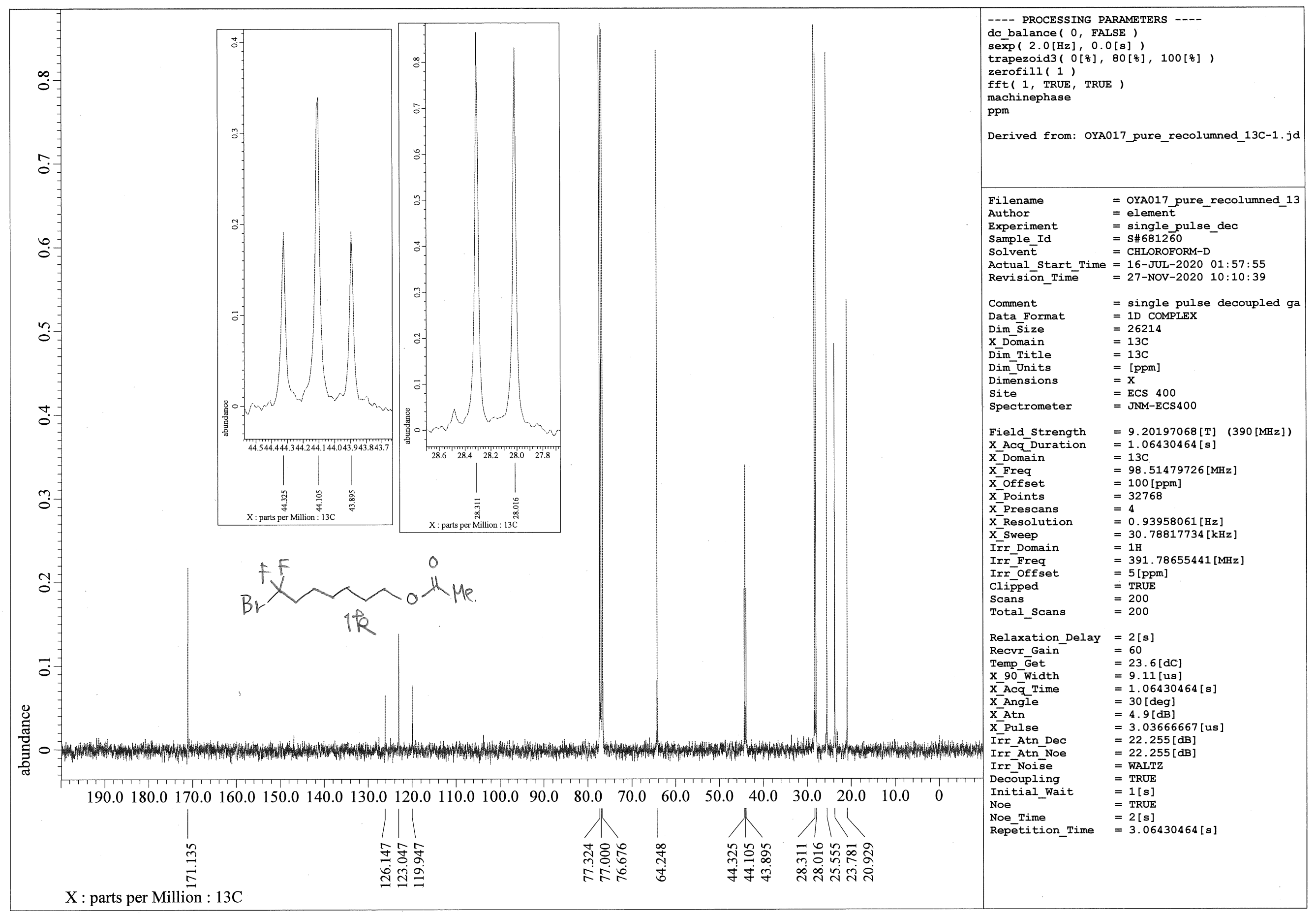




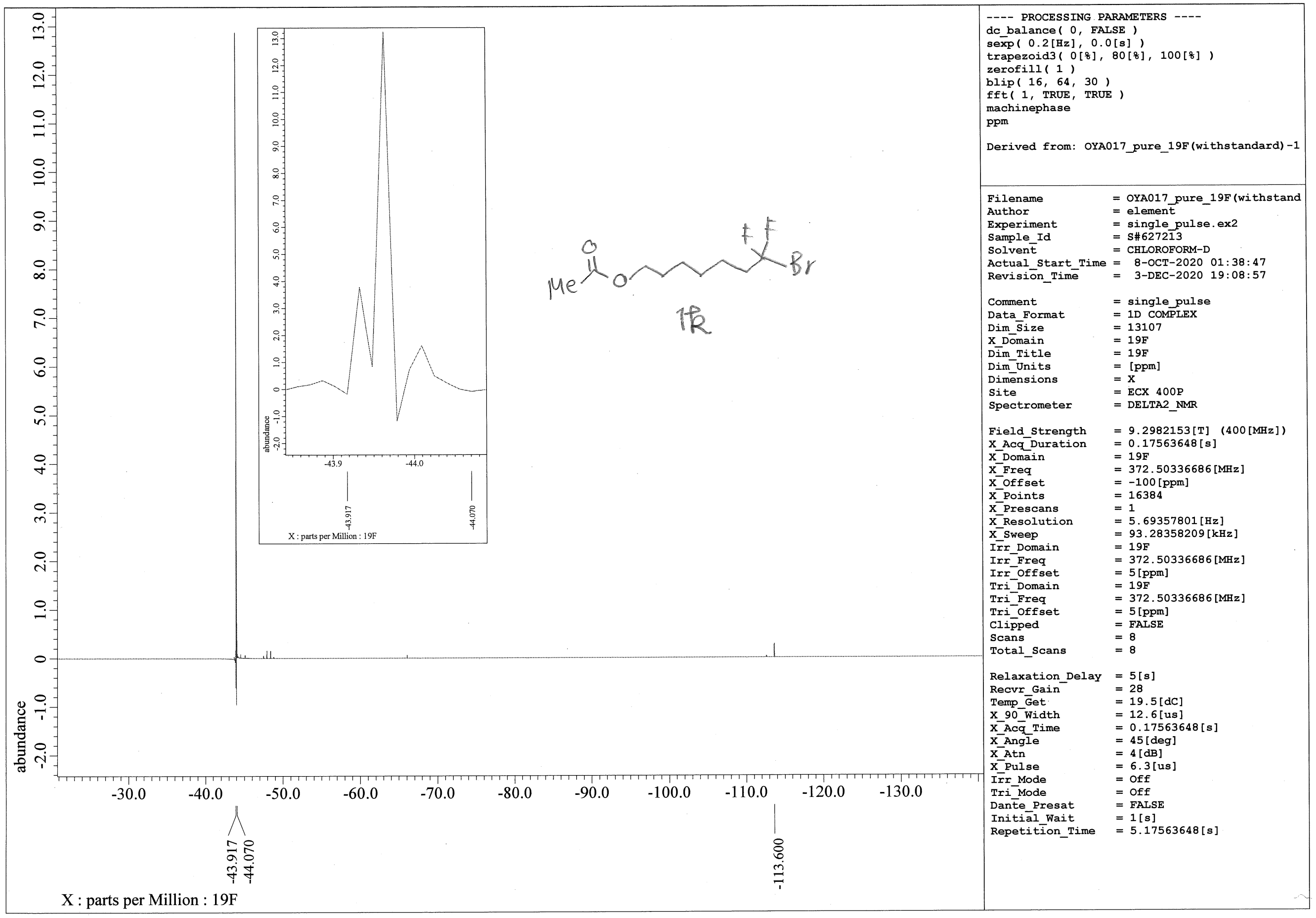




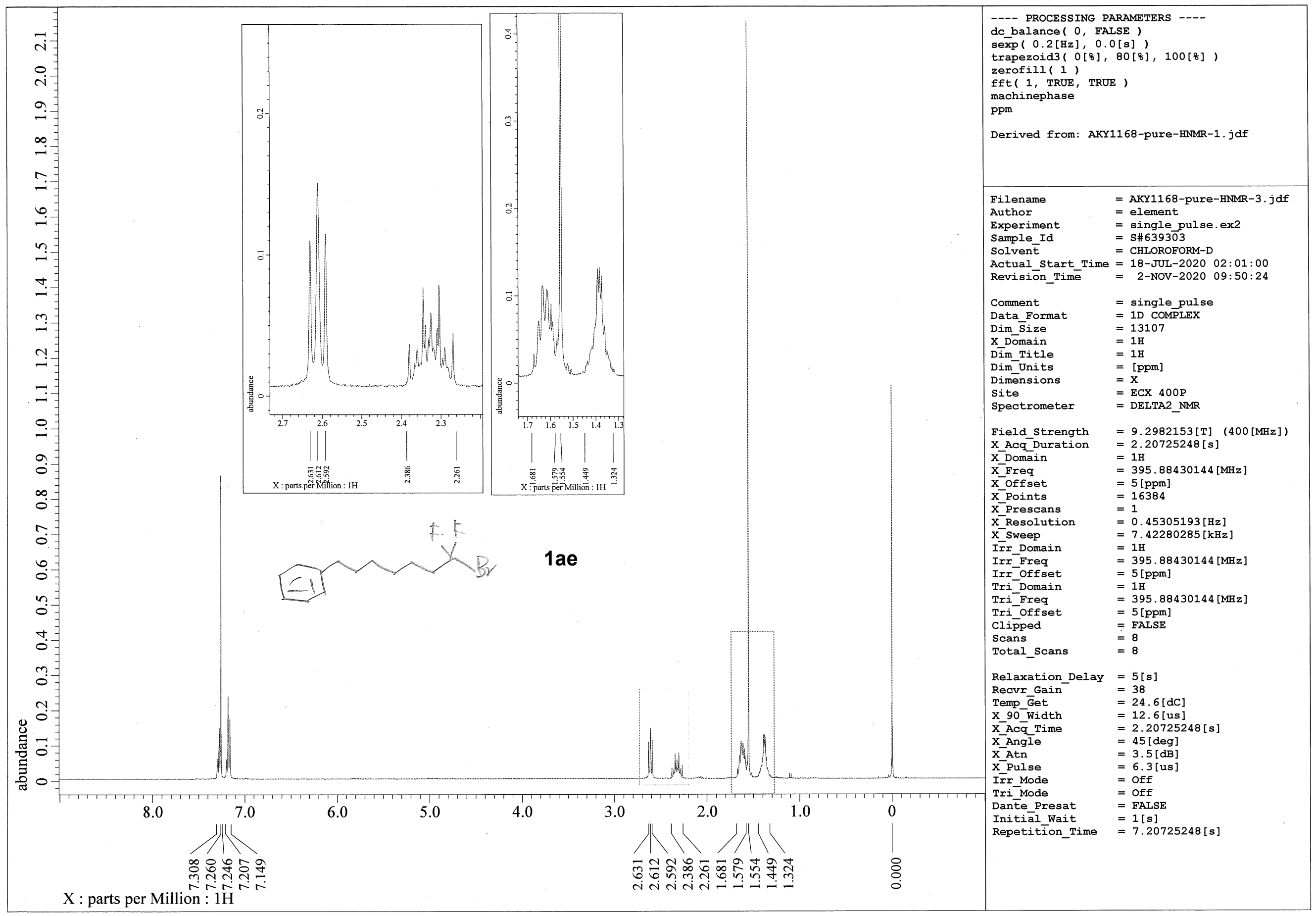




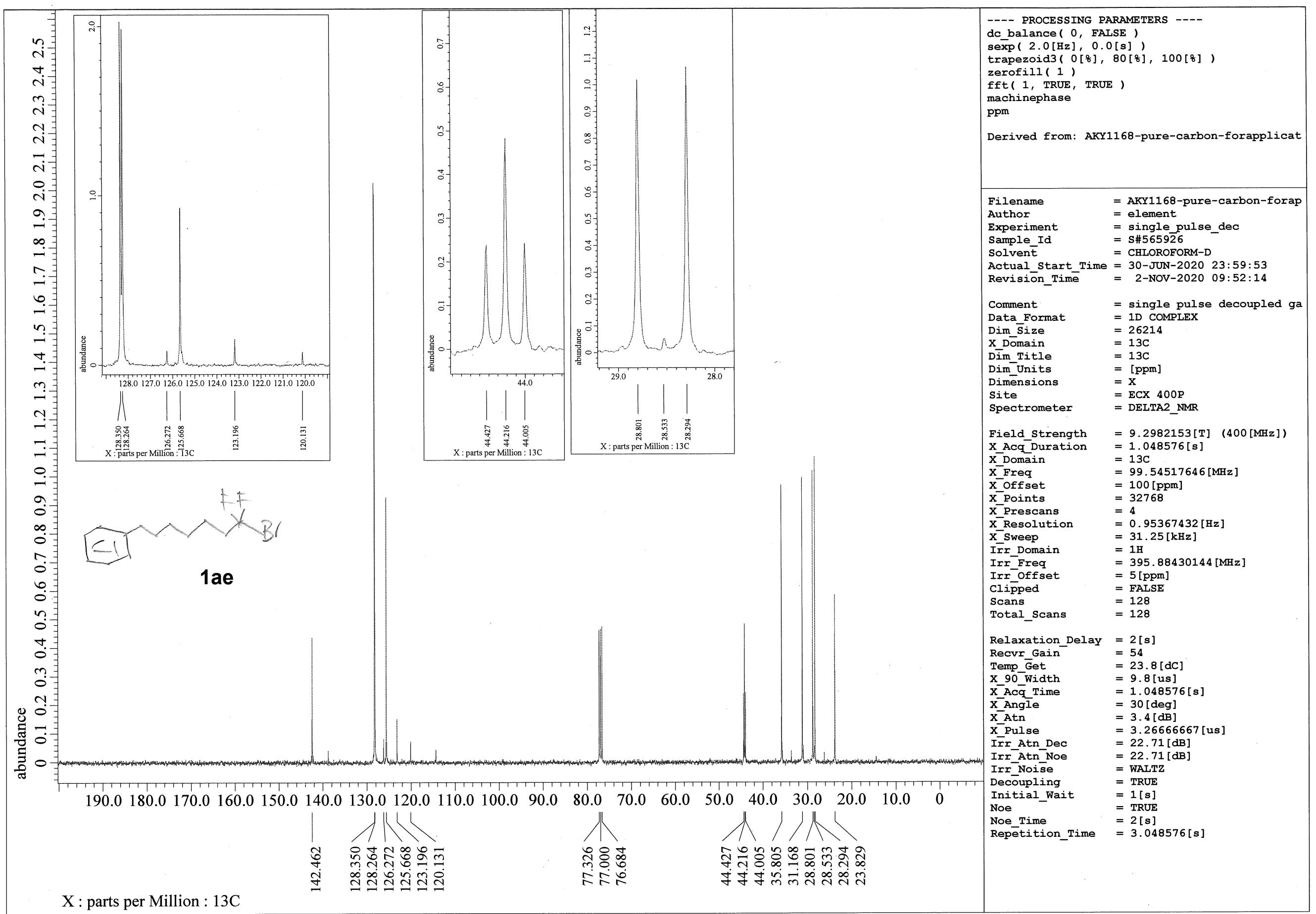




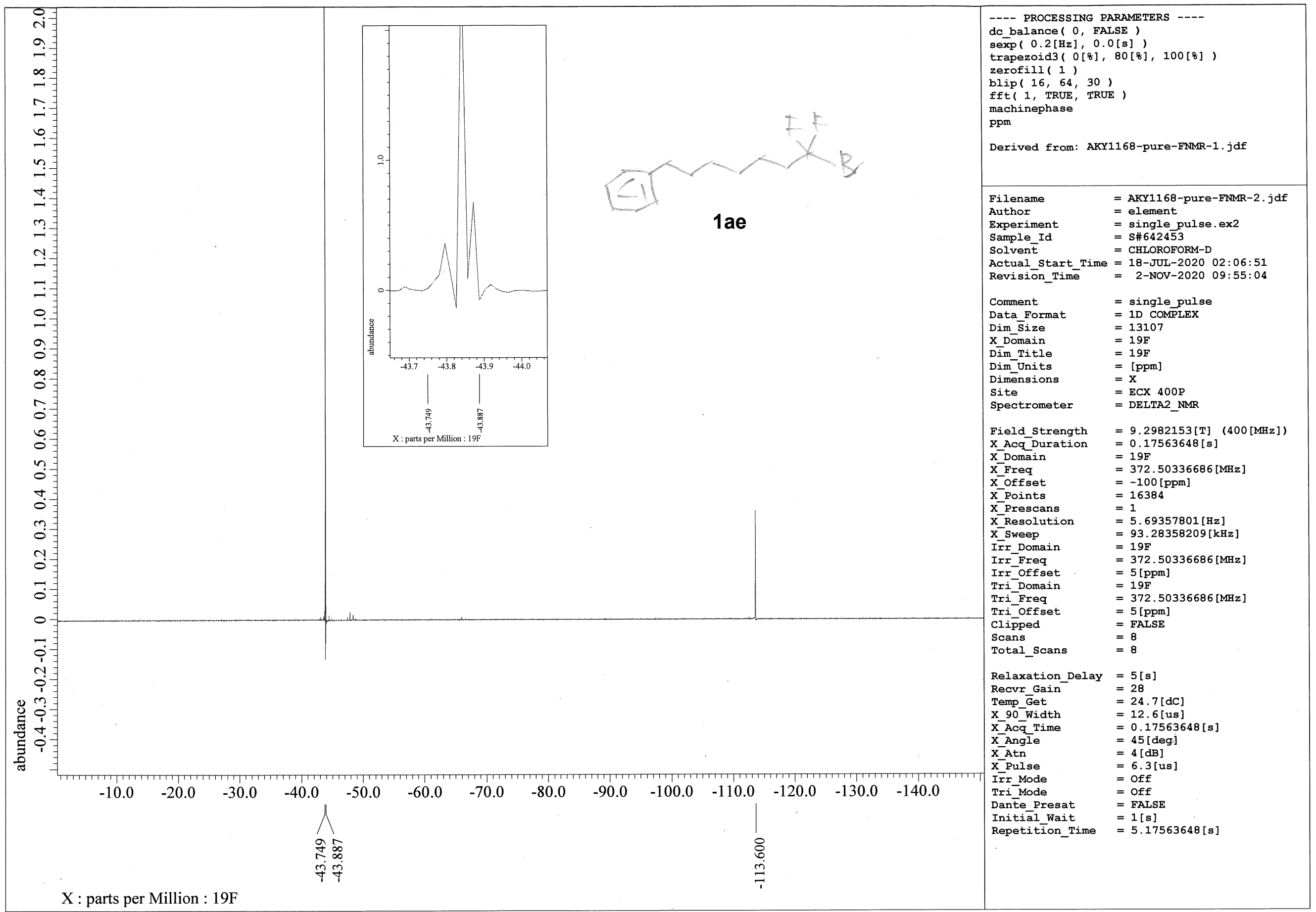




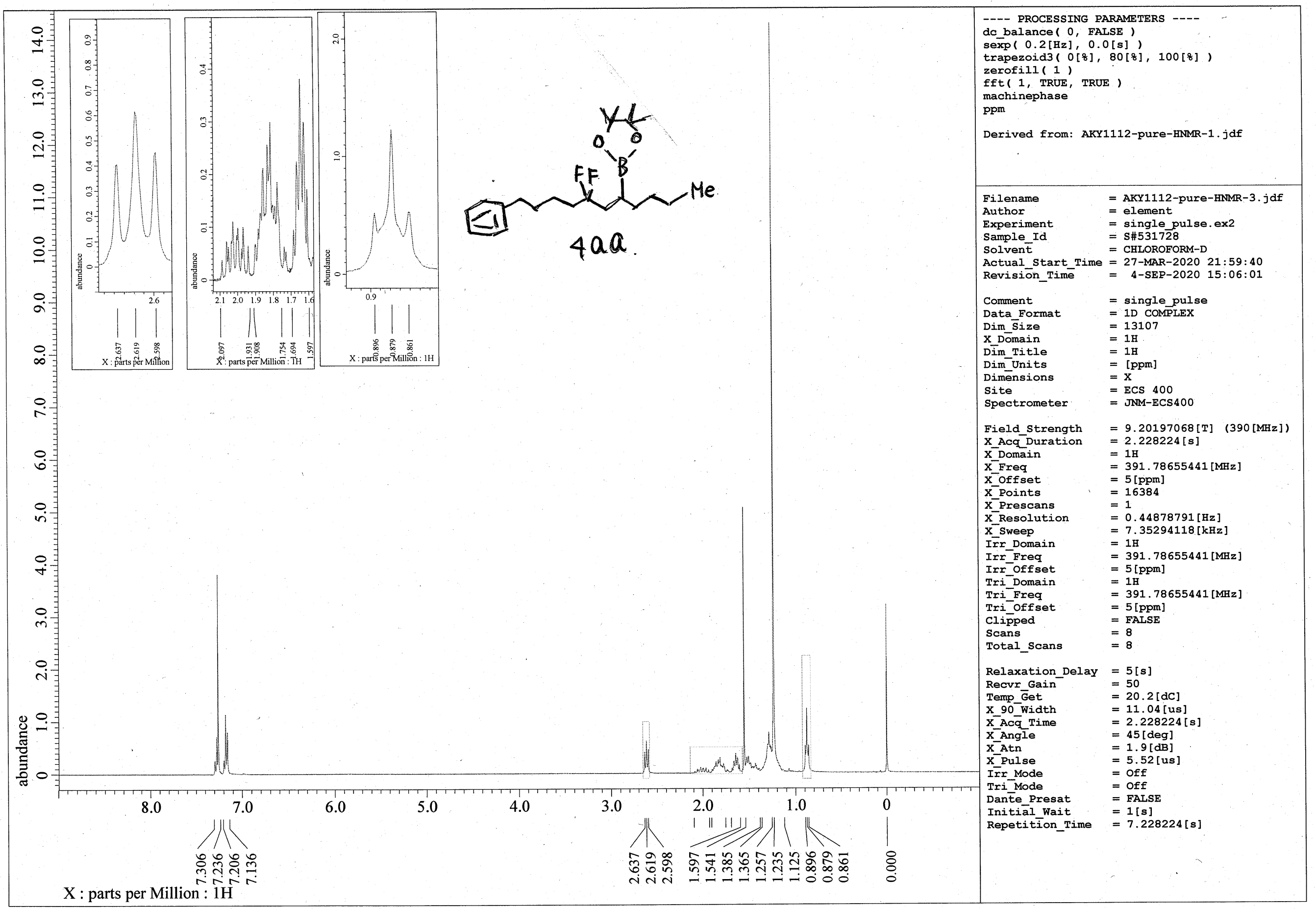




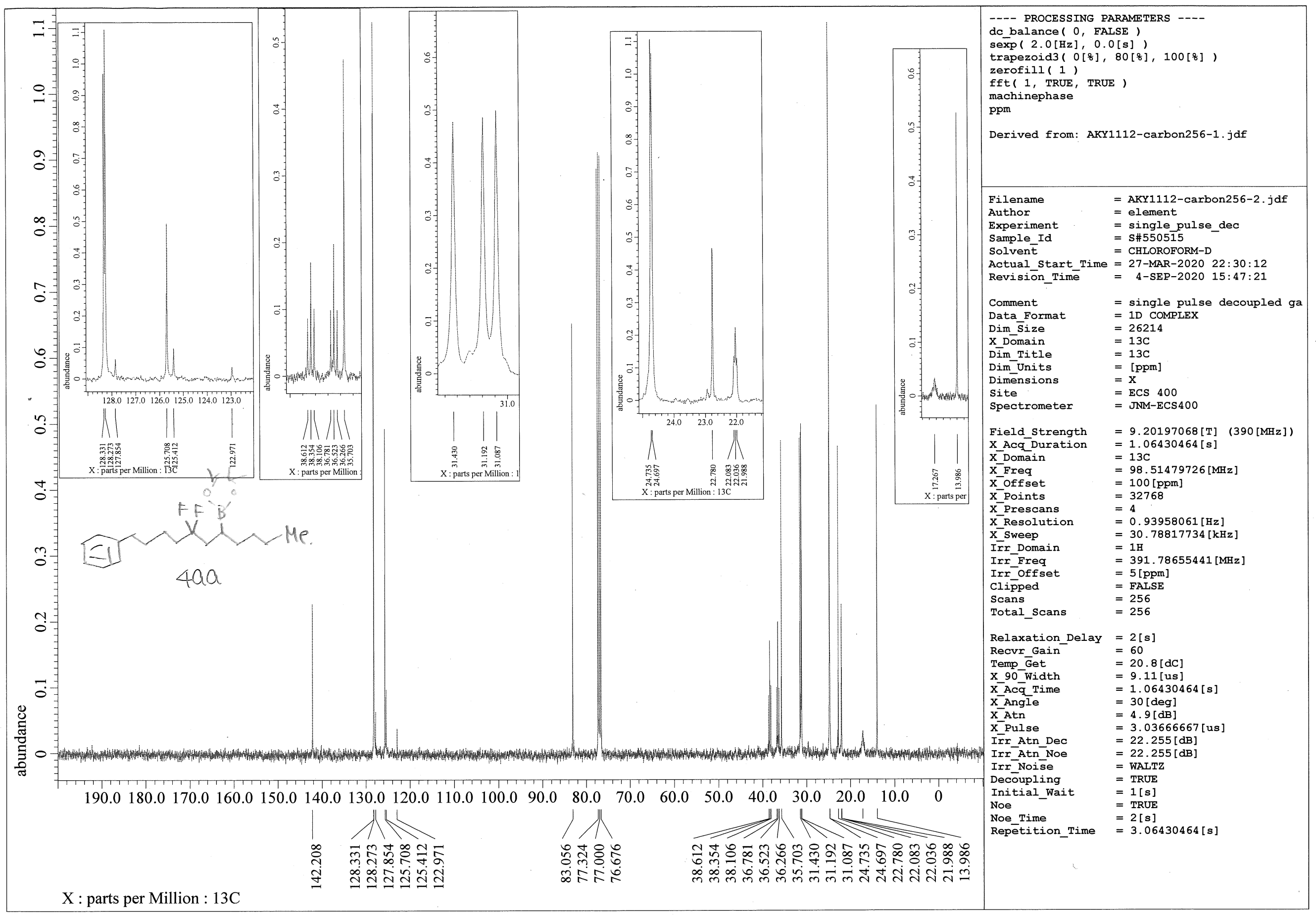




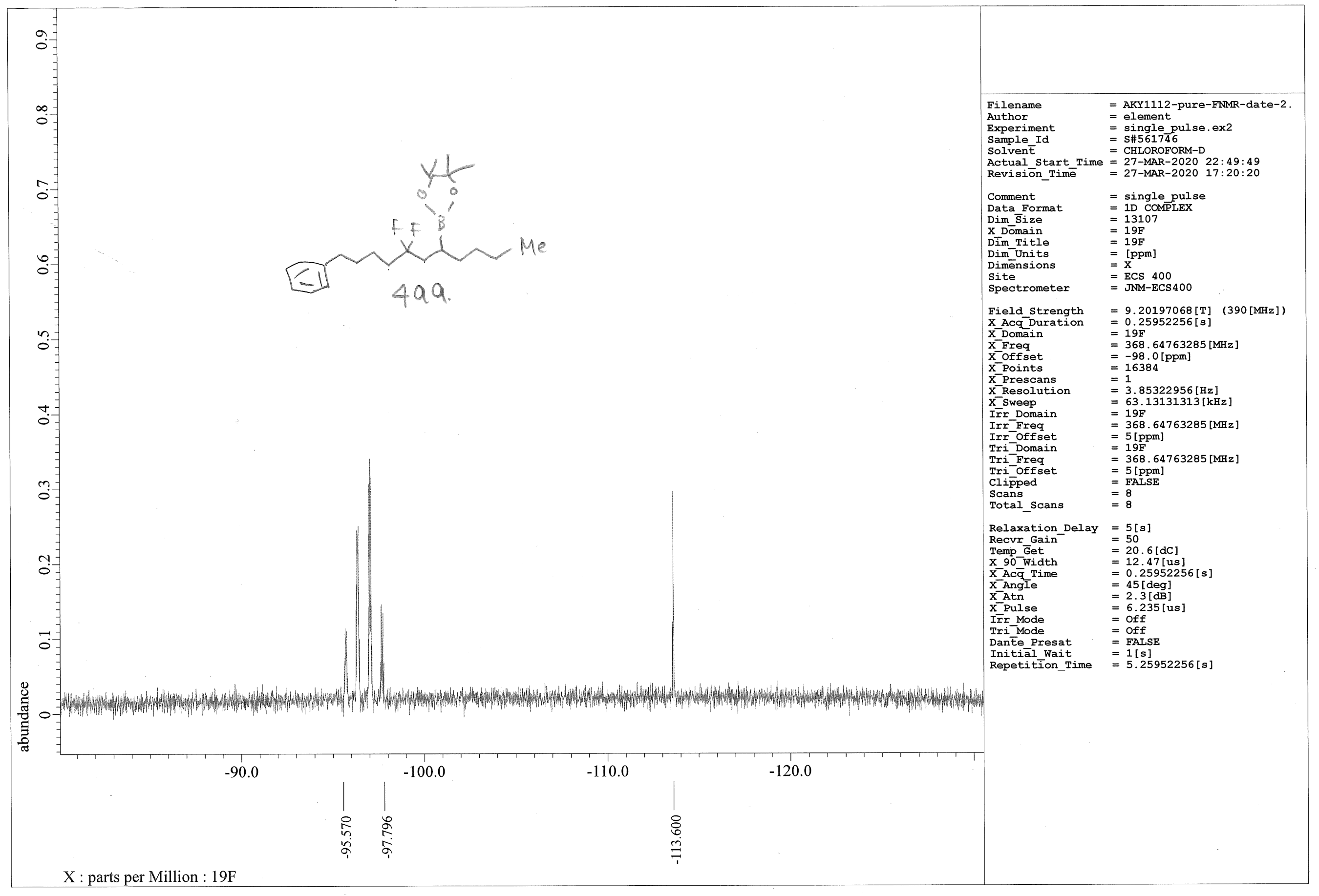




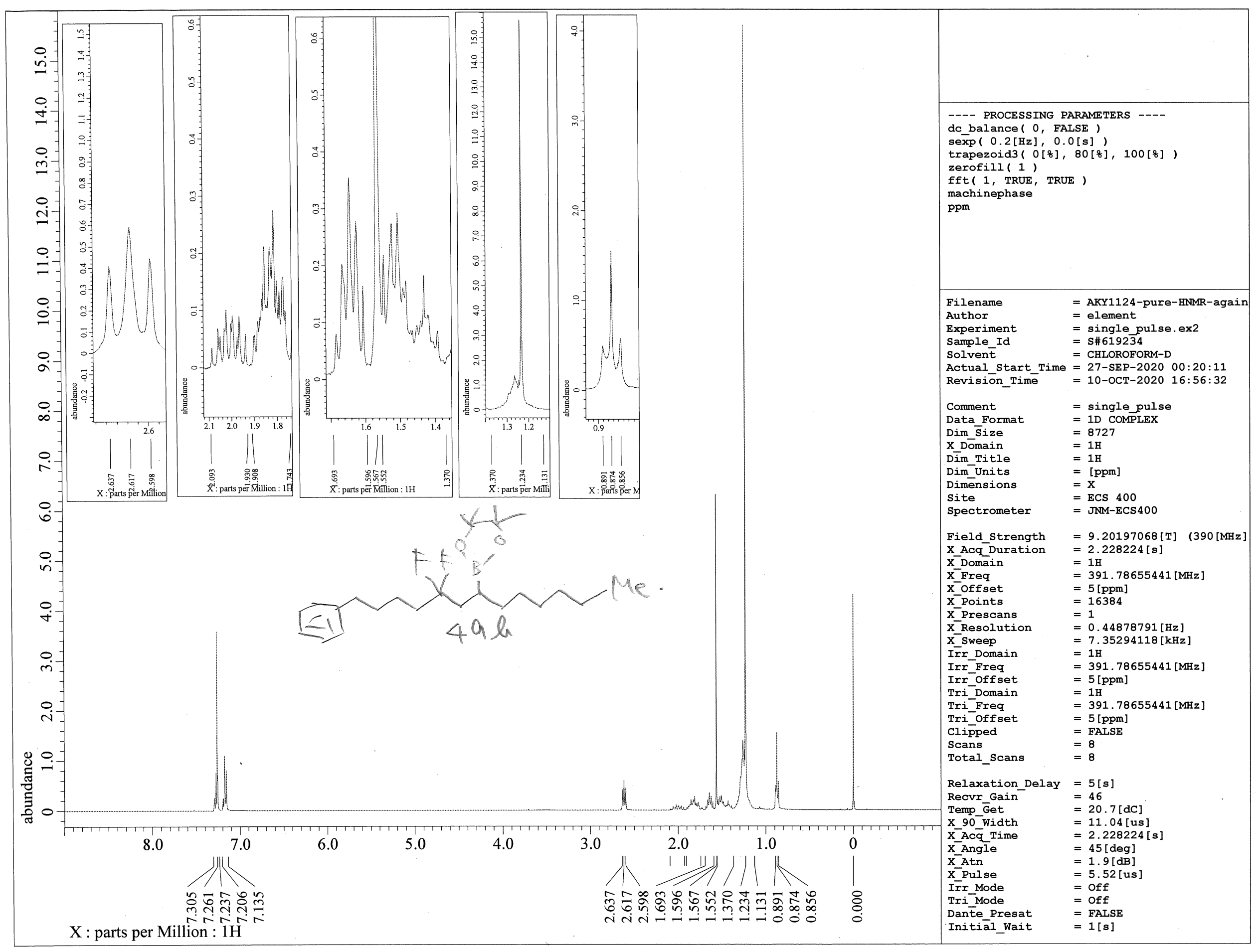




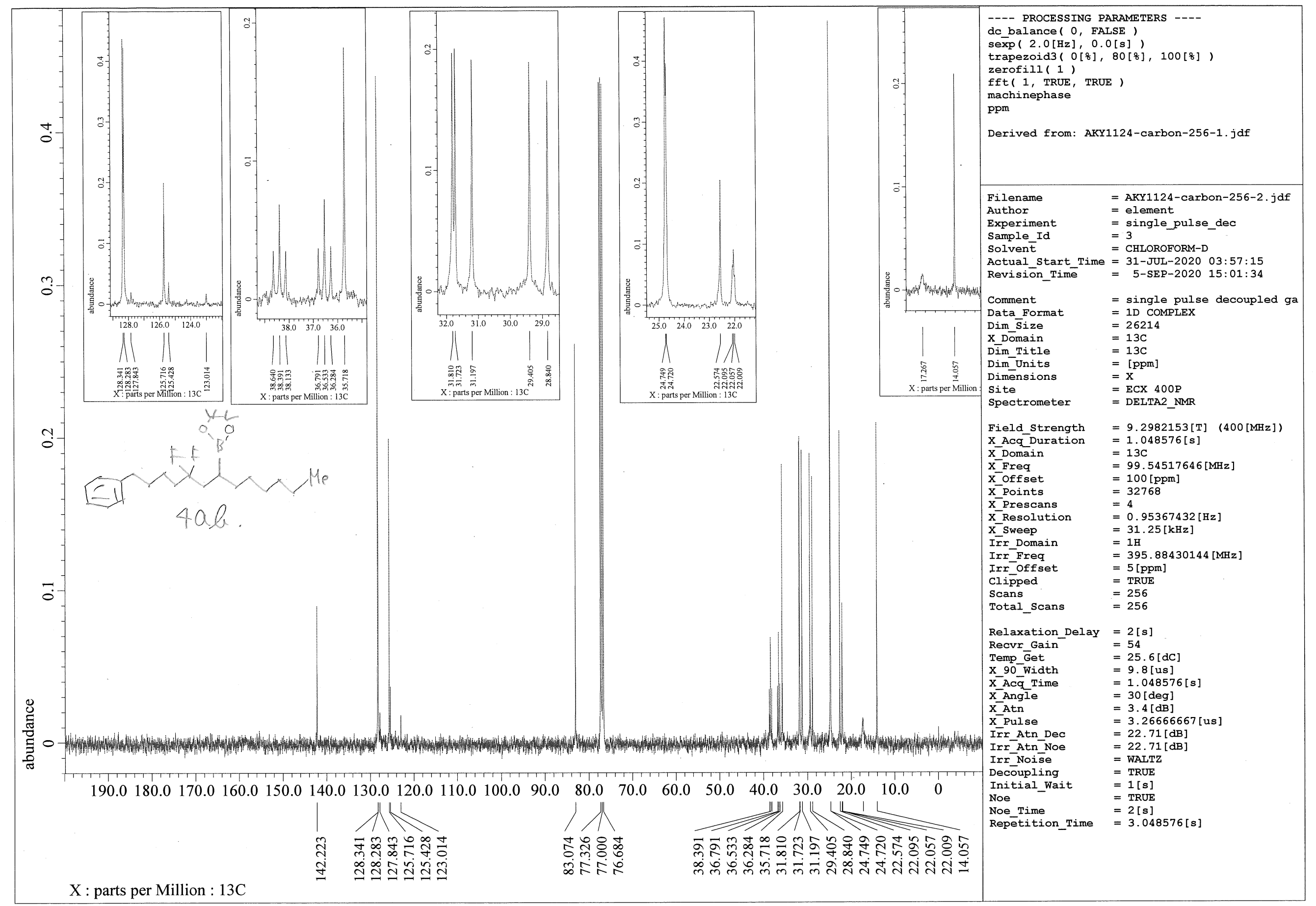




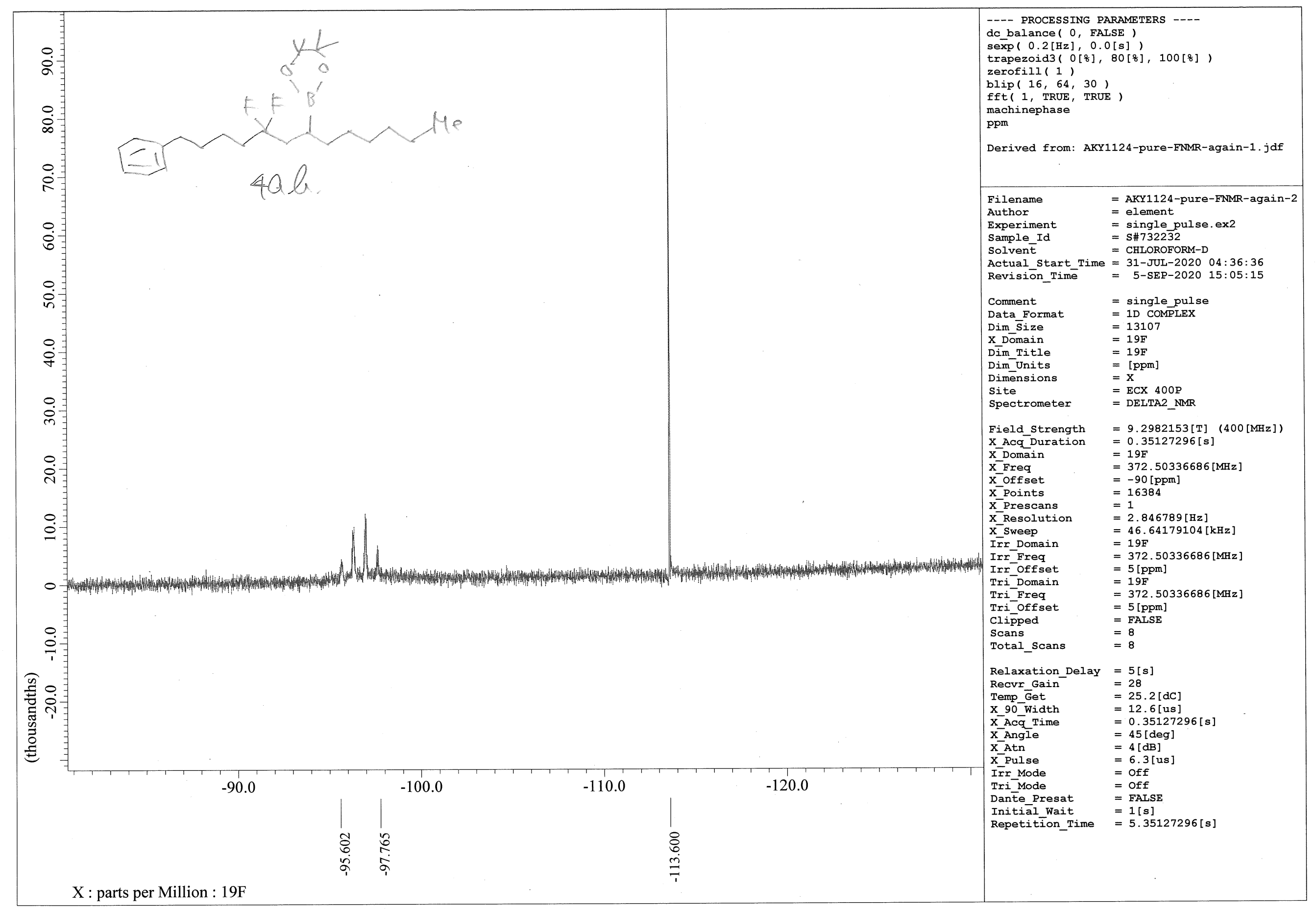




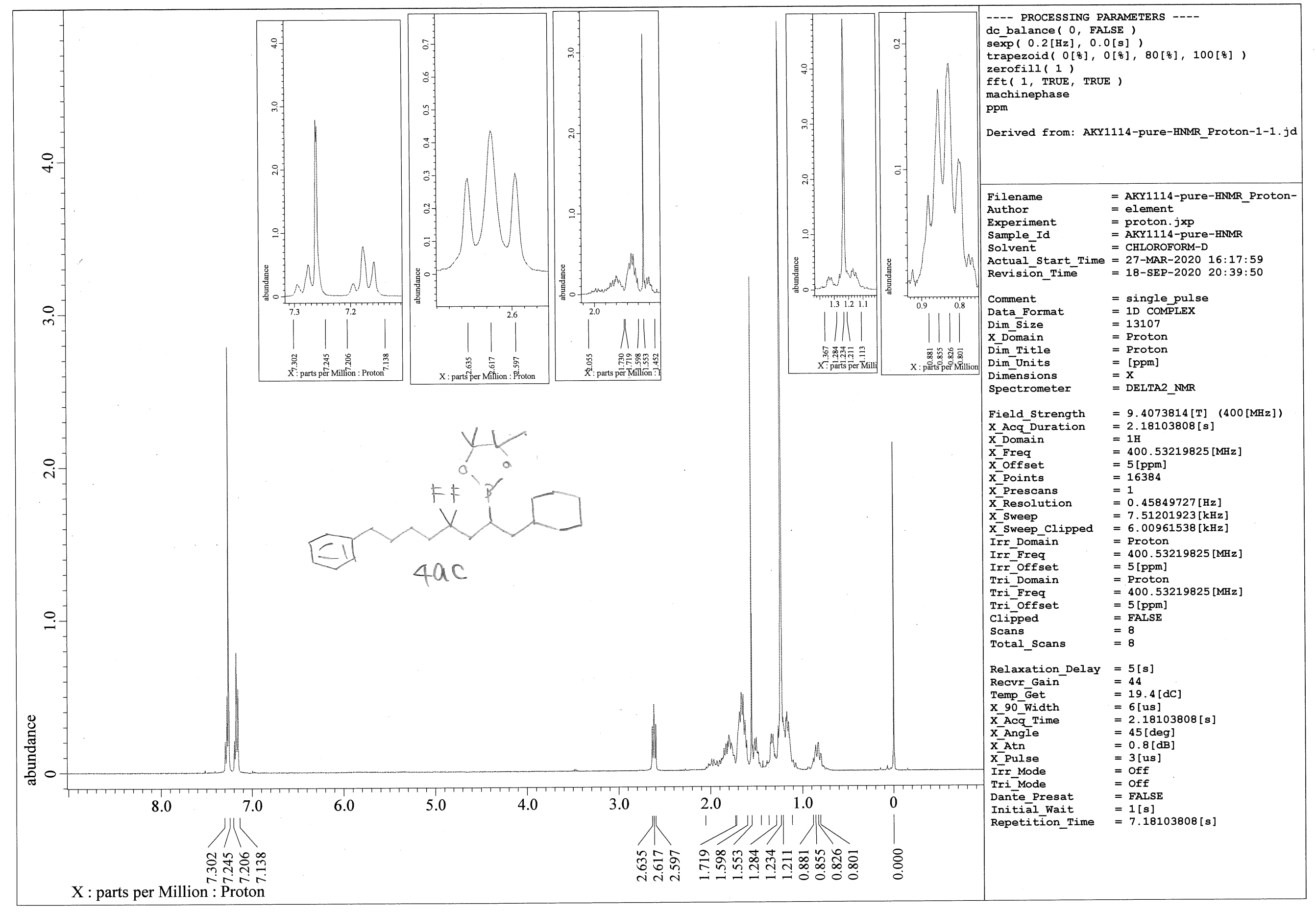




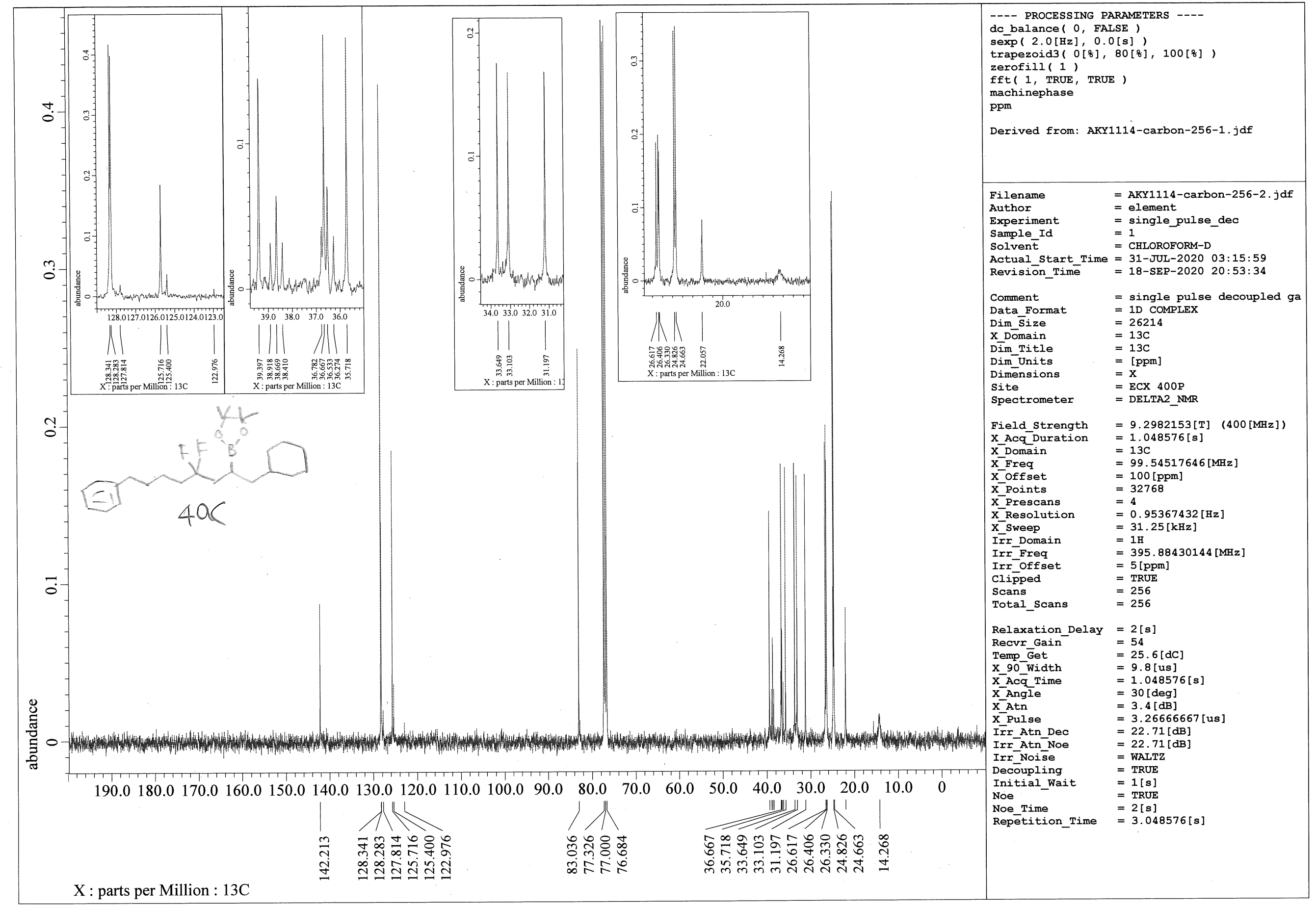




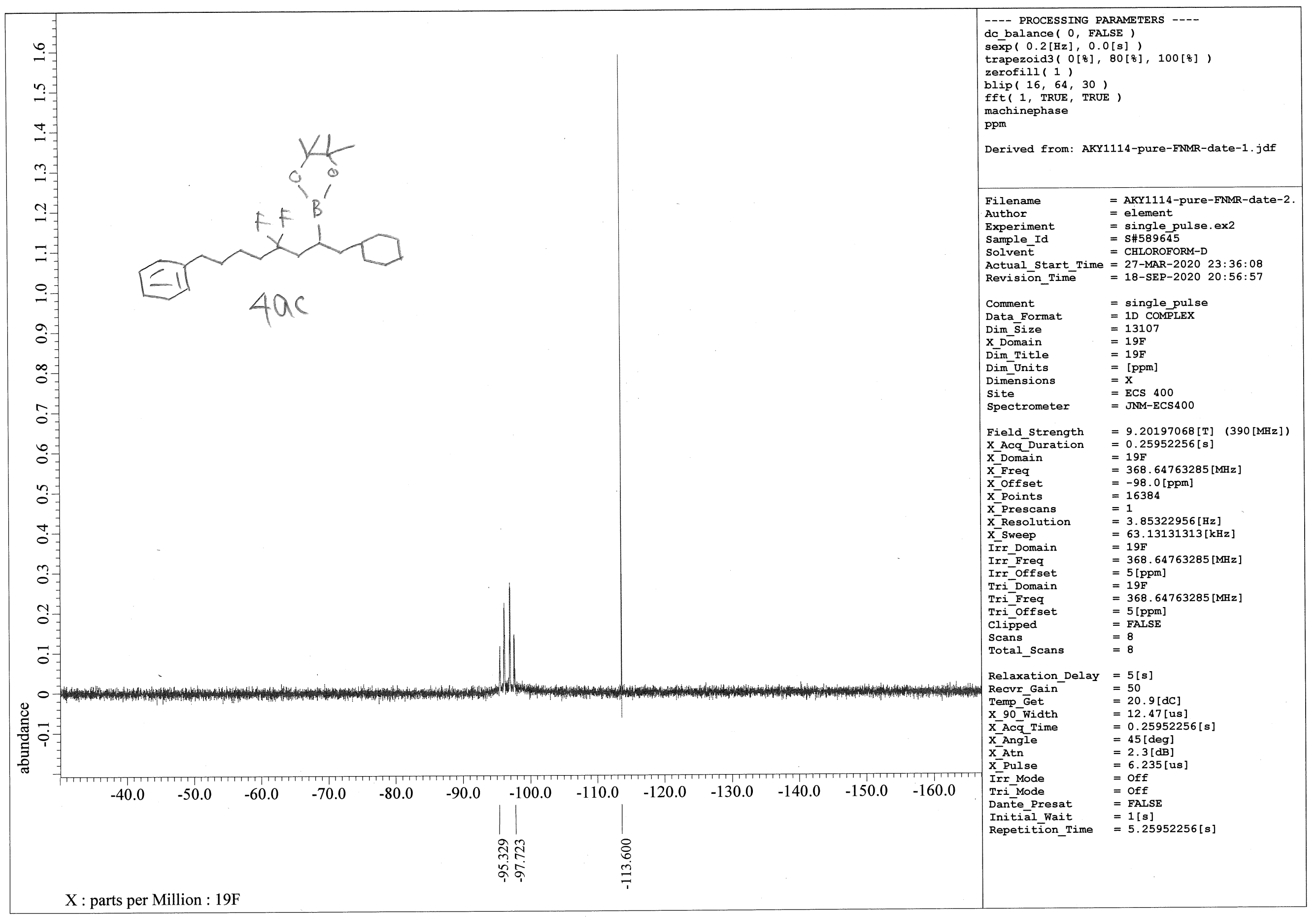




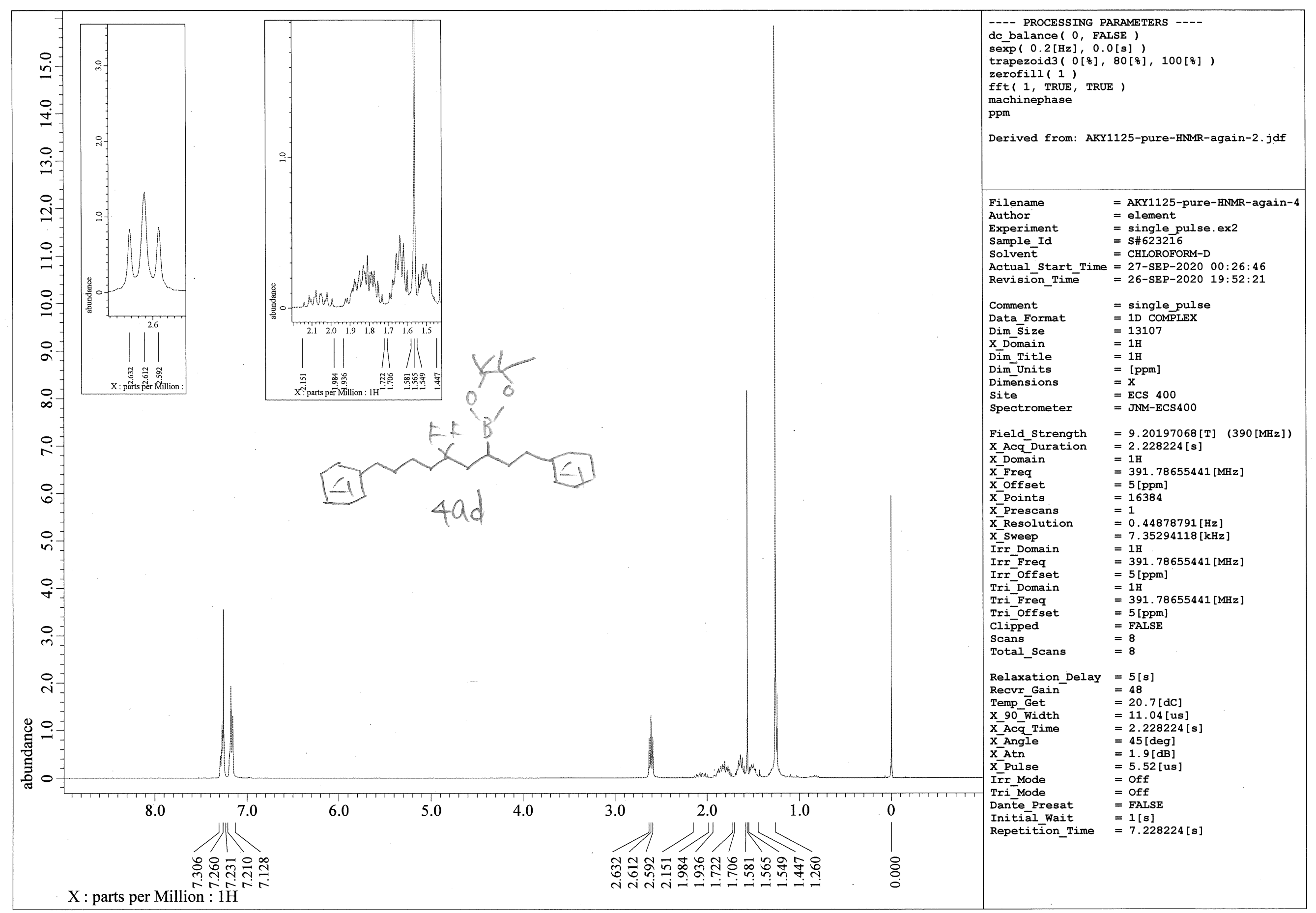




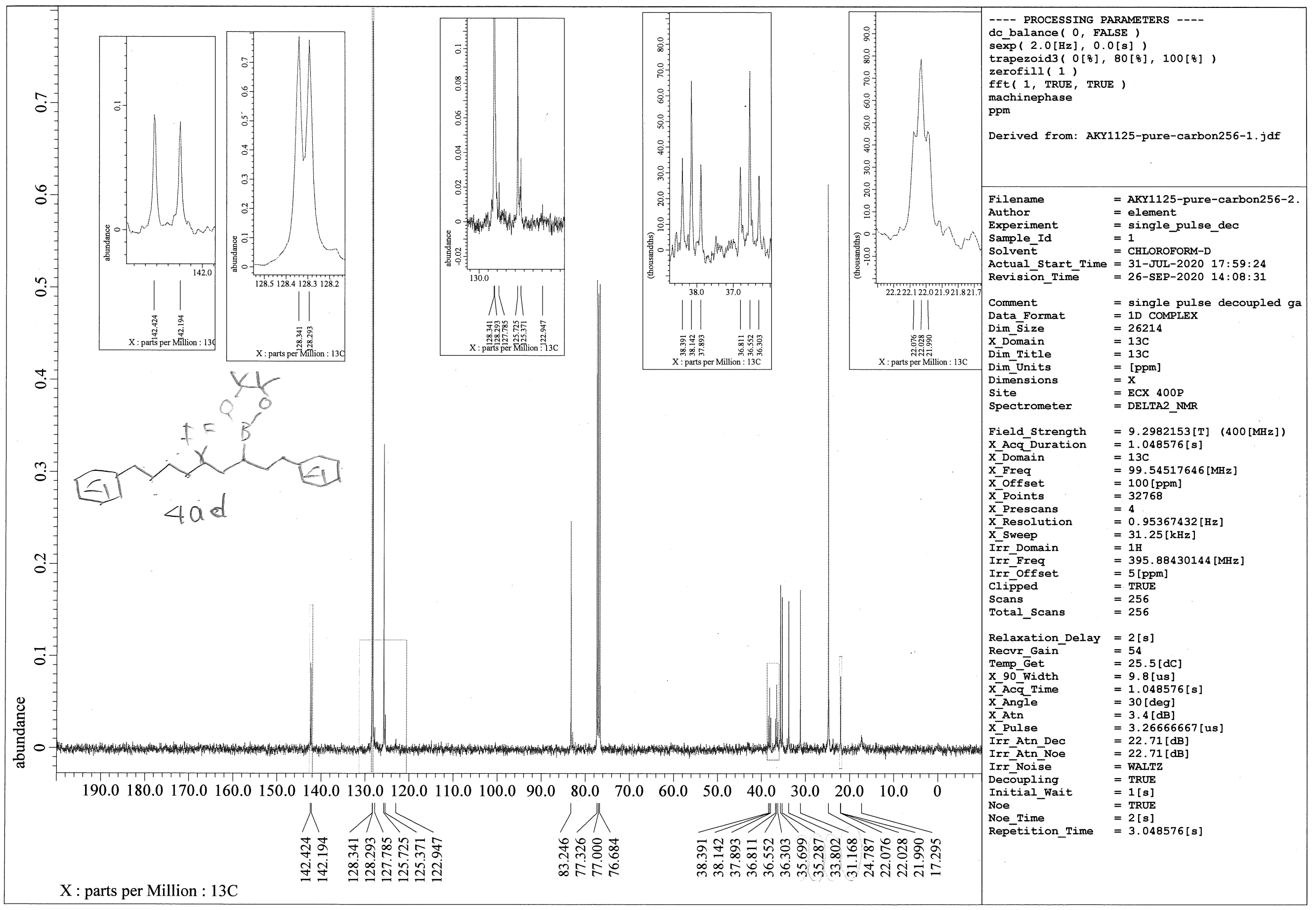




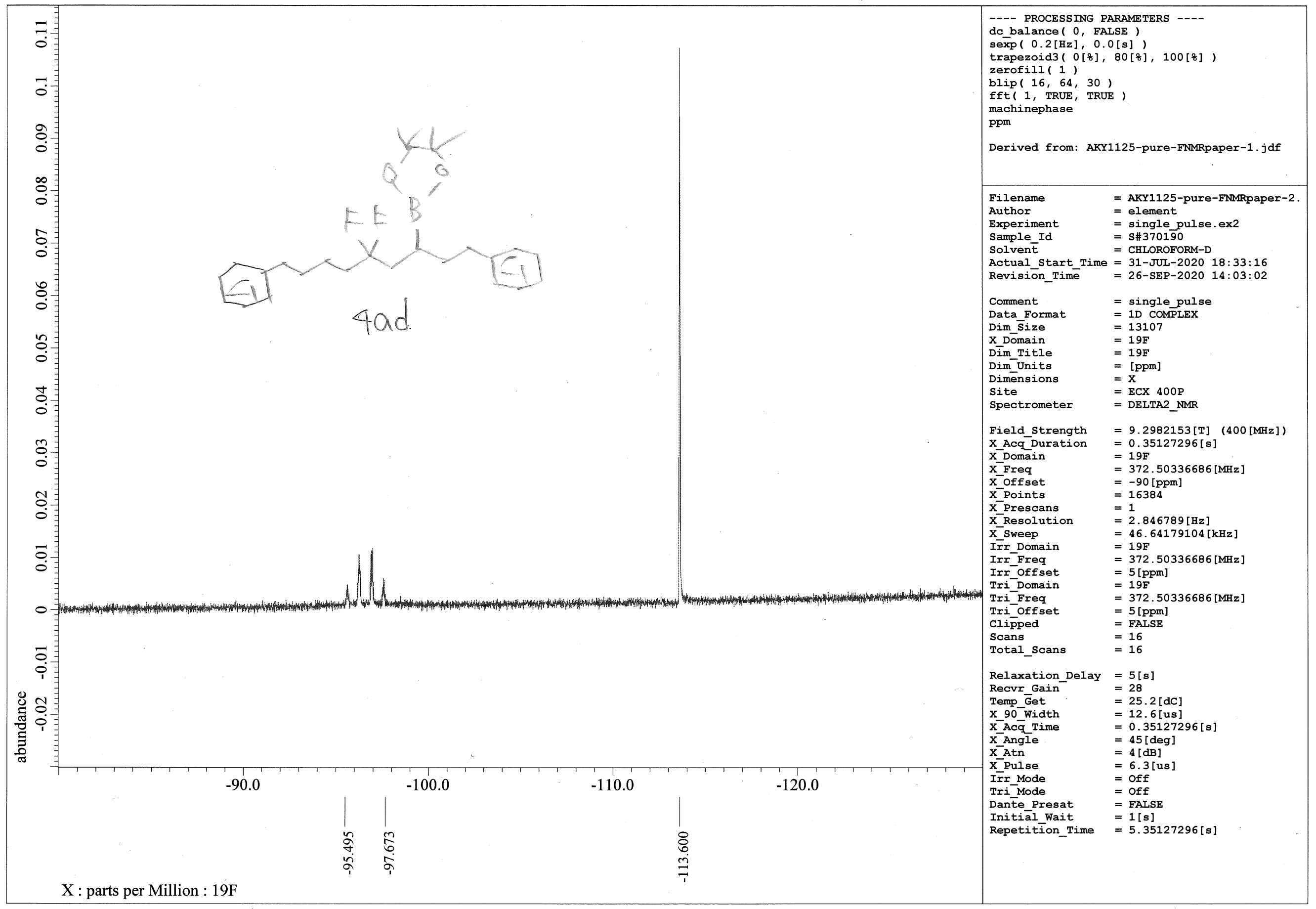




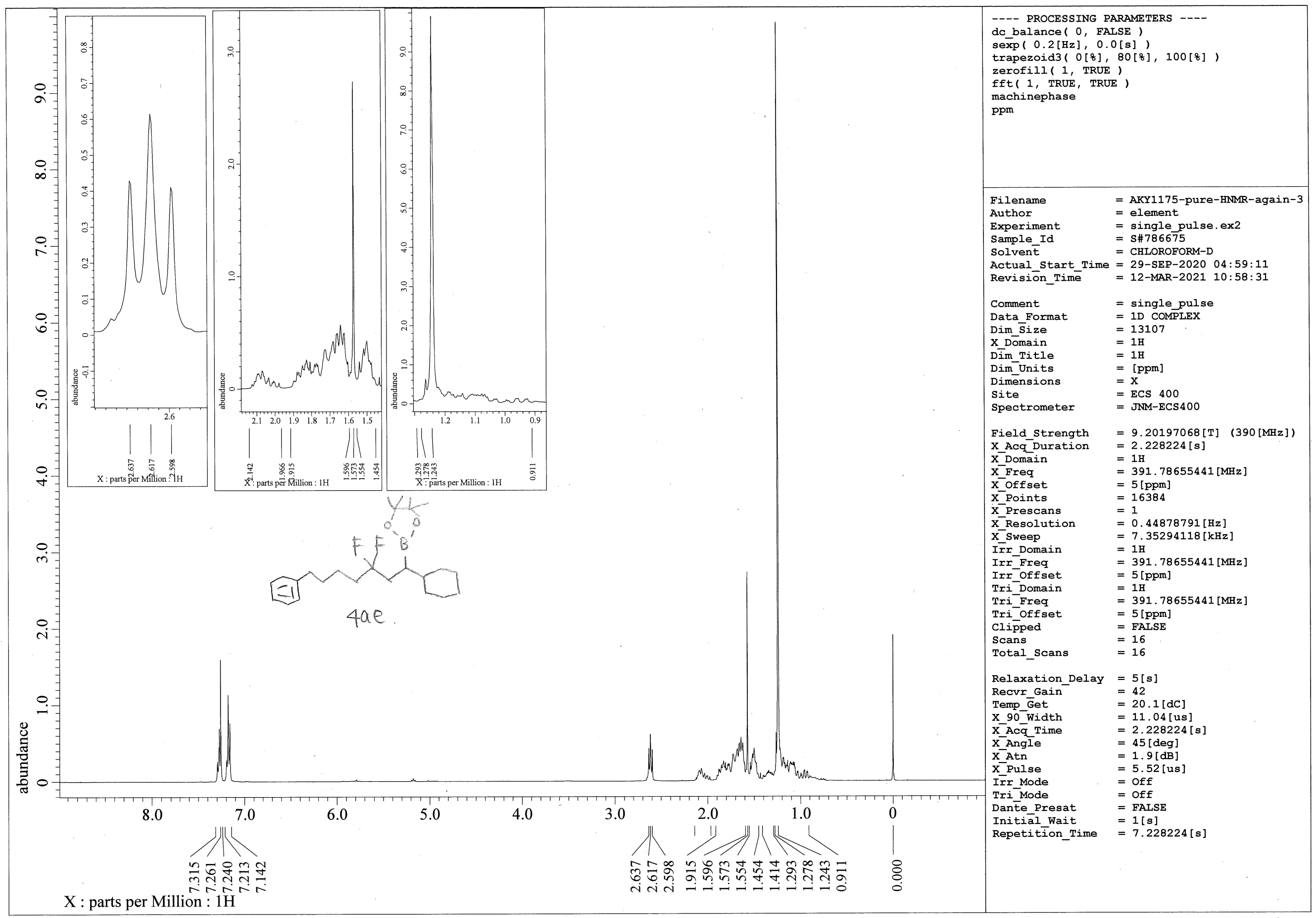




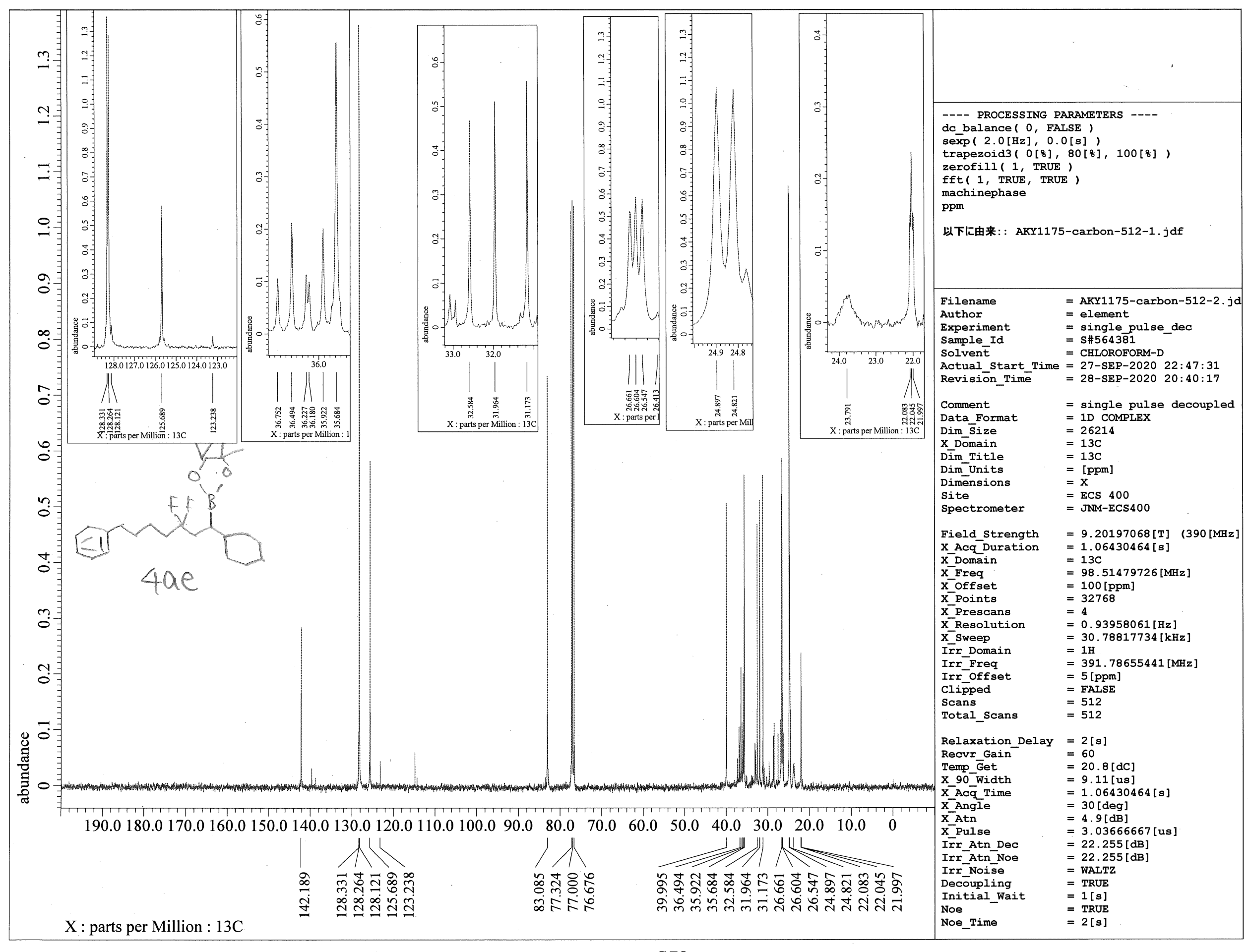




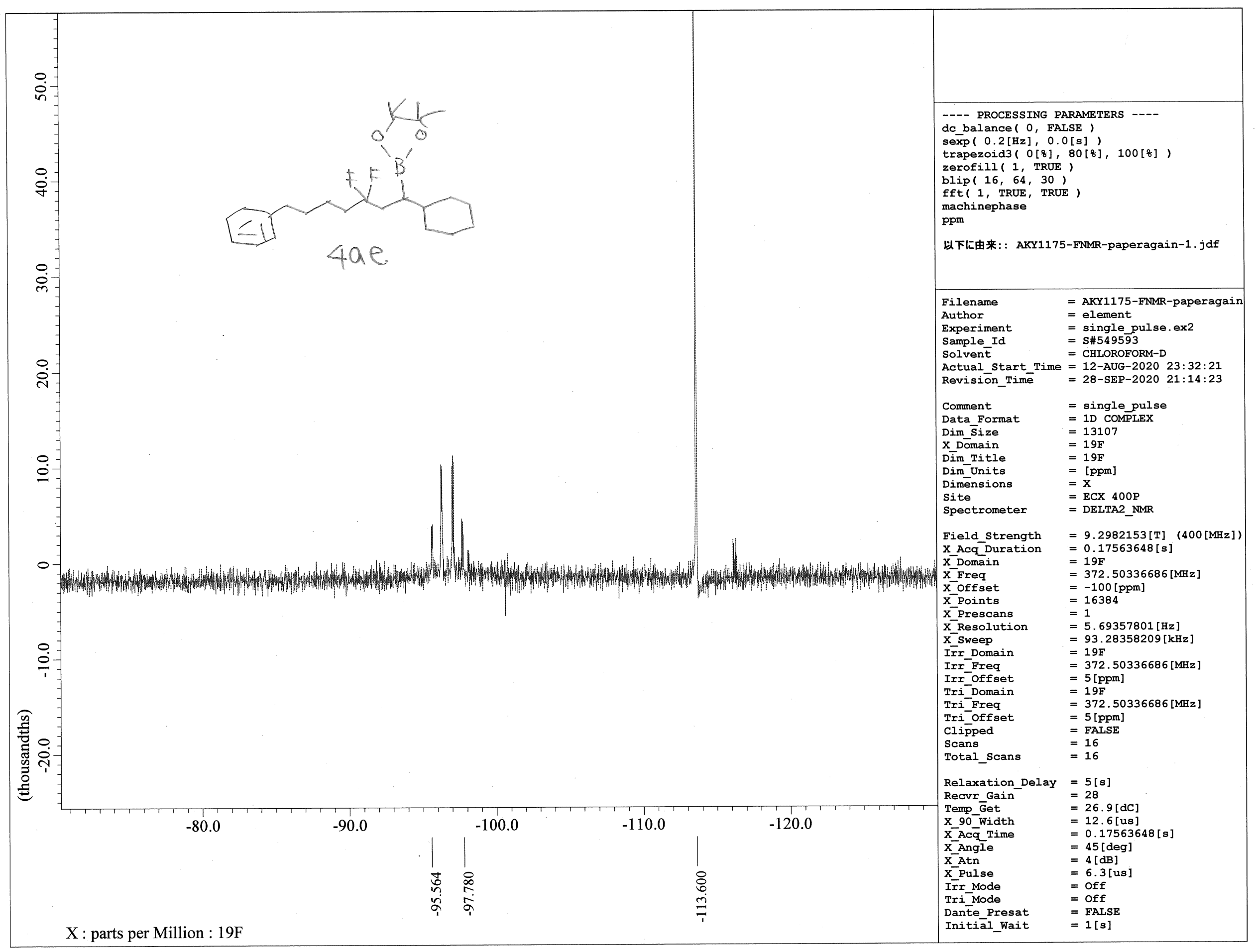




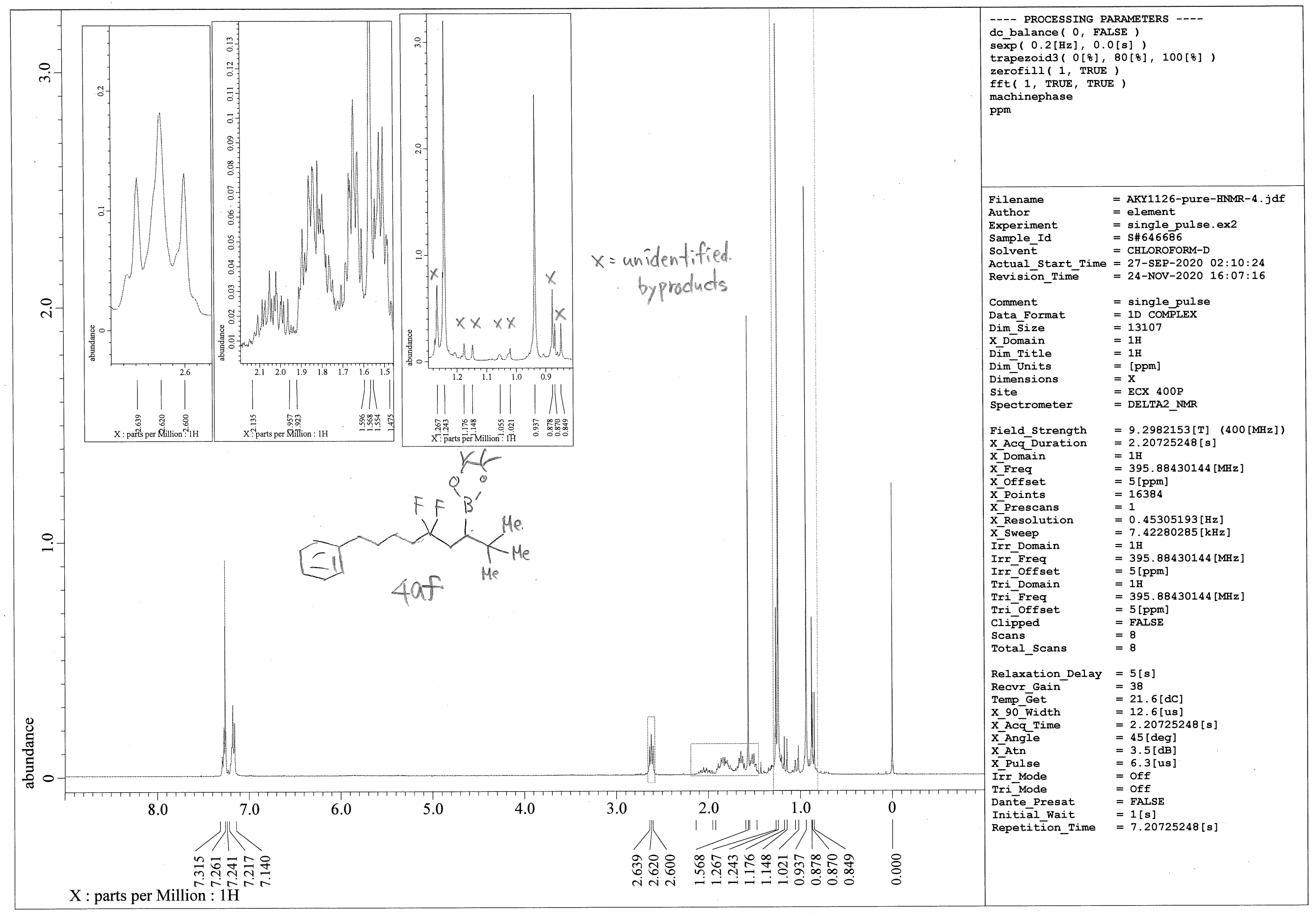




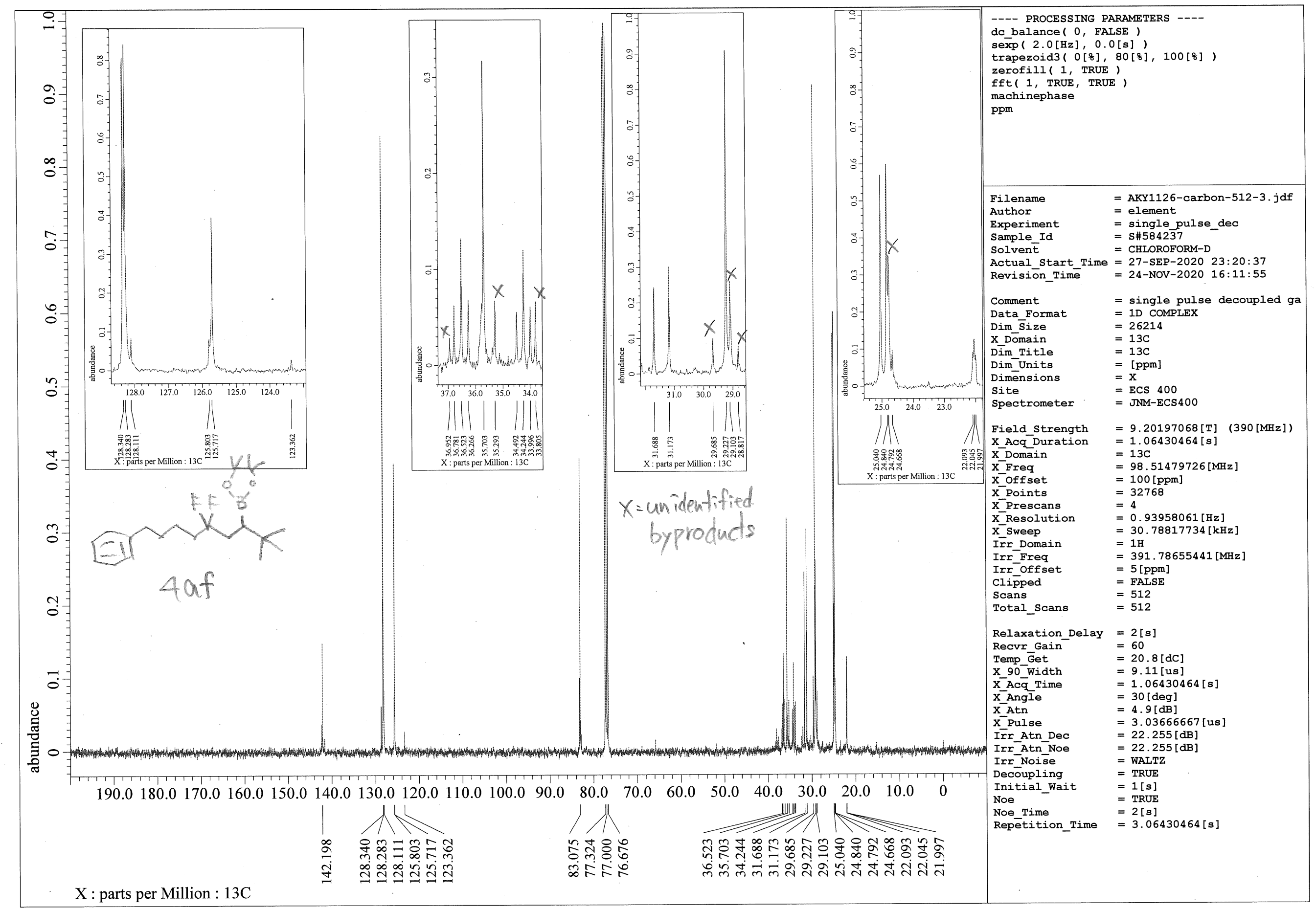




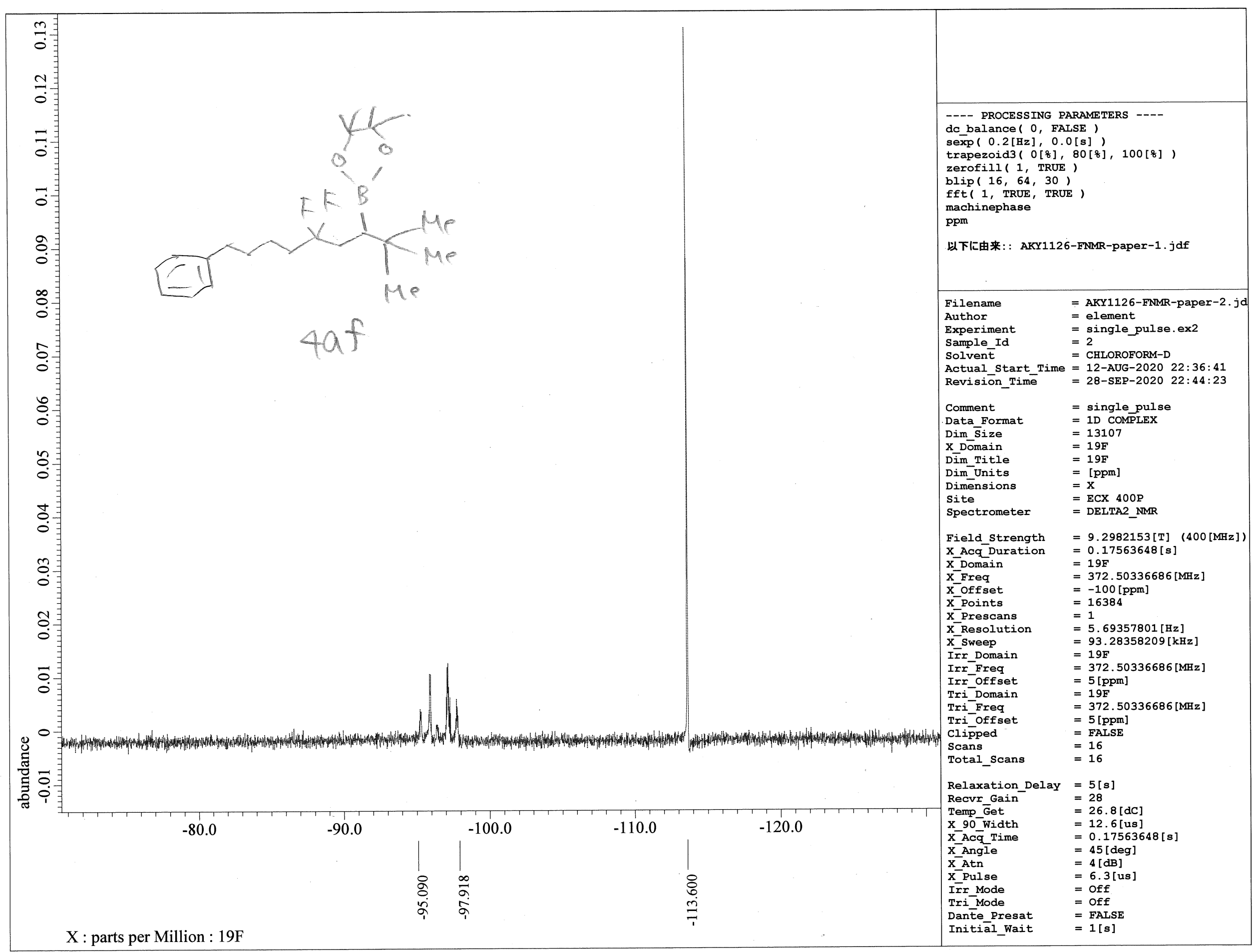




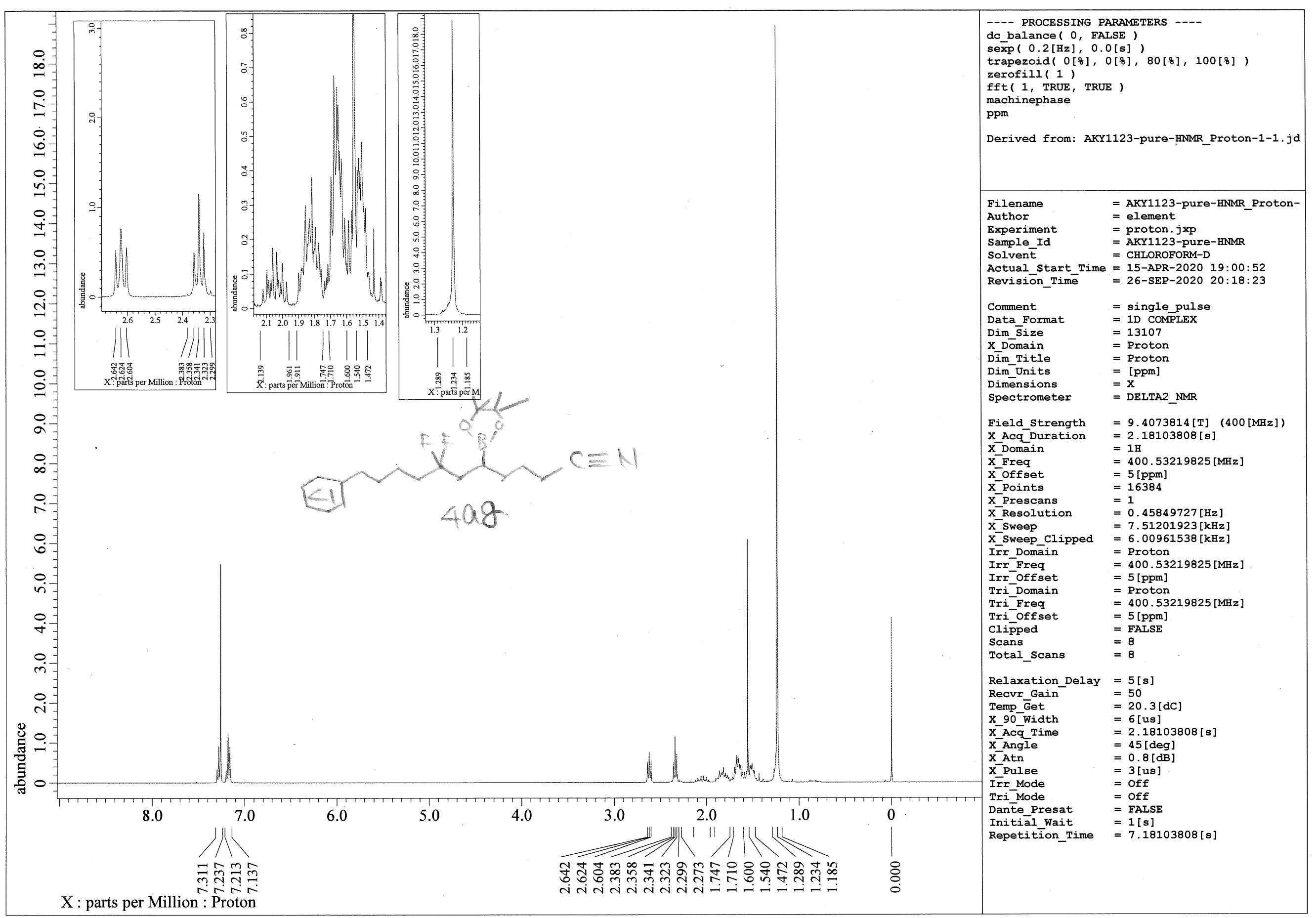




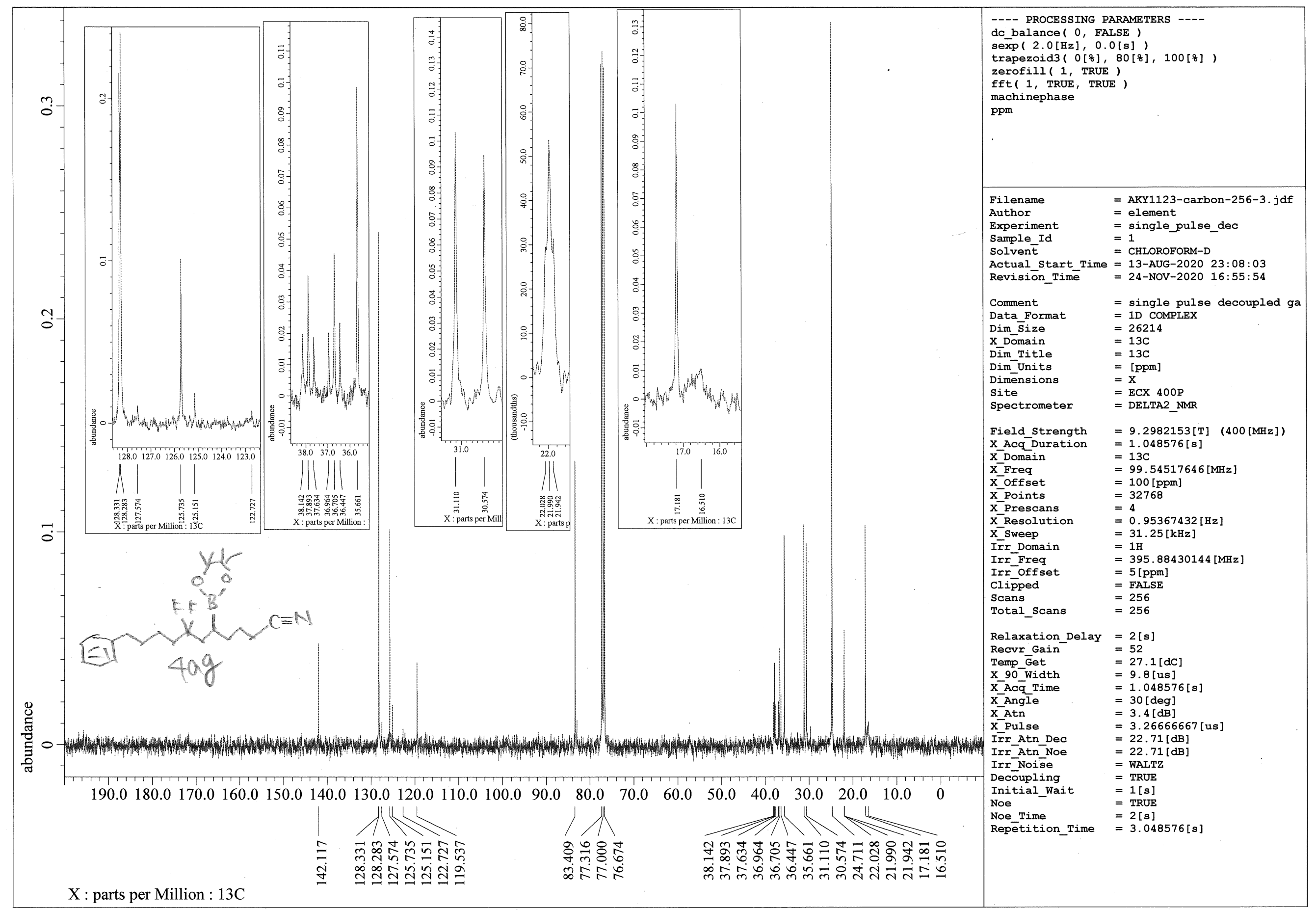




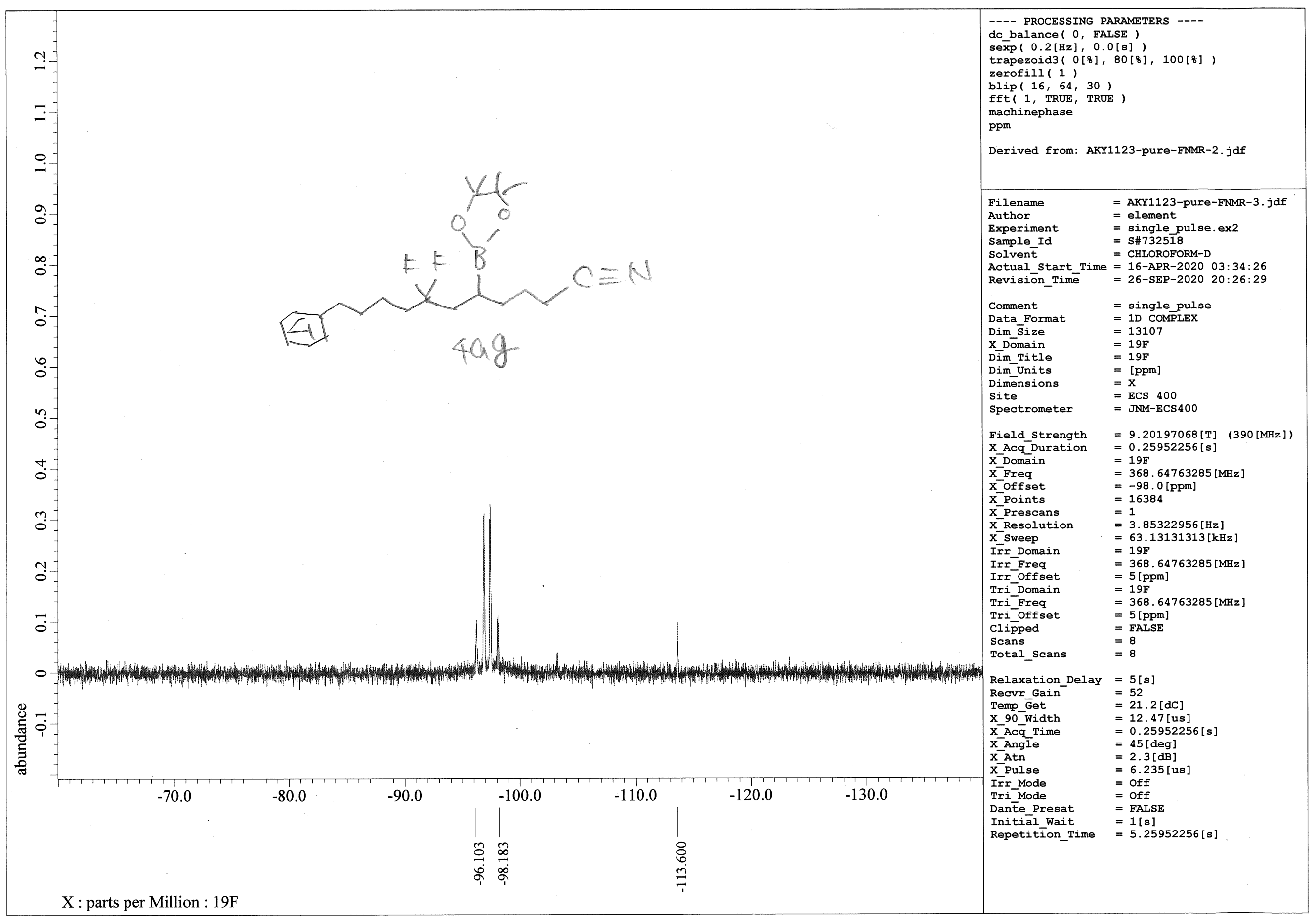




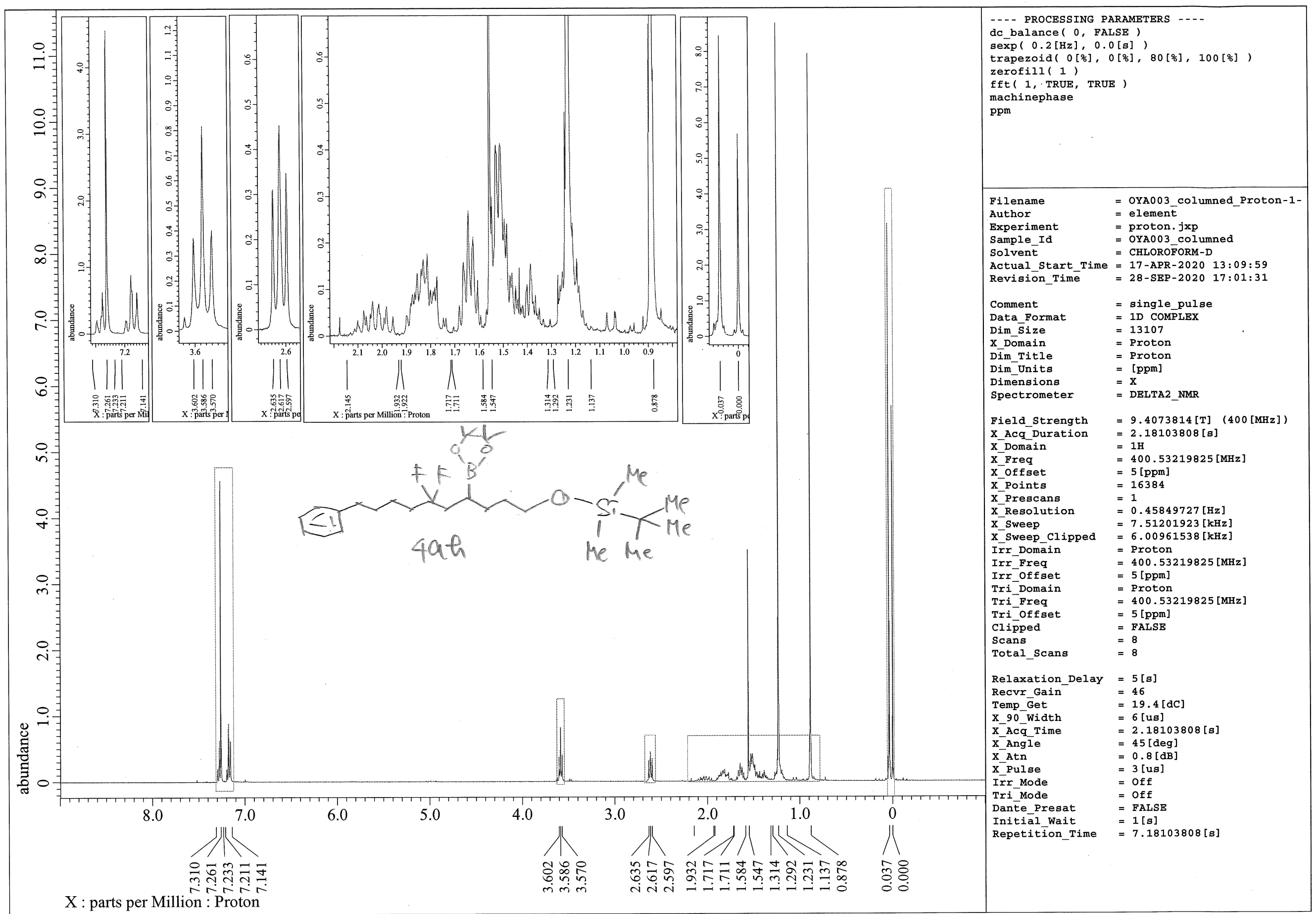




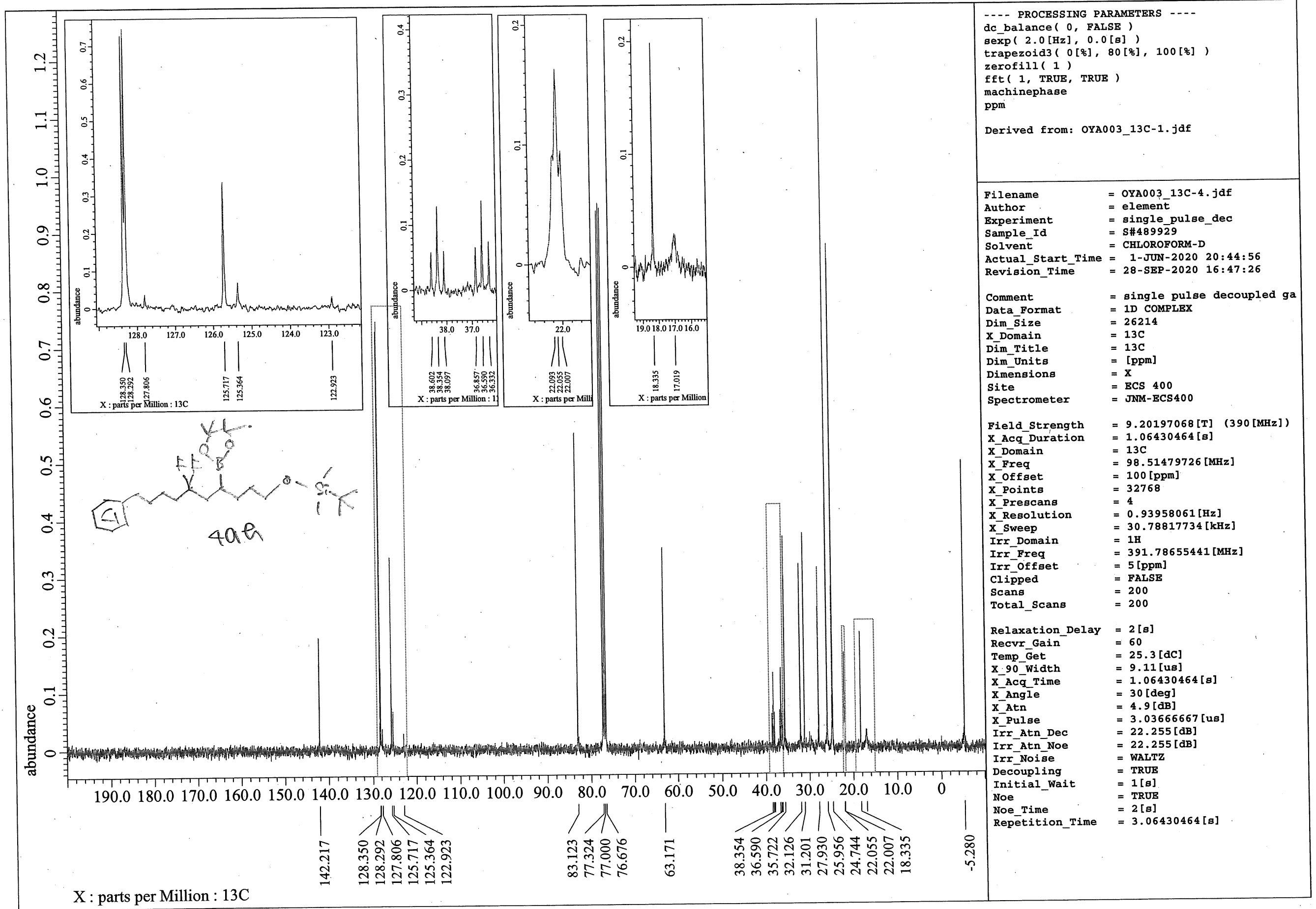




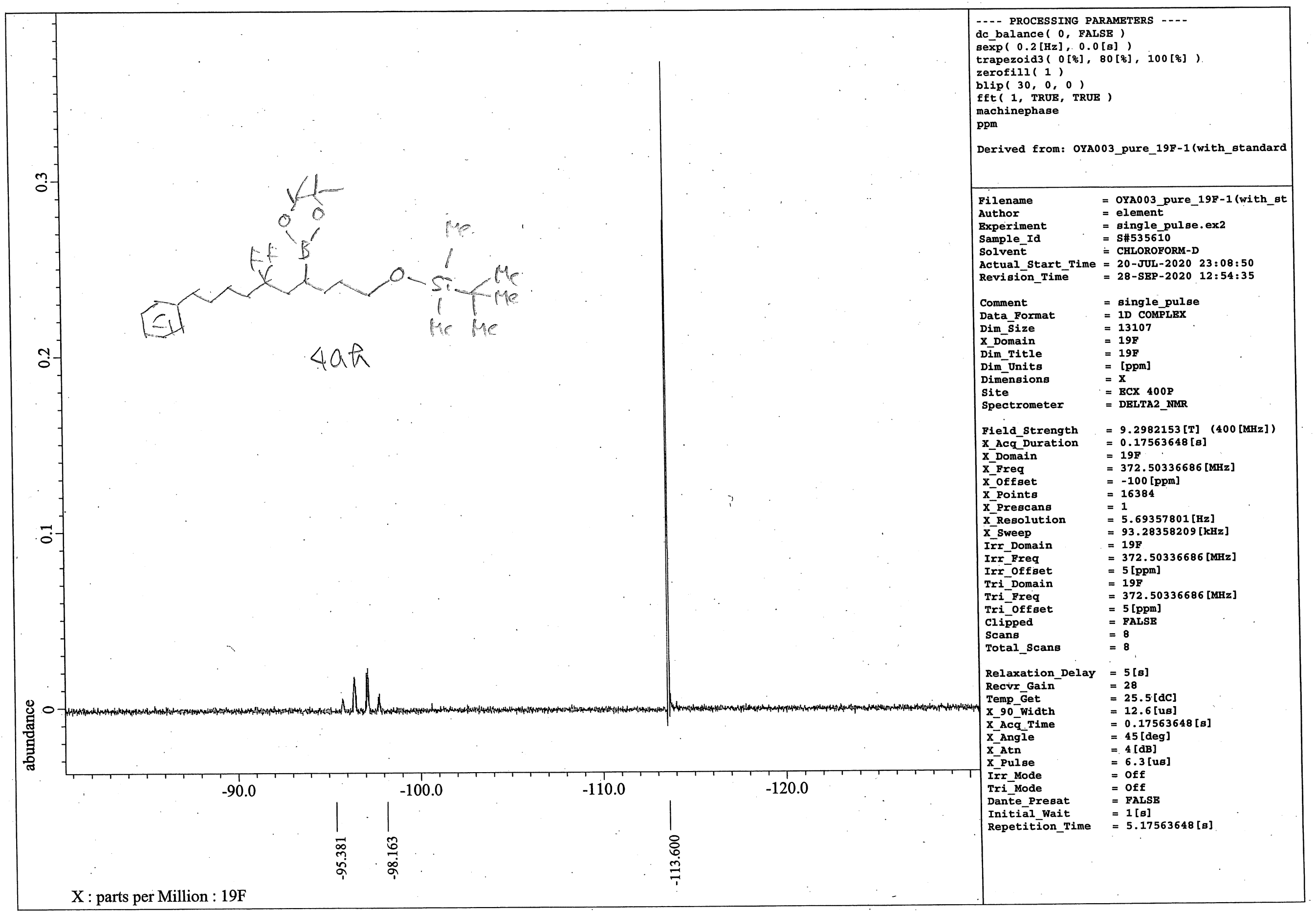




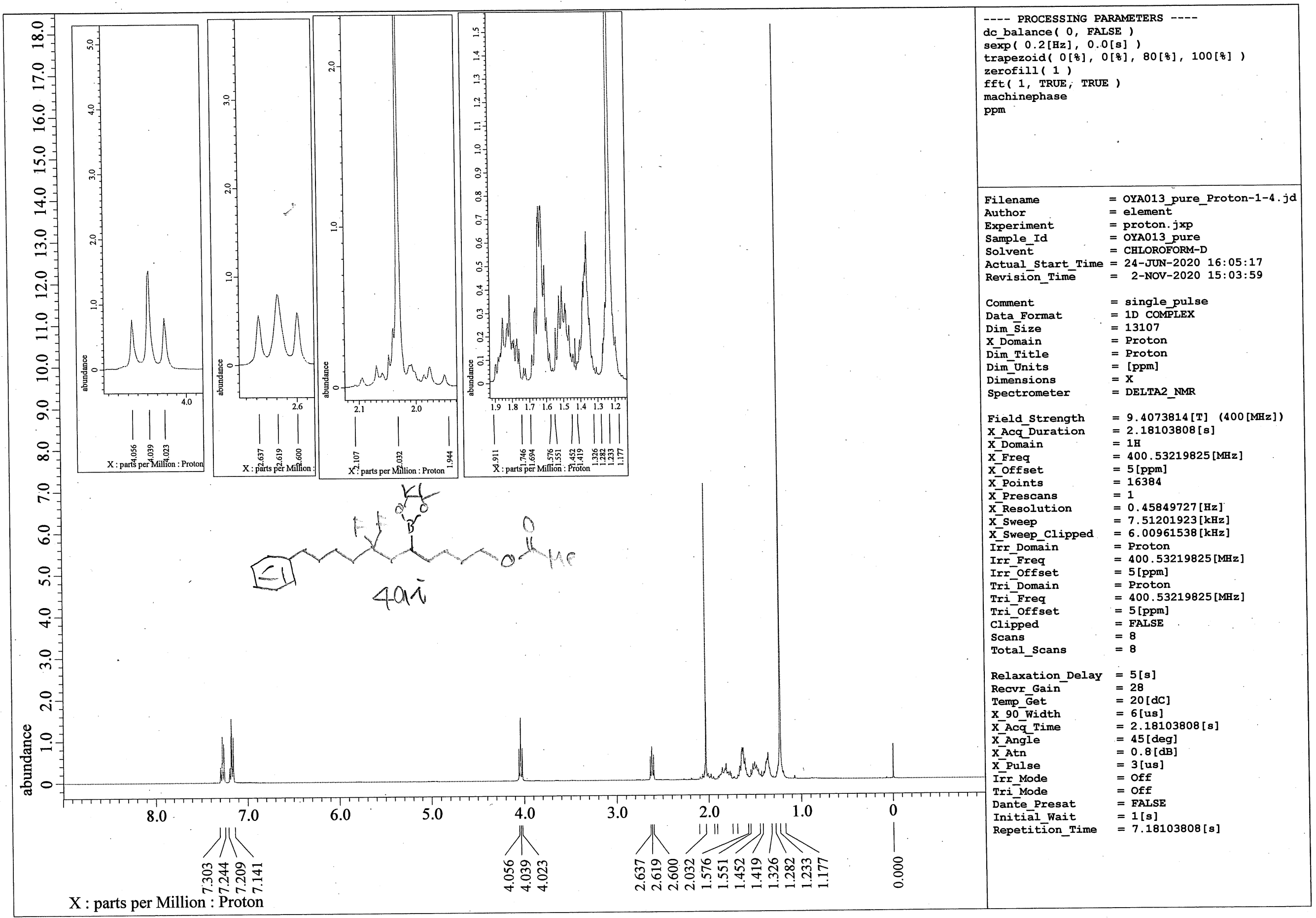




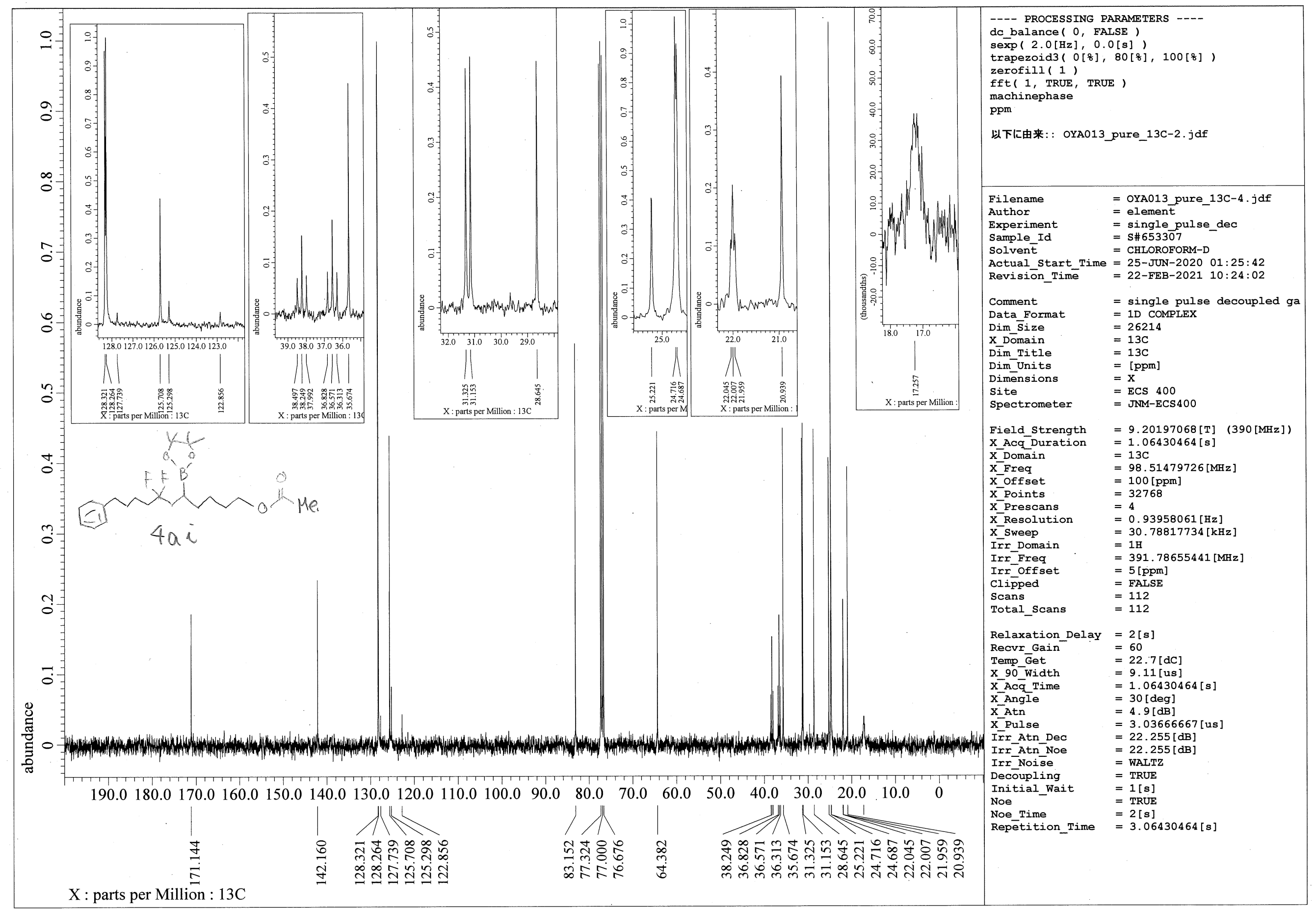




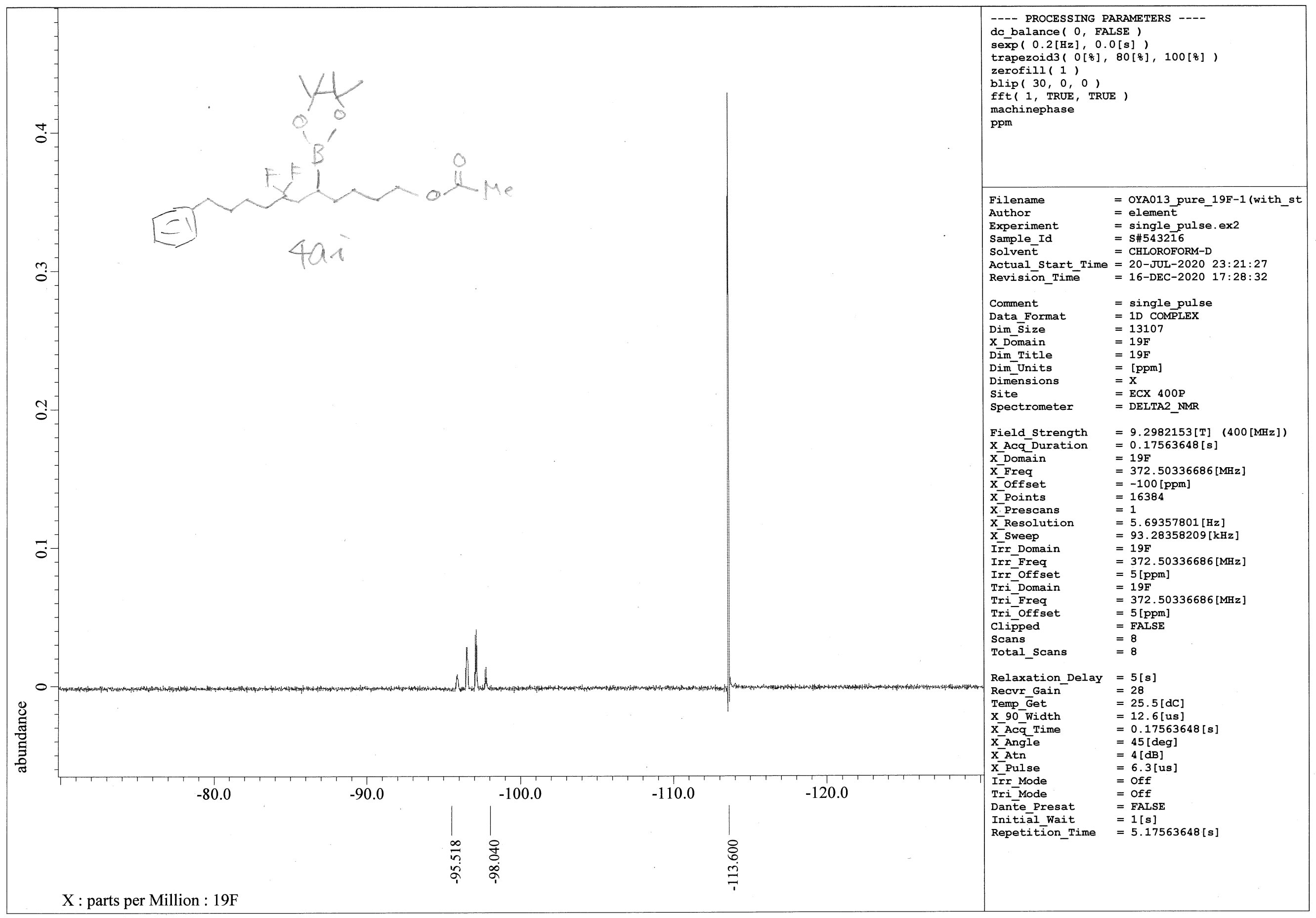




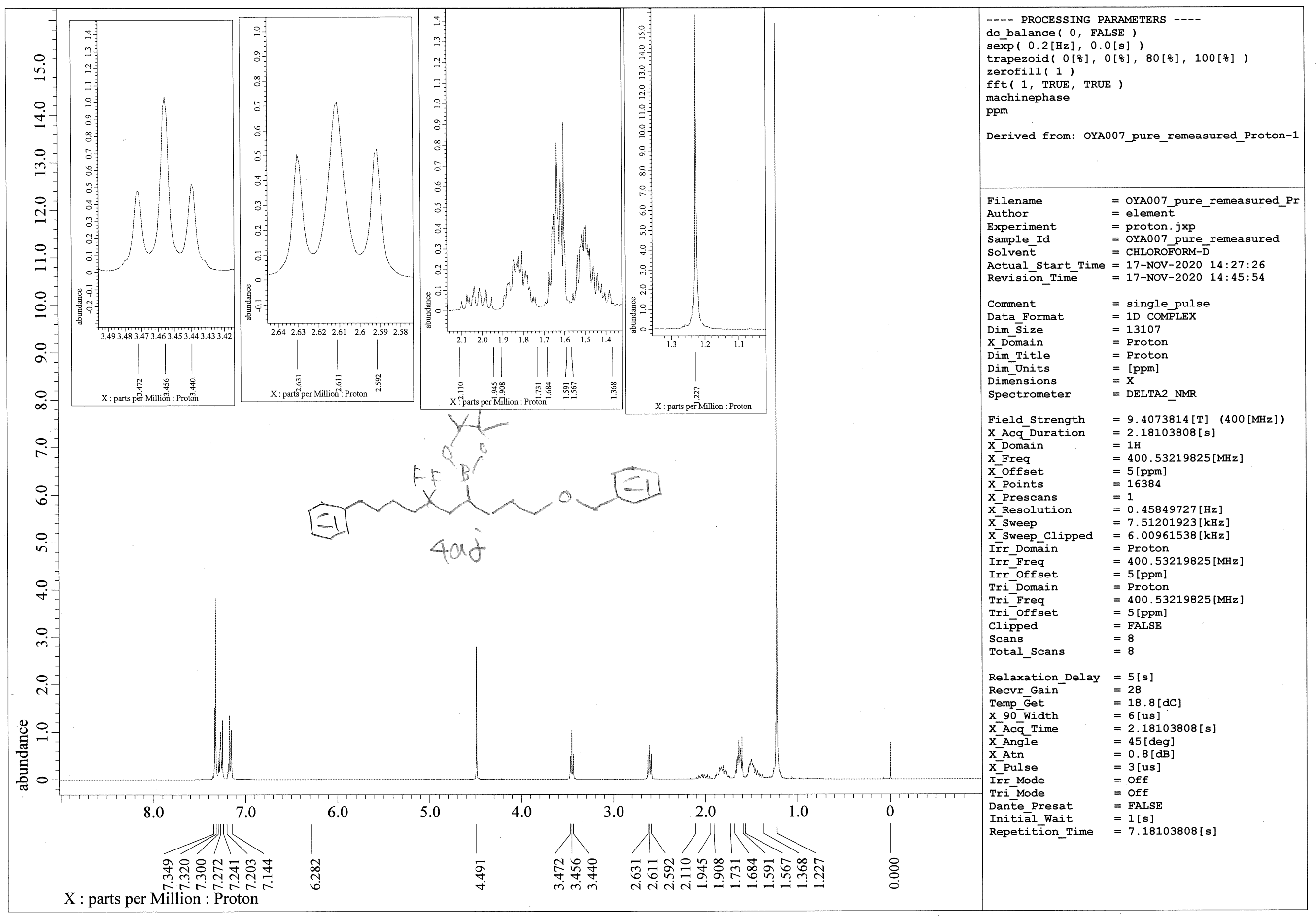




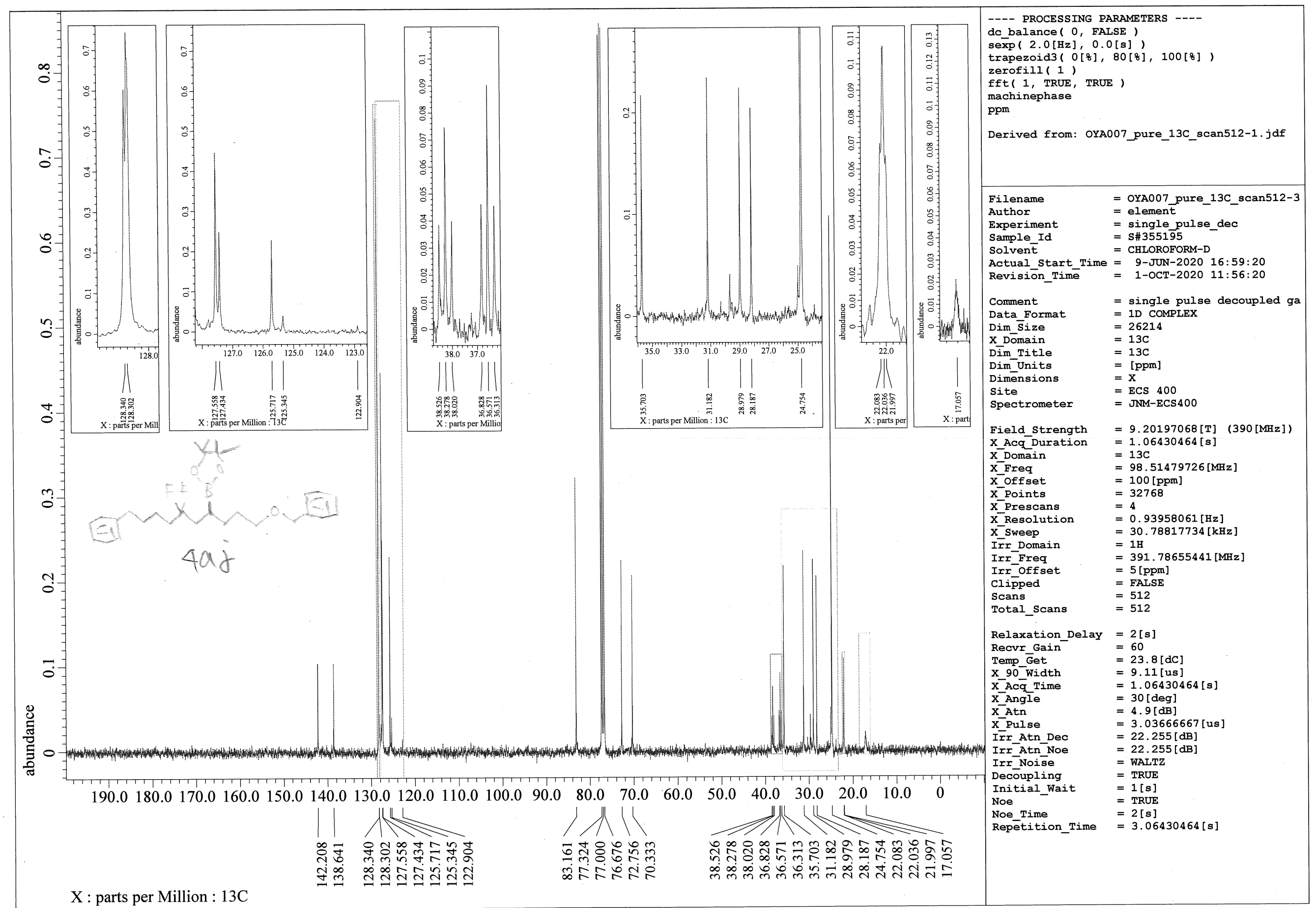




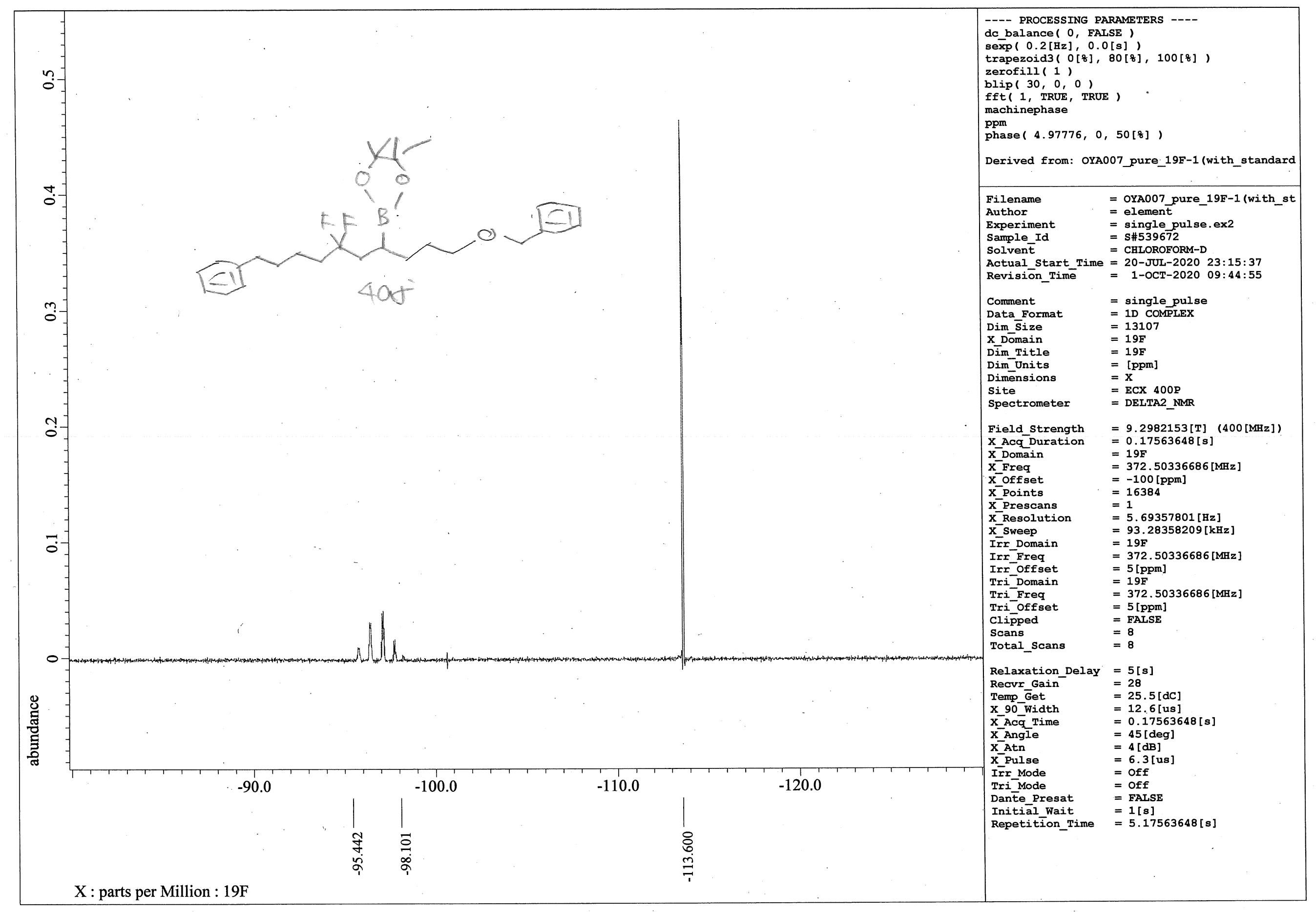




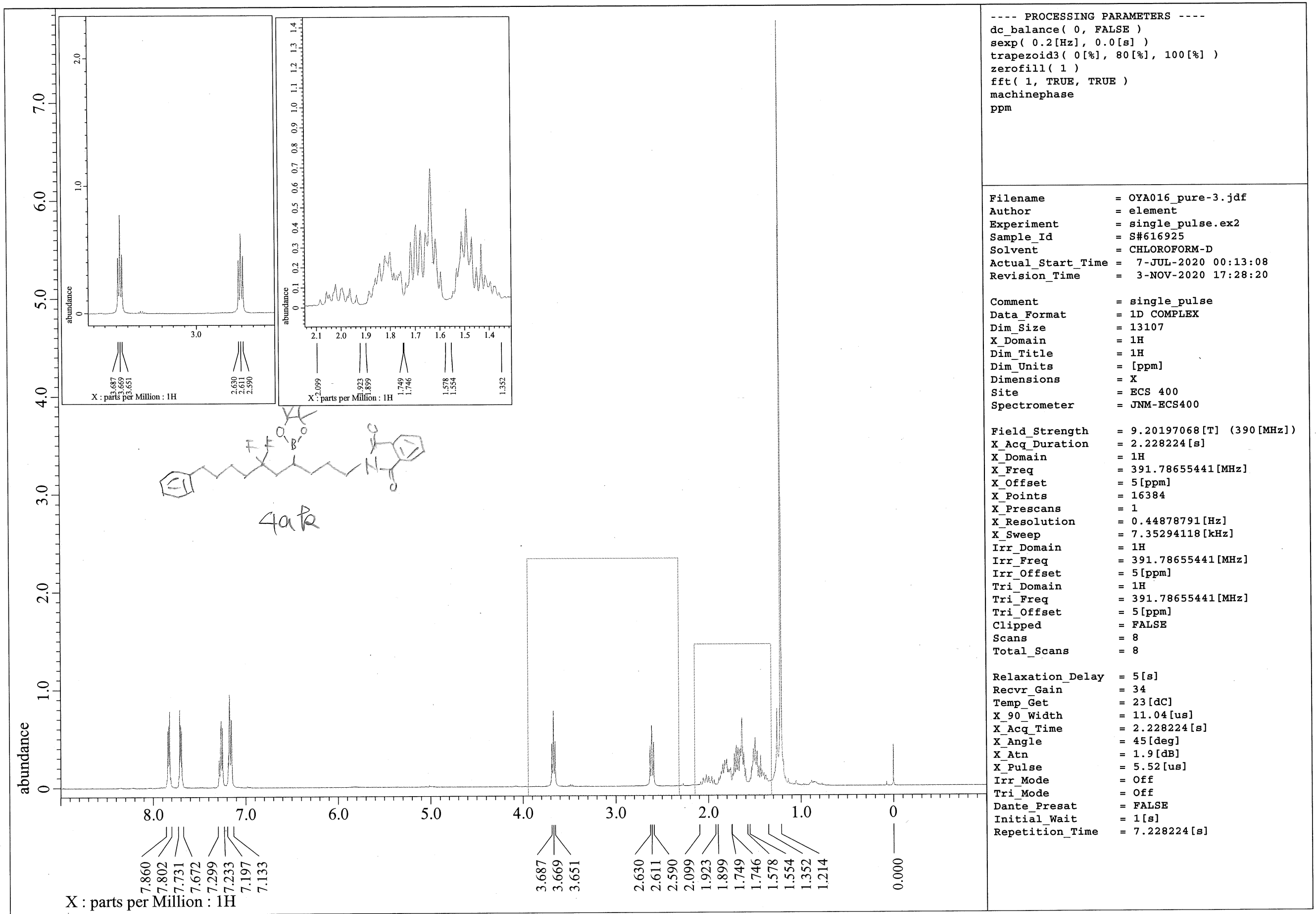




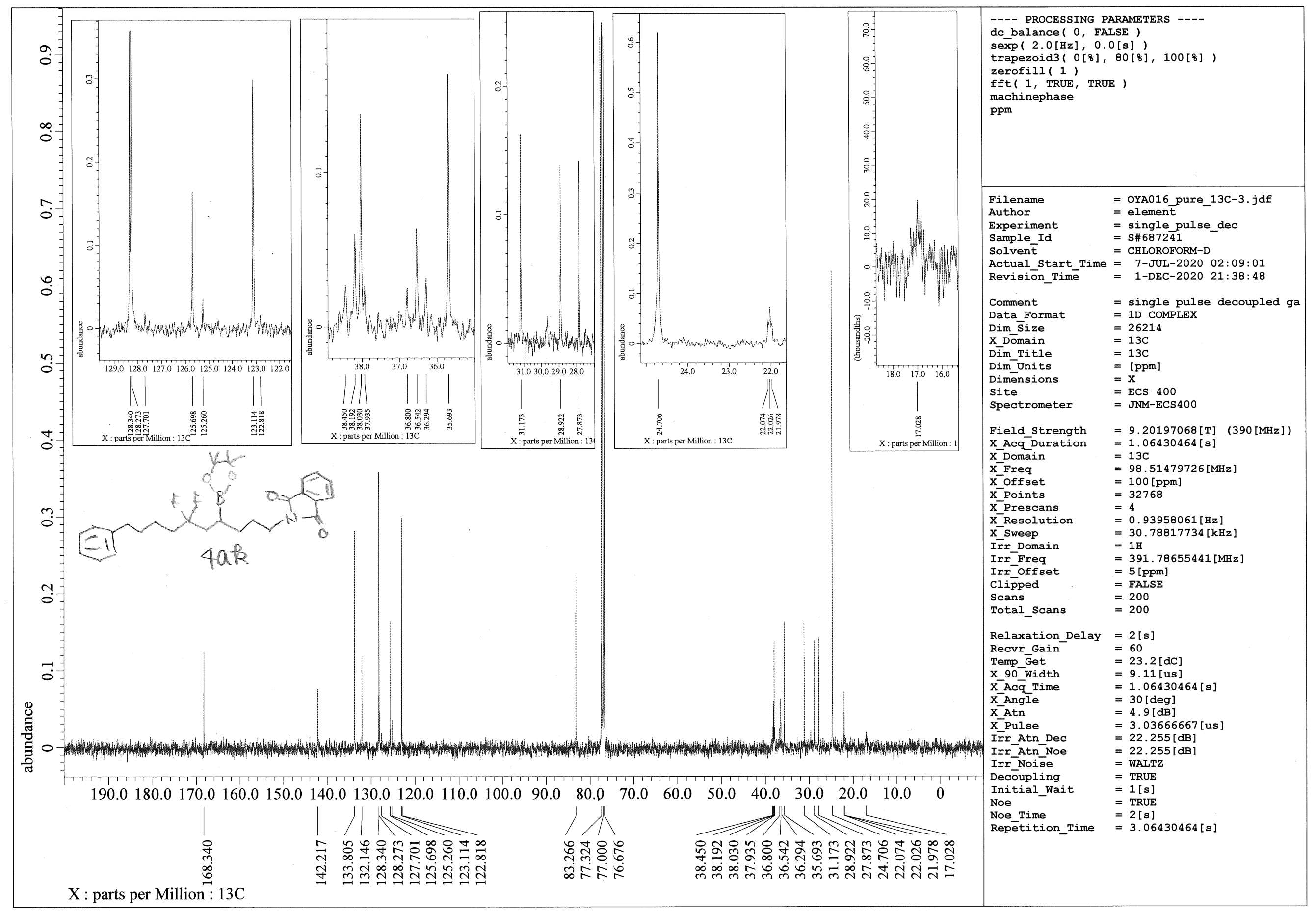




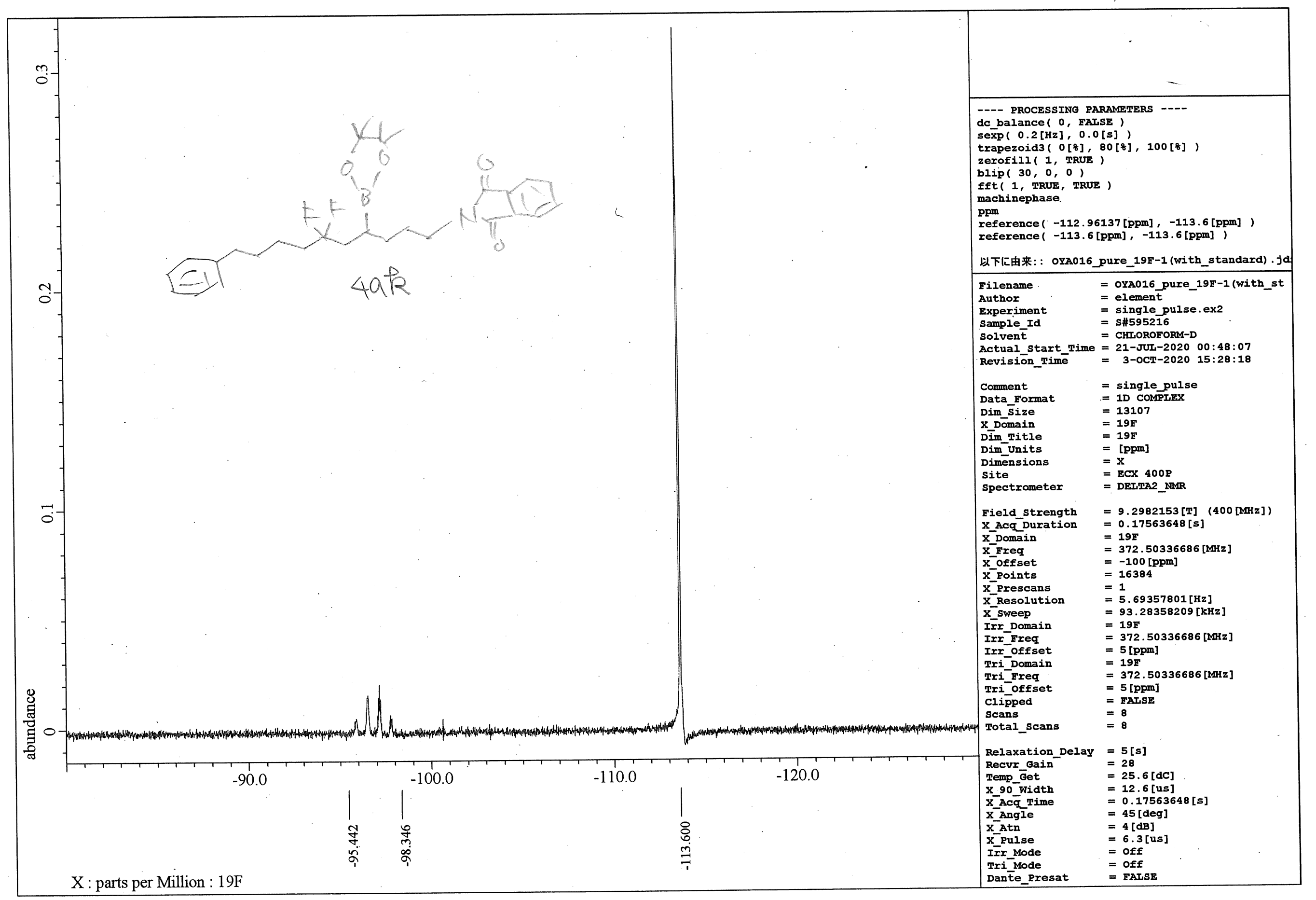




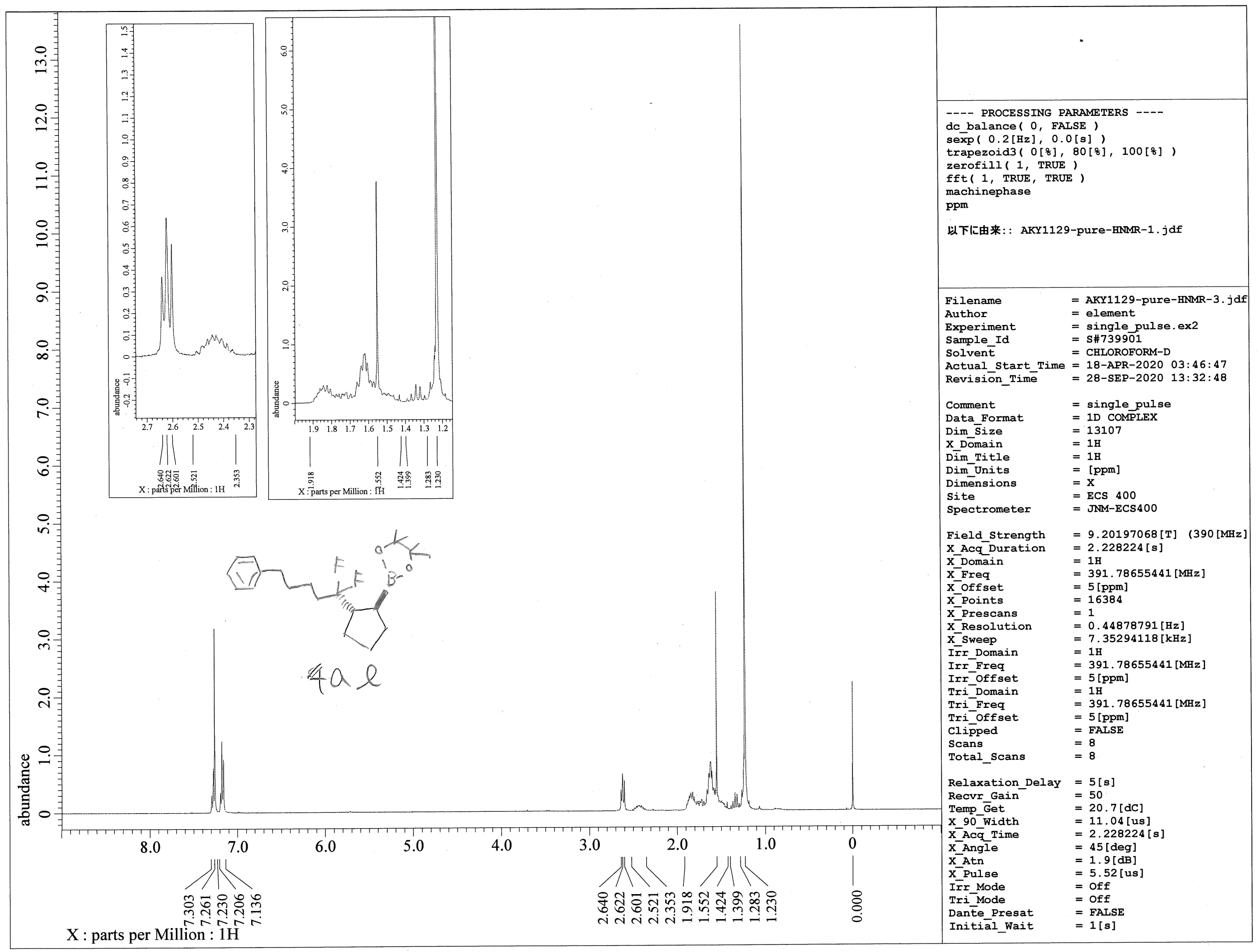




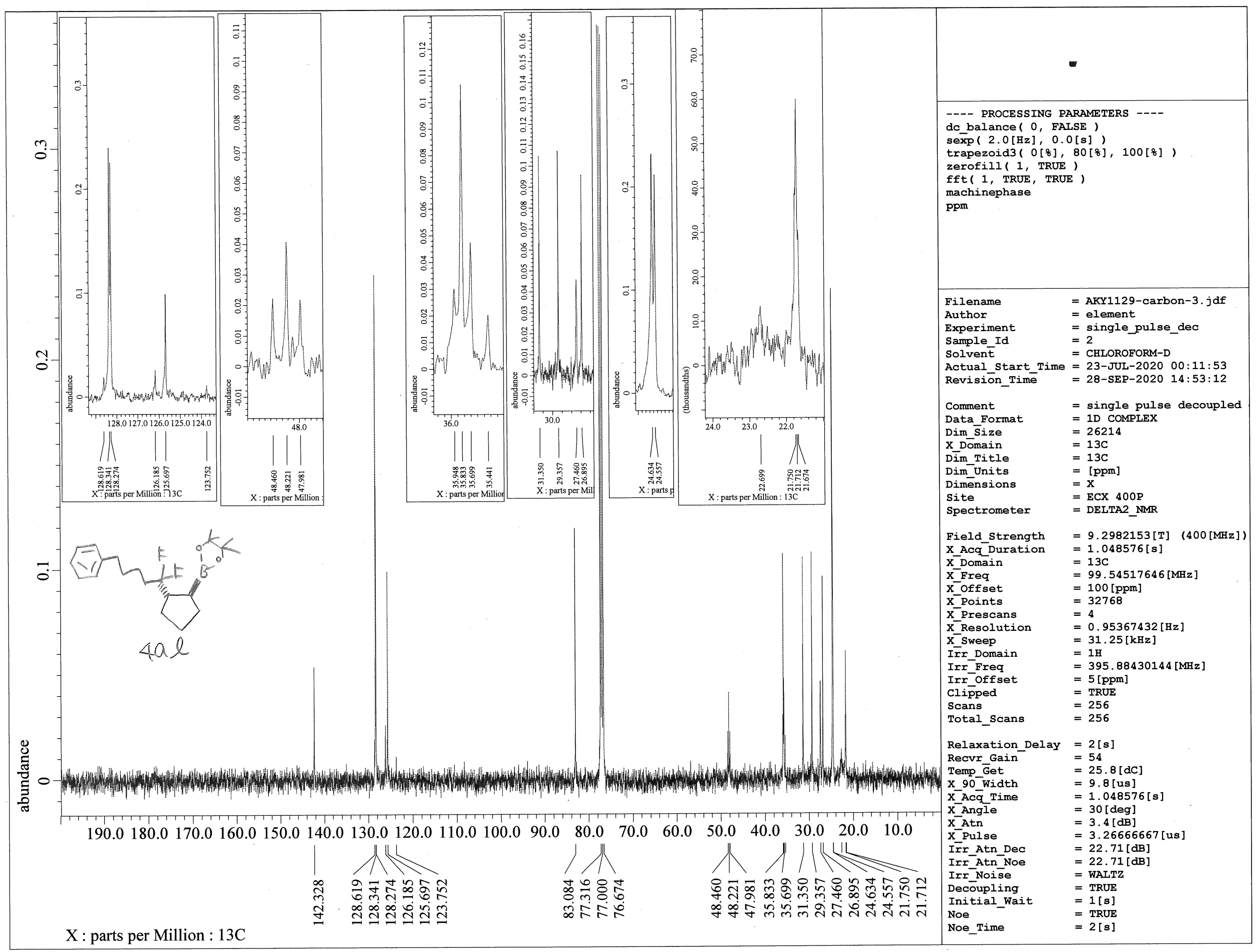




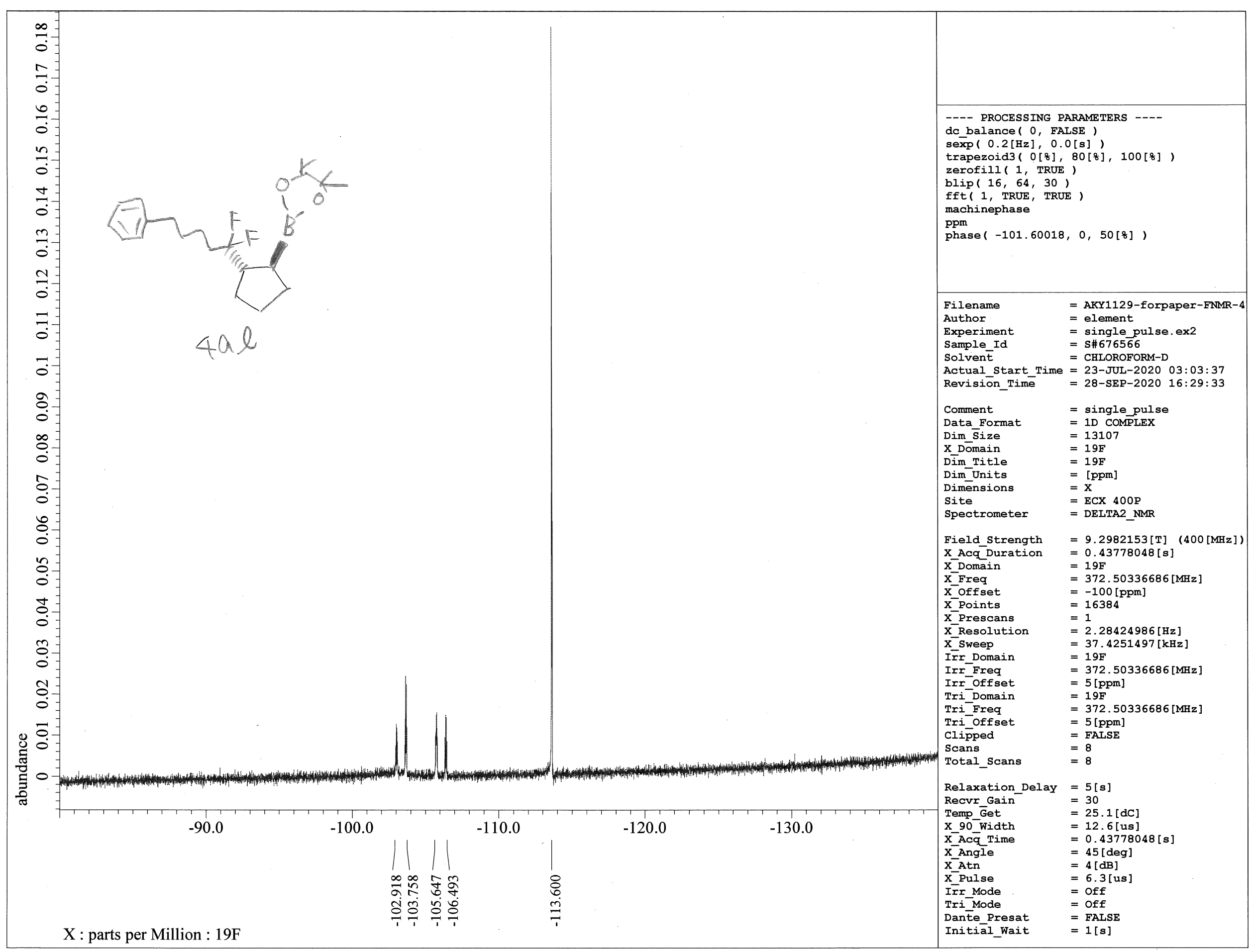




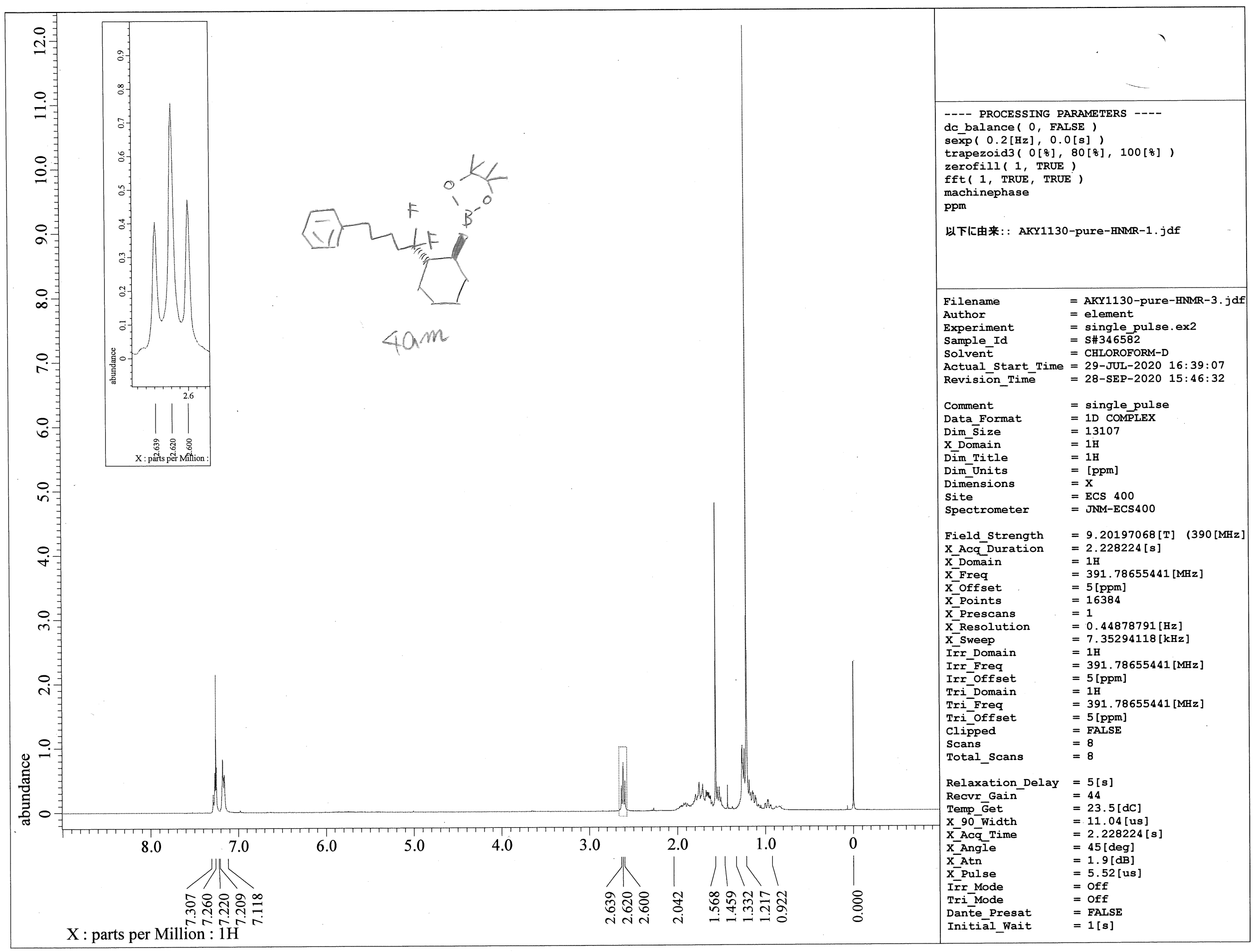




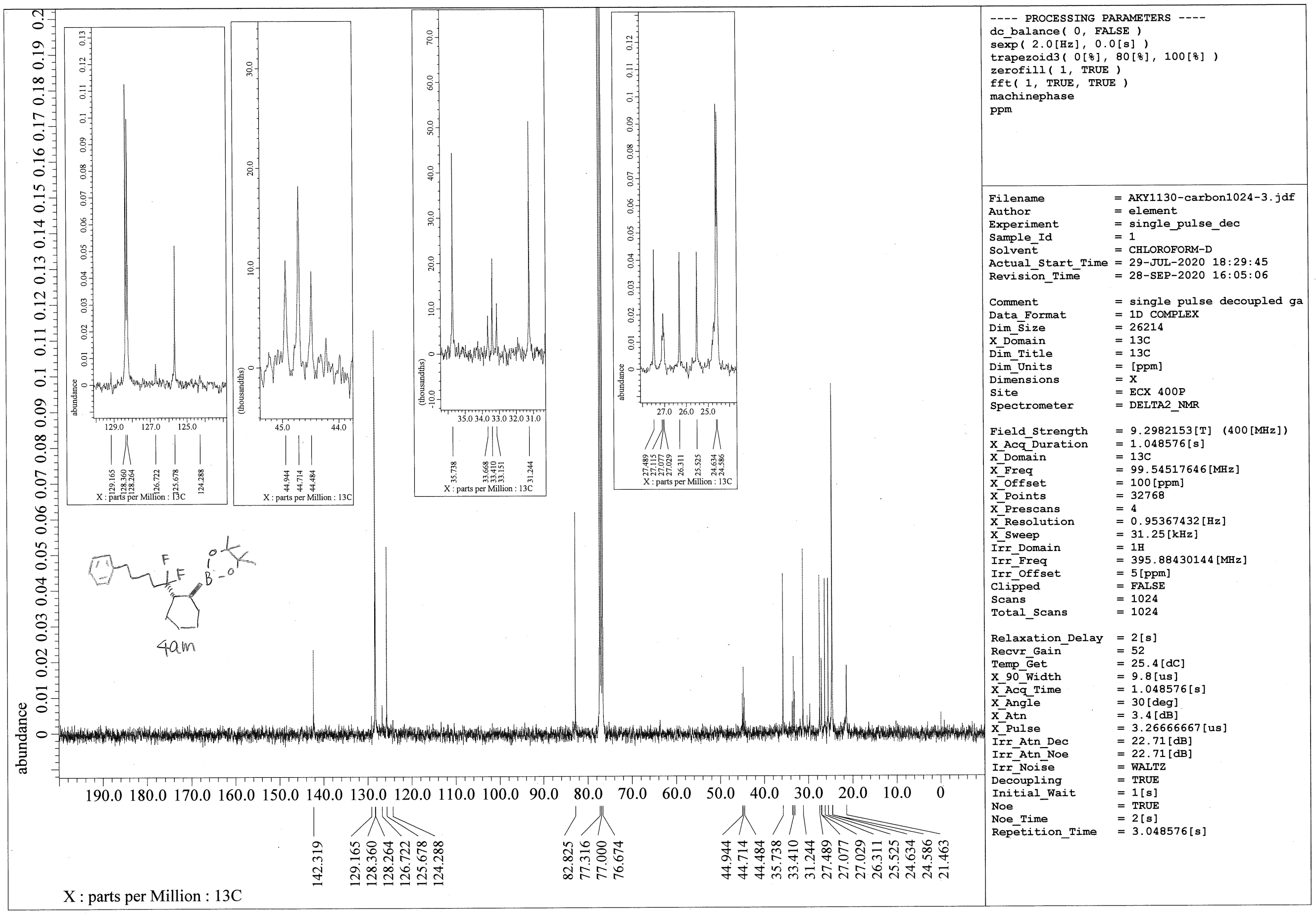




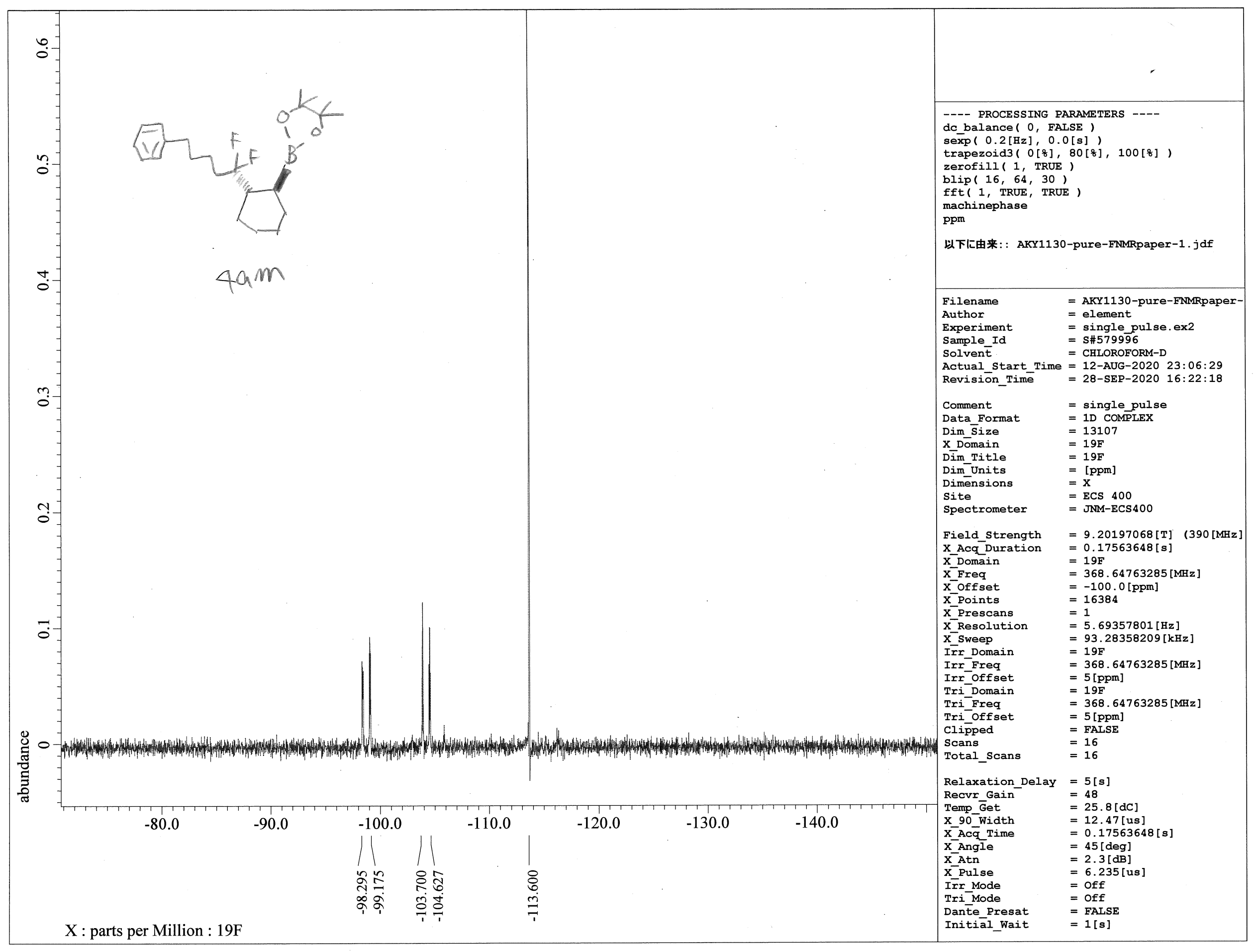




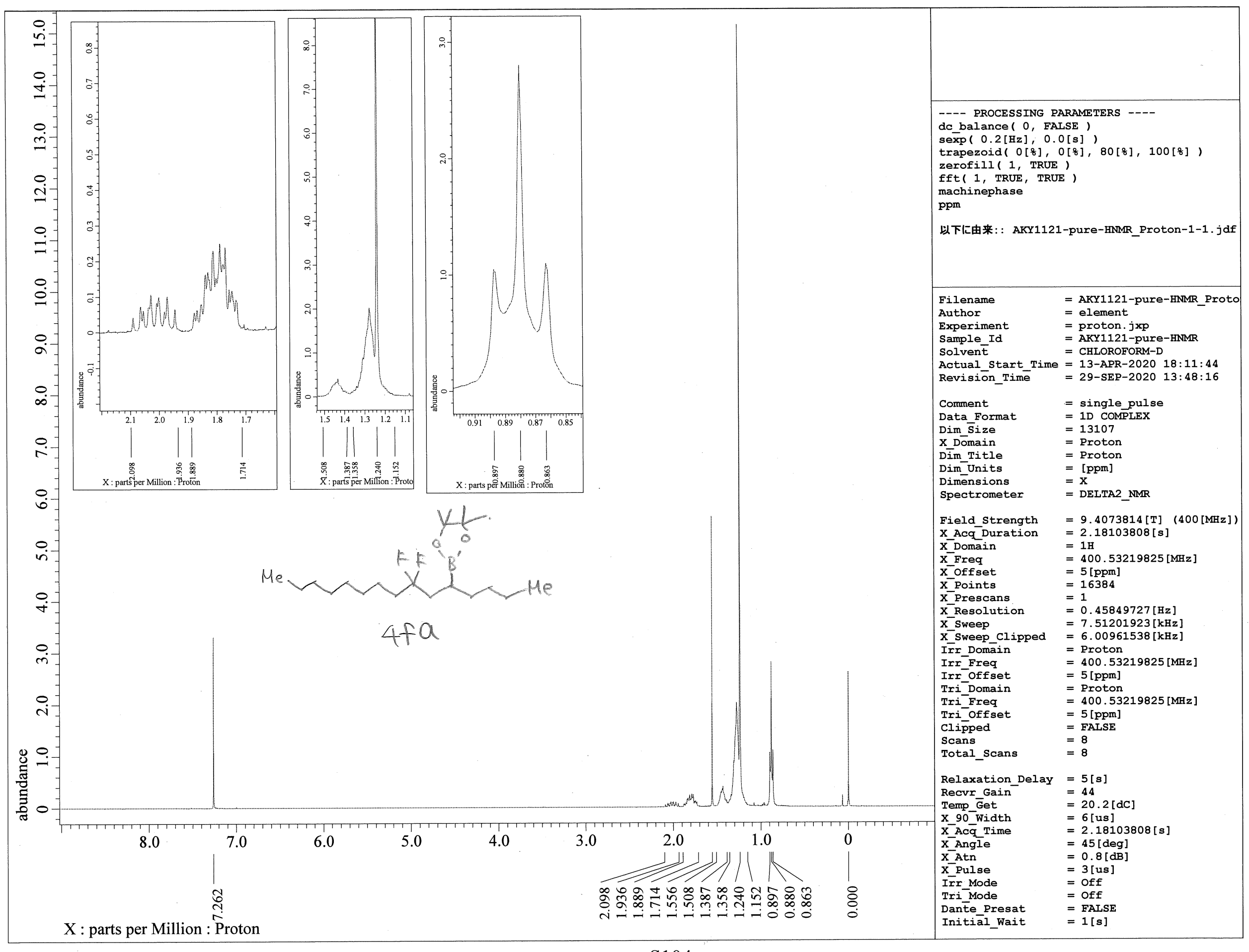

S104 


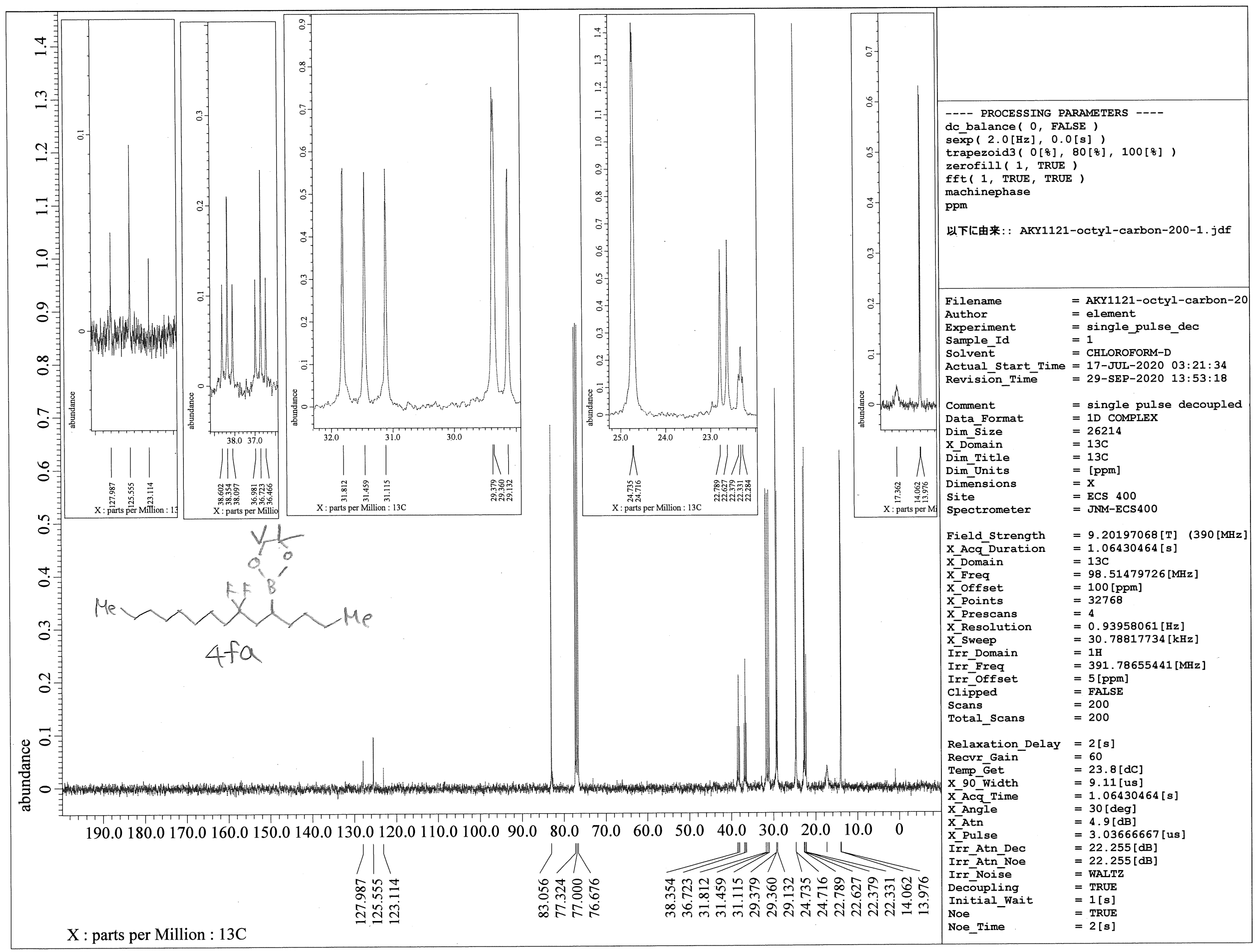

S105 


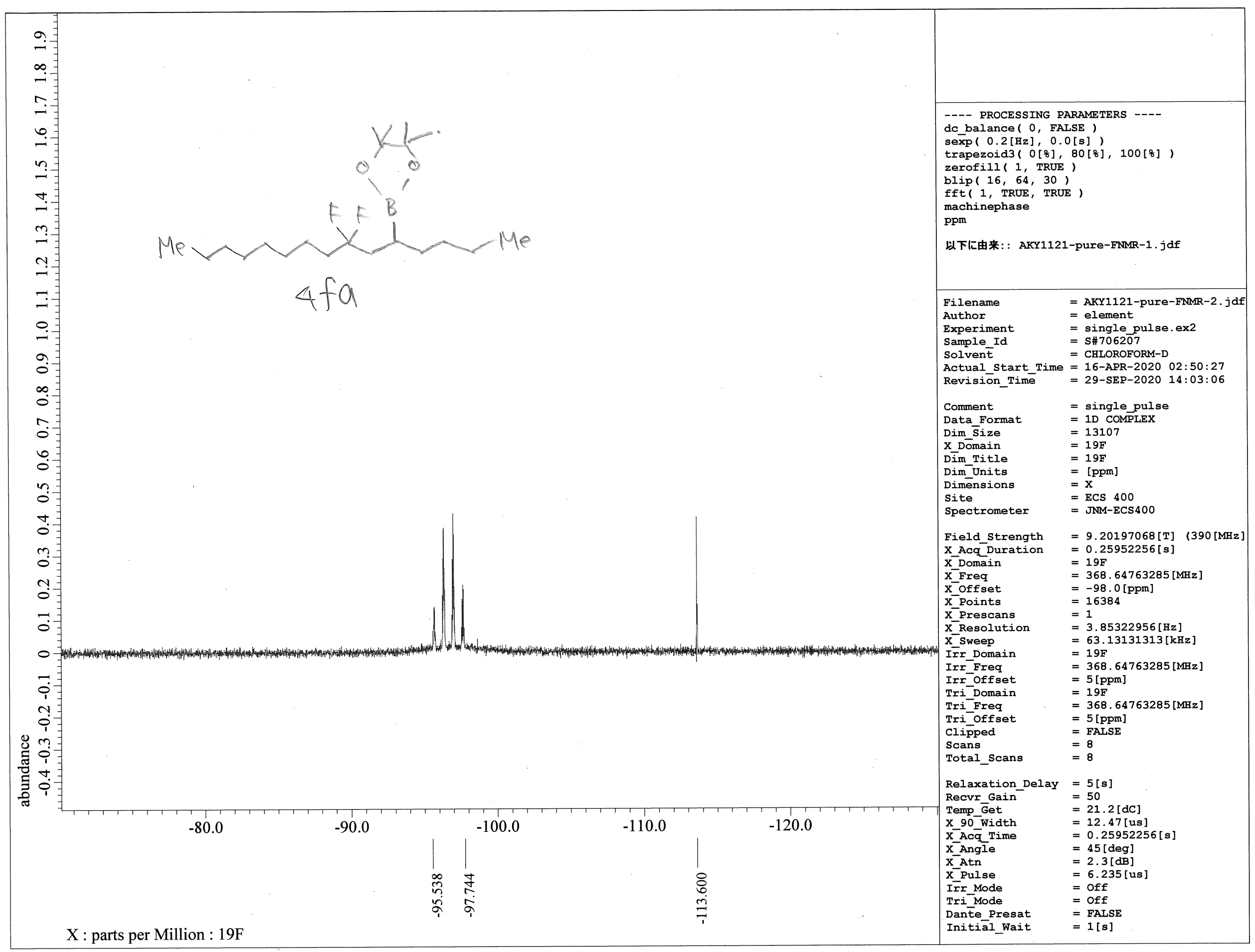




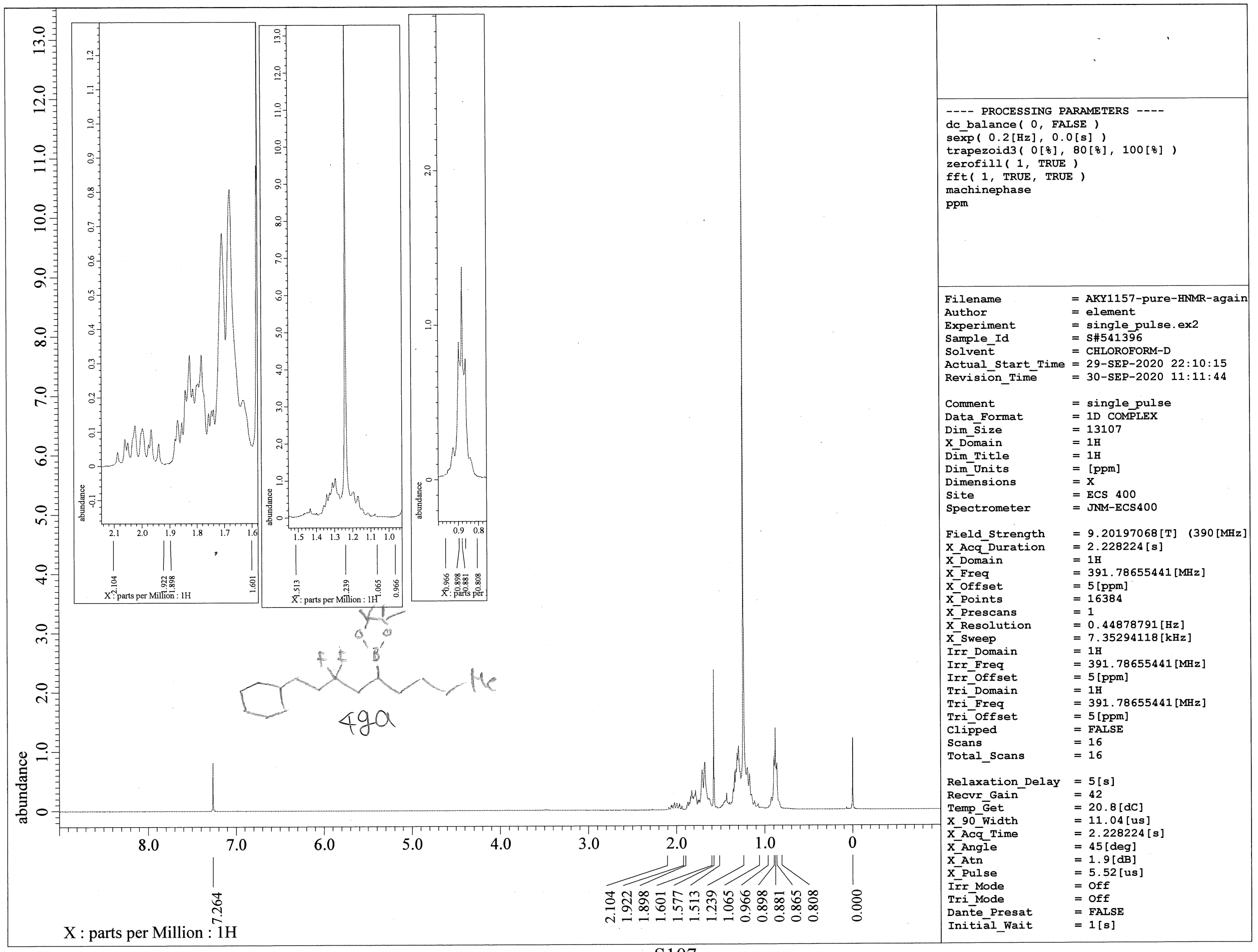




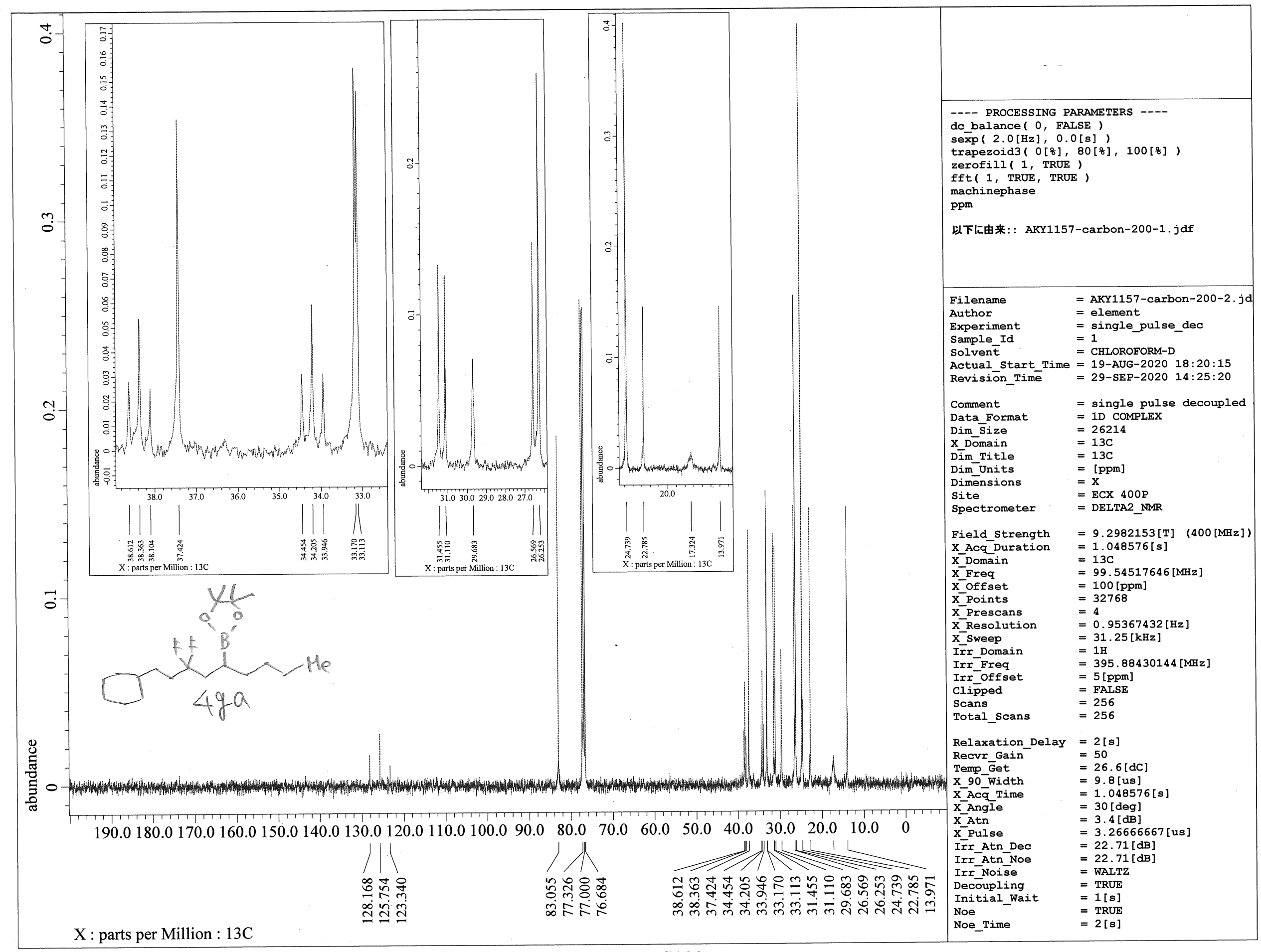

S108 


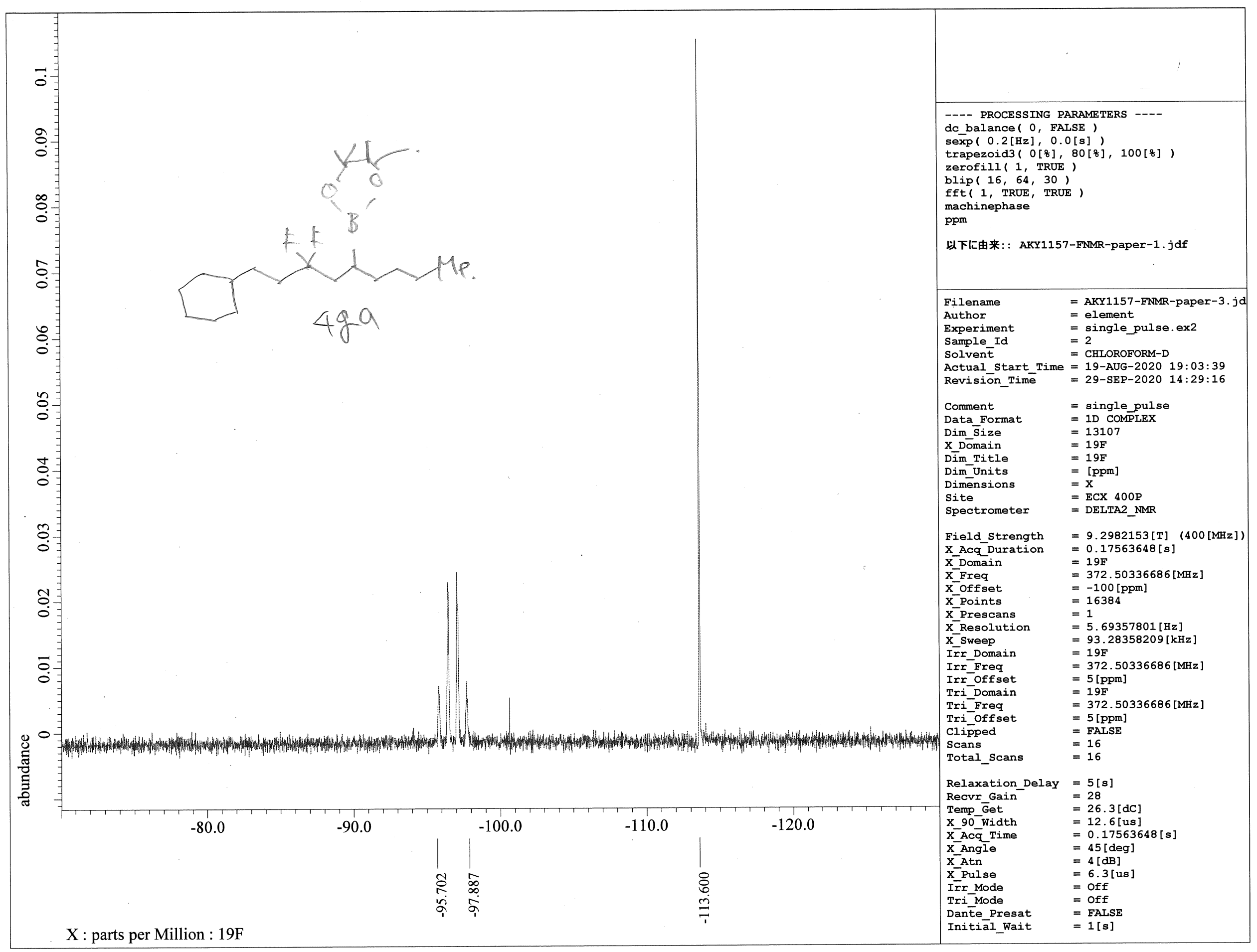




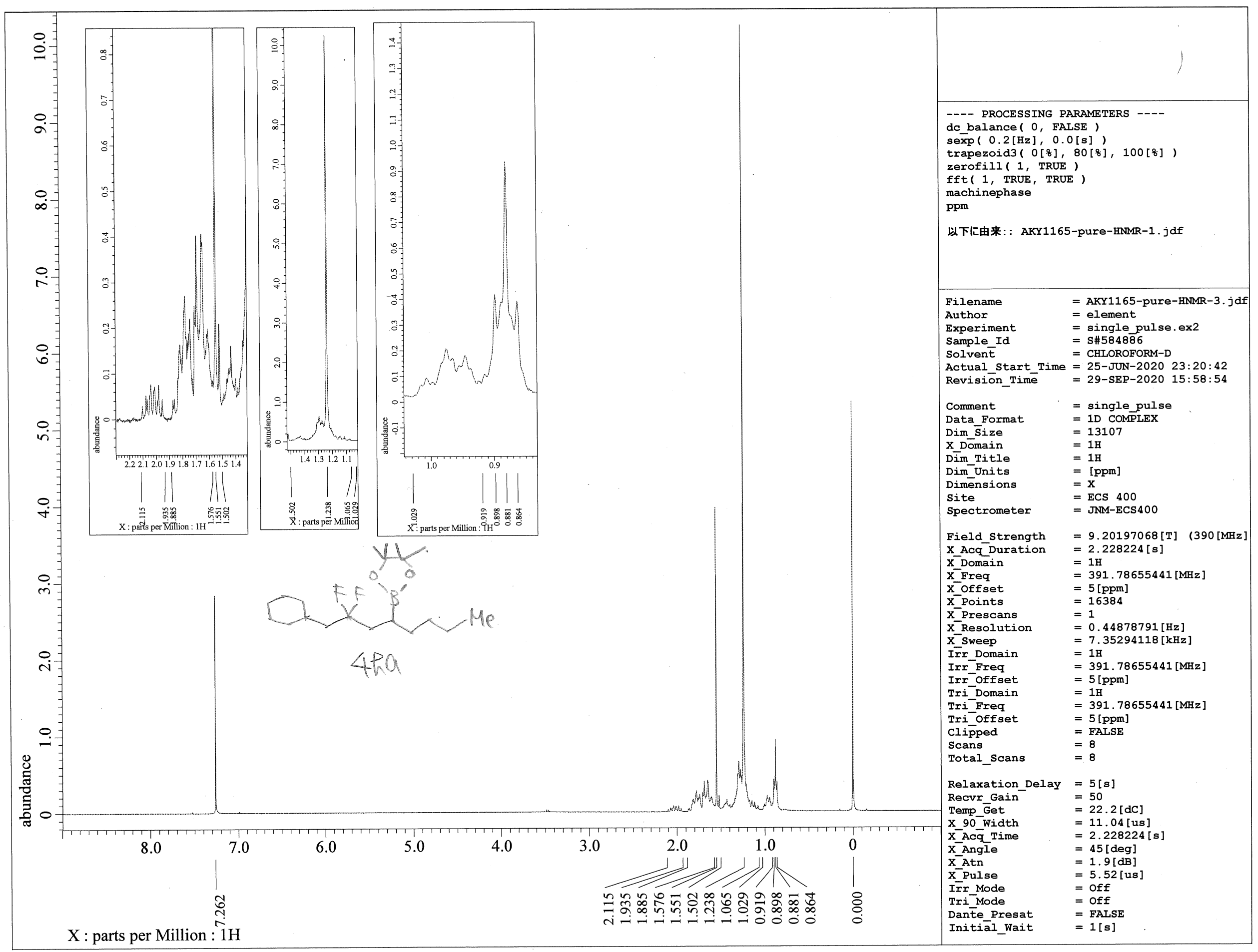

S110 


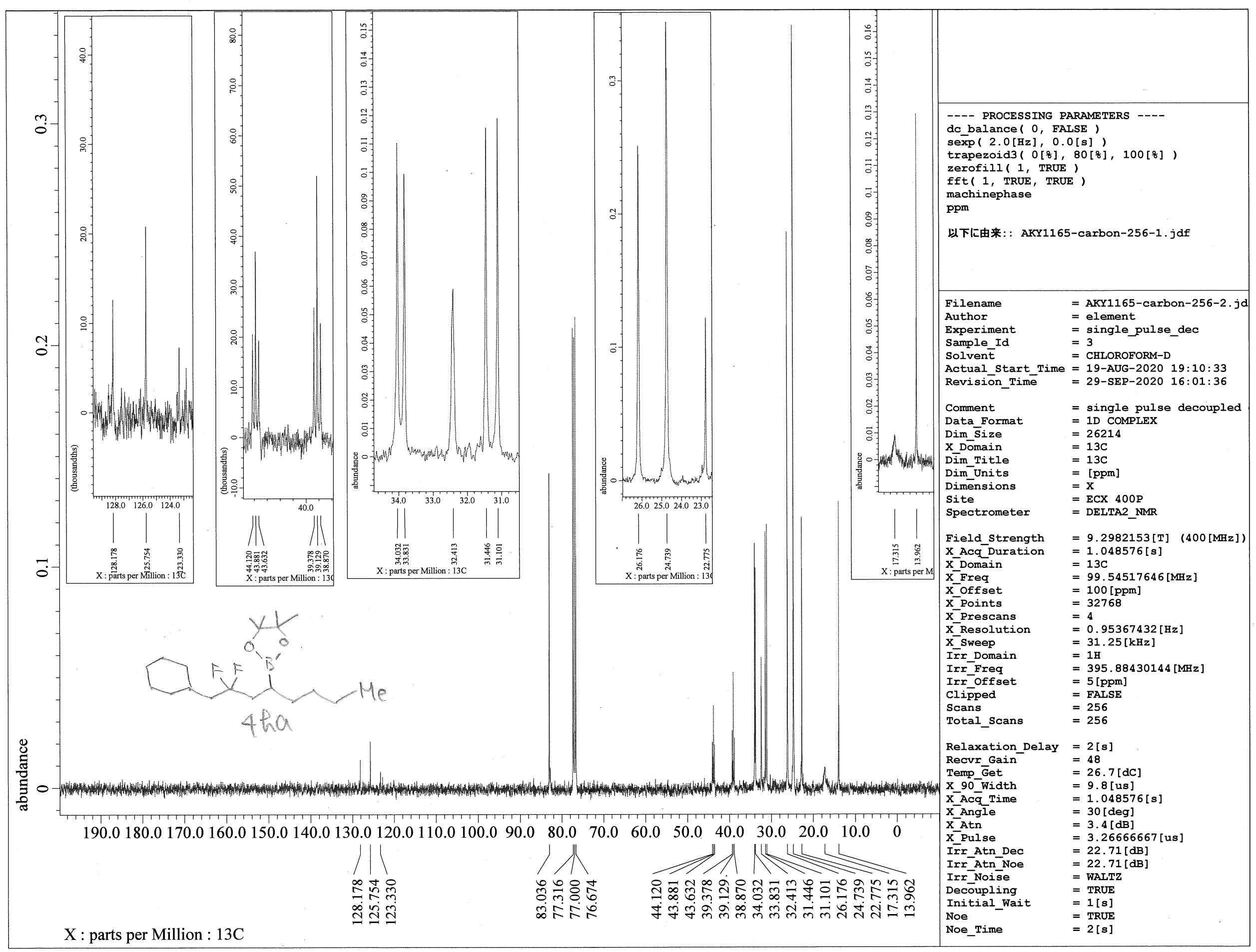

S111 


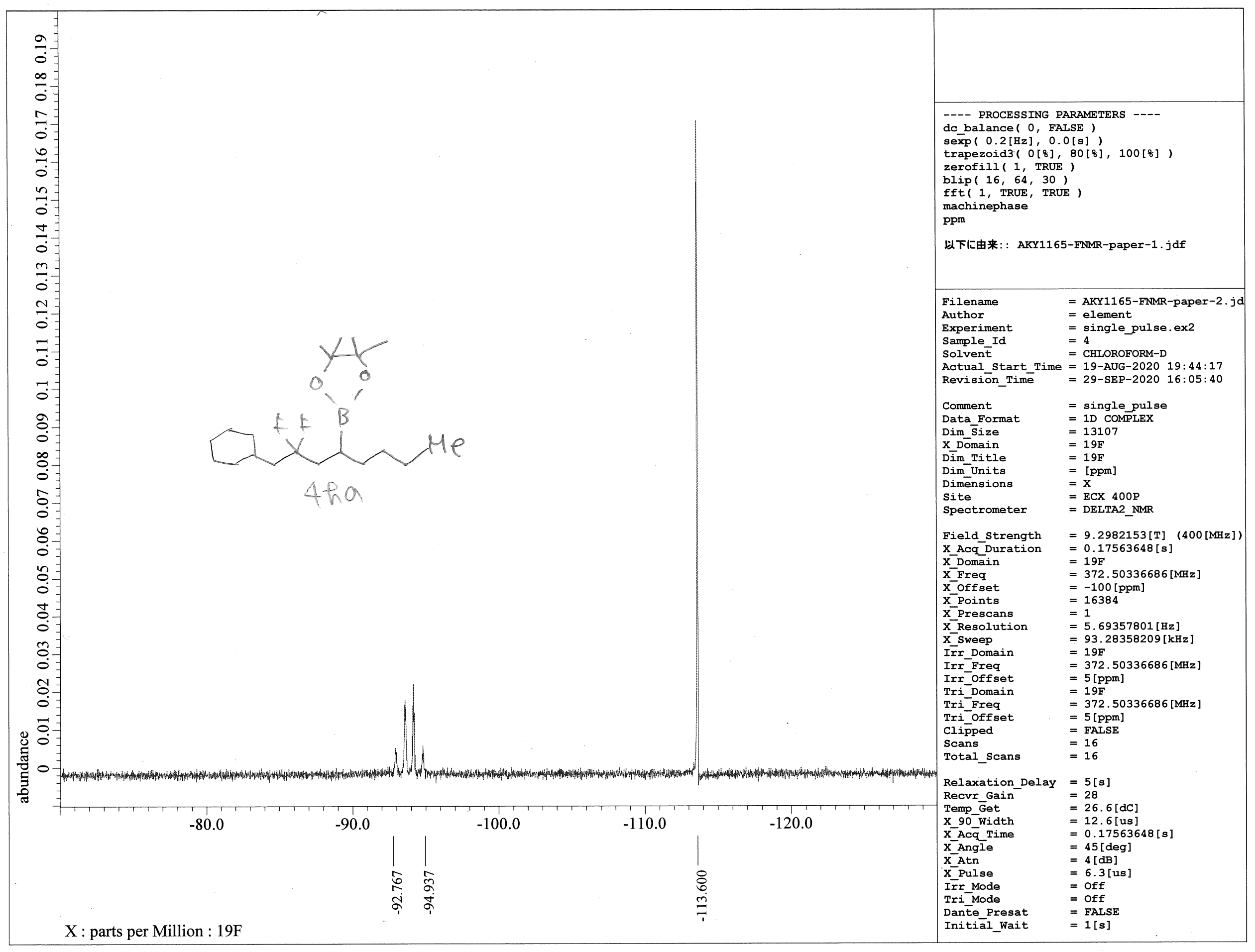




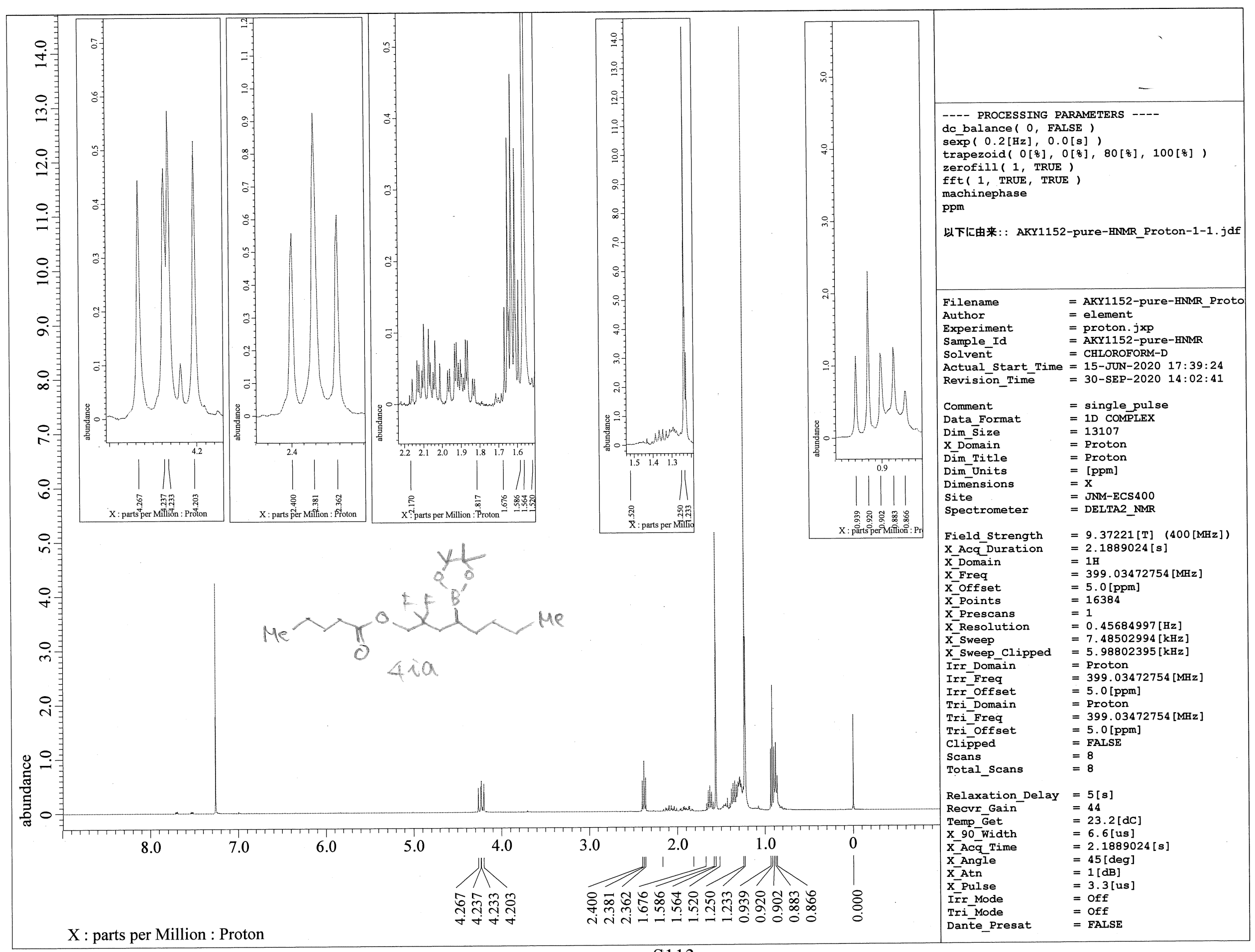




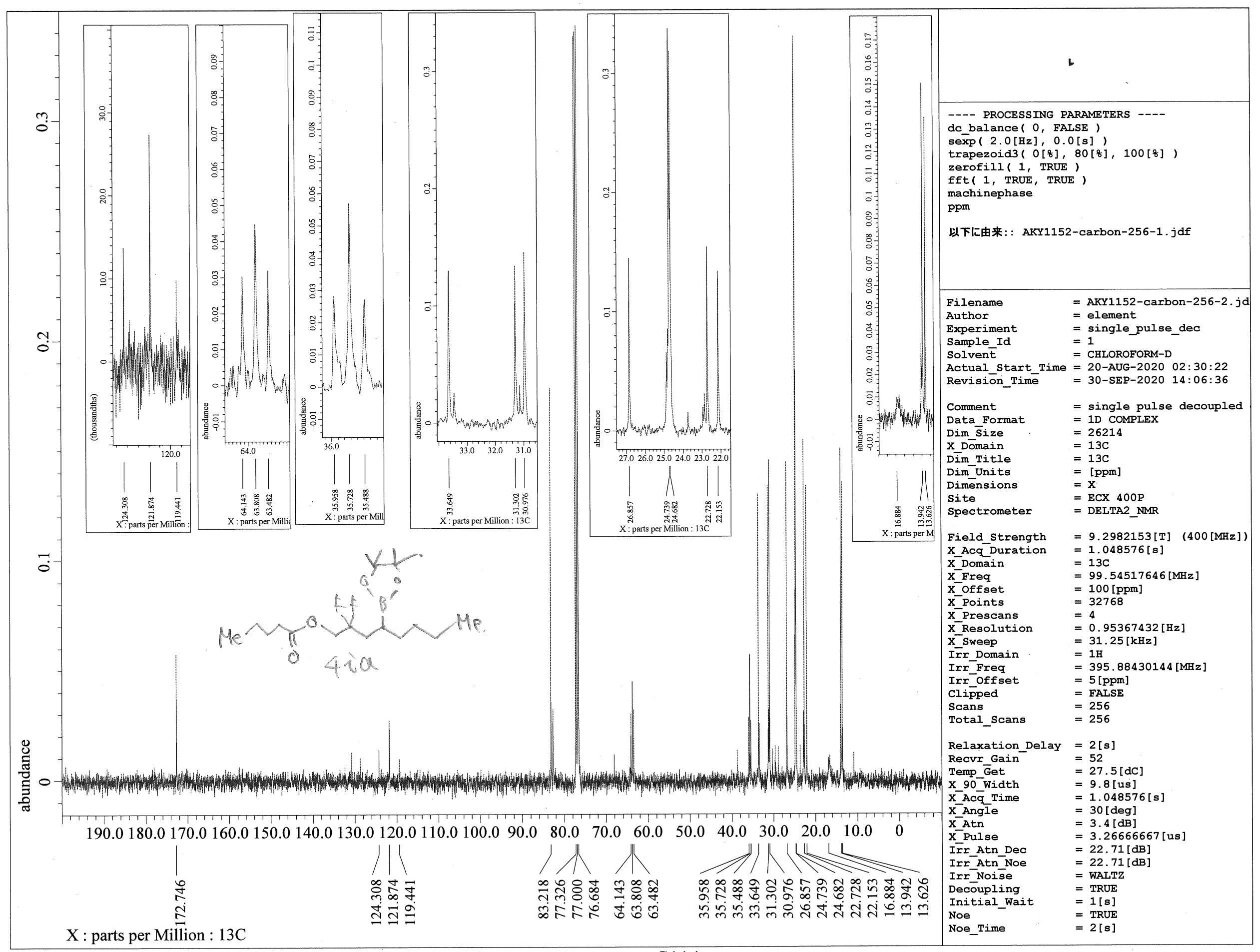




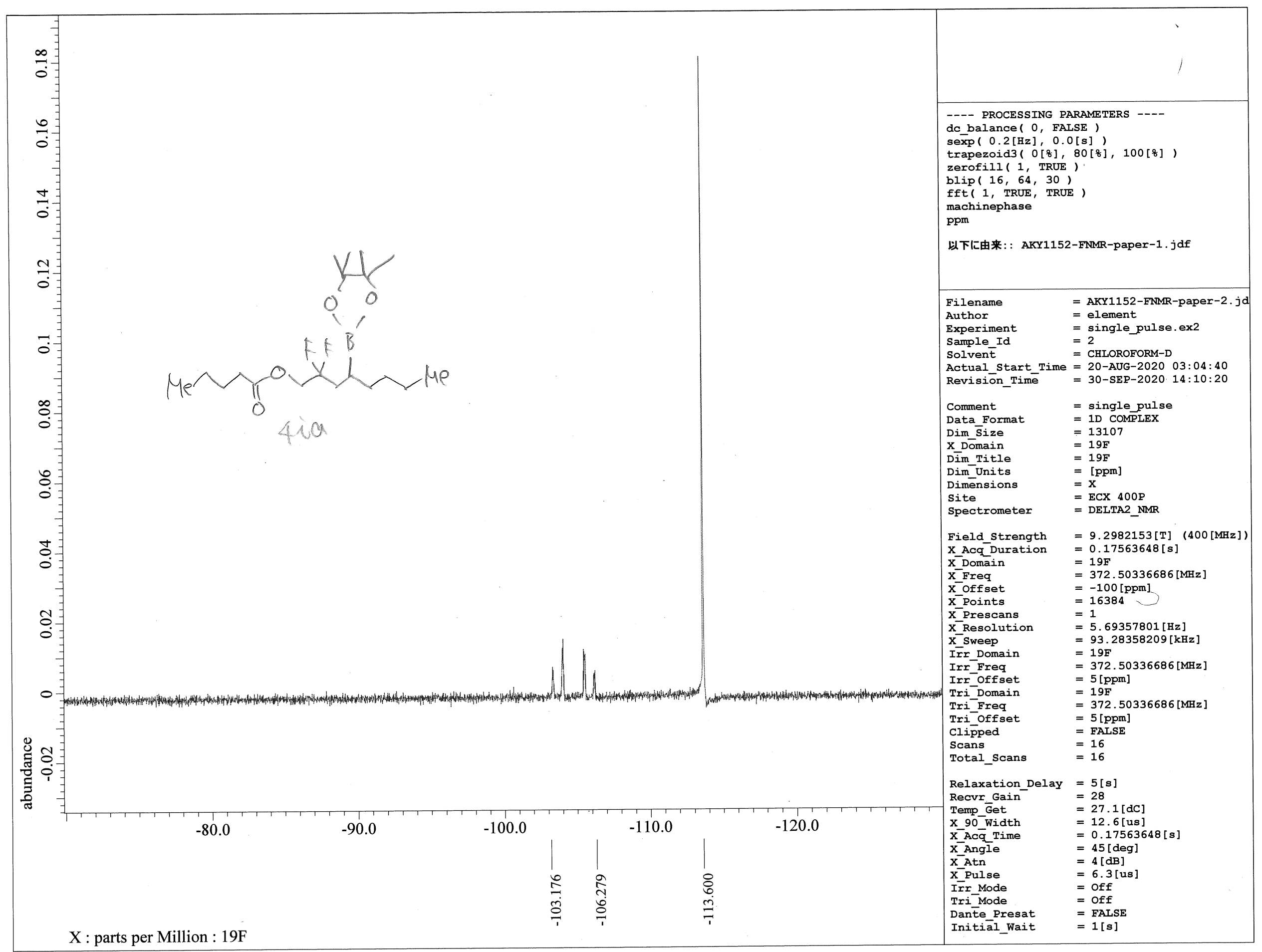

S115 


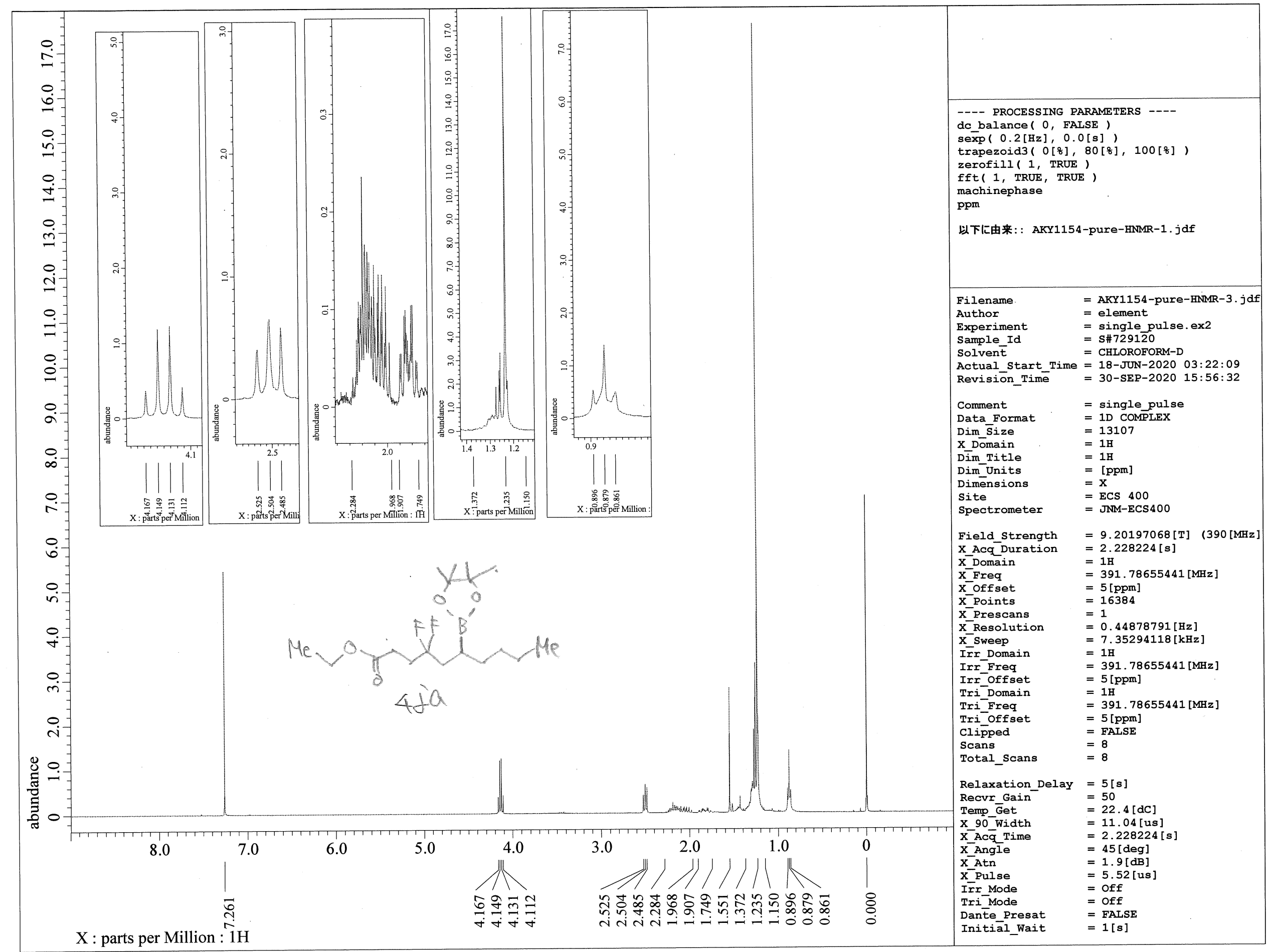

S116 


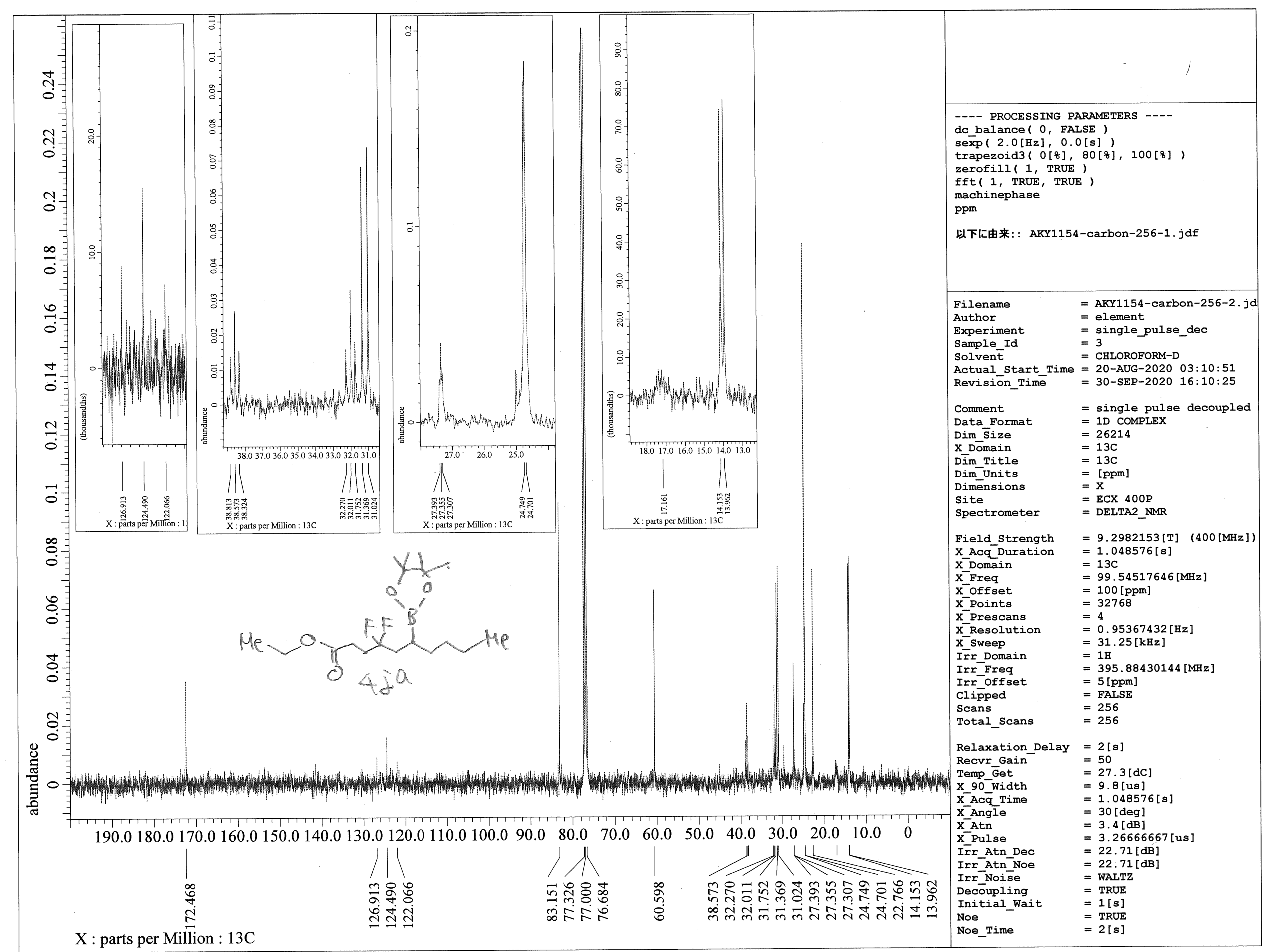

S117 


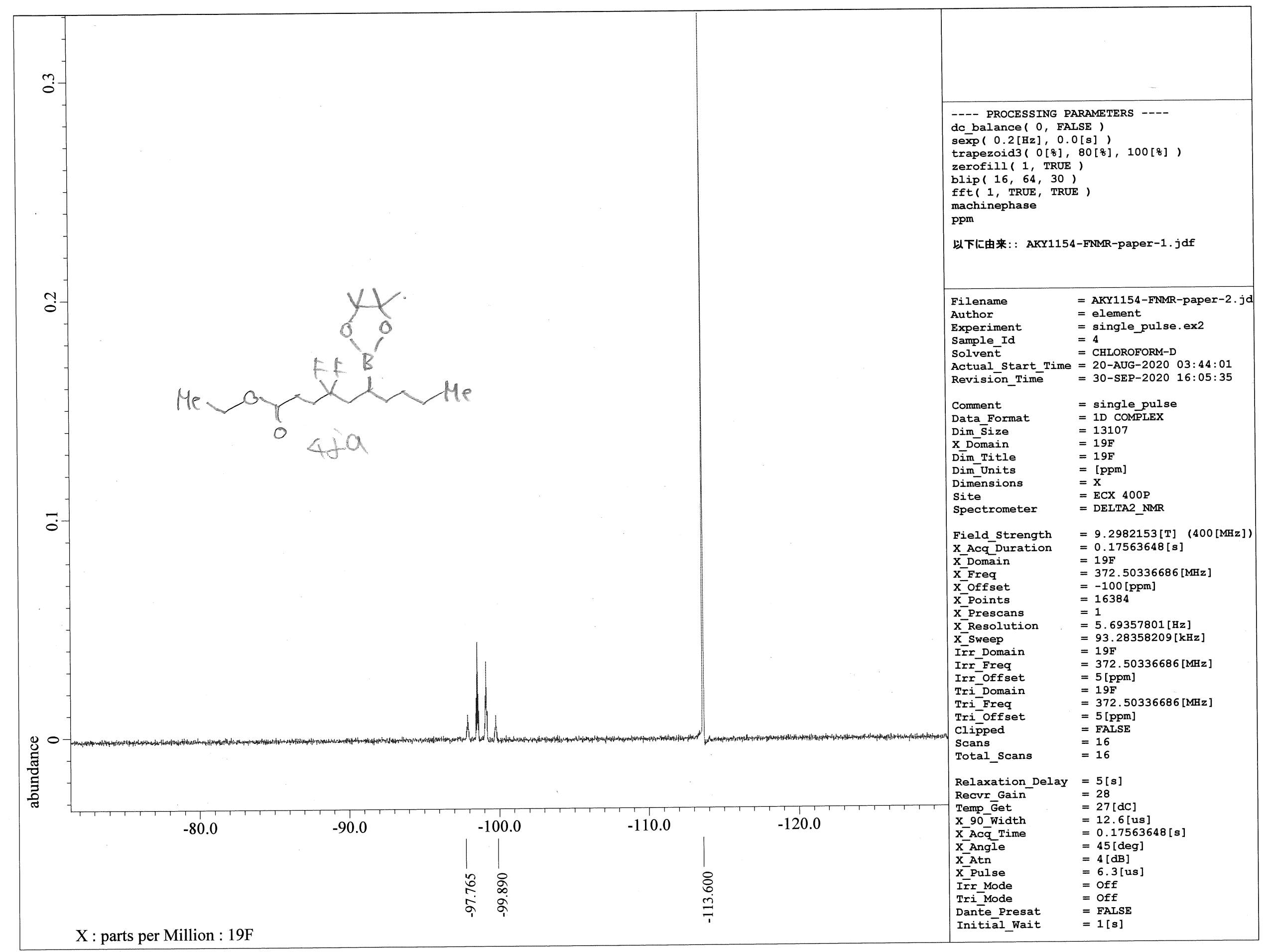




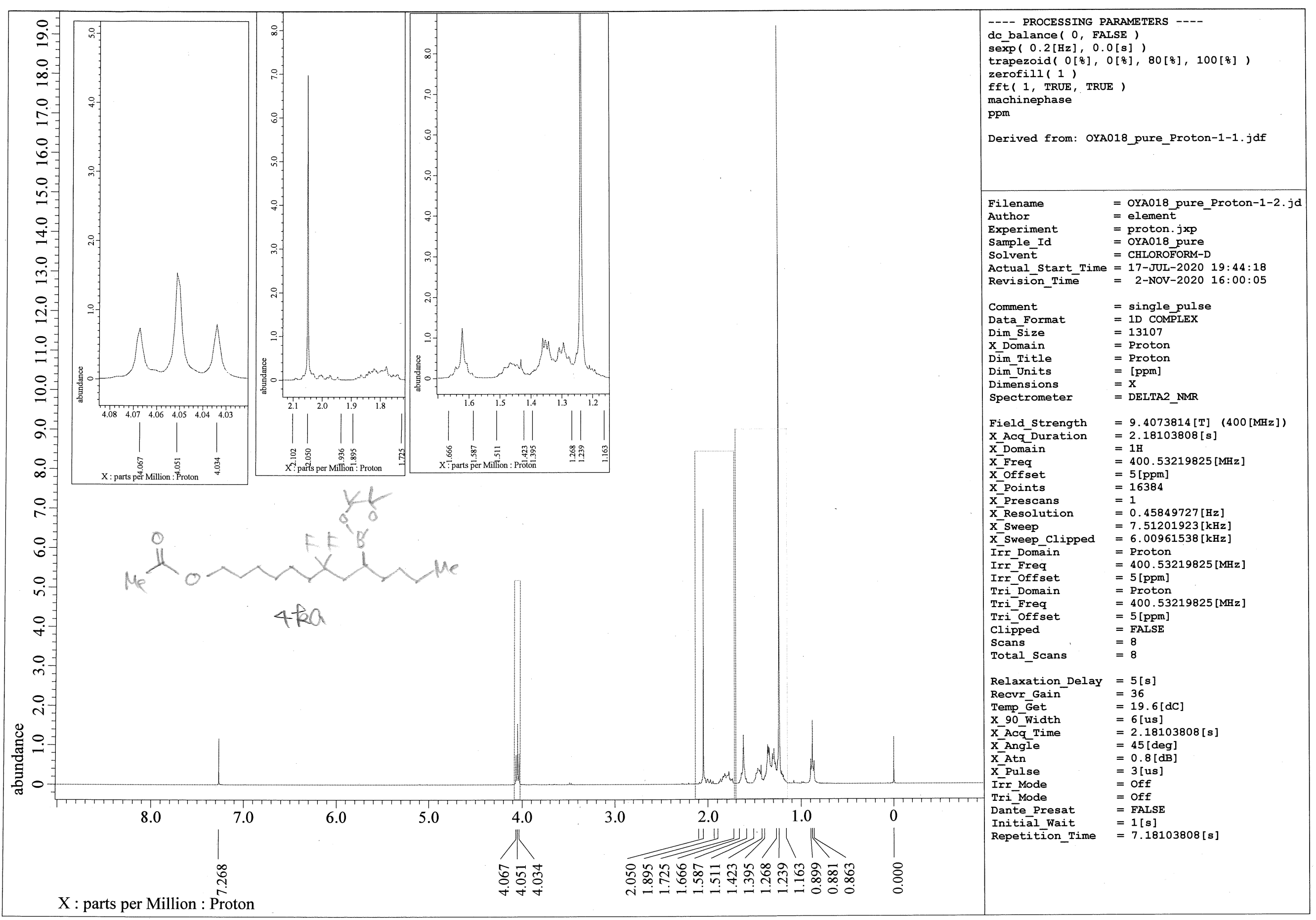




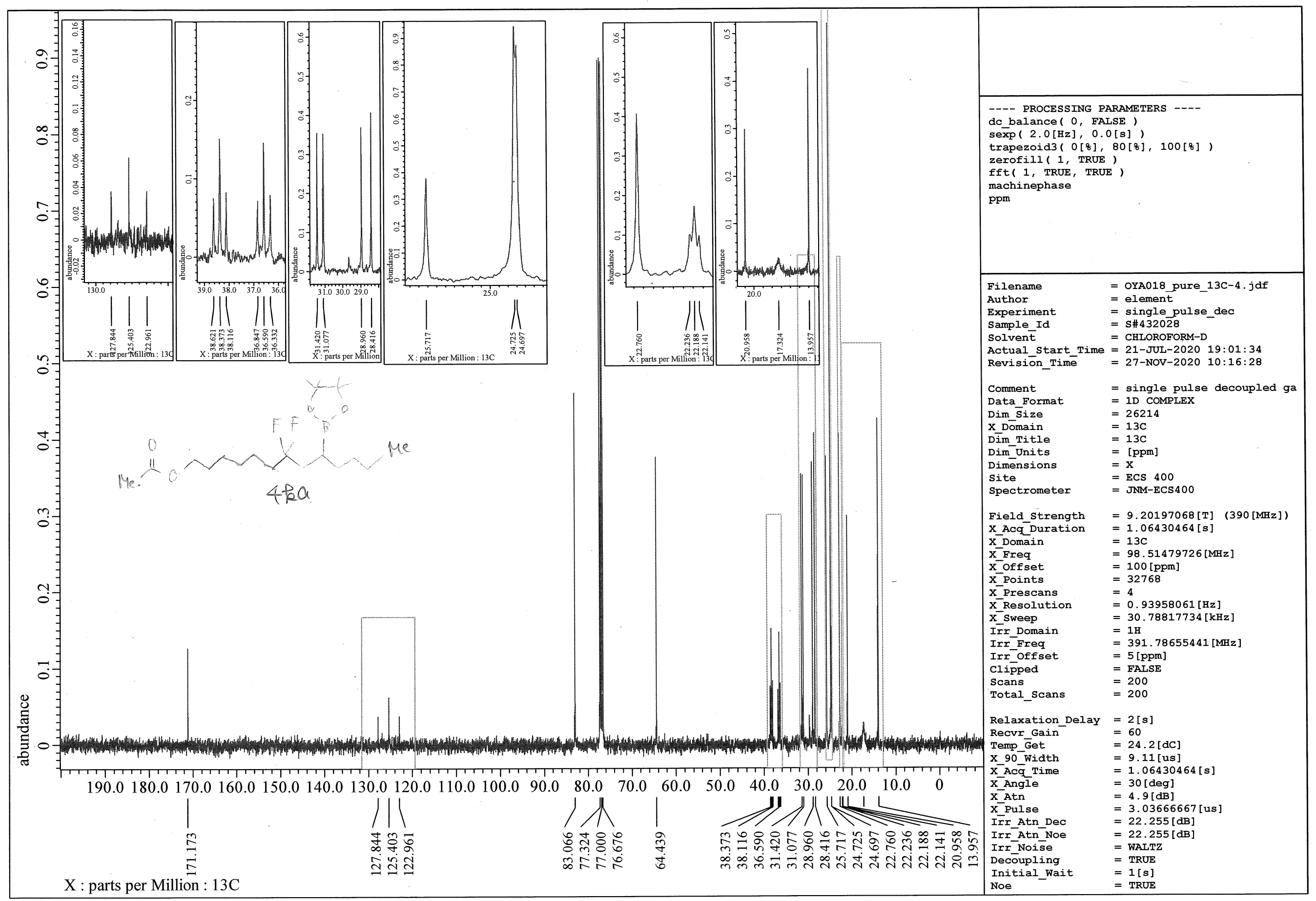




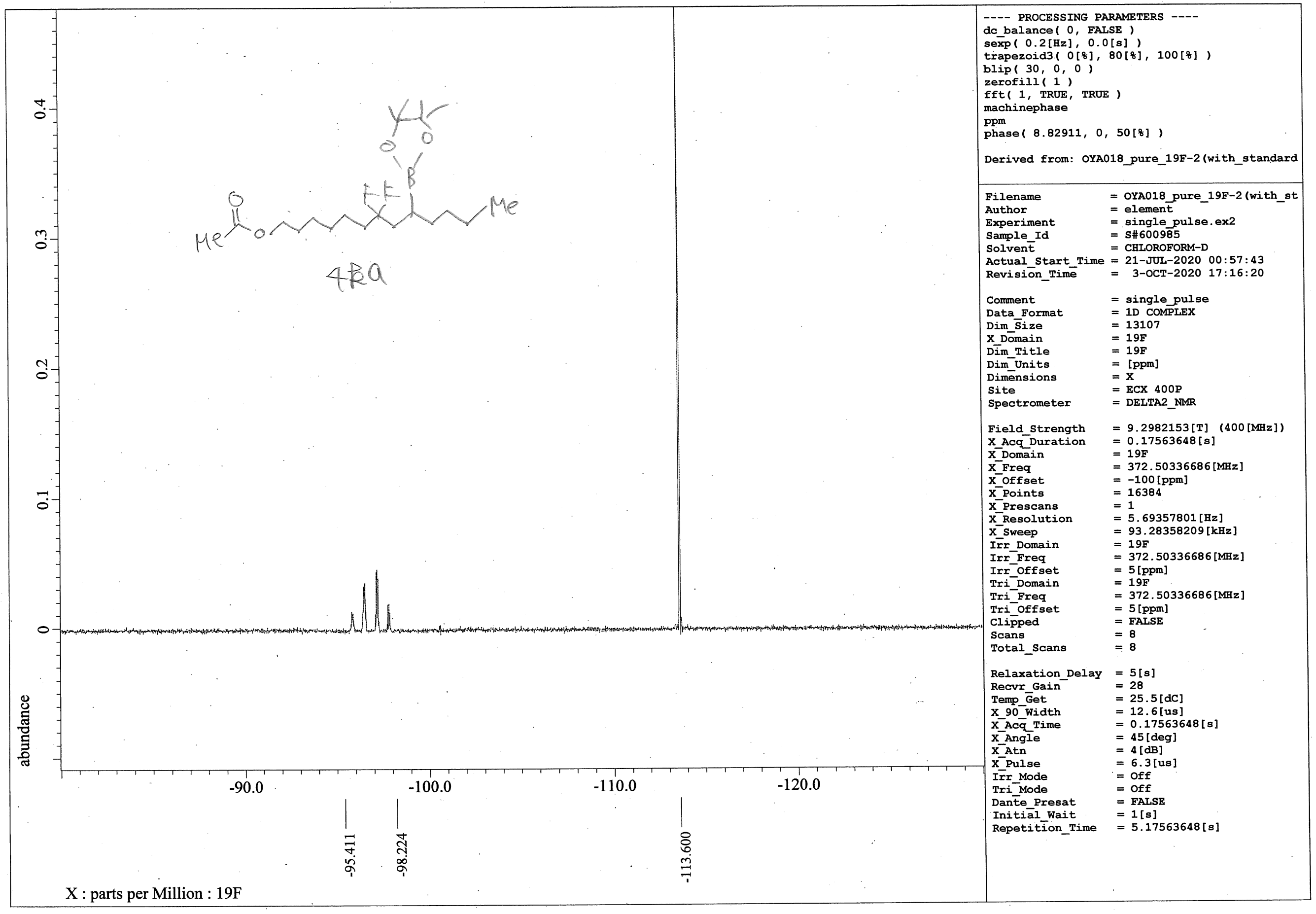




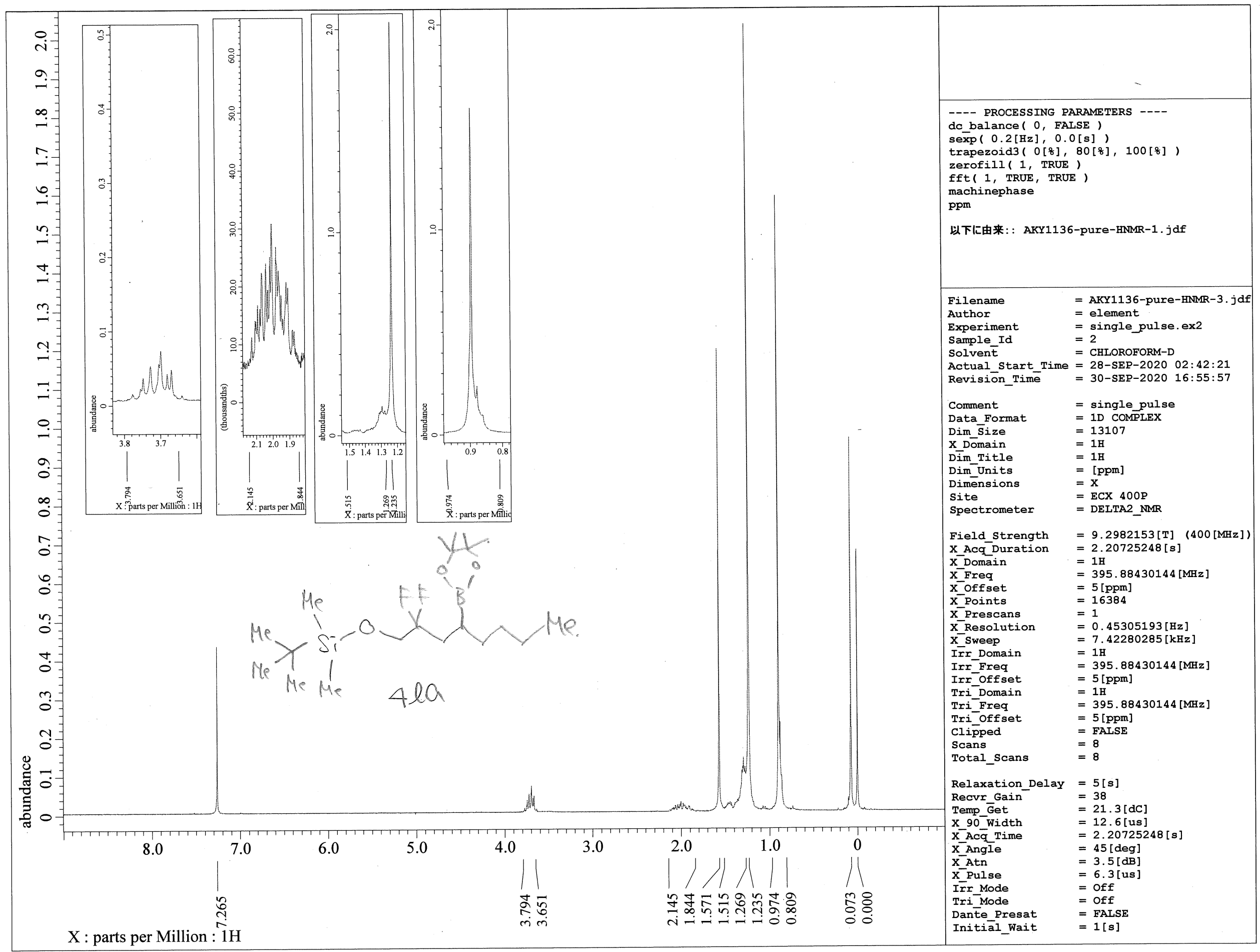




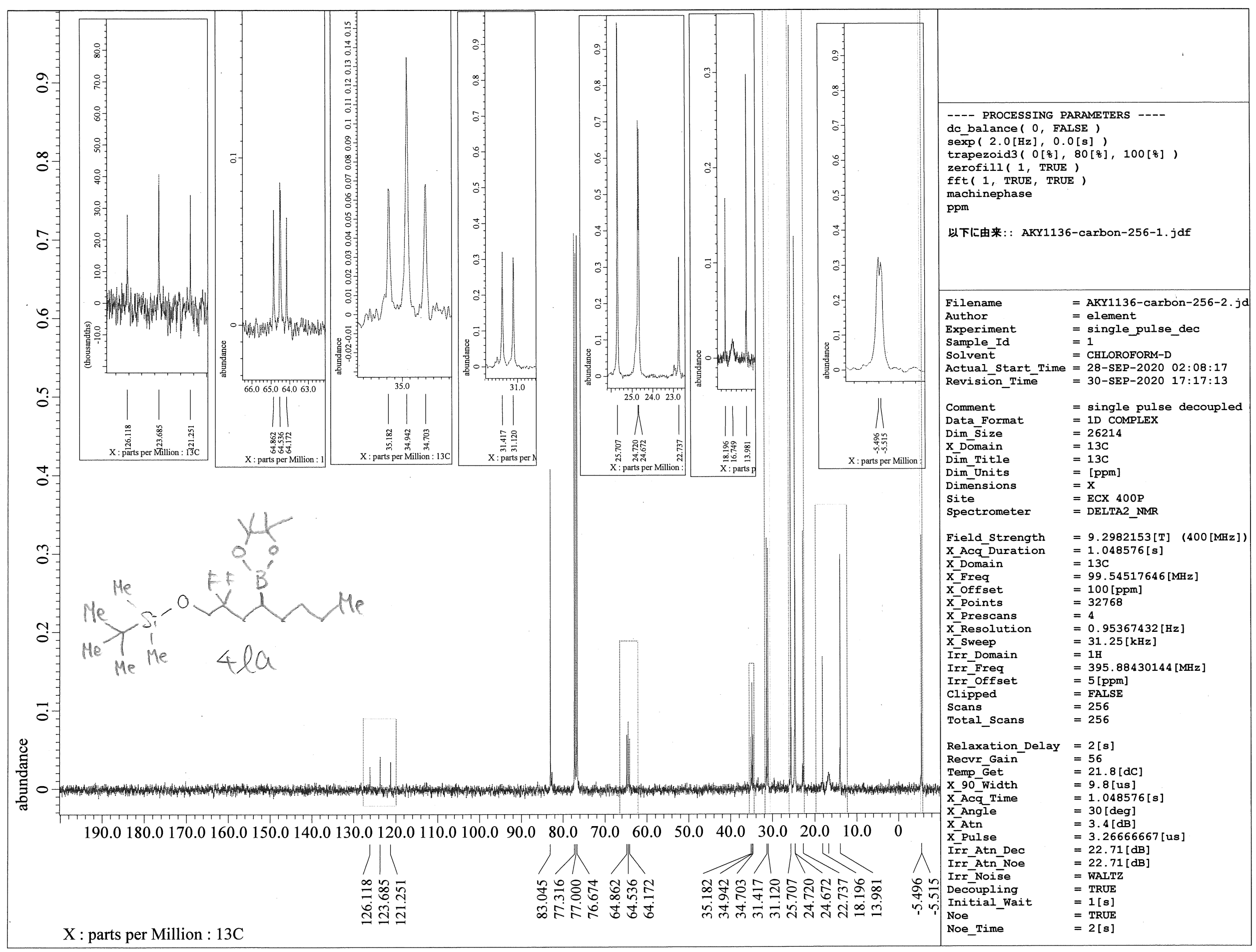




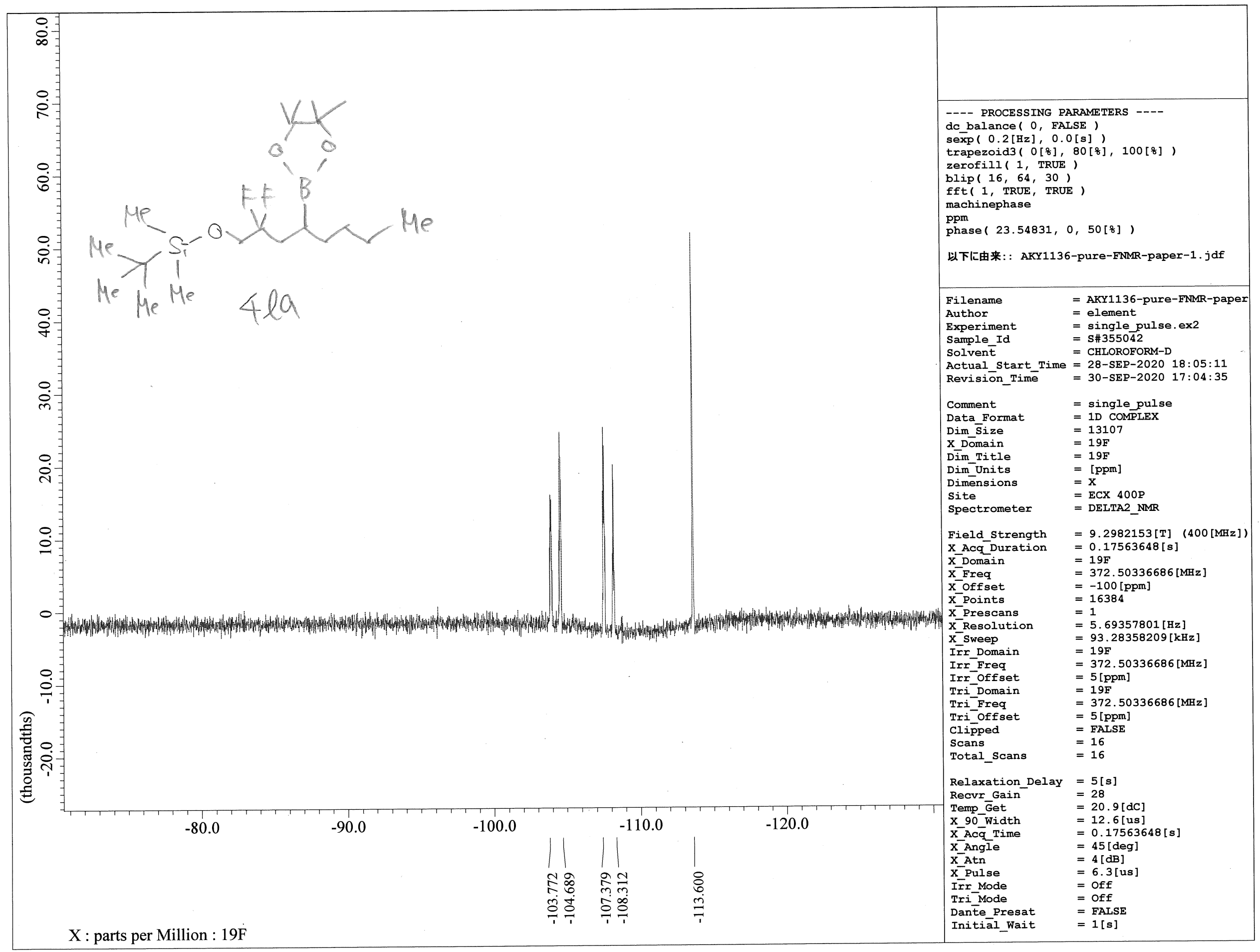




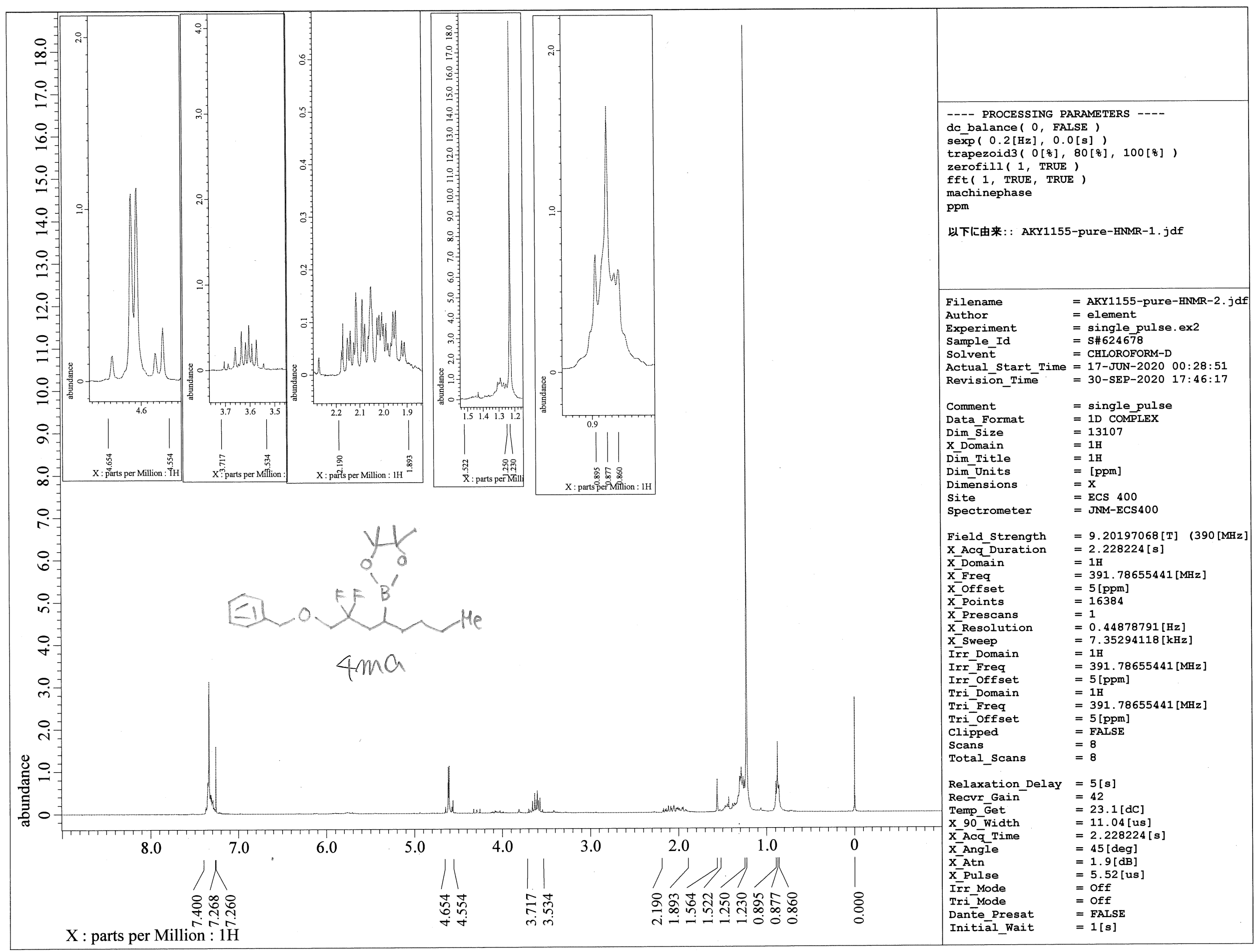




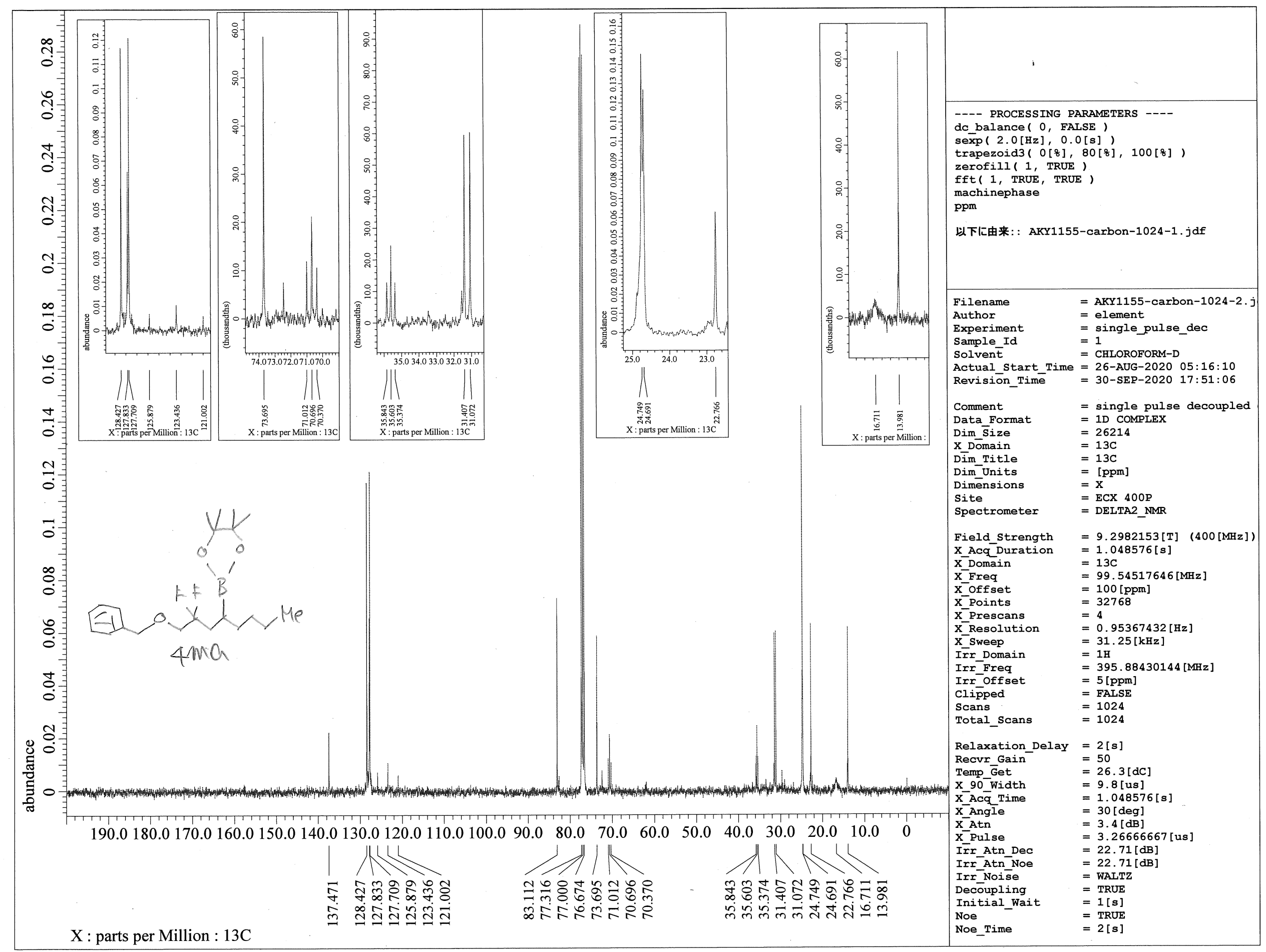




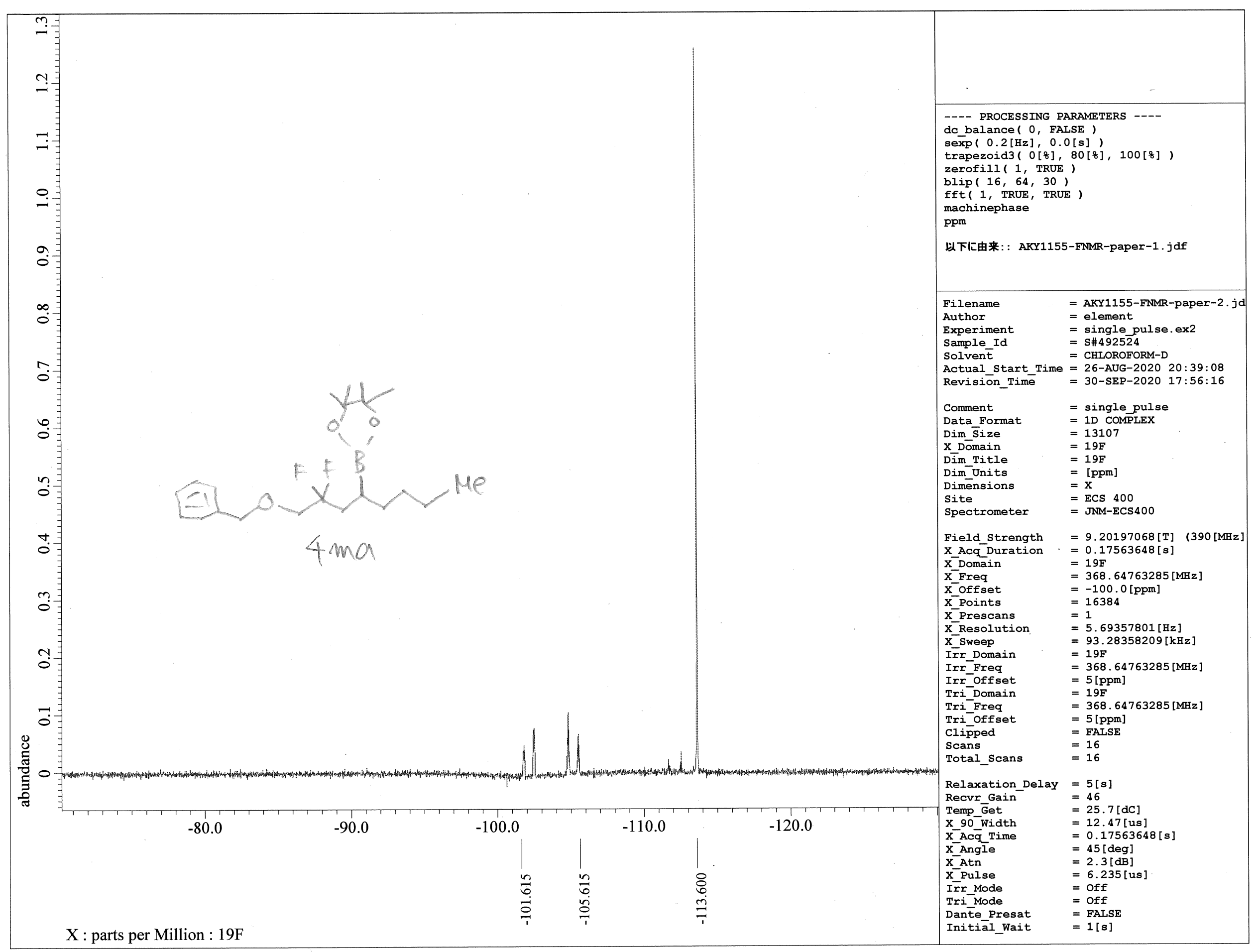




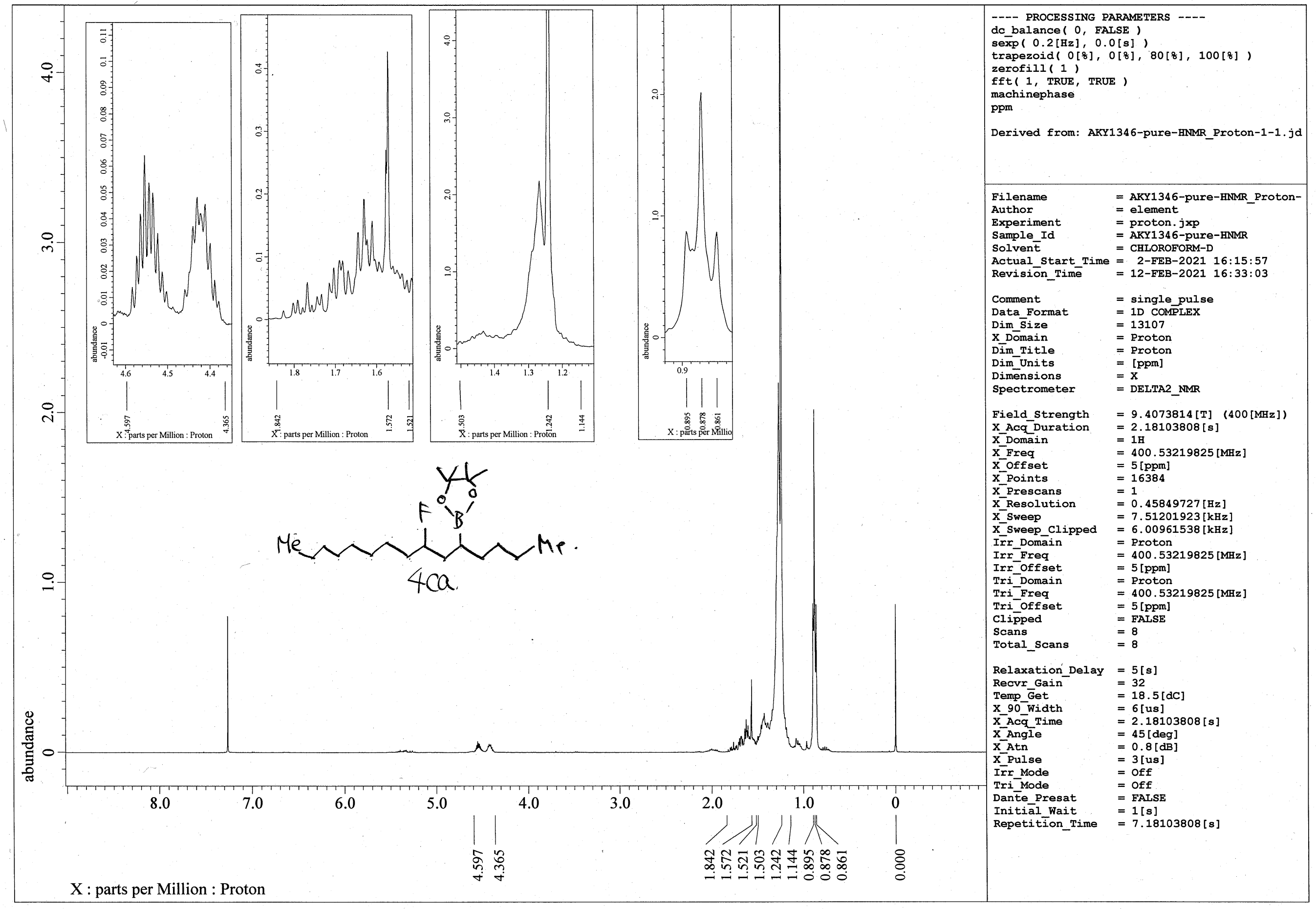




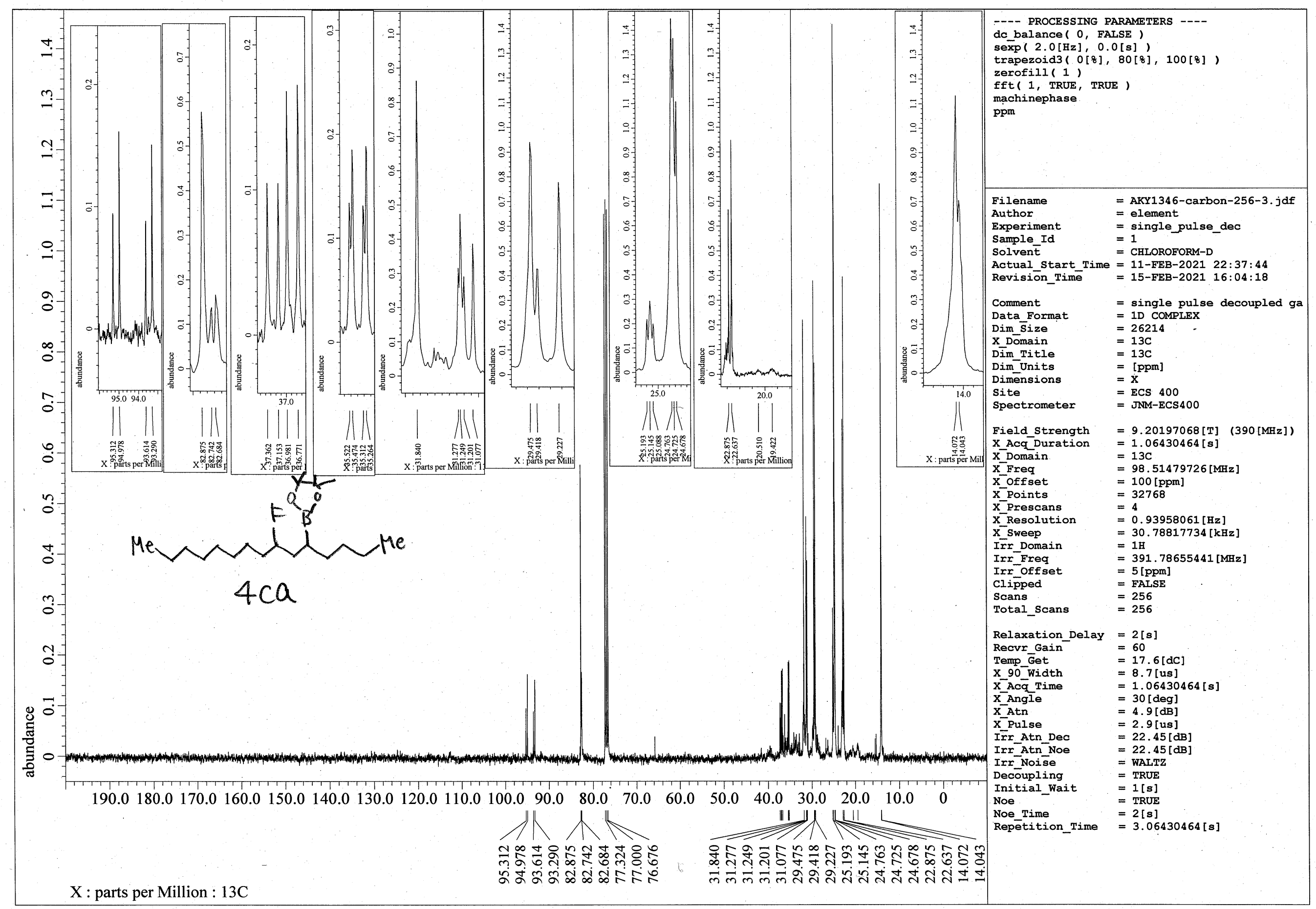




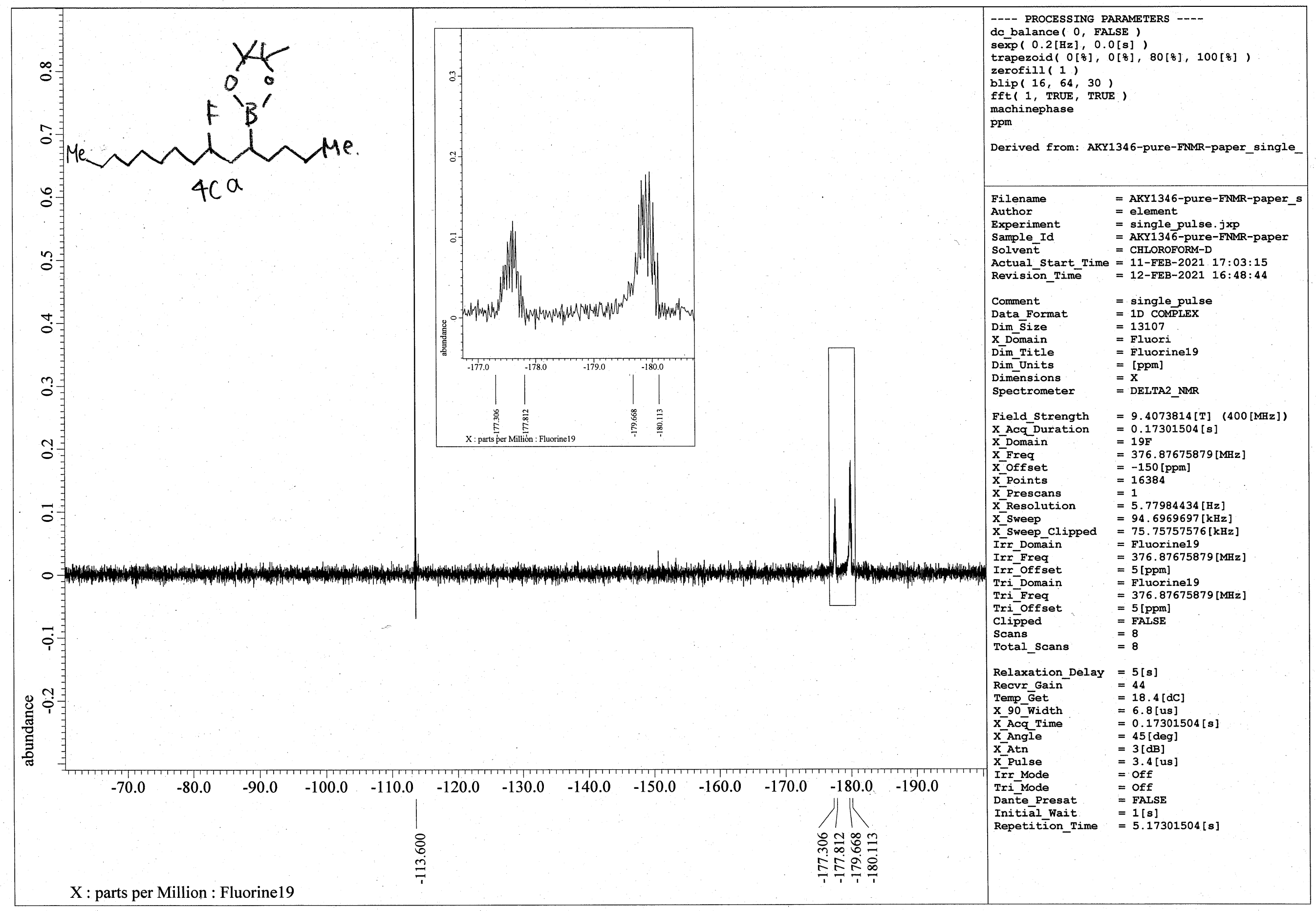




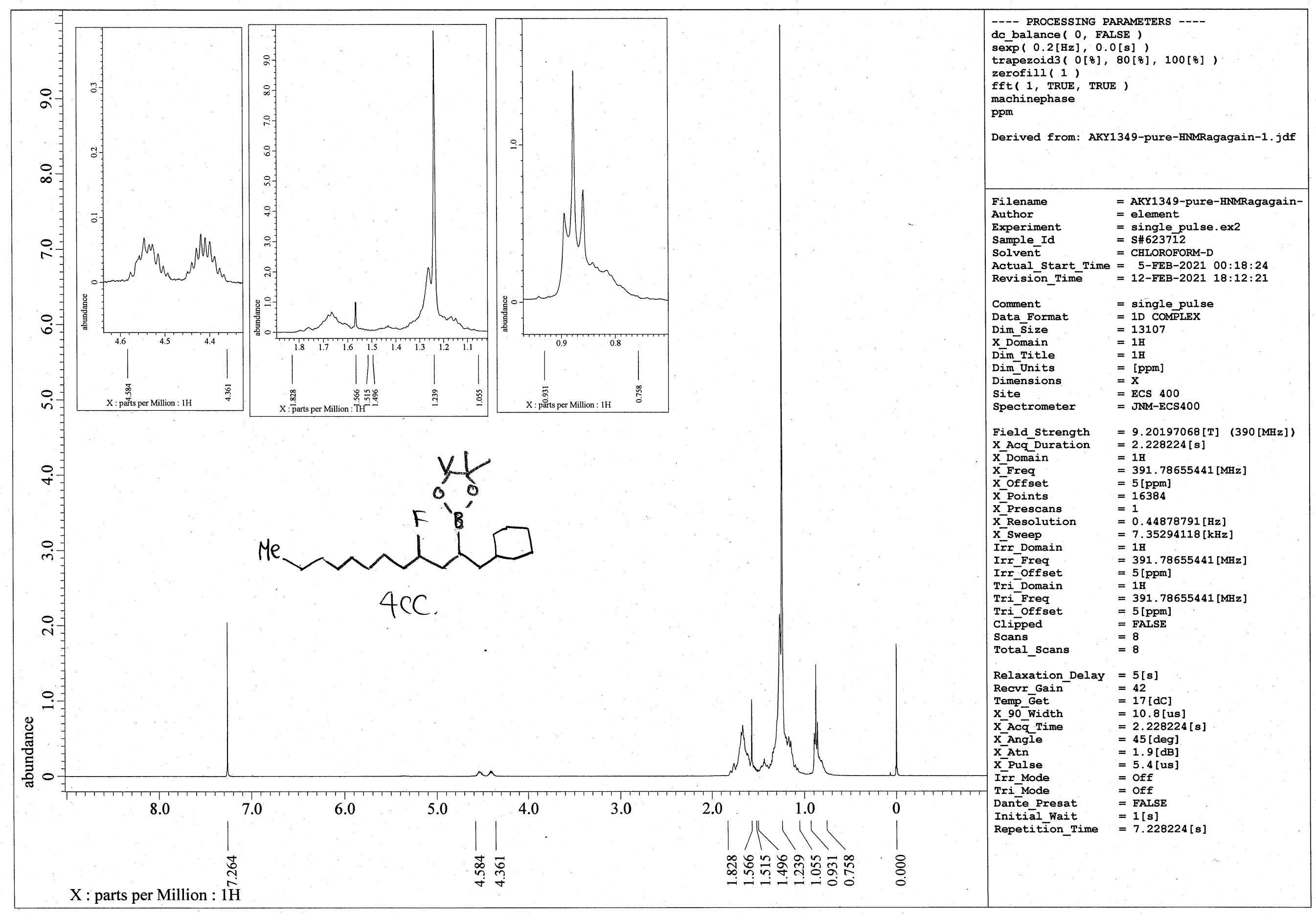




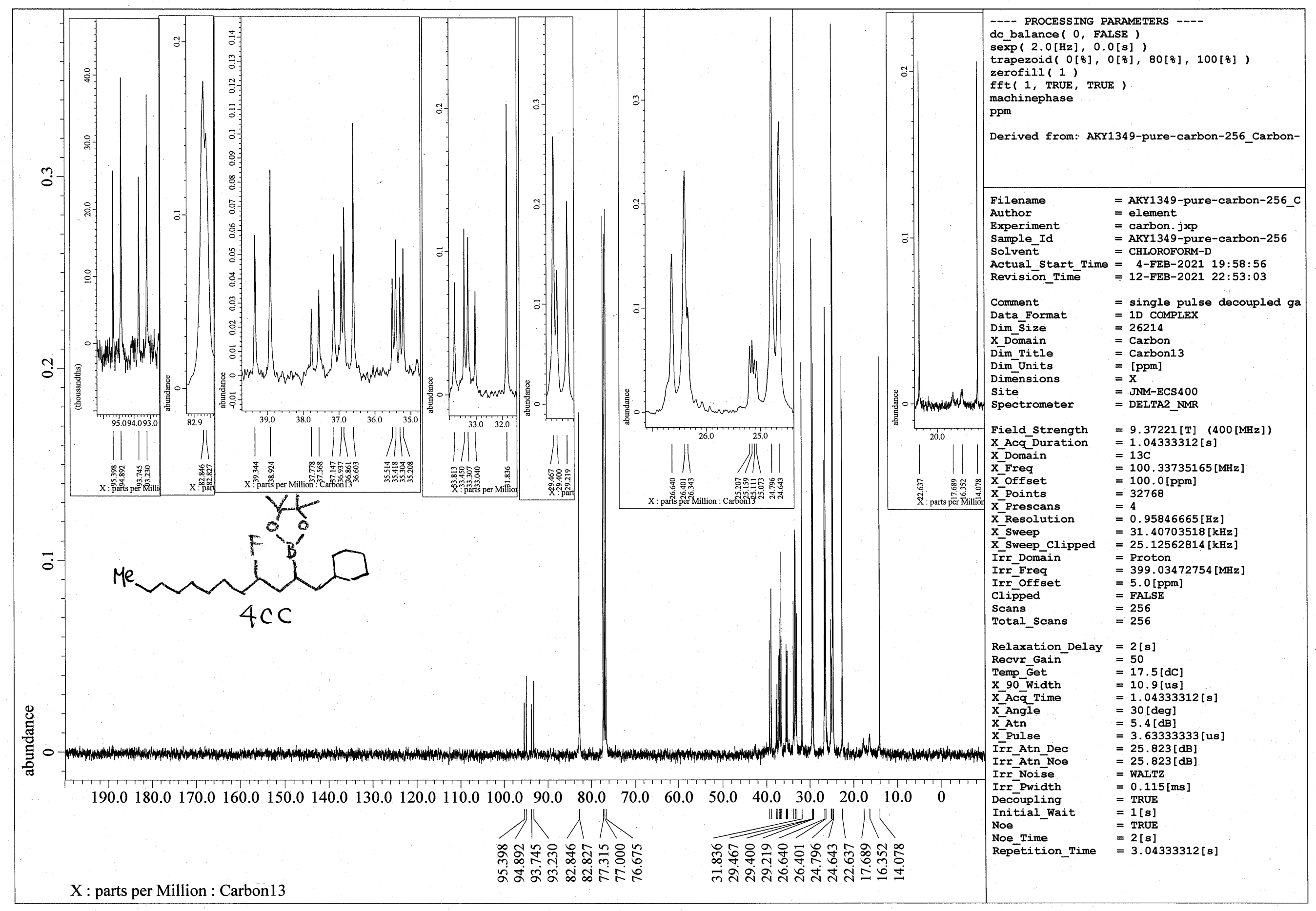




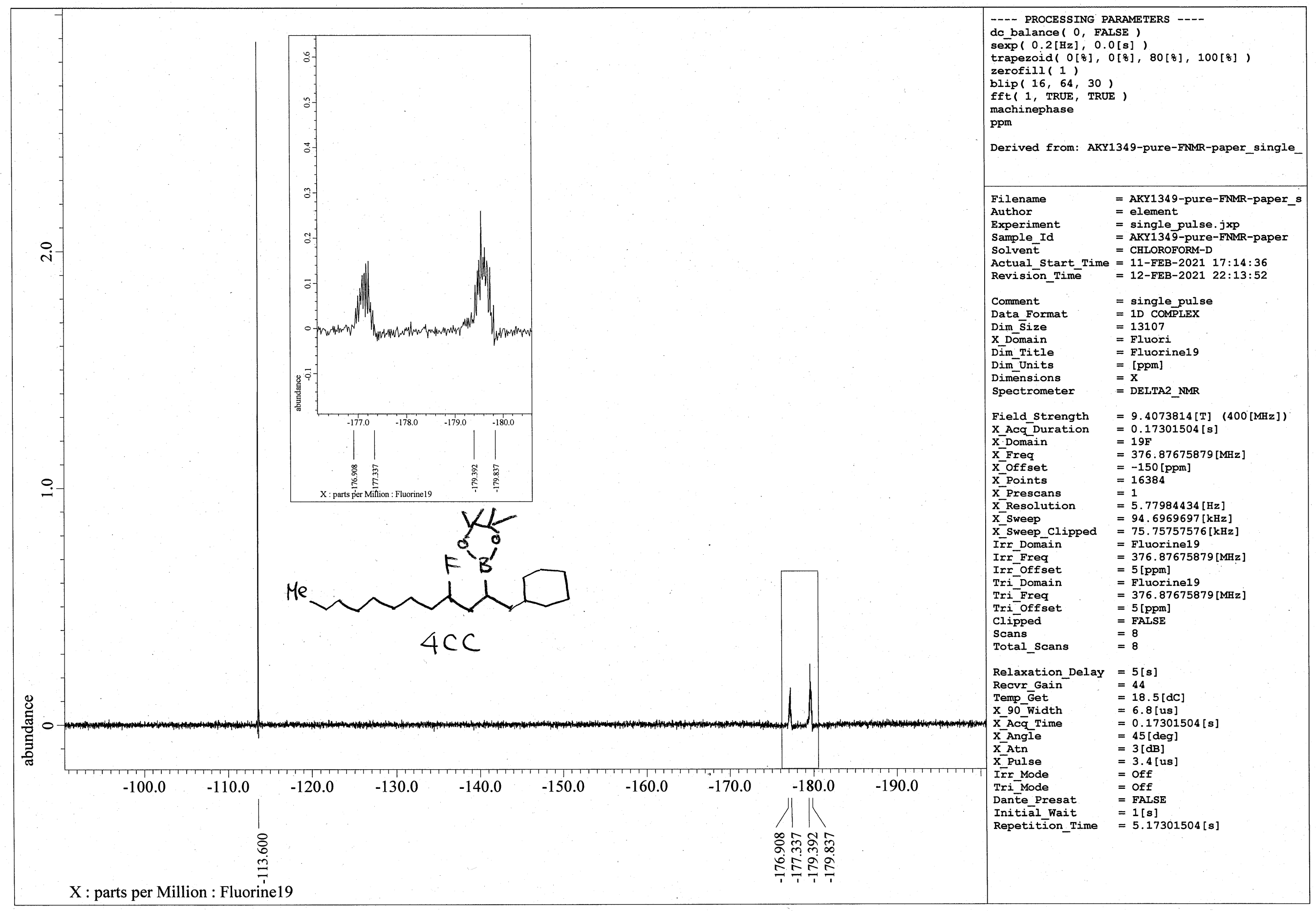




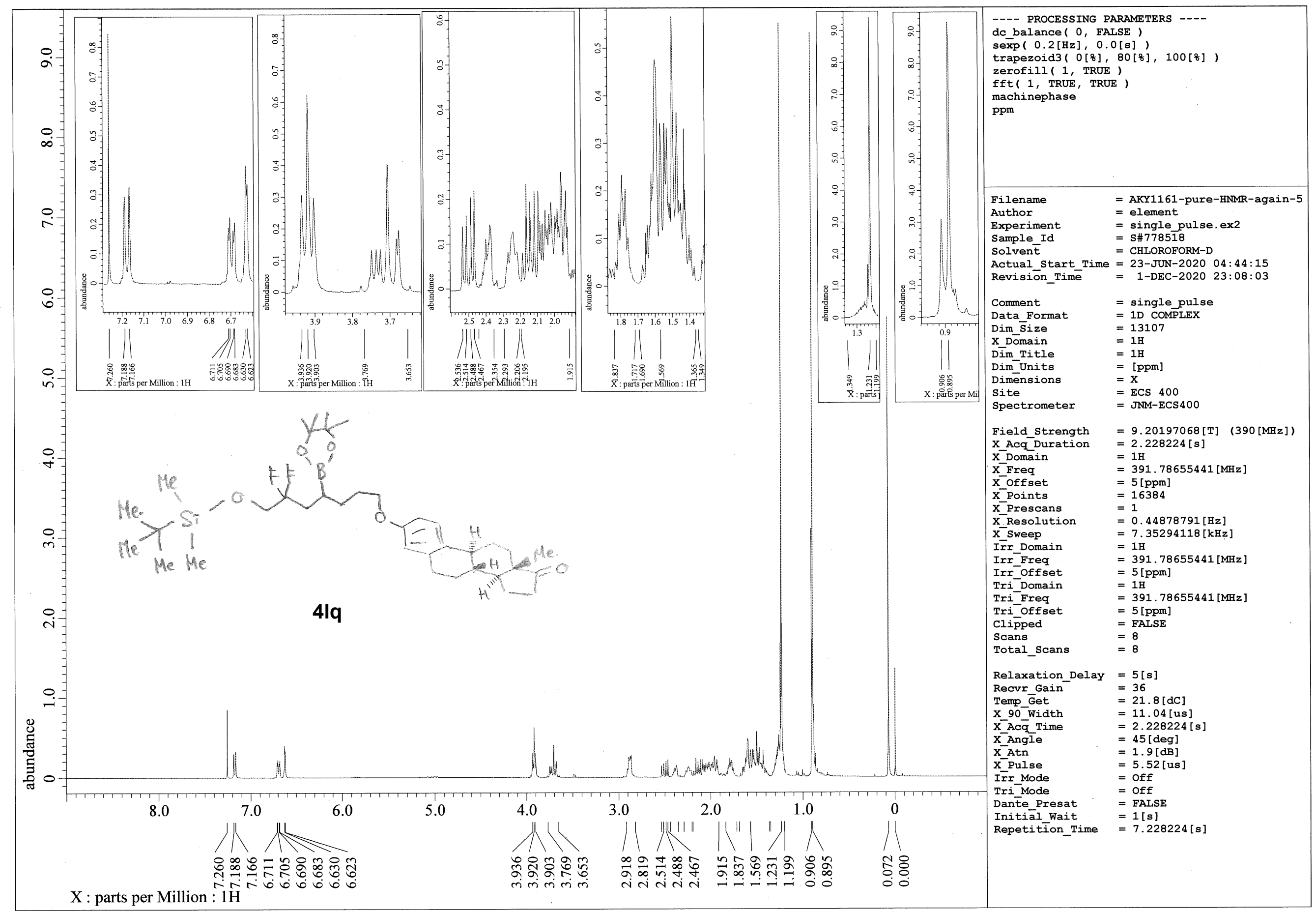




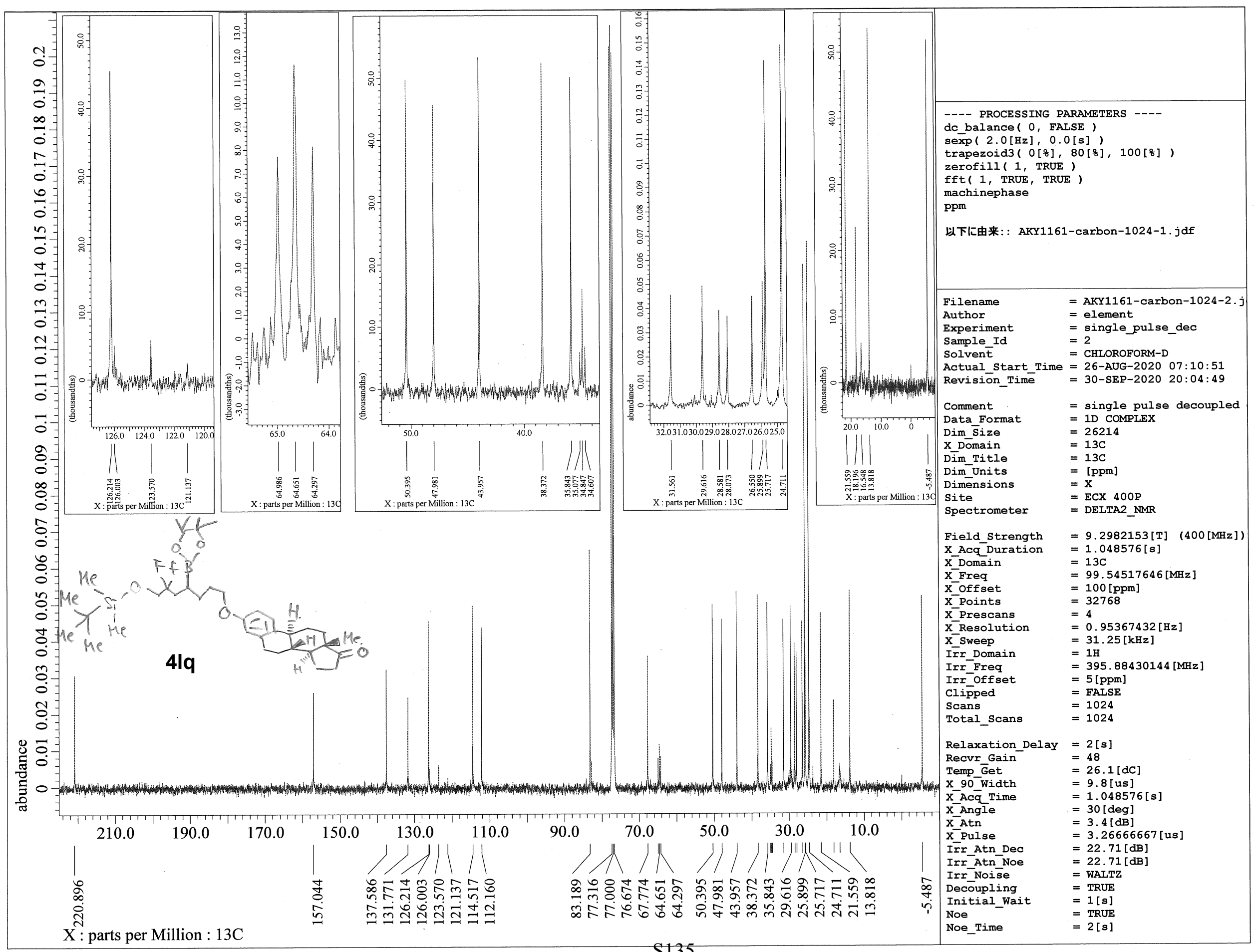




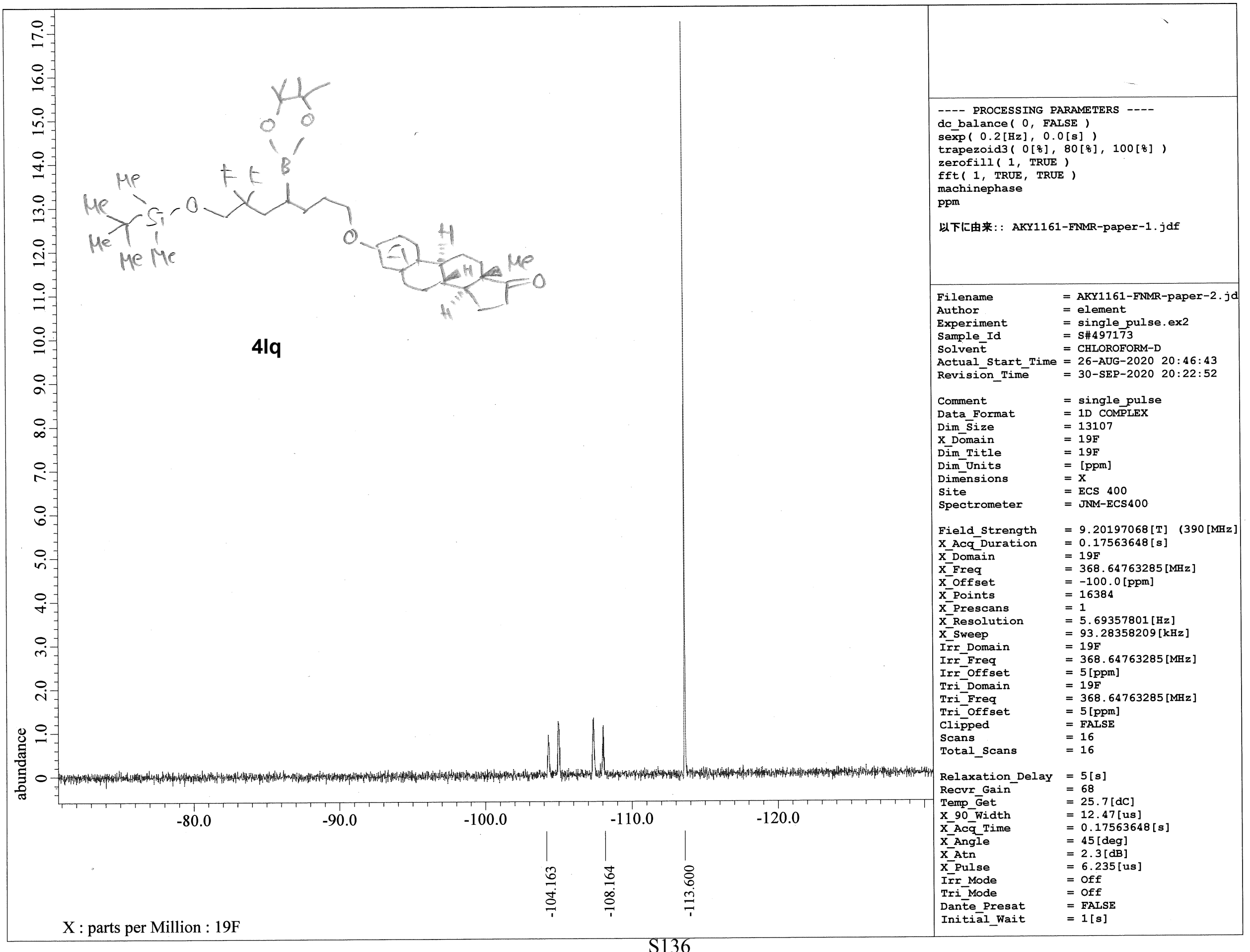




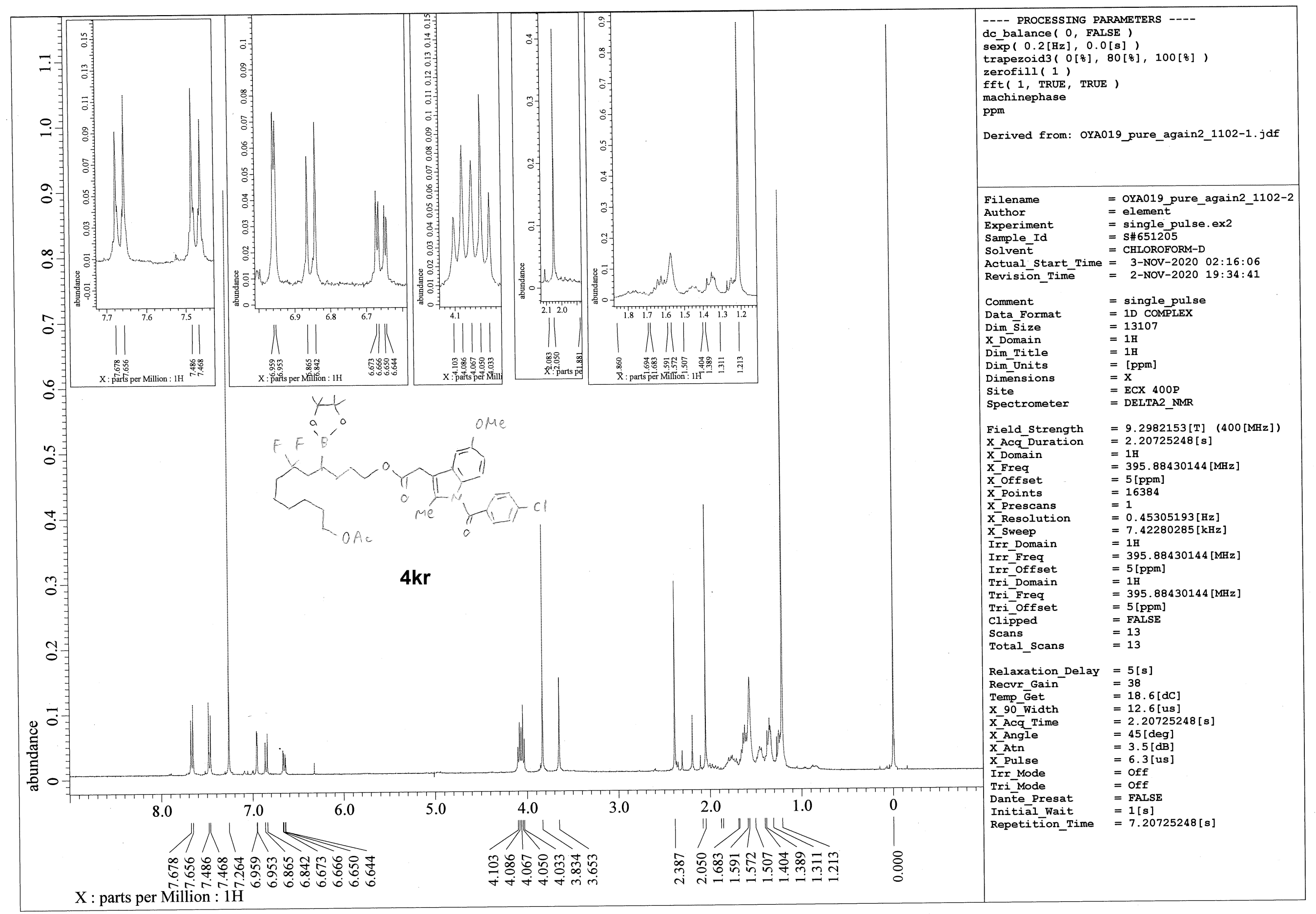




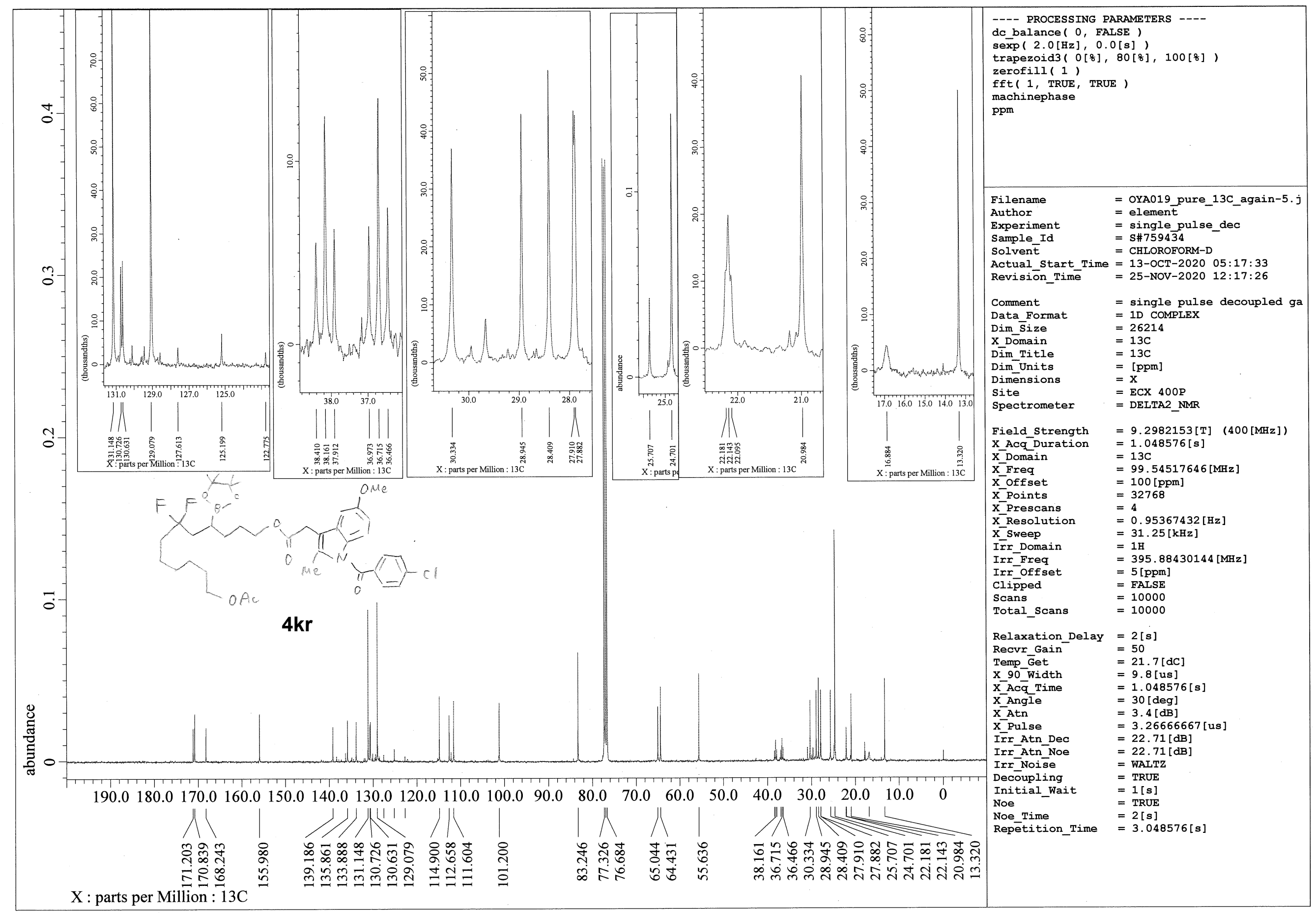




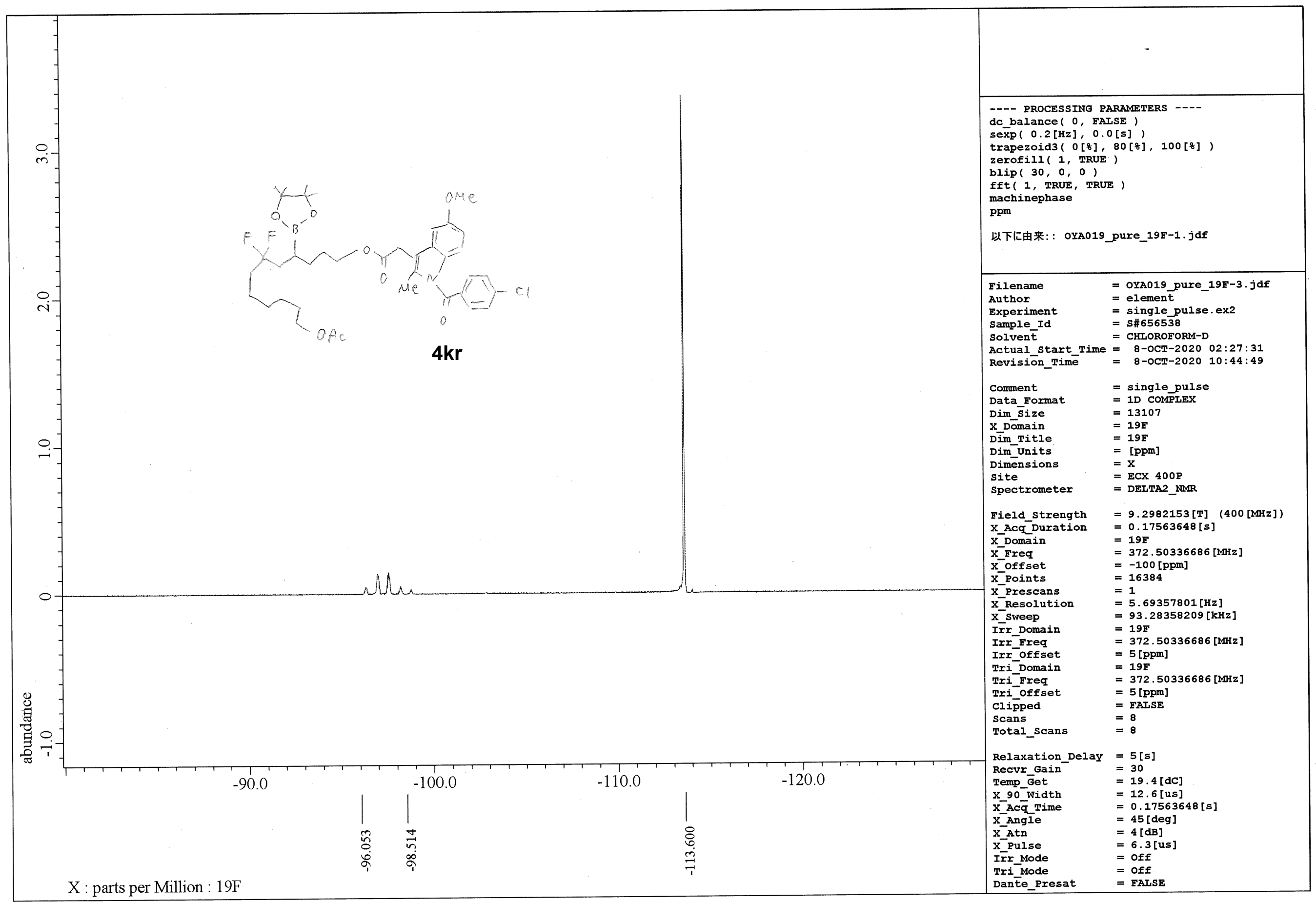




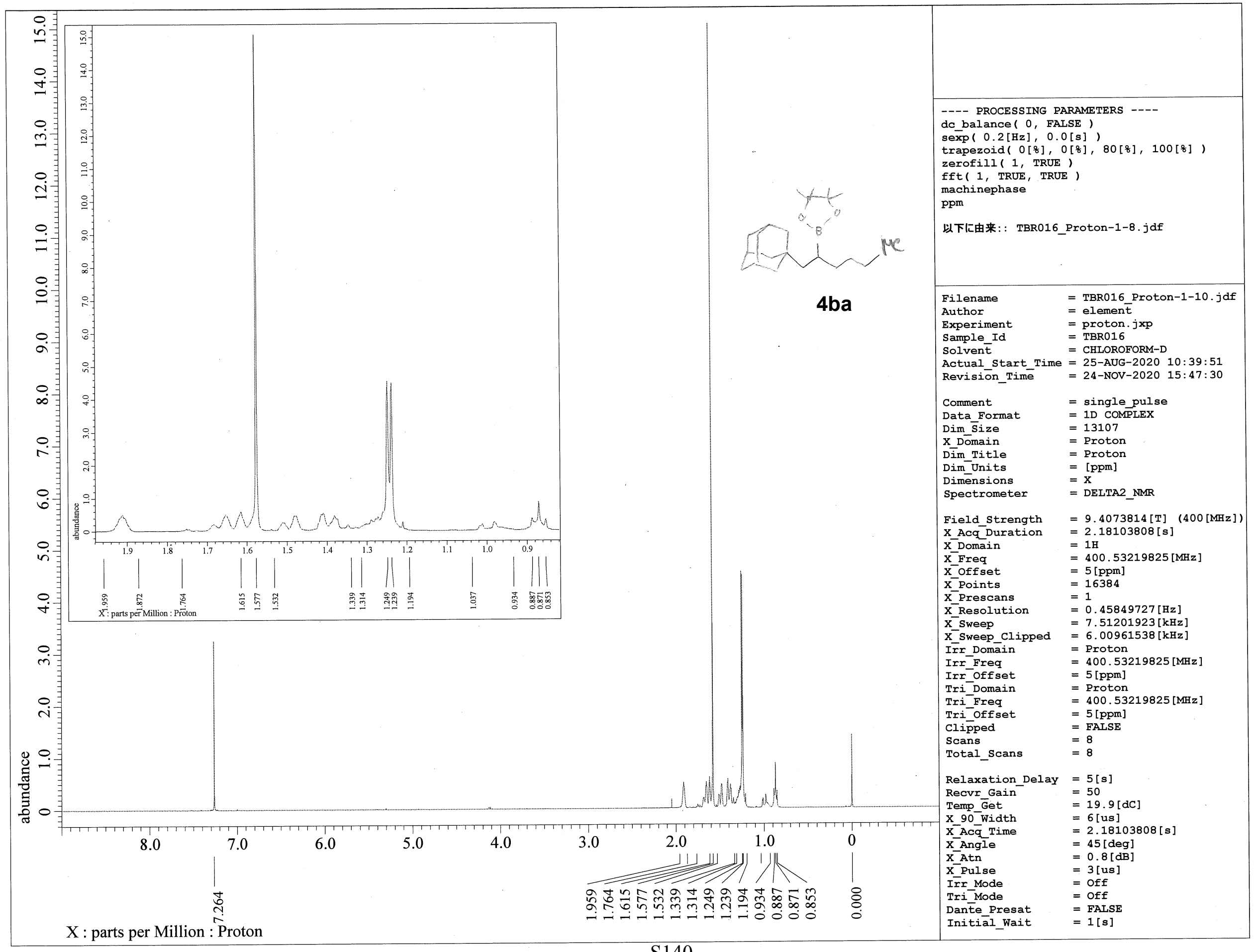




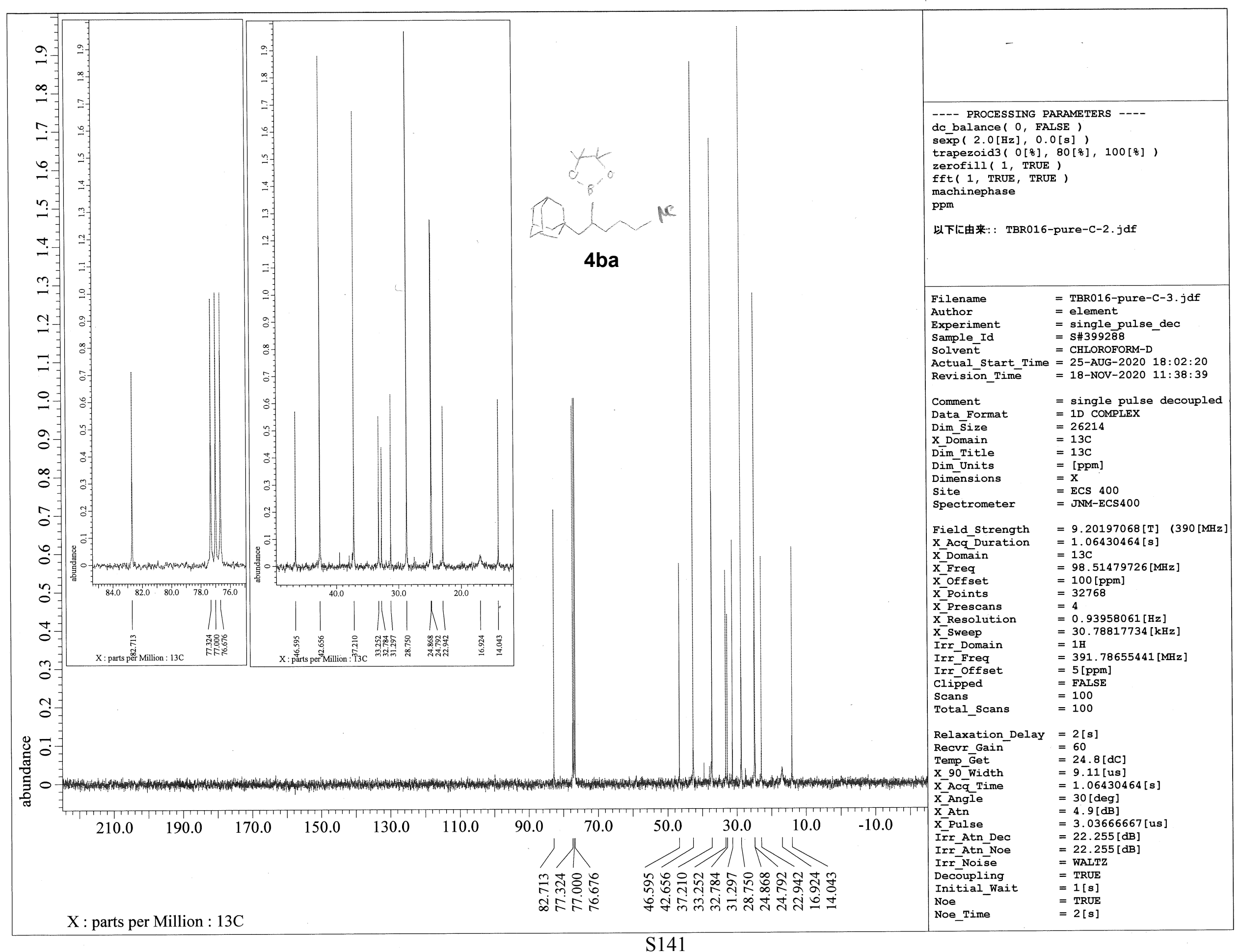




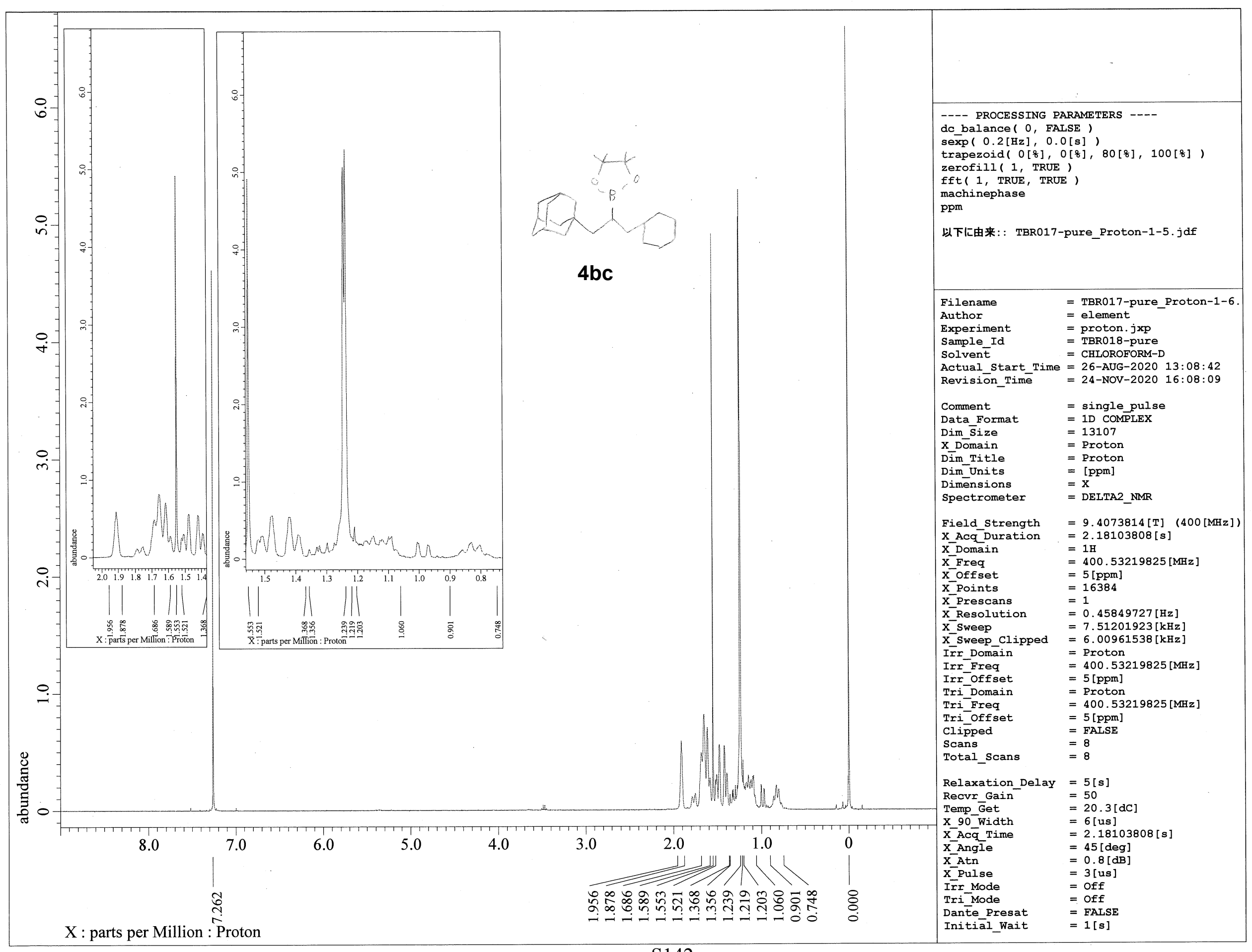




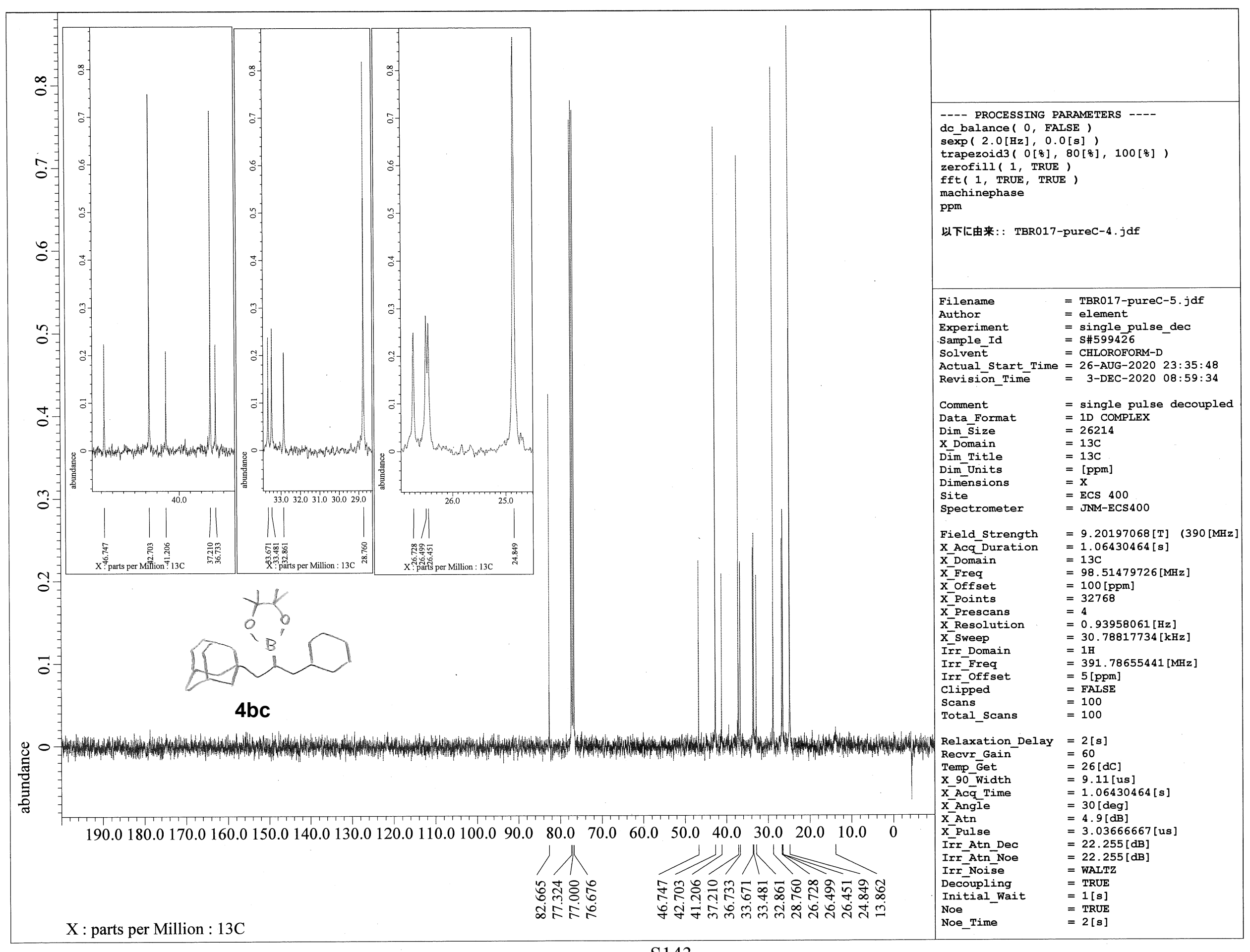




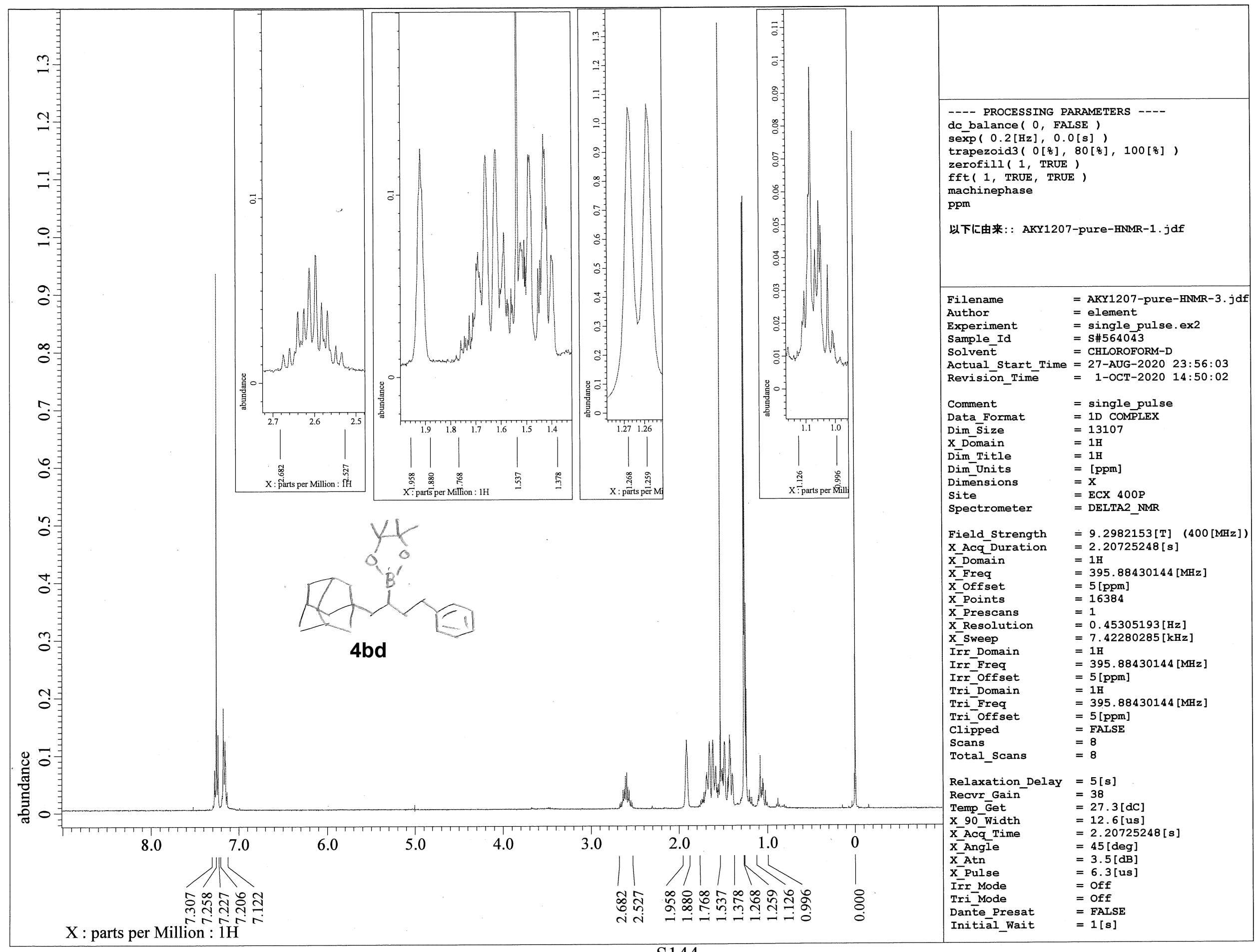




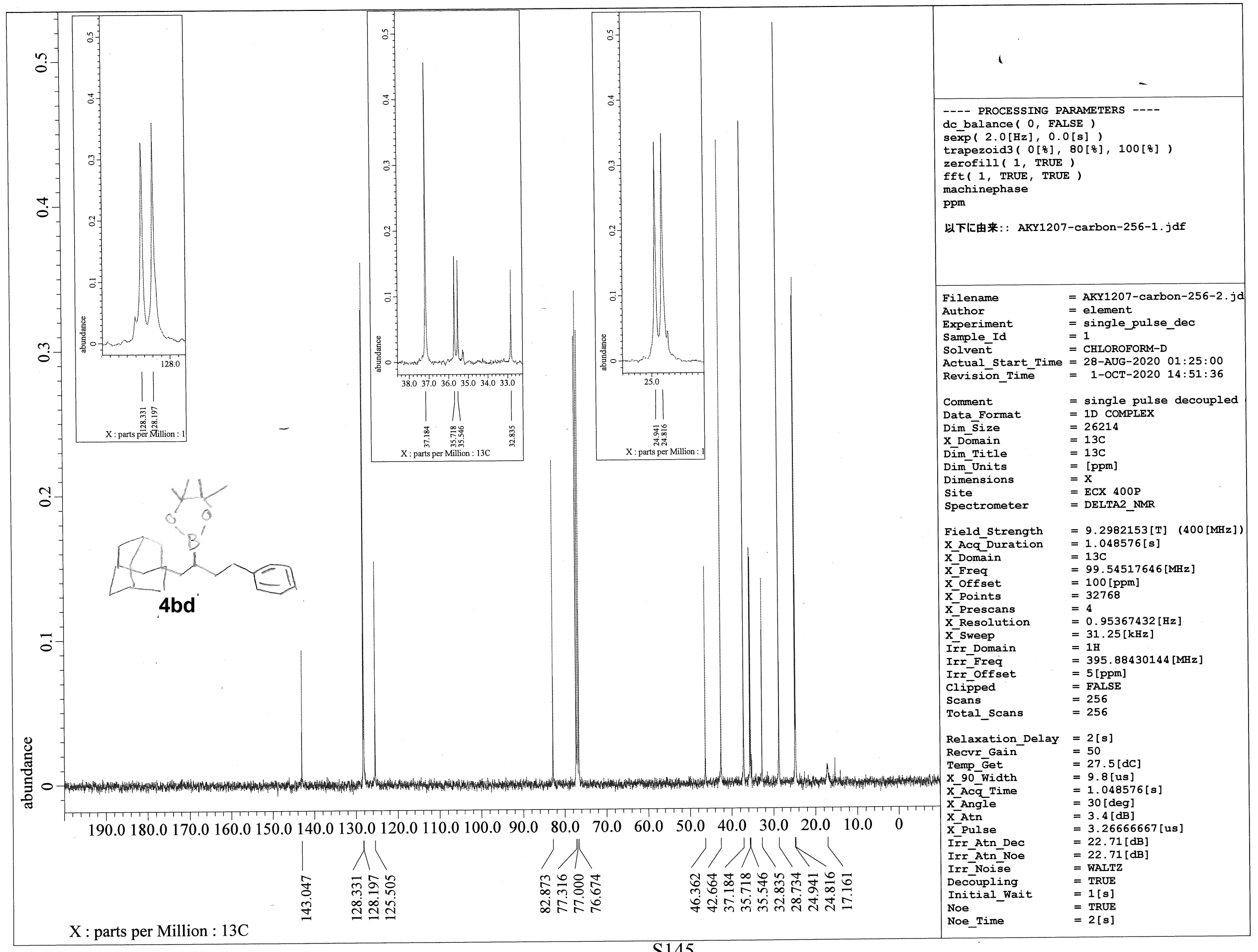




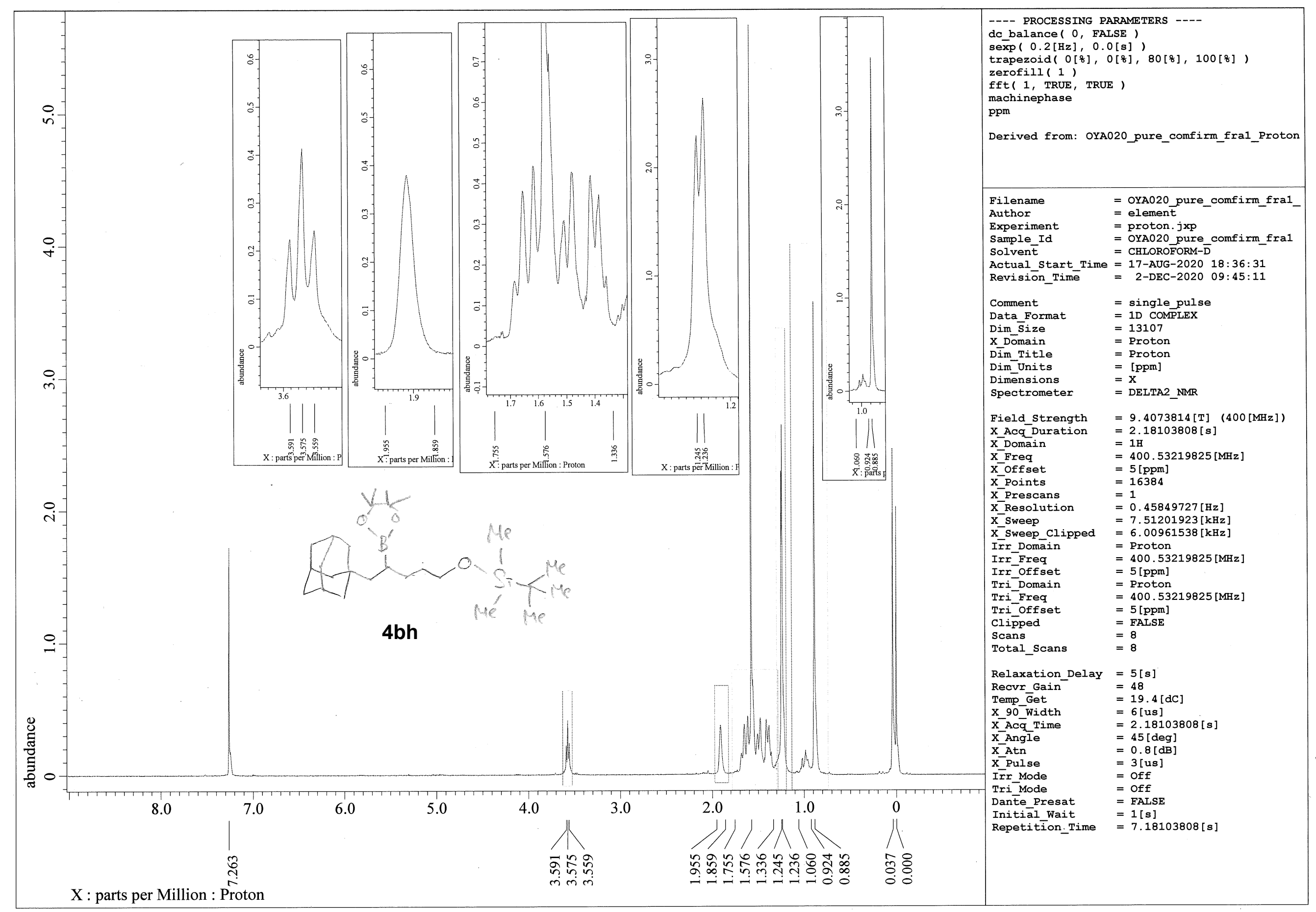




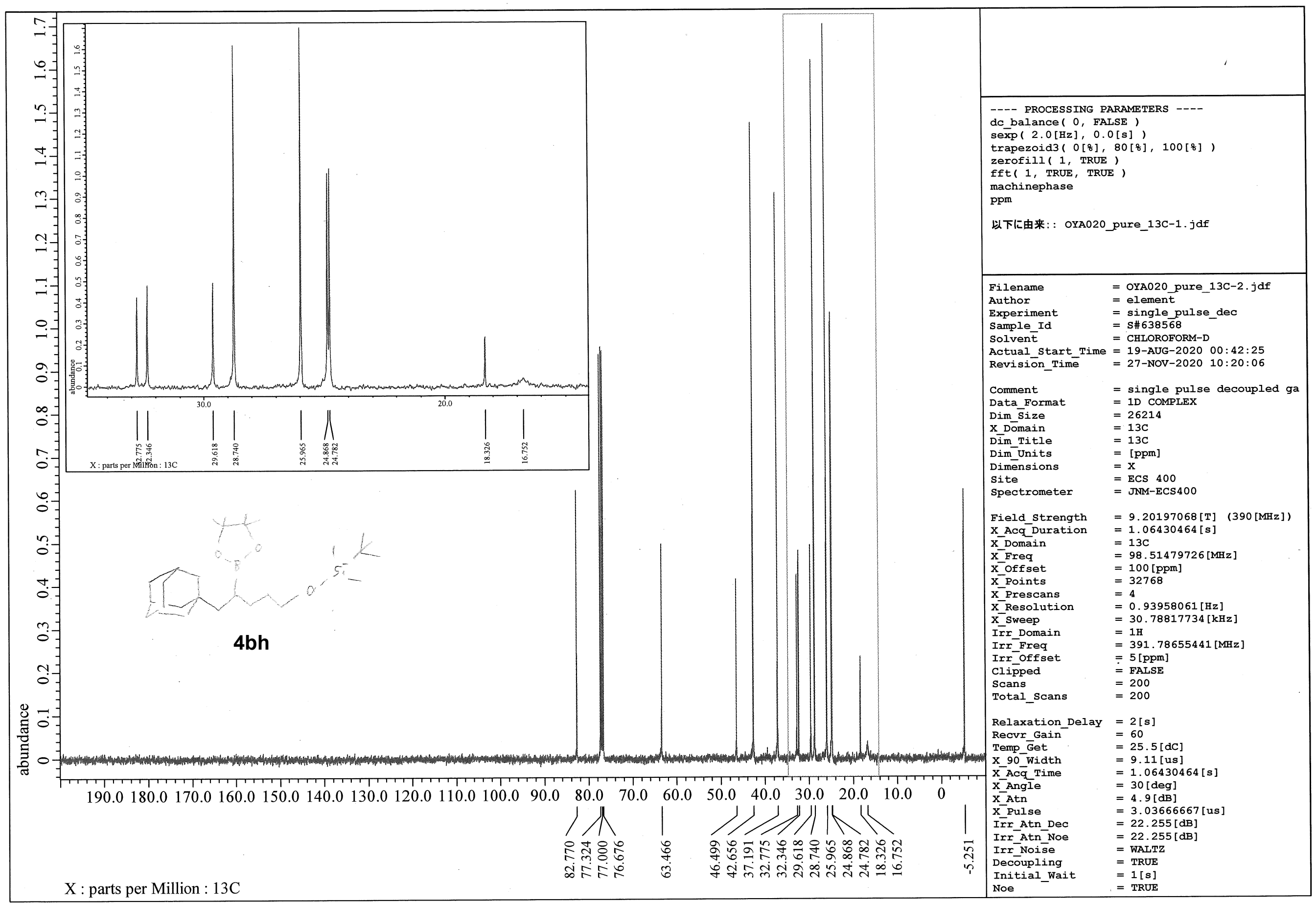




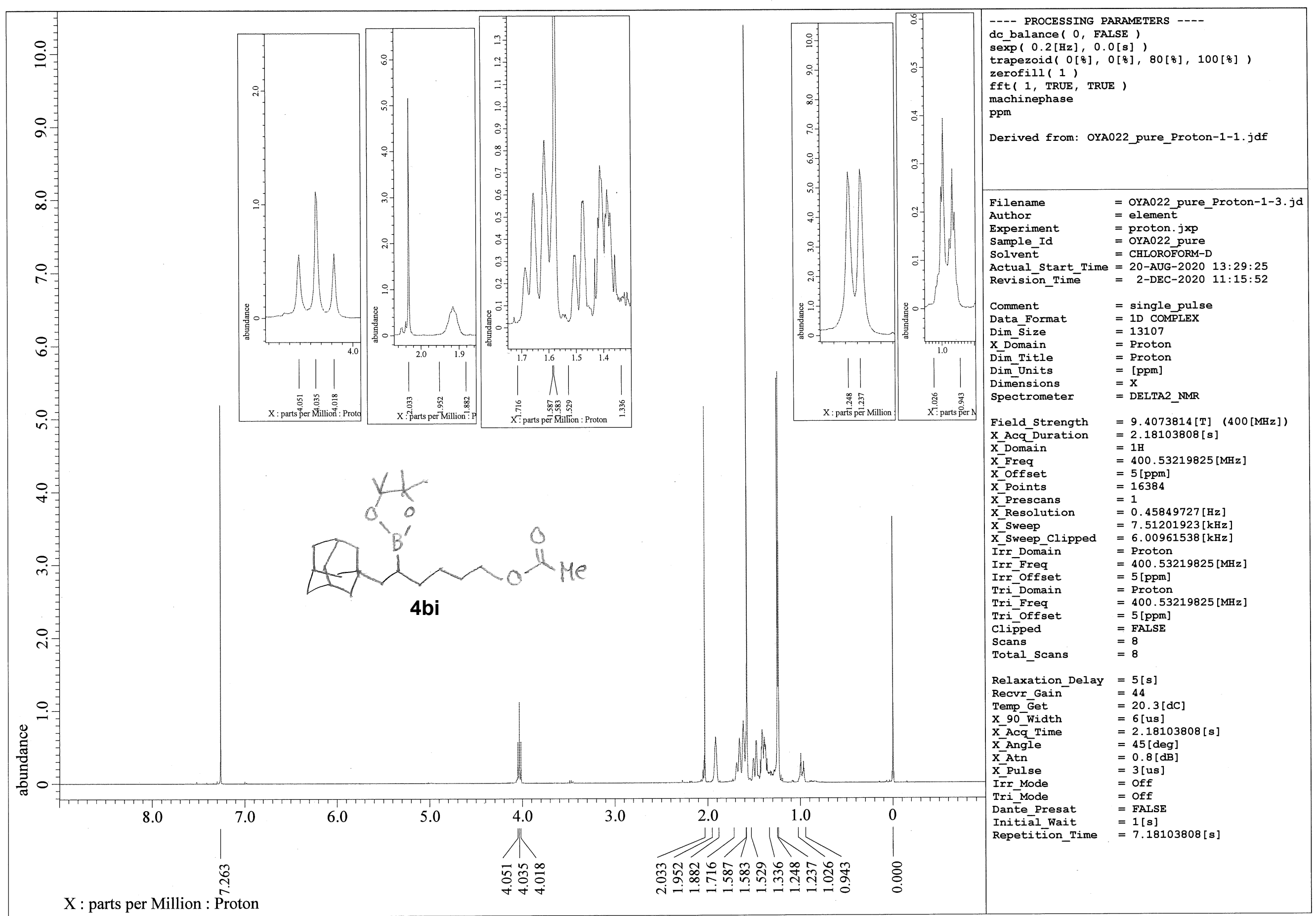




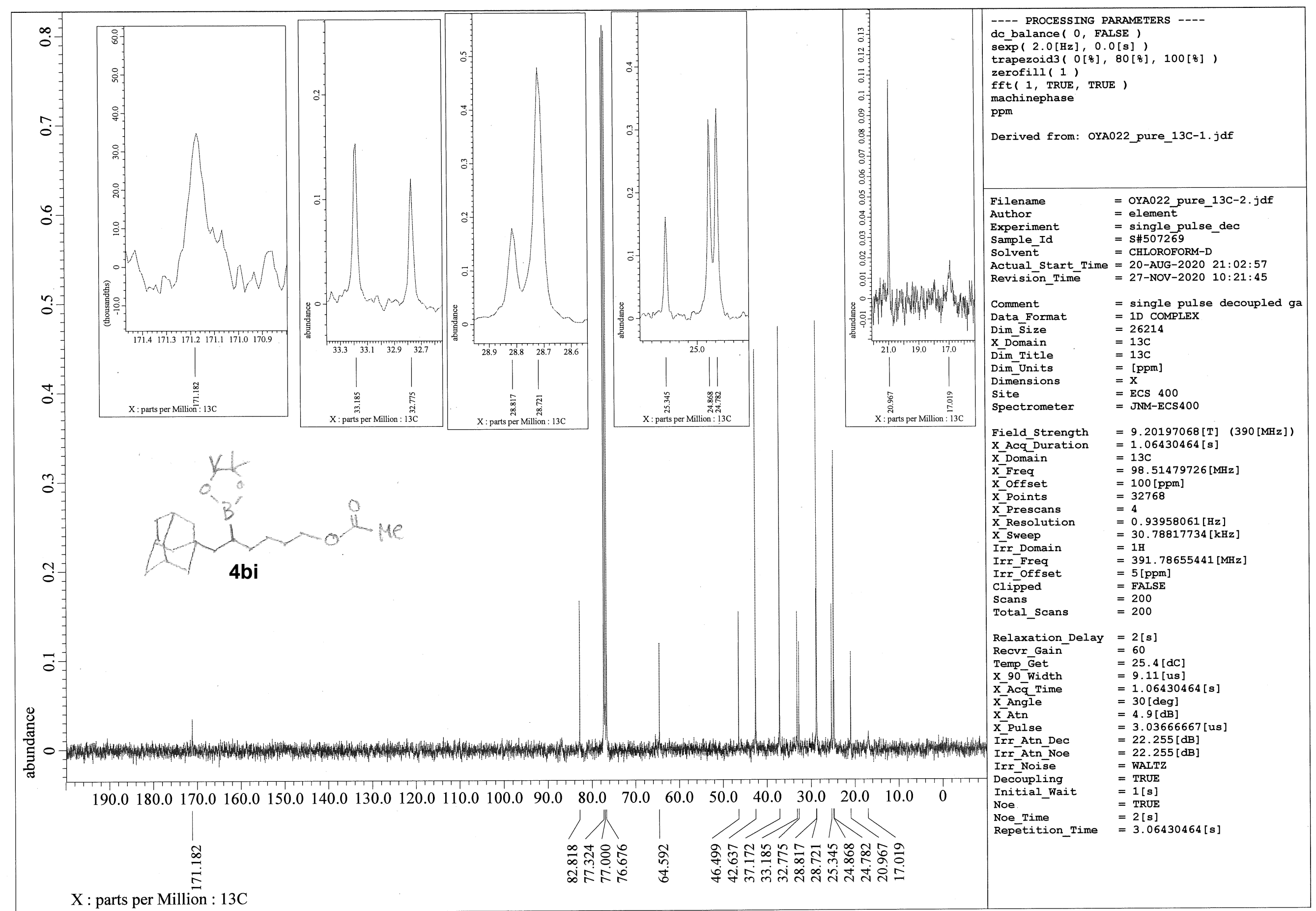




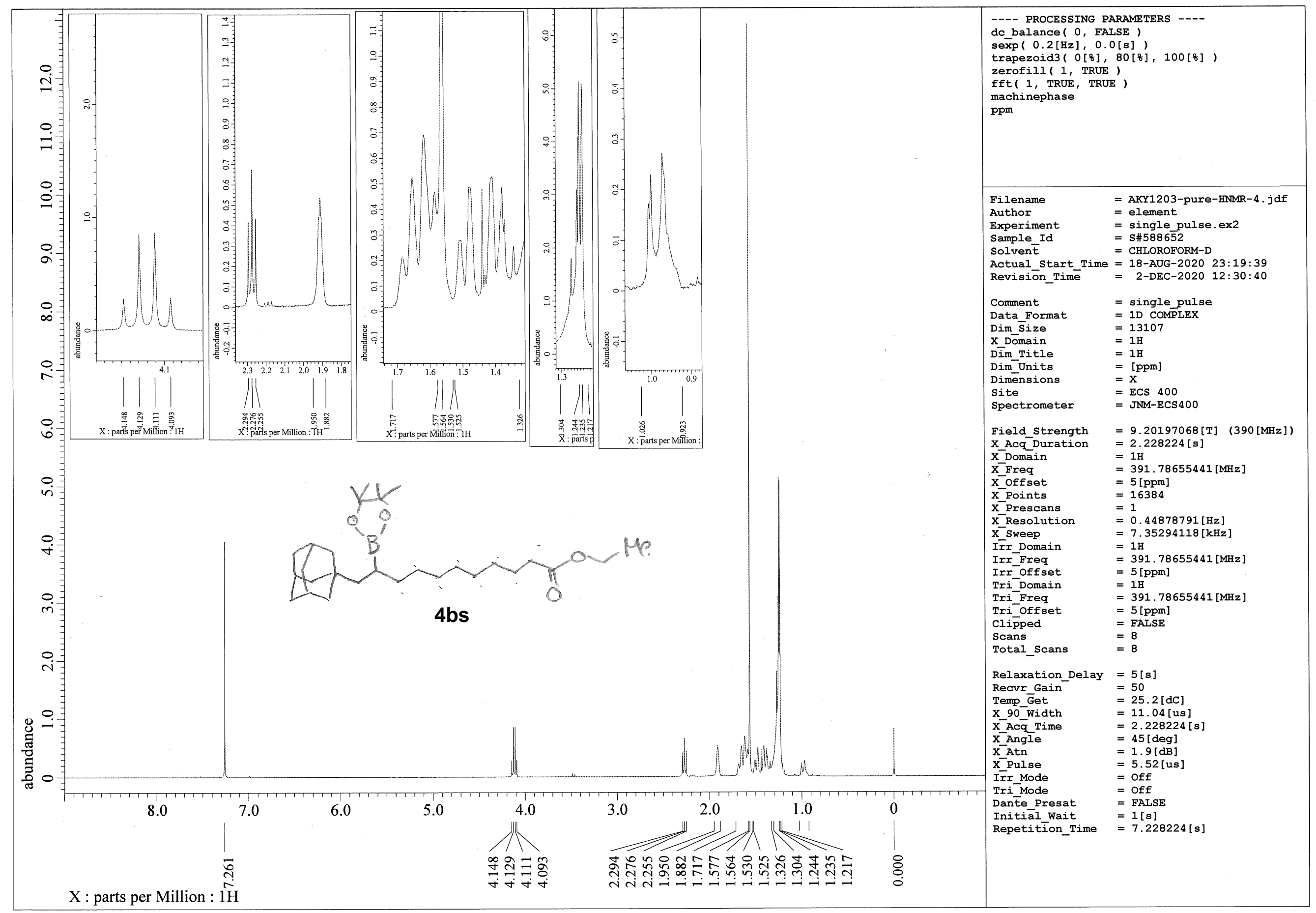




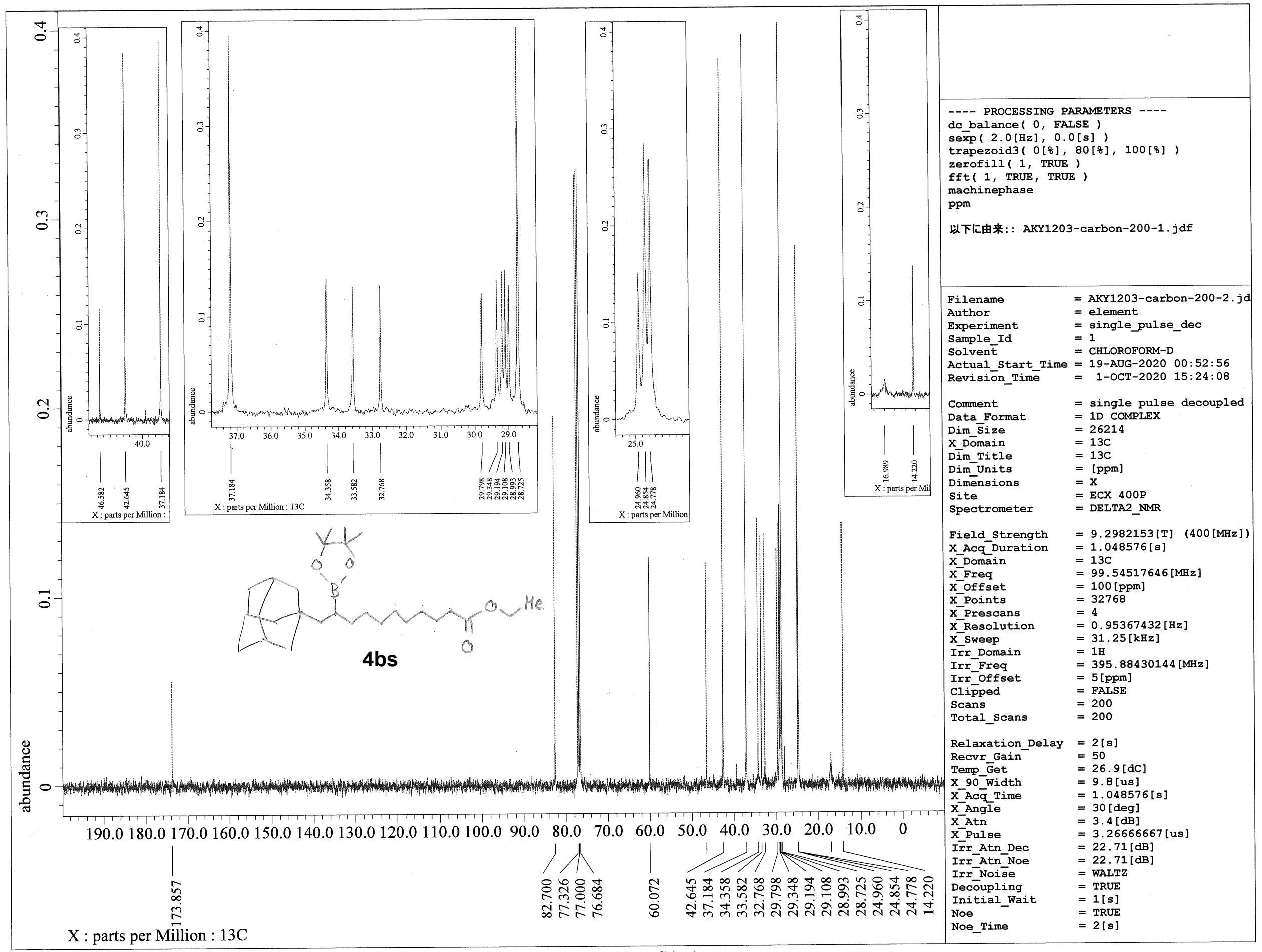




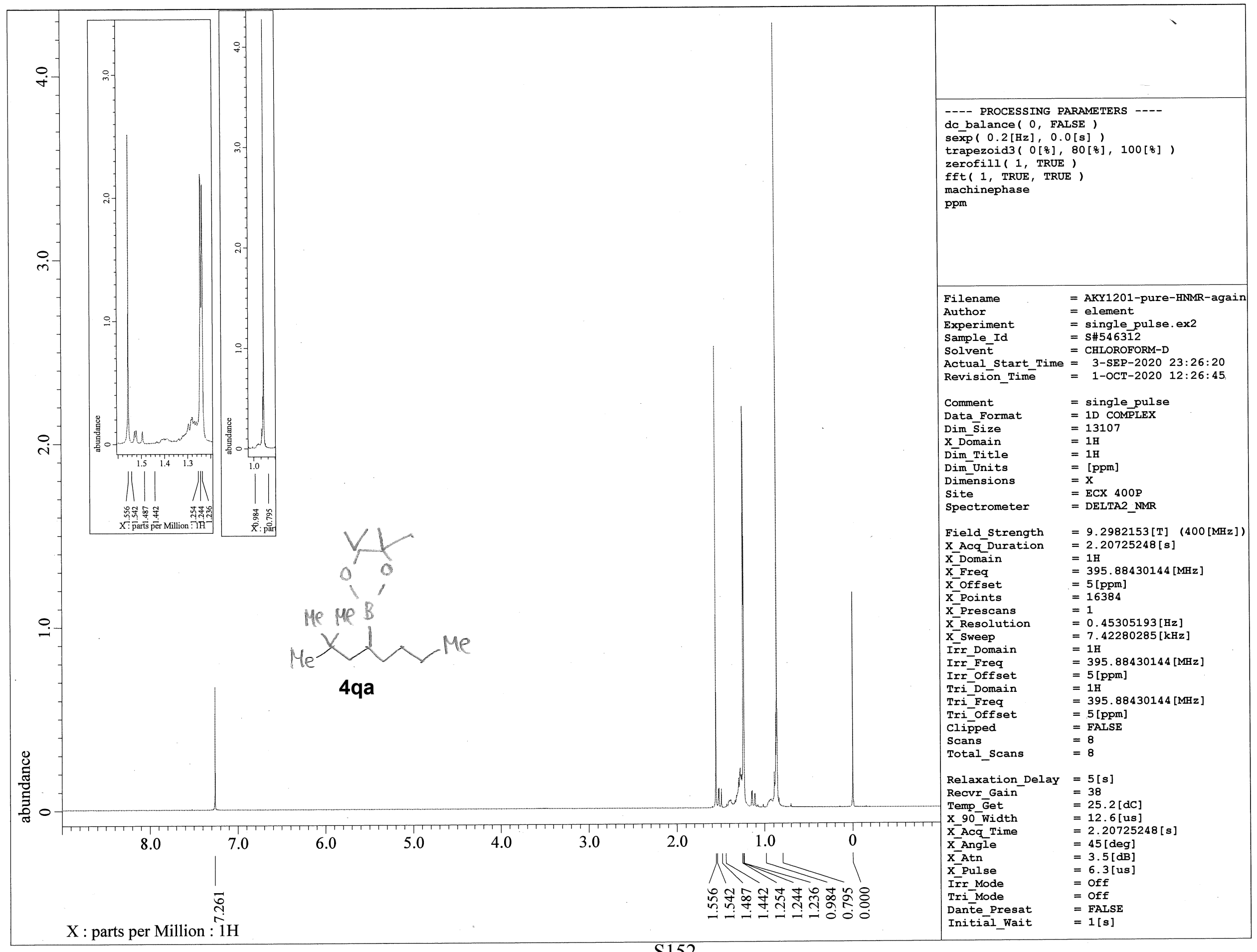




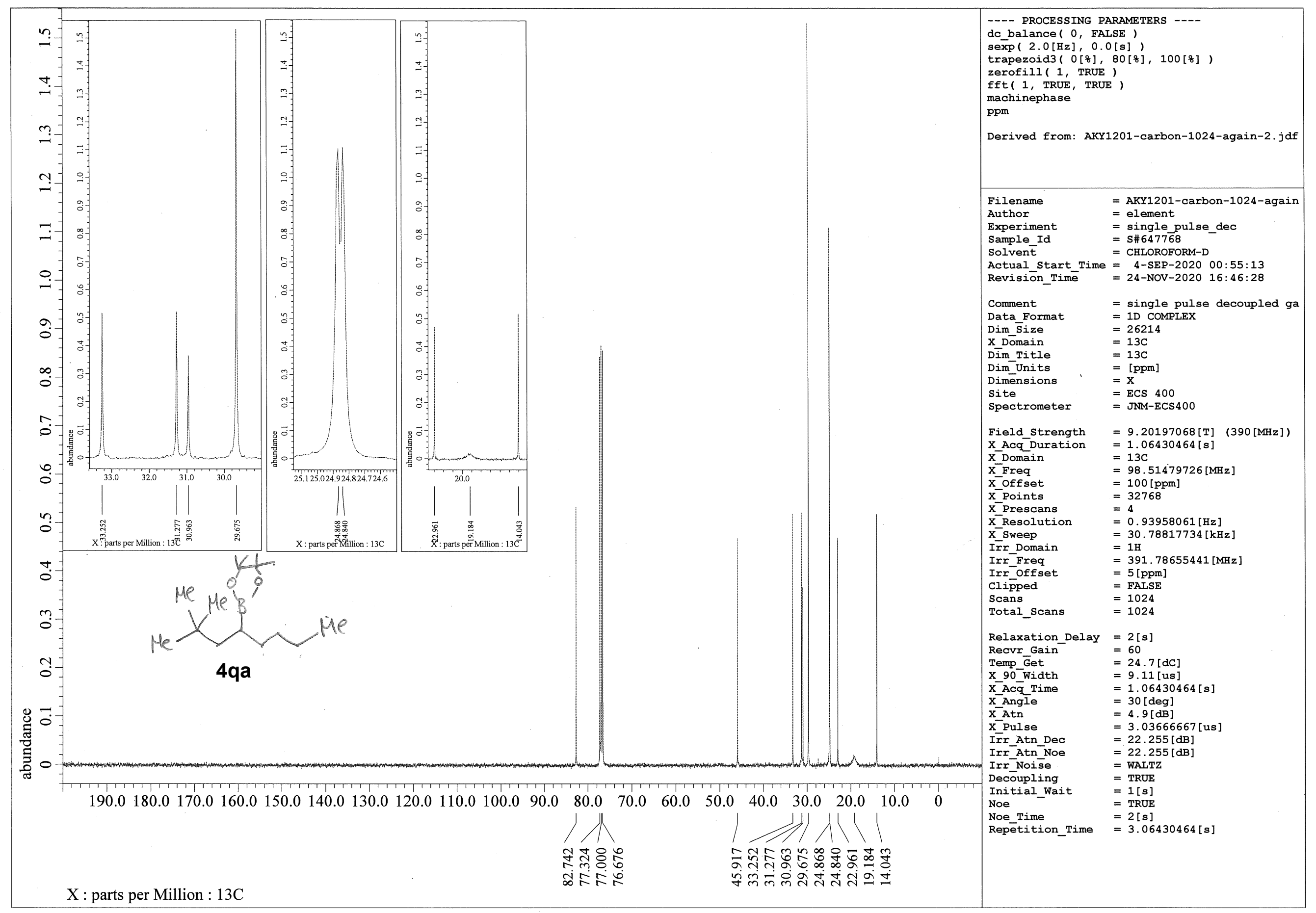




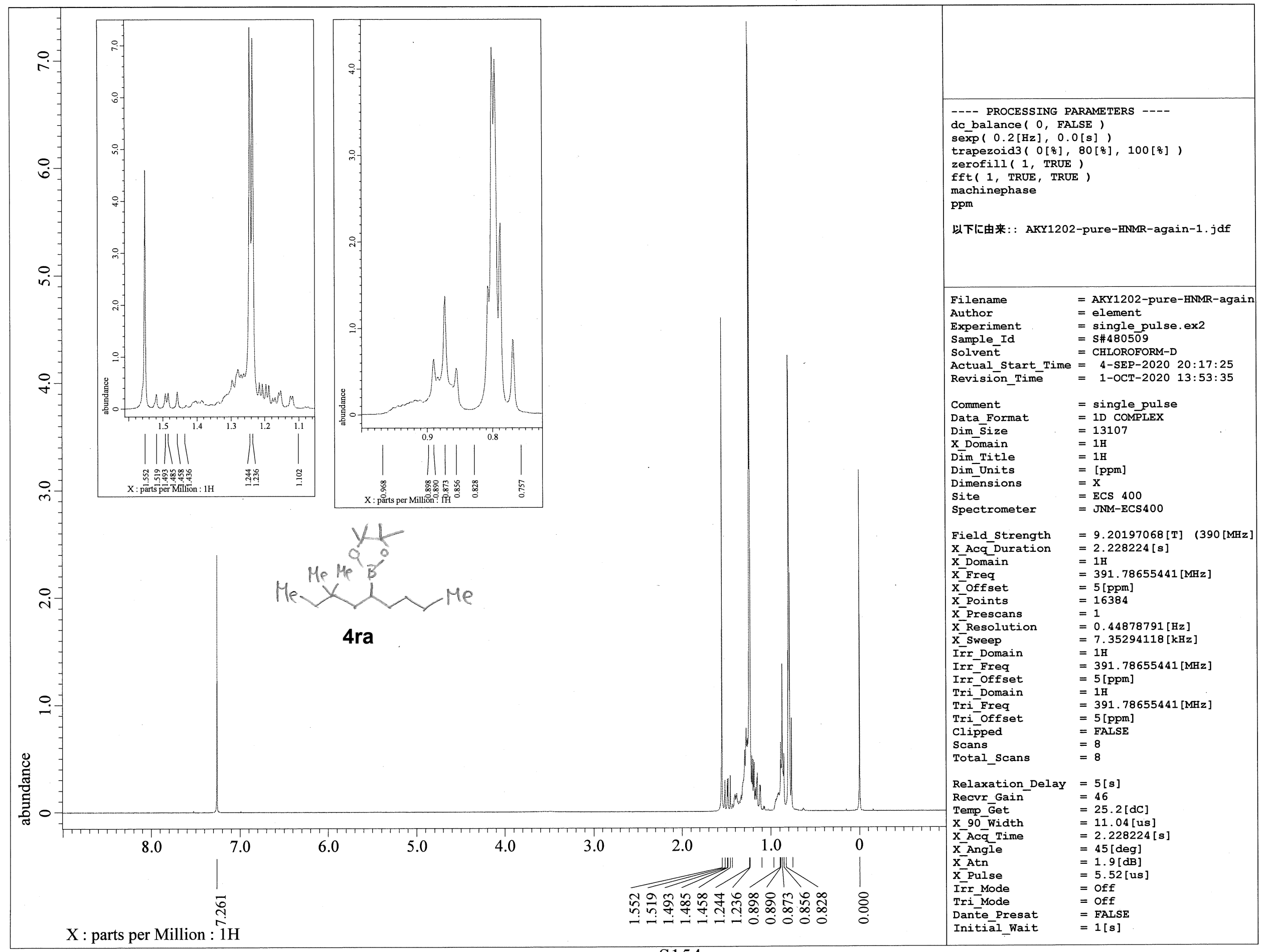




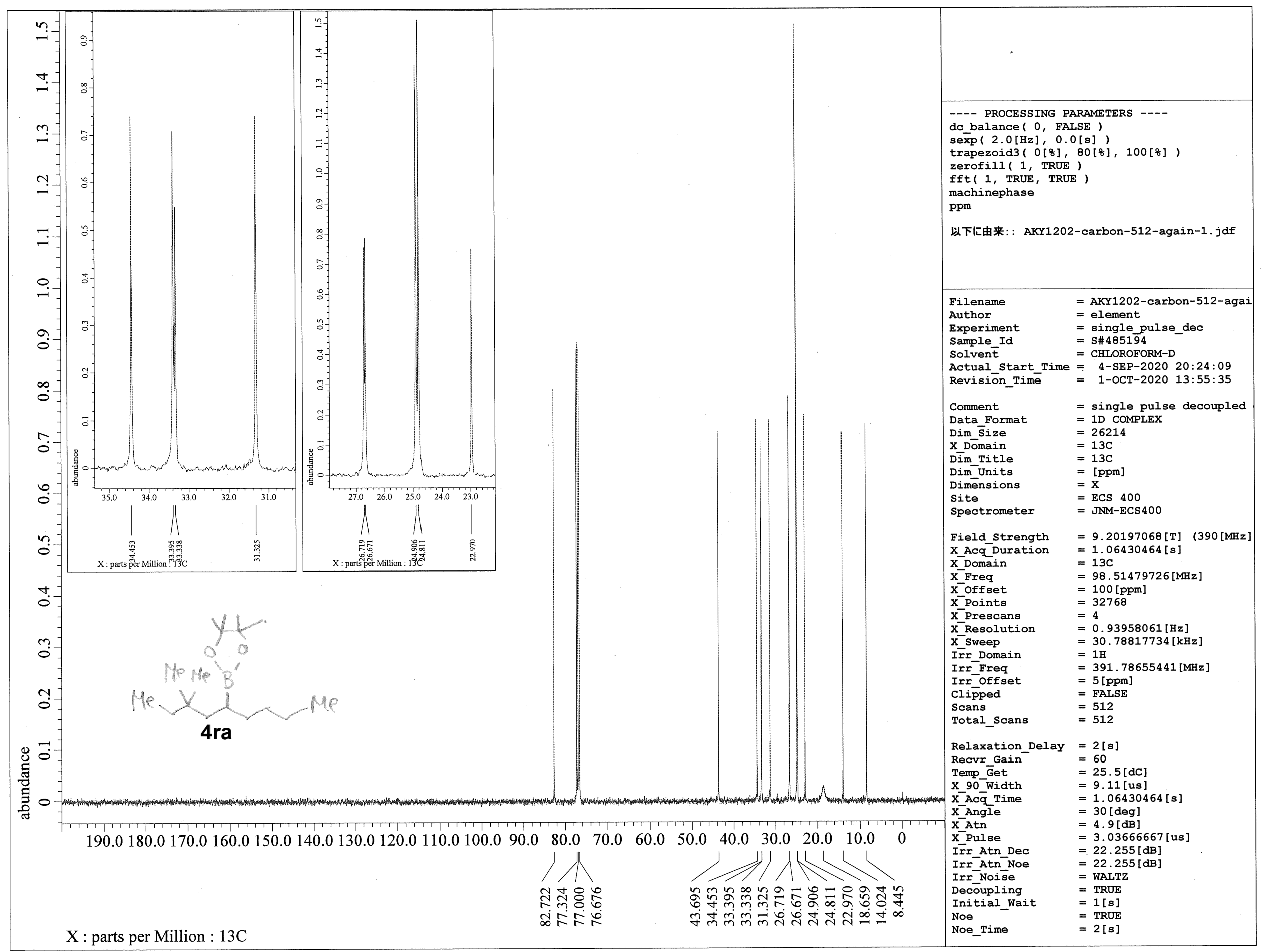




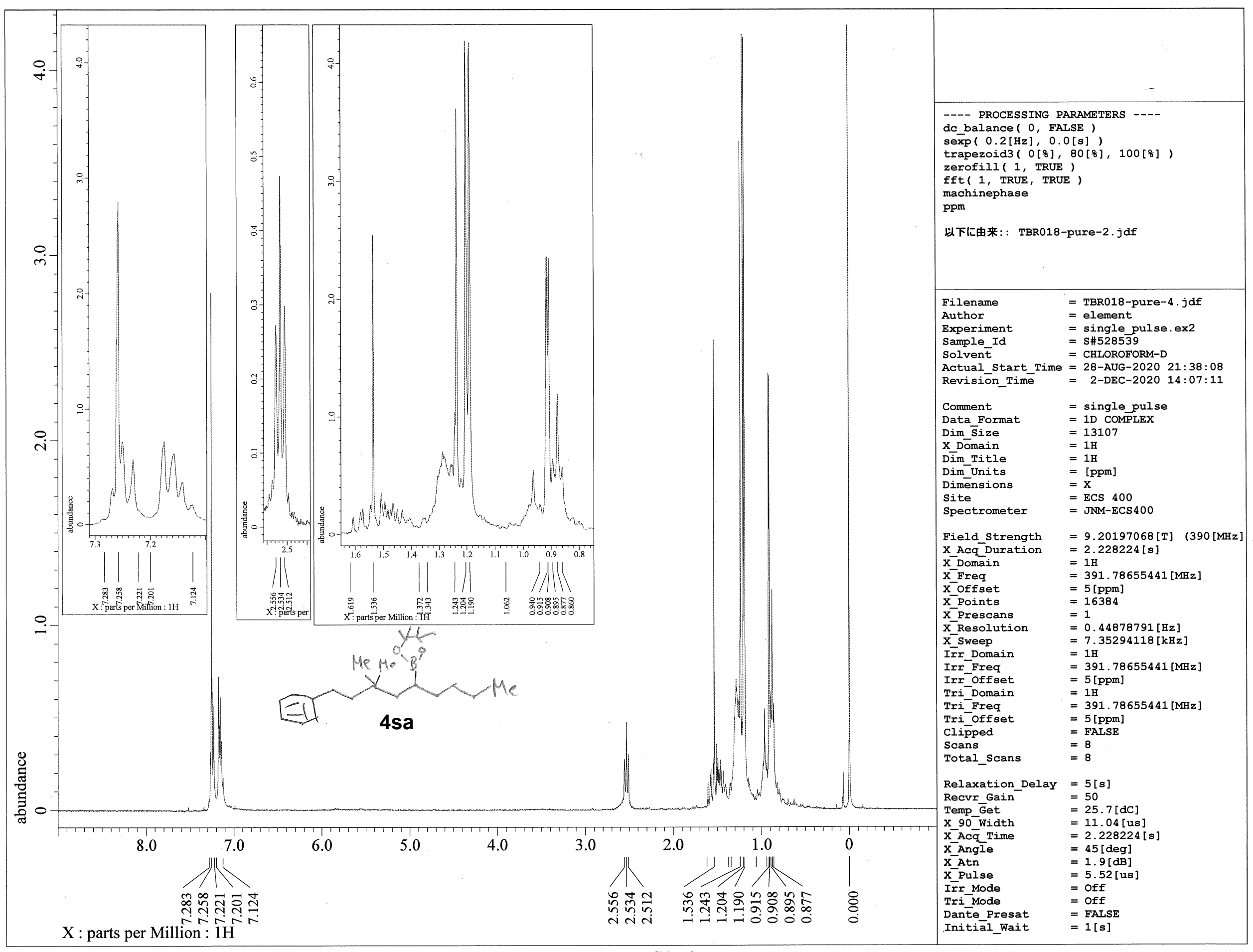




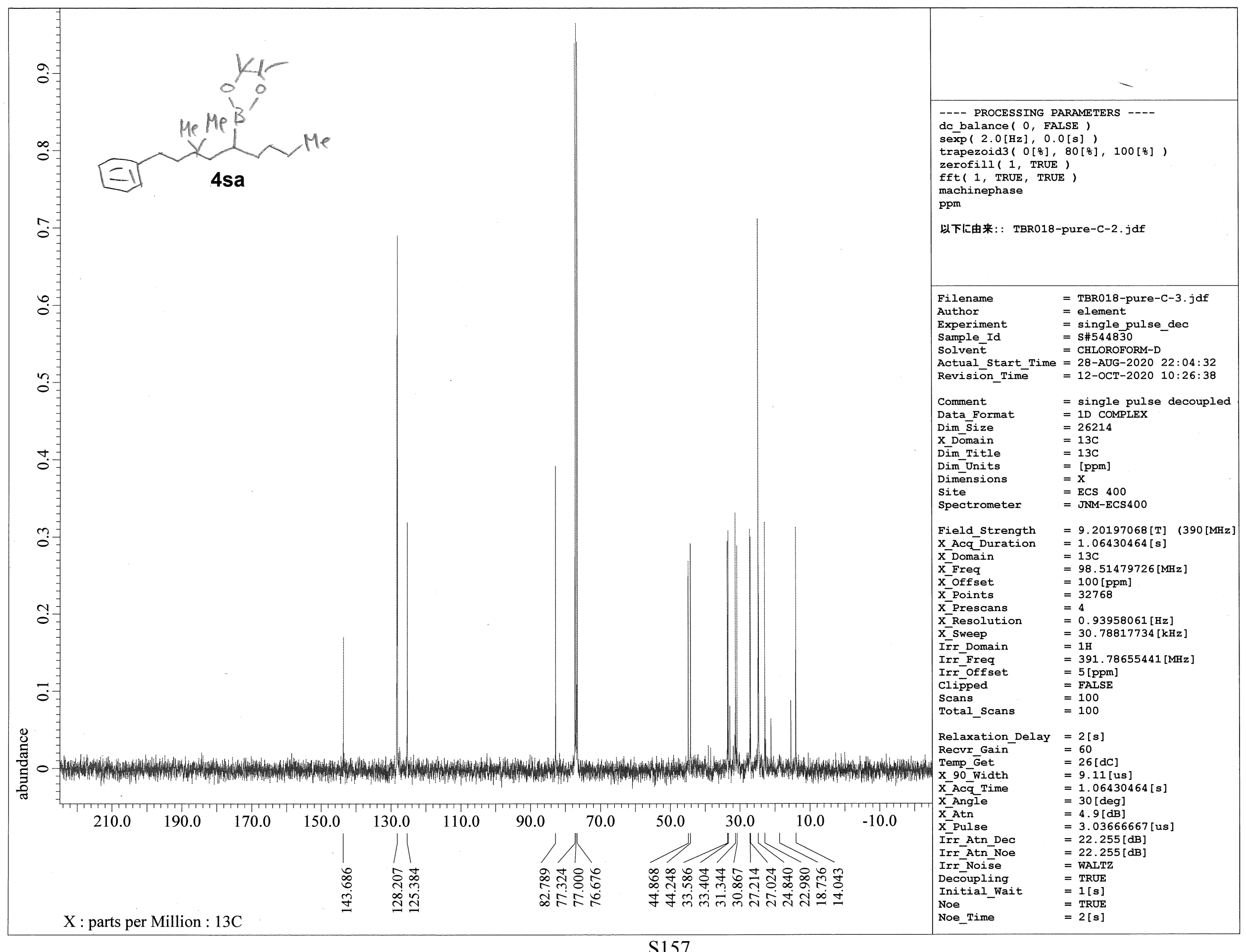




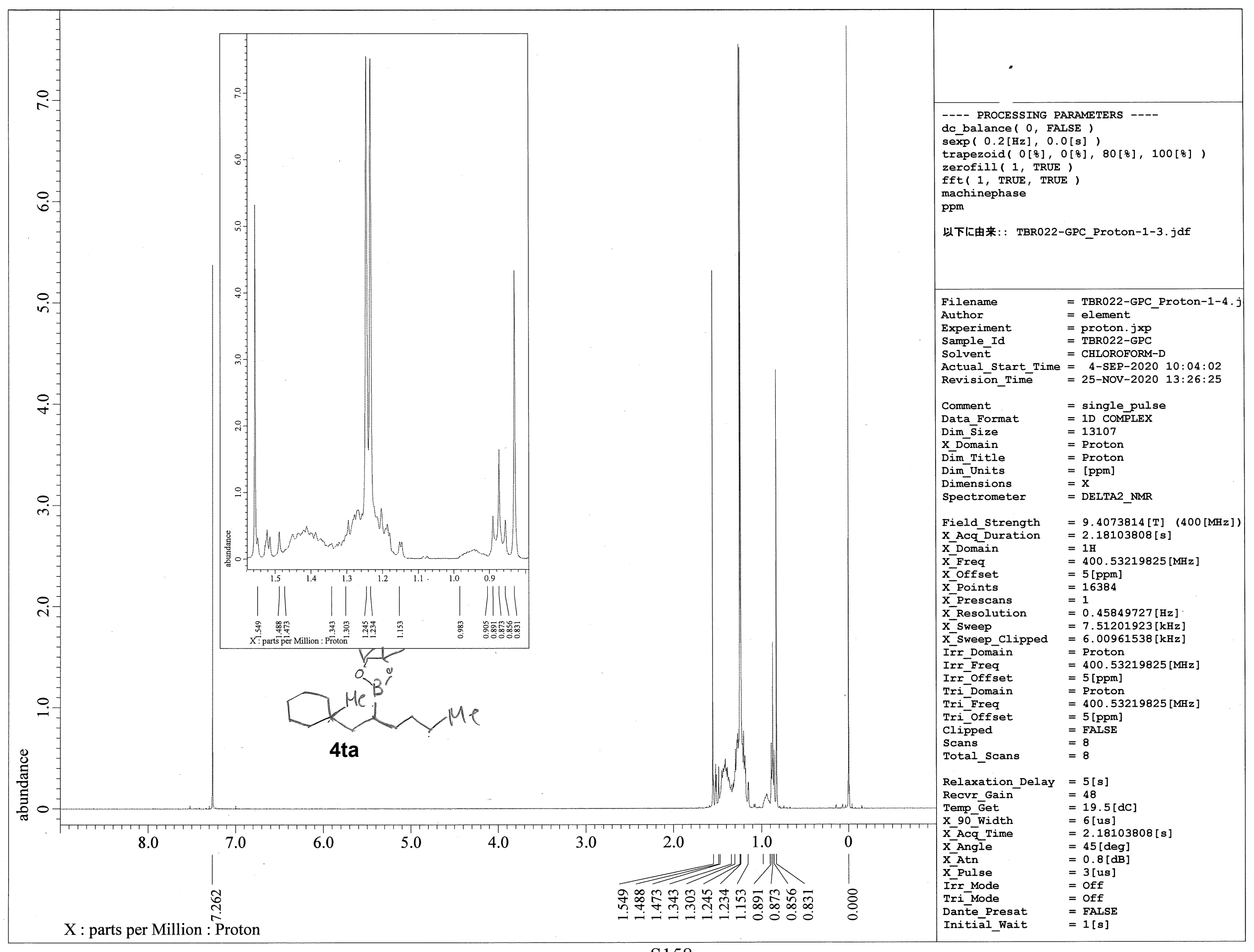




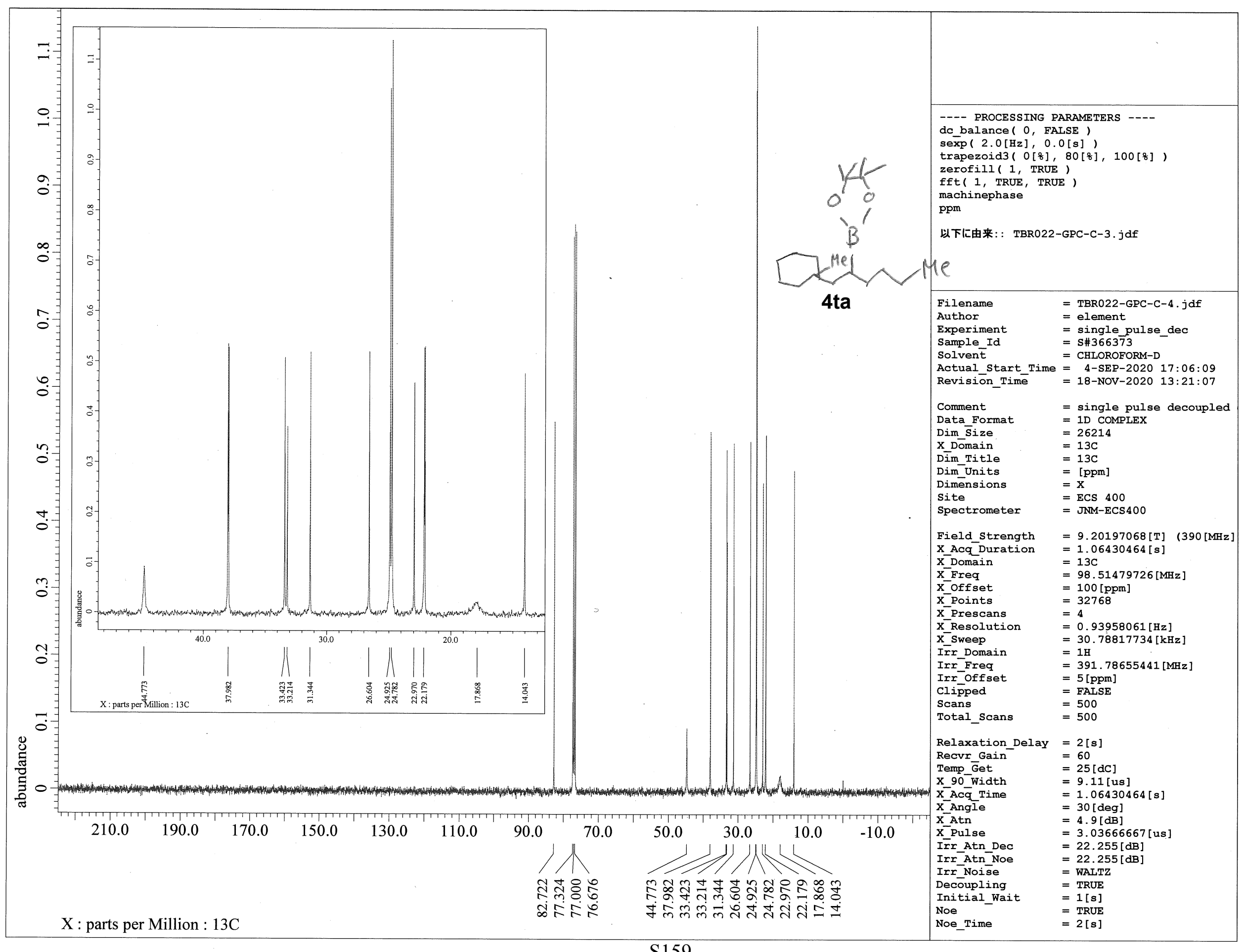




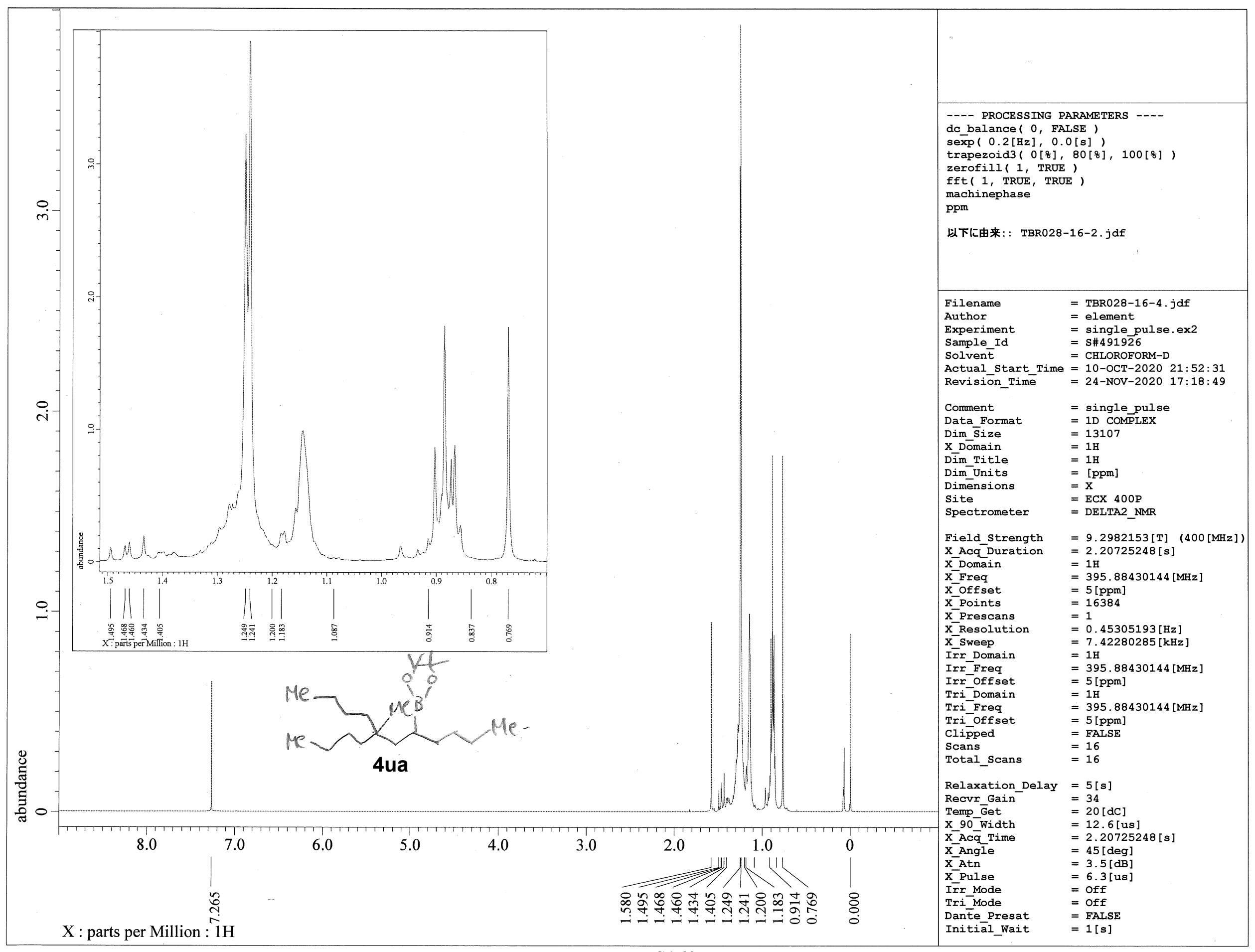




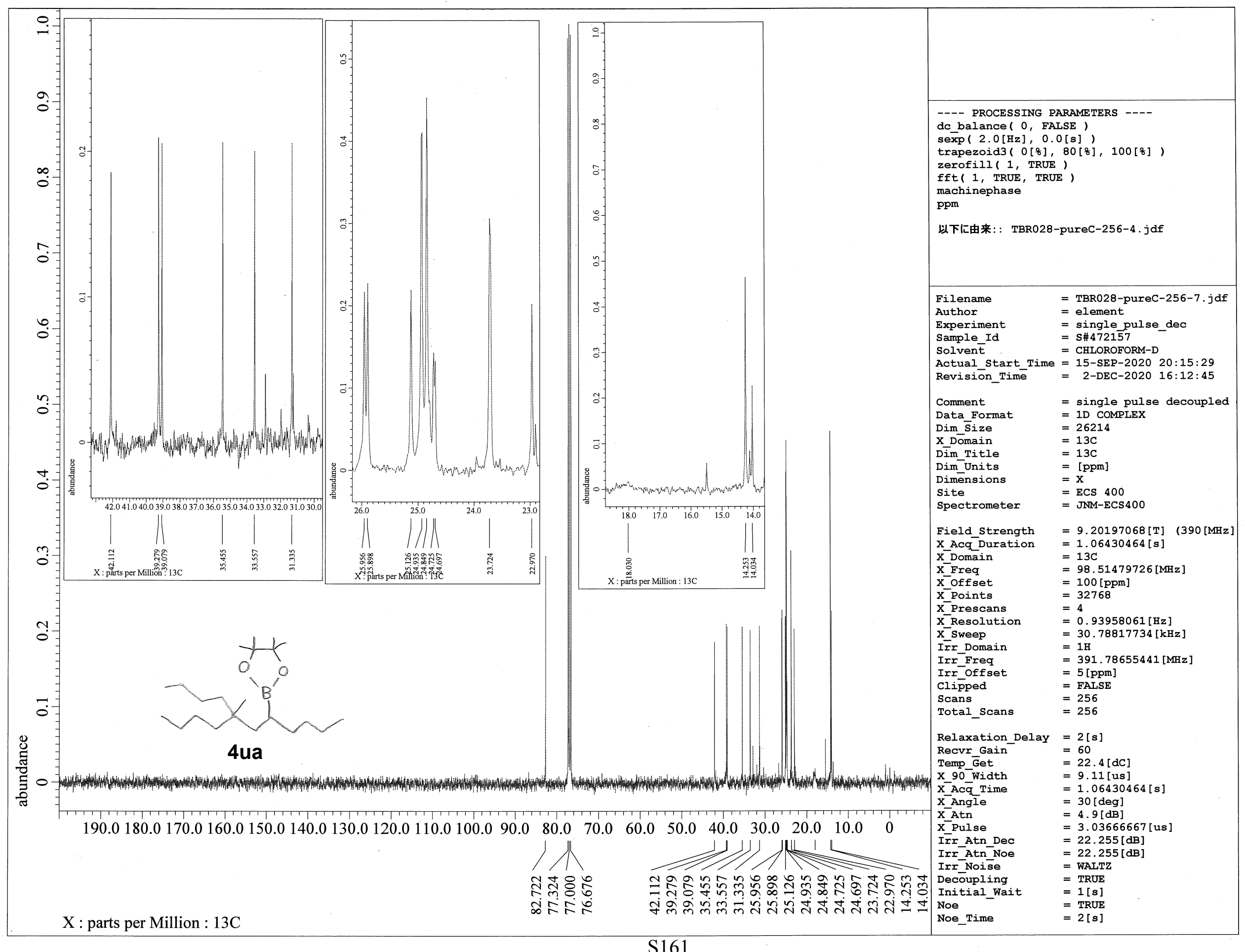




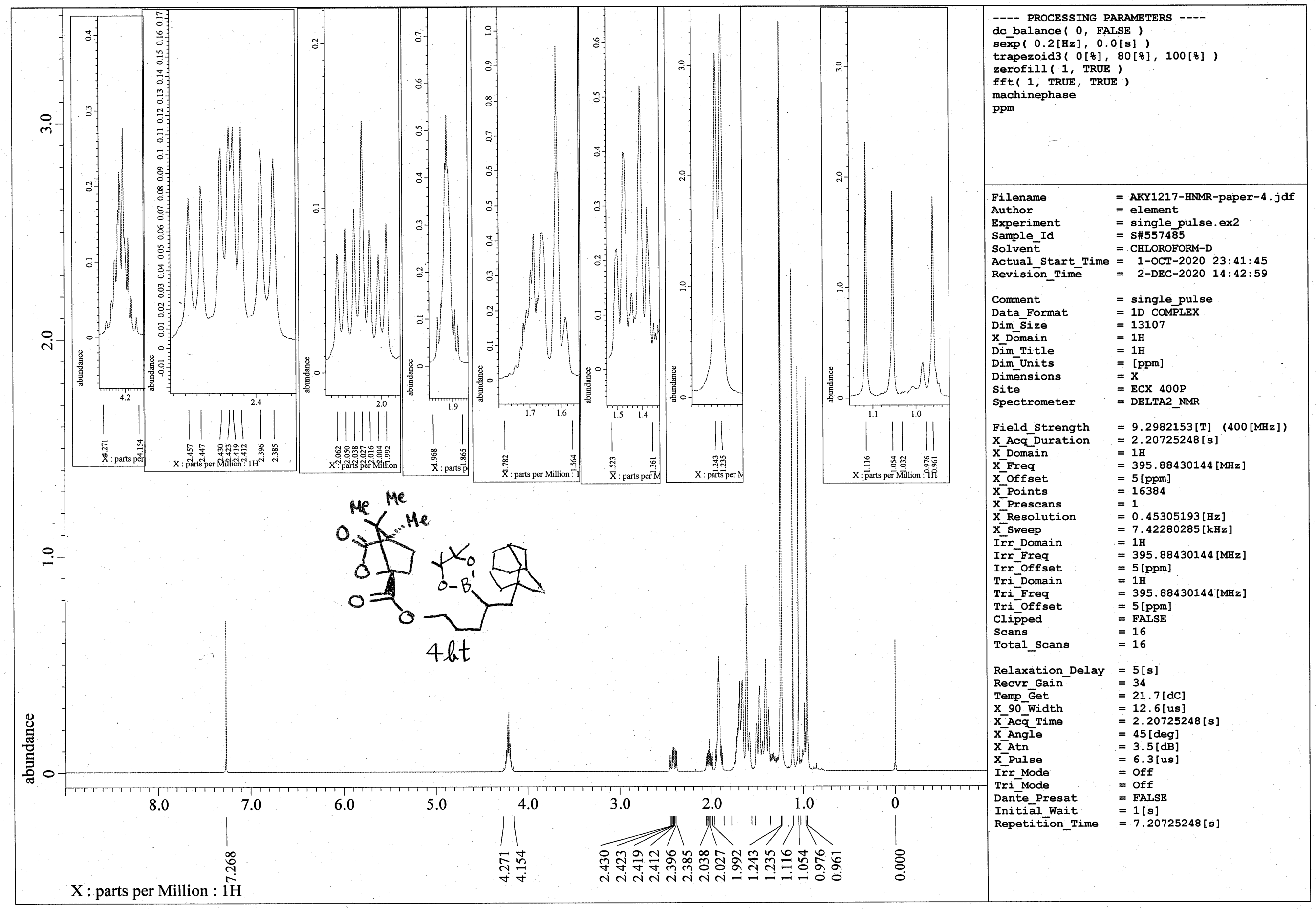




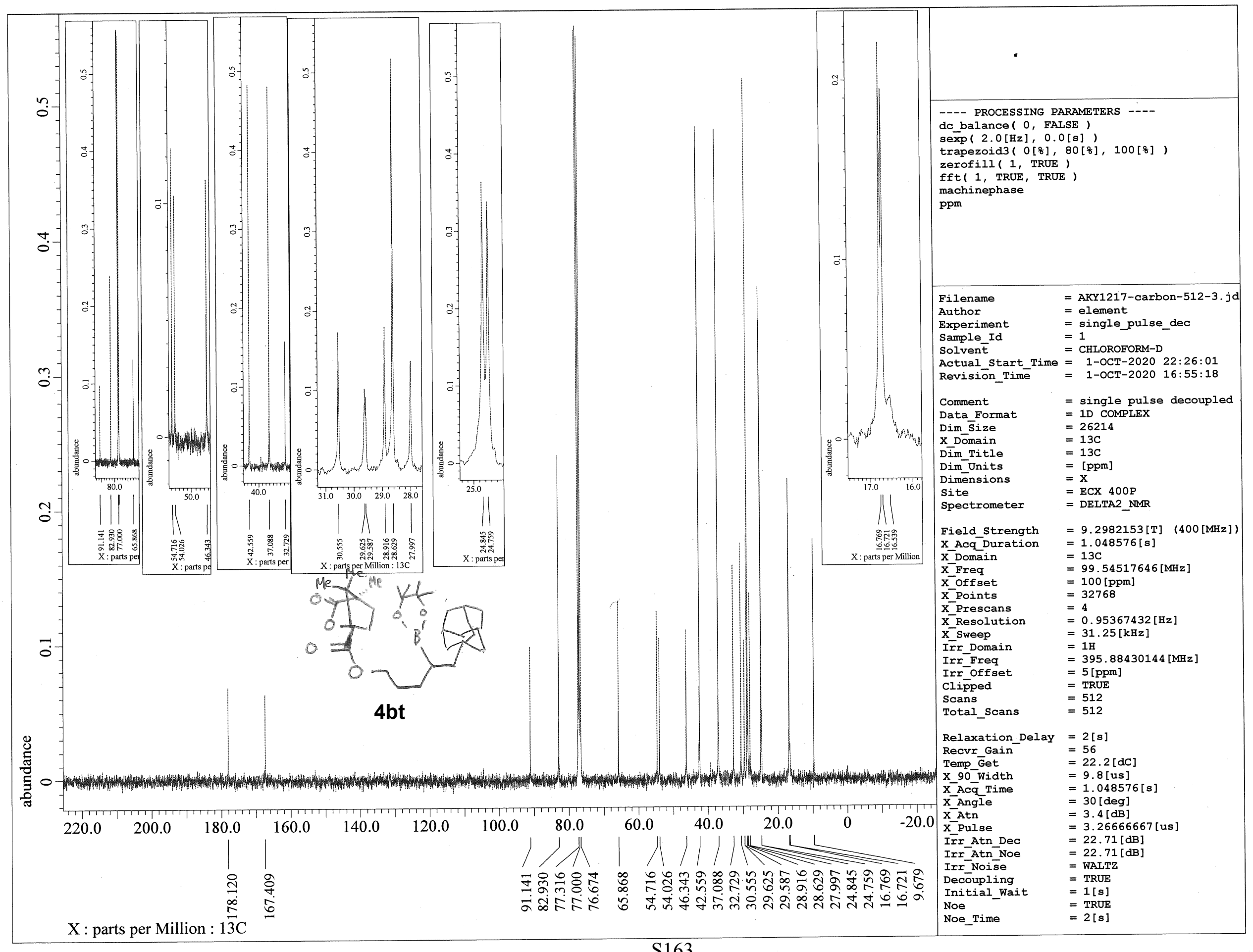




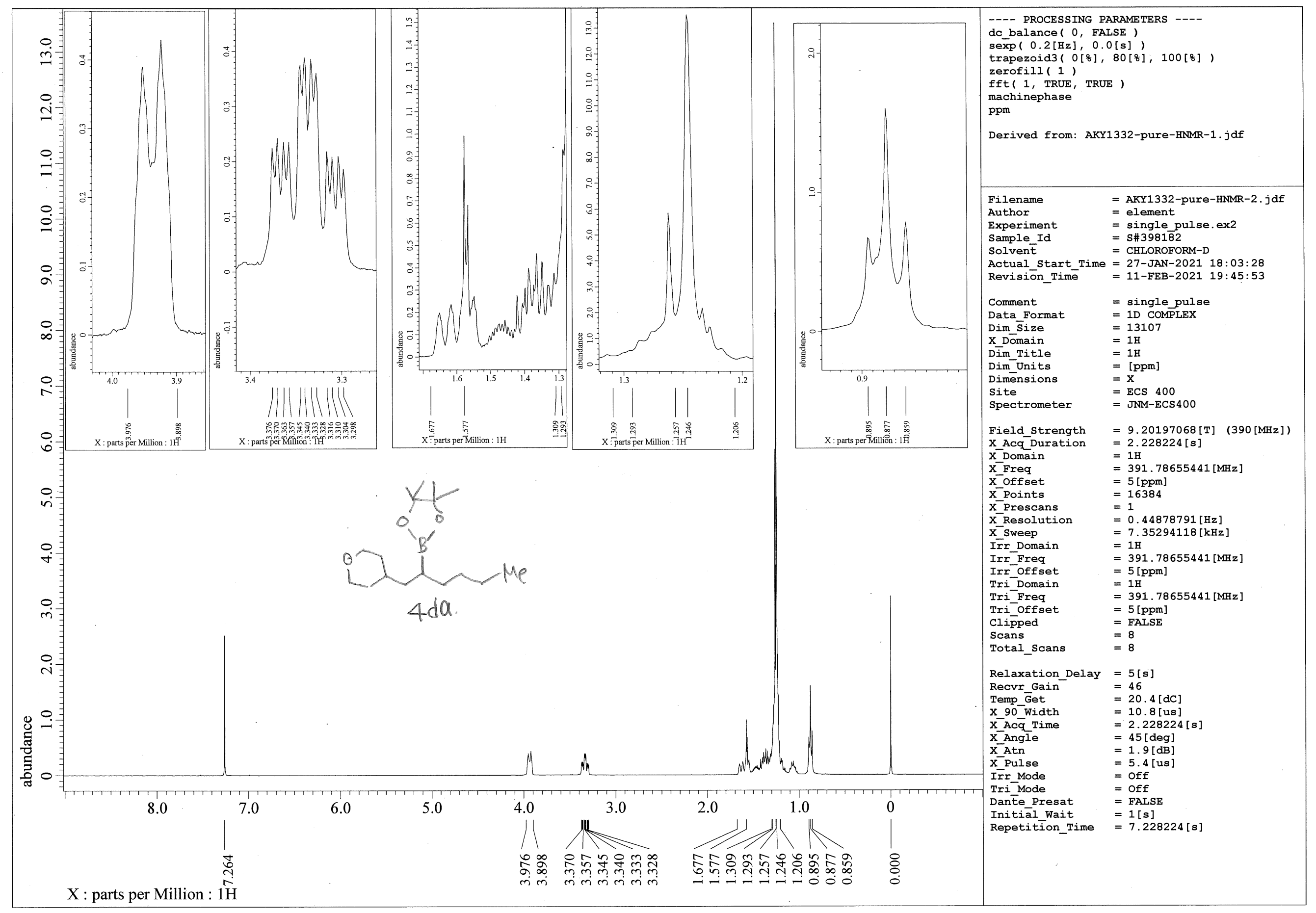




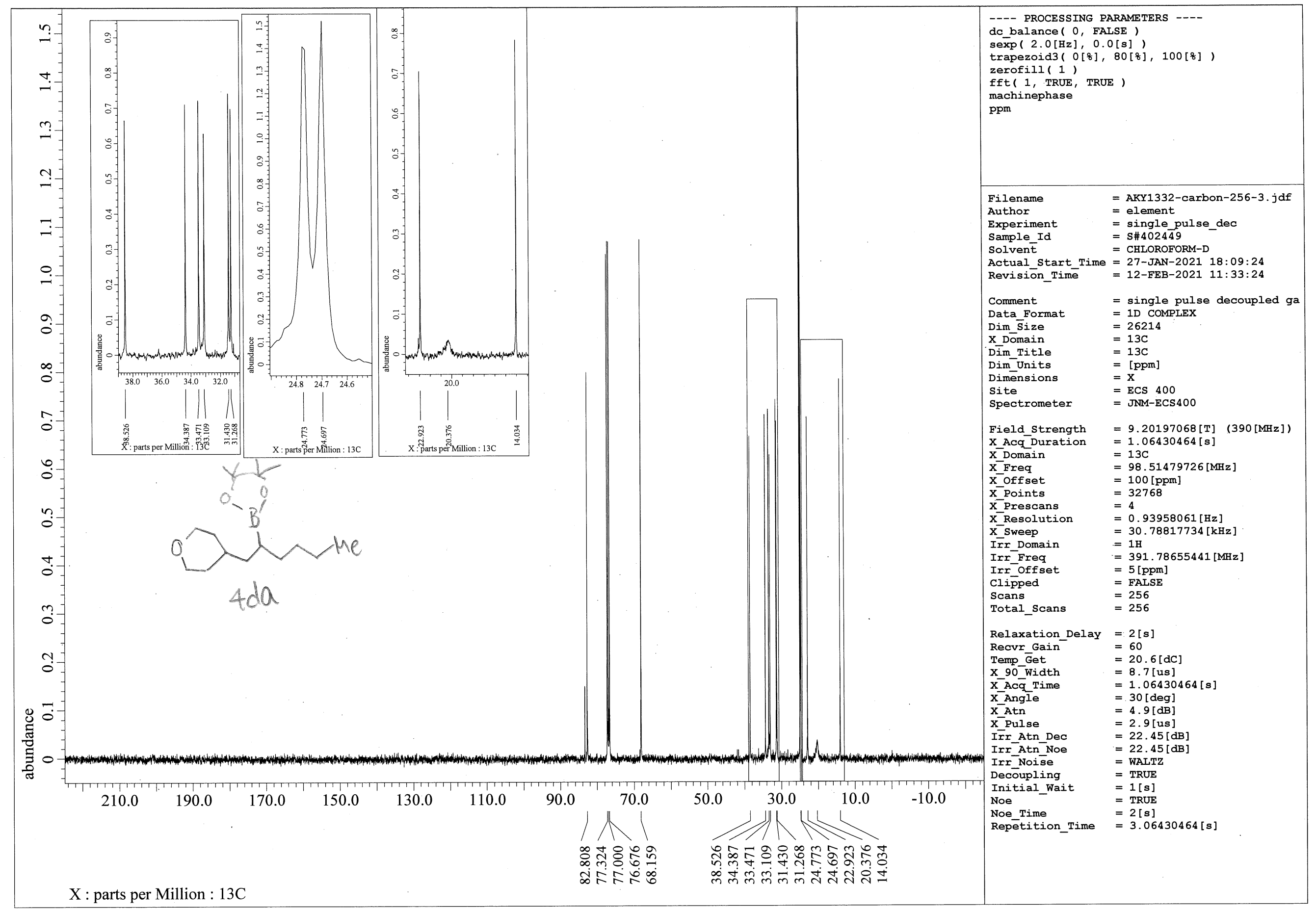




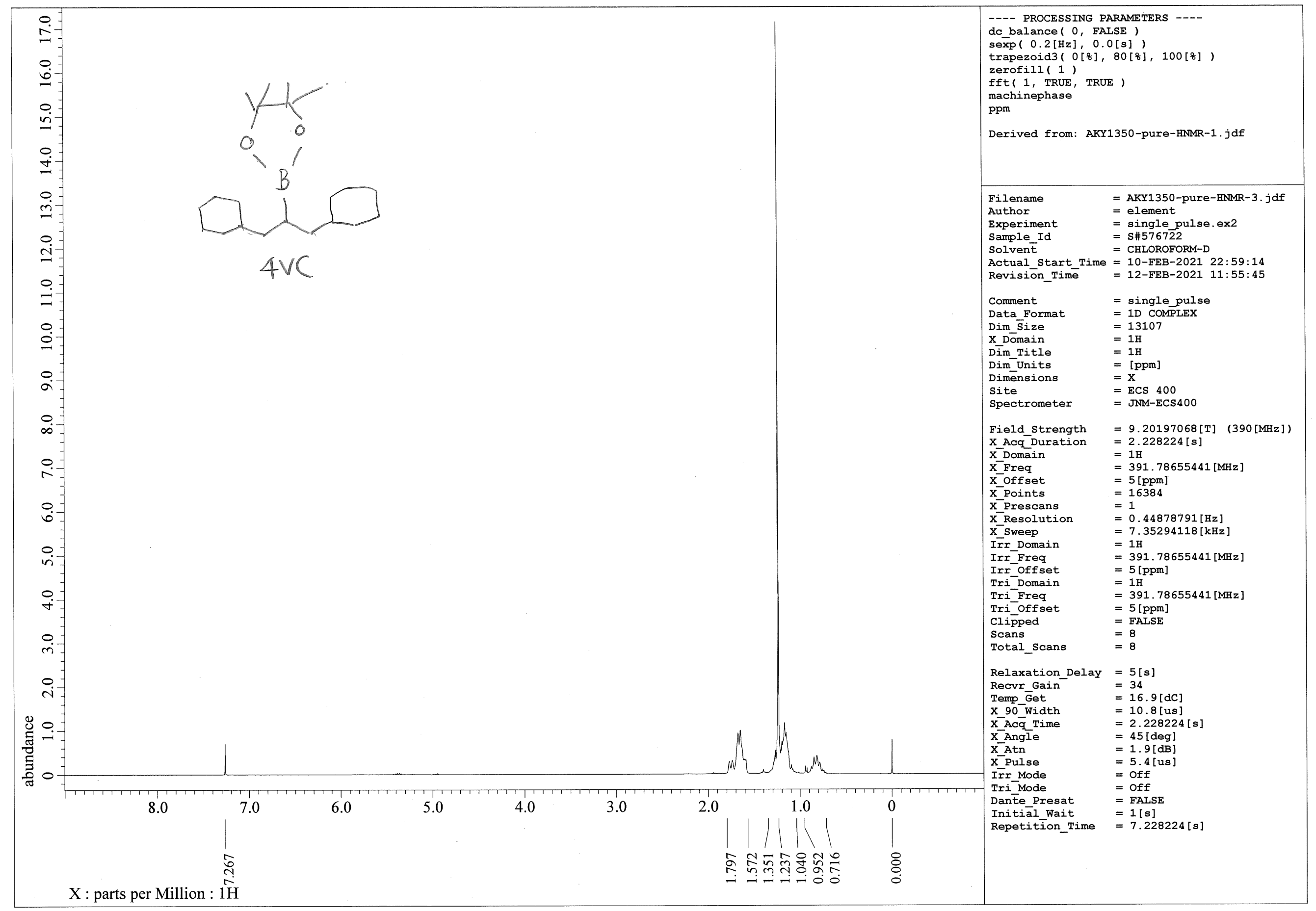




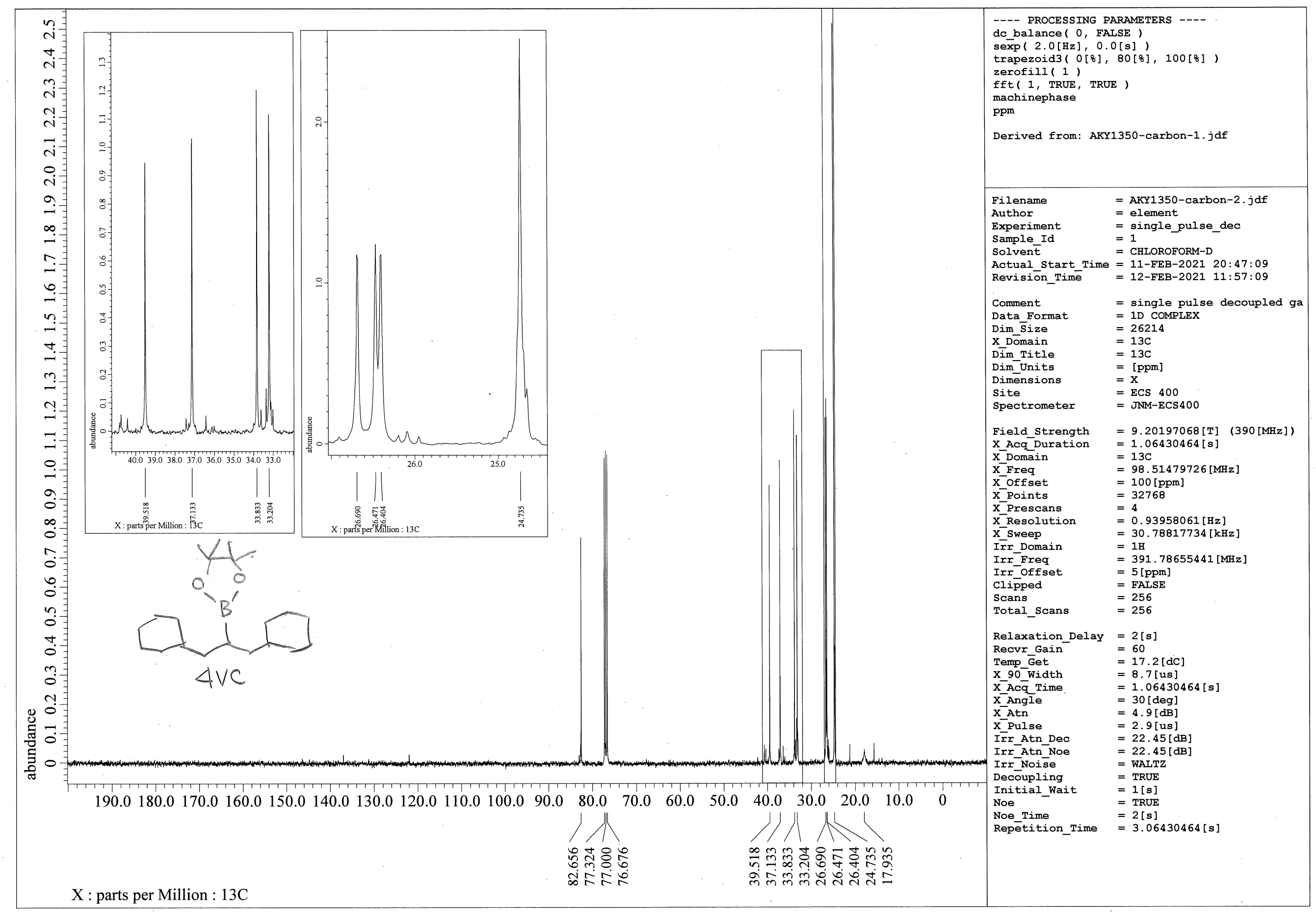




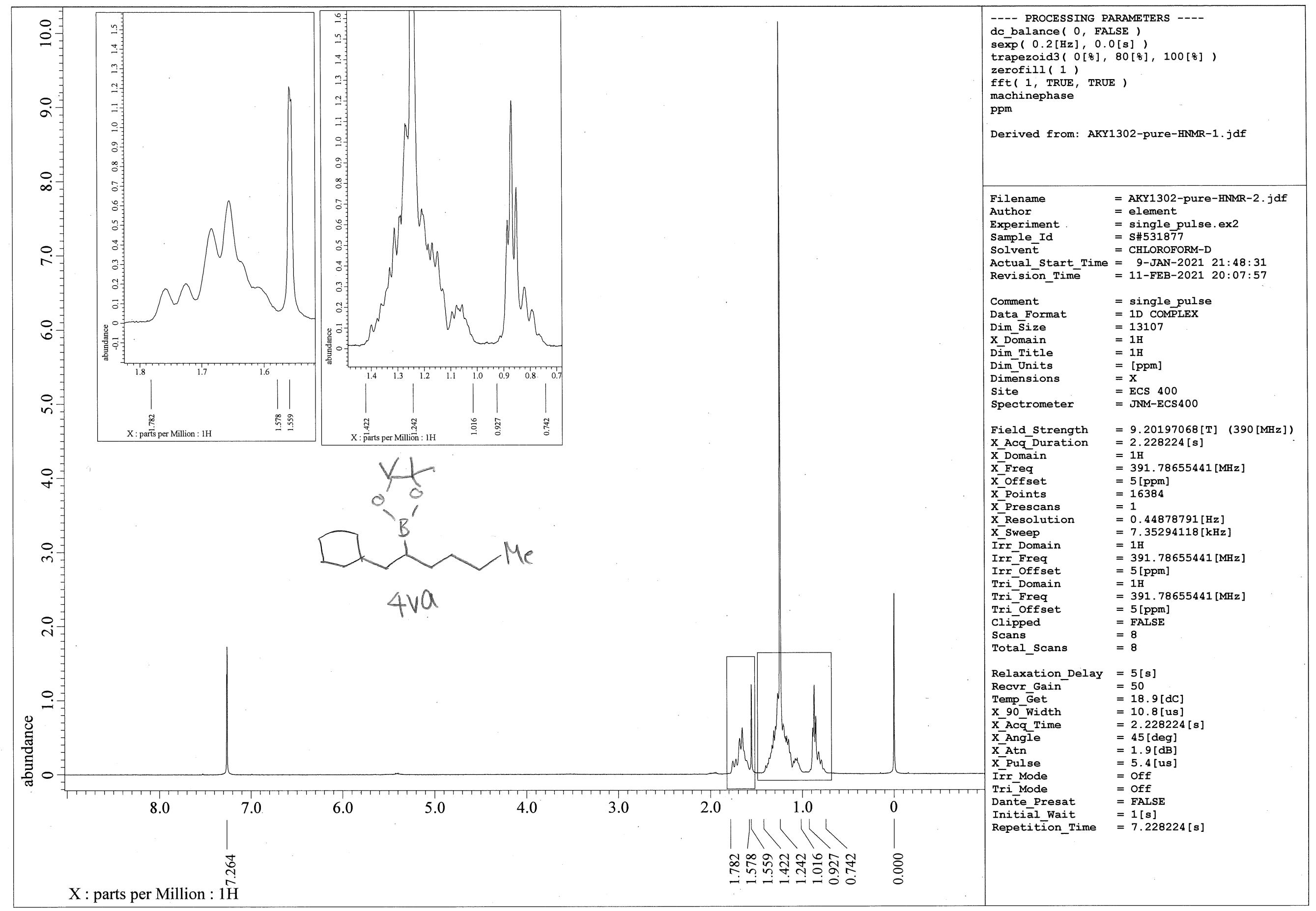




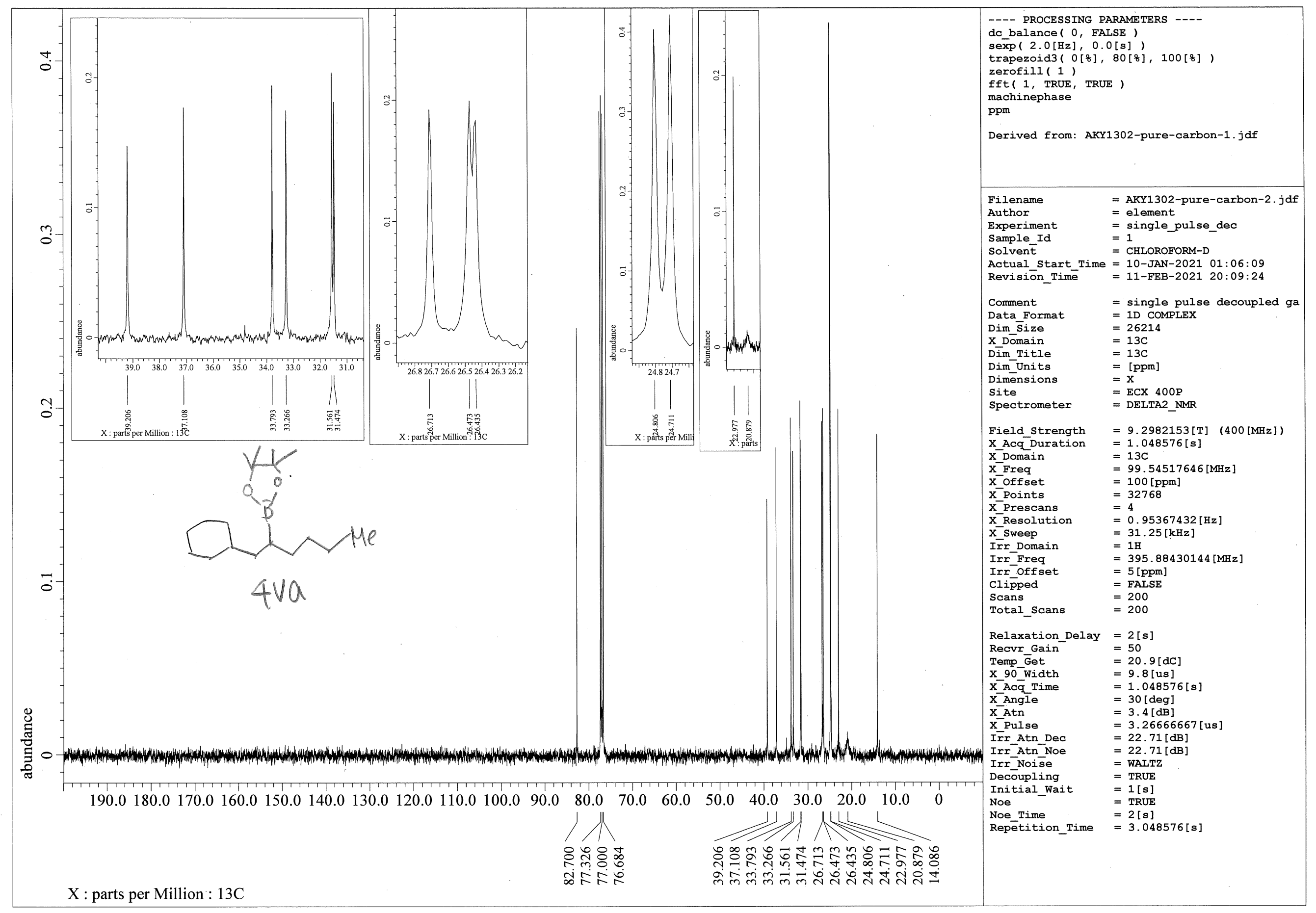




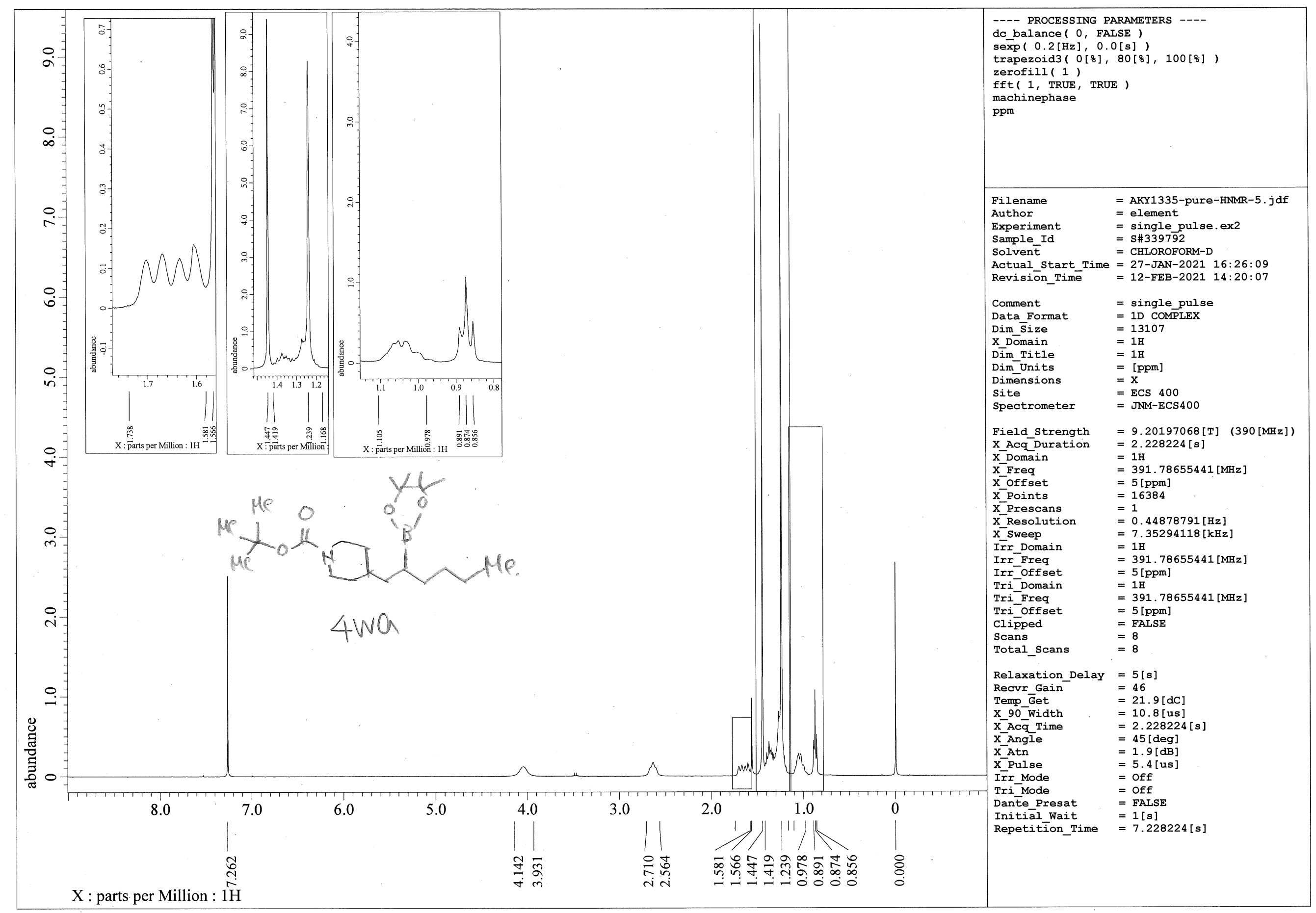




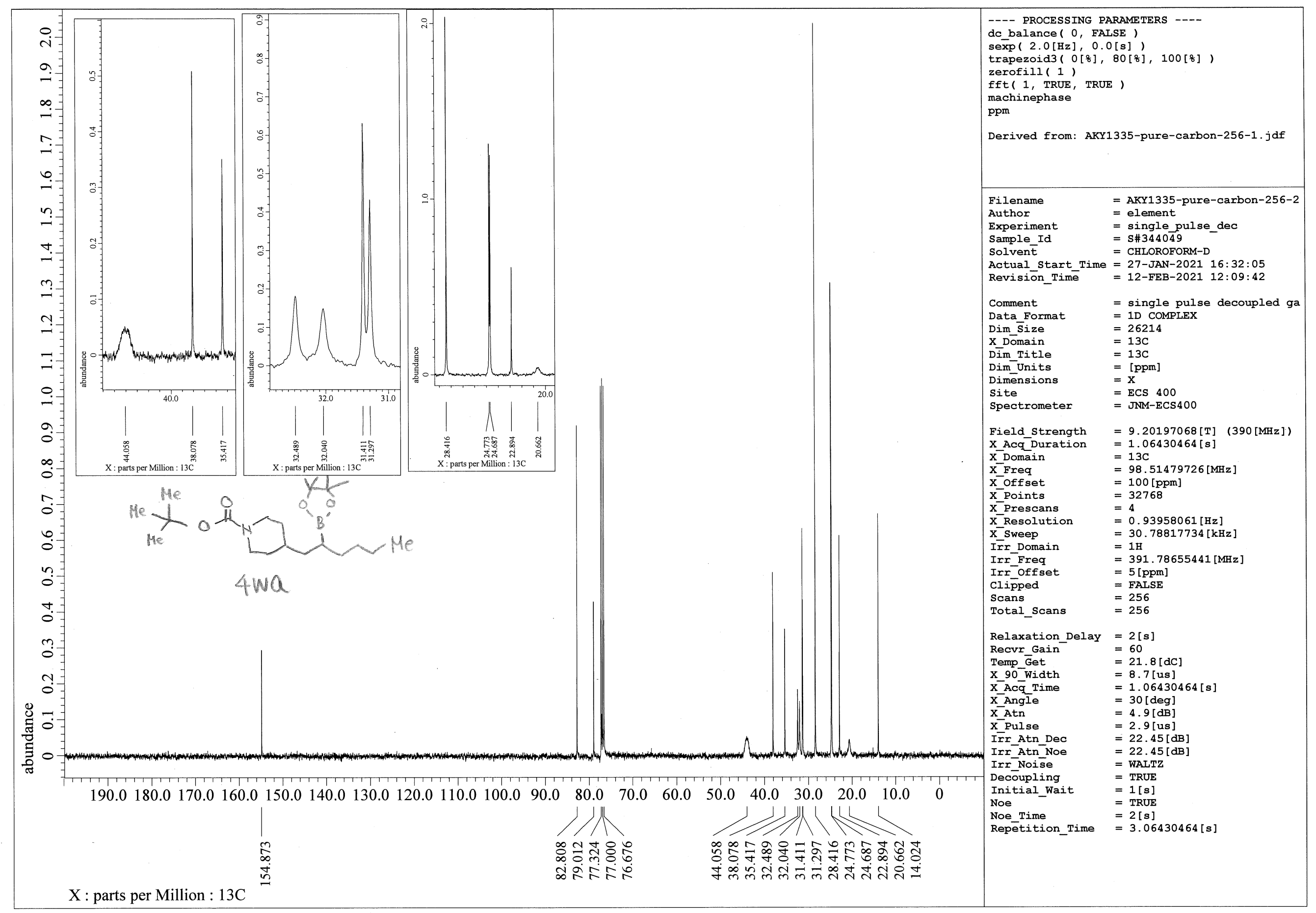




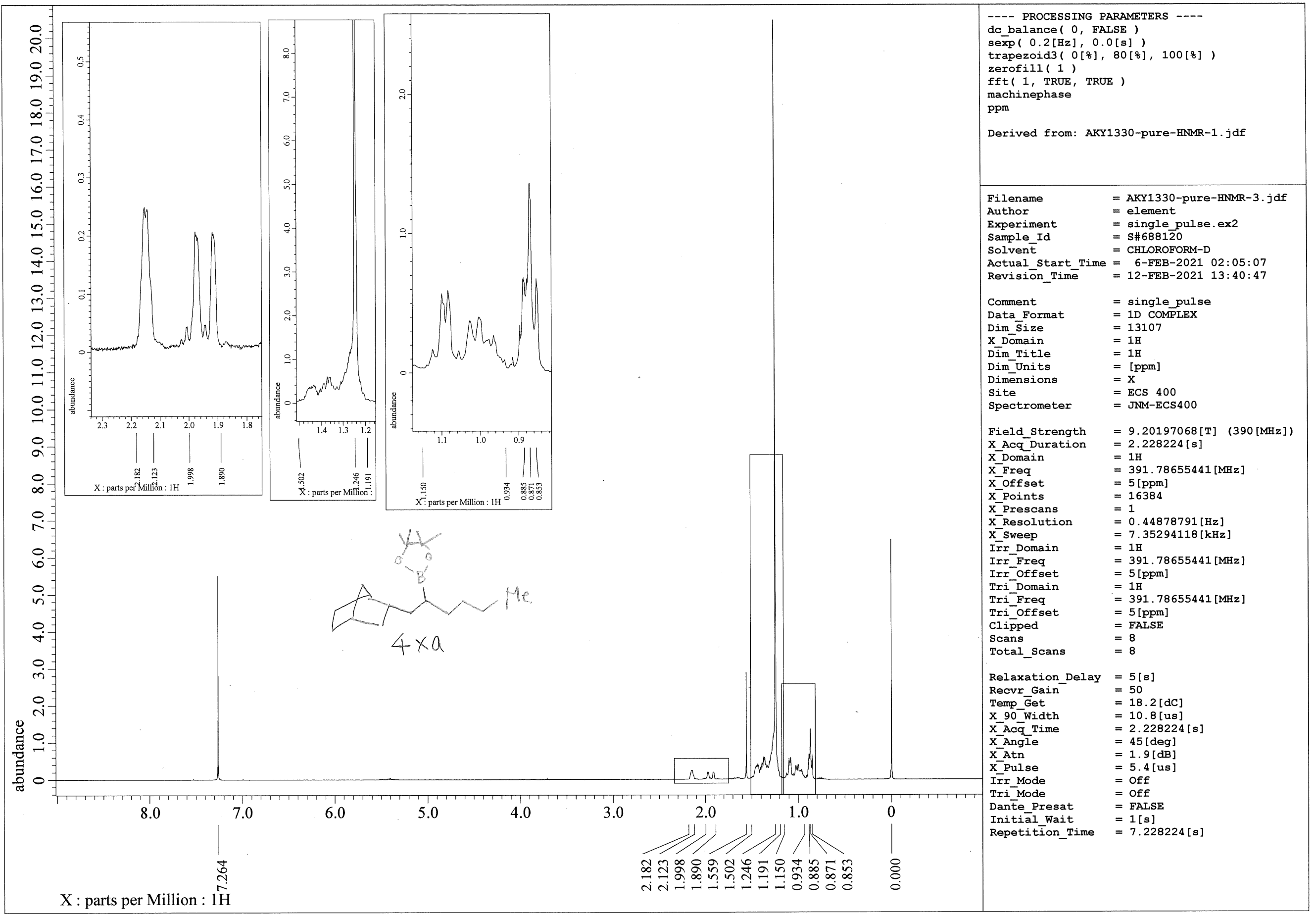




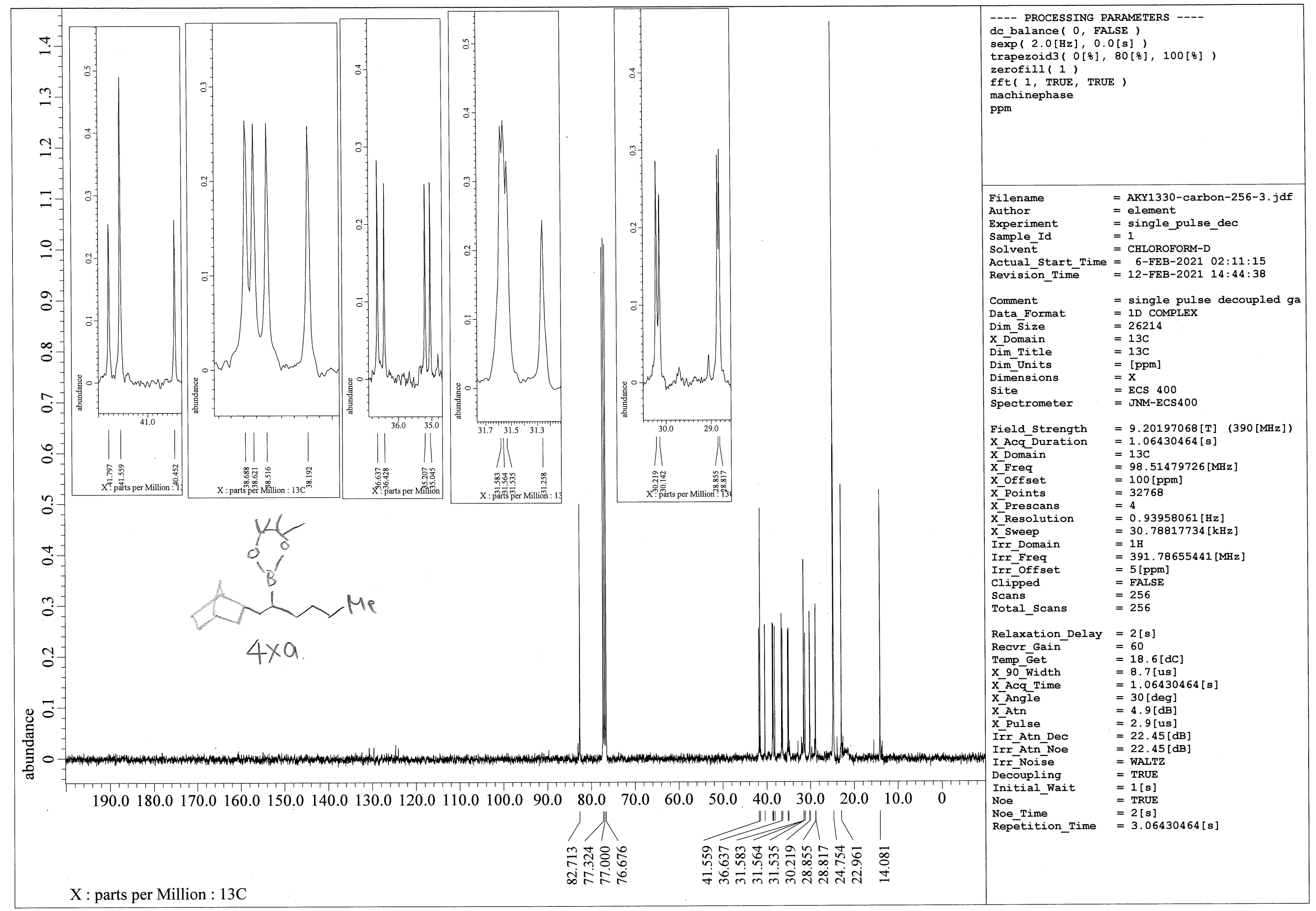




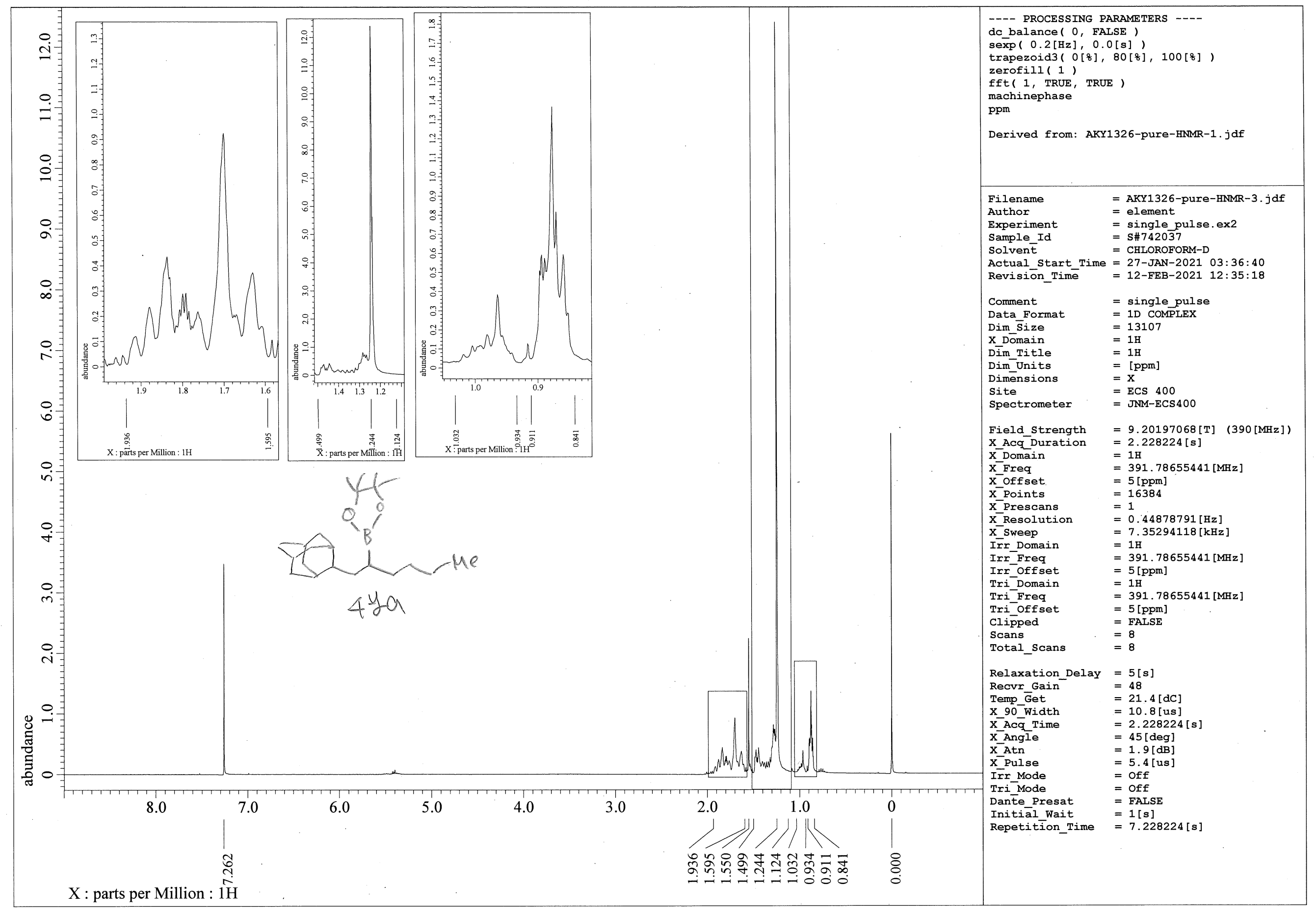




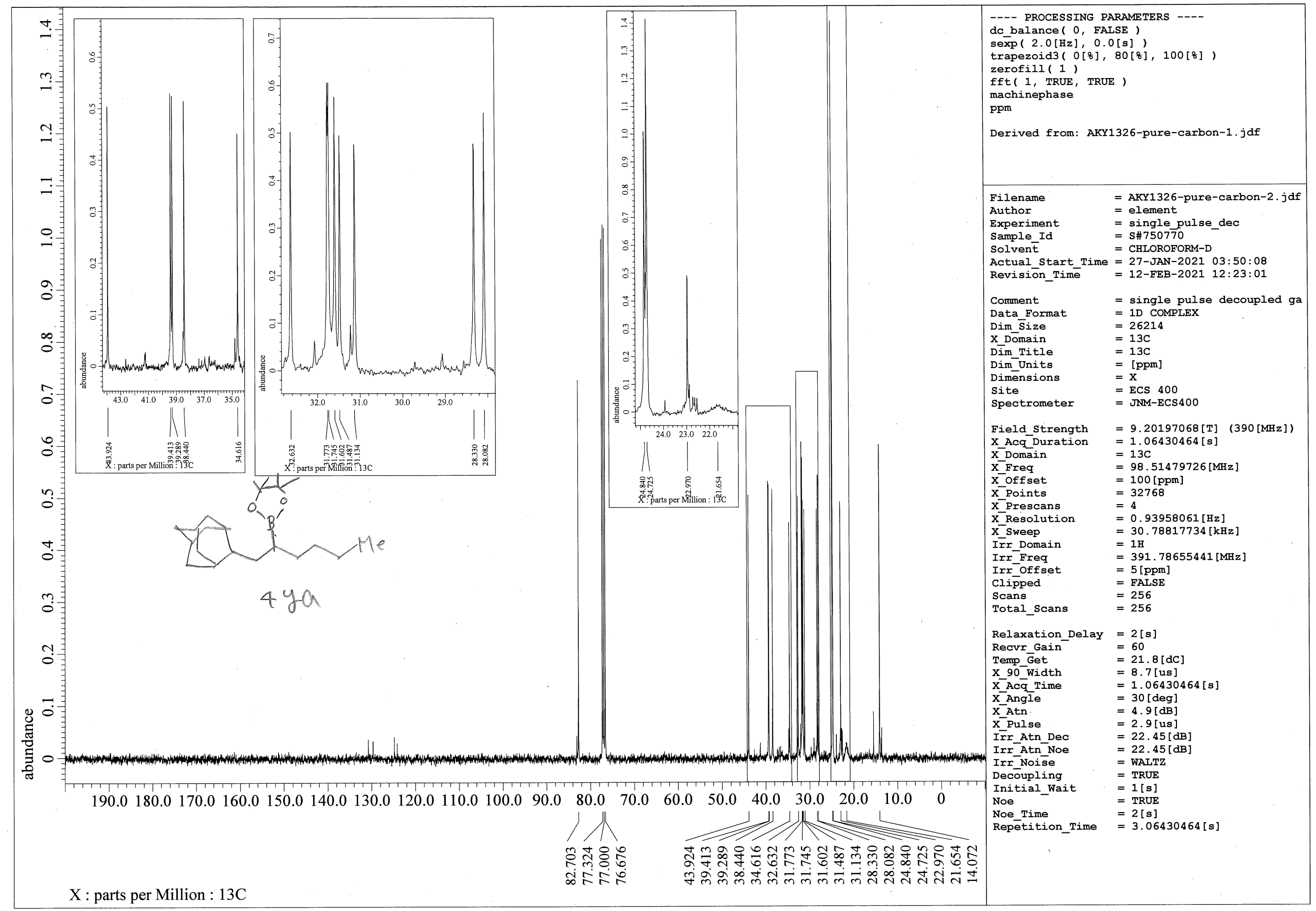




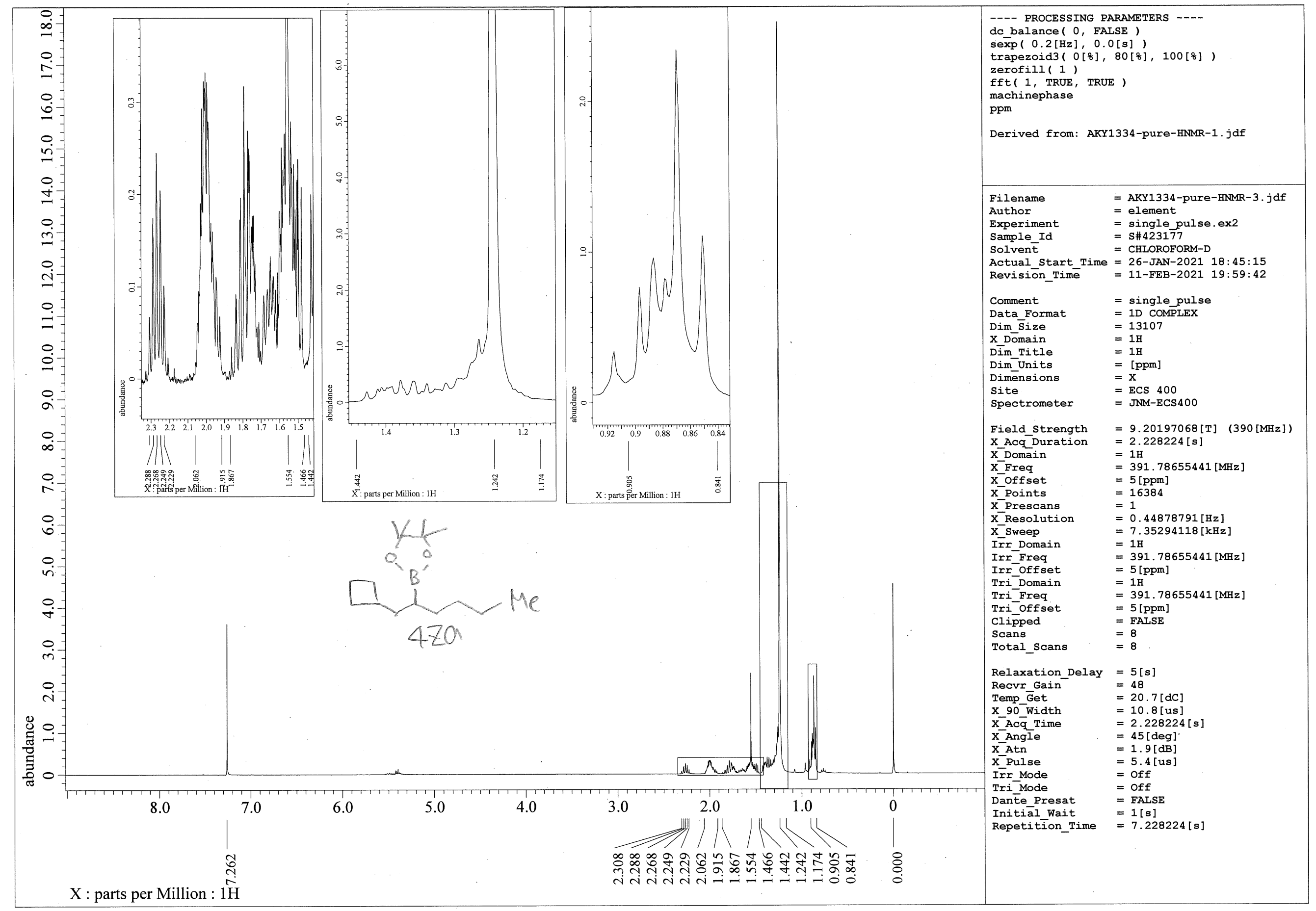




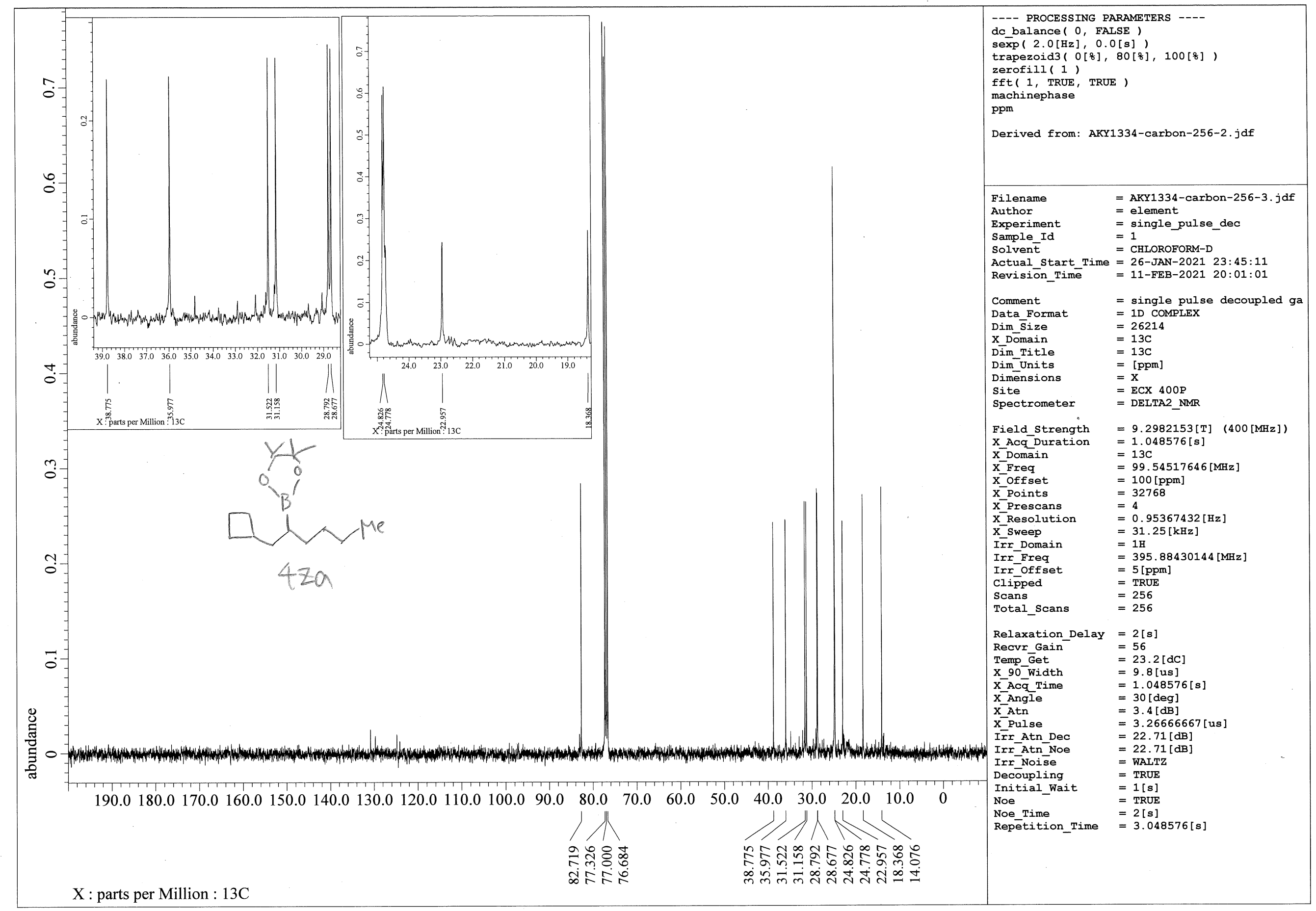




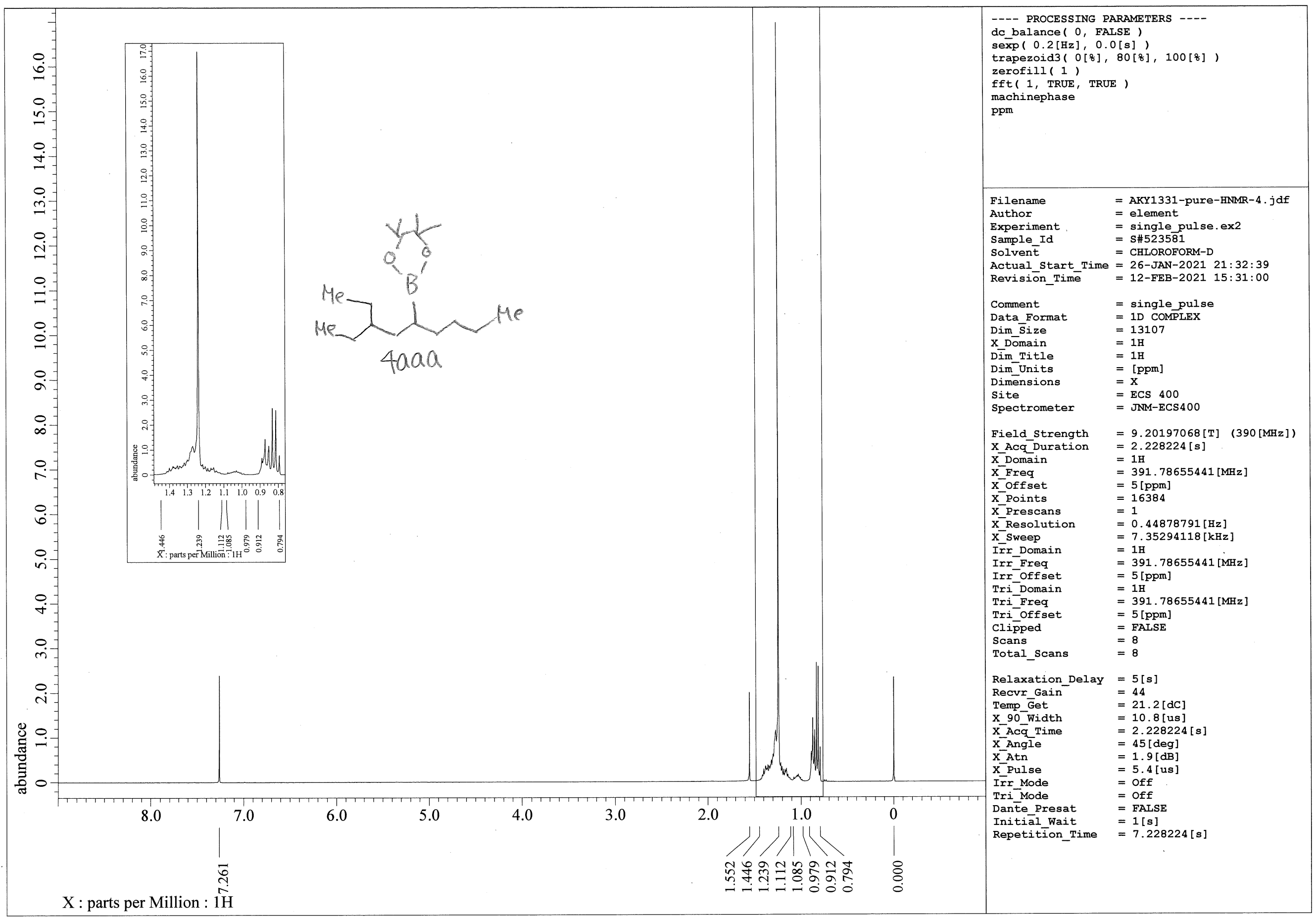




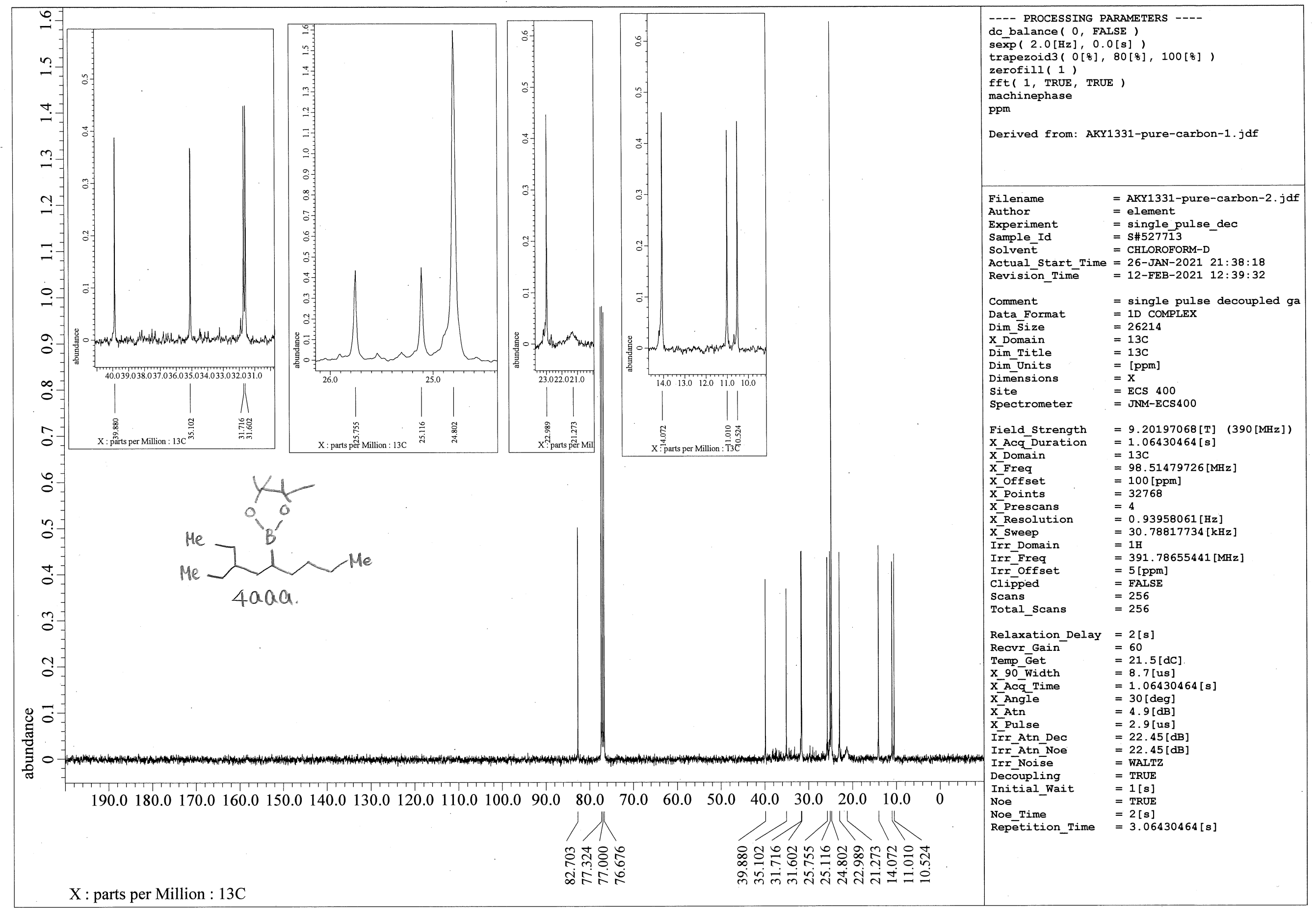




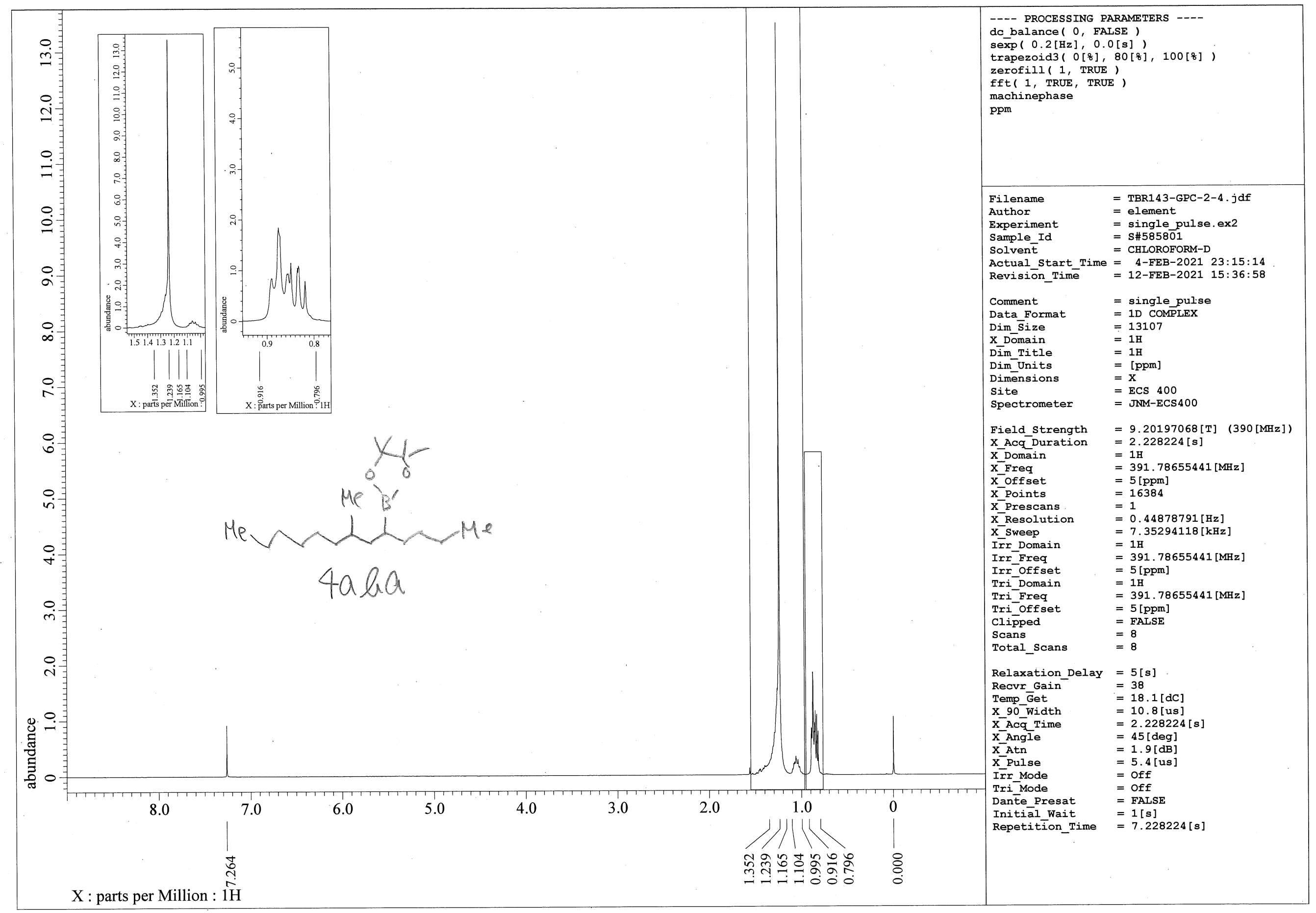




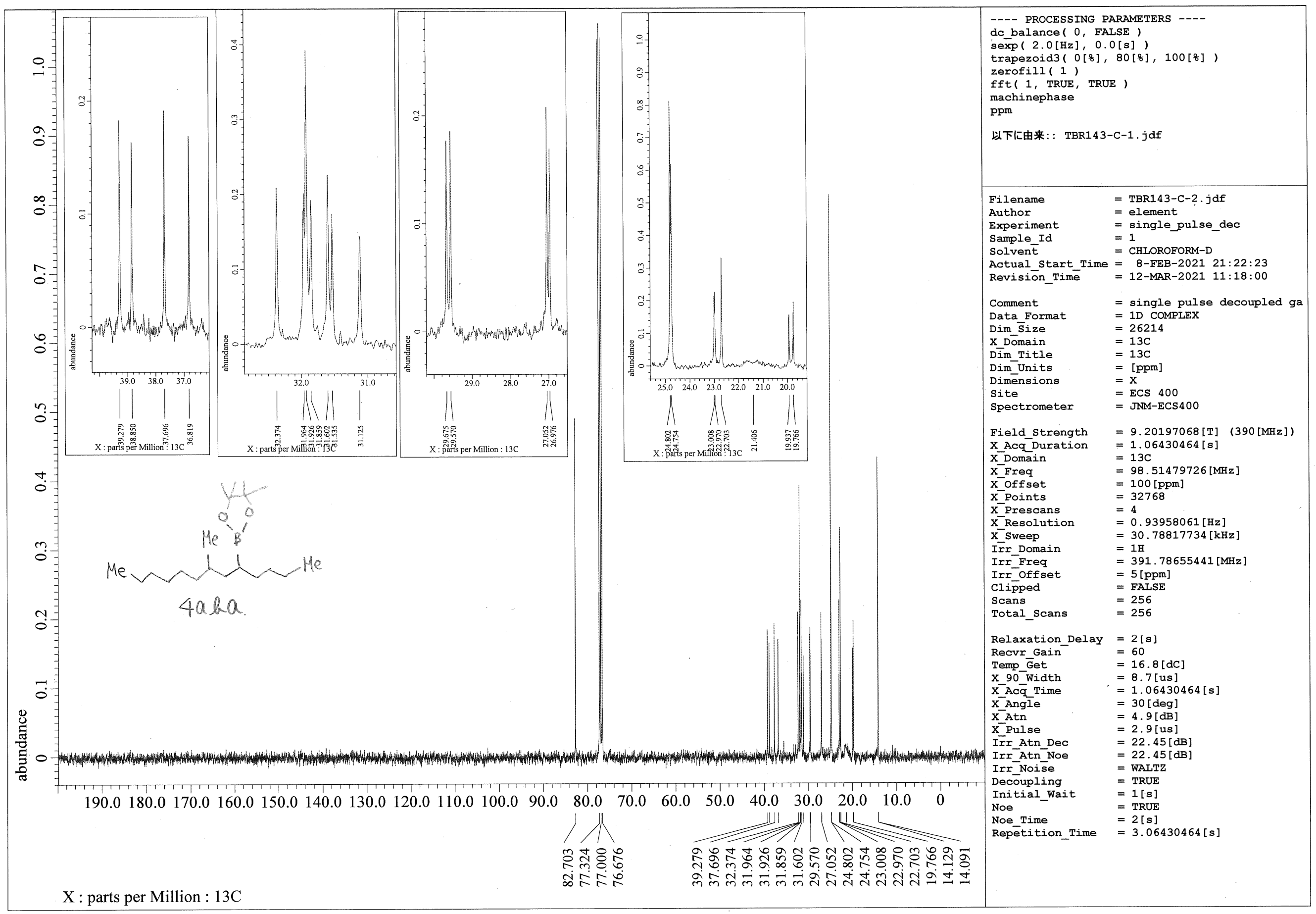




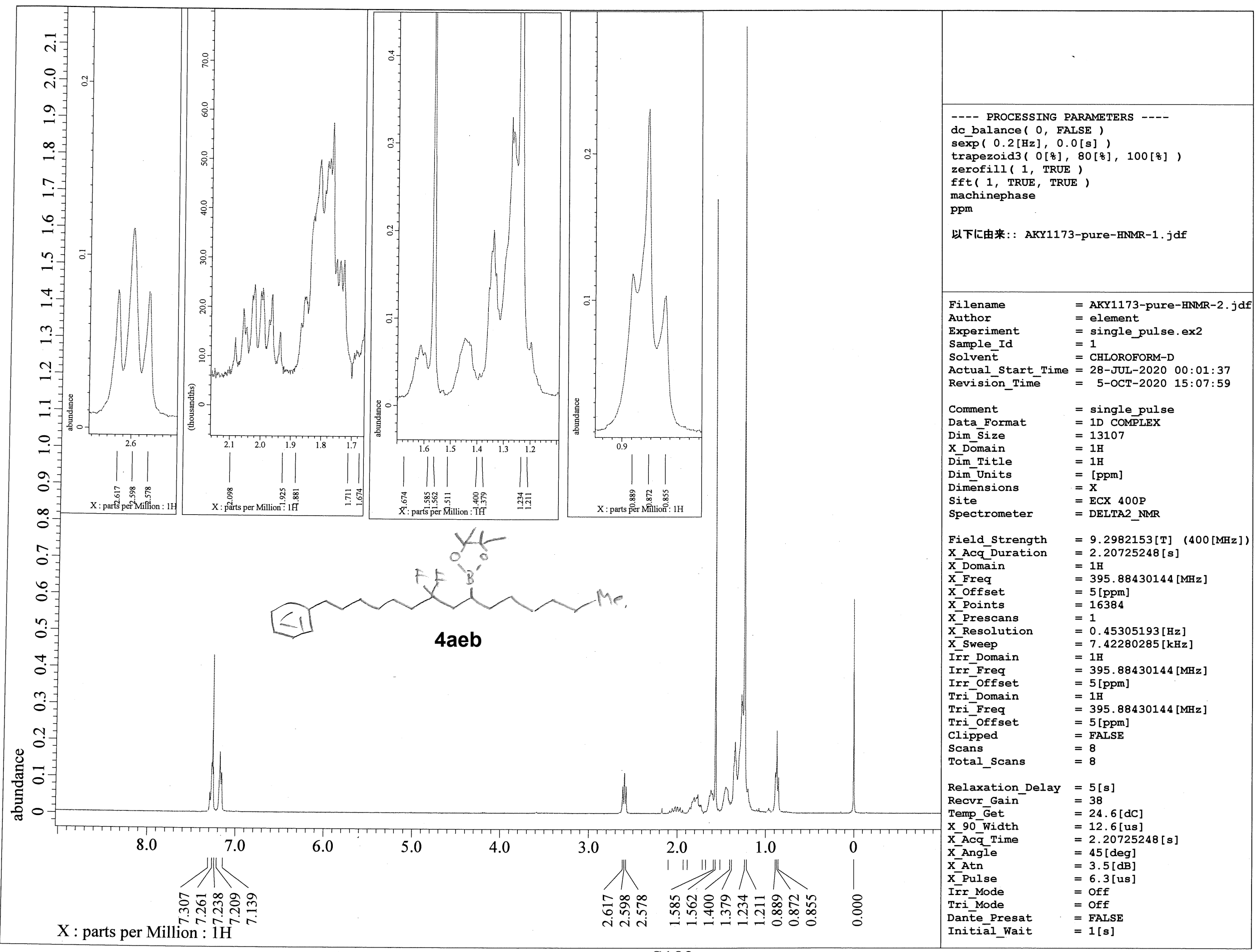




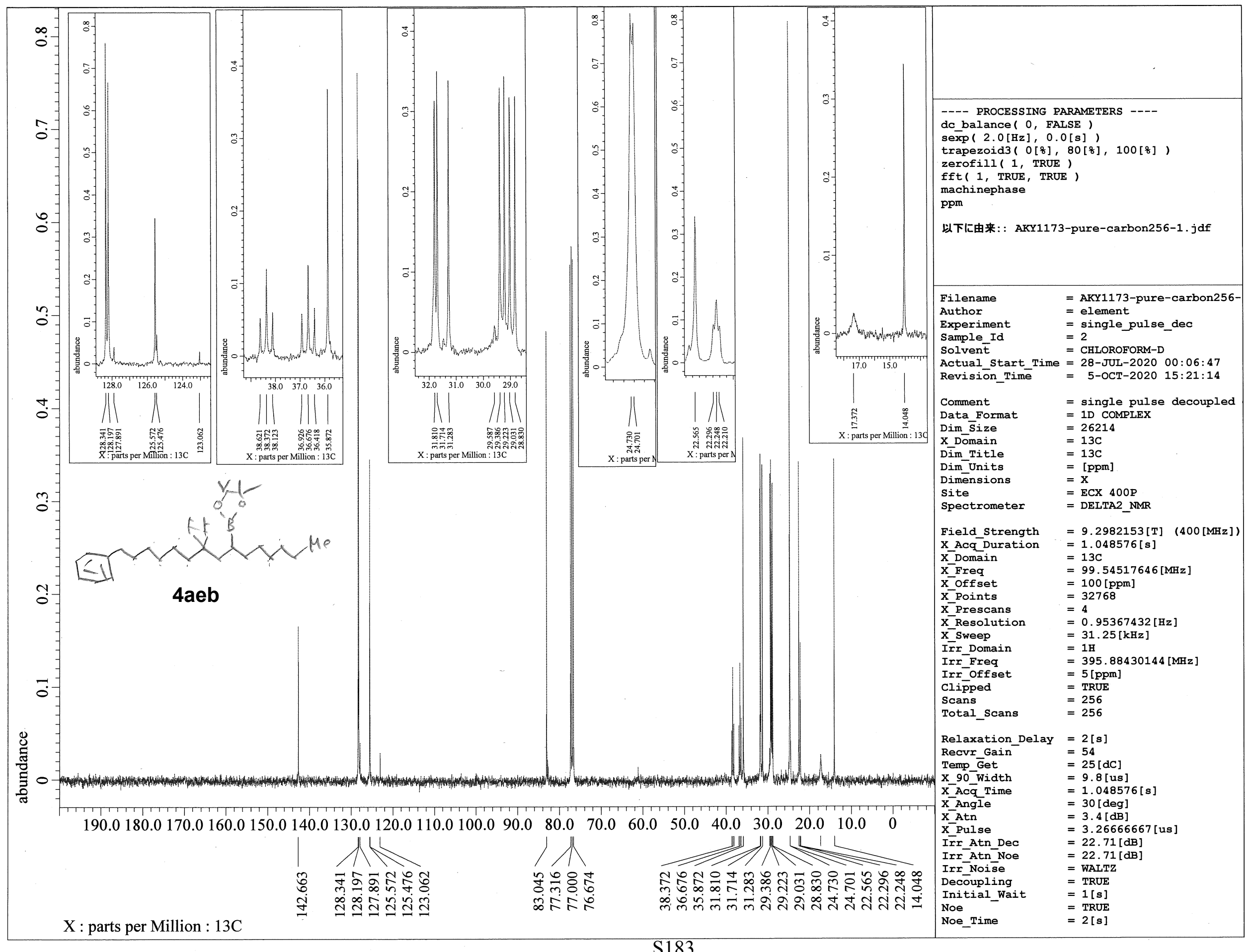




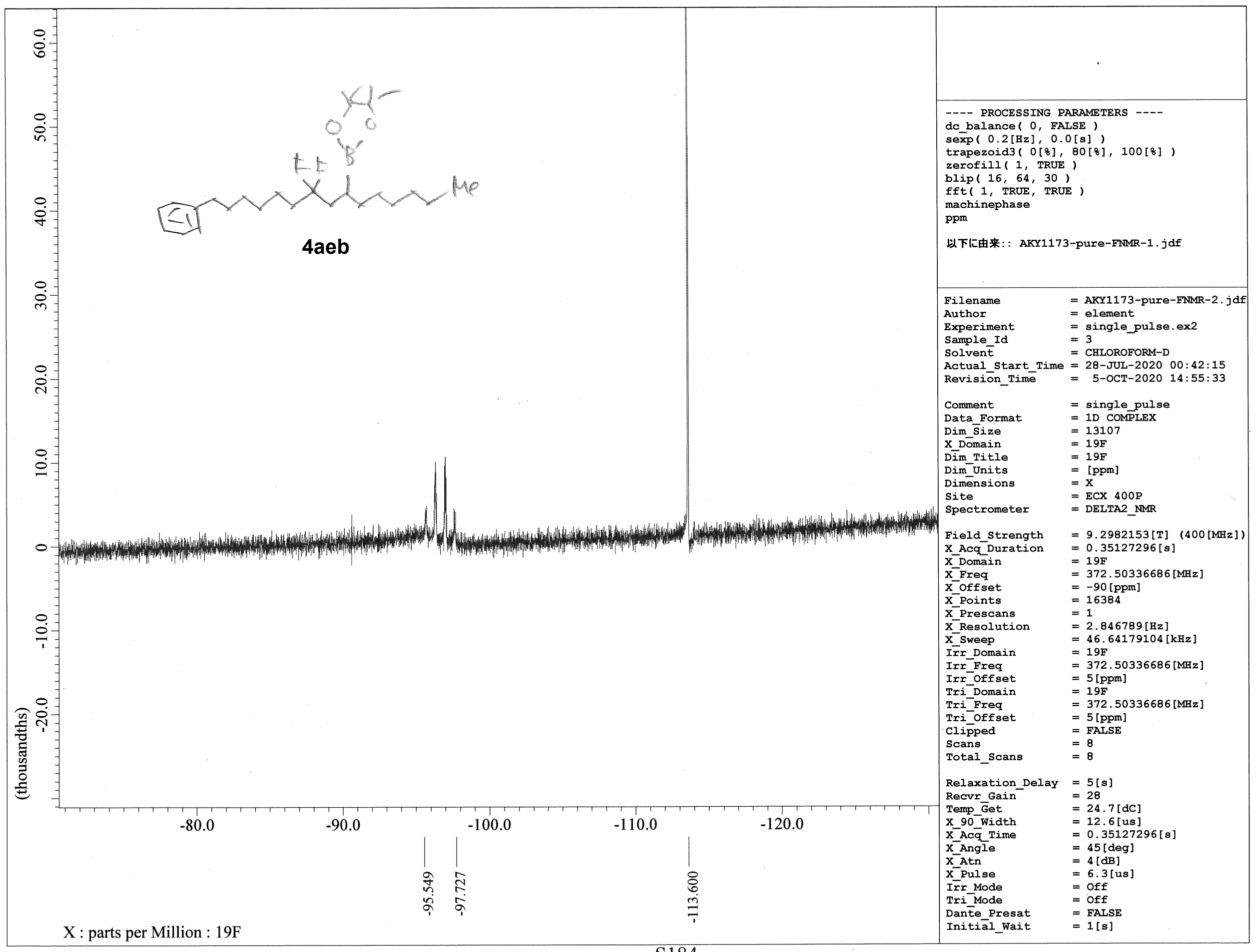




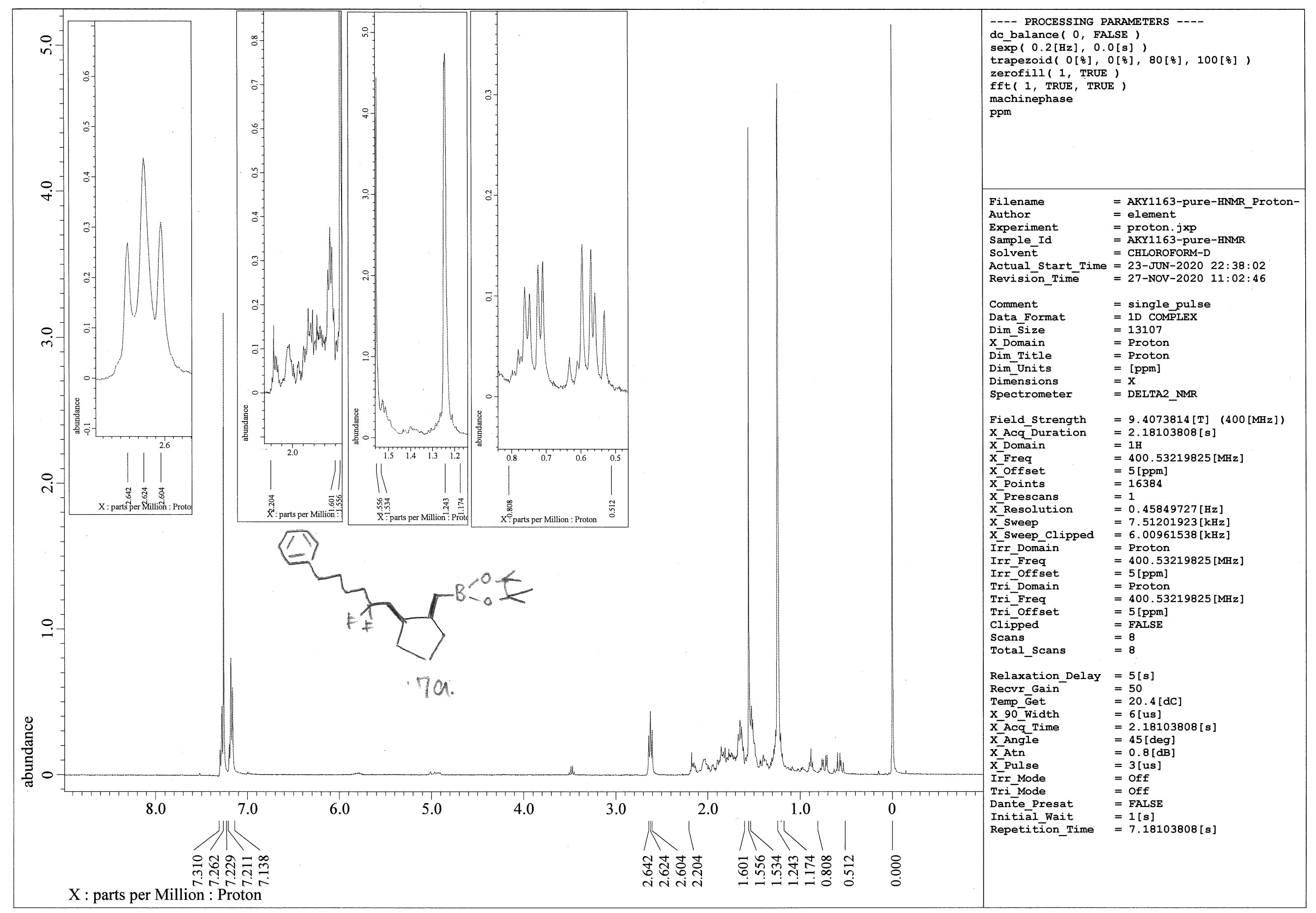




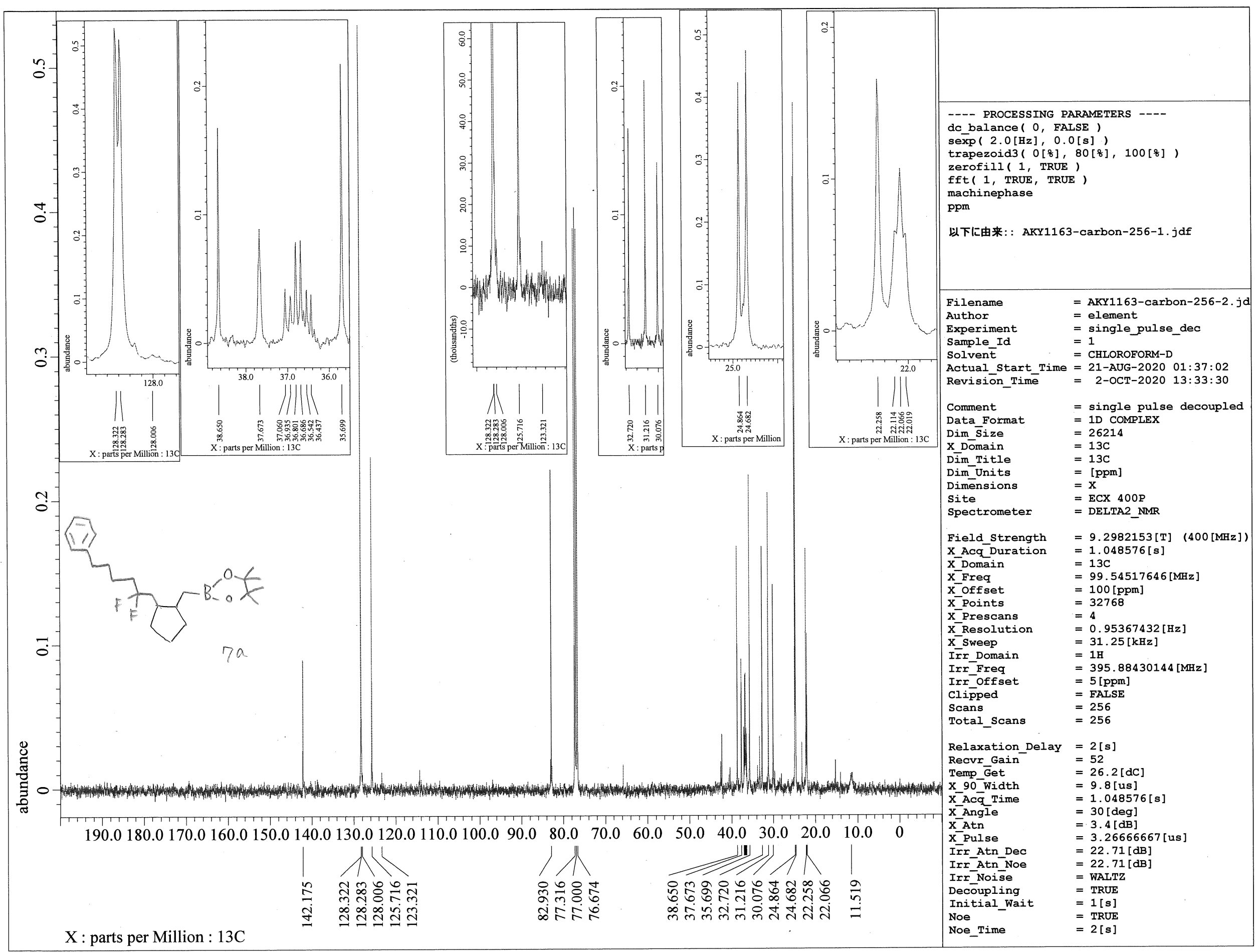




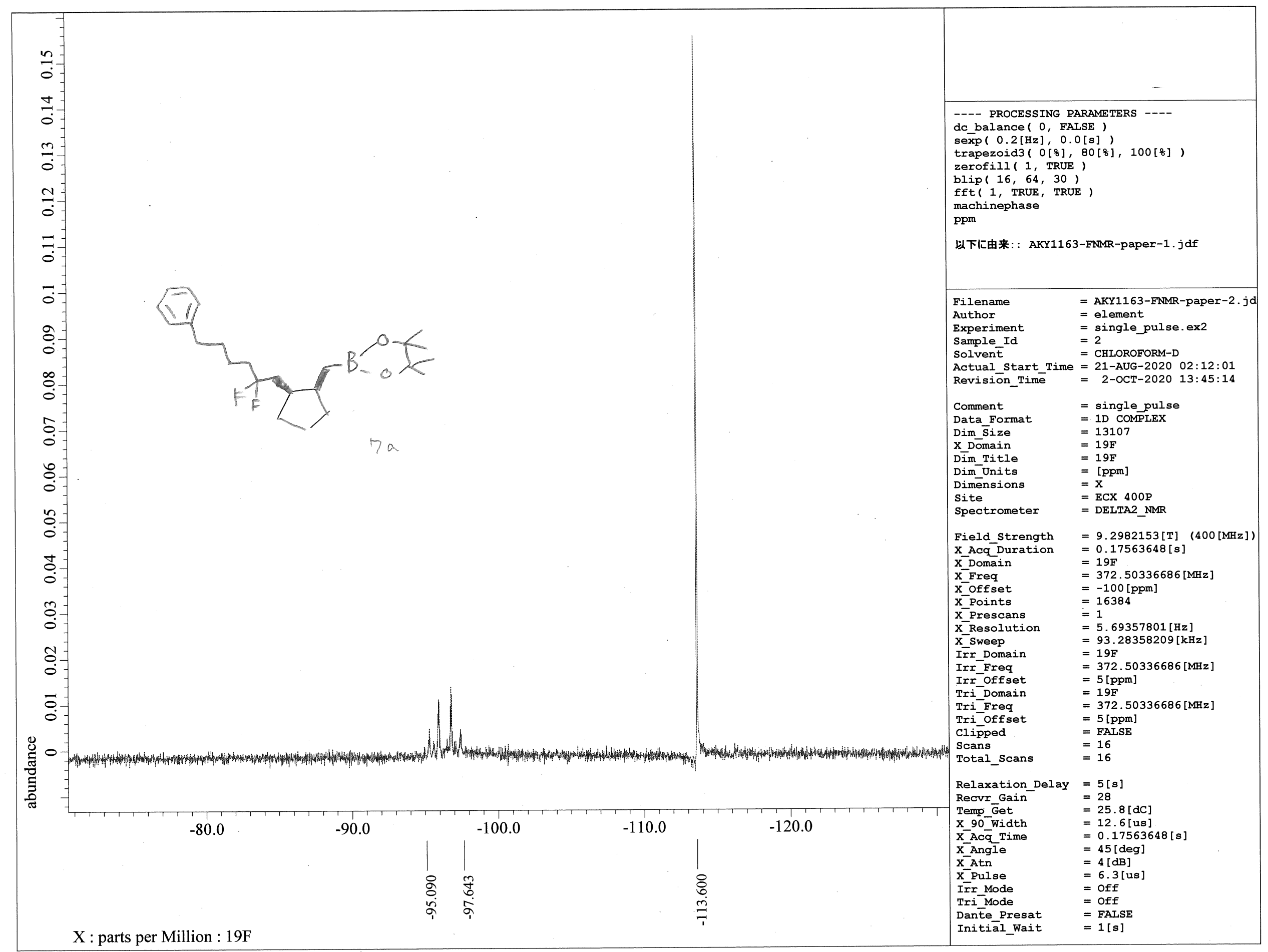




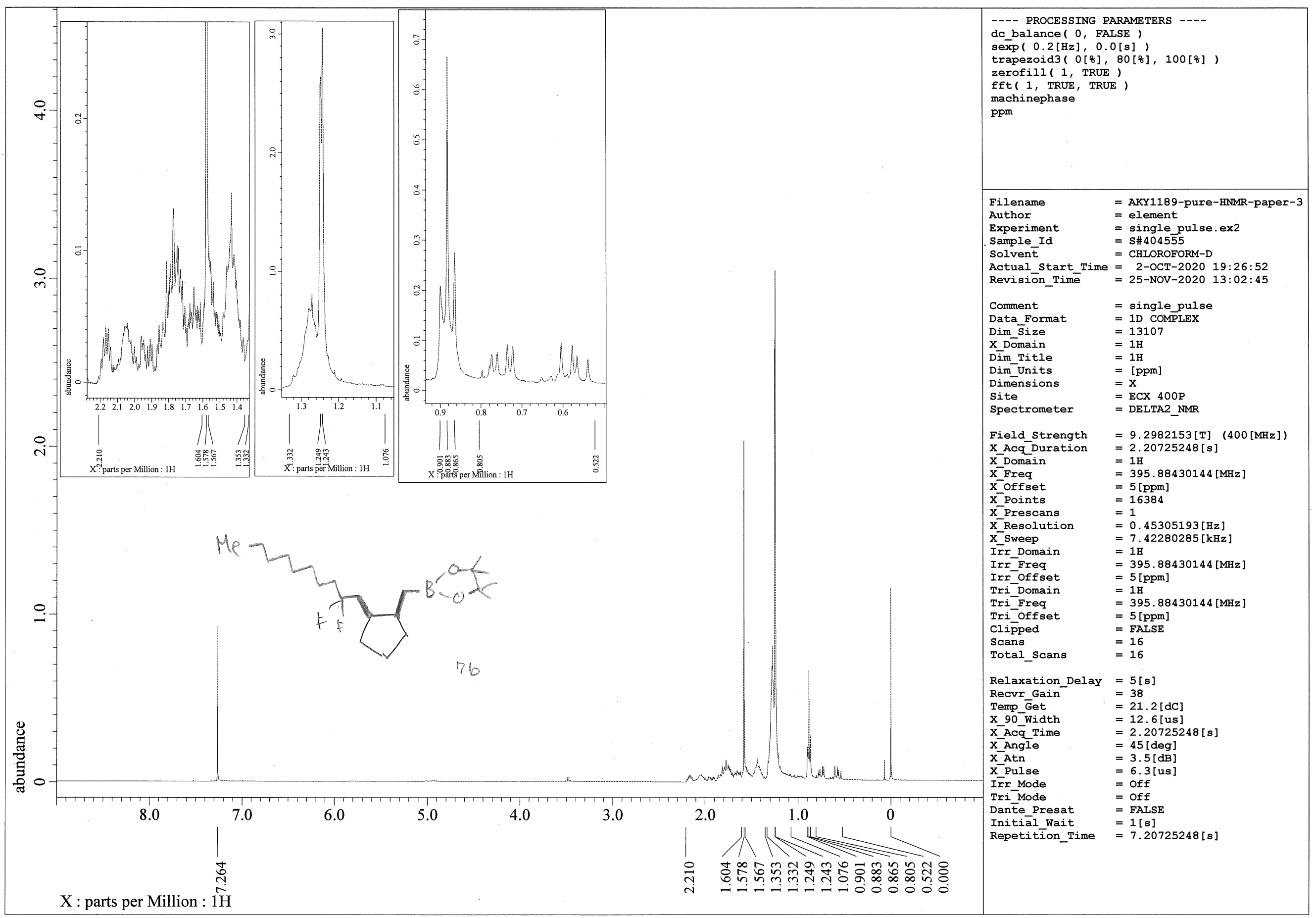




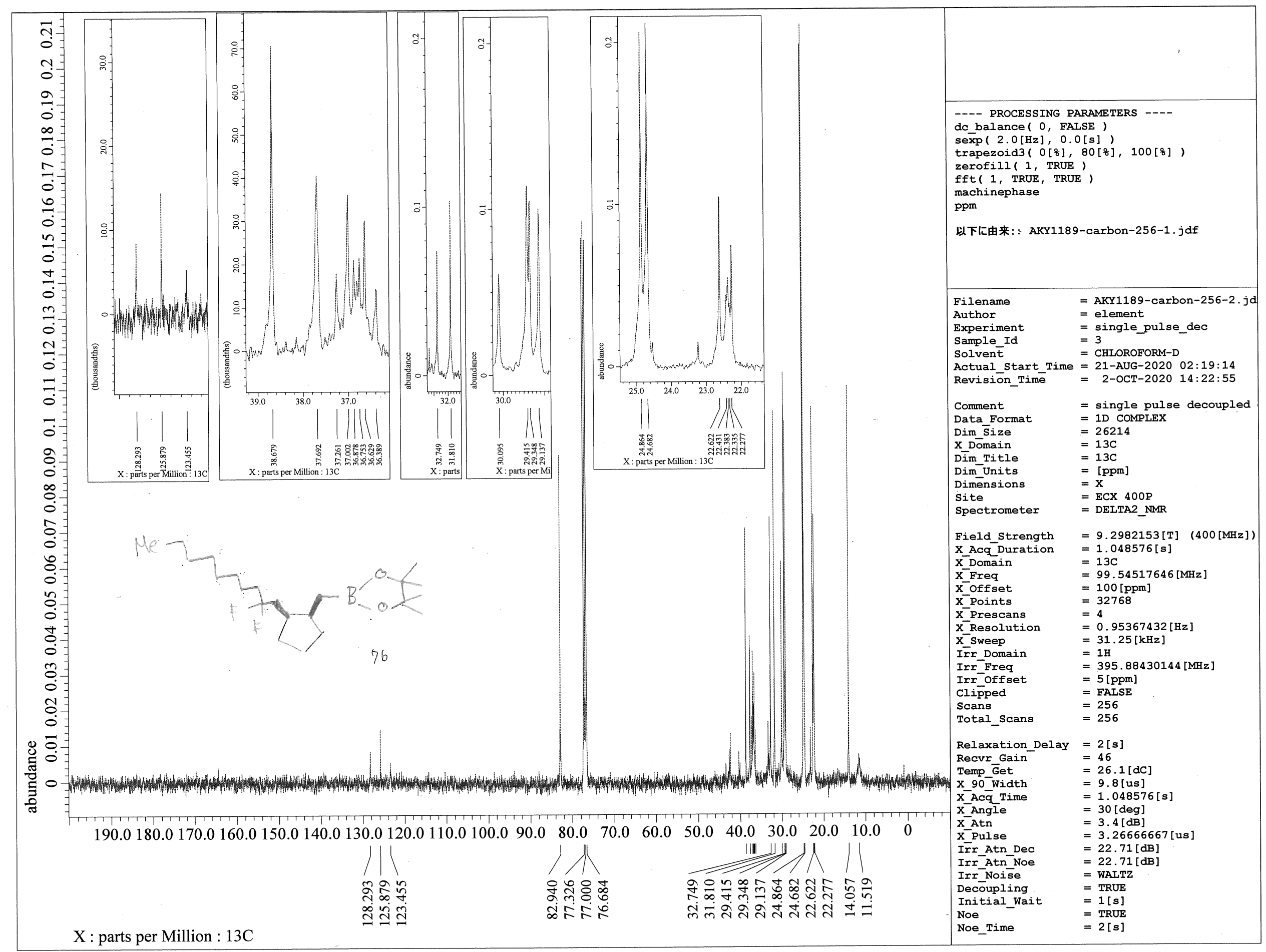




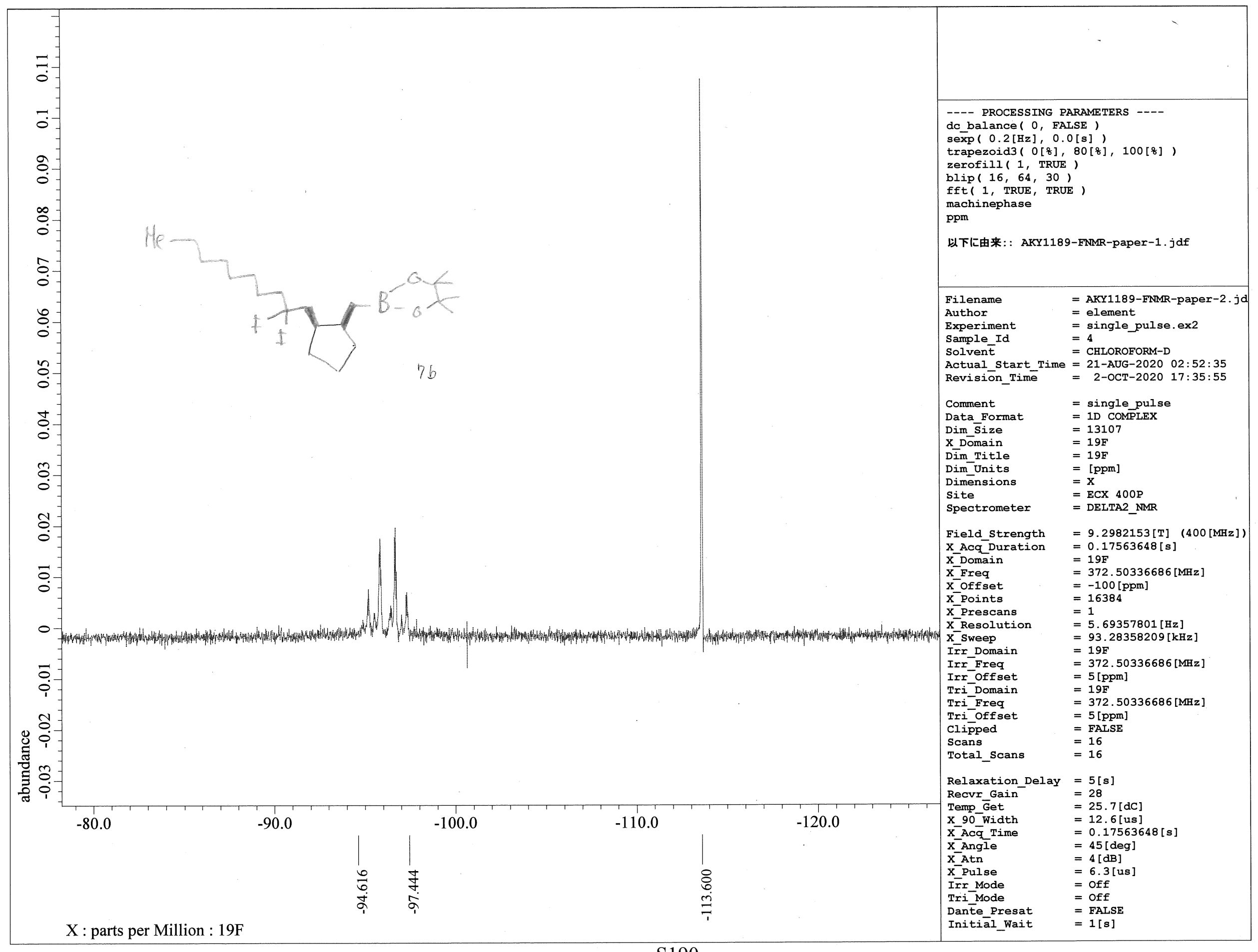




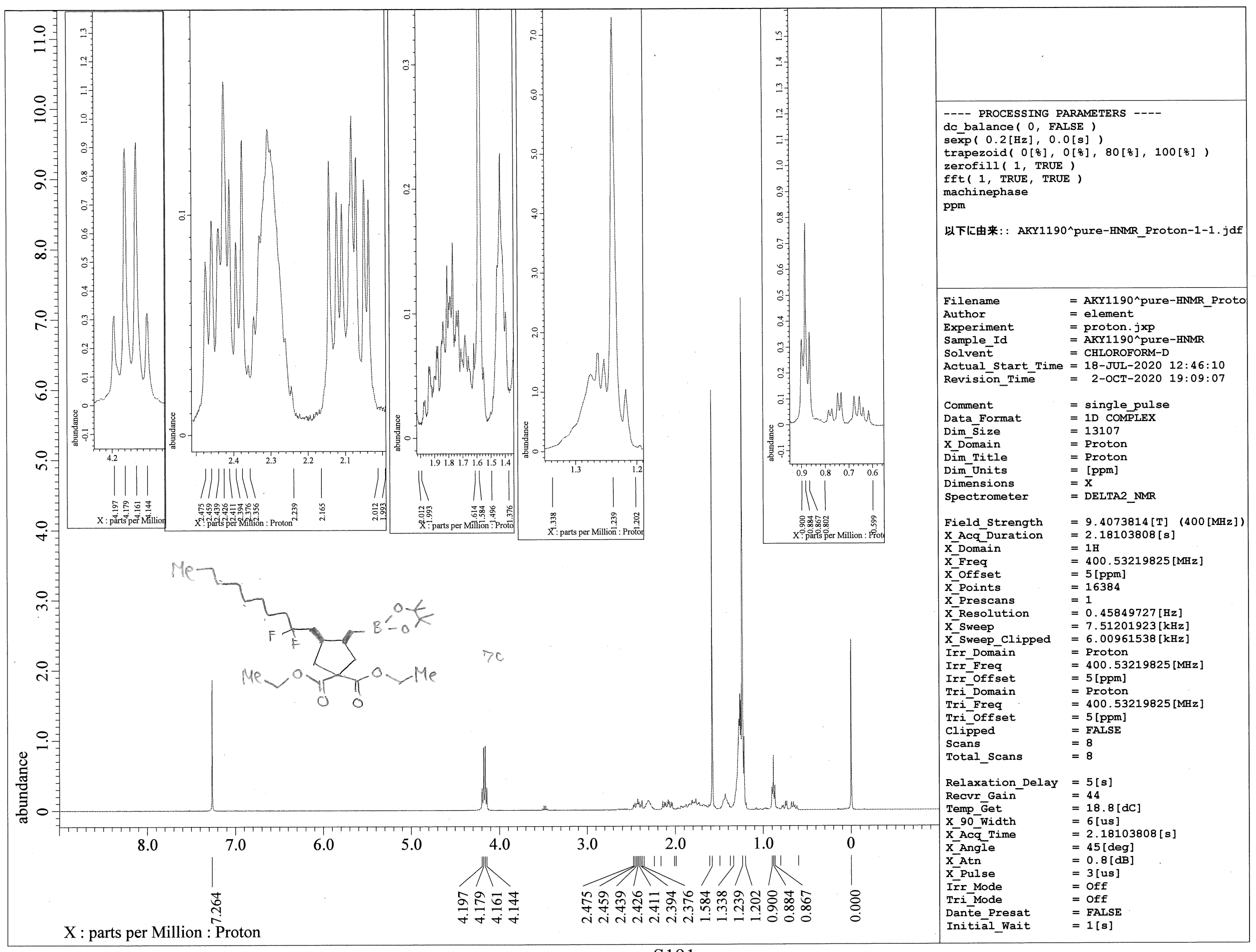




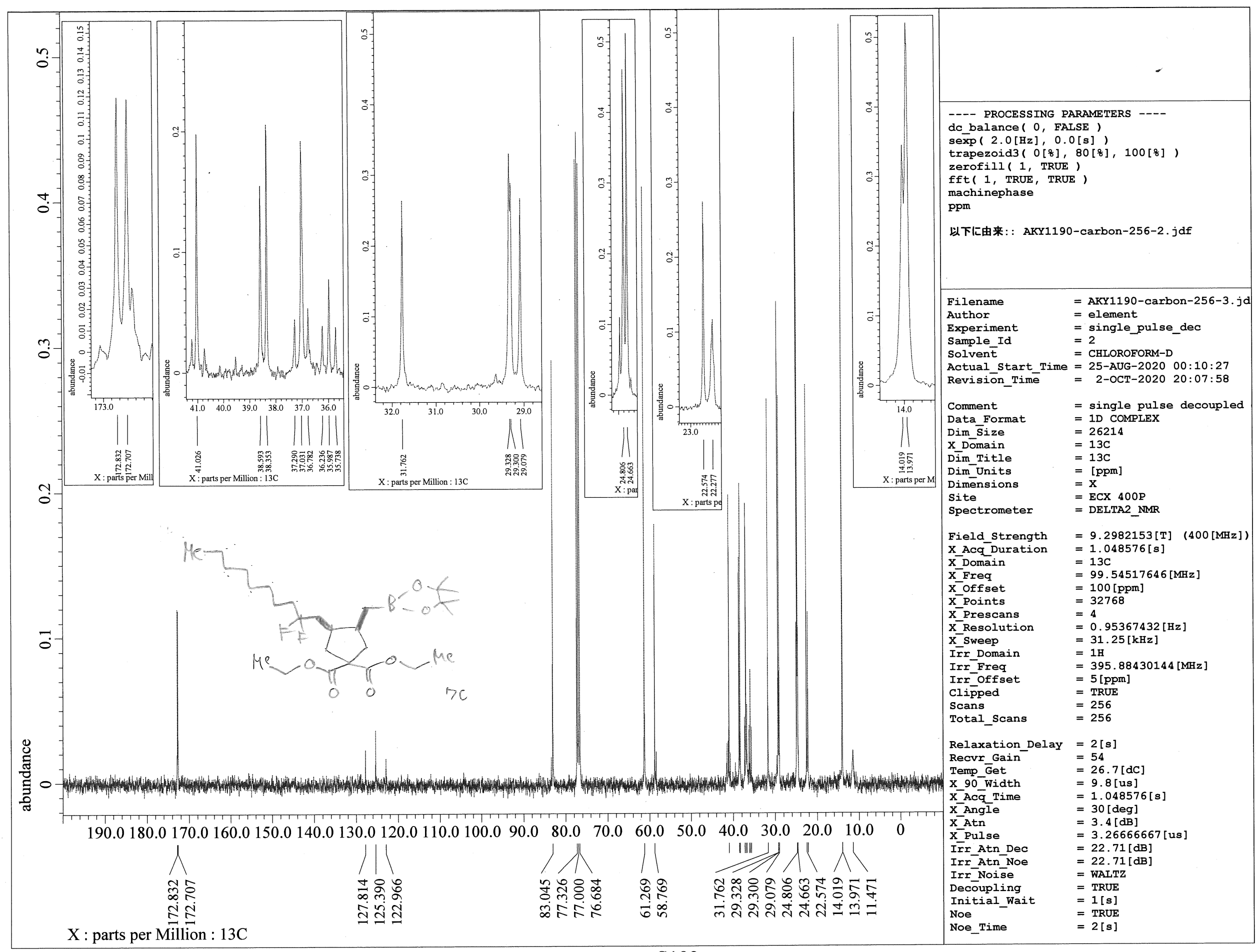




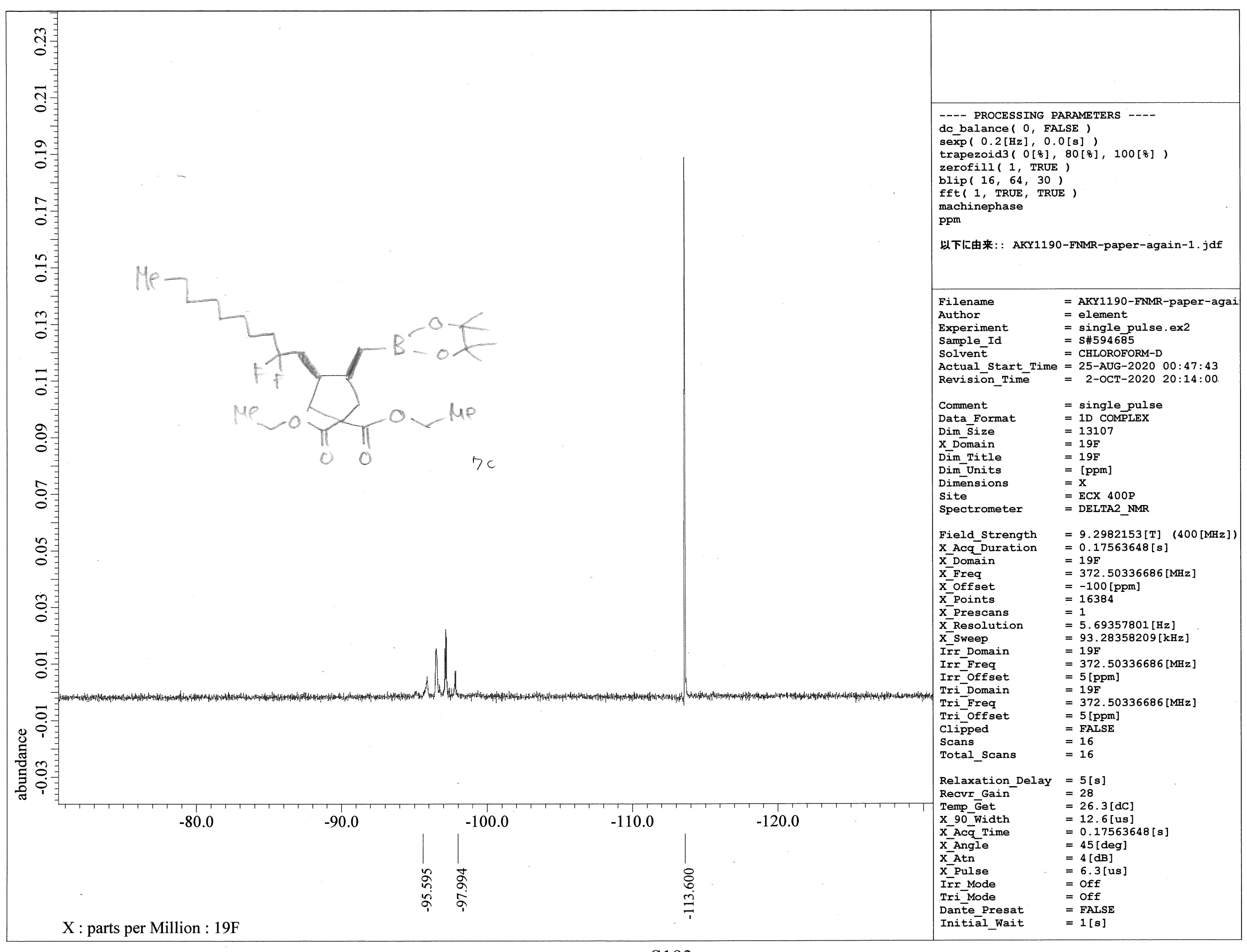




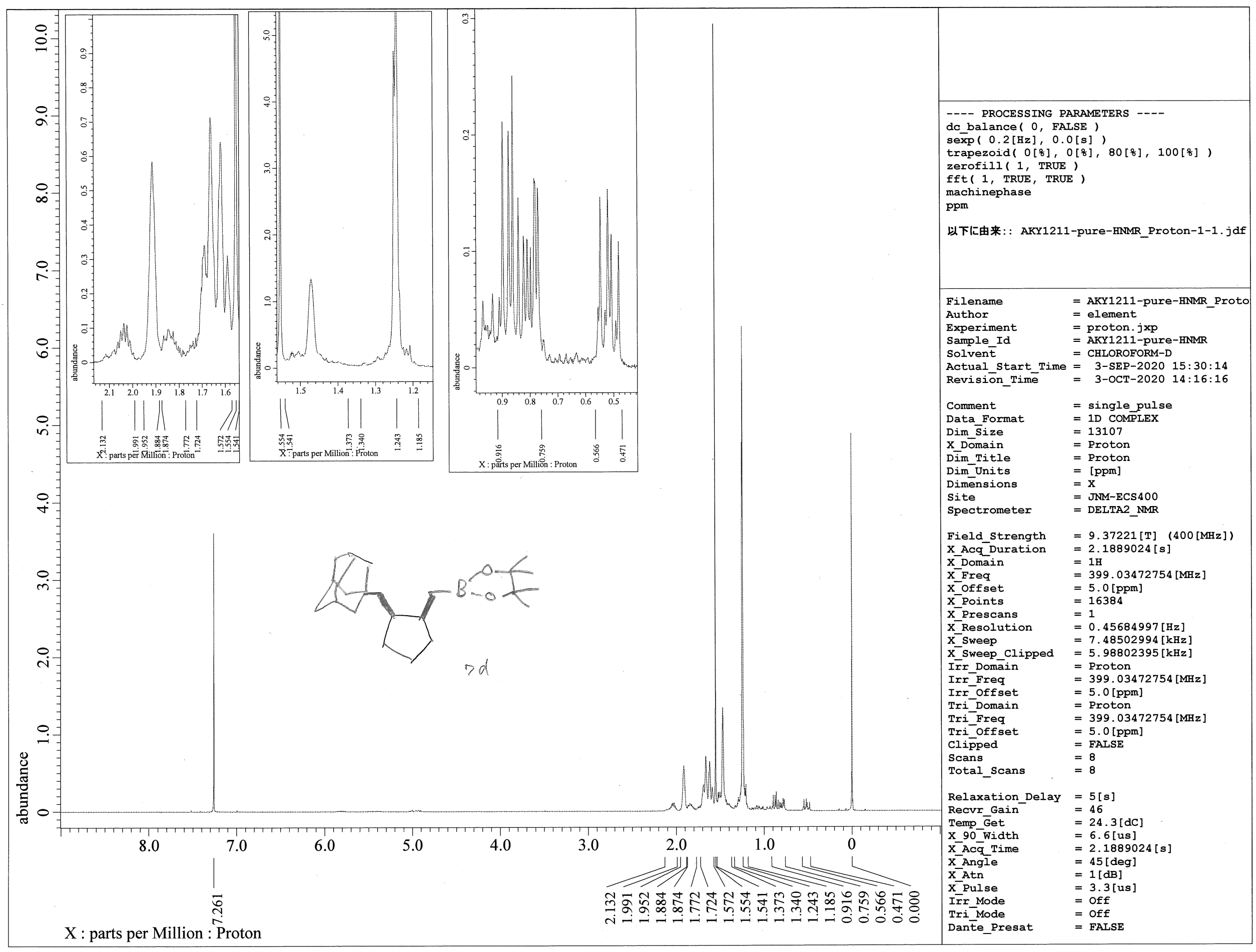

S194 


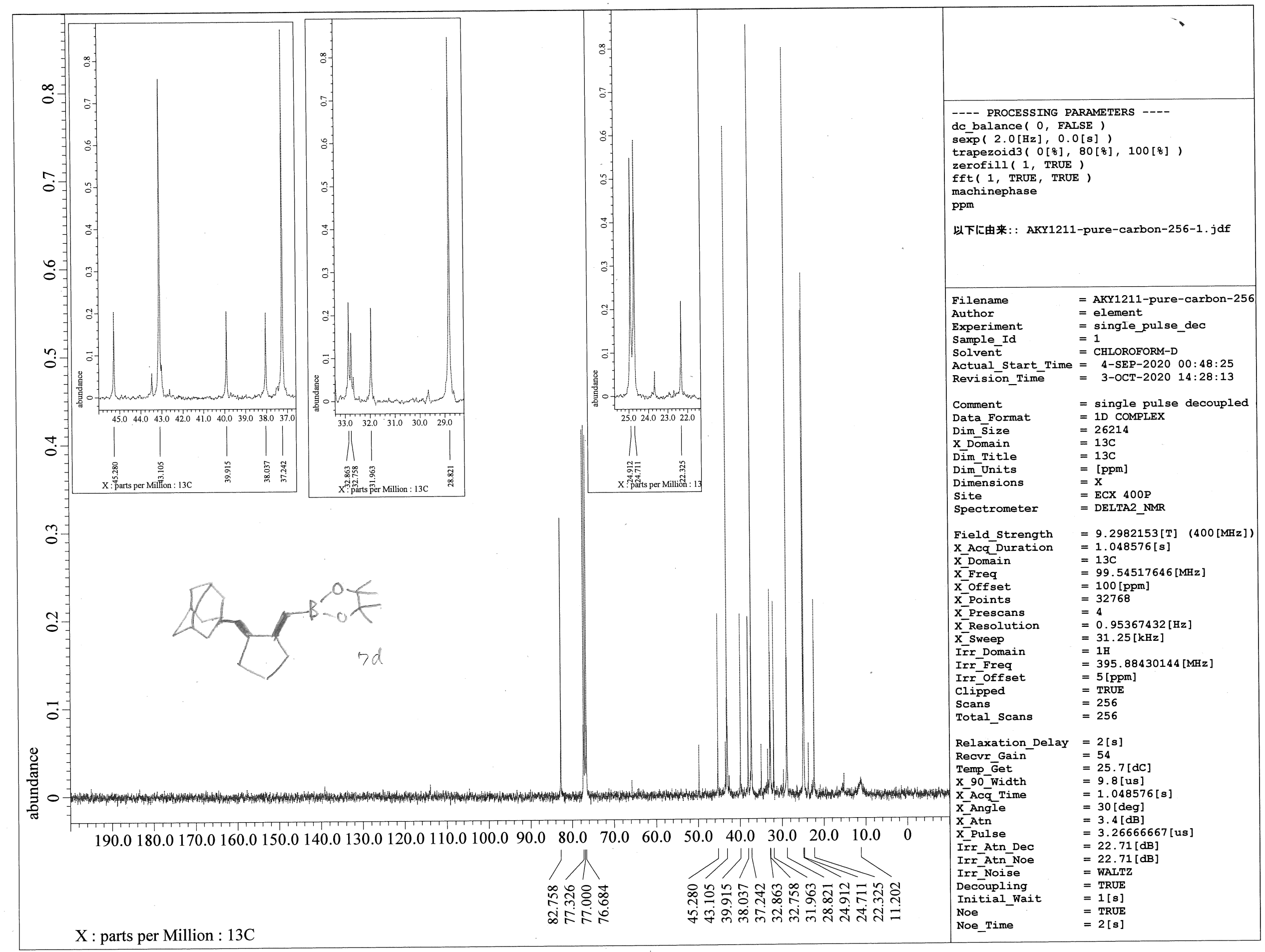




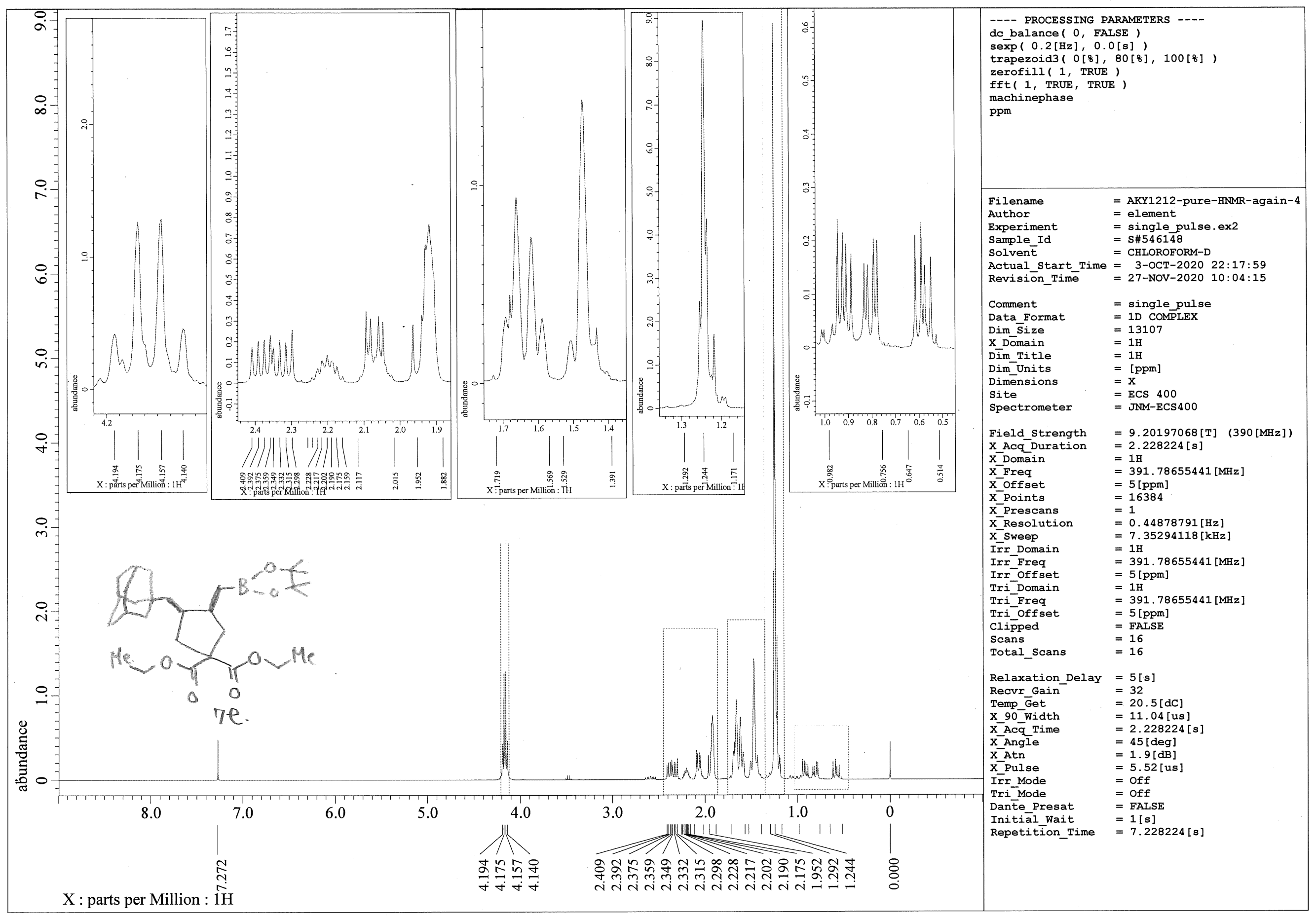




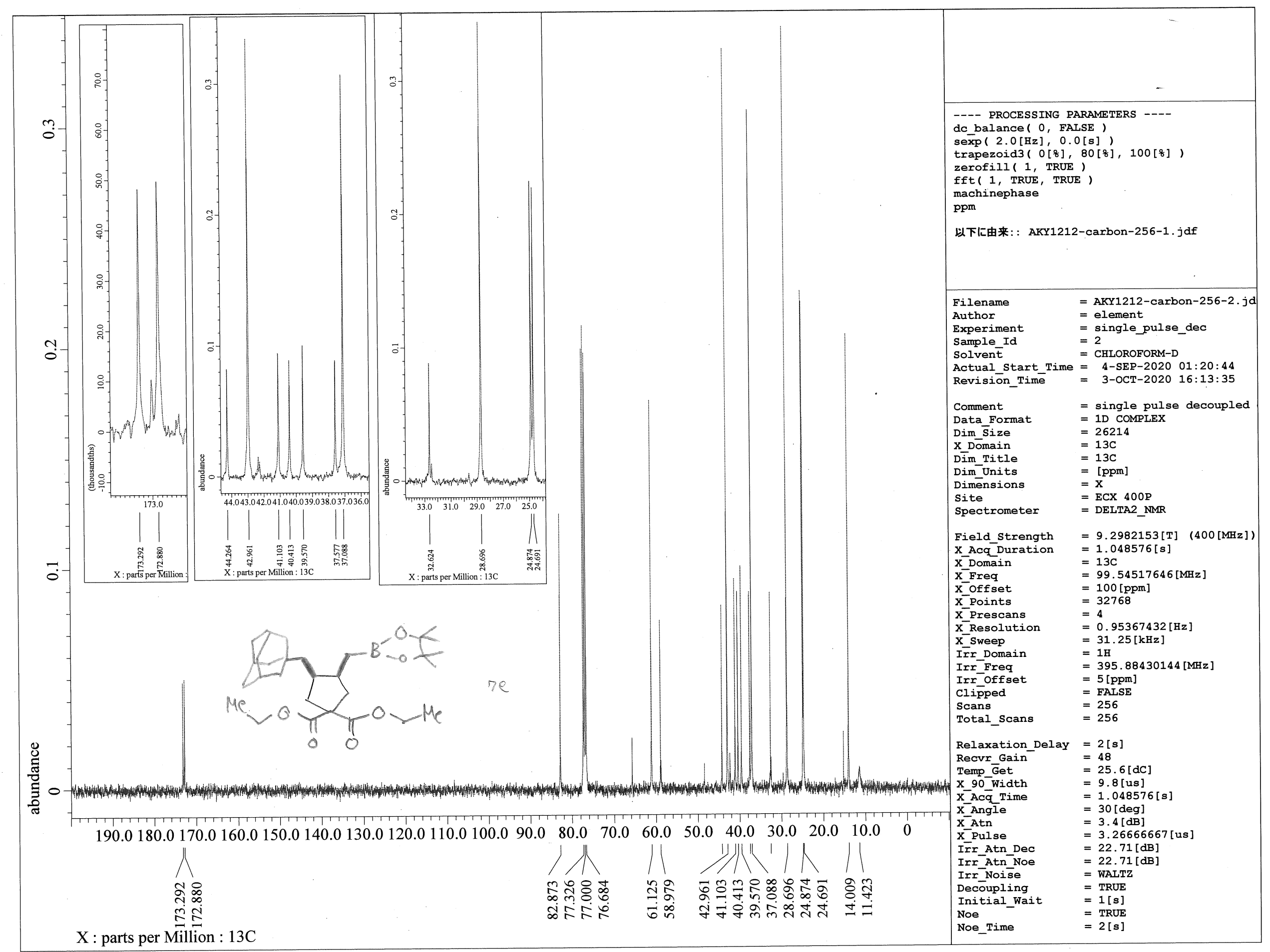




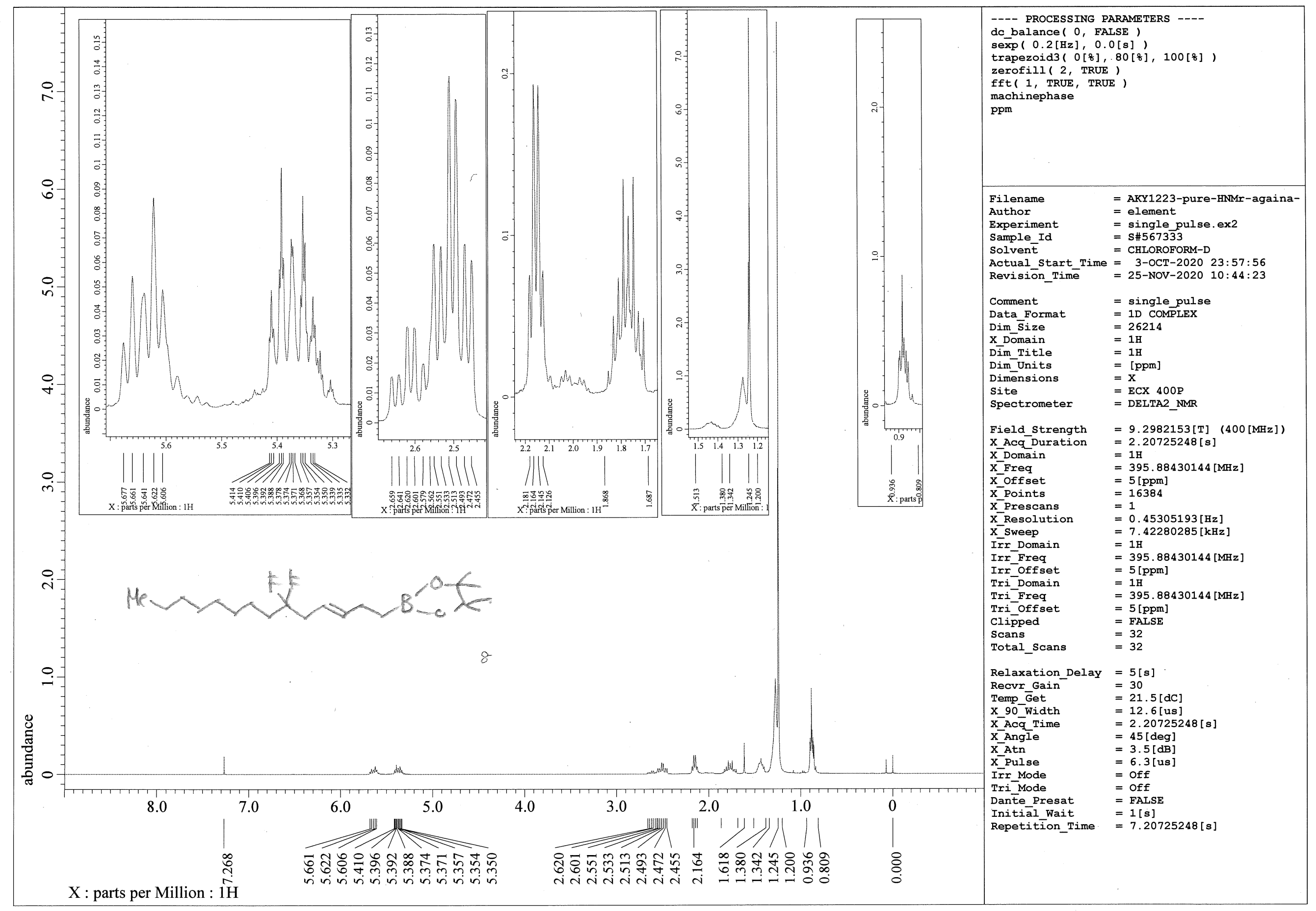




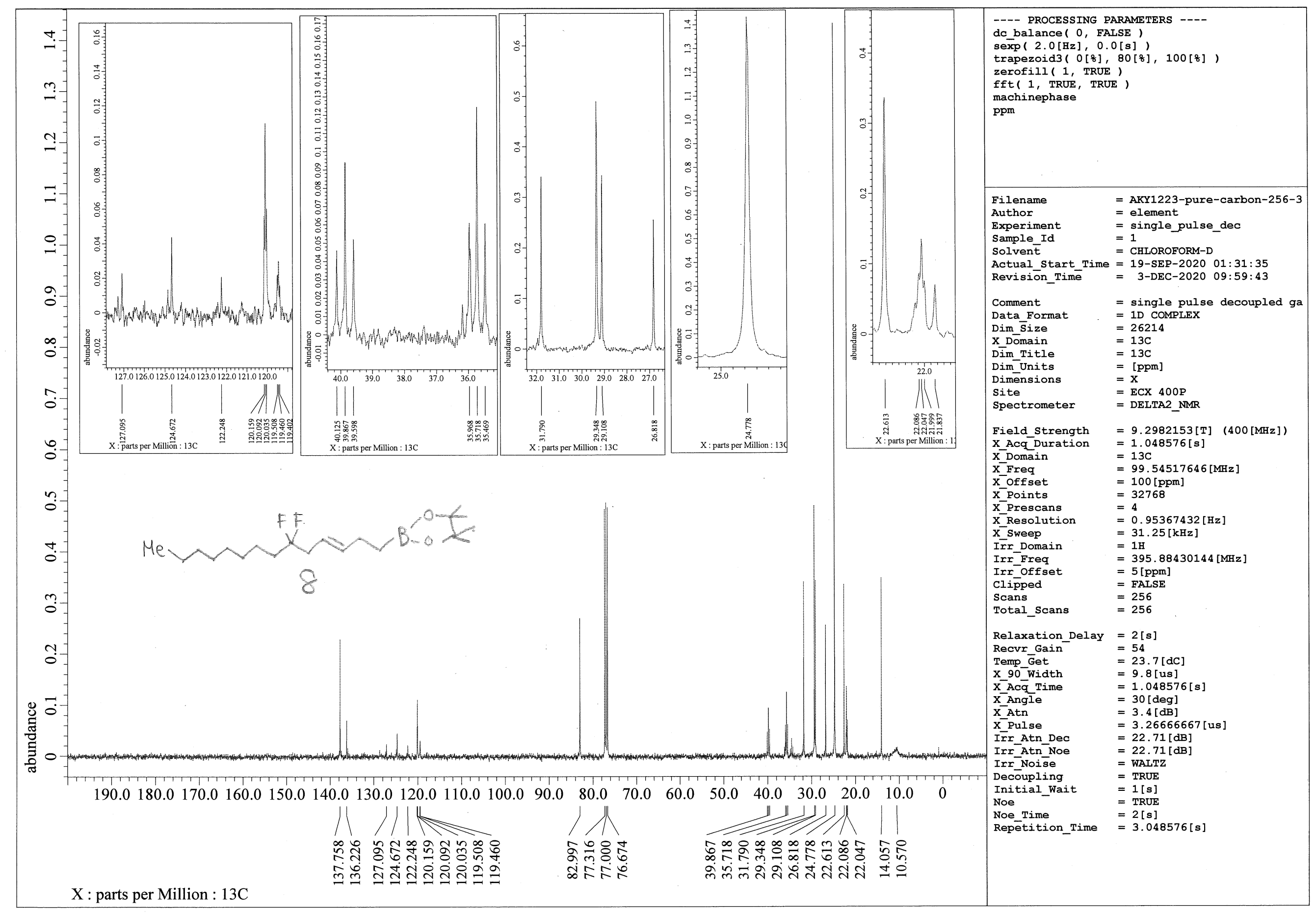




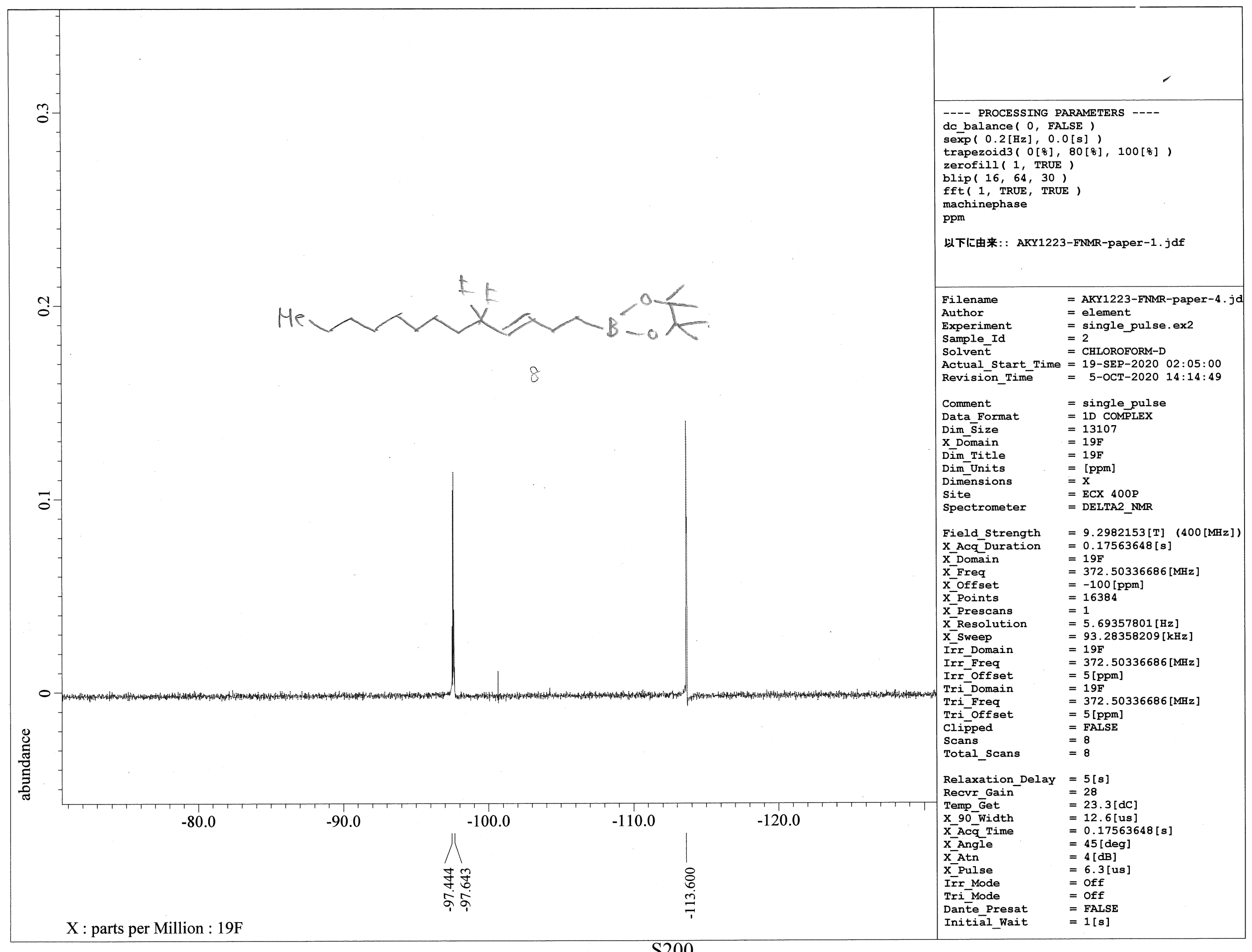




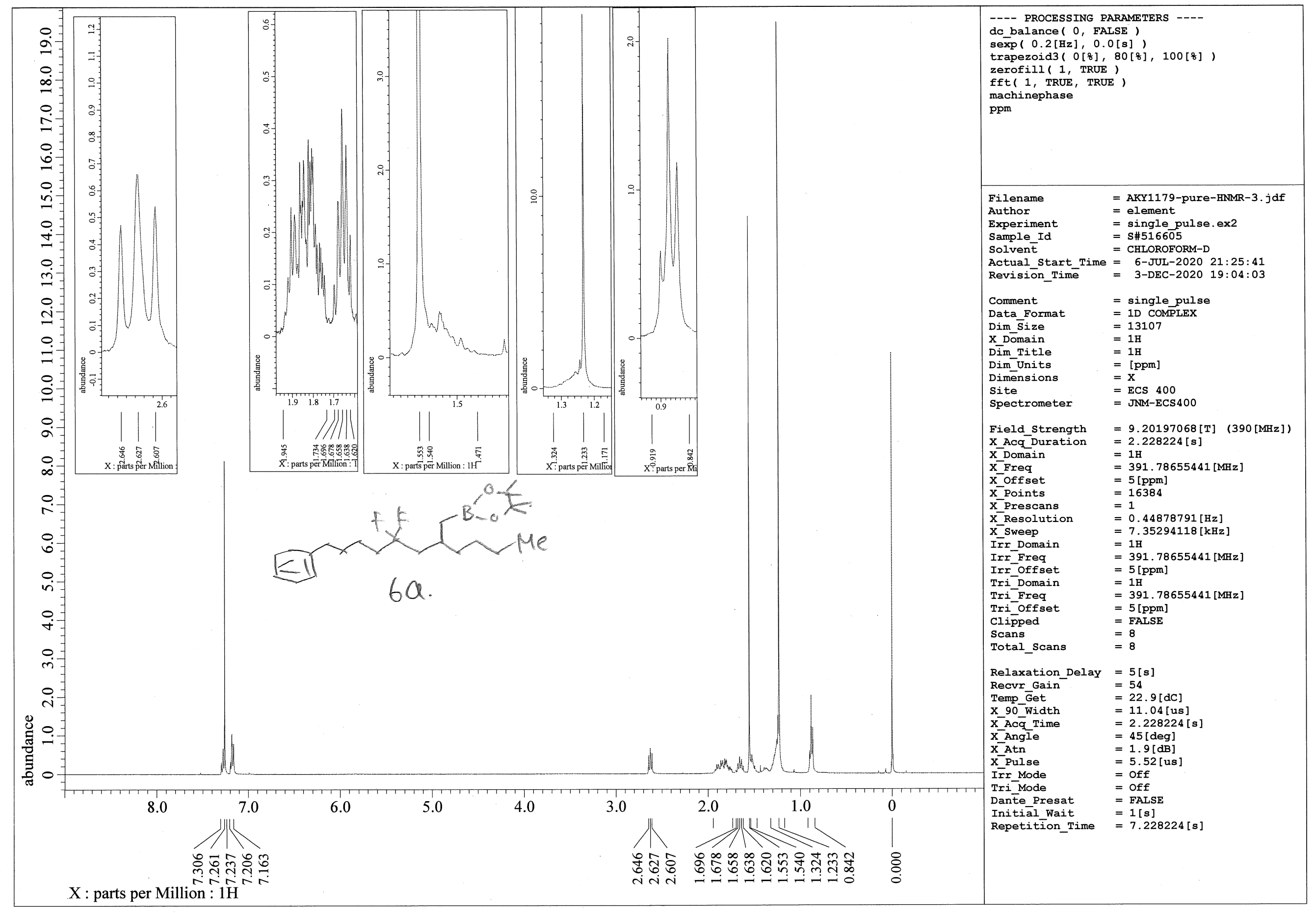




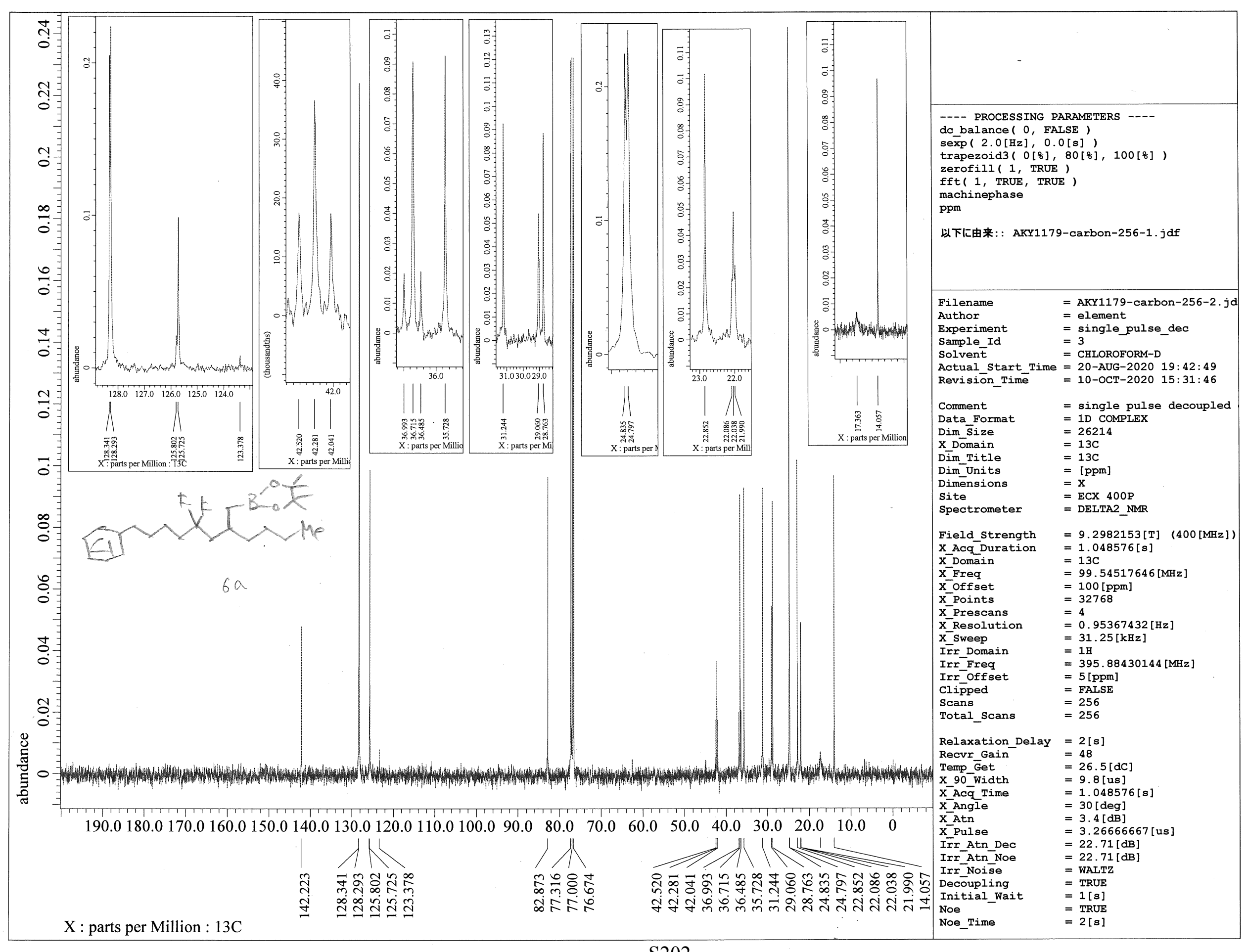




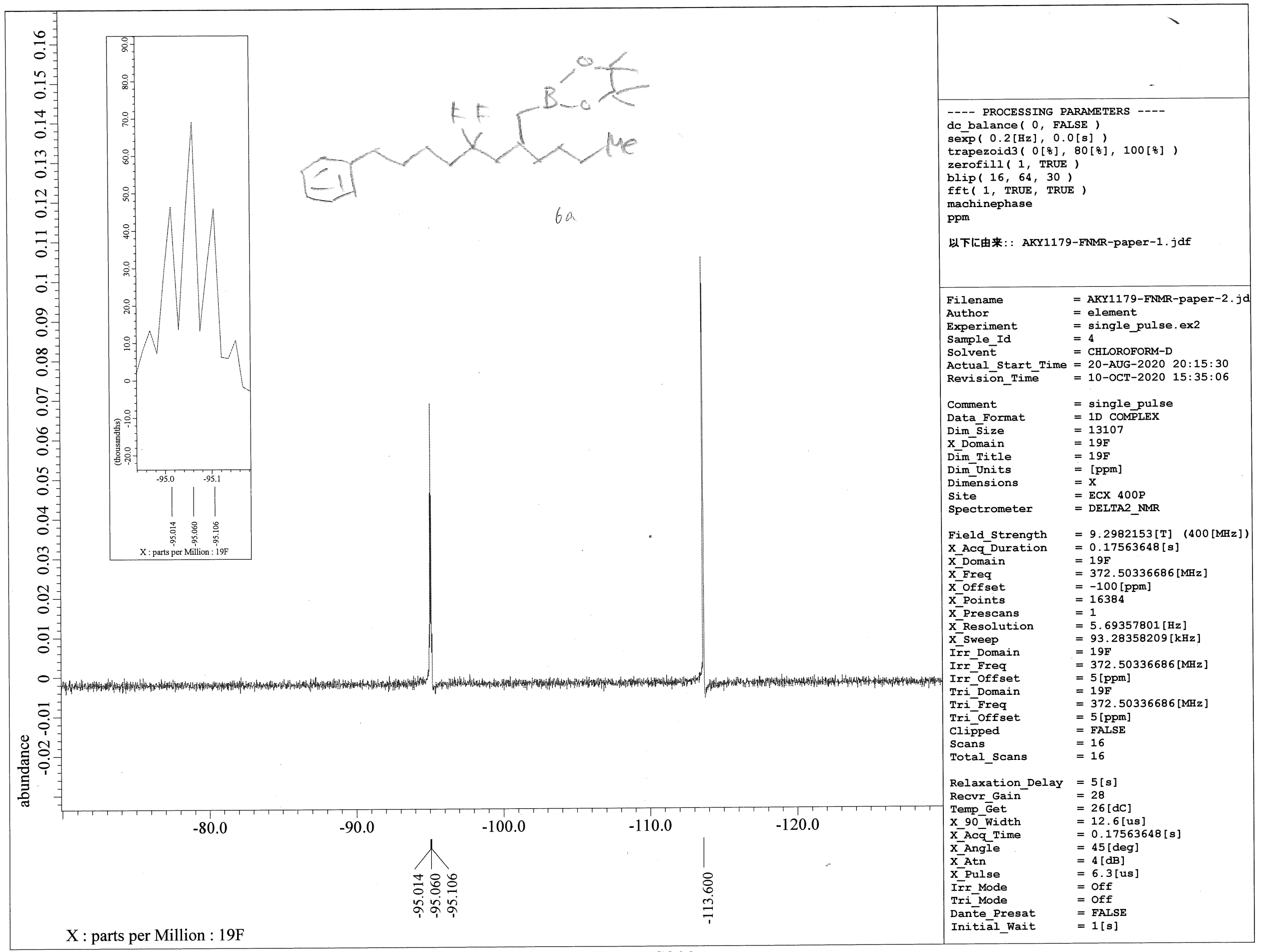




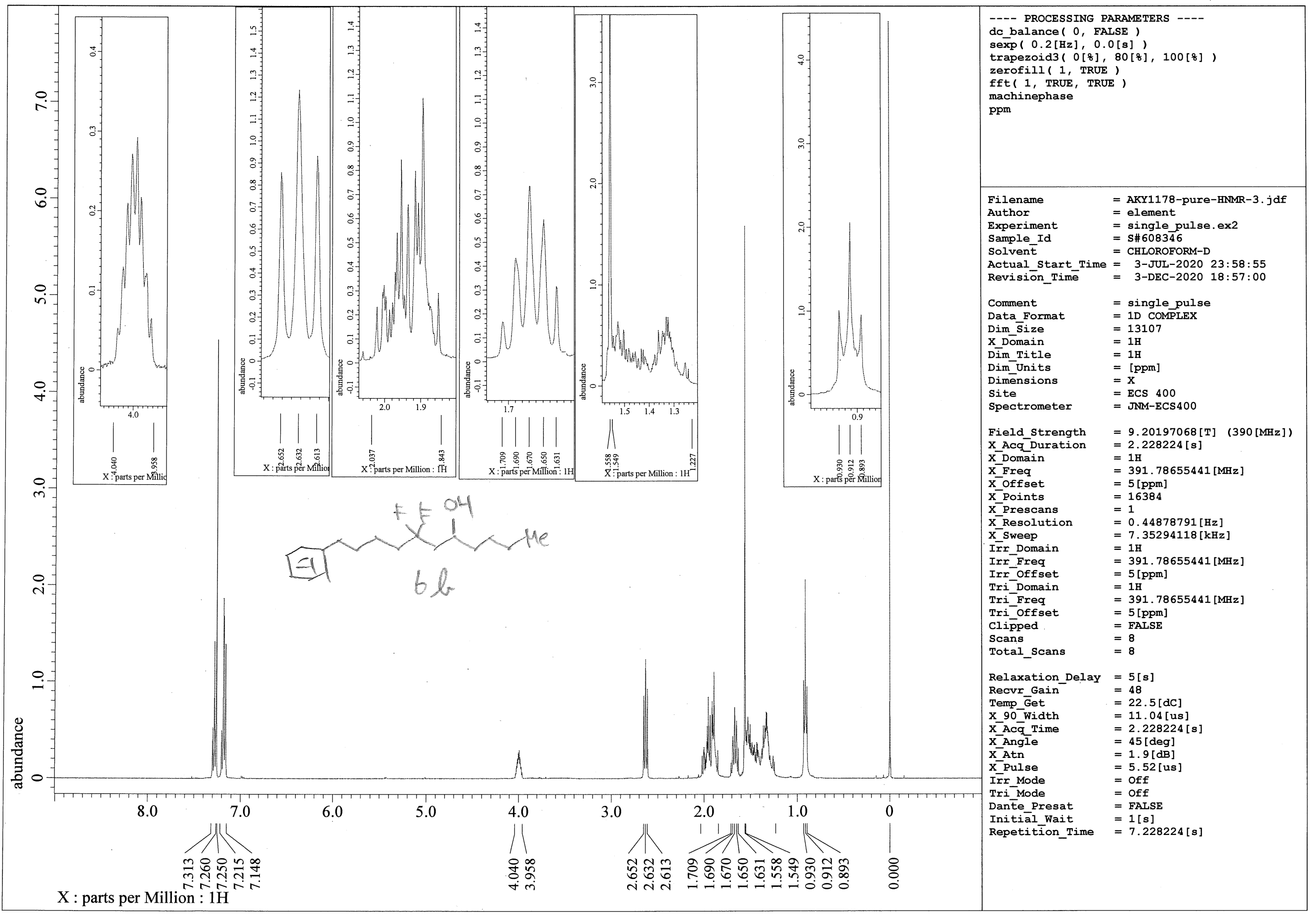




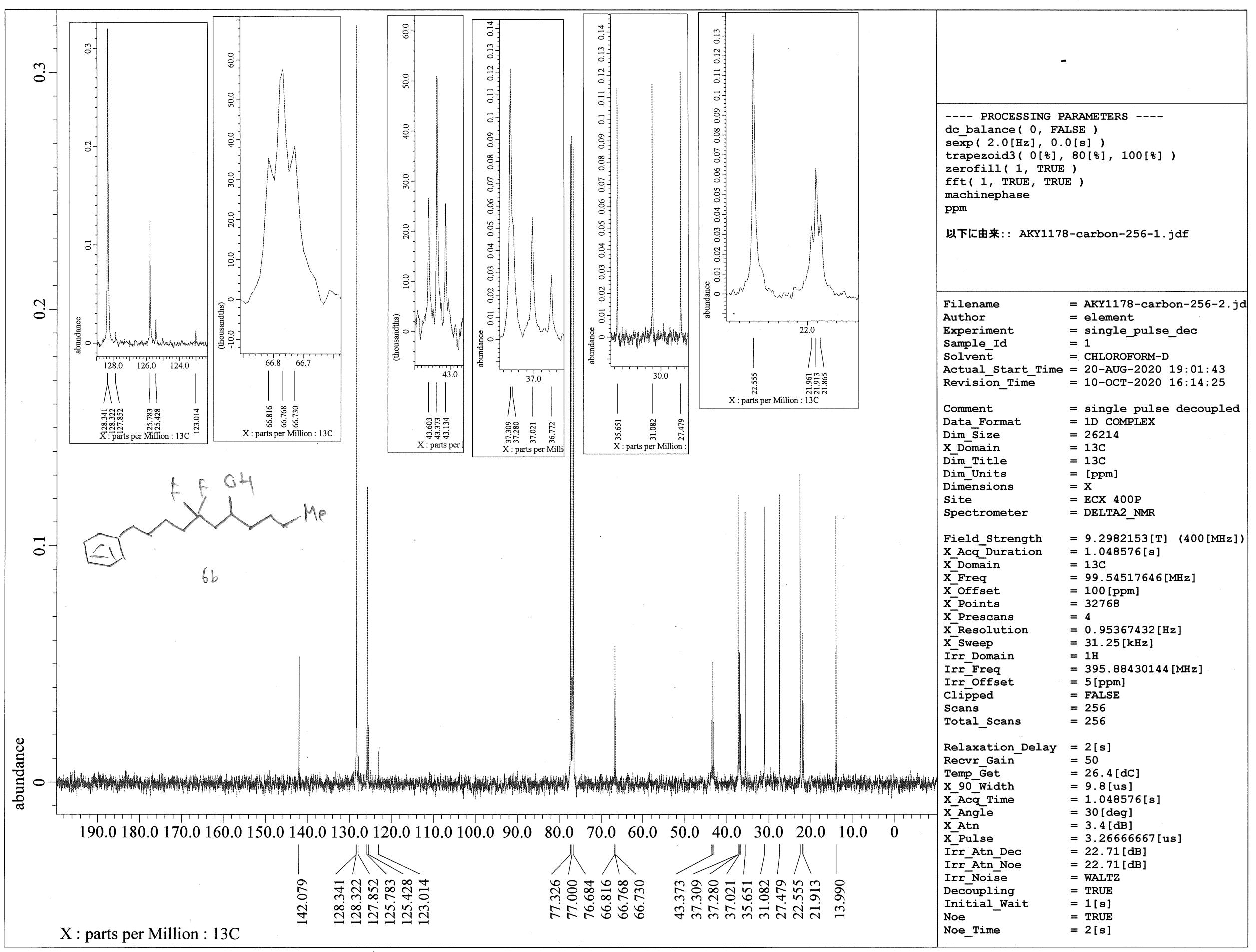




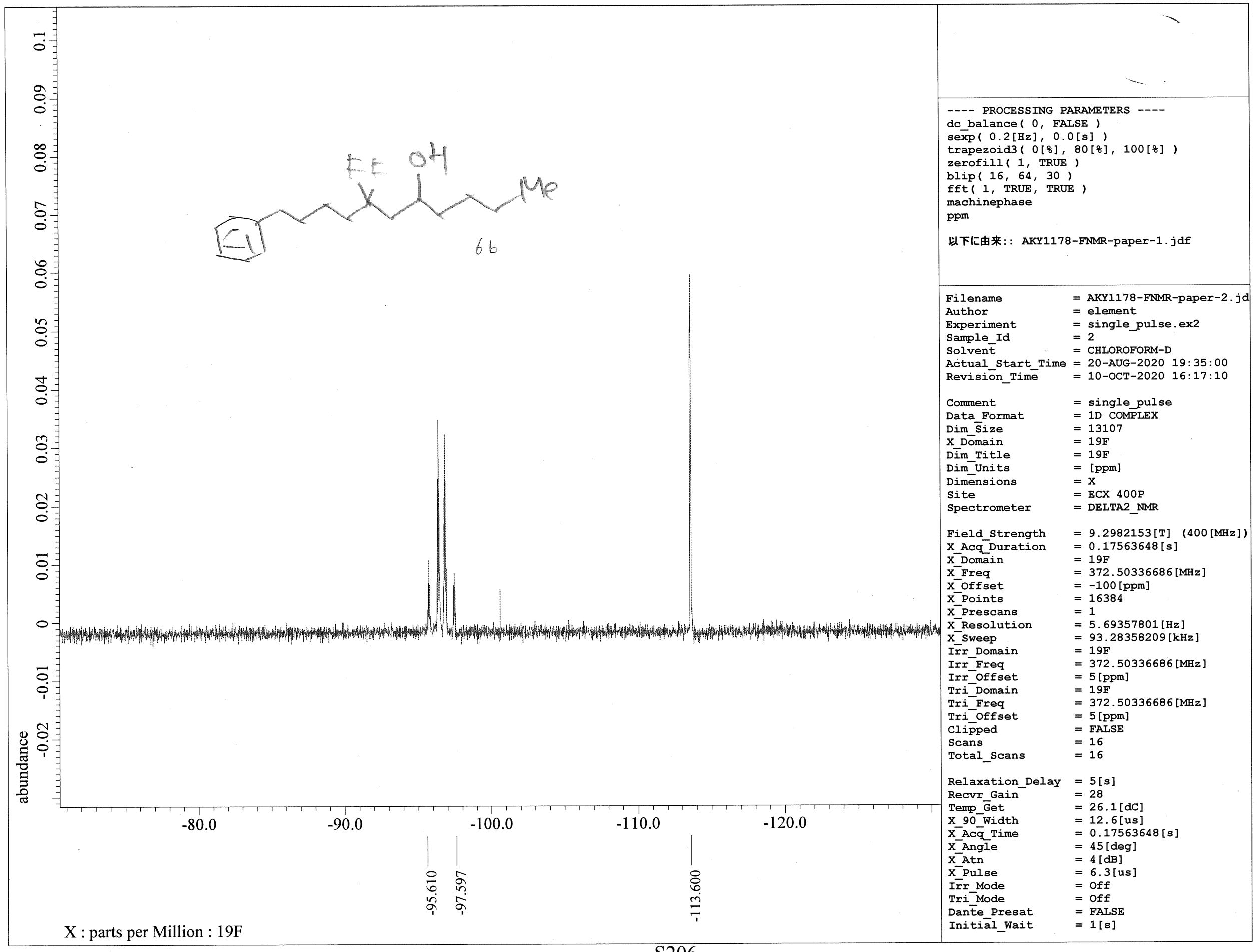




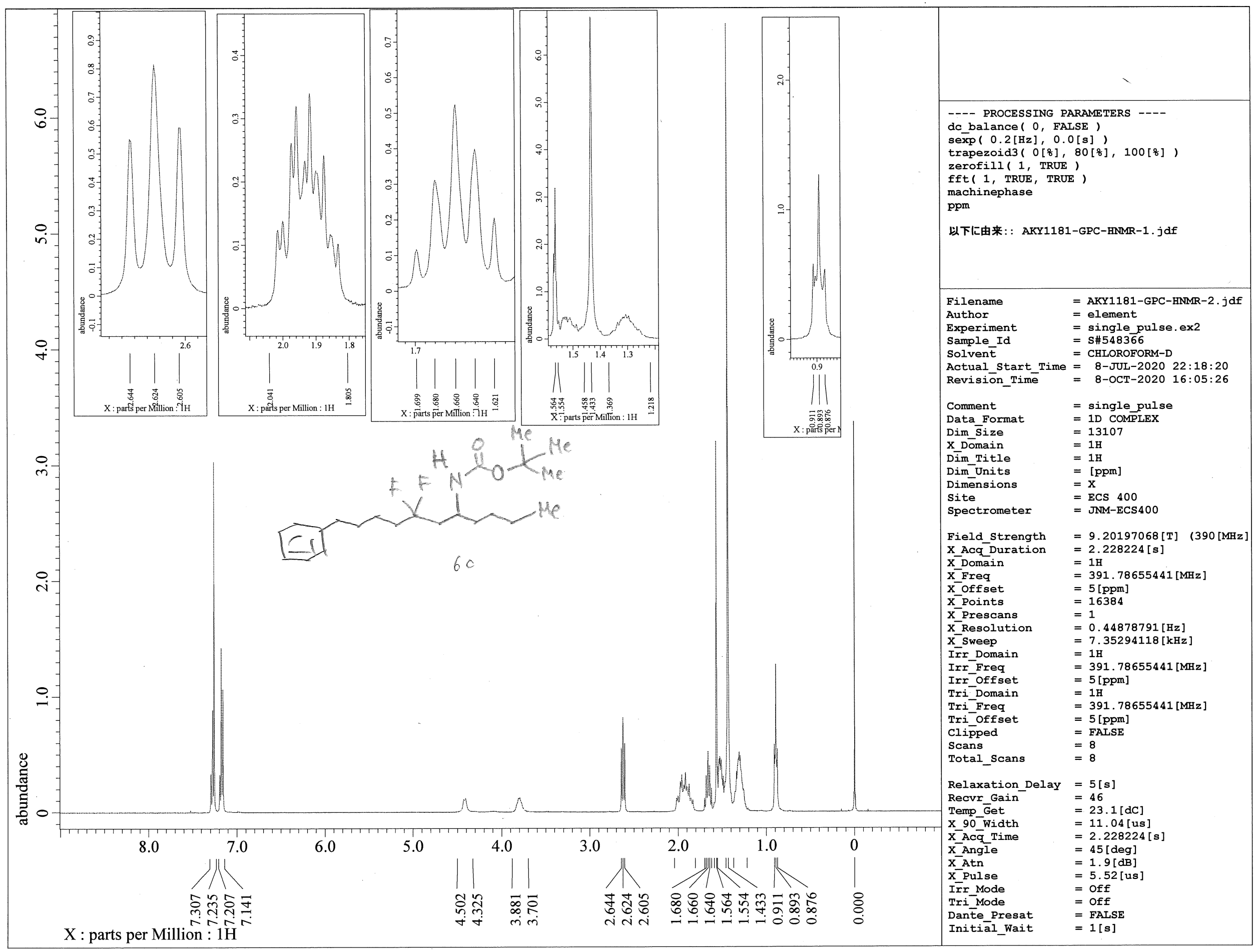




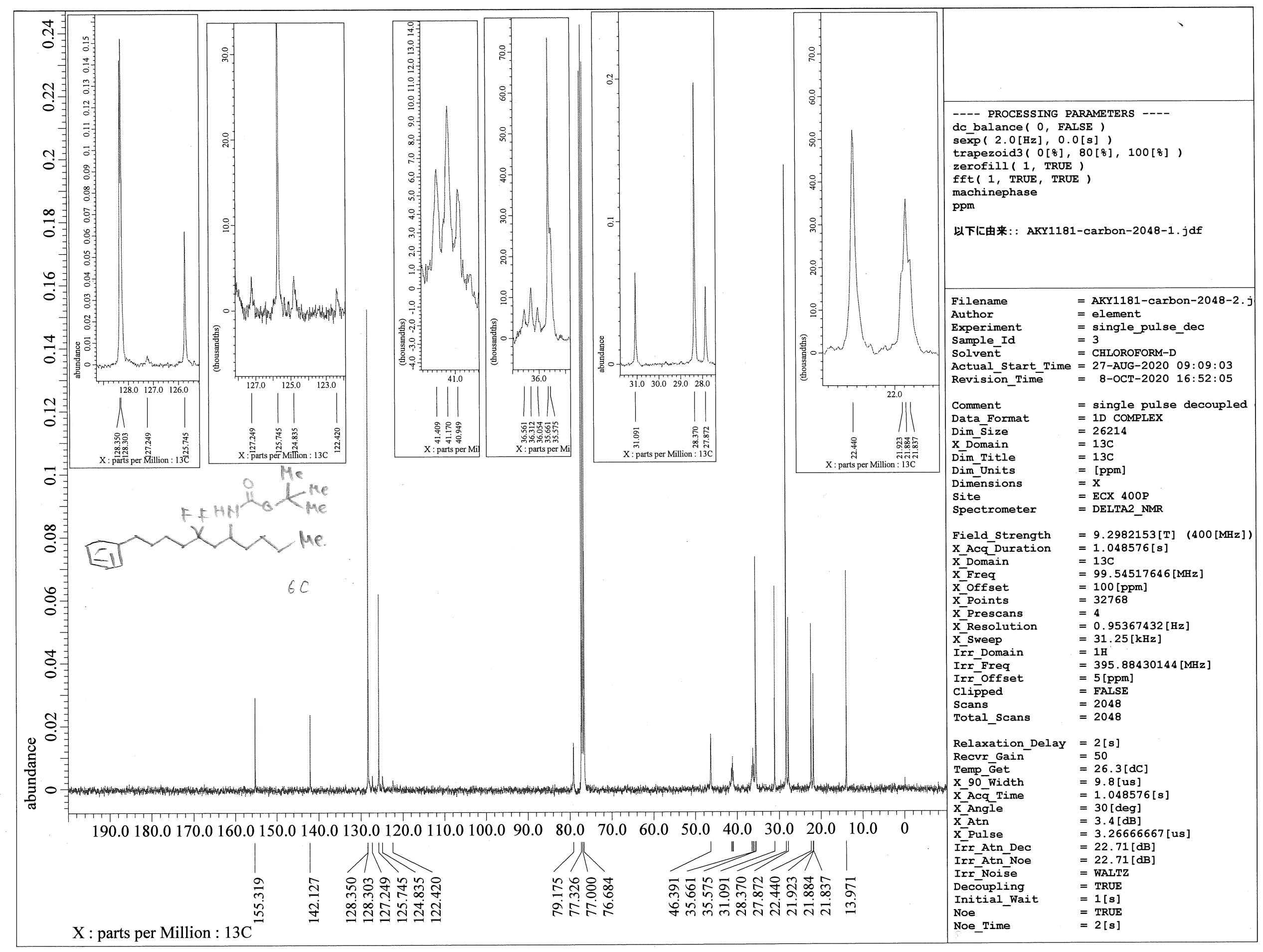




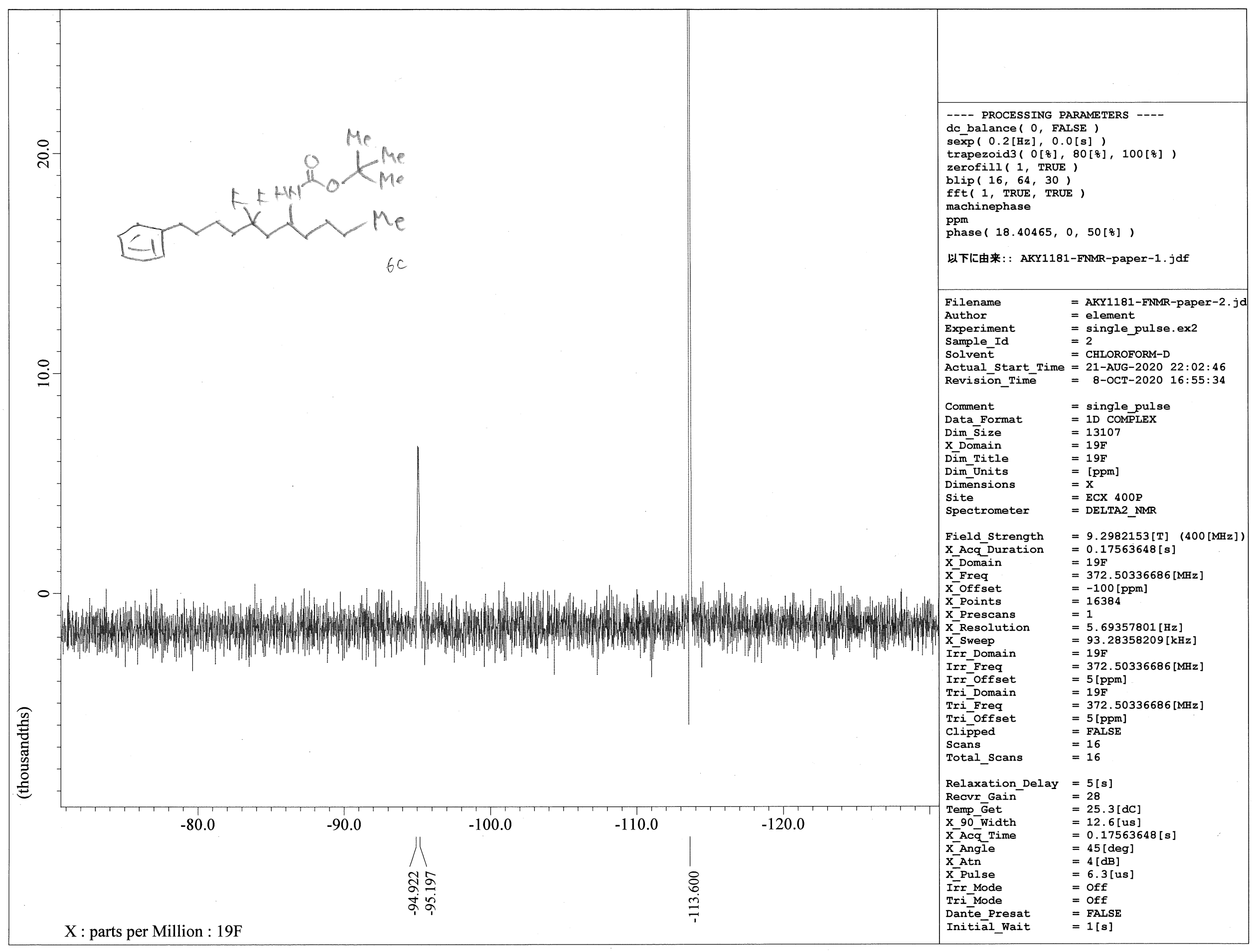




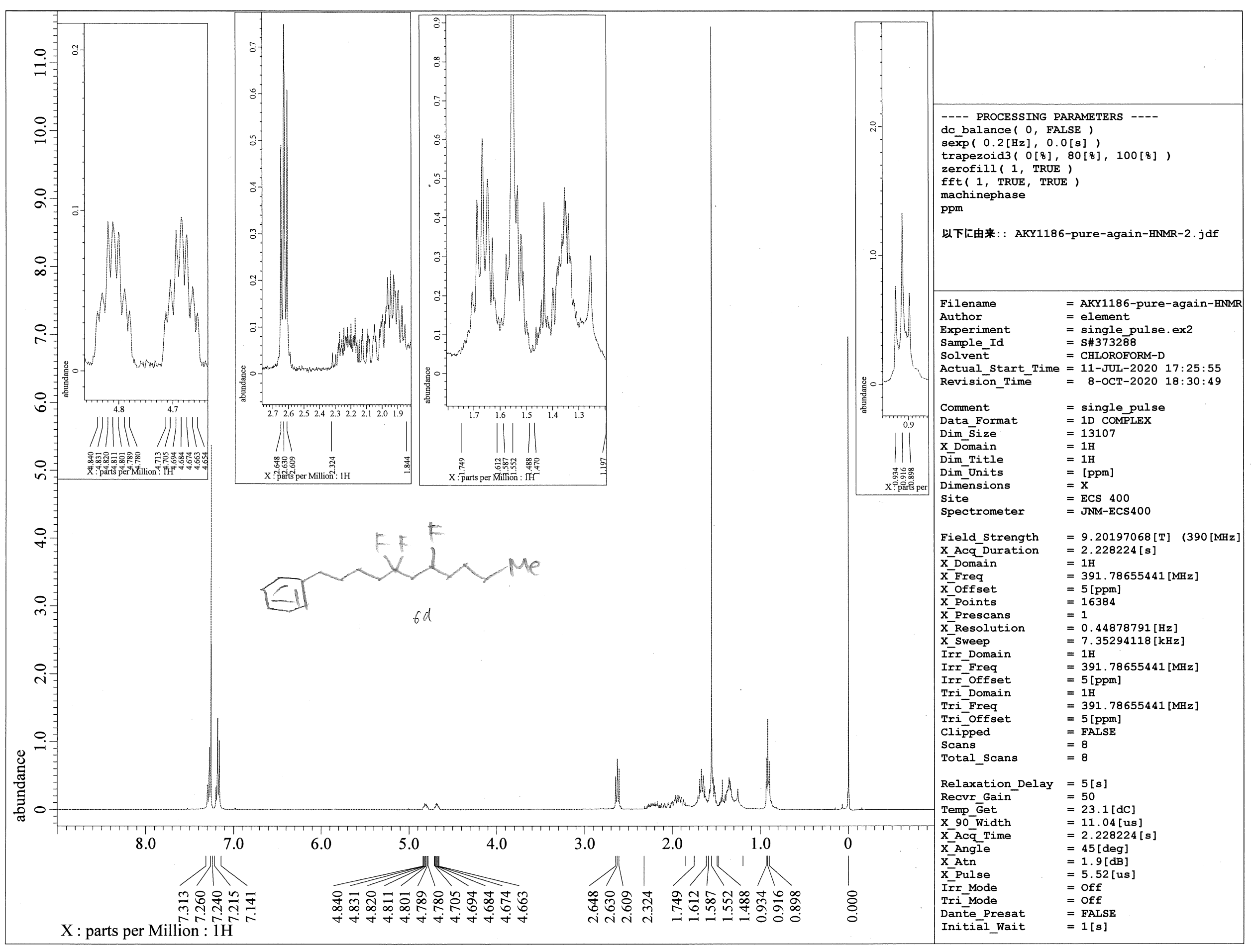




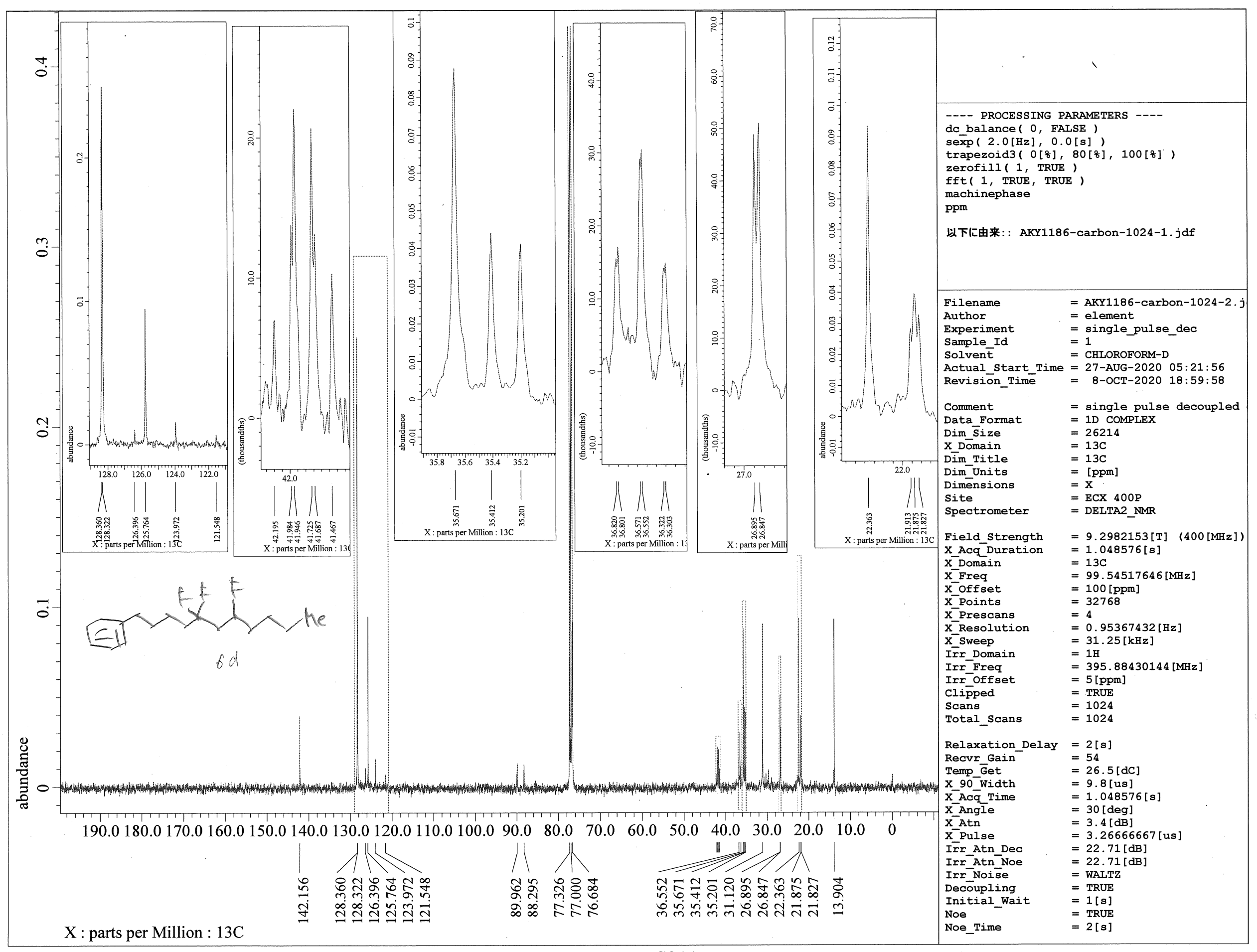




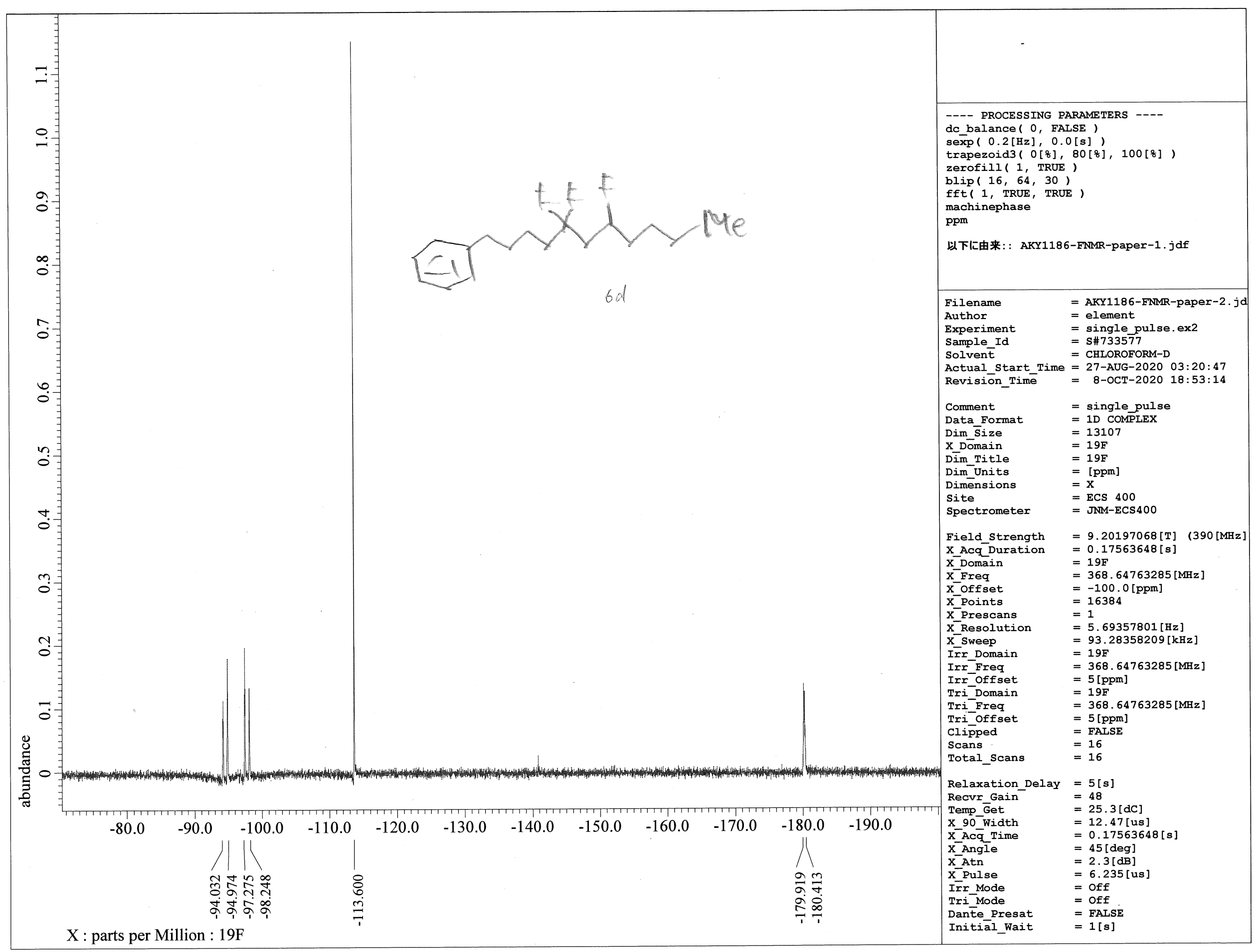

S212 


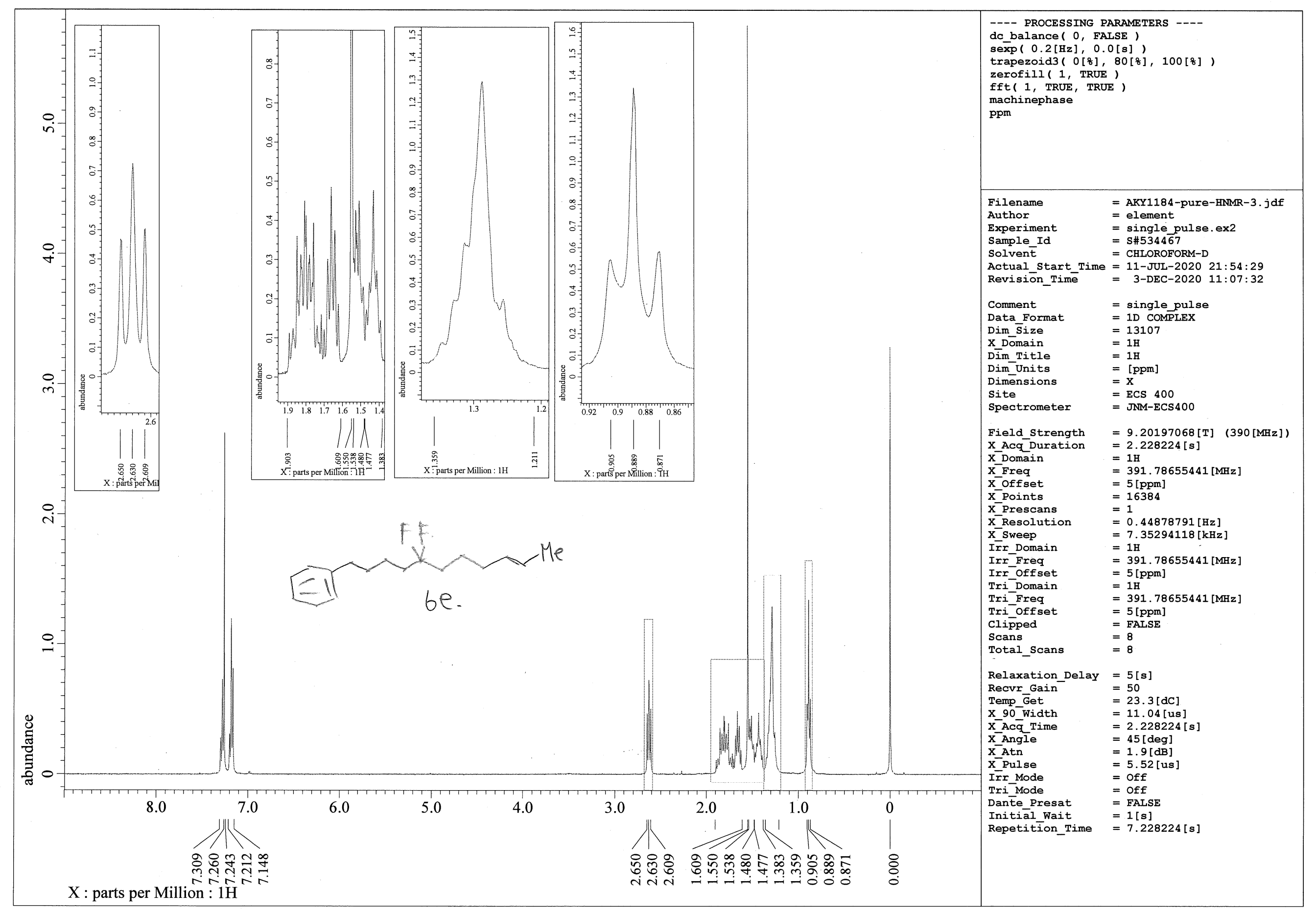




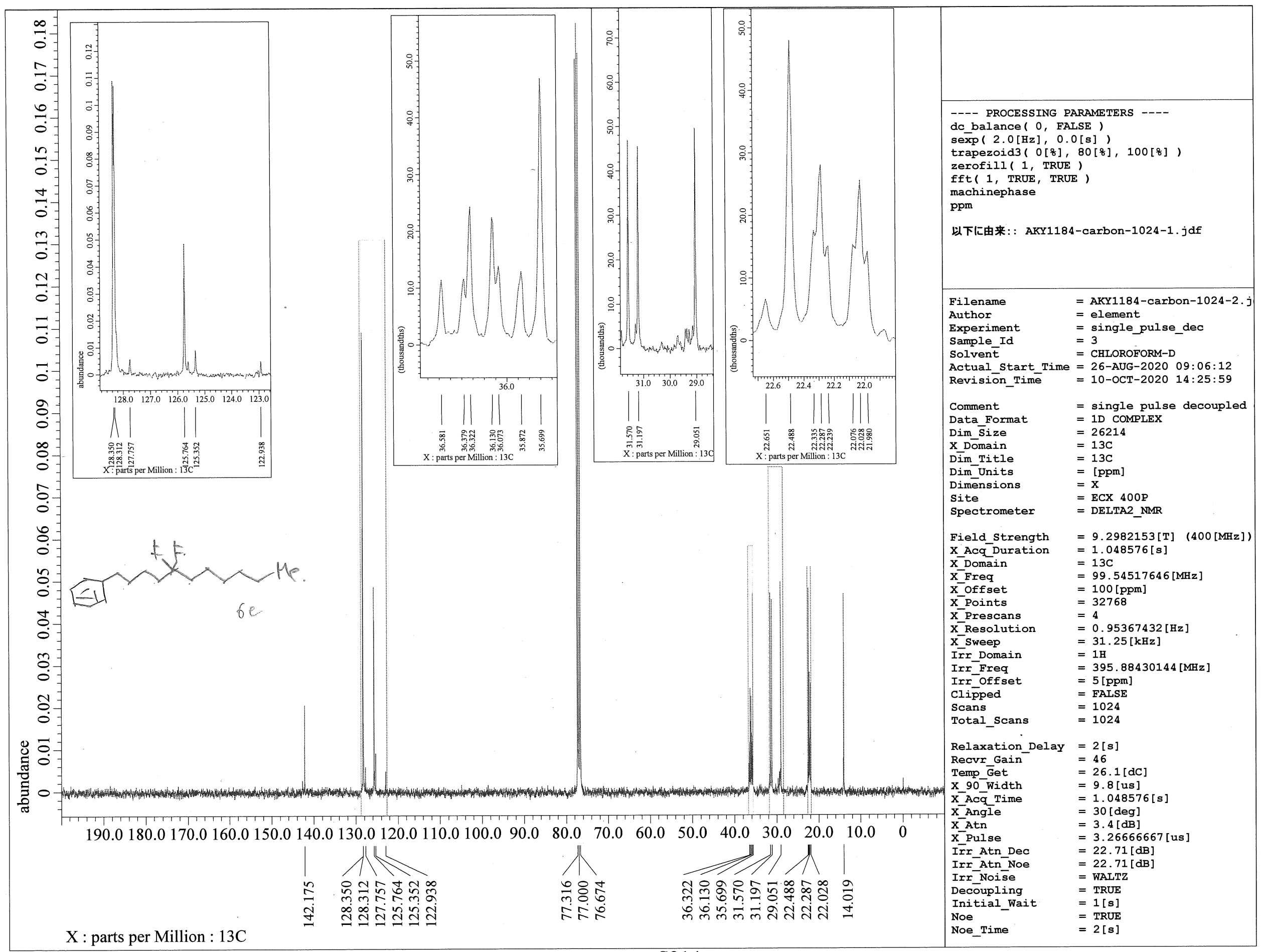




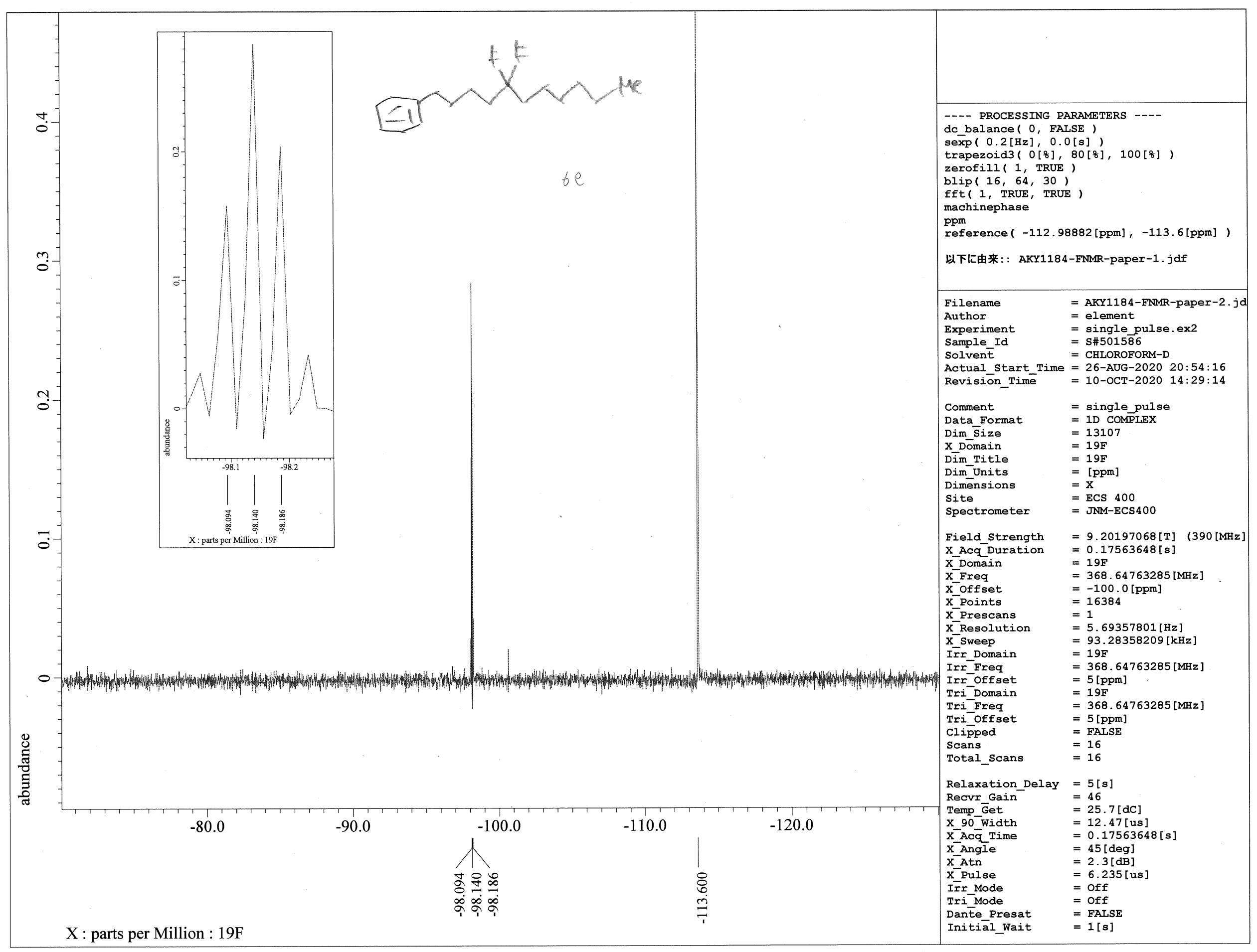




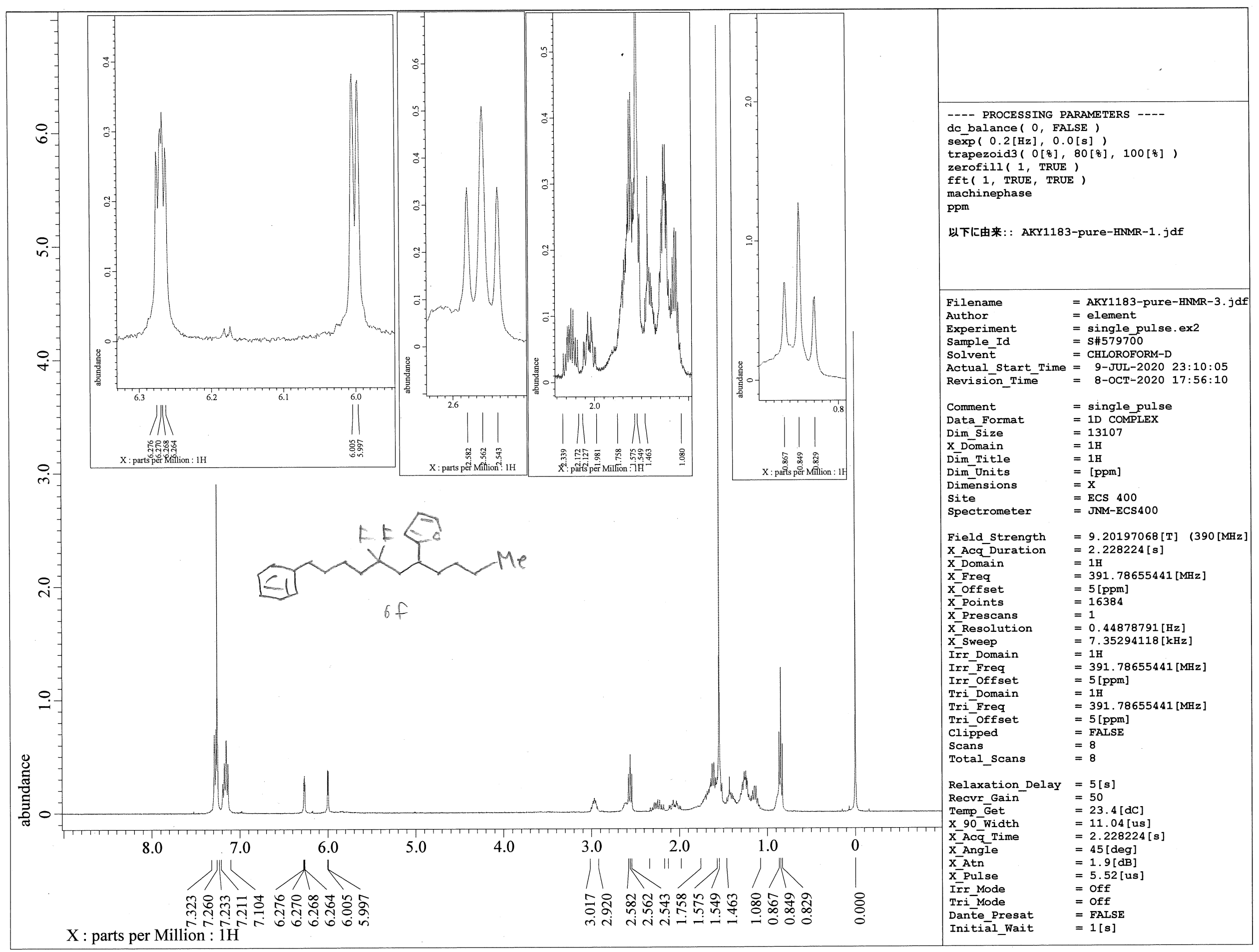

S216 


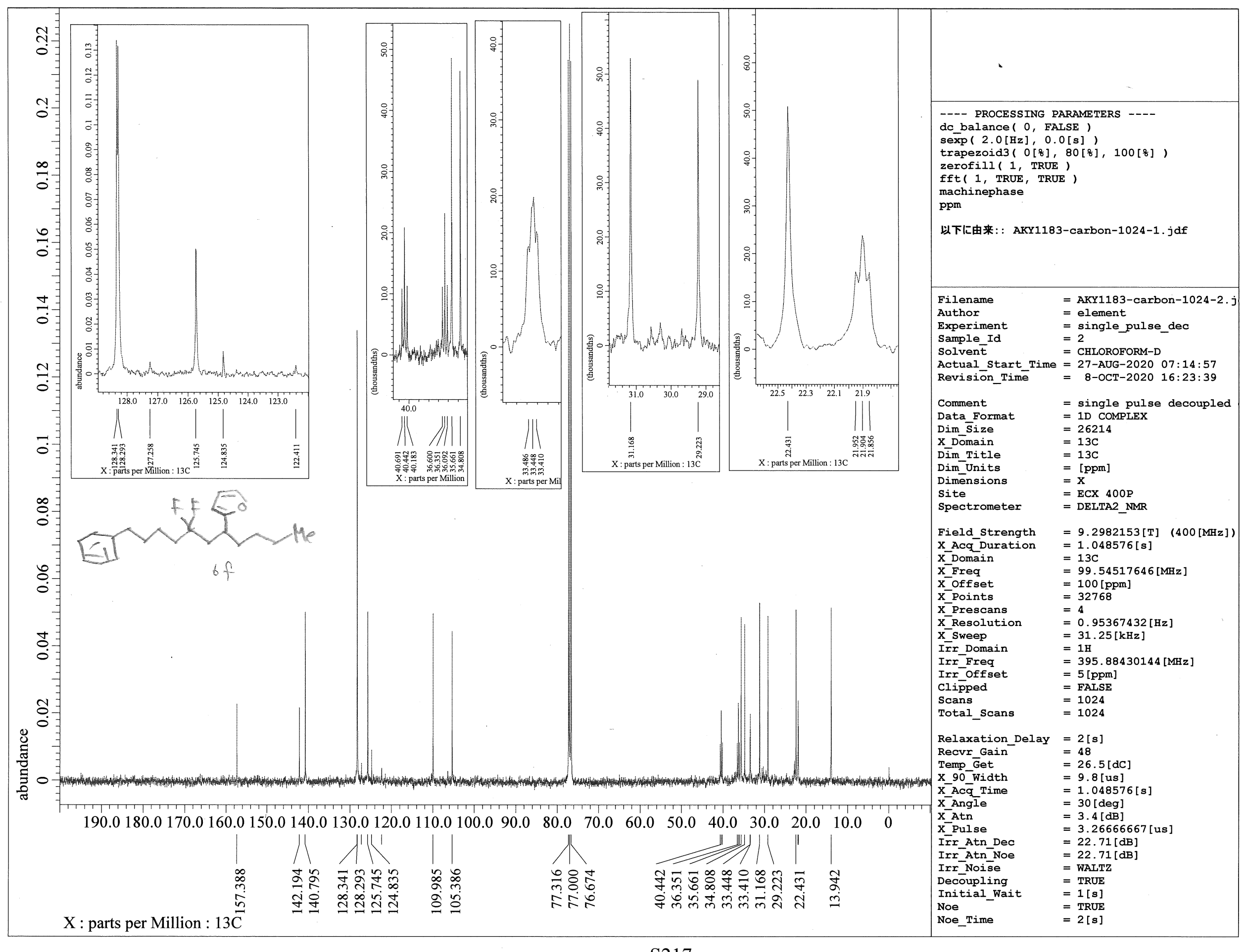

S217 


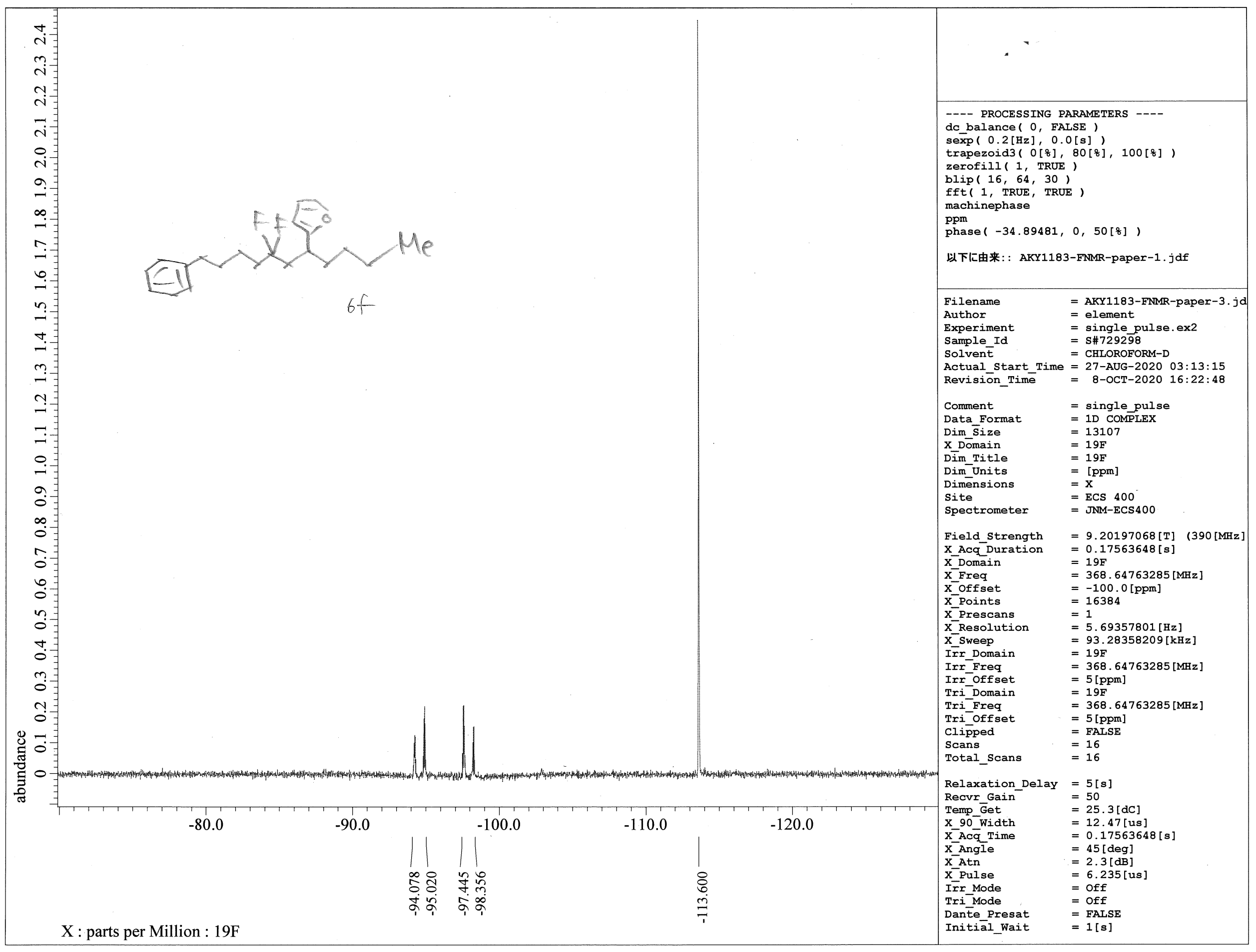




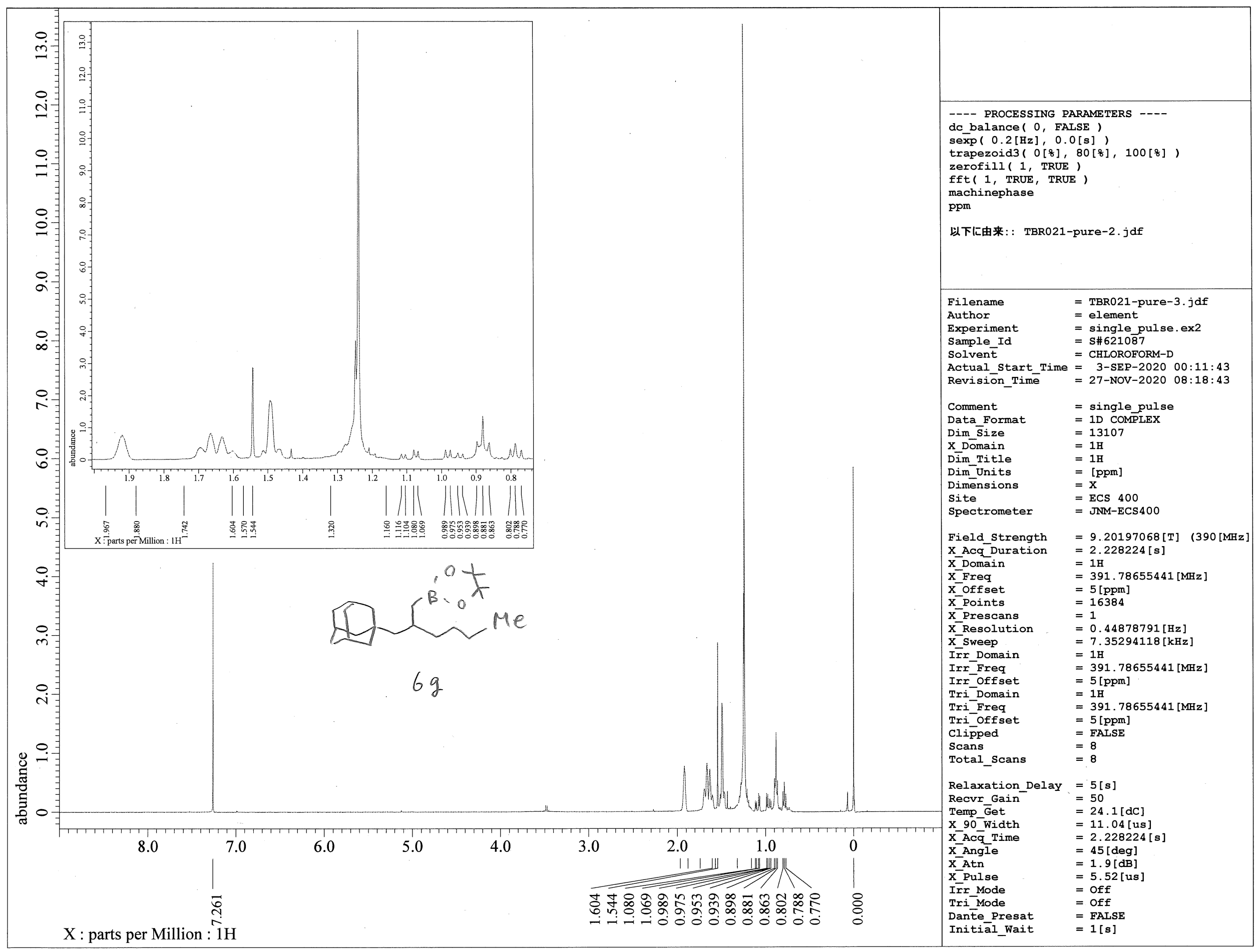

S219 


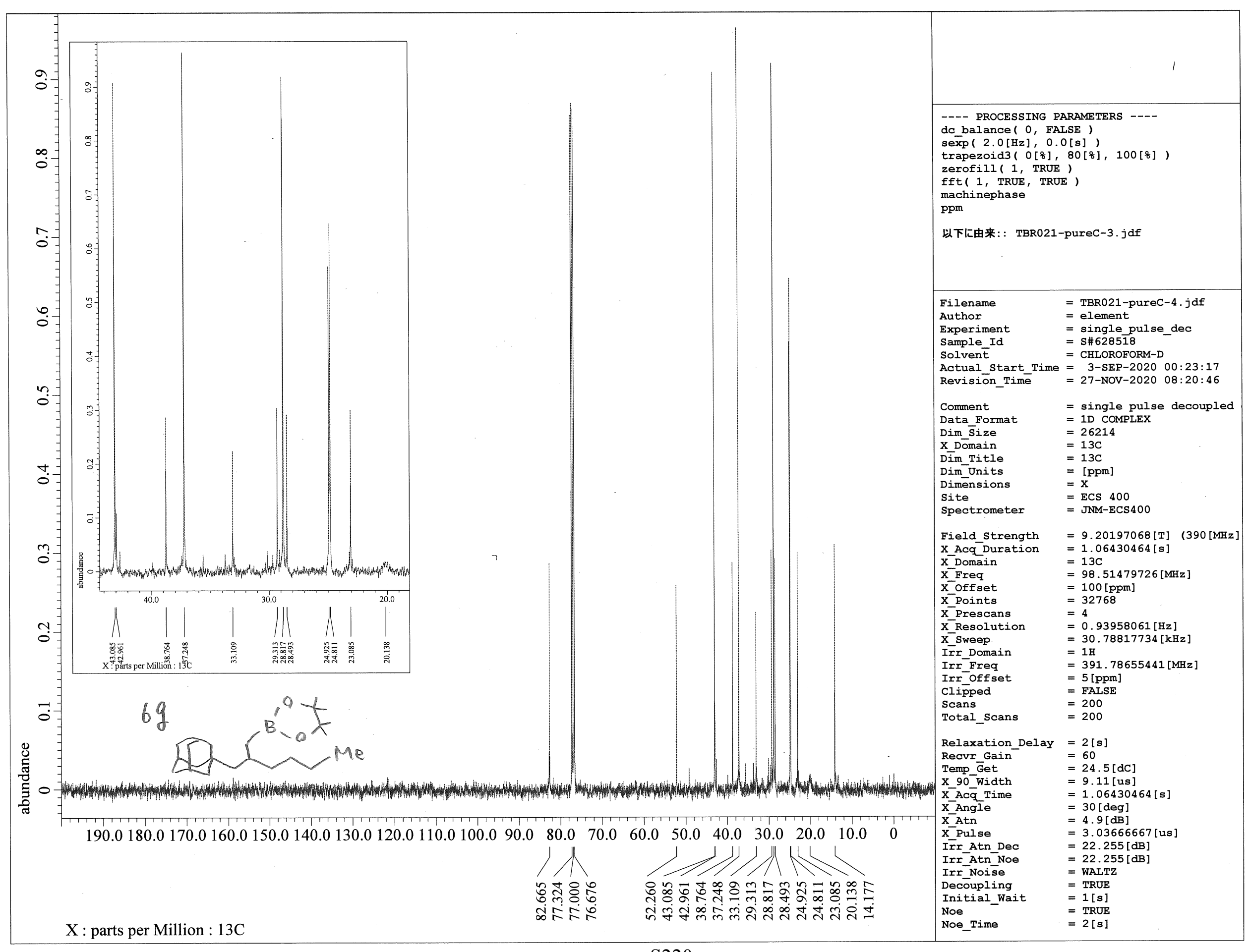




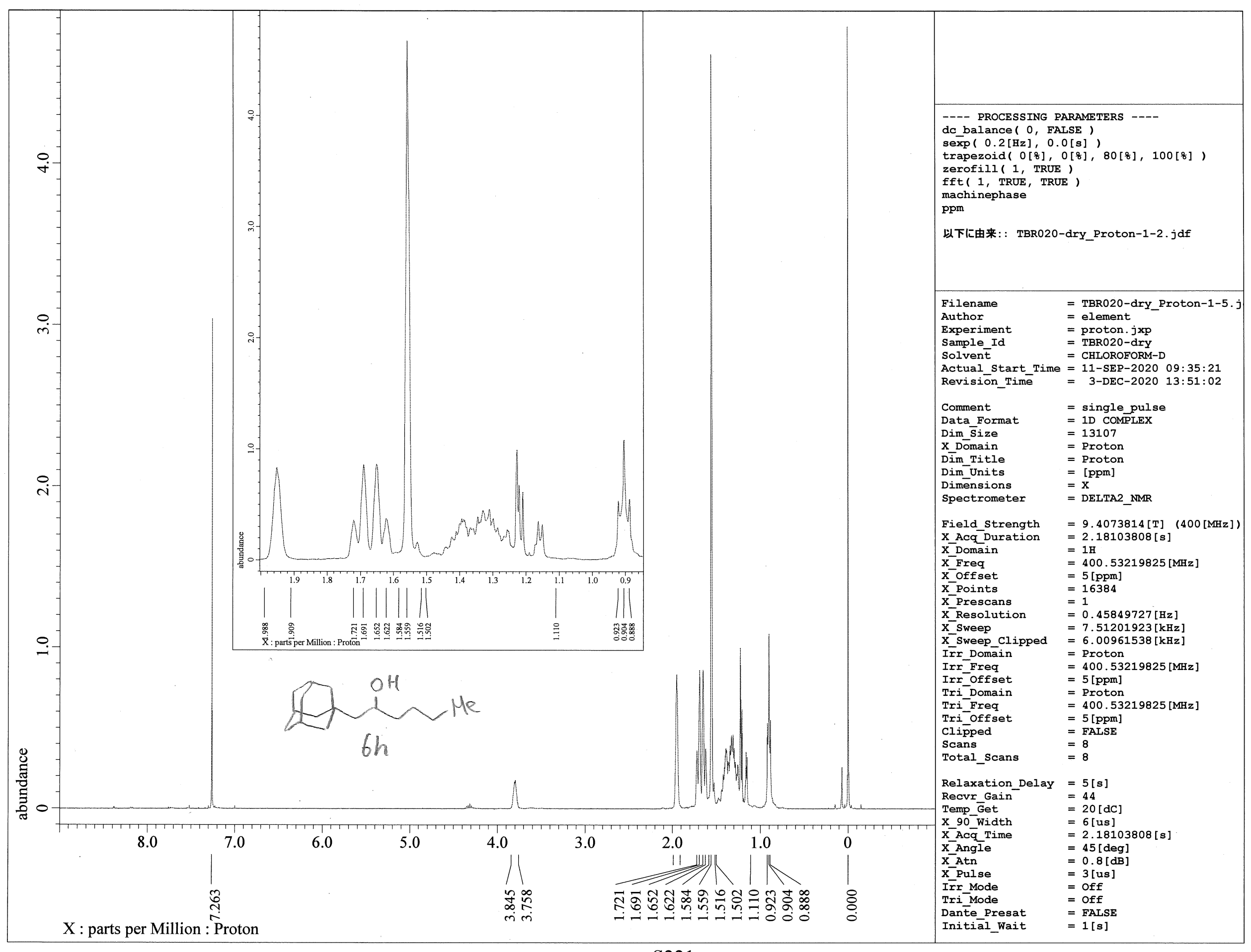

S221 


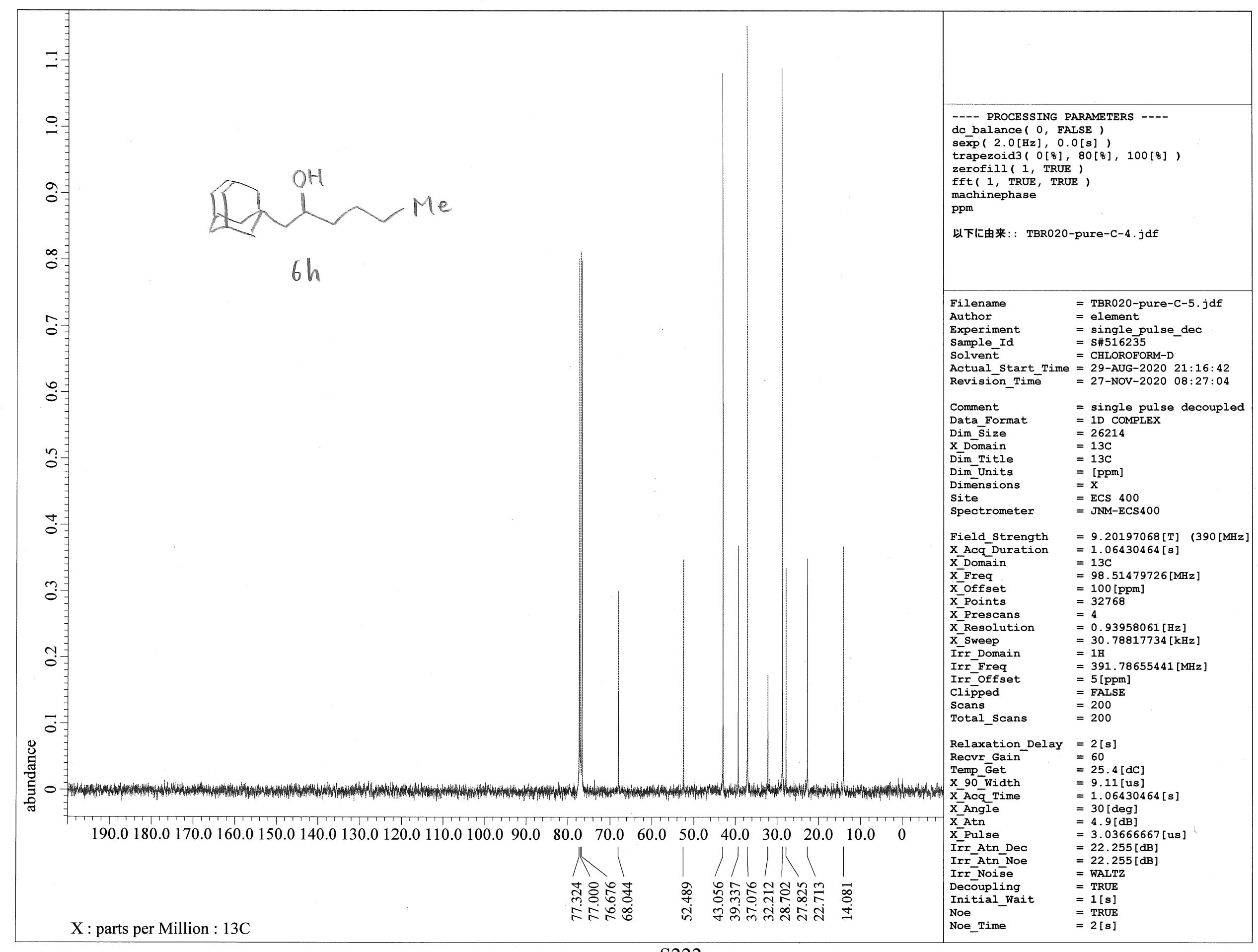




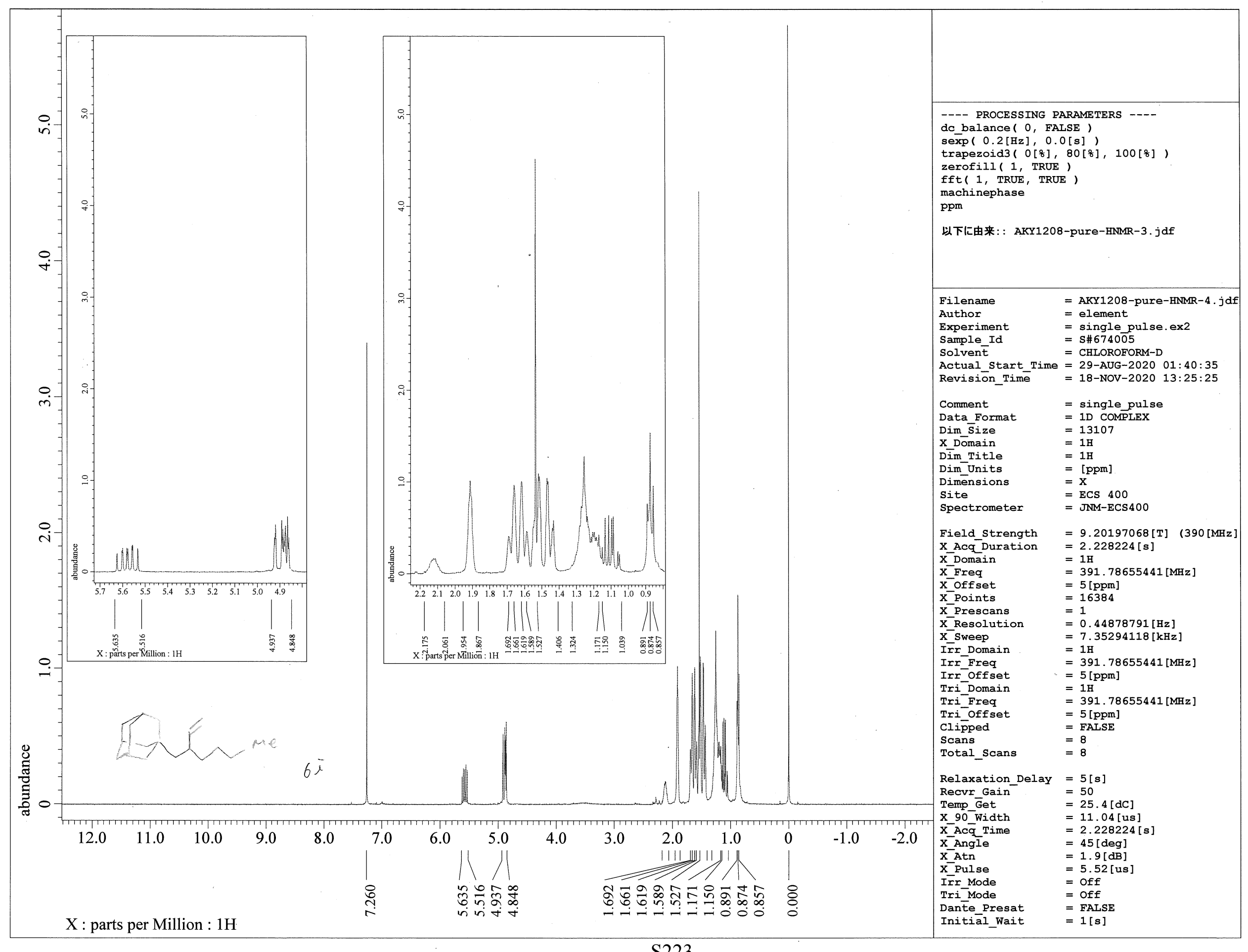




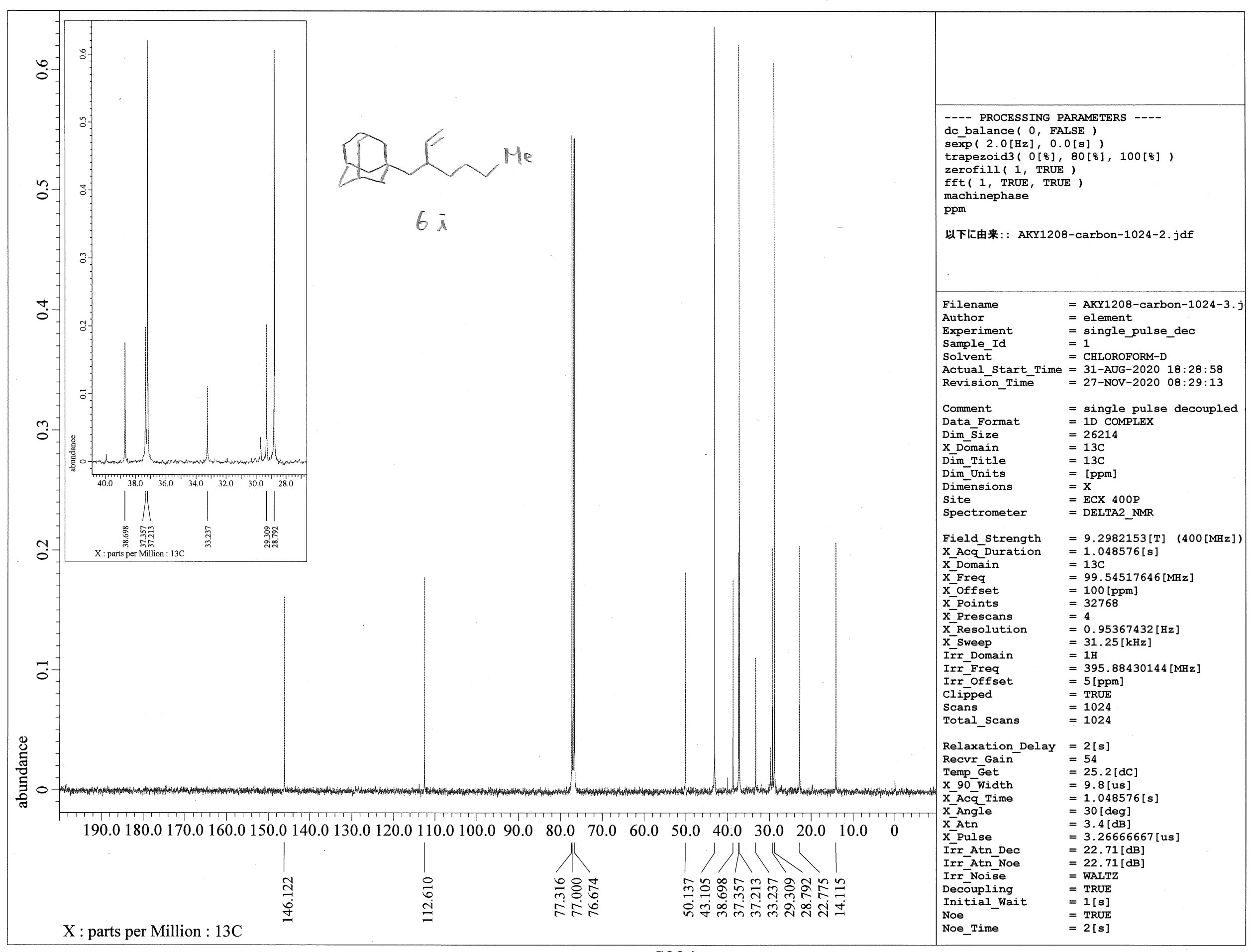




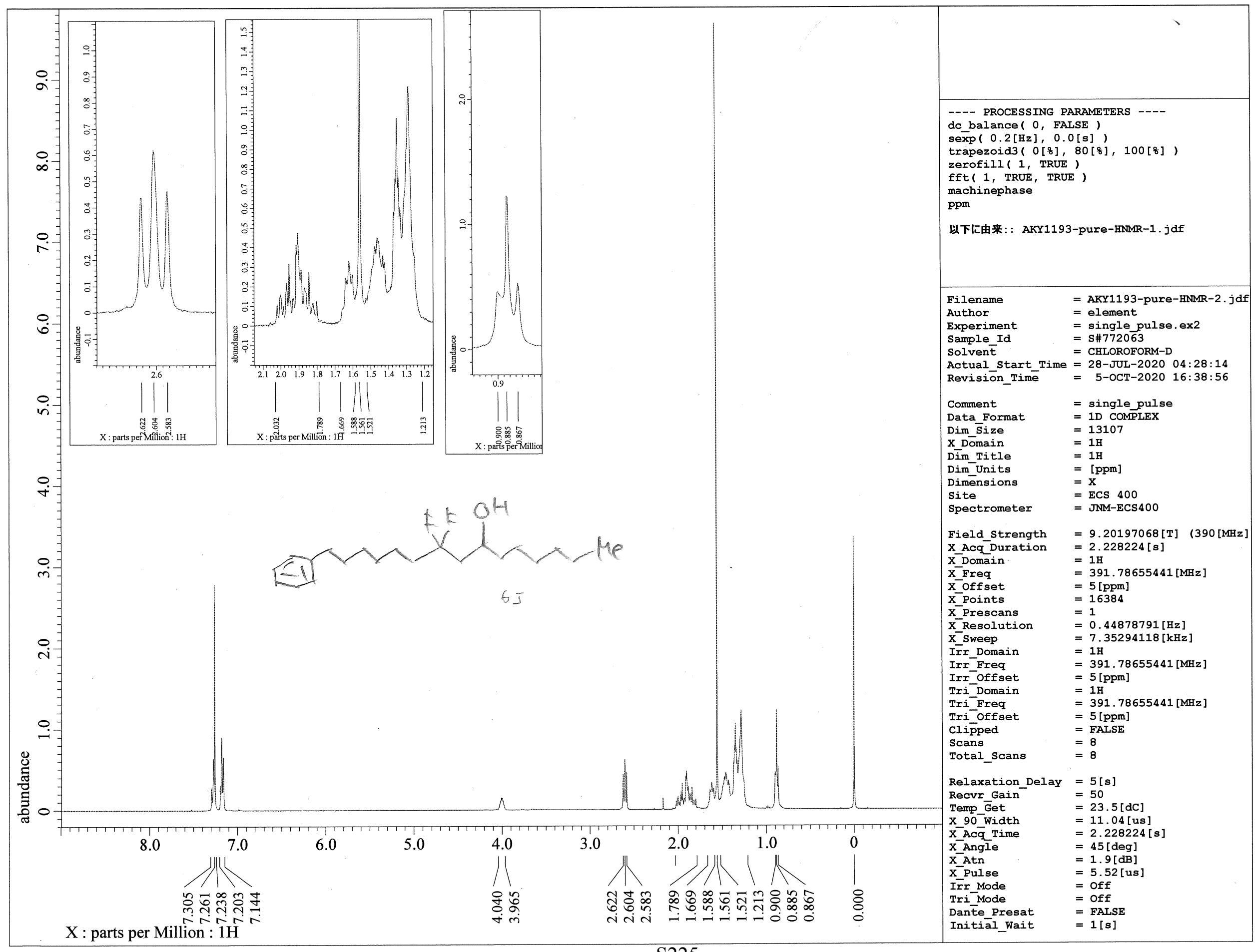




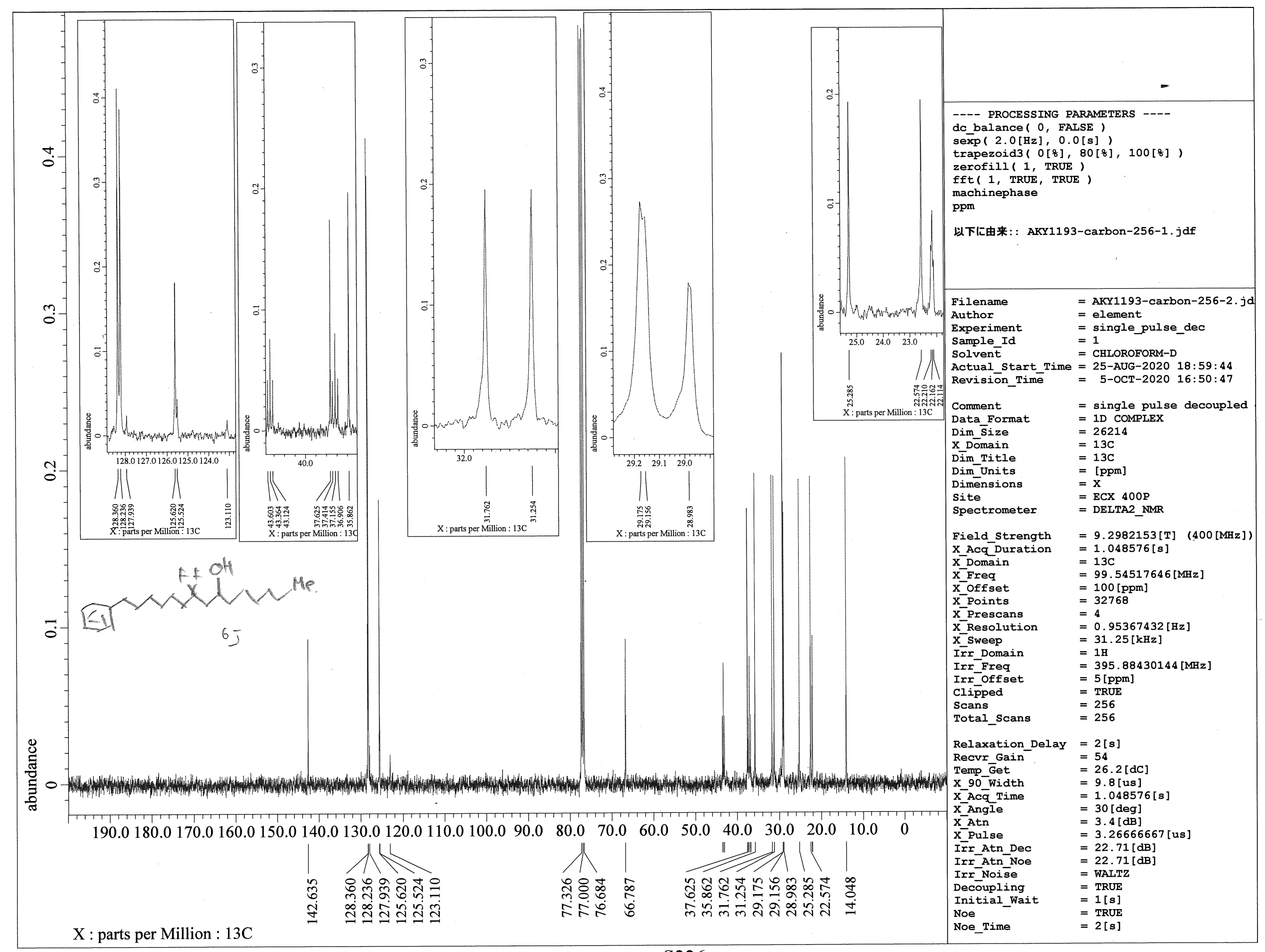




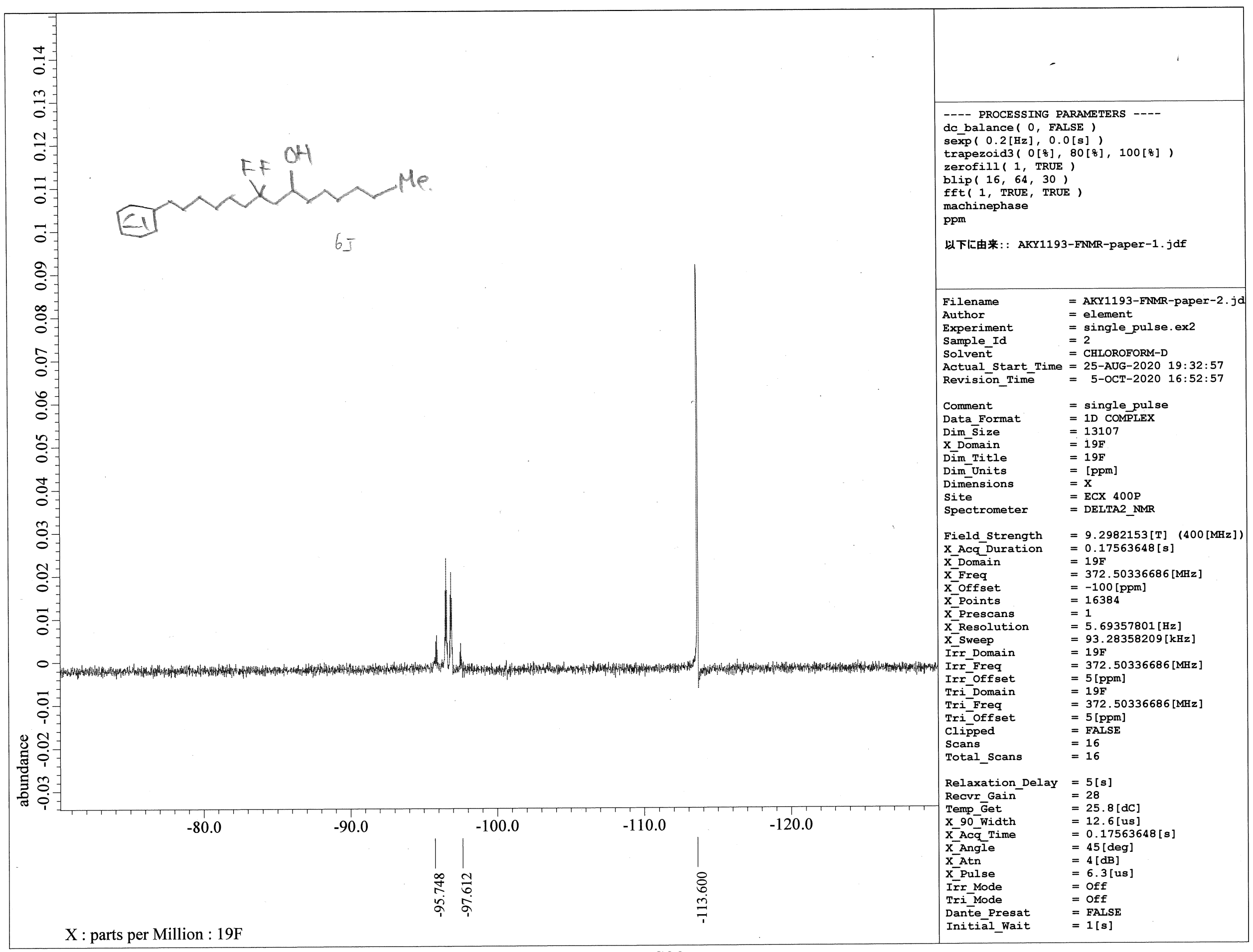




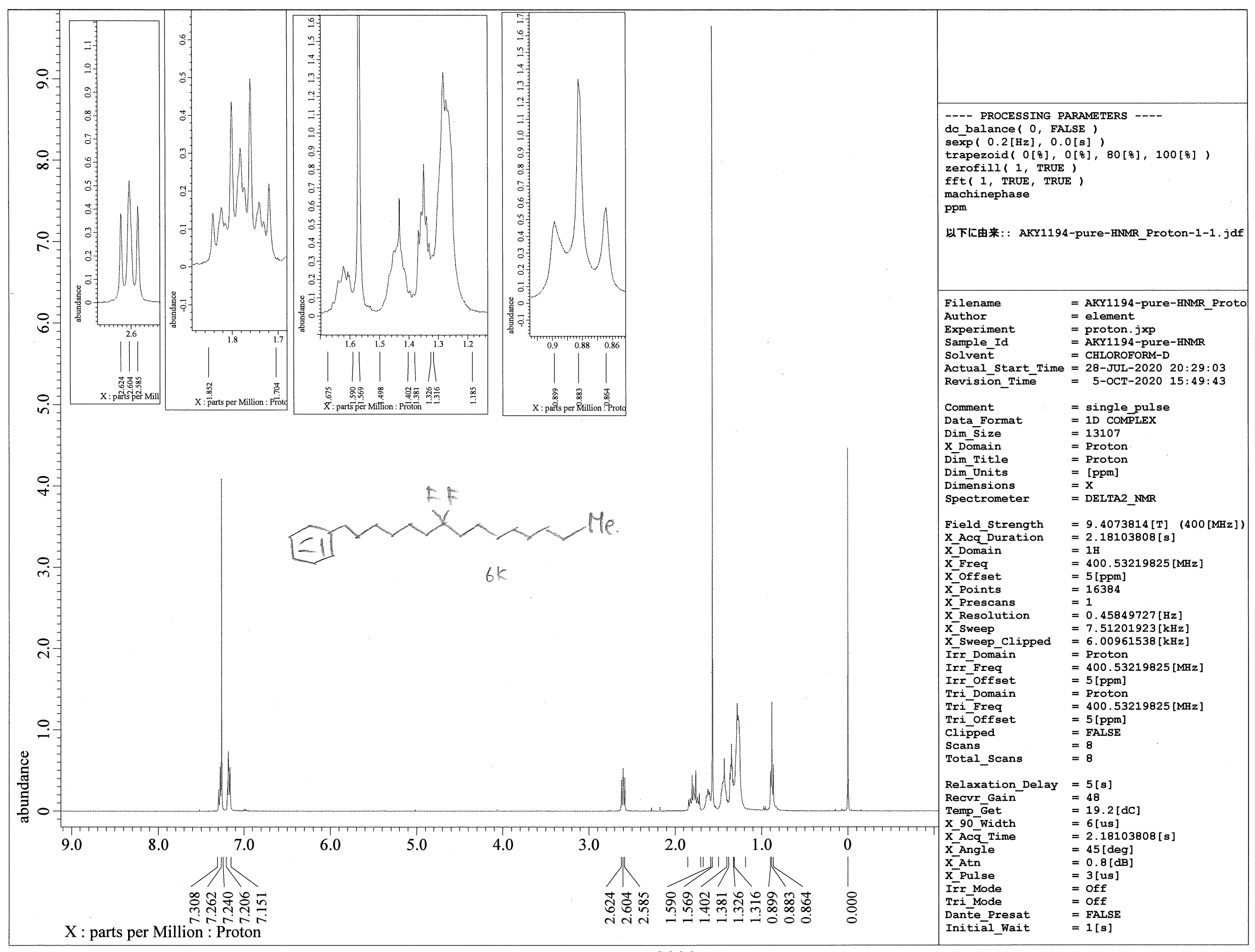

S228 


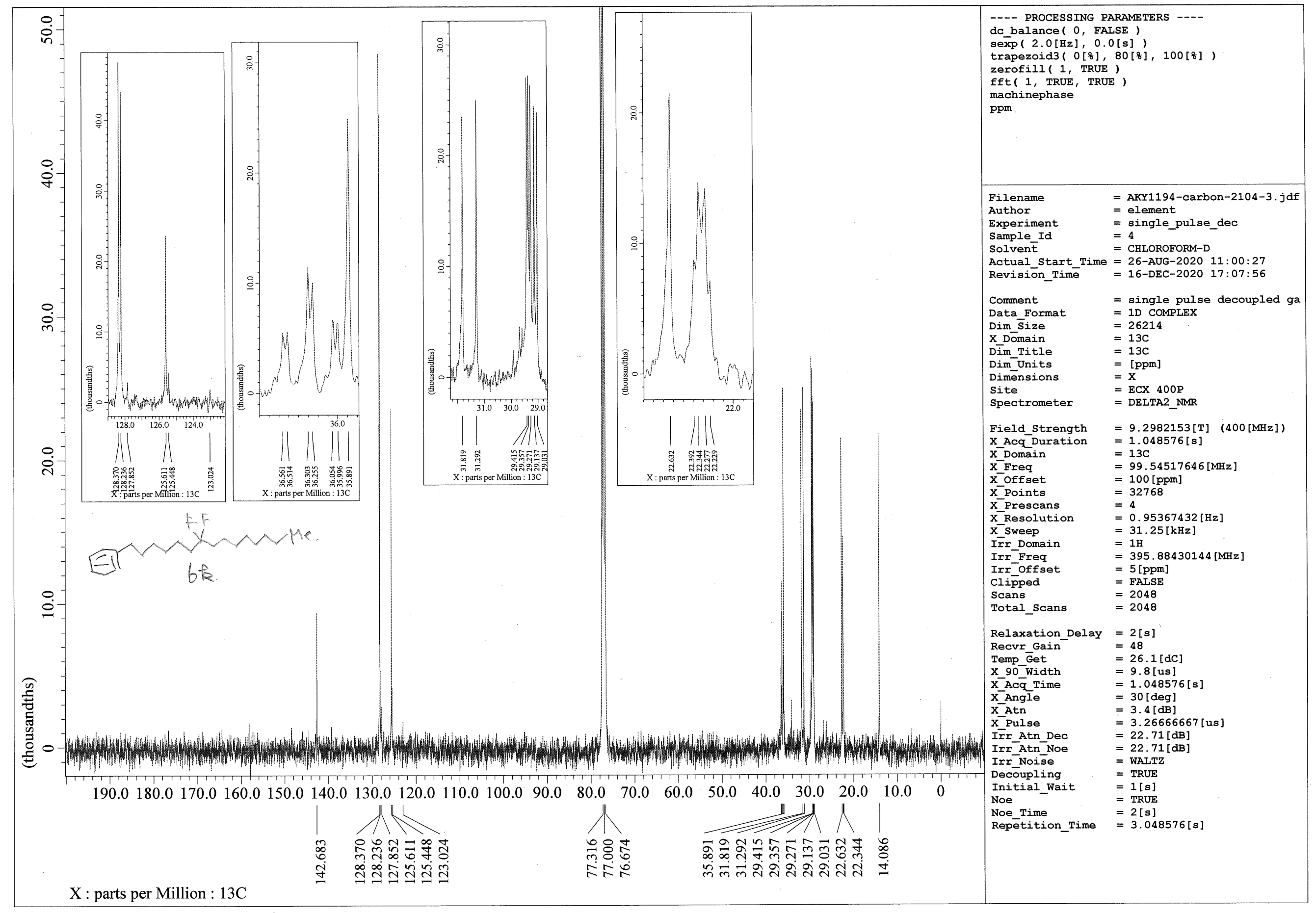




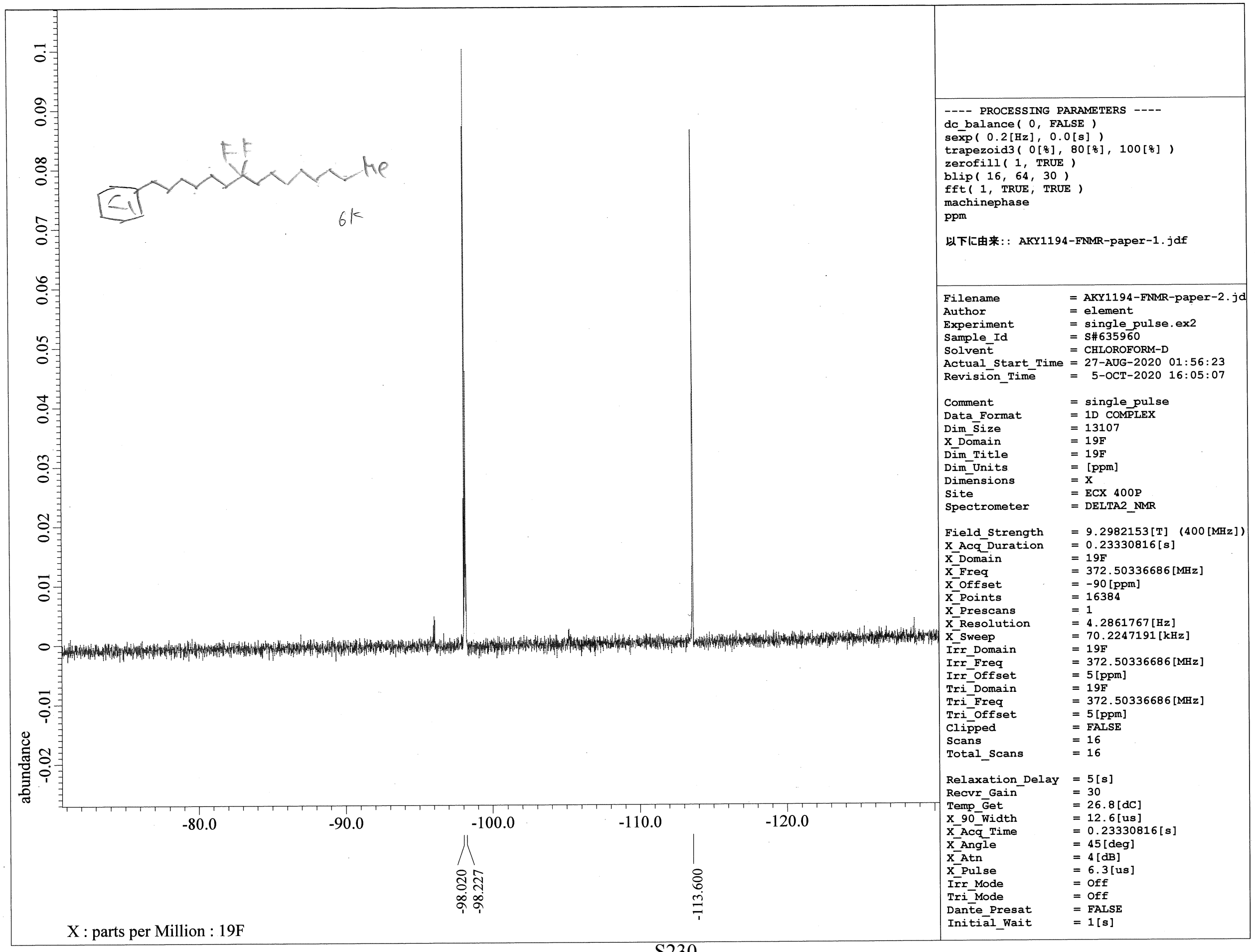

$\mathrm{X}$ : parts per Million : 19F 


\section{Chromaster System Manager Report}

Analyzed Date and Time: 2021/02/10

Reported Date and Time: 2021/02/10

$$
13: 11
$$

Processed Date and Time: 2021/02/10

$$
15: 04
$$

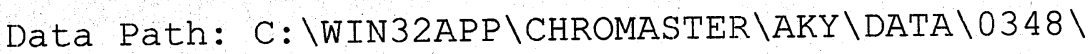

Processing Method: IB_OZW

System (acquisition): Sys 1

Series: 0348

Application (data): AKY

Sample Name: TBR146-chiral-OH-5\%

Injection from this vial: 1 of 1

Vial Number: 10

Vial Type: UNK

Sample Description:

Volume: $10.0 \mathrm{ul}$

Chrom Type: Fixed WL Chromatogram, 207 nm

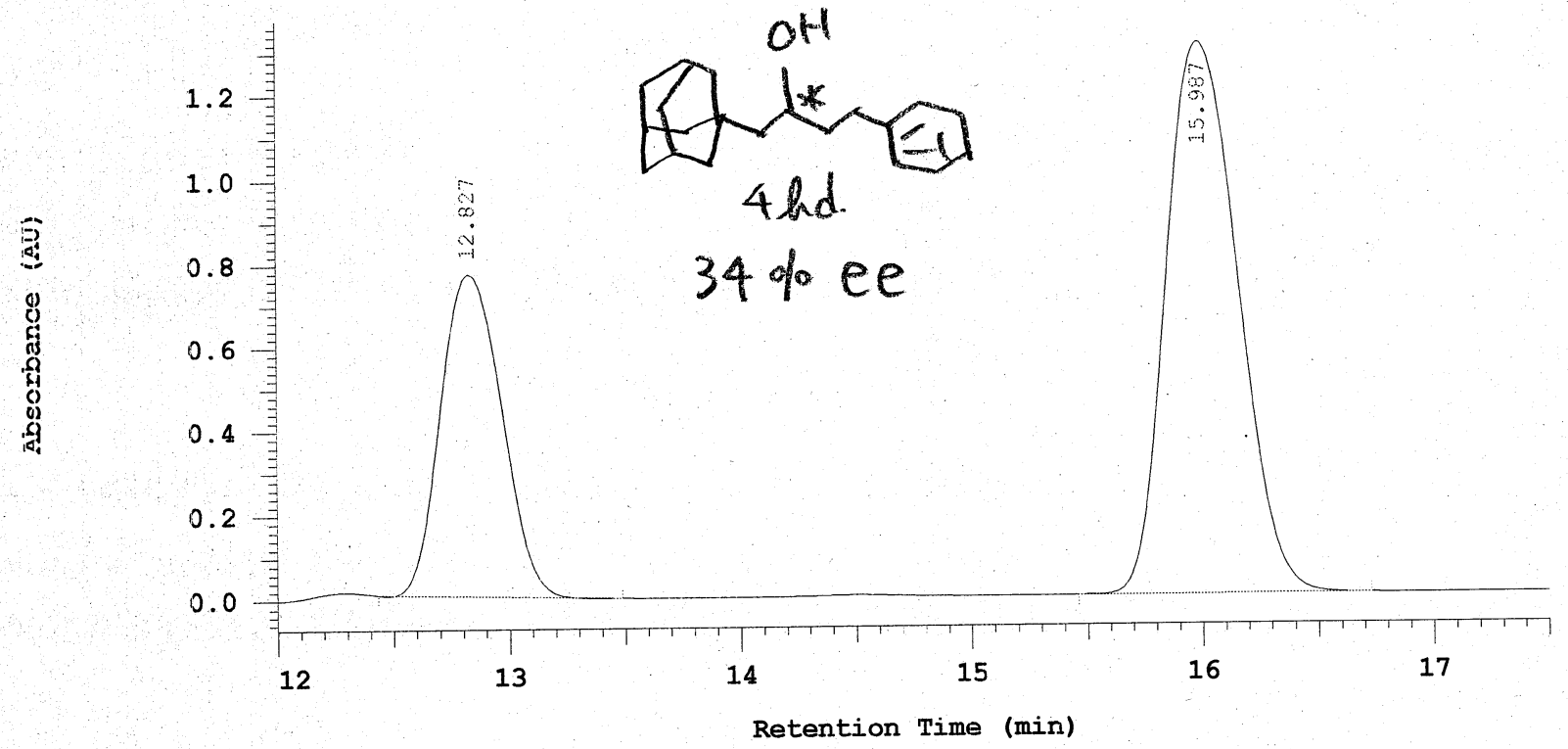

Erocessing Method: IB_OZW

Method Developer:

Pump 1: 5110

Pump 1 Solvent $A$ : hexane

Pump 1 Solvent B: 2-propanol

Pump 1 Solvent C:

Pump 1 Solvent D:

Method Description:

Chrom Type: Fixed WL Chromatogram, $207 \mathrm{~nm}$

Peak Quantitation: AREA

Calculation Method: AREA\%

\begin{tabular}{ccccc} 
No. & RT & Area & Conc 1 & BC \\
\hline 1 & 12.827 & 7011905 & 33.113 & MC \\
2 & 15.987 & 14163622 & 66.887 & MC \\
\hline & & 21175527 & 100.000 & \\
\hline
\end{tabular}

Peak rejection level: 0 


\section{Chromaster System Manager Report} Analyzed Date and Time: $\begin{aligned} & 2021 / 02 / 10 \\ & 12: 15\end{aligned}$

Reported Date and Time: 2021/02/10

$15: 05: 48$

Processed Date and Time: 2021/02/10

Data Path: C: \WIN32APP \CHROMASTER \AKY \DATA \0347\

Processing Method: IB_OZW

System (acquisition): Sys 1

Application (data): AKY

Sample Name: OYAXXX-rac-OH-5\%

Injection from this vial: 1 of 1

Series: 0347

Vial Number: 9

Vial Type: UNK

Sample Description:

Chrom Type: Fixed WL Chromatogram, 207 nm

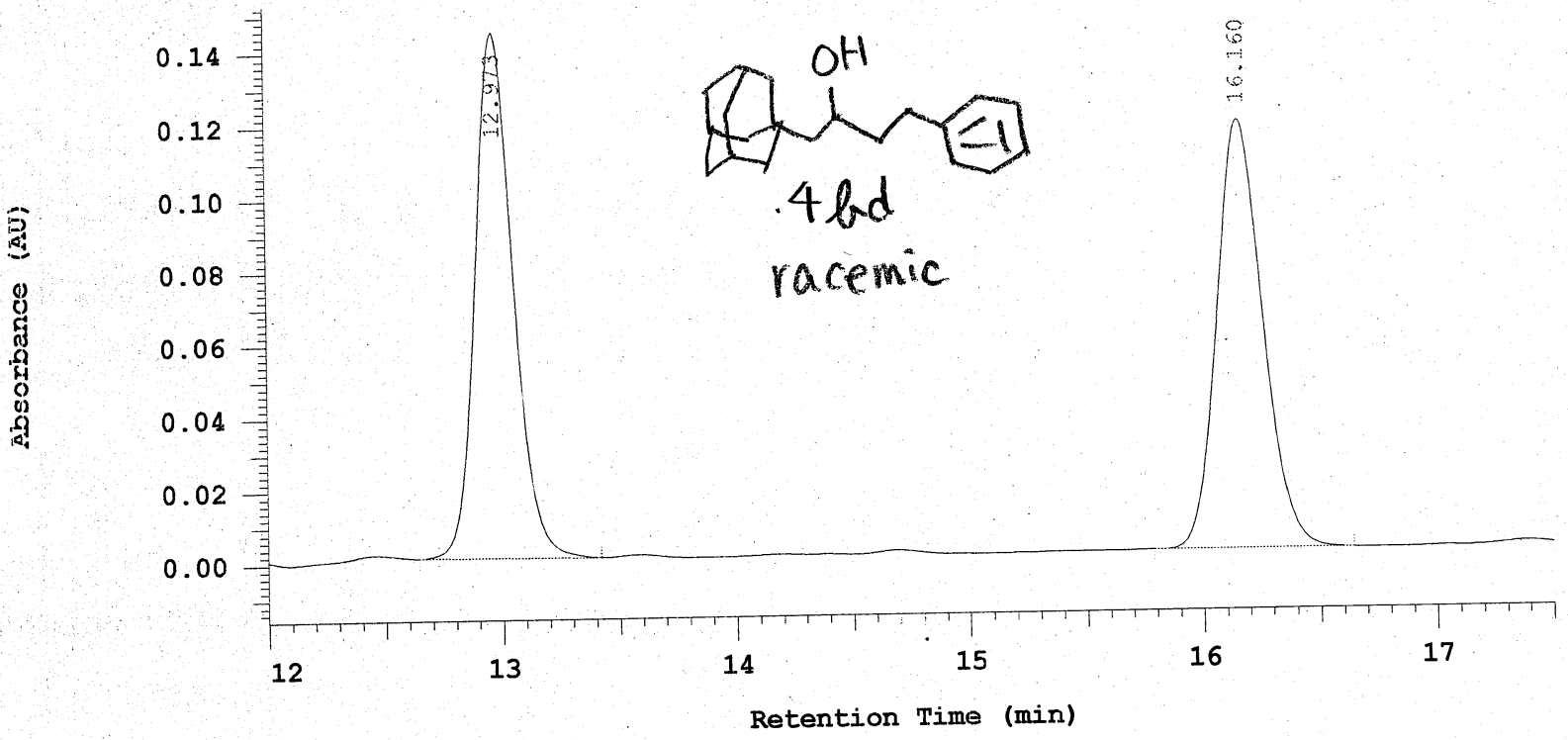

Erocessing Method: IB_OZW

Method Developer:

Pump 1: 5110

Pump 1 Solvent $A$ : hexane

Pump 1 Solvent B: 2-propanol

Pump 1 Solvent C:

Pump 1 Solvent D:

Method Description:

Chrom Type: Fixed WL Chromatogram, 207 nm

Peak Quantitation: AREA

Calculation Method: AREA\%

\begin{tabular}{ccccc} 
No. & RT & Area & Conc 1 & BC \\
\hline 1 & 12.973 & 812648 & 50.246 & MC \\
2 & 16.160 & 804685 & 49.754 & MC \\
\hline & & 1617333 & 100.000 & \\
\hline
\end{tabular}

Peak rejection level: 0 